\title{
Post-Translational Protein Modifications involved in Exo- and Endocytosis of Synaptic Vesicles
}

\author{
Dissertation \\ for the award of the degree \\ “Doctor rerum naturalium" \\ of the Georg-August-Universität Göttingen
}

within the doctoral program

"Biomolecules: Structure-Function-Dynamics"

of the Georg-August University School of Science (GAUSS)

submitted by

Ivan Silbern

from Tschebarkul, Russia

Göttingen, 2021 


\section{Thesis Committee}

Prof. Dr. Henning Urlaub

Prof. Dr. Reinhard Jahn

Prof. Dr. Silvio O. Rizzoli
Bioanalytical Mass Spectrometry

Max-Planck Institute for Biophysical Chemistry,

Göttingen; Institute for Clinical Chemistry

University Medical Center, Göttingen

Laboratory of Neurobiology

Max-Planck Institute for Biophysical Chemistry,

Göttingen

Department of Neuro- and Sensory Physiology, University Medical Center Göttingen

\section{Members of the Examination Board}

Reviewer

Prof. Dr. Henning Urlaub

Bioanalytical Mass Spectrometry

Max-Planck Institute for Biophysical Chemistry,

Göttingen; Institute for Clinical Chemistry

University Medical Center, Göttingen

Second Reviewer

Prof. Dr. Reinhard Jahn

Laboratory of Neurobiology

Max-Planck Institute for Biophysical Chemistry,

Göttingen

\section{Further members of the Examination Board}

Prof. Dr. Silvio O. Rizzoli

Prof. Dr. Nils Brose

Prof. Dr. Tobias Moser

Dr. Alexander Stein
Department of Neuro- and Sensory Physiology, University Medical Center Göttingen

Department of Molecular Neurobiology, Max Planck Institute of Experimental Medicine, Göttingen

Institute for Auditory Neuroscience and InnerEarLab, University Medical Center Göttingen

Research Group Membrane Protein Biochemistry, Max Planck Institute for Biophysical Chemistry, Göttingen

Date of oral examination: $13^{\text {th }}$ of October 2021 

Dedicated to my daughter Margarita 



\section{Abstract}

Neurotransmitter release is a key step that enables information flow between the pre- and post-synapse. However, regulation of the neurotransmitter release remains an intricate and widely unexplored matter despite recent advances in the understanding of the neurotransmitter release machinery and the analysis of the synaptic proteome and protein modifications. Indeed, post-translational protein modifications such as phosphorylation are suitable to quickly fine-tune the neurotransmitter release "in place" via affecting tertiary protein structures and protein-protein interactions, and globally, via modulating signaling pathways. Here, the investigations were focused on the dependence of protein phosphorylation in synaptosomes on the synaptic vesicle (SV) cycling, determining kinase-substrate interactions, and modulatory effects of selected sites on exo- and endocytosis.

The analysis of synaptic phosphoproteome was conducted using $\mathrm{TiO}_{2}$-based enrichment of phosphorylated peptides with subsequent chemical labeling by isobaric mass tags (TMT) and a mass spectrometry-based quantification. Synaptosomes were employed as a functional model of a synapse as they contain the required neurotransmitter release machinery and respond to stimulation. First, the applicability of electrical stimulation was tested. The fieldstimulation evoked reproducible glutamate release that was significantly suppressed in the absence of $\mathrm{Ca}^{2+}$, though it remained uncertain, to which degree the release is governed by exocytosis. Therefore, another approach using a $\mathrm{KCl}$-induced depolarization and treatment with botulinum neurotoxins (BoNTs) was used to identify phosphorylation events that depend on SV cycling. BoNTs cleave specifically SNARE proteins and thus block exocytosis and SV cycling, but do not impede $\mathrm{Ca}^{2+}$-influx evoked by the plasma membrane depolarization. Comparison of phosphorylation events in synaptosomes stimulated in the presence of $\mathrm{Ca}^{2+}$, EGTA $\left(0\right.$ net $\left.\mathrm{Ca}^{2+}\right)$ or pre-treated with BoNTs identified sites that were differentially phosphorylated following BoNT treatment, i.e., SV-cycling-dependent sites, and sites that were differentially phosphorylated when comparing $\mathrm{Ca}$ and EGTA conditions, but did not change under BoNT treatment, i.e., primarily $\mathrm{Ca}^{2+}$-dependent sites. Further differential expression analysis revealed that BoNT-treatment mostly caused de-phosphorylation of synaptic proteins. A kinase-substrate analysis showed that $>25 \%$ of BoNT-responsive sites are predicted MAPK substrates and $<9 \%$ are putative CaMKII targets. In contrast, $>20 \%$ of primarily $\mathrm{Ca}^{2+-}$ dependent sites are presumably regulated by $\mathrm{CaMKII}$, which corroborates $\mathrm{Ca}^{2+}$ dependence of these phosphorylation events. SV-cycling-dependent phosphorylation sites on syntaxin-1 (T21/T23-Stx1), synaptobrevin (S75-Vamp2), and cannabinoid receptor-1 (S314/T322-Cnr1) were further investigated for their impact on exo- and endocytosis. In collaboration with Dr. Eugenio Fornasiero and Prof. Dr. Silvio O. Rizzoli, corresponding phosphomimetic and non-phosphorylatable variants of the proteins were expressed in 
cultured hippocampal neurons. Imaging of the $\mathrm{pH}$-sensor pHluorine coupled to synaptobrevin-2 revealed that the expression of phosphomimetic and non-phosphorylatable sites affected exo- and endocytosis in neurons.

This work is first to investigate the electrical stimulation in relation to the $\mathrm{Ca}^{2+}$-dependent neurotransmitter release and exocytosis in synaptosomes. It further provides a comprehensive draft of synaptosomal phosphoproteome and is first to demonstrate its global dependence on an active SV cycling. The analysis of cultured hippocampal neurons expressing non-phosphorylatable and phosphomimetic mutants of pre-synaptic proteins syntaxin-1, synaptobrevin-2, and cannabinoid receptor-1 further demonstrates that the identified SV-cycling-dependent sites affect exo- and endocytosis. 


\section{Table of contents}

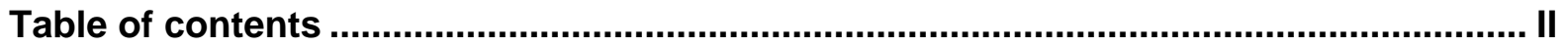

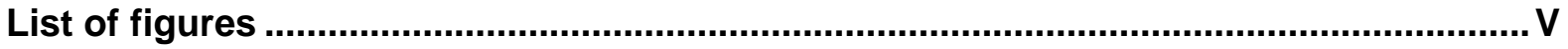

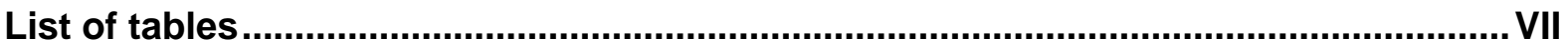

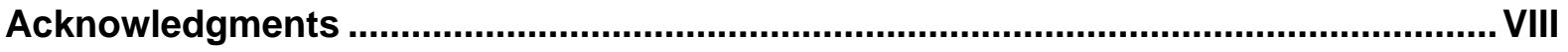

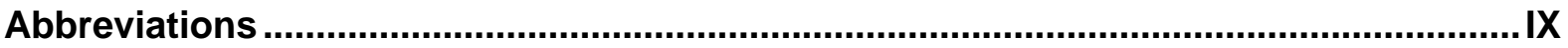

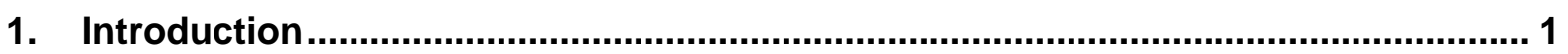

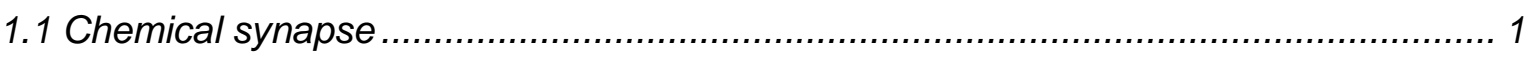

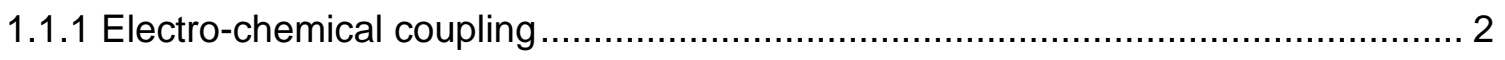

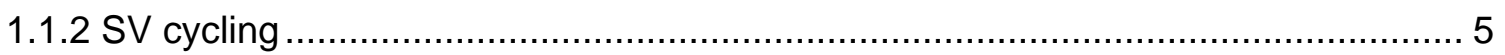

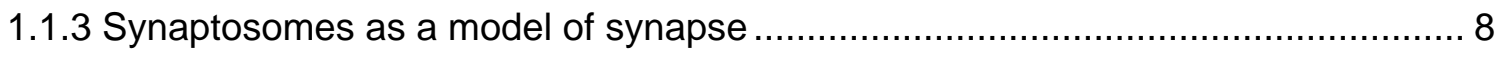

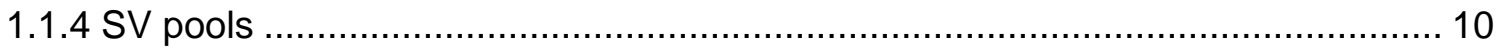

1.2 Post-translational modifications of proteins ........................................................... 11

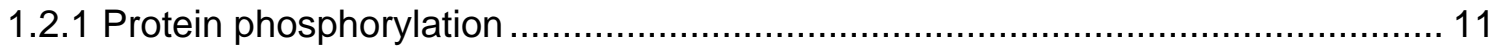

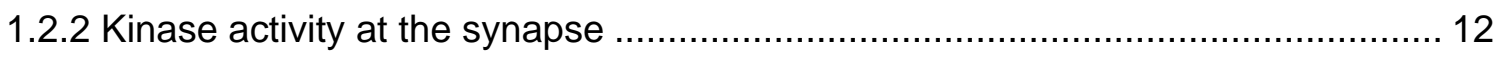

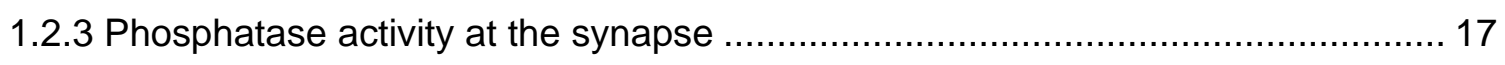

1.3 Mass-spectrometric analysis of peptides, proteins, and PTMs ............................... 19

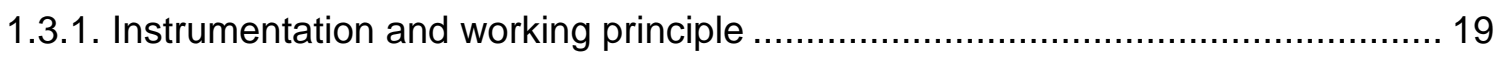

1.3.2. Sample Preparation for Mass Spectrometry (MS) ........................................... 21

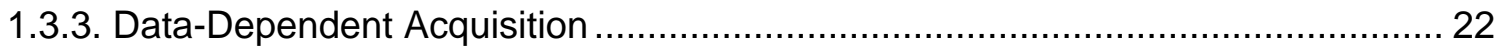

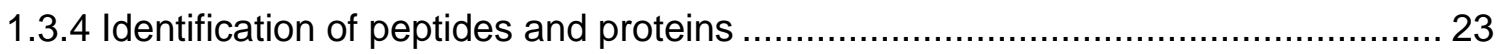

1.3.5 Protein quantification using a label-free and an isobaric-tag labeling approach... 24

1.3.6 Post-processing of quantitative proteomics data ............................................. 25

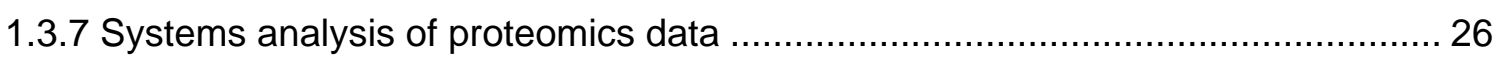

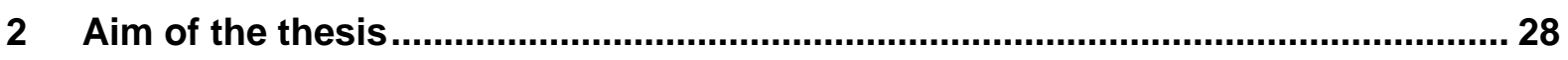

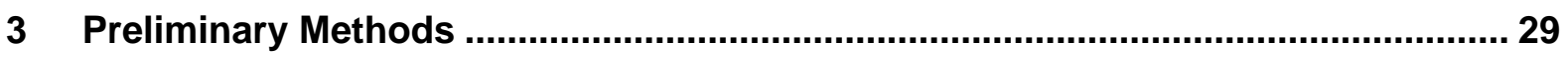

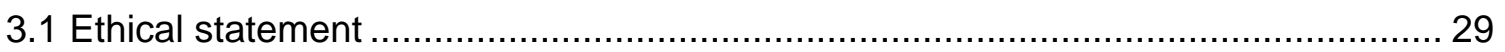

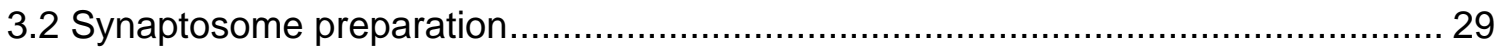

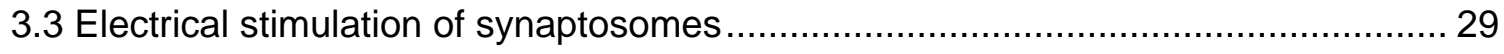

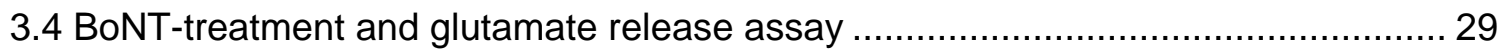

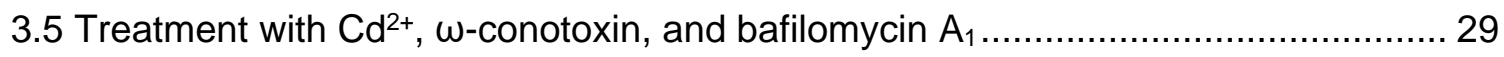

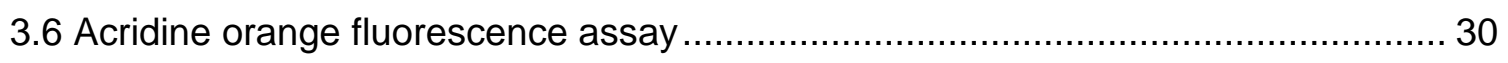

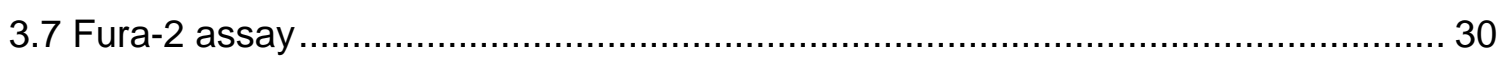

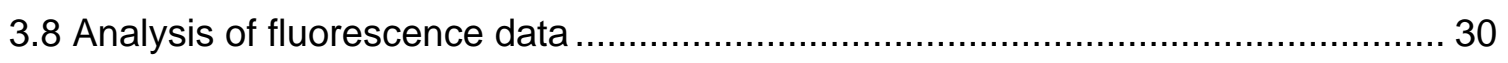

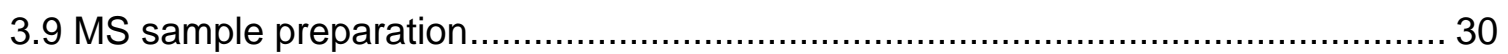

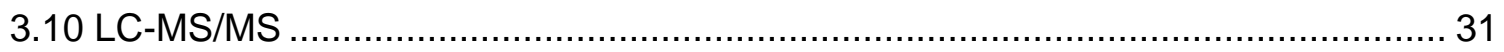


3.11 Data Analysis.

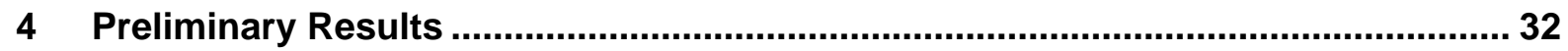

5 Protein phosphorylation in depolarized synaptosomes: Dissecting primary effects

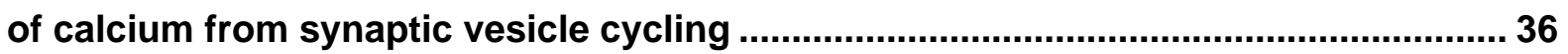

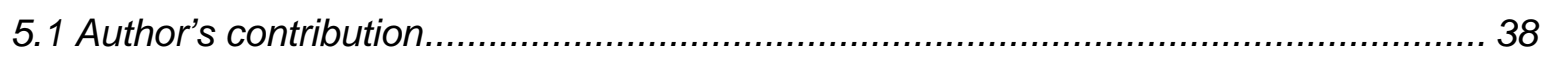

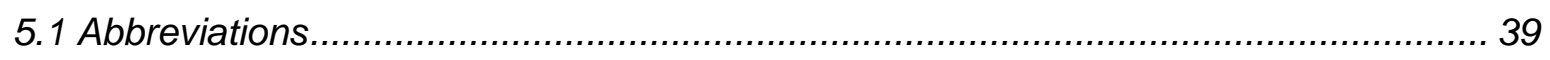

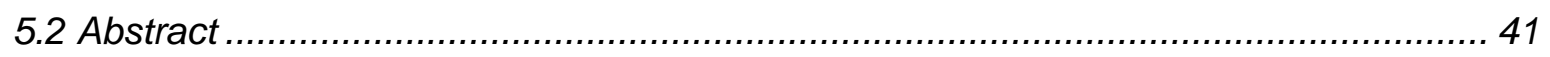

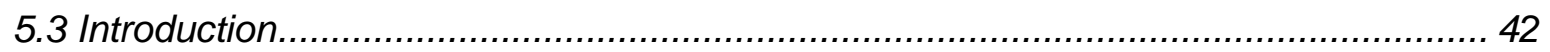

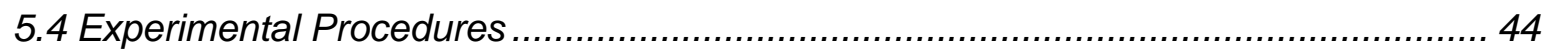

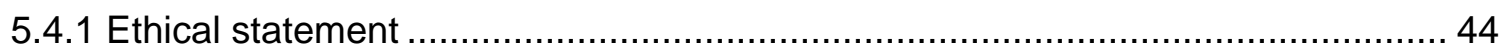

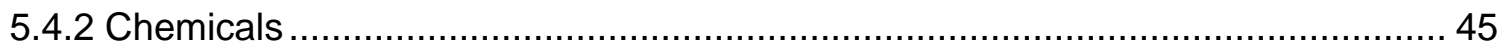

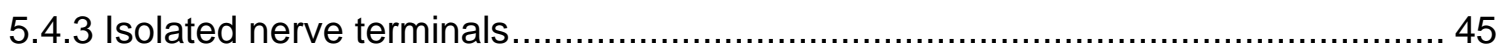

5.4.4 BoNT-treatment and glutamate release assay ............................................... 45

5.4.5 Phosphopeptide enrichment and TMT-Labeling ............................................. 46

5.4.6 Basic reversed-phase (bRP) chromatography............................................. 47

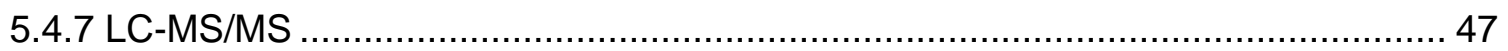

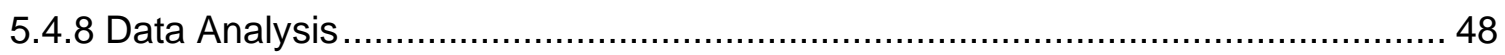

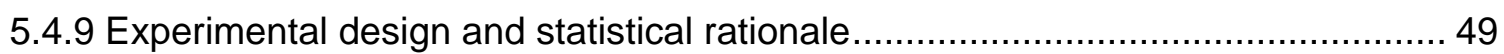

5.4.10 Defining primary $\mathrm{Ca}^{2+-d e p e n d e n t ~ a n d ~ S V-c y c l i n g-d e p e n d e n t ~ p h o s p h o r y l a t i o n ~}$

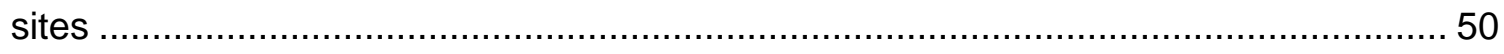

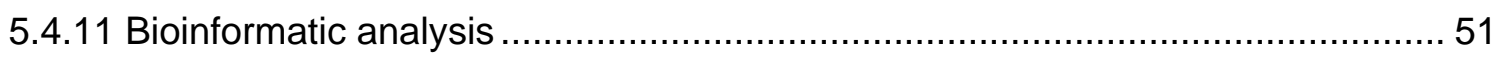

5.4.12 C. botulinum cell culture supernatants profiling ............................................ 52

5.4.13 Calcium influx in synaptosomes following BoNT-treatment and $\mathrm{KCl}$-stimulation 53

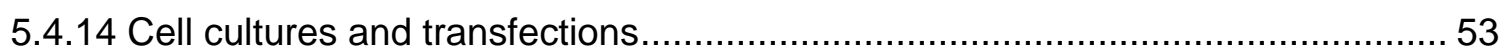

5.4.15 DNA cloning of wild type proteins and respective phosphomimetic mutants...... 54

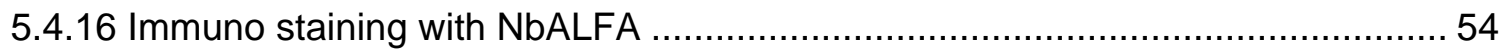

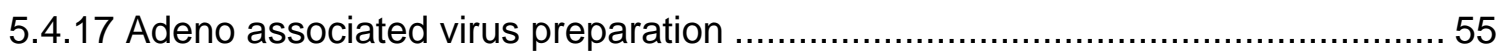

5.4.18 Synaptic vesicle exo-/endocytosis measurements ........................................ 55

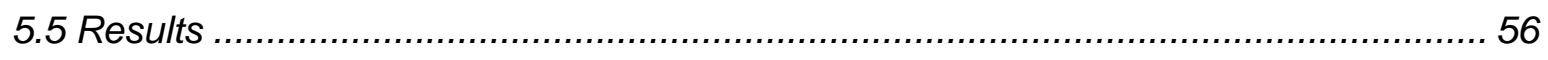

5.5.1 Synaptosome depolarization by $\mathrm{KCl}$ in the presence of $\mathrm{Ca}^{2+}$ results in substantial

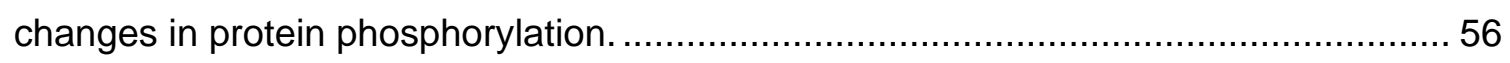

5.5.2 Block of SV cycling with botulinum neurotoxins induces changes in protein phosphorylation.

5.5.3 Dissecting primary $\mathrm{Ca}^{2+-}$ driven phosphorylation events from events linked to SV cycling.

5.5.4 Phosphomimetic sites on SNARE proteins and cannabinoid receptor-1 modulate neurotransmitter release. 


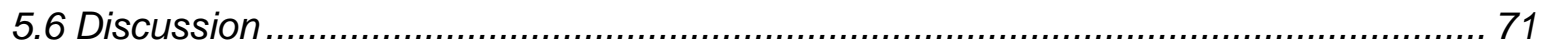

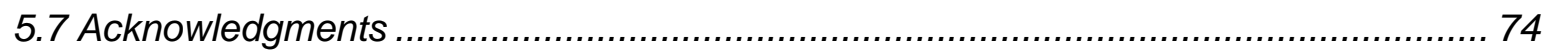

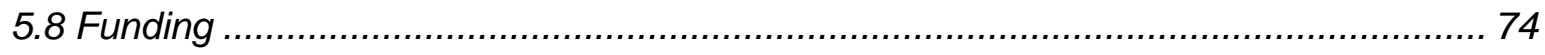

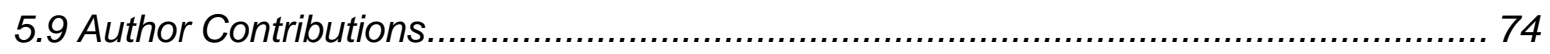

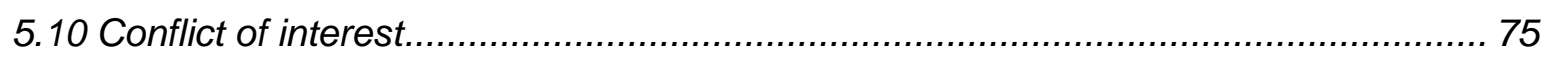

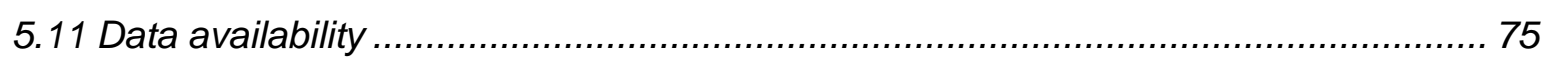

5.12 Overview of the supplementary material (supplementary tables, figures and datasets)

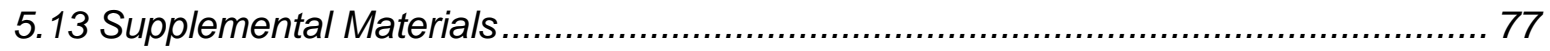

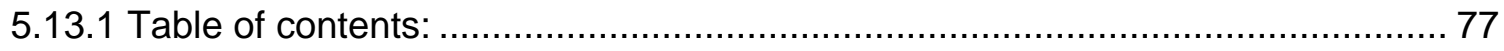

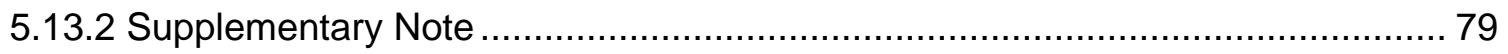

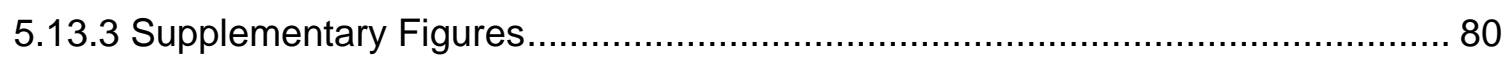

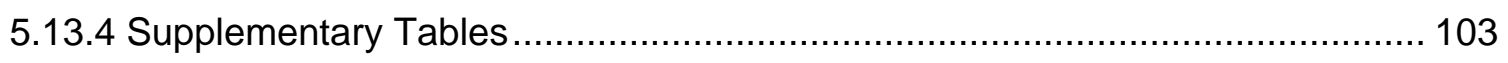

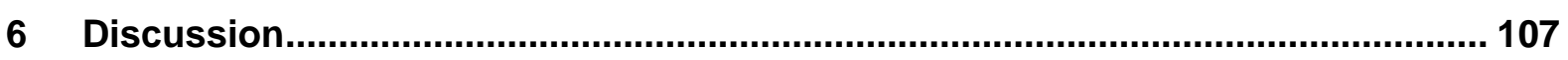

6.1 Electrical field-stimulation of synaptosomes.............................................. 107

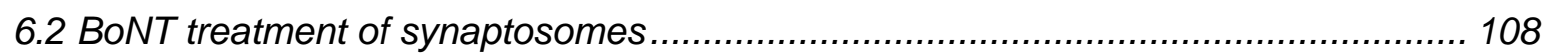

6.3 Categorization of phosphorylation events ...................................................... 109

6.4 Challenges and limitations of MS-based phosphoproteomics............................... 110

6.5 Challenges and limitations of the functional analysis of phosphorylation sites ......... 111

6.6 Regulation of the kinase and phosphatase activity via phosphorylation .................. 112

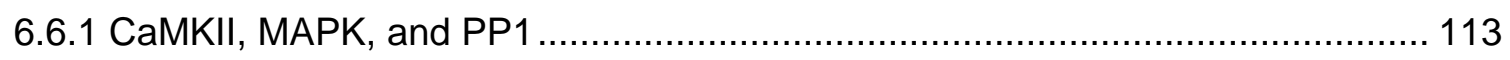

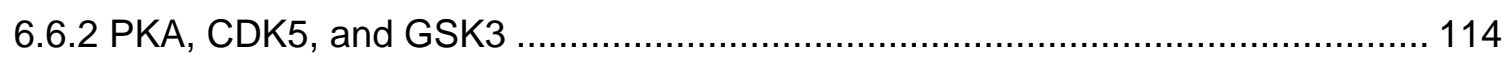

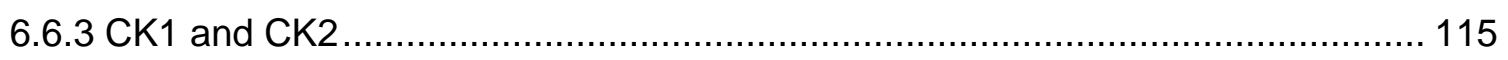

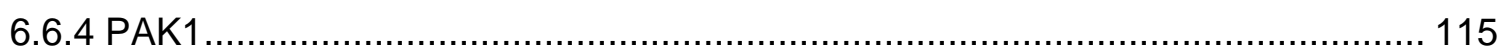

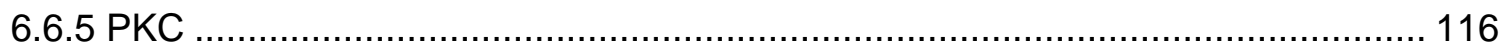

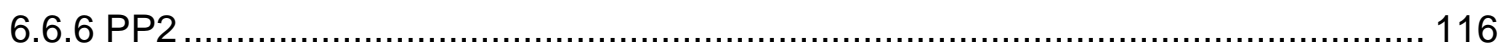

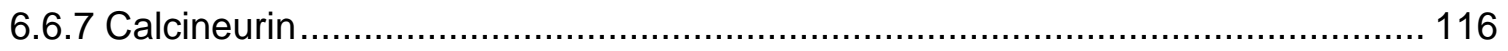

6.8 Primary $\mathrm{Ca}^{2+-d e p e n d e n t ~ s i t e s ~ a n d ~ S V-c y c l i n g-d e p e n d e n t ~ p h o s p h o r y l a t i o n . . . . . . . . . . . . . . ~} 116$

6.8.1 Active zone and exocytosis-related proteins ............................................... 117

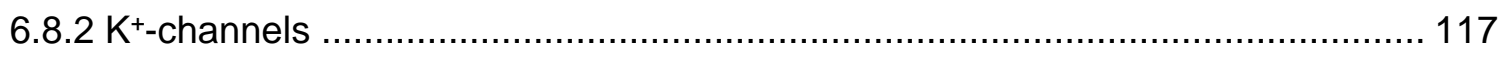

6.8.3 Cytoskeleton-associated proteins ............................................................ 118

6.8.4 Phosphorylation of SNARE and its implication for exocytosis .......................... 119

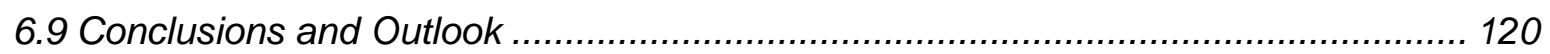

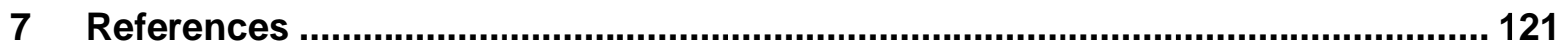

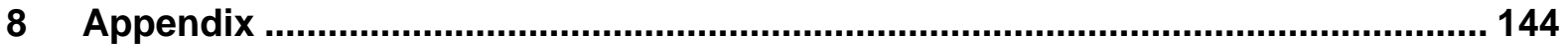

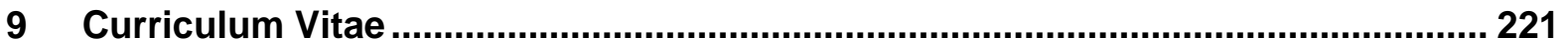




\section{List of figures}

Figure 1.1: Nerve terminal and synaptic vesicle cycling ................................................

Figure 1.2: Electron micrograph of synaptosomal fraction..............................................

Figure 1.3: Ion path of the Q Exactive HF mass spectrometer ........................................19

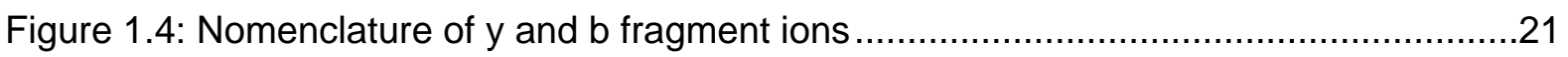

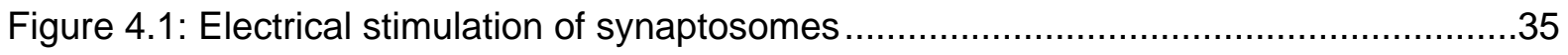

Figure 4.2: Phosphoproteomics analysis of electrically stimulated synaptosomes ...............36

Figure 5.1: Workflow for the phosphoproteome analyses of synaptosomes under different

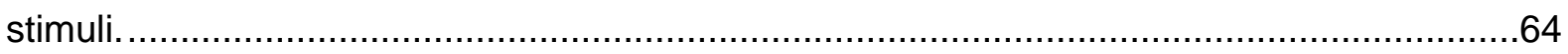

Figure 5.2: Substantial changes in synaptic phosphoproteome following depolarization and responsible kinase groups .65

Figure 5.3: Functional annotation of proteins carrying regulated phosphorylation sites based on Ca vs. EGTA experiments 66

Figure 5.4: Dependence of regulated phosphorylation sites on calcium influx and SV cycling.

Figure 5.5: Schematic representation of presynaptic compartment with a single docked synaptic vesicle, plasma membrane and cytoskeleton elements, based on Chua et al .......68 Figure 5.6: Phosphomimetic modulation of phosphorylation sites found differentially regulated in synaptobrevin (Vamp2), syntaxin-1 and cannabinoid receptor-1 affects SV recycling properties .69

Figure 5.S1 Glutamate release assay and $\mathrm{Ca}^{2+-}$ levels in cytoplasm .80

Figure 5.S2: Kinase groups that might be responsible for the observed phosphorylation changes

Figure 5.S3: Dependence of phosphorylation sites on calcium influx and SV cycling

Figure 5.S4: Site occupancy assessment

Figure 5.S5: Changes in phosphorylation site intensities for Calcium/calmodulin-dependent kinase 2 alpha, beta, gamma, and delta

Figure 5.S6: Changes in phosphorylation site intensities for Mitogen-activated protein kinase 1, 3, and Protein kinase $C$ beta .86

Figure 5.S7: Phosphatases and their putative substrates. .87

Figure 5.S8: Manual annotation of proteins carrying regulated phosphorylation sites.

Figure 5.S9: Changes in phosphorylation site intensities for Regulating synaptic membrane exocytosis (RIM) protein 1, 2, and RIM-binding protein 2. X-axis shows positions of modified amino acids .89 
Figure 5.S10: Changes in phosphorylation site intensities for Dynamin 1, Clathrin coat assembly protein AP180, and Amphiphysin

Figure 5.S11: Changes in phosphorylation site intensities for Synapsin 1, 2, and 3. X-axis shows positions of modified amino acids

Figure 5.S12: Changes in phosphorylation site intensities for Spectrin beta chain. X-axis shows positions of modified amino acids

Figure 5.S13: Changes in phosphorylation site intensities for Adducin alpha, beta, and gamma. X-axis shows positions of modified amino acids

Figure 5.S14: Changes in phosphorylation site intensities for Potassium voltage-gated channel subfamily A member 2 , and subfamily B member 1 and 2 ....

Figure 5.S15: Changes in phosphorylation site intensities for Potassium voltage-gated channel subfamily D member 2 , subfamily KQT member 2 , Potassium/sodium

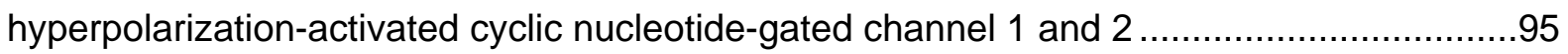

Figure 5.S16: Regulated phosphorylation sites on selected proteins

Figure 5.S17: Changes in phosphorylation site intensities for Calcium-activated potassium channel subunit alpha 1 , Potassium voltage-gated channel subfamily $\mathrm{H}$ member 1 , and Potassium voltage-gated channel subfamily $Q$ member 5 .

Figure 5.S18: Changes in phosphorylation site intensities for Syntaxin 1A, Vesicleassociated membrane protein 2, Syntaxin-binding protein 1, and Cannabinoid receptor 1...99 Figure 5.S19: Proteomic profiling of C. botulinum cell culture supernatants 100

Figure 5.S20: Phosphoproteome analysis of BoNT-treated HeLa nuclear extract .............101

Figure 5.S21: Proteomics analysis of Mock- or BoNT-treated synaptosomes 102

Figure 6.1: $\mathrm{KCl}$ stimulation of synaptosomes: proposed connection of protein phosphorylation and SV cycling activity 


\section{List of tables}

Table 1.1: Ionic concentrations and equilibrium potentials

Table 5.S1: Number of regulated phosphorylation events per predicted kinase group in $\mathrm{Ca}$ vs. EGTA experiment

Table 5.S2: Enriched gene ontology biological function terms based on synapse-specific

SynGO database 104

Table 5.S3 Number of regulated phosphorylation events per predicted kinase group in Mock vs. BoNT experiment 105

Table 5.S4: Number of regulated phosphorylation events per predicted kinase group in Ca vs. EGTA and Mock vs. BoNT experiments 106

Table 8.1: primary $\mathrm{Ca}^{2+-}$ dependent sites, selection from Suppl. Data 01 144

Table 8.2: SV-cycling-dependent sites, selection from Suppl. Data 01 174

Table 8.3: Phosphorylation events from clusters 1 and 2 as presented in Figure 4.2 215 


\section{Acknowledgments}

First of all, I would like to express my gratitude to my supervisor, Prof. Dr. Henning Urlaub for his trust and continuous support during my $\mathrm{PhD}$ time and previously during the Master's studies. I have learnt a lot about mass spec and life while being a part of your group! Here I would also like to thank Dr. Christof Lenz who was the first to introduce me to the peculiarities of working in a proteomics lab and Dr. Kuan-Ting Pan who always helped me to navigate and advance projects I was working on.

Furthermore, I would like to thank cordially Prof. Dr. Reinhard Jahn for advising me during the $\mathrm{PhD}$ time and being open to my questions. Here I would also like to thank all members of the Neurobiology lab and the group of Dr. Alexander Stein for their willingness to share materials, instruments, lab space and, most important, their expertise. Especially, I would like to thank Dr. Mahdokht Kohansal Nodehi, a former PhD student of Prof. Dr. Jahn, who shared her preliminary work on electrical stimulation with me.

I would like to take this opportunity to thank sincerely Prof. Dr. Rizzoli and Dr. Eugenio Fornasiero for their advices and willingness to help, as well as for a great collaboration. I would also like to express my gratitude to Prof. Dr. Tobias Moser, Prof. Dr. Nils Brose, and Dr. Alexander Stein for their willingness to serve on my examination board.

My warmest thanks go to all members of the Bioanalytical mass spectrometry group for the lively environment and (though sometimes heated) discussions. In particular, I would like to thank former, current, and occasional "inhabitants of the cave": Sofia Ainatzi, Dr. Yanlong Li, Dr. Fan Pan, Dr. Christof Lenz, Uwe Plessmann, Dr. Ralf Pflanz, Dr. Kuan-Ting Pan, Fanni Bazsó, as well as Dr. Alexandar Chernev. I would like to address my special thanks to Dr. Momchil Ninov, Dr. Iwan Parfentev, and Sofia Ainatzi for helping me with synaptosomal preparations and with reviewing this thesis. Furthermore, I would like to thank Monika Raabe, Annika Reinelt, Uwe Plessmann, Dr. Sabine König, Dr. Ralf Pflanz, and Dr. Olex Dybkov for an excellent support in all technical (and beyond) questions, as well as Sascha Krause and Thomas Gundlach for taking care of lab animals.

Last but not least I would like to greatly thank my family, my mum, dad, my brother Sascha and my wife Daria for being always on my side, supporting me during my studies and forgiving me my late hours at work. Your sacrifice meant a lot to me! 


\section{Abbreviations}

\begin{tabular}{|c|c|}
\hline AAV & adeno-associated virus \\
\hline ACN & acetonitrile \\
\hline ADBE & activity-dependent bulk endocytosis \\
\hline AGC & automatic gain control (depending on context) \\
\hline AGC & protein kinase $A, G, C$ kinase group \\
\hline AKAP & A-kinase anchoring proteins \\
\hline AMPA & a-amino-3-hydroxy-5-methyl-4-isoxazolepropionic acid \\
\hline AMPAR & a-amino-3-hydroxy-5-methyl-4-isoxazolepropionic acid receptor \\
\hline AMPK & AMP-activated protein kinase \\
\hline AP & action potential \\
\hline AP1 & adaptor protein 1 \\
\hline AP2 & adaptor protein 2 \\
\hline AP3 & adaptor protein 3 \\
\hline AP180 & adaptor protein 180 \\
\hline aPKC & atypical protein kinase $\mathrm{C}$ \\
\hline BAR & Bin-Amphiphysin-Rvs domain \\
\hline BCKDK & branched chain ketoacid dehydrogenase kinase \\
\hline BoNT & botulinum neurotoxin \\
\hline bRP & basic reversed-phase chromatography \\
\hline BSA & bovine serum albumin \\
\hline CAA & chloroacetamide \\
\hline CAMK & calcium-calmodulin dependent kinases \\
\hline CaMKII & calcium-calmodulin kinase 2 \\
\hline cAMP & adenosine 3', 5'-monophosphate \\
\hline CDK & cyclin-dependent kinase \\
\hline CDK5 & cyclin-dependent kinase 5 \\
\hline CDKL & cyclin-dependent-like kinase \\
\hline CK1 & casein kinase 1 \\
\hline CK2 & casein kinase 2 \\
\hline CLK & SRPK1 and Clk/Sty protein kinase \\
\hline CME & clathrin-mediated endocytosis \\
\hline CMGC & CDK, MAP, GSK, CDKL kinase group \\
\hline CNQX & 6-cyano-7-nitroquinoxaline-2,3-dione \\
\hline cPKC & conventional protein kinase $\mathrm{C}$ \\
\hline CREB1 & CAMP responsive element binding protein 1 \\
\hline
\end{tabular}




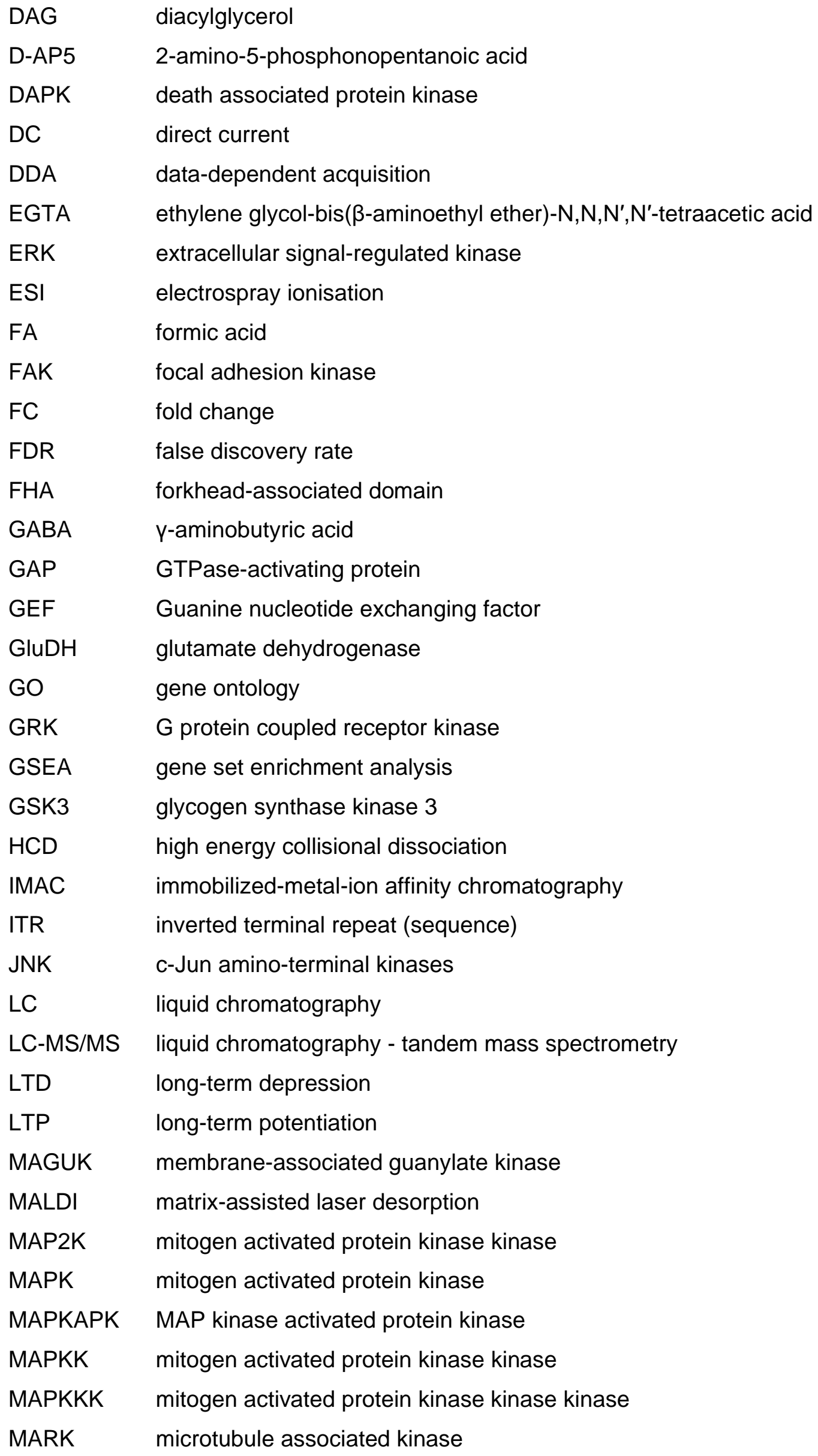




\begin{tabular}{|c|c|}
\hline MAST & microtubule-associated serine/threonine-protein kinase \\
\hline MaxIT & maximum injection time \\
\hline Mnk & MAPK-interacting protein kinase \\
\hline MOAC & metal-oxide affinity chromatography \\
\hline MS & mass spectrometry or mass spectrum, depending on context \\
\hline MS/MS & tandem mass spectrum \\
\hline MS1 & survey mass spectrum \\
\hline MS2 & tandem mass spectrum \\
\hline NCE & normalized collision energy \\
\hline NDR & nuclear dbf2-related kinase \\
\hline NEK & NIMA-related kinase \\
\hline NIMA & never in mitosis $A$ \\
\hline NMDA & methyl-d-aspartate \\
\hline NMDAR & $\mathrm{N}$-methyl-d-aspartate receptor \\
\hline $\mathrm{nPKC}$ & novel protein kinase $\mathrm{C}$ \\
\hline NSF & $N$-ethylmaleimide-sensitive factor \\
\hline PAK & p21 activated kinase \\
\hline PDHK & pyruvate dehydrogenase kinase \\
\hline PFA & paraformaldehyde \\
\hline $\mathrm{Pl}(4,5) \mathrm{P}_{2}$ & phosphatidylinositol $(4,5)$-bisphosphate \\
\hline PKA & protein kinase $A$ \\
\hline PKB & protein kinase $B$ \\
\hline PKC & protein kinase $\mathrm{C}$ \\
\hline PKD & protein kinase $D$ \\
\hline PKGcGK & cGMP-dependent protein kinase \\
\hline PNGase F & peptide- $\mathrm{N}(4)-(\mathrm{N}$-acetyl-beta-glucosaminyl)asparagine amidase \\
\hline POI & protein of interest \\
\hline PP1 & protein phosphatase 1 \\
\hline PP2A & protein phosphatase $2 \mathrm{~A}$ \\
\hline PP2B & protein phosphatase $2 \mathrm{~B}$ \\
\hline PSD & post-synaptic density \\
\hline PSM & peptide-spectrum match \\
\hline PTB & phosphotyrosin-binding domain \\
\hline PTM & post-translational protein modification \\
\hline PTP & protein tyrosine phosphatase \\
\hline QIK & Qin-induced kinase \\
\hline RAS & rat sarcoma gene \\
\hline
\end{tabular}




\begin{tabular}{|c|c|}
\hline $\mathrm{RF}$ & radio frequency \\
\hline RIM & regulating synaptic membrane exocytosis protein \\
\hline ROCK & Rho-associated protein kinase \\
\hline $\mathrm{RP}$ & reserved pool \\
\hline $\mathrm{RRP}$ & readily releasable pool \\
\hline RSK & ribosomal S6 kinase \\
\hline $\mathrm{RT}$ & room temperature \\
\hline SGK & serum and glucocorticoid regulated kinase \\
\hline $\mathrm{SH} 2$ & Src homology 2 domain \\
\hline $\mathrm{SH} 3$ & Src homology 3 domain \\
\hline SHANK & SH3 and multiple ankyrin repeat domains \\
\hline SNAP & soluble NSF-attachment protein \\
\hline SNAP-25 & Synaptosomal-associated $25 \mathrm{kDa}$ protein \\
\hline SNARE & $\mathrm{N}$-ethylmaleimide-sensitive factor-attachment protein receptors \\
\hline SRPK1 & serine/arginine-rich protein-specific kinase 1 \\
\hline STE & "sterile" serine/threonine protein kinases \\
\hline STLK & STE20-like serine/threonine protein kinase \\
\hline SV & synaptic vesicle \\
\hline TANK & TRAF family member-associated NF-kappa-B activator \\
\hline TBK & TANK-binding kinase \\
\hline TCEP & tris(2 carboxyethyl)phosphine \\
\hline TEAB & triethylammonium bicarbonate buffer \\
\hline TFE & trifluoroethanol \\
\hline TK & tyrosine kinase \\
\hline TKL & tyrosine-like kinase \\
\hline TMT & tandem mass tags \\
\hline TMT6 & TMTsixplex isobaric labeling reagents \\
\hline TRAF & tumor necrosis factor receptor-associated factor \\
\hline TTBK & tau-tubulin kinase \\
\hline ULK & Unc-51 like autophagy activating kinase \\
\hline V-ATPase & vacuolar proton ATPase \\
\hline VGLUT1 & vesicular glutamate transporter 1 \\
\hline VT & vesicular transporter \\
\hline Wnk & with no lysine $(\mathrm{K})$ kinase \\
\hline WT & wild type \\
\hline
\end{tabular}




\section{Introduction}

\subsection{Chemical synapse}

Synapses are special types of cellular contacts that enable directed information transfer between cells. Here I refer to the "synapse" meaning specifically the chemical synapse in the central nervous tissue. Other synapse types, e.g., the electrical synapse and the immunological synapse are not considered in this work due to their different organization and function. Although synapses represent highly specialized sites of intercellular communication, synapse-like structures has appeared early during the evolution before the nervous system could be defined as such (1). On electron micrographs a typical synapse appears as an electron-dense structure at the contact zone between two plasma membranes, the pre- and the post-synapse, separated by a thin synaptic cleft (exemplified in Figure 1.1A).
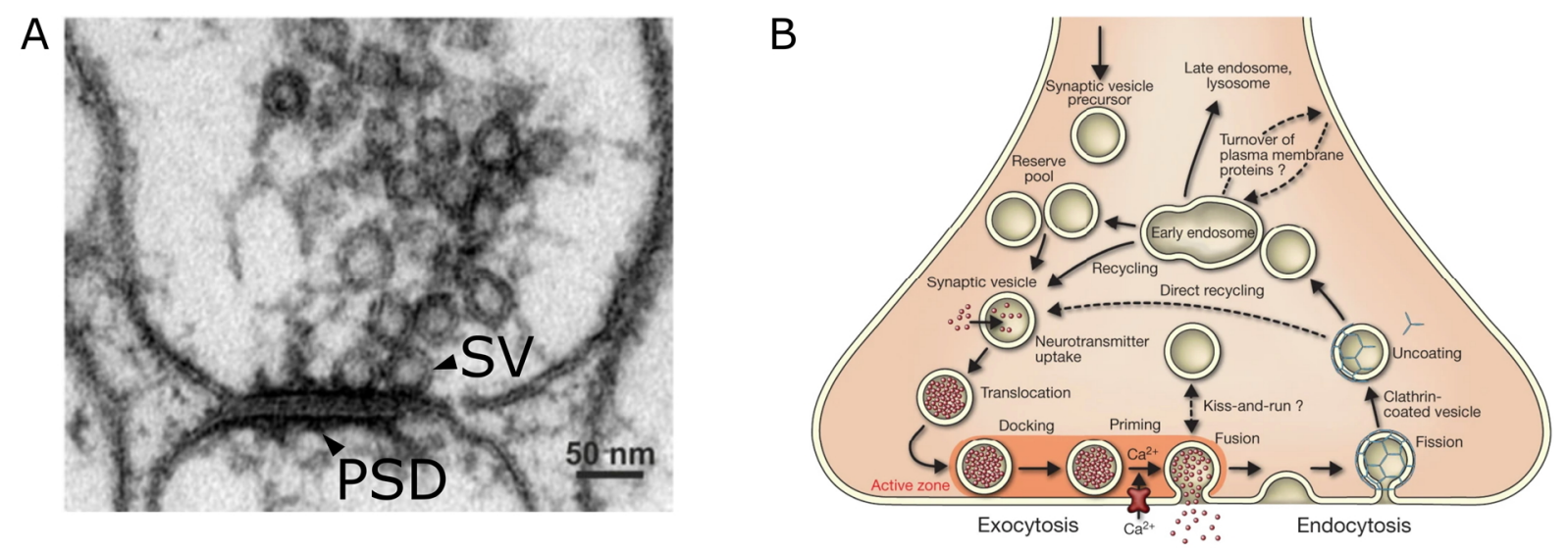

Figure 1.1: Nerve terminal and synaptic vesicle cycling. (A) An electron micrograph of a nerve terminal. The pre-synapse (upper part) can be recognized by the presence of synaptic vesicles (SV) and the post-synapse (lower part) by the post-synaptic density (PSD) at the place of the contact with the pre-synapse. The micrograph is adapted from Guzman et al (2). (B) Proposed steps of SV cycling: i) SVs fuse with the plasma membrane and release their content in the synaptic cleft via exocytosis; ii) SVs are recycled via endocytotic mechanisms and prepared for the next round of endocytosis (refilled with the neurotransmitter, docked and primed at the active zone). The figure is adapted from Jahn and Fasshauer (3).

The pre-synaptic compartment can be readily distinguished by the presence of synaptic vesicles (SVs) - spherical membrane-enclosed structures filled with neurotransmitter. Upon fusion with the plasma membrane, SVs release their content into the synaptic cleft thus mediating chemical signal transmission. SVs are decorated with proteins required for SV trafficking, such as synaptobrevins (Vamp1, Vamp2), small GTP-binding proteins Rab3a-c, etc.; as well as proteins responsible for the composition of intravesicular fluid, such as ion channels (e.g., chloride channels Clcn3, Clcn7), transporters (e.g., glutamate transporters 
VGLUT1 (SIC17a7), VGLUT2 (SIc17a6)), and the vacuolar H+-ATPase. SVs further contain proteins that are engaged in interactions with other proteins and presumably fulfil regulatory functions, such as a $\mathrm{Ca}^{2+-}$-sensor synaptotagmin (Syt1), a chaperone protein Dnaj5 and synapsins (Syn1-3) (4).

The site of SVs release represents a structurally organized electron-dense matrix also known as the active zone $(5,6)$. Large multi-domain proteins such as bassoon (Bsn) and piccolo (Pclo), as well as RIM proteins (Rims1-4), RIM-binding proteins (Rimbp1-3), ERC proteins (Erc1-2), liprins (Ppfia1-4), and Munc13 (Unc13a, b, c) tether different pre-synaptic components such as SVs, cytoskeleton proteins, channels, receptors, and, therefore, orchestrate the turnover of SVs, i.e., SV cycling (7).

The hallmark of the post-synaptic compartment is the electron-dense structure at the plasma membrane also known as the post-synaptic density (PSD) (8). Scaffolding proteins such as PSD-95 (Dlg4), membrane-associated guanylate kinase (MAGUK) proteins (Mpp24), SH3 and multiple ankyrin repeat domains (SHANK) proteins (Shank1-3), etc. hold together proteins of other functional classes, e.g., ionotropic glutamate receptors NMDA (Grin1, Grin2a-d, Grin3a, b) and AMPA (Gria1-4), ion channels, cell adhesion molecules, and cytoskeletal proteins (9). Also, regulatory proteins, such as calcium-calmodulindependent kinases are typically associated with the PSD (10). This definitive organization enables the controlled propagation and the modulation of the signal arriving from the presynaptic part.

\subsubsection{Electro-chemical coupling}

An important moment in the synapse physiology is the transformation of an electrical signal into the chemical, i.e., neurotransmitter release. In a simplified view, biological membranes, e.g., the synaptic plasma membrane, represent an electrical capacitor, which consists of a non-conducting lipid bilayer (membrane) separating two conducting media filling the extraand intracellular space. At rest, the synaptic membrane is negatively charged ( -70 mV) as compared to the extracellular fluid (11). An electric field across the membrane generates the driving force for the positive ions $\left(\mathrm{Na}^{+}, \mathrm{K}^{+}, \mathrm{Ca}^{2+}\right)$ to enter the intracellular space and force negative ions $\left(\mathrm{Cl}^{-}, \mathrm{HCO}_{2}^{-}, \mathrm{H}_{2} \mathrm{PO}_{4}^{-}\right)$in the opposite direction. On the other hand, the uneven distribution of ions between the extra- and intracellular fluids forces the ions to follow their concentration gradient. For each ion, an equilibrium membrane potential at which the electrical and chemical driving forces would cancel each other can be calculated using the Nernst equation (12):

$$
E_{e q}=\frac{R T}{z F} \ln \frac{[\text { Ion }]_{e x}}{[\text { Ion }]_{\text {in }}}(1)
$$


where [lon $]_{e x}$ and [lon] $]_{\text {in }}$ are the extra- and intracellular concentrations of an ion,

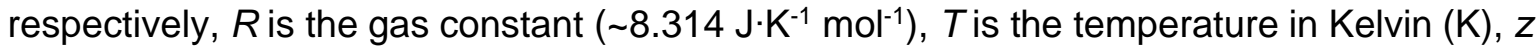
is the ionic charge, and $F$ is the Faraday's constant $\left(\sim 96480 \mathrm{C} \cdot \mathrm{mol}^{-1}\right)$. Ionic concentrations and corresponding equilibrium potentials for $\mathrm{K}^{+}, \mathrm{Na}^{+}, \mathrm{Ca}^{2+}$, and $\mathrm{Cl}^{-}$in a typical mammalian cell are listed in Table 1.1 (adapted from Graziane and Dong (11)).

Table 1.1: Ionic concentrations and equilibrium potentials (adapted from Graziane and Dong (11))

\begin{tabular}{|l|c|c|c|}
\hline Ion & {$[\text { lon }]_{\text {in }} / \boldsymbol{m M}$} & {$[\text { Ion }]_{\text {ex }} / \boldsymbol{m M}$} & $\boldsymbol{E}_{\text {eq }}\left(\mathbf{3} 7^{\circ} \mathbf{C}\right) / \boldsymbol{m} \boldsymbol{V}$ \\
\hline $\mathrm{K}^{+}$ & 140 & 5 & -89.7 \\
\hline $\mathrm{Na}^{+}$ & $5-15$ & 145 & $+61.1-+90.7$ \\
\hline $\mathrm{Ca}^{2+}$ & $1-2$ & $2.2-5$ & $+136-+145$ \\
\hline $\mathrm{Cl}^{-}$ & 4 & 110 & -89 \\
\hline
\end{tabular}

The resting membrane potential $\left(E_{m}\right)$ can be calculated using Goldman-Hodgkin-Katz equation $(13,14)$ when considering the equilibrium potentials and corresponding membrane permeability for each ion. For the monovalent cations $\left(M^{+}\right)$and anions $\left(A^{-}\right)$the equation has the following form:

$$
E_{m}=\frac{R T}{F} \ln \left(\frac{\sum_{i}^{n} P_{M_{i}^{+}}\left[M_{i}^{+}\right]_{e x}+\sum_{j}^{m} P_{A_{j}^{-}}\left[A_{j}^{-}\right]_{i n}}{\sum_{i}^{n} P_{M_{i}^{+}}\left[M_{i}^{+}\right]_{i n}+\sum_{j}^{m} P_{A_{j}^{-}}\left[A_{j}^{-}\right]_{e x}}\right)
$$

where $P$ is the membrane permeability for a given ion.

The difference in the ionic concentrations between the extra- and intracellular space is maintained due to the selective membrane permeability for ions and the activity of transporters (15). The most important transporters in the plasma membrane are:

1. $\mathrm{Na}^{+} / \mathrm{K}^{+}-$ATPase (encoded by Atp1a1-4 (catalytic $\alpha$-subunit) and Atp1b1-4 (auxiliary $\beta$-subunit)), exchanges three $\mathrm{Na}^{+}$inside the cell against two $\mathrm{K}^{+}$from the outside of the cell thus contributing to the charge disparity between extra- and intracellular space (16).

2. $\mathrm{Na}^{+} / \mathrm{Ca}^{2+}$-exchanger (S/c8a1-3) utilizes the energy accumulated in $\mathrm{Na}^{+}$ concentration gradient. It preserves low intracellular $\mathrm{Ca}^{2+}$ concentration as it drives one $\mathrm{Ca}^{2+}$ outwards in exchange for three $\mathrm{Na}^{+}$from the extracellular space (17).

3. $\mathrm{Ca}^{2+}-\mathrm{ATPase}$ is present in the plasma membrane (Atp2b1-4) and endoplasmic reticulum (Atp2a1-3). It actively pumps $\mathrm{Ca}^{2+}$ out of the cytosol into extracellular space or endoplasmic reticulum (18).

4. $\mathrm{HCO}_{3} / \mathrm{Cl}^{-}$-exchangers (S/c4a1-3, S/c4a8, S/c4a10) keep the intracellular concentration of $\mathrm{Cl}^{-}$low. The exchange is $\mathrm{Na}^{+}$dependent (19). 
5. $\mathrm{Na}^{+} / \mathrm{K}^{+} / \mathrm{Cl}^{-}$- co-transporter (Slc12a2) pumps $\mathrm{Na}^{+}, \mathrm{K}^{+}$, and two $\mathrm{Cl}^{-}$into the cytosol, thus contributing to the $\mathrm{Cl}^{-}$-homeostasis (20).

6. $\mathrm{K}^{+} / \mathrm{Cl}^{-}$-co-transporter (S/c12a5-6) drives one $\mathrm{K}^{+}$and one $\mathrm{Cl}^{-}$outwards, thereby maintaining low intracellular $\mathrm{Cl}^{-}$concentration (21).

Specialized ion channels, most importantly, $\mathrm{Ca}^{2+}, \mathrm{Na}^{+}$, and $\mathrm{K}^{+}$-channels, govern the ionic permeability of the membrane. At the pre-synapse, $\mathrm{Ca}^{2+}$-channels clustered at the active zone (represented by the $\mathrm{P} / \mathrm{Q}$ - or $\mathrm{N}$-type $\mathrm{Ca}^{2+}$-channels) trigger the fast neurotransmitter release as discussed below (s. section 1.1.2.1). The pore-forming $\alpha_{1}$-subunits identify the family of $\mathrm{Ca}^{2+}$-channels. For Cav2.1-3 channels, these subunits are encoded by Cacna1a, Cacna1b, and Cacn1e genes, respectively (22).The $\mathrm{Ca}^{2+-i n f l u x}$ is commonly generated through P/Q- (Cav2.1) or N-type (Cav2.2) $\mathrm{Ca}^{2+}$-channels, whereas other types such as R-type (Cav2.3), L-type (Cav1.1-4) $\mathrm{Ca}^{2+}$-channels play a minor role in the triggering of the neurotransmitter release (23). Auxiliary subunits $\alpha_{2} \delta$ (Cacna2d1-4), $\beta$ (Cacnb1-4), and $\gamma$ (Cacng1-9) can be present in different combinations and presumably modulate the function of $\mathrm{Ca}^{2+}$-channels (24). $\mathrm{Na}^{+}$-channels are abundant at the neuron's body and in the axon, while the amount and diversity of $\mathrm{Na}^{+}$-channels at the pre-synapse are rather sparse (25). Nav1.2 (Scn2a) and Nav1.6 (Scn8a) are the most probable channel type at the axon terminals $(26,27)$. The limited number of $\mathrm{Na}^{+}$-channel at the pre-synapse allows to control the shape of arriving signals and consequently the $\mathrm{Ca}^{2+}$-inflow $(27,28)$. In contrast to $\mathrm{Na}^{+}-$ channels, $\mathrm{K}^{+}$-channel family is exceptionally diverse and encompasses $\sim 70$ genes. The channels differ in their structural organization, function and gating mechanisms (29). The most common $\mathrm{K}^{+}$-channel subunits found at the pre-synapse are of $\mathrm{K}_{\mathrm{v}} 1.1$ (Kcna1) and $K_{v} 1.2$ (Kcna2) type $(25,30)$. They belong to low-voltage-gated-channels that activate at modest depolarizations near the resting membrane potential thus preventing aberrant firing (31). The high-voltage-activated $\mathrm{K}_{\mathrm{v}} 3$ channels (Kcnc1-3) located at the pre-synapse become conductive at significant depolarizations (around $0 \mathrm{mV}$ ) and are required to achieve a quick repolarization (32). The role of other types of voltage- and ligand-gated $\mathrm{K}^{+}$-channels present at the pre-synapse is less established, however, their involvement in the regulation of synaptic transmission is generally recognized (33).

In the current view, activity of electrogenic transporters such as $\mathrm{Na}^{+} / \mathrm{K}^{+}-\mathrm{ATP}$ ase and $\mathrm{K}^{+}$ leakage through low-voltage-gated $\mathrm{K}^{+}$-channels maintains the plasma membrane potential at approx. $-70 \mathrm{mV}$ at rest (11). Arrival of a depolarization wave - an action potential (AP) from the proximal part of the axon leads to the depolarization of the pre-synaptic plasma membrane, opening of the voltage-gated $\mathrm{Ca}^{2+}$-channels, $\mathrm{Ca}^{2+}$-influx into the cytosol, SV exocytosis and neurotransmitter release. The involvement of $\mathrm{Na}^{+}$-channels in the active propagation of the action potential at the nerve terminals remains debatable. It is assumed 
that APs spread passively from the preterminal part of the axon to its terminus, while $\mathrm{Na}^{+}-$ channels located in the pre-synapse may modulate this process (27). Opening of the highvoltage-gated $\mathrm{K}^{+}$-channels helps to repolarize the membrane quickly thus allowing for repetitive firing (32).

\subsubsection{SV cycling}

The process of SV cycling can be broadly divided into two functional steps: i) SV fusion with the plasma membrane via the process of exocytosis, and ii) SV recycling (Figure 1.1B). The latter includes SV material retrieval via endocytosis, and the preparation for the next round of SV cycling, i.e., refilling with the neurotransmitter, SV tethering, docking, and priming. Each step of SV cycling is governed by distinct multiprotein complexes, of which the most important members are listed below.

\subsubsection{Exocytosis}

As a part of the SV cycling process, exocytosis is responsible for SV fusion with the plasma membrane and the active release of neurotransmitters. In the current interpretation, $\mathrm{Ca}^{2+}$ ions are the natural trigger of this process (34). Depolarization of the pre-synaptic membrane increases the open state probability of voltage-gated $\mathrm{Ca}^{2+}$-channels clustered at the active zone resulting in the influx of $\mathrm{Ca}^{2+-i o n s}$ into the pre-synapse following the electrochemical gradient (35). SVs near the plasma membrane are "primed" for the fusion via soluble $\mathrm{N}$-ethylmaleimide-sensitive factor-attachment protein receptors (SNARE). SNARE proteins form a core complex, which includes SV membrane-standing protein synaptobrevin (Vamp2) and plasma-membrane-standing proteins syntaxin-1 (Stx1a), and SNAP-25 (Snap25). Interaction of these proteins act as a "molecular zipper" that brings fusing membranes into a close proximity with each other $(36,37)$. Other proteins such as complexins (Cp/x1-3), Munc18 (Stxbp1), and SV membrane-anchored protein synaptotagmin-1 (Syt1) interact with the SNARE and probably regulate the stability of the whole complex (38-40). Moreover, synaptotagmin-1 acts as a $\mathrm{Ca}^{2+}$-sensor that can interact with negatively charged phospholipids of the plasma membrane upon $\mathrm{Ca}^{2+-b i n d i n g ~(41) . ~}$ This interaction may destabilize the complex and lead to complete zippering of the preassembled SNARE complex. The latter may provide required mechanical force to pull the plasma membrane and a SV into close proximity, leading to the formation of a fusion pore or its expansion (42). Other proteins might affect the complex formation. For instance, Tomosyn (Stxbp5) and amysin (Stxbp6) are able to substitute synaptobrevin in the complex thus preventing the formation of functional SNARE complexes $(43,44)$. Similarly, the interaction of synaptobrevin with another SV protein synaptophysin (Syp) may sequester the number of vesicular SNARE molecules available for the fusion (45). 
Following the fusion of the membranes, the SNARE complex remains anchored in the plasma membrane. Vesicle-fusing ATPase NSF (Nsf) and its adaptors $\alpha / \beta / Y$-SNAPs (Napa, $b, g$ ) dissociate the complex under ATP consumption (36). The energy stored in monomeric SNAREs can ignite the next round of SV fusion (46).

\subsubsection{SV Recycling}

The persistent firing of the synapse necessitates recycling mechanisms that replenish the SV pool and maintain the composition and integrity of the pre-synaptic plasma membrane. The recycling process begins with the separation of the membrane material of fused SVs and the plasma membrane via the process of endocytosis. Several endocytotic mechanisms have been described that rely on partially overlapping protein machinery and most probably co-exist in the synapse (47). At least four types of endocytosis have been described: clathrin-mediated endocytosis (CME), "kiss-and-run", "ultrafast endocytosis", and activity-dependent bulk endocytosis (ADBE) (48). The molecular machinery and the key steps are best described for CME (49). It begins with the binding of clathrin adaptor proteins such as adaptor protein (AP) 2 (Ap2a1, Ap2a2, Ap2b1, Ap2m1, Ap2s1), AP180 (Snap91), epsin (Epn1, Epn2), F-BAR domain protein (Fcho2) to cytosolic domains of SV proteins in the context of phosphatidylinositol(4,5)-bisphosphate $\left(\mathrm{PI}(4,5) \mathrm{P}_{2}\right)$-rich plasma membrane (50-54). The adaptors recruit light (Clta, Cltb) and heavy (Cltc) chains of clathrin, which form a basket-like structure around the invaginating membrane also known as "coated pit" (55). In the next step, a GTPase dynamin (Dnm1) mediates the fission of the pit (56). Endophilins $(S h 3 g / 1,2)$ recruit a phosphatase synaptojanin-1 (Synj1) that dephosphorylates $\mathrm{PI}(4,5) \mathrm{P}_{2}$ and thus promotes detachment of the adaptor proteins $(57,58)$. The disassembly of clathrin is further mediated by the ATPase Hsc70 (Hspa8) and its cofactor auxilin (Dnaj6) $(59,60)$. Other proteins are involved at different steps and take part in the control of the CME machinery. For instance, intersectin (Itsn1, Itsn2), Eps15, and dynamin-binding protein (Dnmbp) serve as platforms for attachment of other proteins (6163). Cell division control protein 42 homolog (Cdc42) and neuronal Wiskott-Aldrich syndrome protein (Wasl) regulate the actin cytoskeleton dynamics (64). Myc boxdependent-interacting protein 1 (Bin1), amphiphysin (Amph), and Dnmbp can further be implicated in the membrane curvature formation (65-67).

CME is a relatively slow process in the minute time range (68). In contrast, other types of endocytosis are much faster $(<10 \mathrm{~s})$ and do not require a clathrin coat. SVs that do not fuse completely with the plasma membrane are thought to undergo "kiss-and-run" endocytosis (69). Such SVs release their neurotransmitter content through a transient nanometer-wide pore (70). SVs remain within the active zone and preserve their membrane composition, which makes them quickly available (within $10 \mathrm{~s}$ ) for the next round of SV cycling (71). Even 
a faster mode of endocytosis ("ultrafast endocytosis") was described that happens within $500 \mathrm{~ms}$. It appears as membrane invaginations flanking the active zone and probably represents a mechanism which allows to rapidly restore the membrane surface following a brief but intense stimulus $(72,73)$. Some key proteins of CME seem to participate in the ultrafast endocytosis, e.g., synaptojanin-1, endophilin-A, and dynamin-1 $(72,74)$. Longer stimulation is able to trigger ADBE (75). It retrieves large areas of membranes that form intracellular endosomes within 1-2 s, which is probably a compensatory mechanism to retrieve excessive membrane material (76). The molecular mechanisms of ADBE and SV reformation from endosome-like structures generated via fast endocytosis are not entirely understood. Several reports show that ADBE and SV regeneration require dynamin-1 dephosphorylation by the calcium-activated phosphatase calcineurin (Ppp3ca-c, Ppp3r1, 2) and the lipid-deforming activity of syndapin-1 (Pacsin1) (77-79). Further, the process of SV retrieval from the endosome-like structures relies on SV protein recognition by adaptor protein complexes involved in CME, i.e., AP2 (80), or its endosomal cognates AP1 and AP3 (81). Therefore, it cannot be excluded that, at least some, SVs are recycled via early/sorting endosomes (82).

The regeneration of SVs requires the filling of SV with neurotransmitters. The driving force for this filling is the electrochemical gradient generated by the vacuolar proton ATPase (VATPase). V-ATPase is the largest multi-subunit protein complex of SVs encoded by Atp6v gene family (83). V-ATPase consists of two main domains, $V_{0}$ and $V_{1}$. The peripheral complex $V_{1}$ possesses the ATPase activity, while the transmembrane complex $V_{0}$ translocates $\mathrm{H}^{+}$through the SV membrane into SV lumen (84). Vesicular transporters (VTs) in the SV membrane utilize this proton gradient to fill SVs with respective neurotransmitter molecules. The transporter type determines the type of the neurotransmitter stored in SV, and it is assumed that, in accordance with the Dale's principle (85), the majority of SVs contain a single type of neurotransmitter (86). However, a recent study has shown that $\sim 36 \%$ of SVs carry two distinct VTs, which renders the possibility for neurotransmitter corelease from the same synapse. For example, $\sim 34 \%$ of all SVs contained glutamate transporter 1 (VGLUT1, Slc17a7) and the zinc transporter-3 (Slc30a3), while 2\% accounted for 27 other combination (87).

The regenerated SVs are recruited to the active zone where they are prepared for fusion following a series of successive "priming" reactions. The key role in the SV tethering and docking is attributed to the active zone proteins such as RIM proteins, calcium-dependent secretion activator 1 (Cadps), and Munc13 $(88,89)$. The role of SNARE proteins in the priming reactions is still under investigation. Although some data suggest an existence of 
partially assembled SNARE complexes, the molecular mechanism of the SNARE arrest in the pre-zipped form is yet to be elucidated $(3,90)$.

\subsubsection{Synaptosomes as a model of synapse}

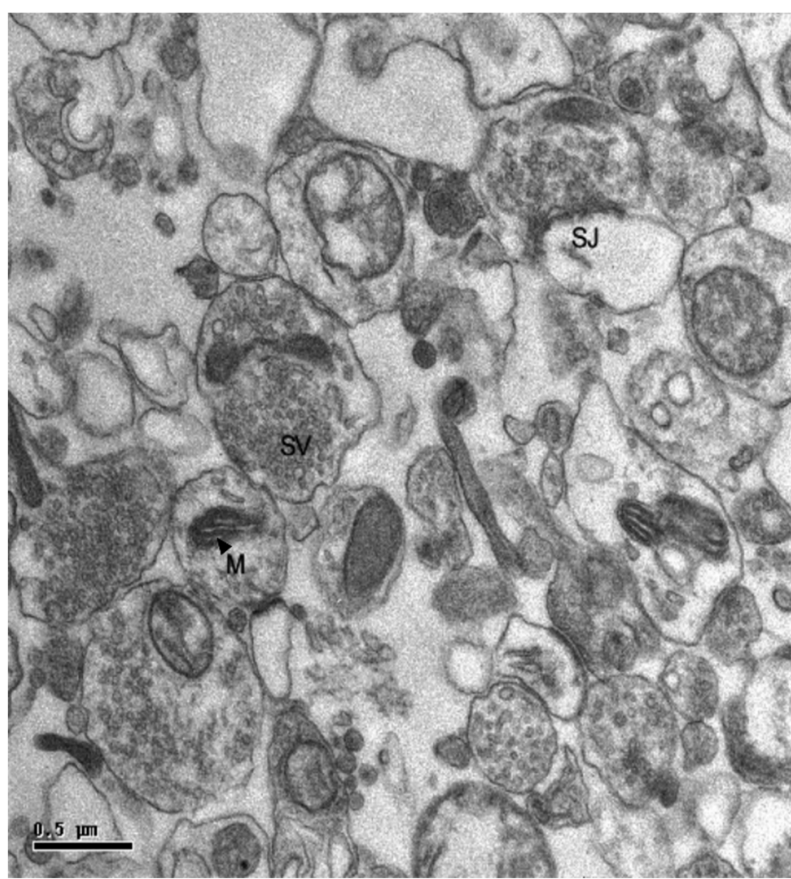

Figure 1.2: Electron micrograph of synaptosomal

fraction. Synaptosomes are membrane-enclosed structures that contain synaptic vesicles (SV), mitochondria (M). In some cases, post-synaptic compartment remains attached via synaptic junction (SJ). Bar, $0.5 \mathrm{~mm}$. Adapted from Schrimpf et al (91).

Synaptosomes represent pinched-off nerve terminals that can be isolated following a gentle homogenization of the brain tissue and differential and gradient centrifugation. First synaptosomes were isolated by Victor Whittaker as reported in 1958 (92). Their subsequent characterization showed that synaptosomes are membrane-enclosed nerve terminals whose membrane reseals during the homogenization process (93). Synaptosomes contain all the machinery needed for the neurotransmitter release (94). Being of $\sim 500-900 \mathrm{~nm}$ in diameter, they contain SVs and mitochondria (Figure 1.2) (93, 95). Over time, multiple protocols for synaptosomes preparation have been developed. The core procedure, however, remained unchanged: i) brain tissue is homogenized using a Teflon/glass homogenizer in an isotonic sucrose/low-salt buffer; ii) the homogenate is clarified from nuclei and debris yielding a crude suspension S1; iii) synaptosomes are precipitated by differential centrifugation yielding a crude synaptosomal pellet P2; and iv) synaptosomes are further purified by differential centrifugation in discontinuous sucrose, Ficoll, or Percoll gradients (96). Of note, purified synaptosomes following the described procedure still contain residuals of neuronal or glial membranes, free mitochondria, or myelin. $\sim 15 \%$ of synaptosomes contain an attached post-synaptic membrane (97). Despite this fact, only self-enclosed axonal terminals are capable of maintaining membrane potential and ATP levels needed for the neurotransmitter release and SV cycling $(98,99)$.

Due to the small size of synaptosomes, they are not amenable to electrophysiological measurements, i.e., patch-clamp. Moreover, synaptosomes most probably lose the most axonal $\mathrm{Na}^{+}$-channels during preparation and are not able to generate classical action potential (96). Nonetheless, fluorimetry-based methods have been found useful in studying 
neurotransmitter release and synaptosomal functionality. In particular, the glutamate release assay became a method of choice to assess the functionality of synaptosomal preparation (100). The assay utilizes the conversion of glutamate to ketoglutarate by glutamate dehydrogenase (GluDH). The concomitant conversion of NADP to NADPH can be monitored as an increase of NADPH-specific emission at $440 \mathrm{~nm}$ wavelength. In order to conduct the assay, GluDH and NADP need to be externally added to the synaptosomal suspension. Glutamate being the most common type of the neurotransmitter in the central nervous system is released from synaptosomes following their stimulation/depolarization. Other fluorescence-based assays utilizing fluorescence dyes allowed to monitor i) cytosolic free $\mathrm{Ca}^{2+}$ (Fura-2 (101)), ii) membrane potential (DiSC 2 (102)), and iii) endocytosis (FMdyes (103) and acridine orange (104)). Accordingly, it was shown that synaptosomes are able to maintain the polarization of the plasma membrane and cover their energy demands via glycolysis and oxidative phosphorylation (98). They hold the cytoplasmic $\mathrm{Ca}^{2+-l e v e l s}$ at 100-200 nM (101) and can release neurotransmitter in a $\mathrm{Ca}^{2+}$-dependent manner via exocytosis and subsequent SV recycling (99).

In order to study the neurotransmitter release, different stimulation protocols have been developed. Unlike neuronal cell cultures, electrical field stimulation of synaptosomes did not find broad acceptance (96) with only a few early studies reporting electrical stimulation of synaptosomes (105-112). Most of them applied a long (>10 $\mathrm{min})$ intense $(100 \mathrm{~Hz})$ stimulation (105-111), while few used $10 \mathrm{~Hz}$ stimulation (112). Despite the observed release of glutamate, aspartate, and GABA by electrically stimulated synaptosomes (105), it remained unclear whether the release is driven by the exocytosis. For example, tetanus toxin (in action similar to botulinum neurotoxins, s. below) reduced the liberation of glycine but not glutamate or other neurotransmitters, which poses a question whether the exocytotic machinery is involved in the neurotransmitter release following the electrical stimulation (113). On the other hand, increased accumulation of an isotope ${ }^{45} \mathrm{Ca}$ by synaptosomes during the course of the stimulation speaks for a possibility of a $\mathrm{Ca}^{2+}$-driven neurotransmitter release $(111,112)$.

Due to the uncertainties associated with the electrical stimulation, currently, chemical methods are commonly used for synaptosomal stimulation. For instance, the addition of $\mathrm{KCl}$ to the external buffer to lift [ $\left.\mathrm{K}^{+}\right]_{\text {ex }}$ to $30-100 \mathrm{mM}$ has been proven a reliable method to achieve strong depolarization of synaptosomal membrane and glutamate release (114). However, the method has been criticized for it causes a persistent depolarization of the synaptosomal membrane leading to a strong increase in intracellular $\mathrm{Ca}^{2+}$-concentration (up to $400 \mathrm{nM}$ ) (114). The latter leads to the activation of $\mathrm{Na}^{+} / \mathrm{Ca}^{2+}-\mathrm{ATPase}$, increased energy demand and reversal of the $\mathrm{Na}^{+}$-coupled transporter of the plasma membrane, e.g., $\mathrm{Na}^{+} /$glutamate 
transporter (115). To overcome the non-physiological clamp-depolarization of the plasma membrane, blocker of potassium channels such as 4-aminopyridine or dendrotoxin were introduced (116). It is hypothesized that the blockage of $\mathrm{K}^{+}$-channels causes a moderate increase in the membrane potential. A spontaneous opening of $\mathrm{Na}^{+}$-channels can further depolarize the membrane leading to the neurotransmitter release. This view is supported by the fact that 4-aminopyridine evoked release is sensitive to $\mathrm{Na}^{+}$-channel blockers, i.e., tetrodotoxin (117).

Apart from tetrodotoxin, other chemicals and toxins can be applied to synaptosomes for studying their effects on the neurotransmitter release. For instance, $\mathrm{Ca}^{2+}$-dependence of the neurotransmitter release can be demonstrated using a metal chelator ethylene glycolbis( $\beta$-aminoethyl ether)-N,N,N',N'-tetraacetic acid (EGTA): incubation of synaptosomes with 0.2-1 mM of EGTA greatly reduced the amount of the released glutamate following $\mathrm{KCl}$ induced depolarization $(100,104,118)$. In a similar way, application of $\mathrm{Ca}^{2+}$-channel inhibitors $\mathrm{Cd}^{2+}$ or $\omega$-conotoxin can efficiently block neurotransmitter release (119). The remaining $\mathrm{Ca}^{2+}$-independent release could be attributed to the leakage of the cytoplasmic glutamate via the glutamate transporter of the plasma membrane (118). The necessity of SNARE proteins for the neurotransmitter release can be demonstrated by the use of botulinum neurotoxins (BoNTs) - a group of exceptionally specific SNARE proteases (120). Application of BoNTs efficiently blocks SV exocytosis without compromising $\mathrm{Ca}^{2+}$-influx or synaptosomal bioenergetics $(121,122)$.

\subsubsection{SV pools}

Despite similar ultrastructure and composition, SVs constitute different populations or pools, which can be distinguished by how fast they release neurotransmitter in response to stimulation (123). Primed SVs compose a so-called "readily releasable" SV pool (RRP) and are the first to release neurotransmitter, i.e., possess the highest release probability (123). An intense stimulation (2 s stimulation at $20 \mathrm{~Hz}$ in hippocampal cell cultures) can deplete this pool, which leads to a significant drop of the neurotransmitter release or the usedependent depression $(124,125)$. However, it seems that RRP is not homogeneous, and primed SVs can exhibit different release probabilities. The later might be interpreted as distinct subpopulations of "fully primed" and "incompletely primed" SVs $(126,127)$. The vacancies in the RRP can be replenished via SV recycling mechanisms such as CME or "kiss-and run" $(128,129)$ or via recruitment from the recycling pool. The latter is responsible for the continuous neurotransmitter release under moderate (physiological) stimulation (123). In contrast to a stimulation-reluctant "reserve" pool, the recycling pool is considered to contain mobile, recently endocytosed SVs $(130,131)$. As indicated above, the reserve pool (at least $\sim 50 \%$ of total SVs) contain SVs that are engaged into SV cycling only during 
phases of intense stimulation (123), although experiments using dynamin knockouts indicate that the reverse pool can be recruited for the release even under low-frequency stimulation if the other SV pools are depleted (132). Since intense stimulation also triggers bulk endocytosis, it is tempting to speculate that SVs regenerated through budding from endosome-like structures contribute to the reserve pool in the first place. However, the existence of distinct recycling routes for different SV pool has not been sufficiently shown (131). Of note, the balance between the pools determines the releasable amount of neurotransmitter and, therefore, the synaptic strength. The activity-dependent modulation of the neurotransmitter release at the pre-synaptic part and the regulation of the evoked response at the post-synapse constitute what is known as synaptic plasticity. Different forms of pre- and post-synaptic plasticity have been described that are generally referred to as long and short potentiation/depression (133). It is assumed that synaptic plasticity plays key roles in learning and memory formation. Various cellular processes contribute to the plasticity and protein phosphorylation is one of the underlying molecular mechanisms.

\subsection{Post-translational modifications of proteins}

Post-translational modifications (PTMs) of proteins are covalent modifications of the polypeptide chain that occur during or after the synthesis and affect the functioning of the modified protein. Among post-translational protein modifications such as glycosylation, acetylation, methylation etc., phosphorylation of serine $(\mathrm{S})$, threonine $(\mathrm{T})$ or tyrosine $(\mathrm{Y})$ is one of the most prominent PTMs - it is estimated that up to $25 \%$ proteins annotated in the Swiss-Prot database (134) might carry this modification (135).

\subsubsection{Protein phosphorylation}

Reversible phosphorylation/dephosphorylation of proteins has been long recognized regulating protein activity, localization in the cell, and protein-protein interactions (136). The first in vitro phosphorylation of casein by a liver enzyme was described by Burnet and Kennedy (137). The authors also noted that the incorporation of a phosphate into the protein could be almost solely attributed to the phosphorylation of serine hydroxyl groups. The latter indeed aligns with the fact that serine is the most frequently phosphorylated amino acid, followed by threonine and tyrosine (135). Other amino acids such as lysine, arginine, and histidine can be also a site of phosphorylation; however, their role in the signaling pathways in eukaryotes is less established $(138,139)$.

Phosphorylation of a protein can have different consequences for the activity of that protein and the whole cell. Thus, introduction of a negative charge has an impact on the conformation and consequently the enzymatic activity of a protein. For example, autophosphorylation of a $\mathrm{Ca}^{2+} /$ calmodulin-dependent protein kinase 2 (CaMKII) renders its 
activity even in the absence of $\mathrm{Ca}^{2+}(140,141)$. Phosphorylation can further affect the interaction with other proteins. Thus, specialized proteins and protein domains that can recognize phosphorylation sites play a central role in the intracellular signaling pathways. Among them are 14-3-3 proteins (Ywhb, Ywhe, Ywhg-h, Ywhq, Ywhz ), WW domains, forkhead-associated (FHA) domains, and WD40 repeats/LRR modules that bind phosphorylated serine or threonine residues (142). Phosphorylated tyrosine residues are recognized by Src homology $2(\mathrm{SH} 2)$, phosphotyrosin-binding (PTB), and C2 domains of protein kinase $C \beta / \theta(P K C \beta, \theta)$, etc. (143).

\subsubsection{Kinase activity at the synapse}

Protein phosphorylation is extensively involved in the cellular signaling pathways - almost every signaling pathway involves kinase and/or phosphatase. In Humans, more than $\mathbf{4 0 0}$ proteins (potentially) possess kinase activity, i.e., the ability to transfer a y-phosphate group of an ATP molecule onto its substrate. Kinases are able to recognize the amino acid sequences - linear motifs - surrounding the phosphorylation site. Consensus sequences of the kinase linear motifs as well as sequence similarities in the kinase domain can be used for kinase classification (144, 145). AGC (Protein kinases A, G, and C (PKA, B, C)), CMGC, (cyclin-dependent kinase (CDKs), mitogen-activated kinase (MAPK), glycogen synthase kinase (GSK), and CLK) CAMK $\left(\mathrm{Ca}^{2+} /\right.$ calmodulin-dependent kinases (CAMKI and CAMKII)), CK1 (casein kinase 1), STE ("sterile" serine/threonine protein kinases), TK and TKL (tyrosine- and tyrosine-like kinases) constitute the main groups of kinases.

In the synapse, it has been proposed that the kinase activity is associated with changes in synaptic signal transmission or synaptic strength, such as long-term potentiation and depression (LTP and LTD, respectively). Although LTP and LTD can be both, pre- and postsynaptic phenomena, these processes were best studied in the post-synapse (146). A brief overview of the most prominent kinases located in the synapse is given below.

\subsubsection{Calcium/calmodulin-dependent protein kinase II (CaMKII)}

CaMKII is a serine/threonine kinase, which is very abundant (1-2\% of total protein in the brain) in the pre- and, especially, post-synapse. Camk2a, $b, d$, and $g$ genes encode $\alpha, \beta, \delta$, and $y$ forms of CaMKII, respectively. The catalytically active enzyme is formed by twelve subunits (147). The protein sequence contains four main regions, the catalytic domain, the auto-inhibitory domain, the variable region, and the self-association domain (148). Peculiar is the (auto)regulation of the CaMKII activity: binding of $\mathrm{Ca}^{2+} /$ calmodulin to the autoinhibitory domain releases the active site of the enzyme from binding the pseudo-substrate sequence of the auto-inhibitory domain. It further exposes a regulatory site (T286 in Camk2a) that can be autophosphorylated. The latter locks the kinase in the active state $(140,141)$. Two other 
autophosphorylation sites (T305 and T306 in Camk2a) inhibit re-association of CaMKII and $\mathrm{Ca}^{2+} /$ calmodulin and, therefore, re-activation of CaMKII upon phosphorylation (149).

CaMKII has been extensively studied in the post-synapse, where it is thought to induce LTP via phosphorylation of AMPA (Gria1-4) and NMDA (Grin1, Grin2a-d, Grin3a-b) receptors $(150,151)$, and Ras/Rap GTPase-activating protein SynGAP (Syngap1) (152). In the presynapse, it was shown to phosphorylate an SV-associated protein synapsin-1 (Syn1) at S603 (153). Phosphorylation affects synapsin-1 interaction with actin cytoskeleton, which subsequently regulates the size of SV cycling pool $(154,155)$. Experiments with CaMKII inhibitors and injections of CaMKII itself demonstrated its importance for the pre-synaptic plasticity (156). Moreover, it was also shown that CaMKII may regulate the pool of docked SVs, and this regulation does not depend on its enzymatic activity (157) and might be a result of its F-acting bundling capabilities (158).

\subsubsection{Mitogen-activated protein kinases (MAPK)}

MAPK represent a group of serine/threonine kinases expressed in neuronal and nonneuronal cells. These kinases are often involved into response to extracellular stimuli such as growth factors that affect cell proliferation, differentiation, and survival. MAPK-signaling is organized in cascades: a basic module consists of MAP kinase kinase kinase (MAPKKK) that phosphorylates and activates MAP kinase kinase (MAPKK), which activates the downstream MAP kinase (MAPK). The MAPKKK and MAPKK levels are mainly responsible for the signal transmission, while MAPK phosphorylate a large number of substrates (159). There are four main cascades distinguished by their MAPK component: i) extracellular signal-regulated kinase (ERK) 1 and 2 (Mapk3, Mapk1, respectively), ii) ERK5 (Mapk7), iii) C-Jun amino-terminal kinases (JNK) 1-3 (Mapk8-10), and iv) p38 (Mapk11-14) (160).

The classical cascade starts with the production of an active GTP-bound form of the small protein Ras in response to the extracellular stimuli. GTP-Ras triggers activation of MAPKKK Raf (Raf1), which activates MAPK/ERK kinase (MEK) 1/2 (Map2k1-2), which further phosphorylates ERK1/2 (159). Dual phosphorylation of ERK1 at T203 and Y205 (in mouse/rat) and ERK2 at T183 and Y185 (in mouse/rat) induces their full activation (161). ERK1/2 are "proline-directed" kinases: they phosphorylate serine or threonine adjacent to proline. The most common consensus sequences for substrate recognition are P-X-S/T-P or $\mathrm{S} / \mathrm{T}-\mathrm{P}$, where $\mathrm{X}$ is any amino acid (162).

High levels of ERK $1 / 2$ were found in the adult brain (163) and the and the ERK1/2 activation was observed following glutamate receptor stimulation and synaptic activity $(164,165)$. The ERK activation in the synapse is presumably Ras-dependent and mediated via $\mathrm{Ca}^{2+}$ dependent activation or inhibition of guanine nucleotide exchange factors (GEFs) and 
GTPase activating factors (GAPs), though an exact mechanism is to be elucidated (166168). Studies including MEK inhibitors have demonstrated the importance of MAPK for different forms of synaptic plasticity (reviewed in (169)). ERK substrates that can modulate the synaptic functioning include C-terminal domain of $\mathrm{K}_{\mathrm{v}} 4.2$ (Kcnd2) $\mathrm{K}^{+}$-channel (170) and synapsin-1 phosphorylation at S62, S67, and S549 (positions in rat Syn1) (171). The longlasting effects of MAPK activation are probably mediated via transcriptional activation (172, 173).

\subsubsection{Protein kinase A (PKA)}

PKA is a family of adenosine $3^{\prime}, 5^{\prime}$-monophosphate (cAMP)-activated serine/threonine protein kinases. While inactive, PKA holoenzyme consists of two catalytic and two regulatory subunits. In humans, Prkaca, Prkacb, and Prkacg genes encode for catalytic subunits. Two types of regulatory subunits are encoded by Prkar1a, b (Type I) and Prkar2a, $b$ (Type II), respectively. Binding of CAMP to a regulatory subunit cause its conformational change and release of the catalytic subunit (174). The PKA activity depends, therefore, on the cAMP levels and adenylate cyclase (Adcy1-9) activity, though recent data suggest that the holoenzyme can exhibit kinase activity at basal cAMP levels without subunit dissociation (175). The latter mechanism may rely on the interaction of Type II regulatory subunit with A-kinase anchoring proteins (AKAPs, in particular Akap5). Importantly, AKAPs are able to anchor PKA to cytoskeletal and/or organellar proteins. In the synapse, AKAPs may bring it close to the plasma membrane and SV fusion sites where it may regulate the neurotransmitter release via phosphorylation of Rim1 at S413 $(176,177)$, SNAP25 at T138 (178), and C-terminus of complexins (179) and others (180). Furthermore, a neuron specific signal-transduction cascade from cAMP/PAK to MAPK has been discussed (181).

\subsubsection{Protein kinase $C(P K C)$}

PKC comprises a family of serine/threonine kinases that contain a similar catalytic domain but differ in their regulatory domains (isoenzymes). Three major PKC groups were described: i) conventional PKC (cPKC, including $\alpha, \beta$, and y enzymes encoded by Prkca, Prkcb, and Prkcg, respectively); ii) novel PKC (nPKC, including $\delta, \theta, \epsilon$, and $\eta$ forms encoded by Prkcd, Prkcq, Prkce, and Prkch, respectively); iii) atypical PKC (aPKC, including $\zeta$ and I encoded by Prkcz, Prkci, respectively) (182). All PKC isoforms contain one or two C1 domains that binds to diacylglycerol (DAG), however, only cPKC and nPKC are DAGdependent. Conventional and novel PKC further contain a C2 or a C2-like domain that binds $\mathrm{Ca}^{2+}$ and acts as a $\mathrm{Ca}^{2+-}$ sensor (only in the case of $\mathrm{CPKC}$ ). Atypical PKCs require neither $\mathrm{Ca}^{2+}$ nor DAG, but could be stimulated by phosphatidylserine (183). Following synthesis, PKCs become phosphorylated at conserved amino acid residues: for Prkcb these are T500, T641, and S660 (the last two autophosphorylated) (184). Following the phosphorylation, the 
kinase can adopt an autoinhibited conformation. Elevated concentrations of $\mathrm{Ca}^{2+}$ and DAG induce conformational changes that lead to the PKC translocation to the plasma membrane and releasing of the catalytic domain from the pseudosubstrate binding (182). At the synapse, PKC activity has been found modulating synaptic strength (185). SNAP25 (S187), Munc18-1 (S313), and synaptotagmin-1 (T112) were identified as PKC substrates. The phosphorylation of these and other PKC substrates might contribute to the regulation of the neurotransmitter release (186-188).

\subsubsection{Cyclin-dependent kinase 5 (CDK5)}

CDK5 (Cdk5) is a member of the cyclin-dependent kinase family, but unlike other CDKs, its function is not primarily focused on the regulation of the cell cycle, but rather on the regulation of a variety of other cellular processes in neurons as well as other cell types (189). Another hallmark that distinguishes CDK5 from other members of the CDK family is its dependence on non-cyclin activators, p35 (Cdk5r1) or p39 (Cdk5r2) (190-193). The localization of CDK5 activators might represent the mechanism of spatial restriction of the kinase activity of the ubiquitously expressed CDK5 (193). Further regulation mechanism include p35 phosphorylation at S8 and T138 by CDK5 itself that leads to the proteasomedependent degradation of p35 (194). Moreover, phosphorylation of CDK5 at Y15 by tyrosine-kinases $\mathrm{Abl}(A b)$ and Fyn $(F y n)$ increases its catalytic activity $(195,196)$.

Several synaptic targets were identified that can convey the role of CDK5 in the synapse organization and plasticity. For instance, it has been shown to phosphorylate S549 and S551 of synapsin-1 (rat) which correlates with the reduced actin filament binding of that protein $(171,197)$. CDK5 may regulate endocytosis itself via phosphorylation of Munc18-1 at T574 $(198,199)$ and $P / Q$ type $\mathrm{Ca}^{2+-}$-channels $(200)$. Furthermore, CDK5 phosphorylates amphiphysin at multiple sites (S262, S272, S276, S285, and T310) $(201,202)$, dynamin-1 at S778 (203), and synaptojanin at S1144 (204). The three proteins are also known as "dephosphins" because they undergo dephosphorylation by a phosphatase calcineurin - an essential process for SV endocytosis (205). The re-phosphorylation of dephosphins by CDK5 indicates an important role the kinase plays in the regulation of SV endocytosis (206).

\subsubsection{Glycogen synthase kinase 3 (GSK3)}

GSK3 is a constitutively active and ubiquitously expressed serine/threonine kinase. It exists in two isoforms, GSK3a (Gsk3a) and GSK3 $\beta$ (Gsk3b). GSK3 is a proline-directed kinase that exhibits a weak affinity to substrates containing proline next to serine/threonine (207). However, a common requirement for GSK3-mediated phosphorylation is a prephosphorylated serine/threonine at the +4 position relatively to the to the target site $(\mathrm{S} / \mathrm{T}-\mathrm{X}$ $\mathrm{X}-\mathrm{X}-\mathrm{pS} / \mathrm{pT}$, where $\mathrm{pS} / \mathrm{pT}$ is a pre-phosphorylated serine or threonine) (208). GSK3 can perform a sequential phosphorylation of closely located $\mathrm{S} / \mathrm{T}$ residues since the recently 
phosphorylated serine/threonine acts as a pre-primed site for the following S/T residue. The latter can be observed in the case of glycogen synthase (Gys1-S653, S649, S645, and S641) and $\beta$-catenin (Ctnnb1-T41, S37, S33) (207). GSK3 phosphorylation at an N-terminal serine (Gsk3a-S21/Gsk3b-S9) turns it into a pre-phosphorylated substrate, or pseudosubstrate, thus reducing GSK3 activity (209). Several signaling cascades can mediate this phosphorylation, including CaMKII following an increase in the cytosolic $\mathrm{Ca}^{2+}$ concentration (210).

In the pre-synapse, GSK3 can regulate exocytosis via phosphorylation of P/Q-type $\mathrm{Ca}^{2+}$ channels (211). It participates in re-phosphorylation of dephosphins, whereby CDK5 acts as a pre-priming kinase for GSK3 (203). Furthermore, GSK3 is implicated in the abnormal phosphorylation of microtubule-binding protein tau (Mapt), thus contributing to the neurofibrillary tangles formation in Alzheimer's disease (212).

\subsubsection{Casein kinase 1 (CK1)}

CK1 is a monomeric serine/threonine kinase encoded by six distinct genes in humans, Csnk1a1, Csnk1d, Csnk1e, and Csnk1g1-3. Similarly to GSK3, CK1 prefers prephosphorylated substrates and exhibit affinity to acidic or phosphorylated residues in the 3 position (213). CK1 is implicated in controlling circadian rhythms $(214,215)$, membrane trafficking (216), cytoskeleton maintenance (217), etc. Via interfering with Wnt signaling, it is involved into the regulation of synapse stability and development $(218,219)$. Moreover, it can phosphorylate tau protein (Mapt), which might affect its aggregation (220). Altogether, it renders CK1 importance in neurodegenerative diseases, especially in Alzheimer disease (221).

\subsubsection{Casein kinase 2 (CK2)}

CK2 is a ubiquitously expressed serine/threonine kinase. The holoenzyme consists of two catalytic ( $\alpha$ and $\alpha$ ', encoded by Csnk2a1 and Csnk2a2, respectively) and a non-catalytic $\beta$ subunit (Csnk2b). CK2 is constitutively active and its localization to different compartments and cooperation with other proteins can confer the substrate specificity $(222,223)$. CKII (auto)phosphorylation can further regulate its activity (224). Thus, CKII phosphorylation at Y250 correlates with an increase enzymatic activity (225). Experiments have shown CK2 involvement into synapse organization and plasticity $(226,227)$. Syntaxin-1a (Stx1a-S14), and NMDA receptor (Grin2b-S1480) are among CK2 targets and their phosphorylation may contribute to the synaptic plasticity $(223,228-230)$.

\subsubsection{9 p21-activated kinase 1 (PAK1)}

PAK1 (Pak1) is a serine/threonine kinase which that interact with the activated forms of small GTPases, namely cell division control protein 42 homolog (Cdc42) or Ras-related C3 
botulinum toxin substrate 1 (Rac1) (231). Binding of Cdc42 or Rac1 drives PAK autophosphorylation and subsequent activation. This interaction renders PAK involvement into regulation of cytoskeleton, e.g., loss of actin stress fibers (232), and cell morphology (233). Moreover, PAK is capable of activating MAPK by phosphorylating MAPKKK Raf1 (234).

\subsubsection{Phosphatase activity at the synapse}

Phosphatases catalyze the removal of the phosphate group from the substrate, i.e., proteins or lipids. The human genome encodes $\sim 200$ phosphatases that can be categorized into six phosphatase super-families based on the sequence similarity of the catalytic domain (235). Protein tyrosine phosphatases (PTPs) is the most abundant group (> 100 genes (235)). It contains phosphatases that are more specific in their action and have a weak sequence preference towards their targets. Some members of this family are known as dual-specificity phosphatases (DUSP) and can remove the phosphate group from $\mathrm{Y}, \mathrm{S}$, and $\mathrm{T}$ residues (236). For example, MAPK phosphatases (MKP) represent an important sub-group of PTPs that can dephosphorylate $Y$ and $T$ in the activation loop of MAPK. A much smaller family, PPP, includes only 13 genes (235), but contains important serine/threonine phosphatases for the synapse plasticity, such as Protein phosphatase 1 (PP1), 2A (PP2A), and 2B (PP2B, Calcineurin) (237). In contrast to kinases, the substrate targeting of serine/threonine phosphatases is governed by adaptor proteins or auxiliary domains rather than via docking motifs (235). Absence of clearly defined docking motifs, overlapping substrates and signaling pathways make it challenging to pinpoint functional phosphatase-substrate interaction.

\subsubsection{Protein phosphatase 1 (PP1)}

PP1 is a ubiquitously expressed and a highly abundant phosphatase. Ppp1ca, Ppp1cb, Ppp1cc genes to the four distinct catalytic subunits $(\alpha, \beta / \delta, \gamma 1, \gamma 2)$ via alternative splicing. There is a plethora of regulatory subunits that define the substrate specificity and the subcellular localization of the phosphatase. Prominent regulators of PP1 function are neurabin-1 (Ppp1r9a) and neurabin-2 or spinophilin (Ppp1r9b) that anchor PP1 via modular binding motifs to F-actin - a very abundant cytoskeleton protein of the nerve terminals (238). The PP1-neurabin/spinophilin interaction has been shown to be important for postsynaptic LTD in hippocampus $(239,240)$, which may act via PP1-mediated dephosphorylation of AMPA receptors (241). Phosphorylation of neurabin-1 at S461 by PKA and neurabin-2 at S100 by CaMKII may represent the mechanisms of attenuating PP1 activity (242-244).

Further prominent regulators of PP1 activity are inhibitor-1 (Ppp1r1a) and dopamine- and cAMP-regulated phosphoprotein 32 (DARPP-32, Ppp1r1b). Increased levels of cAMP and 
PKA-mediated phosphorylation makes these proteins to potent inhibitors of PP1. Interestingly, phosphorylation at distinct residues such as Ppp1r1a-S67, T75 or Ppp1r1bT75, which may be accomplished by CDK5, results in the attenuation of PKA activity (245247).

\subsubsection{Protein phosphatase 2A (PP2A)}

PP2A holoenzyme consists of a catalytic subunit (Ppp2ca) associated with a scaffolding PR65 (Ppp2r1a) subunit, which coordinates the association of the catalytic subunit with a variety of regulatory $B$ subunits (248). The number of possible holoenzyme compositions is estimated at 75 (238). A conditional knockout experiment in mice has shown that PP2 loss affects synaptic transmission and plasticity in hippocampus (249). Previous stimulation experiment demonstrated that LTP induction correlates with the decrease of PP2A activity and the phosphorylation of its $55 \mathrm{kDa} \mathrm{Ba}$ subunit (Ppp2r2a) by CaMKII (250). Further reports have shown that B55-PP2A dephosphorylates protein tau (Mapt) at multiple sites (251-253). An intricate mechanism coordinating PKA, PP1, and PP2 activity was proposed by Ahn et al (254). Authors showed that PKA-mediated phosphorylation of B56ठ subunit (Ppp2r5d) has an activating effect on PP2A, which in turns dephosphorylates T75 on PP1 inhibitory subunit Ppp1r1b leading to PP1 inhibition.

\subsubsection{Protein phosphatase 2B (PP2B, Calcineurin)}

Similar to PP1, calcineurin holoenzyme consists of a catalytic subunit (Ppp3ca, Ppp3cb, or $P p p 3 c c)$ and a regulatory subunit (Ppp3r1 or Ppp3r2). Calcineurin is fully activated following binding of $\mathrm{Ca}^{2+} /$ calmodulin to its catalytic subunit and the concurrent binding of $\mathrm{Ca}^{2+}$ to its regulatory subunit $(255,256)$. It is assumed that due to high affinity of calcineurin to $\mathrm{Ca}^{2+} /$ calmodulin, initial increase of the cytosolic $\mathrm{Ca}^{2+}$ concentration activates calcineurin before $\mathrm{Ca}^{2+} /$ calmodulin dependent kinases (257). The latter is particularly important given that the phosphatase activity of calcineurin is required in SV endocytosis (258). Calcineurin acts as a $\mathrm{Ca}^{2+}$-sensor and dephosphorylates dynamin-1, amphiphysin, synaptojanin, AP180, epsin, and eps15 during different steps of SV endocytosis (205). Other cellular effects of calcineurin activation can be mediated via dephosphorylation of PP1 inhibior-1 and DARPP-31 leading to disinhibition of PP1 (259). Due to the calcineurin-mediated PP1 activation, it is not always possible to distinguish a direct and a PP1-mediated effect of calcineurin on protein dephosphorylation. Accordingly, further calcineurin substrates include microtubule-associated proteins Map2, Mapt, and tubulin itself. (260). Their dephosphorylation aid the assembly and stabilization of microtubules $(260,261)$. Calcineurin may further mediate dephosphorylation of known PKC substrates such as the myristoylated alanine-rich C kinase substrate (Marcks), neurogranin (Nrgn) and neuromodulin (Gap43). Dephosphorylation of these proteins enhance their affinity towards 
calmodulin $\left(\mathrm{Ca}^{2+} /\right.$ calmodulin for Marcks) and impact actin cytoskeleton cross-linking by Marcks and synaptogenesis by Ngrn and Gap43 (262). Moreover, calcineurin may be involved in regulation of SV pools via synapsin-1 dephosphorylation (197).

\subsection{Mass-spectrometric analysis of peptides, proteins, and PTMs}

Recent technological advances and development of mass spectrometry (MS) instrumentation have enabled large-scale identification and quantification of proteins and PTMs. In the following, I give a brief overview of the main principles underlying MS-based analysis of peptides, proteins, and PTMs.

\subsubsection{Instrumentation and working principle}

First mass spectrometers have appeared already in the beginning of the $20^{\text {th }}$ century, but for many years, the application of the technique was restricted to studying atoms and small molecules. Only after the invention of the soft ionization techniques electrospray ionization (ESI) (263) and matrix-assisted laser desorption (MALDI) $(264,265)$ in the mid-1980s, mass spectrometers became widely used for the analysis of large labile organic molecules such as proteins and peptides (266). Nowadays, mass spectrometry became an inevitable tool for biochemical studies and studies aimed at a comprehensive system-wide description of protein content, i.e., proteomics studies.

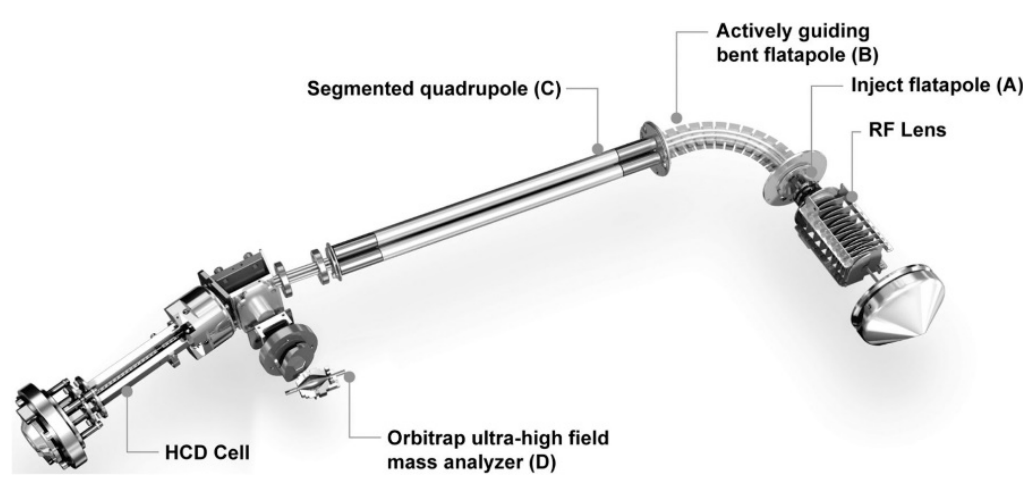

Figure 1.3: Ion path of the $Q$ Exactive HF mass spectrometer. $R F$ lens, inject flatopole (A), and actively guiding bent flatopole (B) guide, focus and pre-filter ions entering the mass spectrometer. Segmented quadrupole (C) functions as a selective mass filter. Masses of intact precursor ions and fragment ions produced in the HCD cell are analyzed by the Orbitrap (D). The figure is adapted from Scheltema et al (267).
A conventional mass spectrometer requires an ion source, mass analyzer and, depending on the implementation, a detector. Usually, it is operated under vacuum, and ionized particles are guided by magnetic and/or electric fields. lons are directed from an ion source to a mass analyzer where the separation of ions according to their mass-to-

charge ratio $(\mathrm{m} / \mathrm{z})$ takes place. A Time-of-flight or quadrupole mass-analyzer further require a detector, which determines the intensity of the ions. A result of the ion detection is a mass spectrum (MS): a list of observed $\mathrm{m} / \mathrm{z}$ and corresponding intensities. (268). An ion path of an ESI - hybrid quadrupole - Orbitrap mass spectrometer as implemented in the Thermo 
Fisher Scientific Q Exactive HF mass spectrometer is illustrated in Figure 1.3 (267). Mass spectrometers applied in this work utilize similar construction principles and elements.

Mass spectrometers are designed to measure charged molecules or ions. In an ESI ion source, an analyte is sprayed under high voltage $(>1 \mathrm{kV})$ through a fine emitter into the inlet of a mass spectrometer. It is assumed that the ionization of larger molecular ions, e.g., peptide ions, is achieved through passive solvent evaporation from small droplets containing only one analyte ion (269). Initially, the high potential difference between the needle and the inlet of the mass spectrometer results in generation of charged droplets with a charge density approaching its theoretical limit, the Rayleigh limit. Solvent evaporation further increases the charge density resulting in the generation of even smaller droplets that are emitted from a Taylor Cone formed on the original droplet. This process leads to a very fine liquid dispersion and a further release of molecular ions due to the solvent evaporation (269).

The initial parts of the ion route, i.e., the RF lens, injection flatopole and the bent flatopole focus the ion beam and act as initial pre-filter to remove neutral molecules, solvent droplets, and ion clusters, as well as to reduce the kinetic energy of ions via collisional cooling (270). Transmitted ions are further filtered by a segmented quadrupole (267). A quadrupole mass analyzer is constructed of four pairwise connected parallel metal rods. By varying the radio frequency (RF) voltage and direct current (DC) voltage applied to the rod pairs, it is possible to stabilize a path of selected ions, while trajectories of other ions end up in collision with the rods (271). The quadrupole is followed by a short octapole that conducts ions into the C-trap connected to the terminal quadrupole that acts as a collision chamber (a high energy collision dissociation or HCD cell (272)). Here, ions become activated through the collision with nitrogen gas molecules filling the collision chamber. A portion of the collision energy is converted into internal energy resulting in the bond breakage and fragmentation. Peptide ions typically break along their amine/peptide bond, which produces a series of ions, i.e., $y$ and $b$ fragment ions as illustrated in Figure 1.4 (273). Fragment ions can be trapped inside the HCD cell, pushed back into the C-trap and ejected into the Orbitrap analyzer (270). 
<smiles>[R]C(N)C(=O)NC(=O)C([R])NC(=O)[C@@H]([R])NC(=O)[C@@H]([R])N</smiles>

Figure 1.4: Nomenclature of $\boldsymbol{y}$ and $\boldsymbol{b}$ fragment ions. In an HCD cell, $y$ and $b$ ions are the common fragment ions that arise due to the breakage of the peptide bond. $B$ ions contain an intact $\mathrm{N}$-terminus, and $y$ ions an intact $C$-terminus of a peptide. The fragments are numbered consecutively with the increasing mass $(274,275) . R$ denotes an amino acid side chain.
Orbitrap acts as both a mass analyzer and a detector providing $\mathrm{m} / \mathrm{z}$ and the signal intensity of an ion. It consists of two cup-shaped outer electrodes and one spindle-like central electrode (276). lons injected into the space between the electrodes oscillate harmonically along the central (axial) electrode. Mass-tocharge ratios and the intensities of ions can be calculated based on the frequency of these oscillations using Fourier transformation. Because the axial frequency does not depend on the

initial energy and the spatial spread of ions, it allows for a high resolution $(>100,000)$ and an accurate mass detection (277).

\subsubsection{Sample Preparation for Mass Spectrometry (MS)}

Currently, there are two main branches in mass spectrometric protein analysis. In a conventional bottom-up experiment, proteins are cleaved by an endoproteinase and resulting peptides are subjected to a mass spectrometry analysis. Information about proteins is inferred from the pool of identified peptides. An alternative workflow known as top-down proteomics aims at analyzing non-cleaved proteins. Since peptides are more amenable for an MS analysis due to a limited set of charge states, uniform fragmentation and better detection limits, the bottom-up approach allows for a large-scale analysis and identification of thousands of proteins in complex samples. (278). Trypsin is a widely used proteinase that cleaves at the C-terminus of arginine $(\mathrm{R})$ or lysine $(\mathrm{K})$. Tryptic peptides often contain 7-20 amino acids and carry one of the basic amino acids at their C-terminus. At low $\mathrm{pH}$, the basic residues are positively charged, which improves the ionization properties and the "flyability" of a peptide. Therefore, it is common to operate the liquid chromatography (LC) coupled to a mass spectrometer at low pH. Supplements such as formic acid (FA) act as $\mathrm{H}^{+}$donors and facilitate the ionization of peptides. During ESI, peptides typically acquire two or more $\mathrm{H}^{+}$, which results in multiply charged peptide ions that can be analyzed by a mass spectrometer operated in a positive ionization mode. Moreover, specific cleavage sites simplify the subsequent peptide identification, since it reduces the number of theoretical peptides that can fit to an MS spectrum (273).

When analyzing PTMs, additional enrichment methods are usually required, since posttranslationally modified peptides, e.g., phosphopeptides, are underrepresented in complex 
samples. Common enrichment strategies employ immobilized-metal-ion affinity chromatography (IMAC (279)) and metal-oxide affinity chromatography (MOAC (280)). The enrichment is conducted at low $\mathrm{pH}$, which facilitates the protonated state of carboxyl groups of acidic amino acids ( $D$ and $E$ ) and of the peptide $C$ terminus. In contrast, phosphate groups remain deprotonated due to their different pKa values. As a result, phosphate groups become complexed by the metal oxide or metal ion on the solid phase forming an enrichable complex (281).

When speaking about identifying and quantifying proteins in a biological sample, one often has to deal with thousands of different proteins. To decrease the complexity of the sample and improve coverage of identified proteins and peptides in a bottom-up MS experiment, different protein and peptide fractionation approaches have been developed. In particular, chromatographic techniques using a C18-based reversed-phase stationary phase has proven useful for efficient peptide separation (282). Off-line pre-fractionation at basic $\mathrm{pH}$ (bRP) can be successfully combined with an on-line (directly coupled to a mass spectrometer, LC-MS/MS) reversed-phase chromatography at acidic $\mathrm{pH}(283,284)$. Thus, the general scheme for the sample preparation includes the following steps: i) protein digestion using specific endoproteinase (e.g., trypsin); ii) optional: enrichment of modified peptides for PTM analysis; iii) off-line pre-fractionation using bRP to reduce sample complexity; iv) sample analysis using LC-MS/MS.

\subsubsection{Data-Dependent Acquisition}

After passing an ion source, ionized peptides are subjected to a survey scan (MS1) to determine their $\mathrm{m} / \mathrm{z}$ ratio and intensity. In a top $\mathrm{N}$ mode, $\mathrm{N}$ of the most intense peaks (precursors) are sequentially selected by quadrupole for fragmentation in a collision cell (an HCD cell, s. above). Identification of precursor fragments allows for a confident precursor identification based on its fragmentation pattern (285).

Due to the preselection of precursor ions based on their intensity, this type of MS/MS acquisition is known as data-dependent acquisition (DDA). Acquisition in this mode produces pairs of MS1-precursors and corresponding MS2-fragment spectra, and a narrow $\mathrm{m} / \mathrm{z}$ window used for precursor selection assures that fragments observed in MS2 originate from a selected precursor ion. The fragmentation patterns can be matched against theoretical MS/MS spectra, resulting in peptide-spectrum matches (PSMs) (273).

However, the drawback of DDA is that by selecting $\mathrm{N}$ precursors, it introduces a stochastic and irreproducible component into MS/MS acquisition. Furthermore, as the decision which MS/MS spectra will be acquired is mostly based on the precursor intensity, peptides present 
in low amounts (such as phosphorylated peptides) might be completely masked by highlyabundant peptides resulting in precursor undersampling (286).

\subsubsection{Identification of peptides and proteins}

An acquired tandem MS/MS spectrum is a record of $m / z$ of fragment ions and corresponding ion intensities. An MS/MS spectrum further contains the information about its parent or "precursor" ion, i.e., its $\mathrm{m} / \mathrm{z}$ and charge (deduced from the isotopic distribution of the precursor ion). A common approach for peptide identification based on MS/MS spectra is the automated database search (287). This approach utilizes protein sequence databases - collections of amino acid sequences of proteins that extensively covers proteins potentially present in the analyzed sample. Protein sequences are in silico digested in accordance with specified cleavage rules (cleavage after $\mathrm{K}$ or $\mathrm{R}$ for trypsin), and the mass of the peptide precursor and its theoretical fragments ( $b$ - and $y$-ions) are calculated. In the case of possible (variable) modifications such as phosphorylation, a precursor mass and masses of theoretical fragment ions are computed separately considering all possible positions of the modification within the peptide sequence. Acquired MS/MS spectra are matched against the theoretical spectra. Certain mass deviations (tolerances) between the observed $\mathrm{m} / \mathrm{z}$ of an ion and calculated $\mathrm{m} / \mathrm{z}$ are allowed for the match to be reported. Each match is scored, and the best peptide-spectrum match (PSM) is assigned to the spectrum. In the case of phosphorylated peptides, it is important to not only identify the peptide, but also to localize the phosphate group. The latter is in particular challenging if there are several $S / T / Y$ amino acids in the peptide sequence and a peptide is multiply phosphorylated. Therefore, a confident PTM localization requires rich fragmentation pattern and characteristic fragment ions containing either an intact phosphate (+80 Da) or neutral losses of $\mathrm{H}_{3} \mathrm{PO}_{4}(-98 \mathrm{Da})$ or $\mathrm{HPO}_{3}(-80 \mathrm{Da})$. Based on the observed fragment ions, probabilities for each phosphorylated site are calculated and expressed in percent. Usually, only phosphorylated sites with the localization probability $>75 \%$ are considered for further analysis (first-class phosphorylated sites) (288).

In order to find a minimal acceptable score and to prevent accumulation of false assignments, one makes use of decoy sequences (289). Decoy sequences are non-existing peptide sequences, which are generated, for example, via reversal of the existing peptide sequences in the database. When comparing the distribution of PSM scores for existing peptides $\left(S_{P S M}\right)$ vs. the distribution of scores for a priori false positive matches to decoy sequences $\left(S_{d e c}\right)$, it is possible to derive a cut-off score $S_{c u t}$, such that the list of reported PSMs with $S_{P S M} \geq S_{\text {cut }}$ will contain no more than $N$ false positives (290). The tolerated 
proportion of false positives (also known as the false discovery rate, FDR) is commonly set to $1 \%$.

In the bottom-up proteomics, the information about the proteins is inferred based on the pool of all identified peptides (291). Since there are peptides that are shared between proteins, a common approach is to organize proteins in a minimal number of protein groups according to the principle of parsimony $(291,292)$. Thus, each protein group contains proteins that share one or more peptides. Protein groups are distinguished from each other based on peptides that are unique for a given protein group. A leading protein is a characteristic protein that contains the most peptides assigned to its protein group. Shared peptides are usually distributed in "all-or-nothing" principle, i.e., the protein group with the maximum number of peptides becomes all shared peptides assigned. The peptide scores of the assigned peptides are combined into a protein score and the FDR is calculated again on the protein group level (290). The FDR has to be controlled at each level separately since false assignments at a lower level (e.g., peptide level), tend to inflate identifications at a higher level (293).

\subsubsection{Protein quantification using a label-free and an isobaric-tag labeling approach}

MS based proteomics allows not only identification of proteins, peptides, and PTMs, but it can be applied to compare their abundances in different samples. Quantitative approaches can be broadly categorized in label-free and chemical labeling-based methods (294). I discuss in brief a classical label-free approach and an isobaric-tag labeling, as they are both utilized in this work.

The label-free approach utilized by MaxQuant (295) relies on quantification of the precursor signal in MS1 survey scans (296). In a standard DDA method, MS1 survey scans are repeated after a certain time interval or following a fixed number of MS/MS acquisitions, depending on a chromatographic setup, usually, every 2-3 seconds. If the chromatographic elution of a peptide takes $30 \mathrm{~s}$ and MS1 scans are conducted every $3 \mathrm{~s}$, there are approx. 10 time points that can be used to reconstruct the elution profile of a precursor. Area under the curve correlates with the peptide abundance and is suitable for quantitative comparisons (297).

An alternative approach to peptide quantification utilizes isobaric labels such as tandem mass tags (TMT) (298). TMT reagents consist of an amine-reactive NHS-ester group, a mass normalizer, and an MS/MS reporter. Each reagent has the same nominal mass but differs in the distribution of ${ }^{13} \mathrm{C}$ and ${ }^{15} \mathrm{~N}$ isotopes between the mass reporter and the mass normalizer. TMT reagents are highly reactive towards primary amines, i.e., $\mathrm{N}$-termini and lysine side chains of peptides. After labeling with TMT reagents, different peptide samples 
are pooled and analyzed as a single sample. Due to the isobaric nature of the TMT reagents, precursor signals are indistinguishable in MS1. Only the precursor fragmentation leads to generation of reporter ions with distinct masses. Intensities of the reporter ions in the MS/MS spectrum are used for quantitative comparison of peptide abundances in the labeled samples (299).

The advantages of TMT-labeling technique vs. label-free approach is in that i) it reduces the technical variance and minimizes the problem of missing values because the peptide samples are processed as one following the labeling and pooling; ii) it is better compatible with pre-fractionation techniques resulting in a deeper proteome coverage, since the reporter ion intensities and not the elution profiles of peptide ions are used for quantification; iii) it boosts sensitivity because intensities of low-abundant peptides are combined in MS1, which increases their chance to be selected for MS/MS. The disadvantages of the labeling approach lie in the cost of the reagents, the lower dynamic range, and the inaccuracy in depicting large changes due to the co-isolation of other precursor ions leading to "ratio compression" (300). A development on an SPS-MS3 acquisition method (301) allowed to alleviate the "ratio compression" problem, although at the cost of the reduced sensitivity (300). Therefore, an experimenter can select a suitable method to prioritize the quantification accuracy or the sensitivity depending on the scientific question.

It has to be noted that in a bottom-up proteomics the lowest quantitative level is either the precursor (MS1 peak area, label free quantification) or a PSM (reporter ion intensity, TMT), while the desired quantification levels are often peptides or proteins. Several precursors or PSMs may identify the same peptide and several peptides may originate from a single protein. This requires, therefore, a summarization of quantitative values obtained at the lowest quantitative level. Different sophisticated algorithms have been suggested that propagate quantitative values up to peptide or protein level $(302,303)$, though simple mean, median or sum of precursor/reporter ion intensities being still common summarization methods (304).

\subsubsection{Post-processing of quantitative proteomics data}

Label-free or label-based protein quantitative data enable, in the first place, a relative quantification of peptide and/or protein abundances (294). The peptide intensity observed in an ESI-LC-MS/MS setup is dependent not only on the peptide abundance, but also on its physical and chemical properties. Therefore, a direct comparison of LC-MS/MS intensities of different peptides/proteins would require additional correction steps and use of calibration curves of standards in pre-defined amounts (305). In contrast, intensity comparison of the same peptide/protein allow for a direct estimation of changes in peptide/protein abundance 
in different samples. Such differences are conveniently expressed as $\log _{2}\left(I_{2} / I_{1}\right)$ and known as $\log _{2}$-fold changes $(\log 2 \mathrm{FC})$, where $I$ is a peptide/protein intensity in the sample 1 or 2 , respectively (306). Accordingly, Log2FC of 1 means a two-fold increase in the intensity in the sample 2 as compared to the sample 1, while a value of -1 corresponds to a two-fold intensity decrease in the sample 2 vs. sample 1.

When comparing peptide/protein abundances in two or more samples, it is important to correct for possible systematic errors that arise during sample preparation, i.e., errors in the estimation of the protein concentration and errors associated with liquid handling, and result in unequal protein amounts being analyzed (307). Normalization strategies using median subtraction and its extended version, Tukey's median polishing, proved useful and robust methods for intensity normalization in proteomics (308). These normalization strategies are based on the assumption that abundancies of the majority of peptides/proteins are not changed between the samples.

In the following, log2FC are tested to be significantly different from 0 (no abundance difference), e.g., using a one-sample t-test. Other methods were developed to improve differential analysis in large genomic or transcriptomic data such as microarrays (309), and were further adapted to the analysis of proteomics data (310). For example, limma (311) offers an extended functionality, as it supports complex experimental designs via linear models. Further, it augments the statistical power of a test via correcting individual peptide/protein variances by the global variance within the data set utilizing a Bayesian framework, which is in particular beneficial for experiments with low number of replicates $(311,312)$.

While performing a differential expression analysis, it is important to control the number of false discoveries, since given the number of peptides and proteins quantified in a typical proteomics experiment, some of them might appear differentially expressed just by chance. For large-scale experiments such as a proteomics experiment, statistical methods were developed that estimates a false discovery rate among a list of differentially expressed peptides/proteins (313-315). FDR is commonly expressed in a form of $q$-values, which can take values between zero and one and are similar in this way to $p$-values. One can define a $q$-value threshold, e.g., 0.01, that can be used to list significant tests with the desired FDR level (here 1\%). This approach is convenient, as defining a $q$-value threshold resembles hypothesis rejection based on a $p$-value.

\subsubsection{Systems analysis of proteomics data}

A differential expression analysis commonly produces a list of differentially expressed peptides or proteins. In a case of a phosphoproteomics study, this might be a list of 
differentially phosphorylated amino acid positions (sites) of proteins. A systems analysis is a next step towards understanding how observed changes might impact cellular processes, pathways, etc. (316). For this, proteins are assigned to hierarchically structured terms (gene ontologies) that concisely describe a molecular or biological function or a cellular localization of proteins (317). By using statistical testing (usually a Fisher's exact test) it is possible to assess which categories are particularly enriched among differentially expressed proteins as compared to some background (usually, a whole proteome or its subset). A fold enrichment and a $p$-value are returned for each term. $P$-values can be corrected for multiple testing using one of the approaches described above. A result is a list of significantly enriched terms that can be further refined analytically depending on the biological context (316). A similar approach can be applied for pathway analysis $(318,319)$ or analysis of protein domains (320) and sequence motifs (321). Furthermore, using specialized databases such as String (322), one can query interaction partners of regulated proteins and explore how they are interconnected. Together with the sequence motif analysis, this information, for example, is utilized to predict kinase-substrates relationships for identified phosphorylated sites $(144,323)$. Nonetheless, one needs to be aware that the analysis is not unbiased (316). Potential sources of bias are the automated annotation and lack of detailed inspection by an expert; overlapping, redundant, too general or too specific categories; problems with the data transfer from one biological system to another; domination of a few extensively studied and, therefore, well annotated proteins over the majority of a rather poorly described proteins, etc. To partially alleviate these problems, a curated pathway database Reactome (319) or a synapse-specific expert-curated database SynGO (324) have been developed. Moreover, consulting experimentally validated data such as provided in the PhosphoSitePlus (325) database can further enhance the credibility of the analysis. 


\section{Aim of the thesis}

An accumulating evidence suggests that kinases and phosphatases play an important role in the synapse physiology and that protein phosphorylation may modulate the neurotransmitter release, and, therefore, the synaptic strength. It is widely accepted that the increase in the cytosolic $\mathrm{Ca}^{2+}$ concentration is the main trigger of the phosphoproteome changes in the synapse. The main goal of this thesis is to demarcate phosphorylation events that depend on active SV cycling from those that are primarily triggered by the $\mathrm{Ca}^{2+}$-influx due to the membrane depolarization. Such separation would offer a new point of view at the protein phosphorylation in the synapse and propose sites that exert a direct modulatory role on the neurotransmitter release. To achieve this, several steps had to be made. First, a stimulation protocol had to be established that would either allow for an active SV cycling with low net $\mathrm{Ca}^{2+}$-influx or abolish SV cycling while preserving the $\mathrm{Ca}^{2+}$-influx. For this, the applicability of electrical field stimulation as a potentially milder stimulation in comparison to chemical stimulation protocols and BoNT as a potent and very specific blocker of exocytosis and, therefore, of a complete SV cycling, were investigated. Second, a selection and optimization of suitable sample preparation techniques for MS-based analysis were carried out to achieve a comprehensive identification and quantification of the synaptic phosphoproteome. Thus, application of TMT-labeling techniques together with prefractionation techniques and MS2 and SPS-MS3-based quantifications allowed for a comprehensive analysis of the synaptosomal phosphoproteome. Third, based on the differential expression analysis and predicted substrate-kinase interactions, the demarcation of the SV cycling dependent and primarily $\mathrm{Ca}^{2+}$-dependent phosphorylation sites and their association with different kinase groups was achieved. Finally, selected SVdependent phosphorylated sites had to be tested for their modulatory effects on the exoand endocytosis. In cooperation with Prof. Silvio O. Rizzoli and Dr. Eugenio Fornasiero, modulatory effects of SV cycling dependent phosphorylation sites on syntaxin-1, synaptobrevin-2, and cannabinoid receptor-1 were demonstrated in cultured hippocampal neurons. 


\section{Preliminary Methods}

\subsection{Ethical statement}

All animal experiments were conducted in accordance with the national law of the Federal Republic of Germany. For details s. section 5.4.1

\subsection{Synaptosome preparation}

Synaptosomes were prepared as described in section 5.4.3.

\subsection{Electrical stimulation of synaptosomes}

$1.5 \mathrm{~mL}$ of synaptosomal suspension in a sodium-containing buffer ( $1 \mathrm{mg}$ of synaptosomal protein per $1 \mathrm{~mL}$ buffer) were kept at $37^{\circ} \mathrm{C}$ in a thermostated quartz cuvette (119F-10-40, $3.5 \mathrm{~mL}$, Hellma Analytics, Mühllheim, Germany) under constant stirring as described for the glutamate release assay in section 5.4.4. Platinum electrodes of $1 \mathrm{~mm}$ diameter were inserted at the two opposite corners (distance $\sqrt{2} \mathrm{~cm}$ ) reaching the bottom of the cuvette. Stimulation was applied using square pulses of alternating polarity generated by A385 Stimulus Isolator in a combination with an A310 Accupulser Stimulator (both from World Precision Instruments, Sarasota, USA) and monitored using an oscilloscope (PCSGU250, Velleman, Gavere, Belgium). Following stimulation parameters were applied if not stated otherwise: $15 \mathrm{~ms}$ pulse width, one pulse per $100 \mathrm{~ms}(10 \mathrm{~Hz}), 15 \mathrm{~mA}$ nominal current, voltage at a peak $\sim 2.5 \mathrm{~V}$, stimulus duration $120 \mathrm{~s}$.

\subsection{BoNT-treatment and glutamate release assay}

BoNT-treatment and glutamate release assay were performed as described in section 5.4.4. Electrical stimulation was used instead of $\mathrm{KCl}$-stimulation.

\subsection{Treatment with $\mathrm{Cd}^{2+}$, w-conotoxin, and bafilomycin $\mathrm{A}_{1}$}

Chemicals/toxins were added to synaptosomes resuspended in sodium buffer and preincubated at $37^{\circ} \mathrm{C}$ for at least $5 \mathrm{~min} .20 \mathrm{mM} \mathrm{CdCl}$ stock solution (Merck, Taufkirchen, Germany) in water was added to synaptosomes 5 min prior to the first or the second (repeated) electrical stimulation. A final concentration of 20 or $60 \mu \mathrm{M}$ of $\mathrm{CdCl}_{2}$ was used. $\Omega$-Conotoxin MVCII (Alomone Labs, Jerusalem, Israel) was dissolved in $1 \%(\mathrm{v} / \mathrm{v})$ ethanol/water at the concentration of $1 \mathrm{mM}$. The toxin was added to synaptosomes to a final concentration of 1 or $2 \mu \mathrm{M}, 10$ min prior to the first or the second electrical stimulation. Stock solution of $0.1 \mathrm{mM}$ bafilomycin $\mathrm{A}_{1}$ (Abcam, Cambridge, UK) in 1\% (v/v) DMSO in water was added to synaptosomes to reach a final concentration of $300 \mathrm{nM}$. The toxin was added 10 min before the stimulation, if not stated otherwise. 


\subsection{Acridine orange fluorescence assay}

The acridine orange fluorescence assay was conducted following the previously published protocol (104). In brief, stock solution of $1.5 \mathrm{mM}$ acridine orange in water was added to synaptosomes to reach a final concentration of $5 \mu \mathrm{M}$. Synaptosomes were incubated for $10 \mathrm{~min}$ at $37^{\circ} \mathrm{C}$ in a thermostated quartz cuvette as described for the glutamate release assay. The AO fluorescence was monitored at $490 \mathrm{~nm}$ excitation and $530 \mathrm{~nm}$ emission wavelengths. For stimulations including bafilomycin $A 1$ treatment, the toxin was added $\sim 5 \mathrm{~s}$ prior the stimulation (electrical stimulation or $\mathrm{KCl}$ ).

\subsection{Fura-2 assay}

Fura-2 assay was set up as described in section 5.4.13. The Fura-2 fluorescence was measured in synaptosomes pre-incubated in sodium buffer without extra $\mathrm{Ca}^{2+}$ (zero net $\mathrm{Ca}^{2+}$ or "background"). Then, $\mathrm{CaCl}_{2}$ was added to reach a final concentration of $1.3 \mathrm{mM}$ and the fluorescence was recorded for further $3 \mathrm{~min}$. Synaptosomes were then stimulated using a repeated electrical stimulation separated by a 2 min pause, and then stimulated with $50 \mathrm{mM} \mathrm{KCl}$. The maximum and minimum Fura-2 signal was determined following lysis with $2 \%$ SDS and subsequent quenching with 100 mM EGTA, respectively. Cytoplasmic $\mathrm{Ca}^{2+}$ concentration was calculated as described in (101) assuming $\mathrm{K}_{d}(\mathrm{Ca} / \mathrm{fura}-2)=224 \mathrm{nM}$.

\subsection{Analysis of fluorescence data}

The fluorescence data were analyzed in R programming language for statistical computing. Glutamate release assay and AO fluorescence data were collected as two data points every 3-4 s seconds. A fluorescence signal $(S)$ was corrected by the fluctuations in the lamp current $(R)$ and the corrected signal $S / R$ was used for further analysis. An average of the two data points was computed, and the data were smoothed using rolling truncated mean of seven data points (trim parameter of 0.3 ) to remove excessive fluctuations of the signal. Fluorescence $f$ was normalized by the fluorescence prior to the first electrical stimulation, $f_{0}$. The change in the normalized fluorescence before and after stimulation were assessed as $f / f_{0}$ (after) $-f / f_{0}$ (before), where $f / f_{0}$ (before) is the normalized fluorescence at the start of the stimulation, and $f / f_{0}($ after $)$ is the median fluorescence of the first $60 \mathrm{~s}$ after the stimulation was aborted. In the case of $\mathrm{KCl}$ stimulation, the fluorescence at 2 min after adding $\mathrm{KCl}$ was used.

\subsection{MS sample preparation}

$1.7 \mathrm{mg}$ synaptosomes were resuspended in sodium buffer at a concentration of $1 \mathrm{mg}$ of synaptosomal protein per $1 \mathrm{~mL}$. The synaptosomes were prepared for electrical stimulation as described before. Aliquots of the synaptosomal suspension corresponding to $200 \mu \mathrm{g}$ of 
synaptosomal proteins were collected during, prior, or after the stimulation and immediately lysed in equal volume of lysis buffer (4\% (wt/v) SDS, $1 \mathrm{mM}$ EDTA, $20 \mathrm{mM}$ TCEP, $80 \mathrm{mM}$ CAA, 100 mM HEPES, in water, supplemented with $2 \times$ Halt protease and phosphatase inhibitor cocktail), pre-warmed at $95^{\circ} \mathrm{C}$. Four kinetic experiments were conducted and six samples were collected for each kinetic at the following time points: 1) $0 \mathrm{~s}$ (before stimulation), 2-4) $10 \mathrm{~s}, 30 \mathrm{~s}, 60 \mathrm{~s}$ after the stimulation started, 5) $\sim 5 \mathrm{~s}$ after the stimulation ended, 6) $60 \mathrm{~s}$ after the end of the stimulation. The samples were further processed as described in section 5.4.4 and section 5.4.5. Following the digestion and prior to enrichment of phosphorylated peptides, samples were labeled with TMT6 labeling reagents. After the labeling, materials of each two kinetics were combined, resulting in two TMT6-labeled samples. Subsequent sample preparation followed protocols in the sections 5.4 .5 and 5.4 .6

\subsection{LC-MS/MS}

The LC-MS/MS acquisition followed the LC set up and parameters described in section 5.4.7. BRP fractions of the enriched phosphorylated peptides and the whole proteome were acquired in duplicates or as single injections, respectively, using Fusion Tribrid mass spectrometer (Thermo Fisher Scientific, Waltham, USA). Phosphorylated peptides were eluted using a linear gradient consisting of the following steps: i) increase from $2 \%$ to $7 \%$ buffer B for $4 \mathrm{~min}$; ii) increase from $7 \%$ to $32 \%$ over $86 \mathrm{~min}$; iii) from $32 \%$ to $50 \%$ over $28 \mathrm{~min}$; iv) wash at $90 \%$ over $5 \mathrm{~min}$ and v) equilibration at $2 \%$ over $5 \mathrm{~min}$. The elution gradient for the whole proteome was as follows: i) 5-8\% over $4 \mathrm{~min}$; ii) $8-34 \%$ over $86 \mathrm{~min}$; iii) 34-50\% over $28 \mathrm{~min}$; iv, v) wash and equilibration as described above. MS was operated in positive data-dependent SPS-MS3 (301) mode. MS1 survey scans of $350-2000 \mathrm{~m} / \mathrm{z}$ range were acquired at the resolution of 120,000, automated gain control target (AGC) of $1 \mathrm{e} 6$ and the maximum injection time (MaxIT) of $50 \mathrm{~ms}$. Precursor ions of charge states 2-7 were selected for fragmentation using normalized collision energy (NCE) of $38 \%$. MS2 scans were acquired in Orbitrap at the resolution of 30,000 (phosphorylated peptides) or 15,000 (whole proteome), AGC target of $5 e 5$ and MaxIT of 200 ms (phosphorylated peptides) or $54 \mathrm{~ms}$ (whole proteome). 10 most intense fragment ions were subjected to HCD-fragmentation using NCE of $50 \%$. SPS-MS3 spectra were acquired the resolution of 50,000 , using AGC target of $2.5 \mathrm{e} 5$ and the MaxIT of $200 \mathrm{~ms}$ (phosphorylated peptides) or $86 \mathrm{~ms}$ (whole proteome).

\subsection{Data Analysis}

Data analysis was conducted as described in section 5.4 .8 with a few exceptions. In MaxQuant, the quantification was based on reporter ion intensities in SPS-MS3 scans. The reporter ion intensities were $\log _{2}$-transformed and normalized using median polishing, and 
the intensity of the first channel ( $0 \mathrm{~s}$ time point) was subtracted from the other channels. Phosphorylation sites showing a Pearson correlation of the two kinetic measurements $>0.6$ and a minimum absolute $\log _{2}$ intensity fold change at any time point of 0.585 were subjected to hierarchical clustering using hclust function and Euclidean distances in R.

\section{$4 \quad$ Preliminary Results}

As mentioned before, chemical agents such as $\mathrm{KCl}$ and 4-AP are commonly used to stimulate neurotransmitter release from synaptosomes. One of the drawbacks of this stimulation is that the stimulating agent cannot be easily removed from the system. This mode of stimulation is non-physiologically strong and results in a significant increase in the cytoplasmic $\mathrm{Ca}^{2+}$ concentration. The latter activates $\mathrm{Ca}^{2+}$-dependent kinases and phosphatases, however, it remains unclear, to which extent this activity is relevant for a normal SV cycling process. In order to study phosphorylation events that are closely related to SV cycling, the applicability of electrical stimulation to evoke neurotransmitter release was investigated.

According to early reports, field-stimulation of synaptosomes in suspension caused glutamate liberation monitored by the glutamate release assay. However, high frequency stimulation with short pulses similar to one used in earlier synaptosomal studies (0.4 ms pulses at $100 \mathrm{~Hz}(105))$ or the one commonly applied in neuronal cell cultures (1-2 ms pulses at $20 \mathrm{~Hz}(123))$ did not produce a measurable glutamate release using moderate currents $(<15 \mathrm{~mA} / \mathrm{mL})$. When applying stronger currents, glutamate release in $1.3 \mathrm{mM} \mathrm{Ca}^{2+-}$ containing incubation medium (in the following referred to as $\mathrm{Ca}$ ) almost did not differ from glutamate release in $\mathrm{Ca}^{2+}$-free incubation media containing $0.5 \mathrm{mM} \mathrm{EGTA} \mathrm{(in} \mathrm{the} \mathrm{following}$ referred to as EGTA). Reducing the stimulation frequency and prolonging the pulse duration allowed for a reproducible $\mathrm{Ca}^{2+-}$ dependent glutamate release (Figure 4.1A). The release was proportional to the pulse width and applied current: when using shorter pulses, a stronger current was required to evoke similar glutamate release (Figure 4.1B). Following stimulations were conducted using $15 \mathrm{~ms}$ pulses of alternating polarity delivered every $100 \mathrm{~ms}(10 \mathrm{~Hz})$. Glutamate release halted after the stimulation was aborted. Repeated stimulation of the same synaptosome suspension evoke a reproducible release (s. repeated stimulations in Figure 4.1D-F). These observations imply that the membrane remained intact after the electrical stimulation. Accordingly, an earlier study did not find any significant development of heat or reactive oxygen species at the electrodes that might cause synaptosome damage and thus explain the neurotransmitter release (109). However, a Fura-2 assay could not reveal any increase in the cytoplasmic $\mathrm{Ca}^{2+}$ concentration that is attributable to electrical stimulation (Figure 4.1C). More strikingly, treatment of 
synaptosomes with a combination of botulinum neurotoxins (BoNT) A and D did not hamper the glutamate release; though the toxin treatment caused a significant suppression of the release following $\mathrm{KCl}$-induced depolarization (Figure 4.1D). Application of $\mathrm{Ca}^{2+}$-channel blockers $\mathrm{Cd}^{2+}(20 \mu \mathrm{M})$ or $\omega$-conotoxin MVCII (1 or $\left.2 \mu \mathrm{M}\right)$ caused a decrease of $11 \%, 29 \%$, and $12 \%$, respectively (Figure 4.1E, F). Interestingly, no decline was observed after the application of $60 \mu \mathrm{M} \mathrm{CdCl}_{2}$ or $2 \mu \mathrm{M}$ of the $\omega$-conotoxin in the first stimulation replicate. When using acridine orange (AO) that is trapped in SVs and illuminate after the release, electrical stimulation did not prevent the decay of AO-fluorescence due to the gradual accumulation of the dye inside SVs. In contrast, $50 \mathrm{mM} \mathrm{KCl}$ caused a spike-like increase in the fluorescence (Figure 4.1G). Application of electrical stimulation together with $300 \mathrm{nM}$ bafilomycin $A_{1}$ that inhibits V-ATPase and thus prevents $A O$ trapping in SVs, showed the same rate of $A O$ leakage as the application of bafilomycin $A_{1}$ alone, meaning that electrical stimulation did not accelerate the liberation of $A O$ (Figure 4.1H). When using the glutamate release assay, pre-incubation of synaptosomes with $300 \mathrm{nM}$ bafilomycin $\mathrm{A}_{1}$ resulted in a $15-16 \%$ lower glutamate release, while it decreased the $\mathrm{KCl}$-evoked release by $54 \%$ (Figure 4.1I). Taken together, these observations do not allow to exclude that the glutamate release evoked by electrical stimulation may be generated by some non-exocytotic mechanisms. In contrast, the proportion of the glutamate released via exocytosis is probably below $30 \%$. The reversal of the glutamate transporter of the plasma membrane might be the non-exocytotic pathway that constitutes the observed glutamate release.

Phosphoproteomics analysis of electrically stimulated synaptosomes revealed that a few phosphorylation sites undergo up- (Figure 4.2A, B, cluster 1) or down-regulation (cluster 2) during the stimulation. Among affected proteins, there are proteins involved in the cytoskeleton regulation (Figure 4.2C), e.g., microtubule-associated protein 1a (Map1a), and tau (Mapt), tau tubulin kinase (Ttbk1), Protein kinase $C$ and casein kinase substrate in neurons protein 1 (Pacsin1), and Erythrocyte membrane protein band 4.1-like 3 (Epb41/3). Another prominent group contain proteins involved into signaling pathways, kinases, ubiquitin-ligases and their modifiers: Adenomatous polyposis coli protein (Apc); Serine/threonine-protein kinase BRSK2 (Brsk2); CaMKII (Camk2a, b, g); Serine/threonineprotein kinase DCLK2 (Dclk2); A-kinase anchor protein 12 (Akap12); MAP kinase-activating death domain protein (Madd); PKA (Prkaa1); Protein phosphatase 1 regulatory subunit 7 (Ppp1r7); Neural precursor cell-expressed, developmentally down-regulated 4-like, E3 ubiquitin protein ligase (Nedd4I); etc. The latter group implies regulation through signaling pathways operating via CaMKII, MAPK, PKA, Wnt, PP1, and ubiquitination. Next, phosphorylated sites on $\mathrm{Ca}^{2+}, \mathrm{K}^{+}$, and $\mathrm{Na}^{+}$-channel proteins are affected (Cacnb4, Kcna2, and Scn1a, respectively). Last but not least, several proteins involved in SV cycling are 
affected, including, protein bassoon (Bsn), piccolo (Pclo), caskin-1 (Caskin1), DnaJ homolog subfamily C member 5 (Dnja5), DnaJ heat shock protein family (Hsp40) member C6 (Dnaj6), clathrin light chain A (Clta), AP2 complex subunit alpha (Ap2a1), amphiphysin (Amph), etc. Interestingly, S449/S551 sites on synapsin-1 follow the down-regulation pattern (Figure 4.2D). These sites on synpasin-1 were previously shown to be a target of MAPK and their dephosphorylation increased a pool of available SVs (171). These observations imply that electrical stimulation might induce adaptation processes in synaptosomes. However, it remains to be elucidated, to which degree this effect is achieved due the stimulation of SV cycling. In the following chapter 5, I introduce another approach to study SV cycling dependent phosphorylation events, which is based on KCl-stimulation and BoNT-treatment. The results of this approach are presented in the following manuscript (326), which constitutes a significant part of this thesis, and my own contribution is stated in detail in Author's contribution statement. 
A
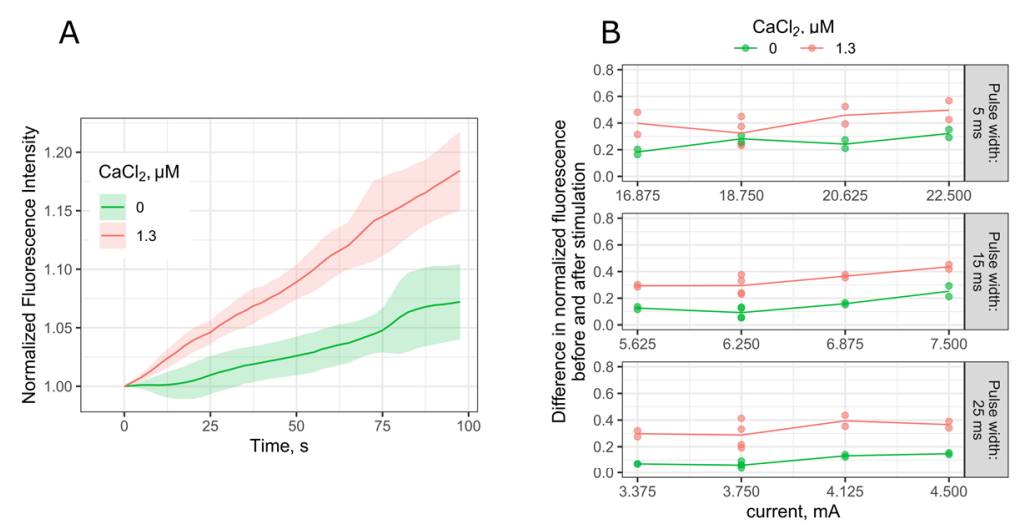

$\mathrm{D}$

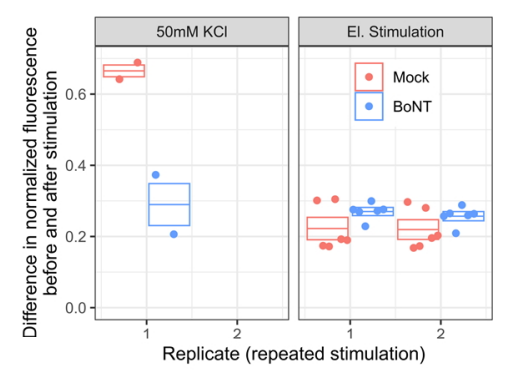

G

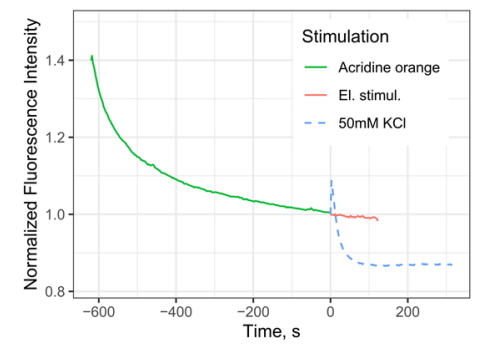

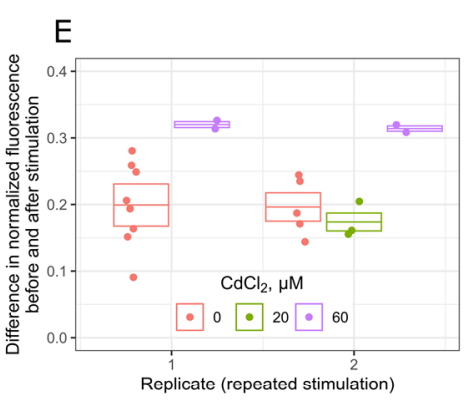

$\mathrm{H}$

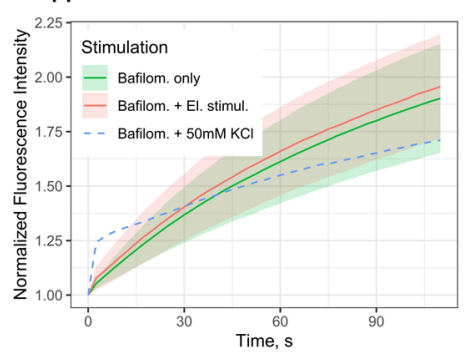

$\mathrm{C}$
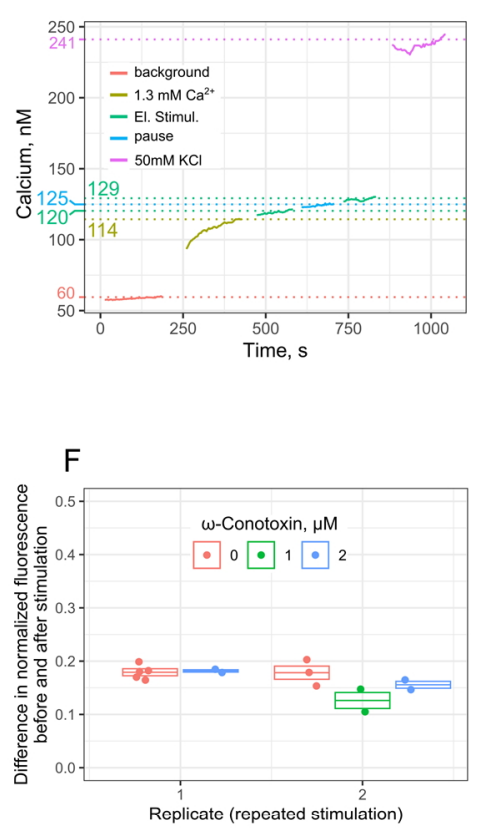

I

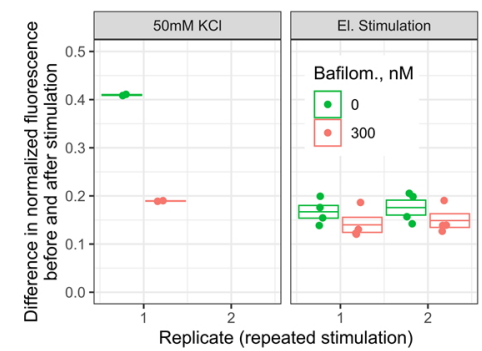

Figure 4.1: Electrical stimulation of synaptosomes. Synaptosomes in suspension (1.5 mg synaptosomal proteins, at a concentration of $1 \mathrm{mg} / \mathrm{mL}$ ) were electrically stimulated for 2 min using $15 \mathrm{~ms}$ square pulses of alternating polarity at a frequency of $10 \mathrm{~Hz}$ and $15 \mathrm{~mA}$ current if not stated otherwise. (A) Glutamate release during the stimulation in the presence of $0(0.5 \mathrm{mM} E G T A)$ or $1.3 \mathrm{mM} \mathrm{Ca}^{2+}$. Lines represent means and areas represent standard deviations $(N=4)$. (B) Testing stimulation parameters. Conditions same as in (A). (C) Fura2 assay, a representative replicate. "Background" corresponds to $0 \mathrm{mM} \mathrm{Ca}^{2+}$ (no $\mathrm{CaCl}_{2}$ added). (D) Glutamate release following treatment with heat-inactivated (Mock) or active BoNT A and D. Stimulation using $50 \mathrm{mM} \mathrm{KCl}$ or electrical stimulation. (E) Glutamate release following treatment with 0,20 , or $60 \mu \mathrm{M} \mathrm{CdCl}$ and electrical stimulation. (F) Glutamate release following treatment with 0 , 1, or $2 \mu \mathrm{M} \omega$-conotoxin MVIIC and electrical stimulation. (G) Acridine orange (AO) fluorescence, a representative replicate. Synaptosomes were preincubated with $A O$ (green solid line) and then stimulated with $50 \mathrm{mM} \mathrm{KCl}$ (blue dashed line) or electrically (red solid line). (H) AO fluorescence following addition of $300 \mathrm{nM}$ bafilomycin $\mathrm{A}_{1}$ and $50 \mathrm{mM} \mathrm{KCl}$ (blue dashed line), electrical stimulation (red solid line) or no stimulation (green solid line). Synaptosomes were pre-loaded with $A O$ as in (G). Lines represent means and areas represent standard deviations ( $N=7$ (Bafilom. only), 6 (El. Stimul.),

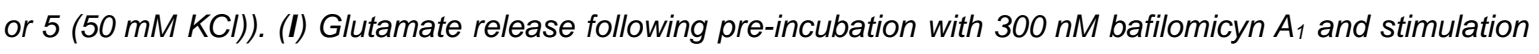
using $50 \mathrm{mM} \mathrm{KCl}$ or the electrical stimulation. 
A
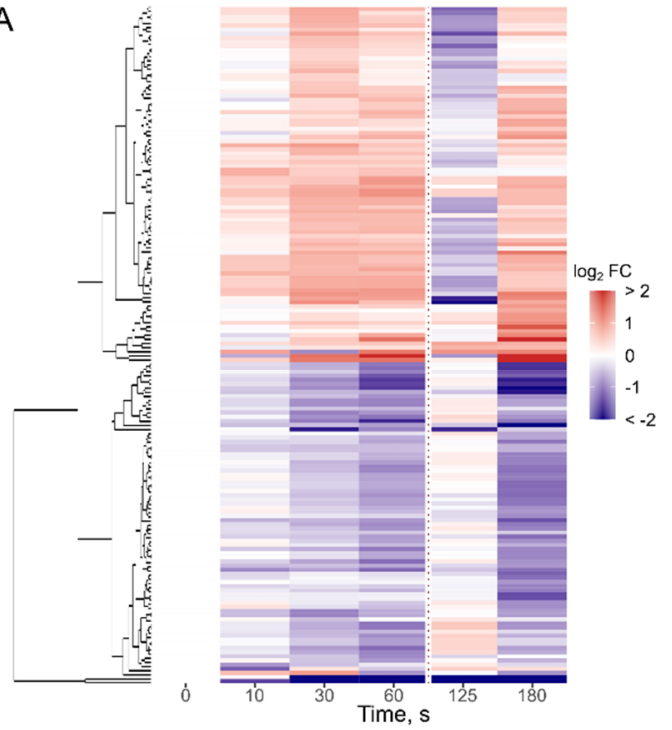

C

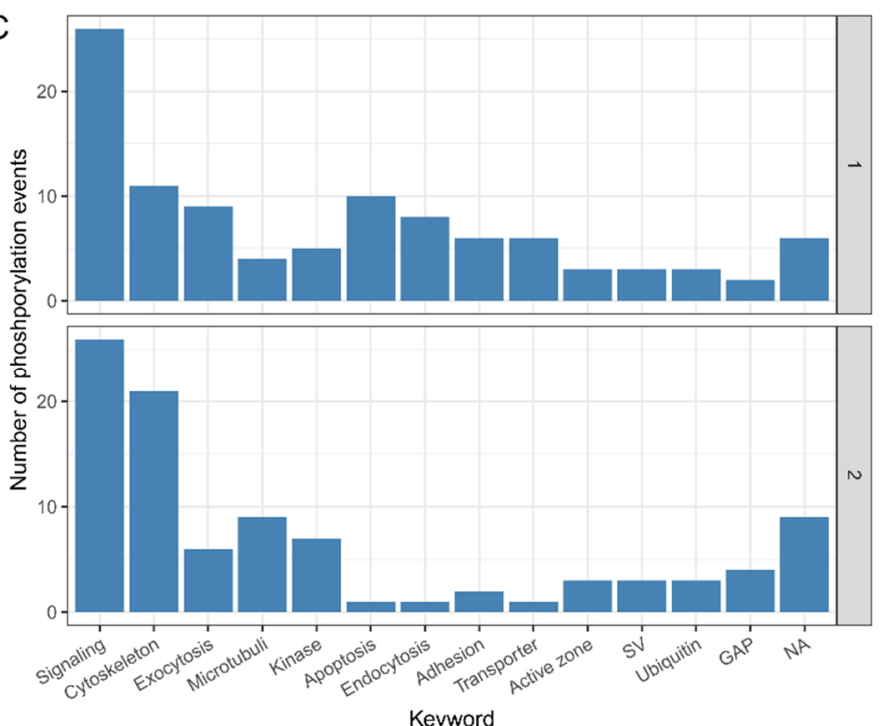

B
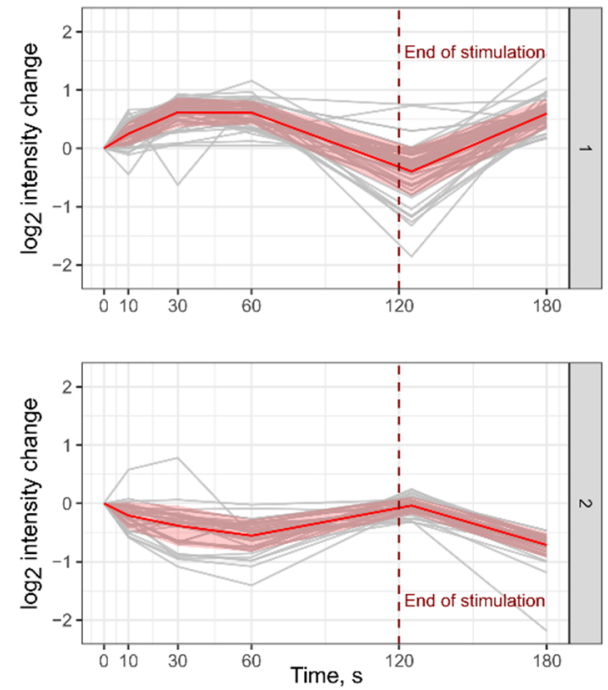

$\mathrm{D}$

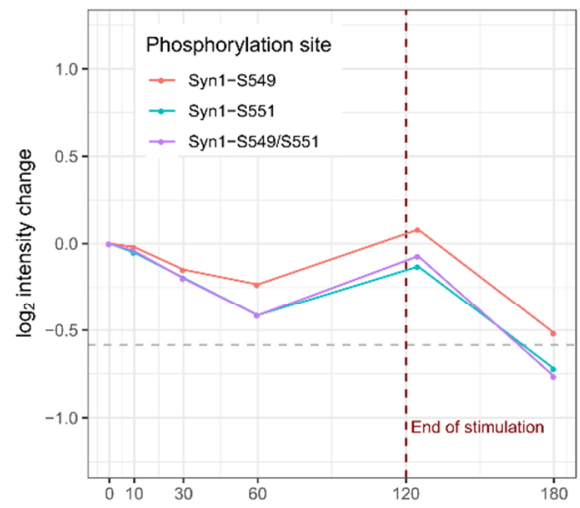

Figure 4.2: Phosphoproteomics analysis of electrically stimulated synaptosomes. Synaptosomes in suspension (1.5 mg synaptosomal proteins, at a concentration of $1 \mathrm{mg} / \mathrm{mL}$ ) were electrically stimulated for $2 \mathrm{~min}$ using $15 \mathrm{~ms}$ square pulses of alternating polarity at a frequency of $10 \mathrm{~Hz}$ and $15 \mathrm{~mA}$. Six time points were collected: right before stimulation ( $0 \mathrm{~s}$ ), during the stimulation ( $10 \mathrm{~s}, 30 \mathrm{~s}$, and $60 \mathrm{~s}$ ), right after the stimulation ( 125 s), and one minute after the stimulation (180 s). The data are based on two kinetic measurements. Each kinetic is an average of two independent stimulation experiments. (A) Heat map of phosphorylation events showing Pearson correlation of $>0.6$ and a minimum absolute $\log _{2} F C$ of 0.585 at any time point as compared to the 0 s time point. A dotted line represents an end of the electrical stimulation. Color code represents $\log _{2} F C$ as compared ot the $0 \mathrm{~s}$ time point. The events are hierarchically clustered (s. dendrogram) based on the Euclidian distance. (B) Representation of the two main clusters from (A). Grey lines depict single phosphorylation events $\left(\log _{2} F C\right)$. Red line represents an average of all events in the cluster. Red area is a mean $\pm S D$. (C) Keywords describing protein function/localization were assigned to proteins containing phosphorylation sites in the clusters 1 and 2. The keyword assignments were performed manually based on the information from available literature. 1-4 keywords were assigned per protein. Bars display a number of phosphorylation events per keyword per cluster, NA not annotated. (D) Kinetics of selected singly (S449 and S551) and doubly (S449/S551) phosphorylated sites on synapsin-1. A grey dashed line represents a minimum $\log _{2} F C$ threshold of 0.585 . 


\section{Protein phosphorylation in depolarized synaptosomes: Dissecting primary effects of calcium from synaptic vesicle cycling ${ }^{1}$}

Ivan Silbern ${ }^{1,2}$, Kuan-Ting Pan", \#, Maksims Fiosins ${ }^{3,4}$, Stefan Bonn ${ }^{3,4}$, Silvio O. Rizzoli5, 6 , Eugenio F. Fornasiero $5, \S$, Henning Urlaub ${ }^{1,2}$, Reinhard Jahn ${ }^{7,8, \S}$

${ }^{1}$ Institute of Clinical Chemistry, University Medical Center Göttingen, D-37075 Göttingen, Germany;

2Bioanalytical Mass Spectrometry Group, Max Planck Institute for Biophysical Chemistry, D-37077 Göttingen, Germany;

${ }^{3}$ German Center for Neurodegenerative Diseases, D-72076 Tübingen, Germany;

${ }^{4}$ Institute for Medical Systems Biology, University Medical Center Hamburg-Eppendorf, D-20246 Hamburg, Germany;

${ }^{5}$ Department of Neuro- and Sensory Physiology, University Medical Center Göttingen, D-37073, Göttingen, Germany;

${ }^{6}$ Cluster of Excellence "Multiscale Bioimaging: from Molecular Machines to Networks of Excitable Cells" (MBExC), University of Göttingen, D-37075 Göttingen, Germany;

${ }^{7}$ Laboratory of Neurobiology, Max Planck Institute for Biophysical Chemistry, D-37077 Göttingen, Germany;

8University of Göttingen, D-37073 Göttingen, Germany;

\#Current affiliation: Frankfurt Cancer Institute, Goethe University, D-60596, Frankfurt am Main, Germany; Hematology/Oncology, Department of Medicine II, Johann Wolfgang Goethe University, D-60590 Frankfurt am Main, Germany.

§Corresponding Authors: RJ (rjahn@gwdg.de), HU (henning.urlaub@mpibpc.mpg.de), EF (efornas@gwdg.de)

\footnotetext{
${ }^{1}$ The manuscript is represented as published in the "Molecular \& Cellular Proteomics" with the exception of the reference list, which is merged with the reference list of this thesis. The joint list of references is presented at the end of this work. The references number in the manuscript are updated to match the merged list of references. Similarly, numbers of figures and tables are adjusted to align the figure and table numbering in the thesis.
} 


\subsection{Author's contribution}

\begin{tabular}{|c|c|}
\hline Figure 5.1 & $\begin{array}{l}\text { I.S., K.T.P. H.U and R.J. conceptualized the experiments using } \\
\text { synaptosomes. I.S. performed experiments and data analysis. }\end{array}$ \\
\hline Figure 5.2 & $\begin{array}{l}\text { I.S. performed synaptosome preparation, stimulation experiments, } \\
\text { BoNT-treatment, phosphopepeptide enrichment, and MS } \\
\text { acquisition. I.S. performed differential expression analysis (B, C), } \\
\text { kinase-substrate analysis (E, F) and comparison to previous studies } \\
\text { (A, D) under supervision of K.T.P and H.U. }\end{array}$ \\
\hline Figure 5.3 & $\begin{array}{l}\text { I.S. performed GO term enrichment analysis under the supervision } \\
\text { of H.U and R.J. }\end{array}$ \\
\hline Figure 5.4 & $\begin{array}{l}\text { I.S. categorized data in }(A, B) \text { and performed the enrichment } \\
\text { analysis in }(C) \text { under the supervision of H.U, R.J, and S.O.R. I.S. } \\
\text { performed the enrichment analysis in (D) following pilot analysis by } \\
\text { M.F and S.B. }\end{array}$ \\
\hline Figure 5.5 & I.S. conducted protein annotations under the supervision of R.J. \\
\hline Figure 5.6 & $\begin{array}{l}\text { E.F. and S.O.R. conceptualized experiments based on the } \\
\text { phosphoproteome data. E.F. performed all experiments and } \\
\text { analyzed data. }\end{array}$ \\
\hline Figure 5.S1 & $\begin{array}{l}\text { I.S. isolated synaptosomes and conducted fluorimetric } \\
\text { measurements }\end{array}$ \\
\hline Figure 5.S2-S18 & $\begin{array}{l}\text { I.S. analyzed and categorized data under the supervision of K.T.P } \\
\text { and H.U. }\end{array}$ \\
\hline Figure 5.S19 & $\begin{array}{l}\text { I.S. performed protein extraction, and MS analysis. I.S. analyzed } \\
\text { data under the supervision of K.T.P and H.U. }\end{array}$ \\
\hline Figure 5.S20 & $\begin{array}{l}\text { I.S. performed BoNT treatment, phosphopeptide enrichment, MS } \\
\text { sample preparation and MS analysis. I.S. analyzed data under the } \\
\text { supervision of H.U. }\end{array}$ \\
\hline Figure 5.S21 & $\begin{array}{l}\text { I.S. performed synaptosome preparation, stimulation experiments, } \\
\text { BoNT treatment, MS sample preparation and MS analysis. I.S. } \\
\text { analyzed data under the supervision of H.U. }\end{array}$ \\
\hline
\end{tabular}




\subsection{Abbreviations}
AAV
adeno-associated virus
ACN
acetonitrile
AGC
protein kinase $A, G, C$ kinase group
$A G C$ automatic gain control
AMPAR a-amino-3-hydroxy-5-methyl-4-isoxazolepropionic acid receptor
AP action potential
BoNT botulinum neurotoxin
bRP basic reversed-phase chromatography
CAA
chloroacetamide
CaMKII calcium-calmodulin kinase 2
CDK cyclin-dependent kinase
CK1 casein kinase 1
CK2 casein kinase 2
CLK SRPK1 and Clk/Sty protein kinase
CMGC CDK, MAP, GSK, CDKL kinase group
CREB1 CAMP responsive element binding protein 1
DAPK death associated protein kinase
FA formic acid
GABA $\quad \mathrm{V}$-aminobutyric acid
GluDH glutamate dehydrogenase
GO gene ontology
GRK G protein coupled receptor kinase
GSK3 glycogen synthase kinase 3
ITR inverted terminal repeat (sequence)
MAPK mitogen activated protein kinase
NMDAR N-methyl-d-aspartate receptor
PAK p21 activated kinase
PKC protein kinase $\mathrm{C}$
PNGase $\mathrm{F}$ peptide-N(4)-( $\mathrm{N}$-acetyl-beta-glucosaminyl)asparagine amidase
POI protein of interest
PP1 protein phosphatase 1
RAS rat sarcoma gene
RT room temperature
SNARE N-ethylmaleimide-sensitive factor-attachment protein receptors
STE "sterile" serine/threonine protein kinases 
SV

synaptic vesicle

TCEP tris(2 carboxyethyl)phosphine

TEAB triethylammonium bicarbonate buffer

TFE trifluoroethanol 


\subsection{Abstract}

Synaptic transmission is mediated by the regulated exocytosis of synaptic vesicles. When the presynaptic membrane is depolarized by an incoming action potential, voltage-gated calcium channels open, resulting in the influx of calcium ions that triggers the fusion of synaptic vesicles (SVs) with the plasma membrane. SVs are recycled by endocytosis. Phosphorylation of synaptic proteins plays a major role in these processes, and several studies have shown that the synaptic phosphoproteome changes rapidly in response to depolarization. However, it is unclear which of these changes are directly linked to SV cycling and which might regulate other presynaptic functions that are also controlled by calcium-dependent kinases and phosphatases. To address this question, we analyzed changes in the phosphoproteome using rat synaptosomes in which exocytosis was blocked with botulinum neurotoxins (BoNTs) while depolarization induced calcium influx remained unchanged. BoNT-treatment significantly alters the response of the synaptic phoshoproteome to depolarization and results in reduced phosphorylation levels when compared with stimulation of synaptosomes by depolarization with $\mathrm{KCl}$ alone. We dissect the primary $\mathrm{Ca}^{2+}$-dependent phosphorylation from SV cycling dependent phosphorylation and confirm an effect of such SV-cycling-dependent phosphorylation events on syntaxin-1a-T21/T23, Vamp2-S75, and cannabinoid receptor-1-S314/T322 on exo- and endocytosis in cultured hippocampal neurons. 


\subsection{Introduction}

In the nervous system, synapses represent specialized cellular junctions that are able to transmit signals from the pre- to the postsynaptic neuron. Signaling is mediated by the release of neurotransmitter molecules that are stored in the presynaptic compartment (327). When an action potential arrives in the nerve terminal, the presynaptic membrane depolarizes, which elicits a rapid influx of calcium ions through voltage-dependent calcium channels clustered around the active zone, an electron-dense protein complex connecting the presynaptic release site with the neurotransmitter receptors in the postsynaptic membrane (35). Major protein constituents of the active zone include RIM proteins, RIMbinding protein and Munc13, as well as bassoon, piccolo, and liprins. Among other functions, these proteins are required for the clustering of calcium-channels and govern the priming and docking of SVs. The calcium ions entering the presynapse are recognized by the calcium-sensor synaptotagmin 1 that triggers exocytosis by lowering the energy barrier for fusion. Exocytotic fusion itself is accomplished by soluble $\mathrm{N}$-ethylmaleimide-sensitive factor-attachment protein receptors (SNARE) $(36,38,328)$. This process requires additional cytoplasmic proteins, such as Munc18 and complexins, which modulate the efficiency of SNARE zippering $(39,40)$.

In order to sustain continuous neurotransmitter release and to maintain the structure and composition of the plasma membrane, fused SVs are retrieved by endocytosis, followed by rapid regeneration of $S V$ precursors that are filled with neurotransmitters and prepared for the next round of neurotransmitter release. It is still debated which mechanisms are primarily responsible for endocytosis and recycling, with several occurring in parallel (49, $69,72,75,329-332)$. The classical pathway of clathrin-mediated endocytosis requires coordinated actions of a protein machinery responsible for coat formation, invagination, and formation of a coated pit, followed by scission, removal of the vesicle from the plasma membrane, and disassembly of the clathrin coat. Key proteins involved in these steps include, among others, clathrin adaptor proteins (AP2, AP180), clathrin itself, the GTPase dynamin-1, the phosphatase synaptojanin-1, endophilins, and related proteins, as well as the clathrin uncoating ATPase Hsc70 and its cofactor auxilin (48). Finally, re-endocytosed SVs are filled with the respective neurotransmitter by specific vesicular neurotransmitter transporters that are fueled by the activity of a vacuolar proton pump (333). In this article, we refer to SV cycling as a multistep process, which includes SV fusion with the plasma membrane mediated by the exocytotic machinery (exocytosis) and SV recycling through available endocytotic mechanisms (endocytosis), followed by the steps in which SVs become ready for the next round of SV cycle (i.e., refilling of the neurotransmitter, SV priming and docking). 
An increasing body of data suggests that the basic exo-endocytotic machinery is fine-tuned by regulatory control mechanisms that mainly operate by phosphorylations and dephosphorylations of proteins involved in the SV cycle. In particular, calcium plays an important role not only as a trigger of exocytosis, but also as a modulator of kinase and phosphatase activity in the presynapse (334). Calcium/calmodulin-dependent kinase 2 (CaMKII) residing in synapses is one of the major targets activated by calcium influx (156, 335-337). Its first known substrate was the SV resident protein synapsin-1, and it is now established that its phosphorylation influences the available pool of SV for exocytosis (154, 155, 338). Other kinases such as protein kinase $C$ (PKC) and cyclin-dependent kinase 5 (CDK5) have been shown to be important for SV endocytosis and control, together with the calcium-dependent protein phosphatase calcineurin, the phosphorylation status of many endocytosis-associated proteins such as dynamin-1, amphiphysin, and AP180 (201, 206, 339-346). Mitogen-activated protein kinases (MAPK) appear to operate on parallel pathways as these can regulate synaptic plasticity either by directly modifying synaptic proteins $(347,348)$ or by affecting gene expression $(349,350)$, thereby contributing to both rapid and long-lasting modulatory effects.

Earlier phosphoproteomics studies have shown systematic changes in phosphorylation of synaptic proteins caused by depolarization-evoked calcium influx, and they have pointed to involvement of CaMKII and MAP kinases in this process $(351,352)$. However, it remains elusive, which of the observed phosphorylation changes caused by calcium influx have a direct functional impact on SV cycling. Indeed, following the initial discovery of synapsin-1 phosphorylation, many studies have demonstrated that phosphorylation of specific synaptic proteins at specific sites does have an impact on exo- or endocytosis. For instance, phosphorylation of SNARE proteins (Snap25-S187, Stx1a-S14, S188, Vamp2-T35, S75), as well as of the SNARE-interacting protein Munc18 (Stxbp1-S313), can indeed interfere with the formation of the SNARE complex, which results in altered exocytosis (353-358). Munc18 phosphorylation can further be a result of the MAP kinase activation due to cannabinoid receptor-1 signaling (347); the latter was also shown to bear a phosphorylation site (Cnr1-S317) that is controlled by PKC (359).

However, it remains to be established which of the vast amount of phosphosite changes observed in recent phosphoproteomic studies are dependent on the membrane trafficking steps of the SV cycle and which are, while being targeted by the activated network of kinases and phosphatases, not directly related to exo-endocytotic cycling. Changes in the protein conformation that accompany assembly and disassembly of multimolecular protein complexes during SV cycling may alter the accessibility of phosphorylation sites as well as the availability of docking sites for kinases and phosphatases. To address this issue, we 
conducted a quantitative phosphoproteome analysis using synaptosomes as a functional model of the synapse. Synaptosomes contain the complete SV cycling machinery, can maintain the membrane potential and ATP at physiological levels, and respond to depolarization by $\mathrm{Ca}^{2+}$ dependent neurotransmitter release $(98,99,115,360)$. To differentiate between calcium-induced changes and phosphorylation events that are directly connected to SV cycling, we utilized botulinum neurotoxins. These bacterial protein toxins represent a group of endoproteinases that selectively cleave individual SNARE proteins and thus block exocytosis and, consequently, SV recycling without compromising $\mathrm{Ca}^{2+}$ influx or synaptosomal ATP-levels $(121,122)$. We stimulated mock- and BoNT-treated synaptosomes with potassium chloride, which is known to elicit dose-dependent $\mathrm{Ca}^{2+-i n f l u x}$ and neurotransmitter release $(100,361)$. By quantitatively monitoring changes of phosphorylation sites of synaptosomal proteins, we found that BoNT-treatment affects almost 1500 phosphorylation sites implying that they are directly dependent on SV cycling. Strikingly, most of the sites show reduced phosphorylation intensity in depolarized synaptosomes following BoNT treatment. We further identified SV-cycling-dependent phosphorylation sites on syntaxin-1a (Stx1a-T21, T23), synaptobrevin (Vamp2-S75), and cannabinoid receptor-1 (Cnr1-T314, T322). Finally, we demonstrated that phosphorylation of these sites elicits a pronounced effect on exo- and endocytosis in cultured hippocampal neurons by expressing phosphomimetic and non-phosphorylatable variants of these proteins.

\subsection{Experimental Procedures}

\subsubsection{Ethical statement}

All experiments involving animals complied with the regulations as designated in the section 4 of the Animal Welfare Law of the Federal Republic of Germany (section 4 Tierschutzgesetz der Bundesrepublik Deutschland, TierSchG). All experiments were conducted in the animal facility at the Max-Planck-Institute for Biophysical Chemistry, Göttingen, Germany and registered accordingly to the section 11 Abs. 1 TierSchG as documented by 3920 00_2a Si/rö, dated 11th Dec 2013 ("Erlaubnis, Wirbeltiere zu Versuchszwecken zu züchten und zu halten") by the Veterinär- und Verbraucherschutzamt für den Landkreis und die Stadt Göttingen and examined regularly by the supervisory veterinary authority of the Landkreis Göttingen. All procedures were supervised by the animal welfare officer and the animal welfare committee of the Max-Planck-Institute for Biophysical Chemistry, Göttingen, Germany established in accordance with the TierSchG and the regulation of animal experiments, dated on 31st Aug 2015 (Tierschutz-VersuchstierVerordung, TierSchVersV). 


\subsubsection{Chemicals}

LC/MS-grade water, methanol, and acetonitrile (ACN) were used in this study if not otherwise stated and were purchased together with chloroform, $25 \%$ (v/v) $\mathrm{NH}_{4} \mathrm{OH}, \mathrm{CaCl}_{2}$, $\mathrm{KCl}, \mathrm{KH}_{2} \mathrm{PO}_{4}, \mathrm{MgCl}_{2}, \mathrm{NaCl}, \mathrm{NaHCO}_{3}, \mathrm{NaHPO}_{4}$, sucrose, and glucose from Merck, Darmstadt, Germany. Trifluoroethanol (TFE), triethylammonium bicarbonate buffer (TEAB), formic acid (FA), EGTA, PM400-Ficoll (Ficoll), NADP, L-Glutamic dehydrogenase from bovine liver (GluDH), guanidine hydrochloride, tris(2-carboxyethyl)phosphine (TCEP), chloroacetamide (CAA), Triton-X100 and MS-grade trypsin were obtained from SigmaAldrich, Taufkirchen, Germany. HEPES was purchased from VWR Chemicals, Darmstadt, Germany. SDS was purchased from Serva Electrophoresis $\mathrm{GmbH}$, Heidelberg, Germany. TFA was obtained from Roth, Karlsruhe, Germany. Peptide-N(4)-(N-acetyl-betaglucosaminyl)asparagine amidase (PNGase F) was obtained from Roche, Mannheim, Germany. Rapigest was obtained from Waters, Milford, USA. Pierce $660 \mathrm{~nm}$ protein assay, Halt Protease and phosphatase inhibitor cocktail, and TMTsixplex isobaric labeling reagents (TMT6) were purchased from Thermo Fisher Scientific, Bleiswijk, Netherlands. C. botulinum strain $A-D$ cell culture supernatants (BoNT A, B, C1, D) were obtained from Miprolab, Göttingen, Germany.

\subsubsection{Isolated nerve terminals}

Isolated nerve terminals were prepared from brains of 5-6 weeks old Wistar rats as described previously (362). Briefly, brains were quickly homogenized in ice-cold homogenization buffer ( $320 \mathrm{mM}$ sucrose, $5 \mathrm{mM}$ HEPES is water) using a Teflon/glass homogenizer. The homogenate was cleared by centrifugation for $2 \min$ at $2988 \times g$ in an SS-34 fixed angle rotor (Thermo Fisher Scientific, Waltham, USA). The supernatant was collected and re-centrifuged for $12 \mathrm{~min}$ at $14462 \times \mathrm{g}$. The synaptosomal pellet was resuspended in the homogenization buffer and loaded onto discontinuous Ficoll gradient $(6 \% / 9 \% / 13 \%(w t / v)$ Ficoll in the homogenization buffer) and centrifuged for $35 \mathrm{~min}$ at $86575 \times g$ in an SW-41 swinging bucket rotor (Beckman Coulter, Krefeld, Germany). The synaptosomal band at the interface between $9 \%$ and $13 \%$ Ficoll was collected and washed with the homogenization buffer. Synaptosomal protein concentration was estimated using Pierce $660 \mathrm{~nm}$ protein assay according to manufacturer's instructions. The viability of synaptosomes was confirmed by glutamate release assay (118).

\subsubsection{BoNT-treatment and glutamate release assay}

1-1.5 $\mathrm{mL}$ of synaptosomal suspension was centrifuged at $6900 \times g$ for $3 \mathrm{~min}$ in a table-top centrifuge, and synaptosomal pellet was resuspended in the sodium-containing buffer (10 mM glucose, $5 \mathrm{mM} \mathrm{KCl}, 140 \mathrm{mM} \mathrm{NaCl}, 5 \mathrm{mM} \mathrm{NaHCO}_{3}, 1 \mathrm{mM} \mathrm{MgCl}_{2}, 1.2 \mathrm{mM} \mathrm{NaHPO}_{4}$, 
$20 \mathrm{mM}$ HEPES in water) to achieve a final concentration of $1 \mathrm{mg} / \mathrm{mL}$ of synaptosomal proteins. $\mathrm{CaCl}_{2}$ was added to a final concentration of $1.3 \mathrm{mM}$ and synaptosomes were incubated for $5 \mathrm{~min}$ at $37^{\circ} \mathrm{C}$ before adding BoNT. For Ca vs. EGTA experiments, synaptosomes were pre-incubated with $1.3 \mathrm{mM} \mathrm{CaCl}_{2}$ or $0.5 \mathrm{mM}$ EGTA for $15 \mathrm{~min}$ at $37 \mathrm{C}$ before stimulation with $\mathrm{KCl}(50 \mathrm{mM})$. To block SNARE-assisted neurotransmitter release, $20 \mu \mathrm{L}$ of each BoNT were added per $1 \mathrm{mg}$ of estimated synaptosomal protein (the final BoNT concentration of 100-200 nM was estimated on the basis of the total protein content of the semi-purified BoNT, which amounted to approximately $10 \mu \mathrm{g} / \mu \mathrm{L}$ ). The following combinations of $C$. botulinum cell culture supernatants were used: i) $C$. botulinum $A$ and $D$ or ii) C. botulinum $\mathrm{C} 1$ and B. Alternatively, synaptosomes were treated with the same amount of respective cell culture supernatants inactivated by heating at $95^{\circ} \mathrm{C}$ for $1 \mathrm{~h}$ (mockcontrol). BoNT- and mock-treated synaptosomes were incubated at $37^{\circ} \mathrm{C}$ for 90 min and then directly subjected to the glutamate release assay. In brief, synaptosomal suspension was transferred into a quartz glass cuvette (Hellma, Müllheim, Germany) and kept stirred at $37^{\circ} \mathrm{C}$ during the measurements. $1 \mathrm{mMNADP}$, and $50 \mathrm{U}$ per $1 \mathrm{mg}$ of synaptosomal protein of GluDH were stepwise added to the suspension. Synaptosomes were depolarized by adding $\mathrm{KCl}$ to a final concentration of $50 \mathrm{mM}$. NADPH-fluorescence at $440 \mathrm{~nm}$ was monitored at each step using Fluorolog-3 fluorimeter (Horiba Jobin Yvon, Bensheim, Germany). After 2 min of stimulation, the reaction was quenched with an equal volume of lysis buffer (6 M Guanidine hydrochloride, 200 mM HEPES, 20 mM TCEP, 80 mM CAA, $1 \times$ Halt Protease and phosphatase inhibitor cocktail). The synaptosomal sample was incubated for $5 \mathrm{~min}$ at $95^{\circ} \mathrm{C}$, chilled on ice and then sonicated for $10 \mathrm{~min}$ using $30 \mathrm{~s}$ on/30 s off - cycles at the maximum output of Bioruptor ultrasonication device (Diagenode, Seraing, Belgium). The sample was repeatedly incubated for $5 \mathrm{~min}$ at $95^{\circ} \mathrm{C}$, and proteins were precipitated following methanol/chloroform protein precipitation method (363).

\subsubsection{Phosphopeptide enrichment and TMT-Labeling}

Precipitated synaptosomal proteins (1-2 mg per condition) were re-dissolved in a digestion buffer (100 mM TEAB, 10\% TFE, 0.1\% Rapigest) and sonicated in the Bioruptor for $10 \mathrm{~min}$ at maximum intensity using $30 \mathrm{~s}$ on/30 s off cycles. Trypsin was added at 1:40 trypsin-toprotein ratio (wt/wt) and proteins were digested overnight at $37^{\circ} \mathrm{C}$. Next day, the sample was treated with $2.5 \mathrm{U}$ of PNGase F per $1 \mathrm{mg}$ of the initial protein amount for $1 \mathrm{~h}$. Afterwards, ACN was added to a final concentration of $45 \%(\mathrm{v} / \mathrm{v})$ and the sample was incubated with a second portion of trypsin (1:100 trypsin-to-protein ratio, wt/wt) for $1 \mathrm{~h}$. The sample was centrifuged for $15 \mathrm{~min}$ at $17,000 \times g$ in a table-top centrifuge, and the cleared supernatant was subjected to phosphopeptide enrichment as previously described with modifications (364). Briefly, equal input peptide amounts were assured by measuring 
peptide concentration in Nanodrop-1000 (Thermo Fisher Scientific, Waltham, USA) using an A280 method. $\mathrm{KCl}, \mathrm{KH}_{2} \mathrm{PO}_{4}$, and TFA were added to the peptide sample to reach final concentrations of $228 \mathrm{mM}, 3.9 \mathrm{mM}$, and $38 \%(\mathrm{v} / \mathrm{v})$, respectively. Peptides were incubated with $\mathrm{TiO}_{2}$-beads (GL Sciences, Tokyo, Japan) at 1:10 protein-to-beads ratio for 20 min at $40^{\circ} \mathrm{C}$. Unbound peptide fraction (not-phosphorylated peptides) was collected, and $\mathrm{TiO}_{2}-$ beads were subjected to four washing steps using washing buffer $(60 \%(\mathrm{v} / \mathrm{v}) \mathrm{ACN}, 1 \%(\mathrm{v} / \mathrm{v})$ TFA in water). Bound phosphopeptides were eluted using $3.75 \%(\mathrm{v} / \mathrm{v}) \mathrm{NH}_{4} \mathrm{OH} 40 \%(\mathrm{v} / \mathrm{v})$ $A C N$ in water, snap-frozen in liquid nitrogen and dried in a centrifugal Savant SpeedVac vacuum concentrator (Thermo Fisher Scientific, Waltham, USA). Dried phosphopeptides and peptides in the unbound fraction were subjected to desalting using pre-packed $\mathrm{C} 18$ spin-columns (Harvard Apparatus, Holliston, USA). Desalted and dried peptides were redissolved in $50 \mathrm{mM}$ TEAB $50 \%(\mathrm{v} / \mathrm{v}) \mathrm{ACN}$ in water, and TMT6-labeling reaction was conducted according to manufacturer's instructions. After the labelling, peptide samples were accordingly combined, cleaned using the pre-packed C18 spin columns, and concentrated in a SpeedVac.

\subsubsection{Basic reversed-phase (bRP) chromatography}

TMT6-labeled peptides were fractionated using Agilent 1100 series HPLC system (Agilent, Santa Clara, USA) equipped with a C18-X-Bridge column (3.5 $\mu \mathrm{m}$ particles, $1.0 \mathrm{~mm}$ inner diameter, $150 \mathrm{~mm}$ length; Waters, Milford, USA). The HPLC was operated at the flow rate of $60 \mu \mathrm{L} / \mathrm{min}$ under basic $\mathrm{pH}$ (buffer $\mathrm{A}: 10 \mathrm{mM} \mathrm{NH}_{4} \mathrm{OH}$ in water, $\mathrm{pH} \sim 10$; buffer $\mathrm{B}: 10 \mathrm{mM}$ $\mathrm{NH}_{4} \mathrm{OH}$ and $80 \%$ (v/v) ACN in water, $\mathrm{pH} \sim 10$ ). The column was equilibrated with the $95 \%$ buffer $A$ and $5 \%$ buffer $B$ mixture. Peptides were resolved using a linear gradient ranging from $10 \%$ to $35 \%$ buffer $B$ for 35 min followed by a linear increase to $60 \%$ over 5 min and a washing step at $90 \%$ buffer $B$ for $5 \mathrm{~min}$. One-minute fractions were collected and concatenated into 24 (for Mock/BoNT phosphorylated peptides) or 12 (for Mock/BoNT not

phosphorylated peptides and Ca/EGTA phosphorylated peptides) final fractions as suggested elsewhere (284). BRP-Fractions were snap-frozen in liquid nitrogen and dried in the SpeedVac.

\subsubsection{LC-MS/MS}

Dried peptides were re-dissolved in $2 \%(\mathrm{v} / \mathrm{v})$ ACN $0.1 \%(\mathrm{v} / \mathrm{v})$ TFA in water. Each concatenated bRP-fraction was analyzed in triplicates (phosphorylated peptides) or as a single injection (non-phosphorylated peptides). Dissolved peptides were injected onto a C18 PepMap100-trapping column $(0.3 \times 5 \mathrm{~mm}, 5 \mu \mathrm{m}$, Thermo Fisher Scientific, Waltham, USA) connected to an in-house packed C18 analytical column ( $75 \mu \mathrm{m} \times 300 \mathrm{~mm}$; ReprosilPur 120C18-AQ, $1.9 \mu \mathrm{m}$, Dr. Maisch GmbH, Ammerbuch, Germany). The columns were 
pre-equilibrated using a mixture of $98 \%$ buffer $A(0.1 \%(v / v)$ FA in water), $2 \%$ buffer $B(80 \%$ $(\mathrm{v} / \mathrm{v}) \mathrm{ACN}, 0.1 \%(\mathrm{v} / \mathrm{v}) \mathrm{FA}$ in water) or $95 \%$ buffer $\mathrm{A}, 5 \%$ buffer $\mathrm{B}$ (not-phosphorylated peptides). Liquid chromatography was operated on an UltiMate-3000 RSLC nanosystem (Thermo Fisher Scientific, Waltham, USA). Phosphopeptides were eluted using a 60 minlinear gradient ranging from $10 \%$ to $25 \%$ buffer $B(80 \%(v / v)$ ACN, $0.1 \%(v / v)$ FA in water) followed by a linear increase to $45 \%$ buffer B over 10 min and a washing step at $90 \%$ of buffer $\mathrm{B}$ for $5 \mathrm{~min}$. For not-phosphorylated peptides, a steeper gradient was applied ranging from $10 \%$ to $45 \%$ buffer $B$ followed by an increase to $60 \%$ buffer $B$ and a washing step at 90\% buffer B. Eluting peptides were sprayed into a Q Exactive HF-X or Orbitrap Exploris 480 (Thermo Fisher Scientific, Bremen, Germany). For the analysis of phosphorylated peptides, MS1 scans (350 to 1,600 m/z) were acquired with a resolution of 120,000 at $200 \mathrm{~m} / \mathrm{z}$, 3e 6 automatic gain control (AGC) target, and 50 ms maximum injection time. Precursor ions were isolated using a $0.8 \mathrm{~m} / \mathrm{z}$ isolation window, and a normalized collision energy of $33 \%$ was applied to obtain fragment spectra. Only charge states from $2+$ to $6+$ were considered, and the dynamic exclusion was set to $25 \mathrm{~s}$. The fragment spectra were acquired with a resolution of 30,000, 1e5 AGC target, and 130 ms maximum injection time. For the analysis of not-phosphorylated peptides, following parameters were set differently: MS1 scans were acquired with a resolution of 60,000 at $200 \mathrm{~m} / \mathrm{z}, 300 \%$ normalized AGC target. Fragment spectra were acquired with the resolution of 15,000 at $200 \mathrm{~m} / \mathrm{z}, 50 \%$ of the normalized AGC-target and 54 ms maximum injection time.

\subsubsection{Data Analysis}

Raw files were processed using MaxQuant version 1.6.10.2 (295, 365) under default settings with some exceptions. Specifically, cysteine carbamidomethylation was selected as a fixed modification, whereas methionine oxidation, acetylation of protein $\mathrm{N}$-termini and phosphorylation of serine, threonine, and tyrosine (only for phosphorylated peptides) were set as variable modifications. Up to 5 variable modifications were allowed per peptide. Specific trypsin digestion was selected with up to two missed cleavage sites allowed per peptide. Maximal peptide mass was increased to 6000 . Quantification using reporter ions in MS2 (TMT6plex) and minimal parent ion fraction of 0.75 were selected. MS1 and MS2 mass tolerances were kept at $4.5 \mathrm{ppm}$ and $20 \mathrm{ppm}$, respectively. Canonical amino acid sequences of Rattus norvegicus proteins were retrieved from Uniprot (366) (February 2019, 29951 entries). Reference proteome of C. botulinum (strain Hall / ATCC 3502 / NCTC 13319 / Type A) containing canonical protein sequences (Uniprot, January 2020, 3590 entries) was supplemented with sequences of BoNT B-D (strain Okra / Type B1,

C. botulinum $\mathrm{C}$ phage, C. botulinum $\mathrm{D}$ phage, respectively). Peptide-spectrum matches (PSM) and protein FDR threshold were kept at 0.01. Following steps in data analysis were 
conducted in $\mathrm{R}$ statistical programming language using custom scripts. The scripts are available at github (https://github.com/IvanSilbern/2021_Silbern_etal_Synapt_BoNT_KCl). For each phosphorylation site, a leading protein was selected based on the list of potential candidate proteins for this site reported by MaxQuant. Proteins were ranked based on (listed from the most to the least significant) i) the number of the unique phosphorylation sites assigned to the protein in the data set; ii) the number of all phosphorylation sites per protein in the data set; iii) "reviewed" status and Uniprot-annotation score of the protein sequence; iv) isoform status of the protein sequence. The official gene name and Uniprot accession of the leading protein was used in the subsequent analyses. Impurity-corrected reporter ion intensities for each phosphorylation site identified at one of the three multiplicity levels were extracted from MaxQuant "Phospho(S, T, Y).txt" output table. Potential contaminants, reversed sequences and phosphorylation sites identified with localization probability $<0.75$ as determined by MaxQuant $(295,365)$ were excluded from further analysis. Phosphorylation sites quantified at different multiplicity levels (" 1 " means that the phosphorylation site was quantified on singly, "2" on doubly, and " 3 " on multiply phosphorylated peptides) were considered as separate entities or phosphorylation events. If a phosphorylation event contained $<3$ non-zero intensity values for a given labeling experiment, the event was considered as not quantified in this labeling experiment. Otherwise, missing values were imputed for each TMT-channel/experiment-pair individually by random sampling from a gauss distribution with a mean at the $5 \%$ quantile and a double standard deviation of the log-transformed intensities. Only a minor proportion of phosphorylation events required imputation ( $<1 \%$ per channel). $\log _{2}$-transformed reporter ion intensities were then normalized using Tukey median polishing individually for each labeling experiment and subjected to statistical testing using limma package (311).

\subsubsection{Experimental design and statistical rationale}

In total, 216 .raw files were analyzed in order to quantify changes in phosphorylation site intensities in BoNT- or mock-treated synaptosomes or synaptosomes stimulated in the presence of calcium or EGTA. Out of the 216 .raw files, 144 .raw files were obtained from two TMT6-labeling sets of mock- $v s$. BoNT-treated synaptosomes. These files correspond to 24 concatenated bRP-fractions and three injection replicates per fraction. Remaining 72 .raw files were obtained from another two TMT6-labeling experiments of synaptosomes stimulated in the presence of $\mathrm{Ca}^{2+}$ or EGTA. The files correspond to 12 concatenated bRPfractions and three injection replicates per fraction. Each set of TMT6-labeled samples contained three independent replicates for each of the two treatment conditions, resulting in six independent replicates for each treatment regime (Mock, BoNT, Ca, or EGTA). The choice for this number of replicates was dictated by the constraints of the TMT6-labeling 
and the previous study (351). The phosphorylation site intensities resulting from the three technical replicates were summed in order to obtain a single value reported by MaxQuant (295). We used $\log _{2}$-transformed and normalized reporter ion intensities to assess changes in the phosphorylation site intensities. Specifically, we used the limma package (311) to test the difference in phosphorylation site intensities between mock- and BoNT-treated synaptosomes and synaptosomes stimulated in the presence of calcium or EGTA. Linear models were specified to account for treatment differences (Mock, BoNT, Ca, EGTA) and batch effects between TMT6-sets. Differences in the peptide intensities between mock- and BoNT-treated synaptosomes and synaptosomes stimulated in the presence/absence of calcium were tested. Empirical-Bayes moderated p-values (311) were subjected to multiple testing correction using q-value approach (367). Phosphorylation events (phosphorylation site at a given multiplicity) were considered as significantly regulated when showing at least 1.2 intensity fold change (FC, corresponds to absolute $\log _{2} F C>0.263$ ), and q-value $<0.01$ (corresponds to false discovery rate (FDR) $<1 \%$ ).

\subsubsection{Defining primary $\mathrm{Ca}^{2+}$-dependent and SV-cycling-dependent phosphorylation sites}

Phosphorylation events were defined as "primary $\mathrm{Ca}^{2+}$-dependent" if they show absolute $\log _{2}(\mathrm{Ca} / \mathrm{EGTA})>0.263$ and absolute $\log _{2}($ Mock/BoNT) $<0.263$ or were not quantified in the Mock/BoNT experiment. Conversely, events showing absolute $\log _{2}$ (Mock/BoNT) $>0.263$ were termed as "SV-cycling-dependent". Phosphorylation events that exhibited only small changes in both experiments (absolute $\log _{2}(\mathrm{Ca} / \mathrm{EGTA})<0.263$ and absolute $\log _{2}($ Mock/BoNT) <0.263) were considered as being "not-affected". In the following analyses, only phosphorylation events that surpassed an FDR-threshold of $<1 \%$ were considered. Specifically, "primary $\mathrm{Ca}^{2+-d e p e n d e n t " ~ p h o s p h o r y l a t i o n ~ e v e n t s ~ h a d ~ t o ~ s a t i s f y ~ a ~}$ q-value threshold of $<0.01$ in the $\mathrm{Ca} / \mathrm{EGTA}$ experiment, whereas "SV-cycling-dependent" sites had to meet a q-value threshold of $<0.01$ in Mock/BoNT experiment or a q-valuethreshold of $<0.01$ and an absolute $\log _{2}$ FC threshold of $>0.263$ in Ca/EGTA experiment. Phosphorylation events that did not match these criteria were considered as being "not-affected" as well. If a phosphorylation site was quantified at different multiplicities (phosphorylation events), whereby one of which was classified as being "SV-cyclingdependent", the site was considered as being "SV-cycling-dependent". To define regulation groups at protein level, number of significantly regulated phosphorylation sites were counted per protein (gene). Proteins were distributed in the following groups i) "mostly primary $\mathrm{Ca}^{2+}$-dependent" (> $60 \%$ of sites being categorized as "primary $\mathrm{Ca}^{2+-d e p e n d e n t ") ; ~}$ ii) "mostly SV-cycling-dependent" (> $60 \%$ of sites being categorized as "SV-cyclingdependent"); iii) "mixed" (none of the groups was dominating). 


\subsubsection{Bioinformatic analysis}

Gene enrichment analyses were conducted using gene names of proteins carrying significantly regulated phosphorylation sites in Ca/EGTA or Mock/BoNT experiments. Gene ontology enrichment analysis was performed using David web interface and whole Rattus norvegicus proteome as a background (368). Direct GO term categories surpassing Benjamini-Hochberg-adjusted p-value threshold of 0.001 were considered for further analysis. For Reactome pathway analysis, only enriched (Benjamini-Hochberg-adjusted pvalue < 0.1) "neuronal system" categories were selected (369). Synapse-specific SynGO database was used to retrieve significantly enriched (GSEA "gene cluster" FDR corrected p-value < 0.001) GO-biological function terms (324). Experimentally validated kinasesubstrate interactions were derived from PhosphoSitePlus database ("Kinase_Substrate_Dataset.txt", version of 10.04.2018) (325). In order to account for possible differences in phosphorylation site annotation, sequence windows of the phosphorylation sites in the data set were aligned against sequence windows reported in the PhosphoSitePlus database using BLAST+ software (version: 2.7.1, additional command line parameters: -max_target_seqs 20) (370). Only exact alignments of the phosphorylation sites showing the minimum bitscore of 20 were considered. The best match was selected from filtered alignments ranked based on the alignment bitscore, number of amino acid positions considered identical (nident), number of open sequence gaps, kinase and substrate species (rat $>$ mouse $>$ human $>$ rabbit). Phosphorylation sites with no assignment were subjected to NetworKIN kinase-substrate prediction tool $(144,323)$. Specifically, rat protein sequences were aligned against the reference set of human protein sequences (STRING (322) database version 9.05) by using BLAST+ (additional command line parameters: -max_target_seqs 40 -word_size 6 -gapopen 12 -gapextend 1). The best alignment for each rat protein sequence was selected based on the rat protein sequence coverage, number of amino acid positions considered identical, alignment bitscore, number of open sequence gaps (listed with decreasing importance). Positions of phosphorylation sites in the best matching human protein sequences were subjected to NerworKIN tool. The kinase prediction with the highest NetworKIN score was selected for each phosphorylation site. Predicted kinases were organized in the kinase groups introduced by Netphorest classification (144). The second-level and top-level kinase groups in the kinase hierarchy were used for enrichment analyses and visualizations. The overrepresentation analysis of predicted kinase-substrate interactions for phosphorylation sites significantly regulated in $\mathrm{Ca} / \mathrm{EGTA}$ or Mock/BoNT experiments was conducted in comparison to NetworKIN kinasesubstrate predictions for human proteome. To assess the disbalance in the number of up/down regulated phosphorylation events, the number of up/down regulated 
phosphorylation events controlled by each kinase group was compared to the total number of up/down phosphorylation events among significantly regulated phosphorylated sites. All tests of differential term or kinase enrichment between two groups were done using Fisher's exact test. Benjamini-Hochberg adjusted p-values are reported throughout the study if not specified otherwise. The anticipated phosphatase-substrate interactions rely upon described PP1 and Calcineurin docking motifs (371). To demonstrate possible phosphatase-substrate connections, interaction maps were extracted from protein-protein interactions annotated for Rattus norvegicus (STRING (322) database version 10.5). Shortest paths from phosphatase to target proteins (phosphoproteins involved in endo/exocytosis and containing PP1 or Calcineurin docking motif) were computed using shortest_path function from igraph package (372) (version 1.4.6). Only proteins previously identified in synaptosomes and interactions with experimental evidence or annotated in curated databases were included in the analysis. Phosphorylation site occupancy was calculated using a 3D model presented by Hogrebe et al (300). In brief, a linear model of the form $P h=m_{1} \cdot$ Prot $-m_{2^{*}} N P h$, where $P h$ is the normalized (by median polishing) intensity of the phosphorylated peptide; Prot is the normalized protein intensity and NPh is the normalized intensity of non-phosphorylated peptide. The model was fit by using intensity data from Mock/BoNT experiments. Phosphorylation occupancy was then calculated using the following formula: Occupancy $=a /(1+a)$, where $a=m_{2} \cdot P h / N P h$. "Illegal" stoichiometry values, i.e., values outside the interval $(0 ; 1)$ were excluded from the analysis. Only $m_{2}$ values with a $p$ value below 0.1 were considered.

\subsubsection{C. botulinum cell culture supernatants profiling}

For protein profiling, supernatants of $C$. botulinum cell culture were digested overnight using trypsin. Digested peptides were desalted using pre-packed C18 spin-columns. Samples were analyzed on Q Exactive HF-X and liquid chromatography setup as described above.

To test for unspecific phosphatase activity, C. botulinum cell culture supernatants were incubated with the nuclear extract of HeLa-cells. The nuclear extract was prepared as previously described (373) in Roeder D buffer (20 mM Hepes-KOH pH 7.9, $100 \mathrm{mM} \mathrm{KCl}$, $1.5 \mathrm{mM} \mathrm{MgCl}_{2}, 10 \%$ (v/v) glycerol, $0.5 \mathrm{mM}$ DTT, $0.5 \mathrm{mM}$ PMSF, $0.2 \mathrm{mM}$ EDTA) and was a generous gift of Prof. Dr. Lührmann laboratory. The extract was supplemented with $1.3 \mathrm{mM}$ $\mathrm{CaCl}_{2}$ and $20 \mu \mathrm{L}$ of each $C$. botulinum cell culture supernatant ( $\mathrm{A}$ and $\mathrm{D}$ or $\mathrm{C} 1$ and $\mathrm{B}$ ), heatinactivated cell culture supernatants, or $40 \mu \mathrm{L}$ of the sodium buffer per $1 \mathrm{mg}$ of nuclear proteins. Each treatment condition was performed in triplicate, resulting in a total of 9 samples. The samples were incubated for $1.5 \mathrm{~h}$ at $37^{\circ} \mathrm{C}$. Afterwards, urea in $100 \mathrm{mM}$ HEPES pH 8 was added to the final concentration of $1 \mathrm{M}$ and proteins were digested overnight at $30^{\circ} \mathrm{C}$ using MS-grade trypsin at a trypsin-to-protein ration (wt/wt) of 1:50. Next 
day, peptides were subjected to phosphopeptide enrichment as described above excluding the de-glycosylation and second endoproteinase digestion steps. Enriched phosphopeptides were cleaned using the pre-packed C18 spin columns, re-dissolved in $2 \%$ $(\mathrm{v} / \mathrm{v}) \mathrm{ACN}, 0.1 \%(\mathrm{v} / \mathrm{v})$ TFA in water and analyzed on UltiMate $3000 \mathrm{RSLC}$ nanosystem connected to Orbitrap Exploris 480 mass spectrometer. Raw phosphorylation site intensities as reported by MaxQuant were analyzed as described before using R scripts and the limma package for data normalization, visualization, and statistical testing.

\subsubsection{Calcium influx in synaptosomes following BoNT-treatment and $\mathrm{KCl}$ - stimulation}

Synaptosomes suspensions containing $1 \mathrm{mg}$ of synaptosomal proteins were prepared as described for glutamate release assay. Suspensions were supplemented with $1.3 \mathrm{mM}$ $\mathrm{CaCl}_{2}$ and additional $40 \mu \mathrm{L}$ of the sodium-containing buffer or $1.3 \mathrm{mM} \mathrm{CaCl}_{2}$ and $20 \mu \mathrm{L}$ of each $C$. botulinum cell culture supernatants in two combinations ( $A$ and $D$ or $C 1$ and $B$ ). Synaptosomes were then incubated for $1 \mathrm{~h}$ at $37^{\circ} \mathrm{C}$ under mild agitation. Afterward, synaptosome suspensions were supplemented with $5 \mu \mathrm{M}$ of Fura-2AM dye (Molecular probes, Eugene, USA) and incubated for additional $30 \mathrm{~min}$ at $37^{\circ} \mathrm{C}$. Following steps were conducted as previously described (101). Briefly, synaptosomes were resuspended in $1 \mathrm{~mL}$ of fresh sodium-containing buffer and loaded into the quartz glass cuvette. The synaptosomal suspension was stirred and kept at $37^{\circ} \mathrm{C}$ during the measurements. The fluorescence was continuously measured using Fluorolog-3 at excitation wavelengths of 340 and $380 \mathrm{~nm}$ and emission at $505 \mathrm{~nm}$, for $2 \mathrm{~s}$ at each wavelength. $1.3 \mathrm{mM} \mathrm{CaCl}_{2}, 50 \mathrm{mM} \mathrm{KCl}, 0.4 \%$ (wt/v) SDS, $1 \mathrm{mM}$ EGTA were added stepwise, and the signal was acquired for $3 \mathrm{~min}$ at each step. Fluorescence intensity at $340 \mathrm{~nm}$ excitation was divided by the intensity at $380 \mathrm{~nm}$ excitation and then by the maximum fluorescence ratio following SDS-lysis. Median fluorescence ratio before stimulation with $\mathrm{KCl}$ was subtracted to obtain proportional changes in fluorescence intensity relatively to its maximum.

\subsubsection{Cell cultures and transfections}

Primary hippocampal neuronal cultures were prepared from P2 rats as previously described (374). HEK293T cells were obtained by a commercial supplier (Sirion Biotech $\mathrm{GmbH}$; Germany). HEK293T cells were grown in DMEM supplemented with 10\% FBS, 4 mM L-glutamine and $600 \mathrm{U} / \mathrm{ml}$ penicillin-streptomycin (Lonza). For transfection Lipofectamine 2000 transfection reagent was used following the guidelines of the manufacturer (Thermo Fisher Scientific, Invitrogen, USA). All plasmids for transfection were produced in bacteria 
and purified with the NucleoBond Xtra Midi endotoxin-free plasmid DNA kit (Macherey-Nagel, Germany).

\subsubsection{DNA cloning of wild type proteins and respective phosphomimetic mutants}

All the sequences (including mutants) were designed in silico based on rat genes with the appropriate restriction enzymes for viral cloning and ordered at GenScript (USA). The synaptobrevin (Vamp2) mutants were based on rat Vamp2 (Uniprot: P63045) fused to the optimized version of superecliptic GFP (pHluorin) (375) by mean of a short peptide linker. We used Agel and Sdal (Sbfl) restriction sites for inserting Vamp2-pHluorin and the respective mutants (S75D pseudo-phosphorylated and S75A non-phosphorylatable) in the viral vector (previously described, (376)) allowing neuron-specific expression under the human synapsin-1 promoter. For all the following subcloning steps we relied on commercial chemically competent SURE bacteria from Agilent, to avoid the loss of ITR sequences. Positive clones were confirmed by sequencing, and before transfection in the packaging HEK293T cells, the presence of ITRs and the correct length of all plasmids were checked by restriction enzyme analysis. Syntaxin-1a (Stx1a; P32851) and cannabinoid receptor 1 (Cnr1; P20272) and their mutants (respectively T21E-T23E pseudo-phosphorylated Stx1a, T21A-T23A non-phosphorylatable Stx1a, T314E-T322E pseudo-phosphorylated Cnr1 and T314A-T322A non-phosphorylatable Cnr1) were inserted in the WT Vamp2-pHluorin viral vector by cutting with the enzymes Sgsl (Ascl) and Sbfl (Sdal). Their expression was driven by mean of a second human synapsin-1 promoter from the same viral plasmid to allow consistent coexpression. To distinguish these proteins from the endogenous, an ALFA-tag was added (377). Also in this case, positive clones were confirmed by sequencing, and before transfection in the packaging HEK293T cells, the presence of ITRs and the correct length of all plasmids were checked by restriction enzyme analysis. All synthetic plasmids and their respective sequences are available from the authors upon request.

\subsubsection{Immuno staining with NbALFA}

The expression of Stx $1 a$ and Cnr1 in all viruses and mutants was confirmed by staining with the NbALFA (377). Briefly, neurons were fixed with 4\% paraformaldehyde (PFA; w/v) for $30 \mathrm{~min}$ at room temperature (RT). Cells were quenched in $100 \mathrm{mM} \mathrm{NH}_{4} \mathrm{Cl}$ and permeabilized in PBS containing $4 \%$ bovine serum albumin (BSA; wt/v) and $0.1 \%(\mathrm{v} / \mathrm{v})$ Triton-X 100 for $15 \mathrm{~min}$ at RT. Fluorescently labeled NbALFA (FluoTag-X2 anti-ALFA AbberiorStar635P, NanoTag Biotechnologies \#N1502-Ab635P-L, Germany) was diluted $1: 200$ in blocking solution for $1 \mathrm{~h}$ at RT and subsequently washed three times for 5 min with PBS. Coverslips were mounted on glass-slides using Mowiol solution, dried at and imaged. 
Note that superecliptic GFP (pHluorin) keeps its fluorescence in Mowiol solution since its $\mathrm{pH}$ is $\sim 7.5$ and can thus be imaged with no additional staining.

\subsubsection{Adeno associated virus preparation}

Recombinant AAV particles were prepared as previously described (376). Briefly, vectors were packaged in HEK293T cells using the pDP6 helper plasmid (avoiding adenoviral contamination). Packaging cells were lysed in Tyrode's solution (124 mM NaCl, $5 \mathrm{mM} \mathrm{KCl}$, $30 \mathrm{mM}$ glucose, $25 \mathrm{mM}$ HEPES, $2 \mathrm{mM} \mathrm{CaCl}, 1 \mathrm{mM} \mathrm{MgCl}_{2}, \mathrm{pH} 7.4$ ), passed through $0.22 \mu \mathrm{m}$ syringe filters and viral titer was adjusted by serial dilution on primary hippocampal neurons monitoring pHluorin levels (always expressed under the neurospecific human synapsin-1 promoter).

\subsubsection{Synaptic vesicle exo-/endocytosis measurements}

SV exo/endocytosis assays were performed analogously to what previously described ( 374 , 378). Briefly, neurons were field-stimulated with platinum electrodes in custom-made chambers housing $18 \mathrm{~mm}$ coverslips ( $8 \mathrm{~mm}$ distance between electrodes). We used a Stimulus Isolator combined with an A310 Accupulser Stimulator (both from World Precision Instruments, Sarasota, USA) with a nominal output of $100 \mathrm{~mA}$ for stimulation. In the case of Stx1a and Cnr1, co-expression was confirmed by imaging. Live experiments were performed in Tyrode's solution (124 mM NaCl, $5 \mathrm{mM} \mathrm{KCl}, 30 \mathrm{mM}$ glucose, $25 \mathrm{mM}$ HEPES, $2 \mathrm{mM} \mathrm{CaCl}_{2}, 1 \mathrm{mM} \mathrm{MgCl}_{2}, \mathrm{pH}$ 7.4) supplemented with $10 \mu \mathrm{M}$ 6-cyano-7-nitroquinoxaline2,3-dione (CNQX; Tocris Bioscience, Cambridge, UK) and $50 \mu \mathrm{M}$ 2-amino-5phosphonopentanoic acid (D-AP5; Tocris Bioscience, Cambridge, UK) to avoid spontaneous network activation. At the end of the imaging, $50 \mathrm{mM} \mathrm{NH}_{4} \mathrm{Cl}$ was applied to evaluate total SV content. Live imaging was performed with an inverted Nikon Ti epifluorescence microscope (Nikon, Tokyo, Japan) equipped with a Plan Apochromat 60x 1.4 NA oil-immersion objective, an HBO-100W Lamp, an IXON X3897 Andor camera (Northern Ireland, UK) and an OKOLab cage incubator system (OKOLab, Ottaviano, Italy) to maintain a constant temperature of $37^{\circ} \mathrm{C}$. ND2 Images were imported using the BioFormats (OME) plug-in. For quantifications, the fluorescence intensity was measured with custom-made Matlab (USA) code that can be provided on an individual basis if requested. Briefly, multiple regions containing synaptic boutons were manually selected, in 5 to 8 independent experiments. Between 140 and 345 regions were selected for each condition, over all independent experiments. The Vamp2-pHluorin signal was then measured in the respective regions, and the background intensity (obtained in identically sized regions from the neuron-free adjoining regions) was subtracted. The Vamp2-pHluorin signal curves were then normalized to the maximum intensity obtained during the addition of $\mathrm{NH}_{4} \mathrm{Cl}$. To 
compare easily different conditions and mutant variants, all curves were furthermore normalized to their initial (pre-stimulus) baselines. Statistical differences were tested in Matlab, relying on Kruskal-Wallis tests, followed by Tukey-Kramer post-hoc tests.

\subsection{Results}

We performed a quantitative in-depth analysis of changes in protein phosphorylation events in active synaptosomes. We first quantitatively compared the phosphorylation status of proteins in chemically stimulated synaptosomes under $\mathrm{Ca}^{2+}$-deprived and free $\mathrm{Ca}^{2+}$ conditions; for the latter, depolarization of the plasma membrane of synaptosomes was induced by $\mathrm{KCl}$ in buffer containing EGTA or $\mathrm{CaCl}_{2}$ (Figure 5.1). The viability of the synaptosomes was confirmed by monitoring release of the neurotransmitter (glutamate) (118) (Figure 5.S1A). We applied depolarization for $2 \mathrm{~min}$; this duration was chosen in order (i) to achieve comparability with our previous studies (351) and (ii) to allow the capture of short- and medium-term changes in phosphorylation. Proteins from stimulated and nonstimulated synaptosomes were extracted, digested, and enriched for phosphopeptides in parallel; thereafter, the (phospho)peptides were labeled with unique isotopically labeled TMT6 reagents. Peptides were then pooled, pre-fractionated by basic reverse-phase chromatography and finally analyzed by LC-MS/MS (Figure 5.1). In the second approach, we inhibited SV cycling using BoNT and monitored quantitatively the changes in the phosphoproteome after $\mathrm{K}^{+}$-mediated depolarization, comparing synaptosomes that were treated with active or, respectively, with inactive BoNT (Figure 5.1). Phosphopeptides were enriched and analyzed as described above. This second approach should differentiate phosphorylation events that are dependent on SV exo-endocytotic cycling.

Our analysis shows that phosphorylation is a prominent modification of synaptosomal proteins. Specifically, we identified and quantified phosphorylation under stimulated and non-stimulated conditions on 12021 sites belonging to more than 2600 proteins (unique gene names) with a localization probability of $>75 \%$ as determined by MaxQuant $(295,365)$. This coverage greatly exceeds our earlier one (351) and is comparable to a recent study from another laboratory (352). When the phosphosites quantified from BoNT-treated synaptosomes are included, the number of sites quantified in this study increases to 18981 sites belonging to 3840 proteins (Figure 5.2A, left panel).

\subsubsection{Synaptosome depolarization by $\mathrm{KCl}$ in the presence of $\mathrm{Ca}^{2+}$ results in substantial changes in protein phosphorylation.}

When analyzing synaptosomes depolarized in the presence of calcium ions in comparison with those depolarized in the absence of calcium (EGTA control), we detected substantial 
changes in protein phosphorylation. Specifically, there are 1363 phosphorylated sites mapped to approximately 470 proteins that show intensity changes of at least $20 \%$ at an FDR of $1 \%$ (Figure 5.2B). Using the PhosphoSitePlus database (325) to annotate known kinase-substrate relations and the NetworKIN tool $(144,323)$ to predict possible kinasesubstrate pairs, we observed that certain kinase families were overrepresented including the CaMKII, MAPK and GSK3 kinase groups (Table 5.S1). Importantly, the distribution of up- or down-regulated sites between kinase groups is not random: CaMKII and PKC kinase groups are responsible for phosphorylation sites that appear upregulated, while the majority of sites controlled by MAPK, CDK and CK1 show reduced intensity in the presence of $\mathrm{Ca}^{2+}$ as compared with EGTA controls (Figure 5.2E and Figure 5.S2). The latter observation is in line with previous studies that demonstrated up-regulation of putative CaMKII substrates and down-regulation of predicted MAPK substrates following potassium-triggered depolarization of synaptosomes $(351,352)$.

We also observed changes of phosphorylated sites in response to $\mathrm{KCl}$ treatment that were similar to changes reported in earlier studies (352) for Bassoon (Bsn), Piccolo (Pclo), Synapsin 1 (Syn1), and Dynamin 1 (Dnm1), as well as the microtubule-associated protein Tau (Mapt) and the dihydropyrimidinase-related proteins 2 and 5 (Dpys/2 and Dpys/5), and others (Figure 5.2D). Overall, the overlap of identified and quantified sites with the results of previous studies is approximately $60 \%$ as determined by the overlap in protein sequences flanking phosphorylation sites (sequence windows).

Enrichment analysis of the biological function of proteins carrying regulated phosphorylation sites (DAVID (368)) revealed gene ontology (GO) terms tightly connected with synaptic functioning, cytoskeleton organization, regulation of synaptic functions and neuronal projections development (Figure 5.3A). A similar picture (Figure 5.3B) was obtained when using the synapse-specific SynGO database in which a large set of synaptic proteins was annotated by experts (324). It shows that the significantly enriched terms describe biological functions connected with SV cycling, regulation of neurotransmitter release and synapse organization (for a complete list of enriched terms see Table 5.S2). Interestingly, regulation of some functions might be linked to the activity of certain kinase groups (Figure 5.3A). For example, the number of sites predicted to be substrates for GSK3 and CDK is proportionally higher among proteins involved into the GO term "axonogenesis" as compared with other GO terms. Similarly, many phosphorylated sites on proteins that are involved in the "calcium-dependent regulation of synaptic functions" were also predicted as CaMKII substrates. The proportion of putative MAPK-substrates is higher for proteins associated with "synapse assembly" and "cytoskeleton organization" as compared with other GO terms. 
This analysis reflects, in accordance with recent studies $(351,352)$, the overall changes of phosphorylation in isolated nerve terminals upon stimulation - including the phosphosites that are important for exocytosis and endocytosis, i.e., for SV cycling. However, the observed effects can be a result of both, $\mathrm{Ca}^{2+-i n f l u x}$ and active SV cycling.

\subsubsection{Block of SV cycling with botulinum neurotoxins induces changes in protein phosphorylation.}

As indicated above, our first quantitative analysis does not allow for differentiating phosphorylation changes that are directly dependent on active membrane trafficking in the synapse rather than being caused by the activation of calcium-dependent kinases and phosphatases such as CaMKII (337), calcineurin (379), etc. Of course, these processes are intertwined since these enzymes are known to regulate the phosphorylation status of proteins closely associated with the process of SV cycling $(205,380)$.

We suppressed SV cycling by incubating synaptosomes with BoNT that block exocytosis by cleaving SNARE proteins. Toxin-treated synaptosomes and (as controls) synaptosomes incubated with inactivated toxins were stimulated with the high-potassium buffer in the presence of $\mathrm{Ca}^{2+}$ ions (Figure 5.1). The toxin treatment greatly inhibited neurotransmitter release (Figure 5.S1B) but did not compromise $\mathrm{Ca}^{2+}$-influx (Figure 5.S1C). It led to significant changes in the phosphoproteome of the synaptosomes: 1497 sites showed significant difference with a minimum of $20 \%$ intensity changes at $1 \%$ FDR that can be mapped to approximately 700 proteins. Strikingly, BoNT treatment reduced the intensity of many phosphorylation sites (Figure 5.2C). The phosphorylation status of these sites and their corresponding proteins is apparently dependent on the $\mathrm{Ca}^{2+-i n d u c e d ~ S V ~ c y c l i n g ~}$ activity in the presynapse.

The enrichment analyses for GO terms did not show substantial differences when compared with proteins carrying regulated sites in Ca vs. EGTA experiments (not shown). The impact of BoNT treatment became apparent when we investigated possible substrate-kinase relationships. The enrichment analysis showed significant overrepresentation $(P<0.01)$ of phosphorylation sites that can be attributed to MAPK, PKC and GSK3 kinase groups (Table 5.S3). When comparing the quantitative Cavs. EGTA phosphorylation data, significantly fewer $\left(P \cong 1.0 \times 10^{-5}\right)$ regulated phosphorylation sites could be ascribed to being CaMKII substrates, whereas putative sites for MAPK (1), CLK (2), and PAK (3) were slightly over- $(1,2)$ or under- (3) represented, respectively (Figure 5.2F, Table 5.S4). Therefore, the crucial point in this analysis is the distribution of up- or downregulated phosphorylation sites for the different kinase groups. While depolarization of synaptosomes 
by $\mathrm{KCl}$ in the presence of $\mathrm{Ca}^{2+}$ leads to significantly reduced intensities of phosphorylation sites presumably regulated by MAPK and CDK kinases, the intensities of the putative MAPK, CDK and GSK3 substrates appear higher when comparing mock-treated with BoNTtreated synaptosomes (compare Figure 5.2E and Figure 5.2F). The balance of up- and downregulated phosphorylation sites was also significantly altered $(P<0.01)$ for CLK, CK1 and PAK kinases.

\subsubsection{Dissecting primary $\mathrm{Ca}^{2+}$-driven phosphorylation events from events linked to SV cycling.}

Assuming that the comparison of mock-treated synaptosomes with the BoNT-treated ones reveals the phosphorylation changes caused by $\mathrm{Ca}^{2+}$-induced SV cycling, we were able to identify phosphorylation events that are primarily dependent on $\mathrm{Ca}^{2+}$-influx and are not affected if SV cycling is inhibited (Figure 5.4A and S3, orange dots). In the following, we refer to these sites as "primary $\mathrm{Ca}^{2+-d e p e n d e n t " ~ s i t e s . ~ T h e y ~ a r e ~ p r e s u m a b l y ~ d i r e c t l y ~}$ (de)phosphorylated by $\mathrm{Ca}^{2+-d e p e n d e n t ~ p r o t e i n ~ k i n a s e s ~ a n d ~ p h o s p h a t a s e s ~ u p o n ~} \mathrm{Ca}^{2+}$ influx. Conversely, a large group of phosphorylation events has shown changes when comparing mock- and BoNT-treated synaptosomes. Hence, we termed these sites "SV-cyclingdependent". We noted with interest that the majority of "SV-cycling-dependent" sites did not show significant intensity changes in the Ca/EGTA-experiment. We also noted that many proteins tend to carry regulated phosphorylation sites of one of the two groups introduced above, e.g., mostly "primary $\mathrm{Ca}^{2+-d e p e n d e n t " ~ o r ~ " S V-c y c l i n g-d e p e n d e n t " ~ s i t e s, ~ w h i l e ~ o n l y ~ a ~}$ few contain sites of various categories (Figure 5.4B). Interestingly, the number of proteins that carry mostly "primarily $\mathrm{Ca}^{2+}$-dependent" sites is lower than the number of proteins that carry mostly "SV-cycling-dependent" sites (Figure 5.4B). It indicates the tendency that "primary $\mathrm{Ca}^{2+-d e p e n d e n t " ~ p h o s p h o r y l a t i o n ~ s i t e s ~ a p p e a r ~ c l u s t e r e d ~ o n ~ a ~ f e w ~ p r o t e i n s ~}$ whereas "SV-cycling-dependent" sites are often found as the only significantly regulated phosphorylation site in a single protein. The site occupancy assessment (based on the model of Hogrebe et al. (300)) suggests high phosphorylation levels ( $>60 \%)$ for a number of phosphoproteins, including Bsn, Pclo, Dnm1, Camk2a, Mapt, and Dplys/5, underlining the importance of the observed phosphorylation events (Figure 5.S4). It should be noted that the estimated phosphorylation levels represent only a rough estimate, as the ratio compression, which is a known issue for the TMT-based quantification, might severely affect the occupancy calculation. In terms of probable kinase regulation (Figure 5.4C), sites predicted to be CaMKII-substrates include significantly more "primary $\mathrm{Ca}^{2+}$-dependent" sites than sites classified as "SV-cycling-dependent". This harmonizes well with the strict $\mathrm{Ca}^{2+-d e p e n d e n c e ~ o f ~ C a M K I I-a c t i v i t y ~(381) . ~ C o n v e r s e l y, ~ t h e ~ M A P K ~ g r o u p ~ c o n t r o l s ~}$ significantly more "SV-cycling-dependent" sites than "primary $\mathrm{Ca}^{2+}$-dependent" sites. 
Similarly, predicted PAK-substrates are overrepresented among "primary $\mathrm{Ca}^{2+}$-dependent" sites, whereas predicted PKC, CDK, and CLK-substrates appear more often among "SVcycling-dependent" sites (Figure 5.4C). The majority of phosphorylation sites on CAMKII, MAPK1, MAPK3, and PRKCB themselves show dependence on calcium ions. Increased phosphorylation of the regulatory sites of CaMKII, MAPK1, MAPK3, and PRKCB (Camk2aT286, Camk2b-T287, Camk2d-T287, Mapk1-T183, Y185, Mapk3-T203, Y205, Prkcb-T640) points to the activation of those kinases following depolarization of synaptosomes by $\mathrm{KCl}$ (Figure 5.S5, S6).

We noticed that numerous phosphorylation events on phosphatases and phosphatase regulators were significantly regulated in our study (Figure 5.S7A). Particularly, Neurabin1 (Ppp1r9a), a PP1 regulator, showed increased phosphorylation in the Mock/BoNT experiment, suggesting the involvement of PP1 in regulating SV cycling. Although phosphatase-substrate interactions are more difficult to analyze due to the degenerated nature of phosphatase docking motifs, previous studies led to the proposal of docking motifs of PP1 and the $\mathrm{Ca}^{2+}$-calmodulin-dependent phosphatase calcineurin (371). We therefore examined possible interactions between exo- and endocytosis-related proteins containing PP1 or Calcineurin docking motifs and the respective phosphatase (Figure 5.S7B-C). Available interactome data suggest a partially overlapping set of proteins that can be regulated by the phosphatases (e.g., Rims2, Bsn, Bin1, Stxbp1), which does not allow confident assignment of a phosphatase to a particular phosphorylation event. When considering all potential targets, our results show a minor increase in the percentage of "SVcycling-dependent" sites over "primary $\mathrm{Ca}^{2+}$-dependent" sites on proteins that can be regulated by PP1 (Fisher's exact test, $P=0.075$, Figure 5.S7D).

Because of the tendency of proteins to carry preferentially one of the two groups of significantly regulated phosphorylation sites, we set out to investigate the putative biological functions of these proteins in order to delineate processes that can be controlled by "primary $\mathrm{Ca}^{2+}$-dependent" or "SV-cycling-dependent" phosphorylations. Reactome pathway analysis (369) sheds light on the putative different function of the involved phosphorylated proteins (Figure 5.4D). One obvious difference regarding the two groups are proteins connected to potassium channels ("Voltage-gated potassium channels") which are more enriched in the "SV-cycling-dependent" group. Another striking difference is that phosphorylated proteins termed with "Synaptic adhesion-like molecules" and "Neurexins and neuroligins" are enriched in "SV-cycling-dependent" group. In contrast, terms associated with "Neurotransmitter release cycle", e.g., glutamate, acetylcholine, etc. release, are barely enriched in phosphorylated proteins that carry "SV-cycling-dependent" sites (Figure 5.4D, top). Reactome pathway annotation aligned well with the manual annotation of key-words 
describing protein function or location according to information available in the literature (Figure 5.S8). On the basis of our annotation, we noted that 234 out of 301 phosphorylation sites on proteins directly involved in the processes of exo- and endocytosis belong to the "primary $\mathrm{Ca}^{2+-d e p e n d e n t " ~ g r o u p . ~ F o r ~ e x a m p l e, ~ p h o s p h o r y l a t i o n ~ o f ~ R I M, ~ R I M-b i n d i n g ~}$ protein, synapsin-1, and "dephosphins" such as dynamin-1 (Dnm1), amphiphysin (Amph) and AP180 (Snap91) showed a clear $\mathrm{Ca}^{2+}$-dependent character (Figure 5.S9, S10). Importantly, phosphorylation sites on synapsin-1 followed the previously described pattern of phosphorylation changes when comparing $\mathrm{Ca}$ with the EGTA condition: increased phosphorylation of S9 and S603 and de-phosphorylation of S62, S67, S549, and S551 (153, 171, 348, 382-385) (Figure 5.S11). In contrast, the "SV-cycling-dependent" phosphorylation sites tended to be present on proteins that are described as being associated with cytoskeleton elements and channels, for example, spectrin beta (Sptb, Sptan1, Sptbn1, Sptbn2, Sptbn4) and spectrin-binding proteins adducin alpha (Add1) and beta (Add2), as well as potassium channel subunits that are known constituents or modulators of Kv1 (Kcna2, Kcna4, Kcnab2), Kv2 (Kcnb1, Kcnb2), Kv4.3 (Kcnd2), Kv7.2 (Kcnq2), K2p10.1 (Kcnk10), Hcn (Hcn1, Hcn2, Kctd3) potassium channels (29) (Figure 5.S12-S15). As mentioned above, the observed SV cycling effect on several potassium channels seems to be very specific for the particular proteins and results in increased phosphorylation of the majority of those proteins except Add1 and Kcnma2 (Figure 5.S16). Conversely, the $\mathrm{Ca}^{2+}$-activated potassium channel subunit alpha-1 (Kcnma1) and several other potassium channel subunits and modulators (Konc3, Kcnh1, Kcnip2, Kcnq5) have shown the "primary $\mathrm{Ca}^{2+}$-dependent" character of phosphorylation (Figure 5.S17). We summarize changes in phosphorylation site intensities for protein sequences that contain phosphorylation sites significantly regulated in one of the experiments and provide them as graphics in Suppl. Data 5 and $6\left(\log _{2}(\mathrm{Ca} / \mathrm{EGTA})\right.$ and $\log _{2}$ (Mock/BoNT), respectively). These can be also visualized using a shiny-app accessible at https://s1608-shiny.mpibpc.mpg.de. Figure $\mathbf{5 . 5}$ summarizes our results according to the schematic representation of proteins at the pre-synapse (386). Importantly, the proteins of the core exocytotic machinery including VAMP2, SNAP25, syntaxin-1a, and Munc18 exhibit SV-cycling-dependent sites, while Munc13 reveals one SV-cycling-dependent site which is close to the significance cut-off.

\subsubsection{Phosphomimetic sites on SNARE proteins and cannabinoid receptor-1 modulate neurotransmitter release.}

Since protein phosphorylation can be an epiphenomenon of intracellular signaling, we selected a few of the changing phosphosites for determining directly whether they indeed have a modulatory effect on synaptic transmission. 
We noted with interest that certain phosphorylation sites on SNARE proteins, including synaptobrevin (Vamp2) and syntaxin-1a (Stx1a), have a phenotype which belongs to the "SV-cycling-dependent" group (Figure 5.S18). More intriguing, some of those sites (such as Vamp2-S75) were previously described as being important for the interaction with Munc18-1, a protein that can exhibit a stimulatory effect on trans-SNARE zippering (356). Similarly, we noted that two sites on the cannabinoid receptor-1 (Cnr1), T314 and T322, belong to the "SV-cycling-dependent" group as well. In order to test the effects of phosphorylation at these sites, we expressed the respective wild type (WT) proteins, their phosphomimetic mutants and phosphorylation-null mutants in cultured hippocampal neurons (Figure 5.6A).

With this strategy, we addressed Vamp2-S75, Stx1-S21/S23, Cnr1-T314/T322, and their respective mutants (Vamp2-S75D; Vamp2-S75A; Stx1-S21E/S23E; Stx1-S21A/S23A; Cnr1-T314E/T322E and Cnr1-T314A/T322A). For these proteins, we created adenoassociated viruses (AAVs) that allowed us to mimic the effects of phosphorylation, and we expressed them in neurons together with Vamp2-pHluorin, as a sensor for exo-/endocytosis (Figure 5.6B and 6C).

Our stimulation experiments demonstrated that both phosphomimetic and nonphosphorylatable amino-acid substitutions, in both proteins, influence exo- and endocytosis in the cultured neurons. Specifically, we stimulated neurons with either 60 action potentials (AP) at $20 \mathrm{~Hz}$ (short stimulation) targeting the readily releasable pool of SVs and thus reflecting the immediate exocytotic response, or using $600 \mathrm{AP}$ at $20 \mathrm{~Hz}$ (long stimulation) to mobilize the more inert recycling SV pools, reaching a state in which the entire vesicle cycle is fully activated (123). The expression of phosphomimetic Vamp2-S75D reduced exocytosis following short and long stimulation; it also reduced endocytosis in response to long stimulation. Expression of non-phosphorylatable Vamp2-S75A reduced exo- and endocytosis following both long and short stimulation (Figure 5.6D and 6E). Conversely, pseudo-phosphorylated Stx1a-T21E/T23E enhances exocytosis efficiency during short stimulation, while the non-phosphorylatable variant does the opposite (Figure 5.6F). The effects on exocytosis become negligible during long stimulation, but both mutant variants result in an enhancement of endocytosis under these conditions (Figure 5.6G).

The cannabinoid receptor-1 is known for modulation of the neurotransmitter release (359, 387,388 ), and its activation can also be linked, through the activation of MAP kinases, to the phosphorylation of Munc18 (347). Our experiments show that the pseudophosphorylated variant Cnr1-T314E/T322E reduced exocytosis (most evident during short stimulation), while the non-phosphorylatable variant Cnr1-T314A/T322A increased exocytosis in response to long or short stimulation. The effect on endocytosis was observed 
only for the pseudo-phosphorylatable Cnr1 variant after long stimulation (Figure 5.6H and $6 \mathrm{I})$.

These experiments tested only three of the synaptic phosphorylation sites that we found differentially phosphorylated in this work. Nevertheless, even if these experiments are based on overexpression in a background containing normal levels of wild type proteins, all tested candidates resulted in significant modulations of exo- or endocytosis. Although in the case of Vamp2 both mutants led to a substantial reduction in exocytosis rates, its pseudophosphorylated form (Vamp2-S75D) showed a greater effect on exocytosis than the nonphosphorylatable form (Vamp2-S75A). Presumably, the absence of a charged residue did not fully compensate the steric effect caused by the amino acid substitution at position 75 of Vamp2, which has apparently a stronger impact on protein function than the double mutant at positions $21 / 23$ of Stx $1 a$. The S75 phosphorylation site is located within the RSNARE motif required for forming a coiled-coil complex with Stx1a and SNAP-25 during exocytosis. In contrast, the T21/T23 positions of Stx1a are located in the regulatory $\mathrm{N}$ terminal helical bundle, explaining why mutations show different effects on exocytosis. Overall, these findings support the concept that protein phosphorylation is a key regulatory mechanism in neurotransmitter release that modulates different steps in the SV cycle. 


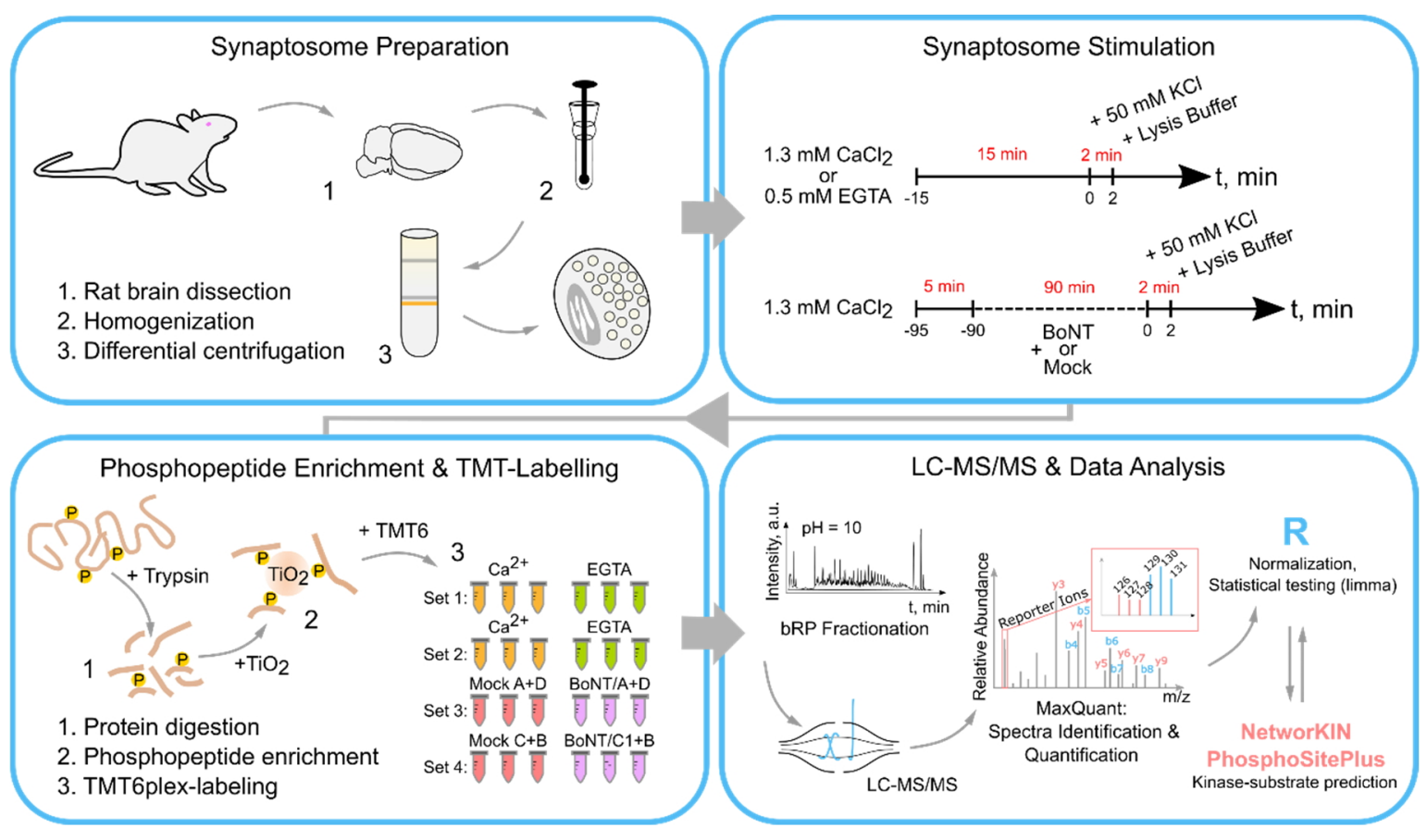

Figure 5.1: Workflow for the phosphoproteome analyses of synaptosomes under different stimuli. Brains of 5-6 weeks old Wistar rats were homogenized and the synaptosomal fraction was enriched by differential centrifugation in discontinuous Ficoll gradient. Two sets of experiments were performed that compared a) stimulation of synaptosomes in the presence of $\mathrm{Ca}^{2+}(1.3 \mathrm{mM})$ or $\mathrm{Ca}^{2+}$-chelator (EGTA, $\left.0.5 \mathrm{mM}\right)$, or b) stimulation of synaptosomes pre-treated with inactivated toxins (Mock) or a combination of two botulinum toxins (BoNT, $A+D$ or $C 1+B$ ). Synaptosomes were depolarized by $50 \mathrm{mM} \mathrm{KCl}$ for $2 \mathrm{~min}$. Each experiment included three independent control stimulations (EGTA or Mock-treated) and three independent test stimulations (Ca or BoNT). Samples were processed in a conventional bottom-up proteomics workflow and phosphorylated peptides were enriched by metal-oxide chromatography $\left(\mathrm{TiO}_{2}\right)$. Replicates within one experiment were labeled with a set of TMT6 isobaric labeling reagents. A pooled peptide sample was fractionated by reversed-phased chromatography at basic $\mathrm{pH}$ and measured in a high-resolution quadrupole-orbitrap mass spectrometer. Reporter ion intensities were utilized to infer relative abundances of phosphorylated peptides. 

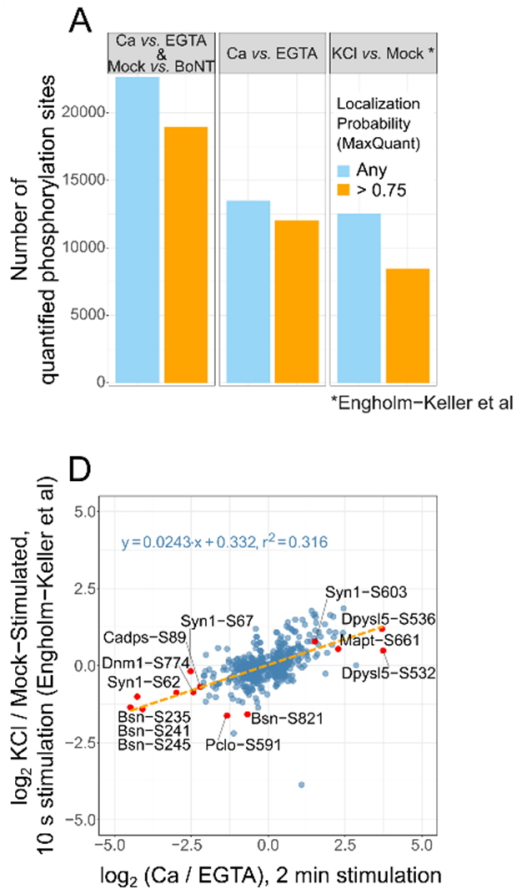

B

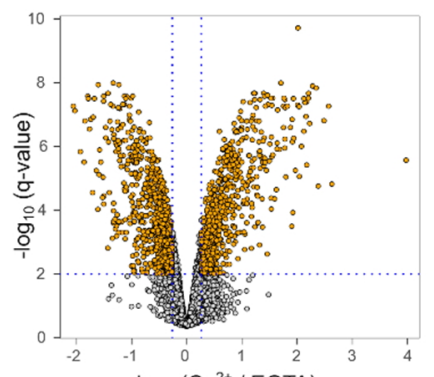

$\log _{2}\left(\mathrm{Ca}^{2+} /\right.$ EGTA $)$

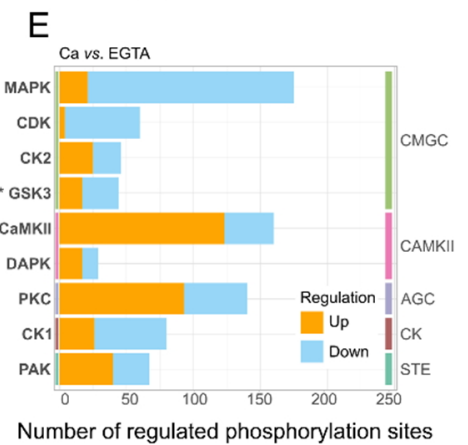

$\mathrm{C}$

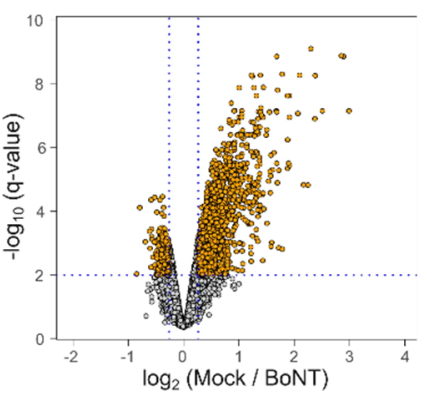

F

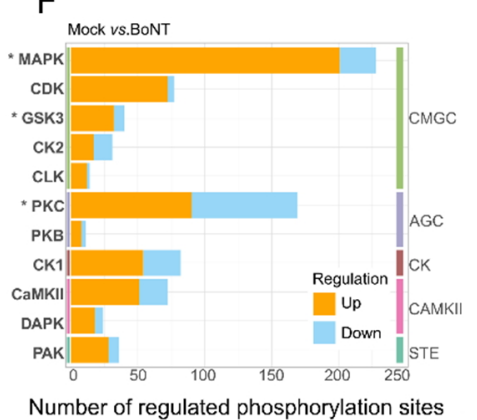

Figure 5.2: Substantial changes in synaptic phosphoproteome following depolarization and responsible kinase groups. (A) Number of quantified phosphorylation sites in Cavs. EGTA and Mock vs. BoNT experiments (panel 1), Ca vs. EGTA experiments only (panel 2) and recently published data set (EngholmKeller (352), panel 3). (B, C) volcano plots depicting - $\log _{10}\left(q\right.$-value) vs. $\log _{2}$ (intensity fold change) of phosphorylation sites quantified within Cavs. EGTA and Mock vs. BoNT experiments, respectively. (D) Comparison of $\log _{2}$ fold changes between our and Engholm-Keller et al (352) data sets. Selected phosphorylation sites are marked as official gene symbol with phosphorylated amino acid and position. (E, F) the stacked bar graphs show the total number of significantly regulated phosphorylation events in Ca vs. EGTA (e) or Mock vs. BoNT (f) experiments that can be a result of known (PhosphoSitePlus database (325)) or predicted (NetworKIN $(144,323))$ kinase-substrate interactions. Orange bars depict the number of phosphorylation events that show significant intensity increase in $\mathrm{Ca}^{2+}$ or Mock-treated synaptosomes versus respective control (EGTA or BoNT). Blue bars correspond to down-regulated events, respectively. The kinase classification (Netphorest Groups) follows one introduced by Netphorest (144) and uses its second-level group annotation. Colored vertical bars delineate the highest-level group in the kinase classification. Asterisks mark kinase groups which are predicted to control significantly more phosphorylation sites than expected as based on predicted kinase-substrate interactions in human proteome (Fisher's exact test Benjamini-Hochberg adjusted p-value < 0.01). Abbreviations: AGC protein kinase A, G, C kinase group; CaMKII calcium-calmodulin kinase 2; $C D K \sim$ cyclin-dependent kinase; $C K \sim$ casein kinase; $C K 1 \sim$ casein kinase 1; $C K 2 \sim$ casein kinase 2; $C L K \sim$ SRPK1 and CIk/Sty protein kinase; CMGC CDK, MAP, GSK, CDKL kinase group; DAPK death associated protein kinase; MAPK mitogen activated protein kinase; GSK3 glycogen synthase kinase 3; PAK p21 activated kinase; PKC protein kinase C; STE "sterile" serine/threonine protein kinases. 


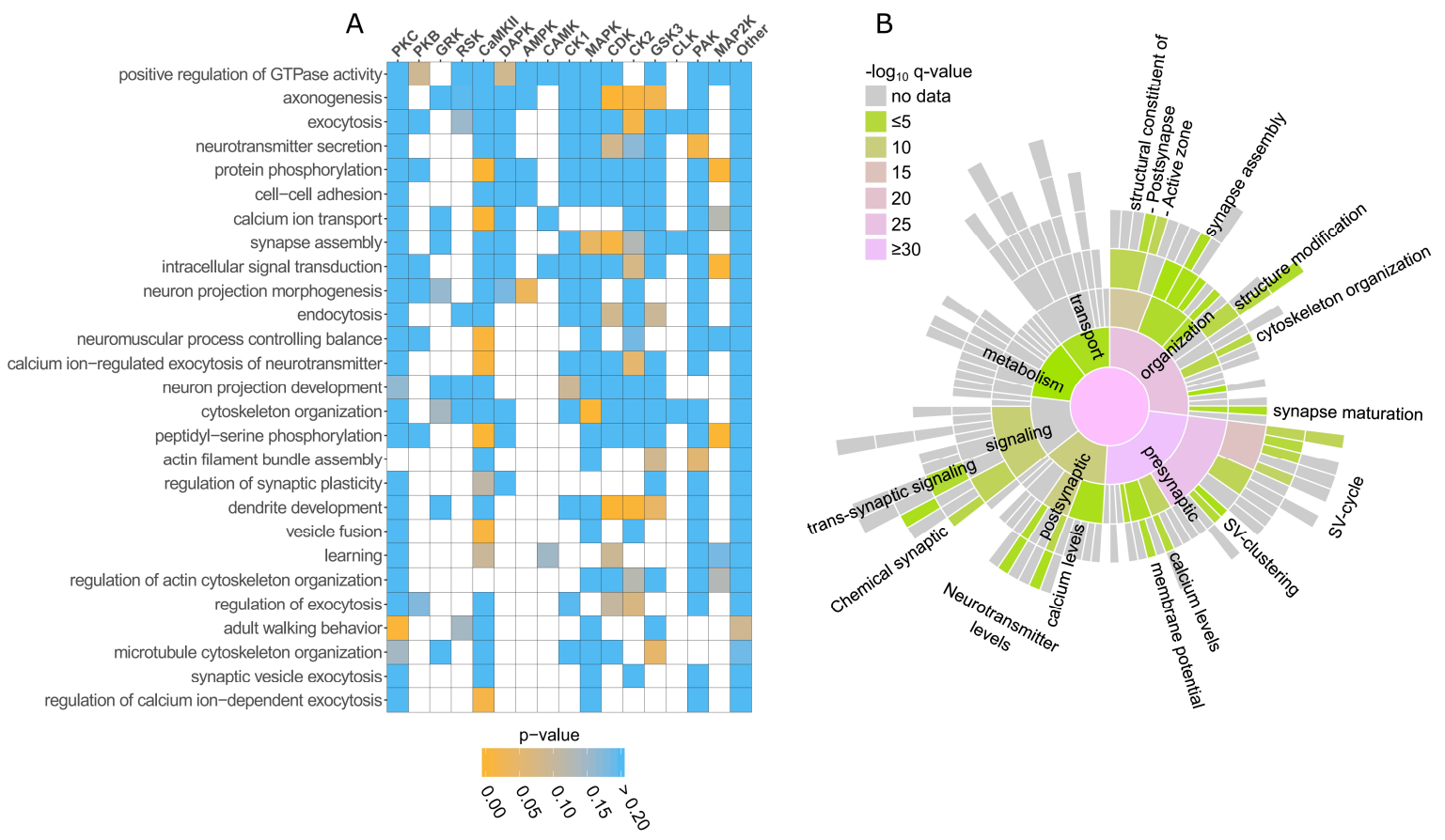

Figure 5.3: Functional annotation of proteins carrying regulated phosphorylation sites based on Ca vs. EGTA experiments. (A) Association between significantly enriched GO terms and kinase groups. The enriched biological function-GO terms (background: Rattus norvegicus, Benjamini-Hochberg adjusted p-value $<0.001)$ were derived using DAVID web service (368) for proteins carrying regulated phosphorylation sites. Overrepresentation of putative kinase-substrates relationships was tested for proteins annotated by each GO term. Frequencies of putative kinase-substrate relationships across all regulated sites for Ca vs. EGTA experiments were taken as a background. The color encodes raw p-values (Fisher's exact test). (B) Sunburst diagram showing enriched biological function terms based on the synapse-specific SynGO database annotation (324). The color encodes the significance of the enrichment (- $\log _{10}(q$-value)). Full list of enriched biological functions can be found in Table 5.S2. 


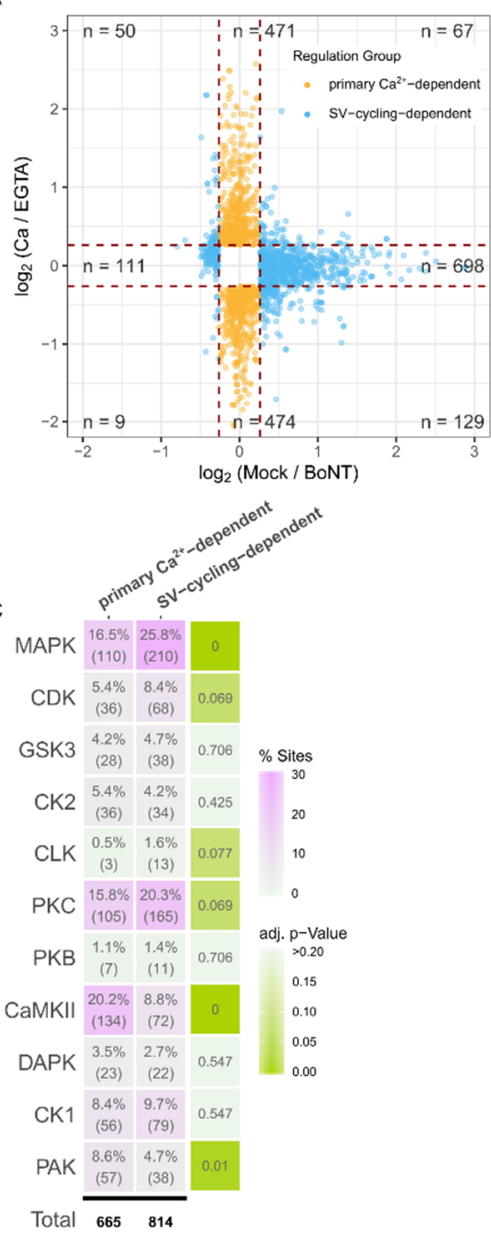

B

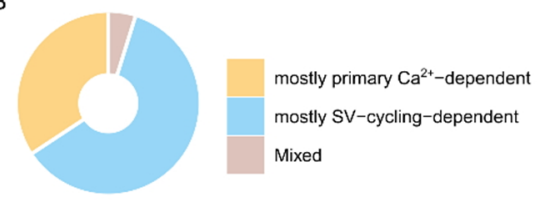

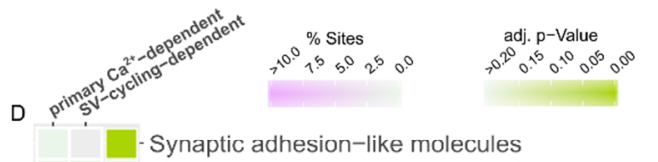

- Receptor-type tyrosine-protein phosphatases

- Voltage gated Potassium channels

- Presynaptic depolarization and calcium channel opening

- Potassium Channels

- GABA synthesis, release, reuptake and degradation

- Norepinephrine Neurotransmitter Release Cycle

- Acetylcholine Neurotransmitter Release Cycle

- Glutamate Neurotransmitter Release Cycle

- Neurexins and neuroligins

- Ras activation upon $\mathrm{Ca}^{2+}$ influx through NMDAR

- Long-term potentiation

- Unblocking of NMDAR, glutamate binding and activation

- Negative regulation of NMDAR-mediated neuronal transmission

- Protein-protein interactions at synapses

- Assembly and cell surface presentation of NMDA receptors

- CREB1 phosphorylation through NMDAR-mediated activation of RAS

- Glutamate binding, activation of AMPA receptors and synaptic plasticity

- Trafficking of AMPA receptors

- Post NMDAR activation events

- Activation of NMDAR and postsynaptic events

- Serotonin Neurotransmitter Release Cycle

- Dopamine Neurotransmitter Release Cycle

- Neurotransmitter release cycle

- Neurotransmitter receptors and postsynaptic signal transmission

- Transmission across Chemical Synapses

Figure 5.4: Dependence of regulated phosphorylation sites on calcium influx and SV cycling. (A) Categorization of phosphorylation events based on the magnitude of the $\log _{2}$ fold changes in Mock vs. BoNT experiment (x-axis) and $\log _{2}$ fold changes in Ca vs. EGTA experiment (y-axis). Only events quantified in both experiments and satisfying a $q$-value threshold of $<0.01$ are shown. The events are classified into two regulation groups: (i) "primary $\mathrm{Ca}^{2+}$-dependent" (sites with no significant or not-quantified SV cycling effect, i.e., absolute $\log _{2}\left(\right.$ Mock/BoNT) < 0.263 and absolute $\left.\log _{2}(\mathrm{Ca} / E G T A)>0.263\right)$, shown as orange dots; and (ii) "SV-cyclingdependent" (absolute $\left.\log _{2}(M o c k / B o N T) ~>0.263\right)$. (B) Proportion of proteins that can be organized in one of the regulation groups based on the categorization of phosphorylation sites they carry. Only sites that surpassed the FDR-threshold of $<1 \%$ were considered. If $>60 \%$ of the significantly regulated sites on a protein belong to one of the two regulation groups ("primary Ca $\mathrm{Ca}^{2+}$-dependent" or "SV-cycling-dependent"), the protein is categorized in "mostly primary Ca ${ }^{2+}$-dependent" or "mostly SV-cycling-dependent", respectively. (C) Enrichment of predicted kinase-substrate interactions for "primary $\mathrm{Ca}^{2+}$-dependent" and "SV-cycling-dependent" regulation groups. The left panel shows the percentage and the absolute number of sites in each category ( $x$-axis) that can be regulated by a particular kinase group ( $y$-axis). The number under the black line provides the total number of sites with predicted kinase-substrate relationship in each category. The right panel shows Benjamini-Hochberg adjusted p-values after a Fisher's exact test. An equal distribution of kinase-substrates relationships between the "primary $C a^{2+-d e p e n d e n t " ~ a n d ~ " S V-c y c l i n g-d e p e n d e n t " ~ c a t e g o r i e s ~ w a s ~ s e t ~ a s ~ a ~ n u l l ~ h y p o t h e s i s . ~}$ 
Figure 5.4: Dependence of regulated phosphorylation sites on calcium influx and SV cycling (continued from previous page). (D) Reactome pathway terms of neuronal origin (369). Reactome pathway terms of neuronal origin were selected based on their enrichment (Benjamini-Hochberg adjusted p-value <0.1) for all proteins carrying significantly regulated phosphorylation sites. Proportion of "primary $\mathrm{Ca}^{2+}$-dependent" and "SVcycling-dependent" sites of all "primary Ca ${ }^{2+-d e p e n d e n t " ~ o r ~ " S V-c y c l i n g-d e p e n d e n t " ~ s i t e s ~ p e r ~ f u n c t i o n a l ~ t e r m ~ i s ~}$ shown as color code (magenta). Fisher's exact test was applied to test equal distribution of "primary $\mathrm{Ca}^{2+}$ dependent" and "SV-cycling-dependent" sites for each term. Benjamini-Hochberg adjusted p-values are represented as a color code (green). Abbreviations: AMPAR stays for a-amino-3-hydroxy-5-methyl-4isoxazolepropionic acid receptor; CREB1 CAMP responsive element binding protein 1; GABA $\sim Y^{-}$ amminobutyric acid; NMDAR N-methyl-d-aspartate receptor; $R A S \sim$ rat sarcoma gene.

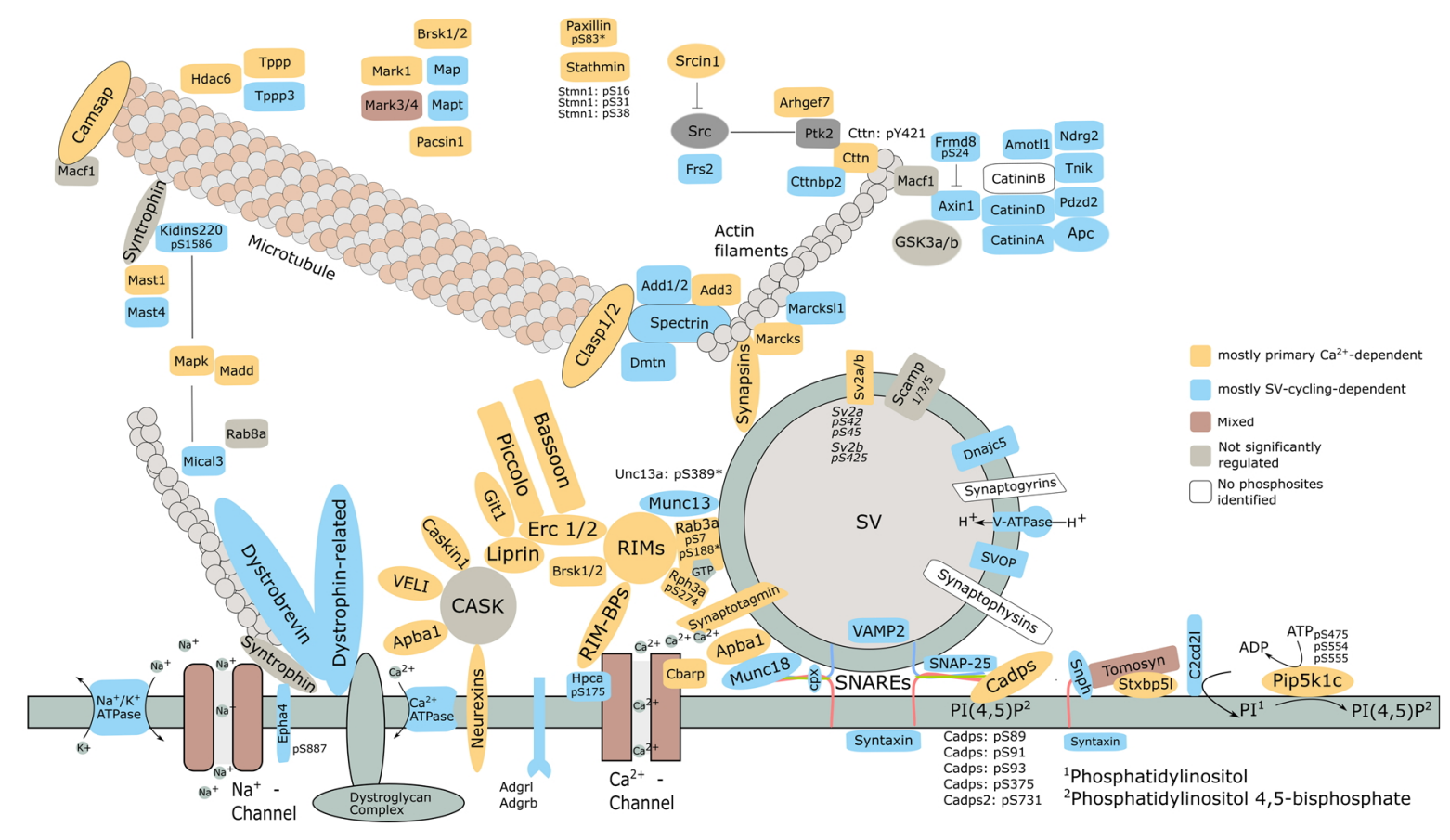

Figure 5.5: Schematic representation of presynaptic compartment with a single docked synaptic vesicle, plasma membrane and cytoskeleton elements, based on Chua et al (386). The represented proteins are categorized in "mostly primary Ca ${ }^{2+}$-dependent" (yellow), "mostly SV-cycling-dependent" (blue), "Mixed" (brown) based on the regulation type of the phosphorylation sites they carry. Proteins with no significantly regulated phosphorylation sites are represented in grey; proteins for which no first-class phosphorylation sites (MaxQuant localization probability < 0.75) were identified are represented in white. In most cases, an official gene symbol was used to name proteins, except: Bassoon stays for Bsn; Ca ${ }^{2+}$-ATPase Atp2b1, Atp2b2, Atp2b3; Camsap Camsap1, Camsap3; CatininA Cttna2; CatininD Ctnnd1, Ctnnd2; Dystrobrevin Dtnb; Dystrophin-related Drp2; Liprin Ppfia1, Ppfia2, Ppfia3, Ppfia4; Map Map1a, Map1b, Map1s, Map2, Map4, Map6, Map7d1, Map7d2; Mapk Mapk1, Mapk3; Na+/K+ ATPase Atp1a1, Atp1a2, Atp1a3; Na+-Channel Scn1a, Scn1b, Scn2b, Scn3a; Neurexins Nrxn1; Piccolo Pcl; RIM-BPs Rimbp1, Rimbp2; RIMs Rim1, Rim2; Spectrin Sptan1, Sptb, Sptbn1, Sptbn2, Sptbn4; Stathmin Stmn1; Synaptotagmin Syt1, Syt6, Syt9, Syt10, Syt17, Styl5, Esyt2; Syntaxin Stx1a, Stx1b, Stx12; Syntrophin Sntb2; Tomosyn Stxbp5; VAMP Vamp2; VATPase Atp6v1d; VELI Lin7a, Lin7b, Lin7c. Asterisks mark sites that do not meet significance criteria of $q-$ value < 0.01: Unc13a-pS389 (q-value $=0.013)$, Rab3a-pS188 (q-value $=0.012)$. 
A

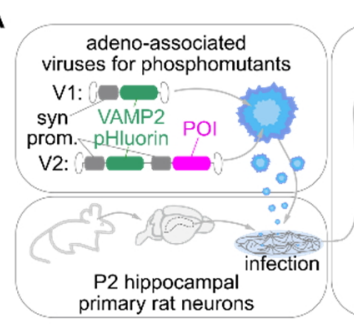

D
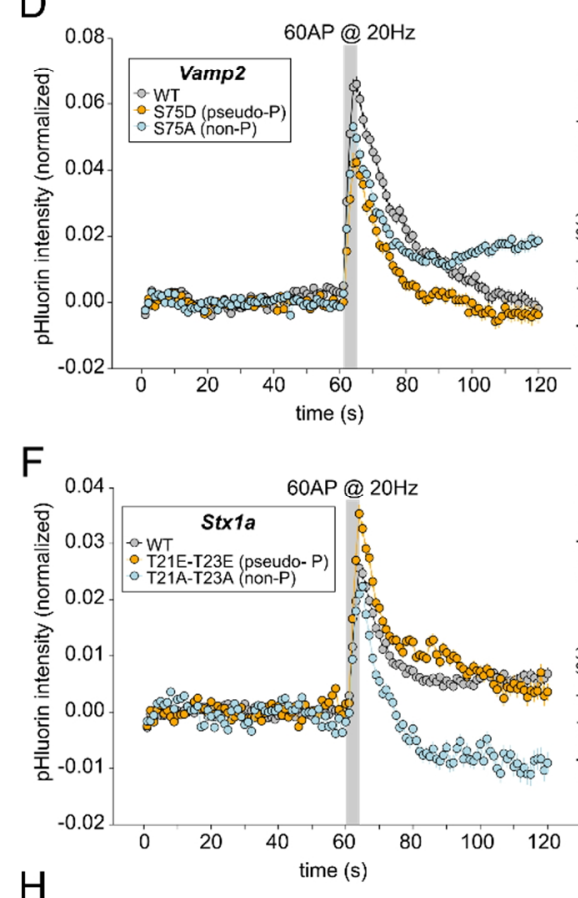

$\mathrm{H}$

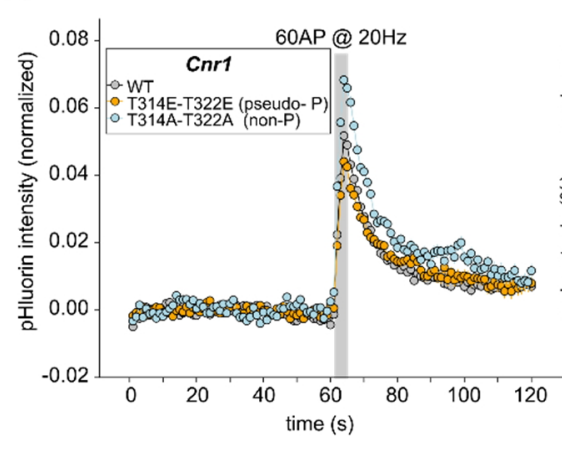

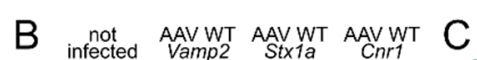
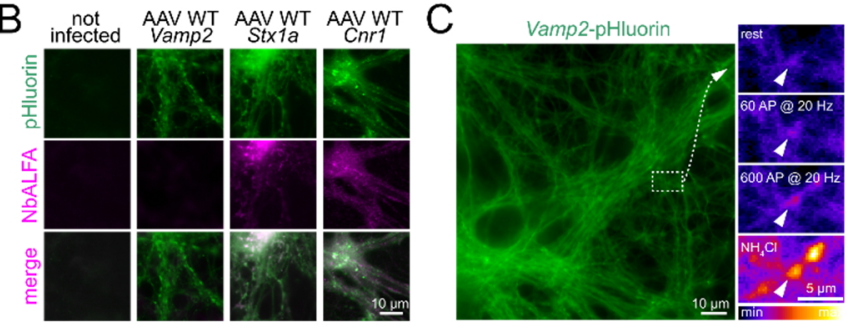

E
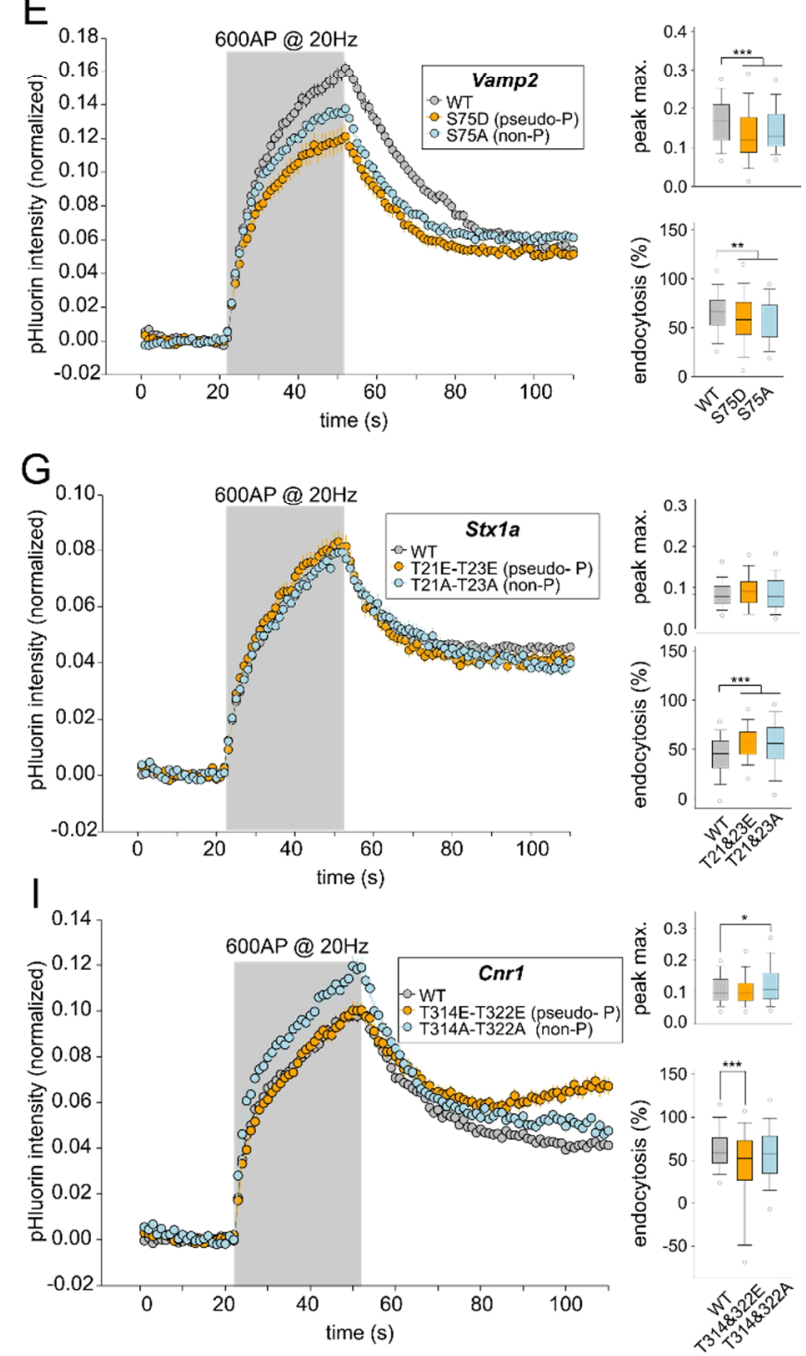

Figure 5.6: Phosphomimetic modulation of phosphorylation sites found differentially regulated in synaptobrevin (Vamp2), syntaxin-1 and cannabinoid receptor-1 affects $S V$ recycling properties (s. next page for details). 
Figure 5.6: Phosphomimetic modulation of phosphorylation sites found differentially regulated in synaptobrevin (Vamp2), syntaxin-1 and cannabinoid receptor-1 affects SV recycling properties (continued from previous page). (A) Experimental scheme. Two versions of the adeno associated viral (AAV) constructs were prepared (V1 and V2). V1 contained Vamp2 wild-type or the respective Vamp2 mutants (Vamp2-S75D and Vamp2-S75A) fused to the superecliptic ( $\mathrm{pH}$-dependent) green fluorescence protein (pHluorin; (375)). The expression of recombinant proteins is driven by the neuron-specific synapsin promoter. In these experiments the Vamp2-pHluorin is used both as the test protein and as a sensor for exo- and endocytosis. V2 contained both WT Vamp2-pHluorin (used as an exo-/endocytosis sensor) and the proteins of interest (POI; respectively Stx1a-WT, Stx1a-T21E-T23E; Stx1a-T21A-T23A; Cnr1-WT; Cnr1-T314E-T322E and Cnr1-T314A-T322A); see methods for details. To distinguish these proteins from the endogenous versions, an ALFA-tag sequence has been added (377). Mutants were expressed in rat primary hippocampal neurons and exo-/endocytosis was measured 4 days post infection (d.p.i.). In order to evaluate exo-/endocytosis, neurons were field-stimulated and changes in fluorescence were measured (375). (B) Immunofluorescence of infected neurons. The high efficiency of infection and the co-expression of the POls (Stx1a; Cnr1) is confirmed by immunofluorescence using a nanobody directed against the ALFA tag (NbALFA; (377)). Scale bar $10 \mu m$ (C) The basal fluorescence of Vamp2-pHluorin (left image) allows to identify single synaptic boutons (inset on the right). Following a defined number of action potentials (AP), SVs fuse with the plasma membrane (middle insets on the right). Upon fusion, the intravesicular $\mathrm{pH}$ increases, resulting in higher pHluorin fluorescence intensity. Ammonium chloride $\left(\mathrm{NH}_{4} \mathrm{Cl}\right)$ is used at the end of each experiment to reveal the localization of SVs. Scale bars: $10 \mu \mathrm{m}$ (left image) and $5 \mu \mathrm{m}$ (right insets). (D, E) Both the phosphomimetic and the phosphorylation-null mutants of Vamp2 showed decreased exocytosis, for both short and long train of stimuli, respectively 60 and 600 action potentials (APS). The maximum peak intensity was significantly lower in both stimuli $(P<0.001)$. The fraction of endocytosed Vamp2 (measured at 30 seconds after the stimulation, as \% of the exocytosed Vamp2) was also decreased, especially for the longer stimulus. ( $\boldsymbol{F}$ and $\boldsymbol{G})$ The phosphomimetic mutant of Stx1a (T21-T23) enhanced exocytosis for short stimuli, while the phosphorylation-null mutant left exocytosis unaffected, while enhancing endocytosis. Under long stimuli the enhancement of exocytosis was no longer significant, but the increase in endocytosis was, for both phosphomimetic and phosphorylation-null mutants. (H and I) The phosphomimetic mutants of Cnr1 (T314E-T322E) reduced exocytosis during short stimuli, while the nonphosphorylatable variant increased exocytosis. The latter effect was also observed during long stimuli. Neither of the Cnr1 variants had a substantial effect on endocytosis. In all panels statistical differences were tested relying on Kruskal-Wallis tests, followed by Tukey-Kramer post-hoc tests ${ }^{*} P<0.05$, ${ }^{* *} P<0.01$ and ${ }^{* * *} P<$ 0.001). 


\subsection{Discussion}

In this study, we have used isolated nerve terminals as a model to differentiate phosphorylation changes that are induced by calcium influx alone from those that in addition require active endo-exocytotic SV cycling. Surprisingly, we found that inhibition of exocytosis by BoNT had a profound effect on the phosphoproteome despite unchanged calcium influx. These findings reveal complex signaling cascades during which regulatory changes in protein phosphorylation are not only dependent on the activation of kinase/phosphatase networks but also on the progression of vesicular trafficking.

By using isobaric tandem-mass tags for labeling of phosphorylated peptides, we were able to substantially increase the phosphoproteome coverage and to achieve reliable quantification for thousands of phosphorylation sites. Despite differences in the synaptosome preparation (Ficoll-purified synaptosomes vs. crude synaptosomal pellet) and stimulation $(50 \mathrm{mM} \mathrm{KCl}$ for $2 \mathrm{~min} v s .75 \mathrm{mM} \mathrm{KCl}$ for $10 \mathrm{~s}$ ), many of the regulated phosphorylation sites observed by us are in excellent agreement with earlier results from another laboratory (Figure 5.2D) (352). Our data show higher magnitudes of changes, which are probably a consequence of the prolonged stimulation time used in our study. The most drastic changes were observed for pacsin (Pacs1-S517, $\log _{2}$ fold change of 3.98), thymosin beta (Tmsb4x-T23, 2.63), ADP-ribosylation factor GTPase activating protein 1 (Arfgap1-S247 2.57), calcium/calmodulin-dependent protein kinase type II subunit delta (Camk2d-S315/S319, 2.49), protein Bassoon (Bsn-S1354, -2.06), septin 5 (Sept5-S17, 1.93), and RIM-binding protein 2 (Rimbp2-S554, -1.86). We also confirm phosphorylation changes in response to stimulation that were reported earlier for synapsin-1, dynamin-1, Tau protein, as well as the multi-site phosphorylations of the giant active zone proteins Bassoon and Piccolo (242, 338, 341, 346).

It seems that several proteins known to function at the postsynapse respond to potassium chloride stimulation and BoNT treatment in synaptosomes (e.g., Shank1, 2, 3; components of NMDAR, Grin1, Grin2a, Grin2b). It would be of interest to investigate whether these proteins are also present at measurable levels in the presynapse and whether they are affected by synaptic activity. Although synaptosomal preparations are known contain fragments of the postsynaptic membrane (97), they usually do not retain metabolic activity, in contrast to functionally active membrane-enclosed nerve terminals that can maintain the ATP levels needed for phosphorylation $(98,99,115,360)$.

Clearly, the primary trigger for the phosphorylation changes is calcium that enters synaptosomes following potassium-induced depolarization. Calcium directly regulates the activity of kinases and phosphatases, including CaMKII, PKC, calcineurin, PP1 etc. (243, 
337, 379, 389). Accordingly, we observe increased phosphorylation of specific sites on CaMKII and MAP kinases that are known to result in activation of the kinases as well as enrichment of possible substrates for CaMKII, MAPK, and GSK3 kinases among regulated phosphorylation sites.

However, the fact that selective inhibition of calcium-activated SV cycling results in major changes of the phosphoproteome documents that the activation status of the kinase/phosphatase networks is directly dependent on the progression of the exoendocytotic cycle. In most cases, BoNT treatment inhibited phosphorylation, and these results are in line with a very early study that used BoNT-treated synaptosomes from the electric organ of Torpedo marmorata and radioactive phosphate for protein labeling (390). Interestingly, phosphorylation events known to be directly regulated by some of the major calcium-dependent kinases and phosphatases such as those on synapsin-1 and dynamin-1, as well as on many of the predicted CaMKII-phosphorylation sites, were almost unaffected by the BoNT treatment. Conversely, predicted sites of MAPK, PKC, and CDK were affected more strongly by the BoNT-treatment. These observations support the view that the activity of MAPK is dependent on active SV cycling, resulting in the phosphorylation of delta catenin, metabotropic glutamate receptor and other proteins in the synapse (391394). Indeed, we observe SV-cycling-dependent phosphorylation changes not only in catenin-delta (Cttnd2) but also in other proteins that might take part in the Wnt pathway such as Apc, Amotl1, and Tnik. Given the connection between the Wnt pathway and the regulation of cell-cell contacts and cytoskeletal elements, it is not surprising that many cytoskeleton- and cell-adhesion-related proteins contain BoNT-responsive phosphorylation sites (e.g., spectrin beta, adducin alpha and beta, neurofascin, neuronal cell adhesion molecule). It is tempting to speculate that these phosphorylation changes represent the activation of pathways directed at increasing synaptic strength (most importantly LTP) by mechanisms such as size increase of the synaptic contact zone, enlargement of the presynaptic bouton and adaptations of the presynaptic cytoskeleton to increase the efficiency of SV cycling (395-398).

How can the wide-ranging neurotoxin effects be explained when considering the extraordinary specificity of the SNARE-proteases, which should have no direct effect on kinases or phosphatases? Given that toxin treatment causes dephosphorylation of many substrates, the observed effect can be explained either by activation of phosphatase or by suppression of kinase activities that are not caused directly by calcium but by changes in proteins that are themselves regulated by the activity of the SV cycle and in turn control kinases or phosphatases. Moreover, it is conceivable that the availability of phosphorylation sites on substrate proteins is coupled to its "activity cycle". Trafficking steps such as SV 
docking, priming, fusion, coat formation, fission, and SV movement are associated with numerous changes in protein conformation and the dynamic assembly and disassembly of multimolecular complexes. Thus, it is to be expected that phosphorylation site accessibility is dependent on these dynamic changes, many of which are likely to be arrested following inhibition of the SV cycle.

An interesting example is given by the protein phosphatase-1 (PP1) whose postsynaptic activation appears to be dependent on synaptic activity (237). Neurabin-1 (Ppp1r9a) can play a crucial role in this, by recruiting PP1 to the synapse by regulating its binding to actin filaments which impacts PP1 activity $(399,400)$. Our observations suggest that PP1 undergoes similar activity-dependent regulation also in the presynapse as we observe "SVcycling-dependent" sites within the actin-binding region of Neurabin-1. It is tempting to speculate whether SV-cycling-dependent activity of PP1 or other phosphatases can be opposed by $\mathrm{Ca}^{2+}$-activated kinases such as CaMKII, MAPK1, MAPK3. This would explain reduced intensities of predicted MAPK-substrates in Ca/EGTA experiments despite apparent activation of MAPK following $\mathrm{K}^{+}$-induced depolarization and $\mathrm{Ca}^{2+}$-influx. Furthermore, it would explain why many phosphosites appear significantly regulated when comparing mock- with BoNT-treated synaptosomes, but do not show significant changes in $\mathrm{Ca} / \mathrm{EGTA}$ experiments, since comparison of $\mathrm{Ca}^{2+}$ with EGTA conditions reveals a composite effect of $\mathrm{Ca}^{2+}$ influx and SV cycling on protein phosphorylation.

Experiments involving botulinum neurotoxins also led us to the discovery of phosphorylation sites on the core SNARE proteins, sites that otherwise did not appear significant. We showed that these sites can affect exo- and endocytosis in hippocampal neurons under stimulation. One possible mechanism is the impaired interaction with Munc18-1, which can facilitate SNARE zippering and thereby promote SV fusion with the plasma membrane (356). The changes of endocytosis are possibly a consequence of altered exocytosis. In contrast to many other BoNT-responsive phosphorylation sites, the phosphorylation sites on Vamp2 and Stx1a that we examined showed increased phosphorylation following toxin treatment, suggesting that the phosphorylation is due to adaptive changes in the synapse rather than a result of SNARE-cleavage by BoNT.

In contrast to the phosphomimetic and nonphosphorylatable variants of the SNARE proteins, the corresponding variants of cannabinoid recepor-1 (Cnr1) had an effect only on exocytosis in cultured hippocampal neurons. The effect can be also linked through the activation of MAPK to Munc18 phosphorylation and regulation of SNARE assembly (387). Similarly, the intensity of phosphorylation of T314/T321 sites was increased following toxin treatment. Although Vamp2-S75, Stx1a-S21/S23, Cnr1-T314/T321 phosphorylation sites were identified in previous studies $(356,357,401,402)$, it is the first time, to the best of our 
knowledge, that phosphorylation of these sites has been shown to have an impact on the exo- and endocytosis in the context of stimulation experiments.

Interestingly, we also found phosphorylation sites on Munc18 that are regulated in SVcycling-dependent manner, namely Stxbp1-S313 and Stxbp1-S506. Both sites were significantly downregulated in the BoNT-treated samples. Although not much known about S506, S313 site was previously described as a possible target of PKC and that its phosphorylation can negatively affect interaction of Munc18 with syntaxin-1 $(187,358)$.

A remarkable aspect of the synapse phosphoproteome regulation has recently been addressed by $F$. Brüning et al. (403). Their analysis shows that phosphorylation sites on many synaptic proteins undergo oscillations during the course of the day. Interestingly, a few of these sites are shown to be regulated upon depolarization by potassium chloride and treatment with botulinum neurotoxins, including phosphorylation sites on proteins previously discussed: Cnr1, Vamp2, Stx1a, Cttnd2, Add1, Add2, Sptbn1, Sptbn4, Kcnb1, Kcnd2, Hcn2, Kcnma1, Tnik, Apc, Camk2a, etc. This also suggests that, despite the caveat that potassium-induced depolarization is non-physiological (352), it allows for identifying regulated phosphorylation sites that are of high functional relevance for normal synapse physiology.

Despite major progress, many aspects of synapse regulation remain to be uncovered. Our study has added another layer of complexity by showing how intricately the phosphorylation-dephosphorylation cascade in the synapse is linked to SV cycling. We believe that the results presented here provide a firm basis for future investigations about how synaptic activity and synaptic plasticity is regulated at the molecular level.

\subsection{Acknowledgments}

We sincerely thank Thomas Gundlach and Sascha Krause for their assistance in animal experiments; Sofia Ainatzi and Dr. Momchil Ninov for help in synaptosome preparations; Monika Raabe, Uwe Plessmann, Dr. Sabine König, and Dr. Ralf Pflanz for technical support; Heiko Ludwig for launching of the shiny-server.

\subsection{Funding}

S.B., S.O.R., H.U., and R.J. are funded by the Deutsche Forschungsgemeinschaft through collaborative research center SFB1286, projects A08, A03, Z02 and Z03.

\subsection{Author Contributions}

R.J., H.U., and I.S. conceptualized the study; E.F. conceptualized and performed phosphomimetic studies in neurons; I.S. performed synaptosomal experiments under the 
supervision of K.T.P., H.U., and R.J.; I.S. analyzed data; M.F. and S.B. contributed to the bioinformatics analysis; initial draft was written by I.S. and H.U., reviewed and edited by H.U, R.J., E.F., S.O.R., and K.T.P.

\subsection{Conflict of interest}

The authors declare that they have no conflicts of interest with the contents of this article.

\subsection{Data availability}

The mass spectrometry data have been deposited to the ProteomeXchange Consortium via the PRIDE(404) partner repository with the dataset identifier PXD020564.

$R$ scripts used for data analysis are available at github: https://github.com/lvanSilbern/2021 Silbern etal Synapt BoNT KCl.

We also provide a visualization tool in a shiny-app format. The source data and code are available at github: https://github.com/IvanSilbern/2021 Silbern etal ShinyApp. The app is deployed and accessible at: https://s1608-shiny.mpibpc.mpg.de.

\subsection{Overview of the supplementary material (supplementary tables, figures and datasets)}

1. 20200901_SupplData_Notes_Figures_Tables.pdf Supplementary Information

2. "SupplData01_Phosphosite_Intensities.xlsx" Phosphorylation site intensities used for the assessment of differential protein phosphorylation in Ca vs. EGTA and Mock vs. BoNT experiments

3. "SupplData02_Proteingroups_Intensities_MockBoNT_AD.xIsx"

Protein group intensities used for the assessment of the differential protein content in depolarized synaptosomes following Mock $(A+D)$ or BoNT $(A+D)$ treatment.

4. "SupplData03_Proteingroups_Intensities_MockBoNT_CB.xlsx"

Protein group intensities used for the assessment of the differential protein content in depolarized synaptosomes following Mock $(\mathrm{C} 1+\mathrm{B})$ or BoNT $(\mathrm{C} 1+\mathrm{B})$ treatment.

5. "SupplData04_Protein_classification.xlsx"

Classification of proteins based on the classification of the regulated phosphorylation sites they carry.

6. "SuppIData05_Phosphoproteins_regulated_CaEGTA.pdf"

Representation of $\log _{2}$ fold changes of the phosphorylation site intensities in depolarized synaptosomes following incubation with $\mathrm{Ca}^{2+}$ or EGTA per protein sequence. Only protein with significantly regulated phosphorylation sites are shown. 
7. "SupplData06_Phosphoproteins_regulated_MockBoNT.pdf"

Representation of $\log _{2}$ fold changes of the phosphorylation site intensities in depolarized synaptosomes following incubation with Mock or BoNT per protein sequence. Only protein with significantly regulated phosphorylation sites are shown. 


\subsection{Supplemental Materials}

\subsubsection{Table of contents:}

\section{Supplementary Notes:}

Supplementary Note 1: C. botulinum cell culture supernatant activity assessment

\section{Supplementary Figures:}

Figure 5.S1: Glutamate release assay and $\mathrm{Ca}^{2+-}$ levels in cytoplasm.

Figure 5.S2: Kinase groups that might be responsible for the observed phosphorylation changes.

Figure 5.S3: Dependence of phosphorylation sites on calcium influx and SV cycling.

Figure 5.S4: Site occupancy assessment.

Figure 5.S5: Changes in phosphorylation site intensities for Calcium/calmodulin-dependent kinase 2 alpha, beta, gamma, and delta.

Figure 5.S6: Changes in phosphorylation site intensities for Mitogen-activated protein kinase 1,3 , and Protein kinase $\mathrm{C}$ beta.

Figure 5.S7: Phosphatases and their putative substrates

Figure 5.S8: Manual annotation of proteins carrying regulated phosphorylation sites.

Figure 5.S9: Changes in phosphorylation site intensities for Regulating synaptic membrane exocytosis (RIM) protein 1, 2, and RIM-binding protein 2

Figure 5.S10: Changes in phosphorylation site intensities for Dynamin 1, Clathrin coat assembly protein AP180, and Amphiphysin.

Figure 5.S11: Changes in phosphorylation site intensities for Synapsin 1, 2, and 3.

Figure 5.S12: Changes in phosphorylation site intensities for Spectrin beta chain.

Figure 5.S13: Changes in phosphorylation site intensities for Adducin alpha, beta, and gamma.

Figure 5.S14: Changes in phosphorylation site intensities for Potassium voltage-gated channel subfamily A member 2 , and subfamily B member 1 and 2 .

Figure 5.S15: Changes in phosphorylation site intensities for Potassium voltage-gated channel subfamily D member 2, subfamily KQT member 2, Potassium/sodium hyperpolarization-activated cyclic nucleotide-gated channel 1 and 2.

Figure 5.S16: Regulated phosphorylation sites on selected proteins 
Figure 5.S17: Changes in phosphorylation site intensities for Calcium-activated potassium channel subunit alpha 1 , Potassium voltage-gated channel subfamily $\mathrm{H}$ member 1 , and Potassium voltage-gated channel subfamily $Q$ member 5 .

Figure 5.S18: Changes in phosphorylation site intensities for Syntaxin 1A, Vesicleassociated membrane protein 2, Syntaxin-binding protein 1, and Cannabinoid receptor 1.

Figure 5.S19: Proteomic profiling of $C$. botulinum cell culture supernatants.

Figure 5.S20: Phosphoproteome analysis of BoNT-treated HeLa nuclear extract.

Figure 5.S21: Proteomics analysis of Mock- or BoNT-treated synaptosomes.

\section{Supplementary Tables:}

Table 5.S1: Number of regulated phosphorylation events per predicted kinase group in Ca vs. EGTA experiment.

Table 5.S2: Enriched gene ontology biological function terms based on synapse-specific SynGO database

Table 5.S3 Number of regulated phosphorylation events per predicted kinase group in Mock vs. BoNT experiment.

Table 5.S4: Number of regulated phosphorylation events per predicted kinase group in Ca vs. EGTA and Mock vs. BoNT experiments.

\section{Supplementary Data:}

"SupplData01_Phosphosite_Intensities.xIsx"

Phosphorylation site intensities used for the assessment of differential protein phosphorylation in Ca vs. EGTA and Mock vs. BoNT experiments

"SupplData02_Proteingroups_Intensities_MockBoNT_AD.xlsx"

Protein group intensities used for the assessment of the differential protein content in depolarized synaptosomes following Mock $(A+D)$ or BoNT $(A+D)$ treatment.

"SupplData03_Proteingroups_Intensities_MockBoNT_CB.xlsx"

Protein group intensities used for the assessment of the differential protein content in depolarized synaptosomes following Mock $(\mathrm{C} 1+\mathrm{B})$ or BoNT $(\mathrm{C} 1+\mathrm{B})$ treatment.

"SupplData04_Protein_classification.xlsx"

Classification of proteins based on the classification of the regulated phosphorylation sites they carry.

"SupplData05_Phosphoproteins_regulated_CaEGTA.pdf"

Representation of $\log _{2}$ fold changes of the phosphorylation site intensities per protein 
sequence in depolarized synaptosomes following incubation with $\mathrm{Ca}^{2+}$ or EGTA. Only proteins with significantly regulated phosphorylation sites are shown.

"SupplData06_Phosphoproteins_regulated_MockBoNT.pdf"

Representation of $\log _{2}$ fold changes of the phosphorylation site intensities per protein sequence in depolarized synaptosomes following incubation with Mock or BoNT. Only proteins with significantly regulated phosphorylation sites are shown.

\subsubsection{Supplementary Note}

\section{C. botulinum cell culture supernatant activity assessment}

BoNT-treatment of synaptosomes negatively affected glutamate release and did not compromise the increase in cytoplasmatic $\mathrm{Ca}^{2+}$-concentration following $\mathrm{KCl}$-stimulation (Figure 5.S1B, C). Proteome profiling of the $C$. botulinum cell culture supernatants used in the study (Figure 5.S19) identified BoNTs among the most abundant proteins. However, we could detect presence of a putative protease and two putative phosphatases in the cell culture supernatants as well. To exclude unspecific phosphatase activity, we treated nuclear extract of HeLa cells with a combination of BoNT/A-D and subsequently performed a total quantitative phosphoproteome analysis. The analysis did not reveal significant phosphatase activity that could be attributed to the treatment with $C$. botulinum cell culture supernatants (Figure 5.S20). To exclude unspecific protease activity, we analyzed peptides in the unbound fraction following $\mathrm{TiO}_{2}$.enrichment of phosphorylated peptides. The analysis did not show strong changes in protein intensities in synaptosomes following BoNT-treatment (Figure 5.S21). Although the intensities of some proteins seem to be slightly affected by BoNT/C1+ BoNT/B treatment, the observed mild effect is not at all comparable with the extreme effect that BoNT-treatment has on the phosphorylation of synaptic proteins. Neither have we seen a significant reduction in the level of SNARE proteins (Figure 5.S4E, F). We, therefore, hypothesized that only a minor pool of SNARE proteins is cleaved following BoNT-treatment. SNARE proteins that reside inside non-functional synaptosomes would be protected from cleavage and mask the reduction in SNARE-proteins. We also cannot exclude some non-proteolytic effects of BoNT since such effects were previously discussed (405). Important for this study, BoNT-treatment dampened glutamate release without compromising calcium influx, which allowed us to investigate calcium and SV cycling effects on protein phosphorylation in synaptosomes. 


\subsubsection{Supplementary Figures}

A

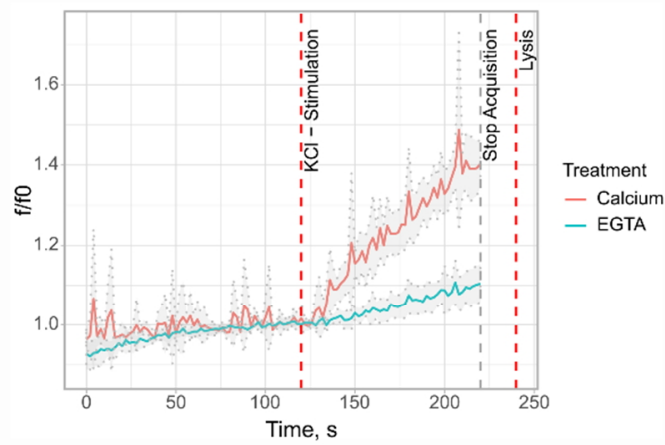

B

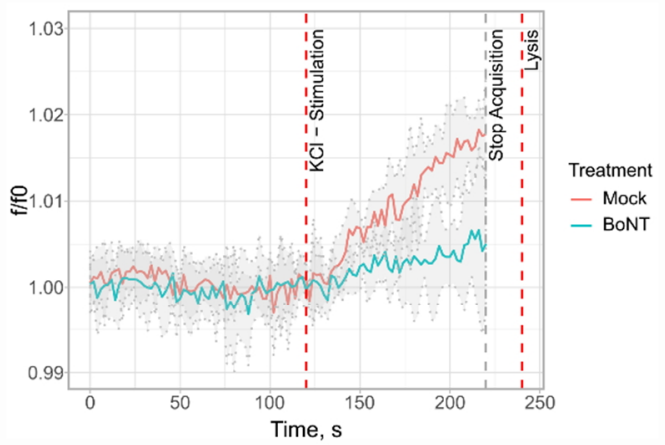

C

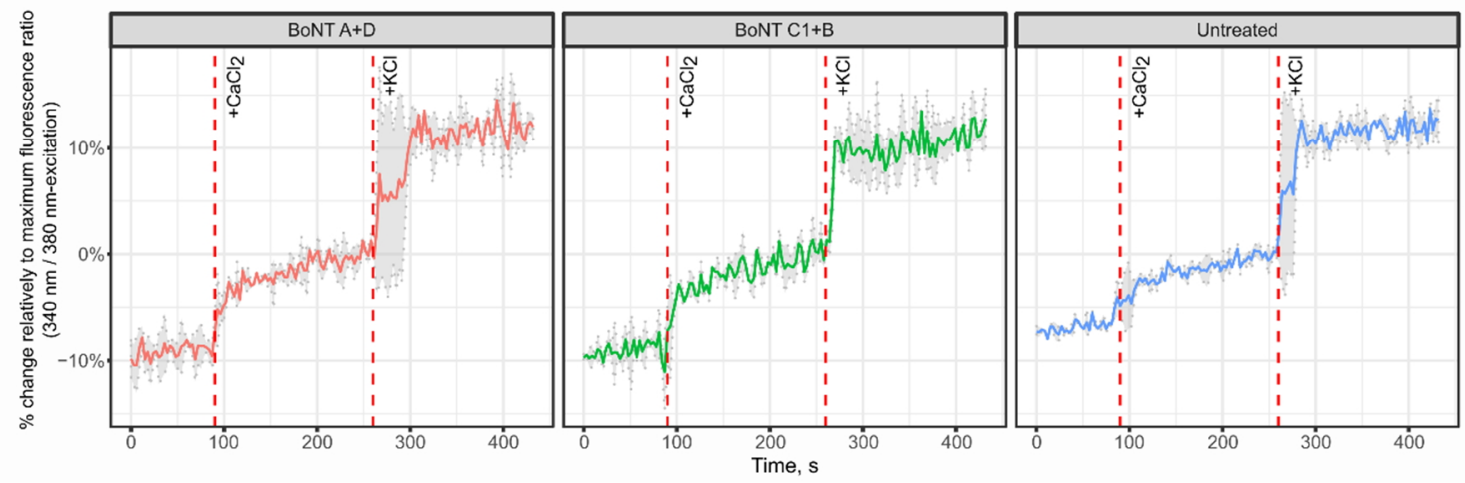

Figure 5.S1 Glutamate release assay and $\mathrm{Ca}^{2+}$-levels in cytoplasm. (A) Glutamate release following depolarization with potassium chloride in the presence of $\mathrm{Ca}^{2+}$ ions or EGTA. X-axis: time in seconds, Y-axis: normalized fluorescence intensity ( $\mathrm{fO}=$ fluorescence intensity before $\mathrm{KCl}$-stimulation). Red line: synaptosomes stimulated in the presence of $\mathrm{Ca}^{2+}$ (average of 6 replicates). Green line: synaptosomes stimulated in the presence of EGTA (average of 6 replicates). Shaded grey area corresponds to the standard deviation at each time point. (B) Glutamate release of Mock- or BoNT-treated synaptosomes. X-axis: time in seconds, $Y$-axis: fluorescent intensity (f) normalized by fluorescence intensity before $\mathrm{KCl}$-stimulation (fO). Red line: mock-treated synaptosomes (average of 6 replicates). Green line: BoNT-treated synaptosomes (average of 6 replicates). Shaded grey area corresponds to the standard deviation at each time point. Due to the presence of an interfering agent in the cell culture supernatants of $C$. botulinum, the magnitude of the fluorescence is reduced and experiences increased fluctuations. (C) Cytoplasmatic calcium levels in BoNT or untreated synaptosomes. Synaptosomes were pre-incubated with BoNT/A and BoNT/D, BoNT/C1 and BoNT/B or an equal volume of sodium-containing buffer (untreated). Changes in cytoplasmic calcium levels were monitored with Fura2-AM dye using an approach previously introduced elsewhere (118). Fluorescence ratios were calculated by dividing fluorescence signal at $340 \mathrm{~nm}$ excitation wavelength by the signal at $380 \mathrm{~nm}$ excitation. Fluorescence ratio was normalized by the maximum fluorescence ratio after synaptosomal lysis. The normalized fluorescence ratio before $\mathrm{KCl}$ stimulation was subtracted to reveal proportional changes in cytoplasmatic $\mathrm{Ca}^{2+-l e v e l s . ~}$ 
A

Netphorest Groups

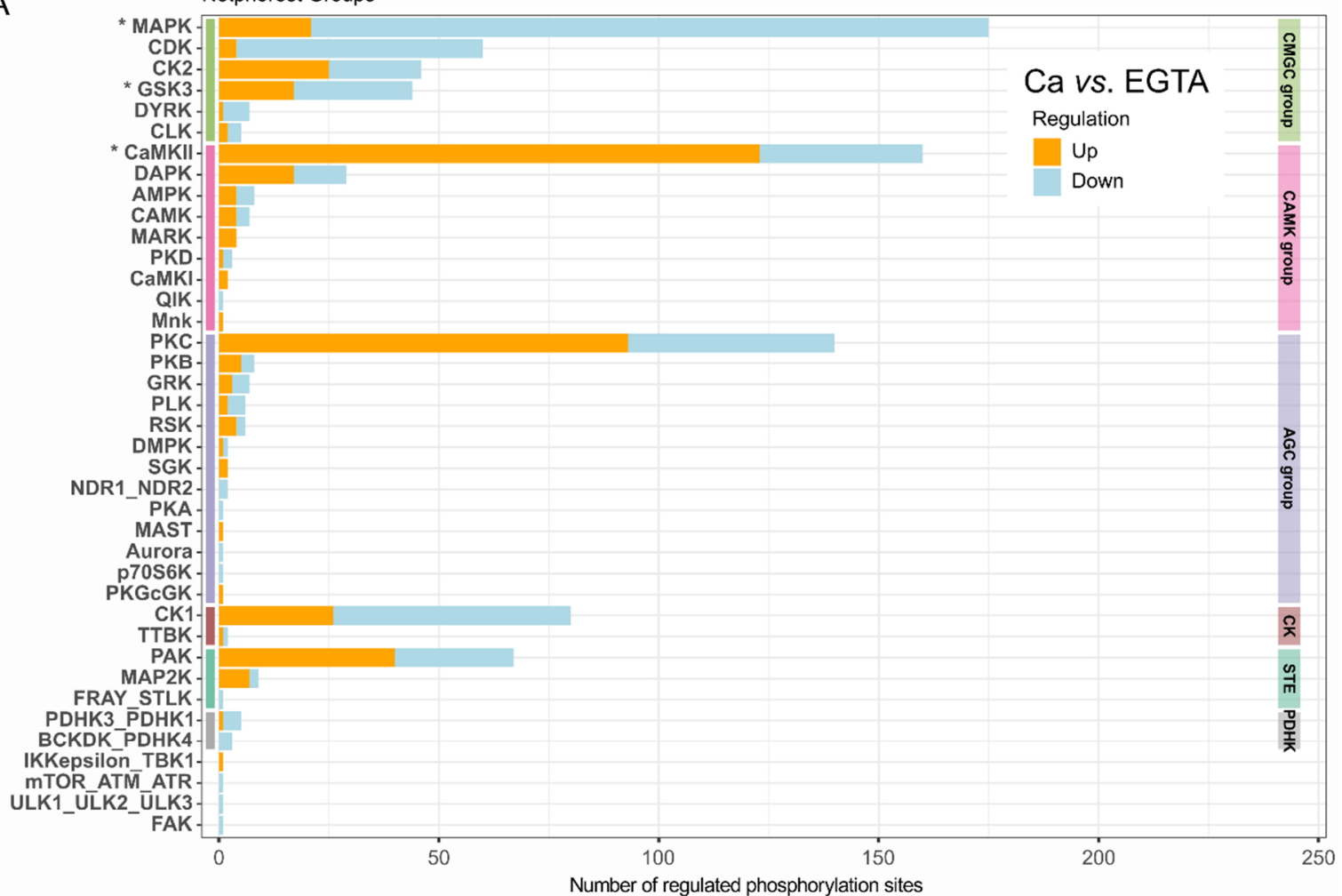

B

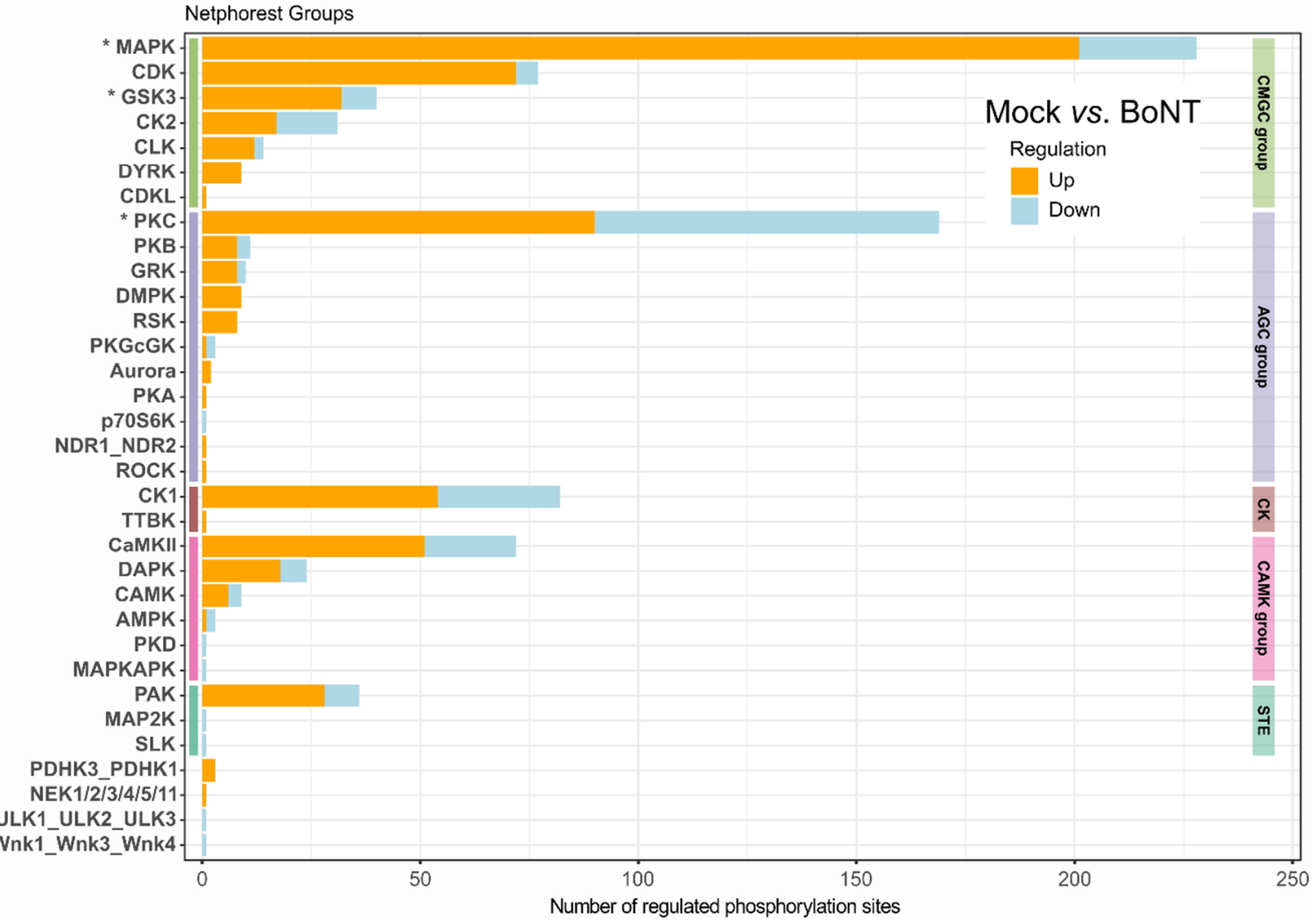

Figure 5.S2: Kinase groups that might be responsible for the observed phosphorylation changes (s. next page for details). 
Figure 5.S2: Kinase groups that might be responsible for the observed phosphorylation changes. $(\boldsymbol{A}, \boldsymbol{B})$ Extended versions of Figure 5.3E and F, respectively. The stacked bar graphs show the total number of significantly regulated phosphorylation events in Ca vs. EGTA (a) or Mock vs. BoNT (b) experiments that can be a result of known (PhosphoSitePlus database (325)) or predicted (NetworKIN $(144,323)$ ) kinase-substrate interactions. Orange bars depict the number of phosphorylation events that show significant intensity increase in $\mathrm{Ca}^{2+}$ or Mock-treated synaptosomes versus respective control (EGTA or BoNT). Blue bars correspond to down-regulated sites, respectively. The kinase classification (Netphorest Groups) follows one introduced by Netphorest (144) and uses its second-level group annotation. Colored horizontal bars delineate the highestlevel group in the kinase classification. Asterisks mark kinase groups which are predicted to control significantly more phosphorylation sites than expected as based on predicted kinase-substrate interactions in human proteome (Fisher's exact test Benjamini-Hochberg adjusted p-value <0.01). Abbreviations: AMPK, AMPactivated protein kinase; BCKDK, branched chain ketoacid dehydrogenase kinase; CaMKII calcium-calmodulin kinase 2; $C D K$, cyclin-dependent kinase; CDKL, cyclin-dependent-like kinase; CK1, casein kinase 1; CK2, casein kinase 2; CLK, SRPK1 and Clk/Sty protein kinase; DAPK, death associated protein kinase; DMPK, DYRK, dual-specificity tyrosine (Y) phosphorylation-regulated kinase; FAK, focal adhesion kinase GRK, G protein coupled receptor kinase; GSK3, glycogen synthase kinase 3; MAPK, mitogen activated protein kinase; MAP2K, mitogen activated protein kinase kinase; MAPKAPK, MAP kinase activated protein kinase; MARK, microtubule associated kinases; Mnk, MAPK-interacting protein kinases; NDR, nuclear dbf2-related kinase; NEK, NIMA (never in mitosis A)-related kinase; PAK, p21 activated kinase; MAST, microtubule-associated serine/threonine-protein kinase; PKA, protein kinase A; PKB, protein kinase B; PKC, protein kinase C; PKD, protein kinase D; PDHK, pyruvate dehydrogenase kinase; PKGcGK, cGMP-dependent protein kinase; QIK, Qininduced kinase; ROCK, Rho-associated protein kinase; RSK, ribosomal S6 kinase; SGK, serum and glucocorticoid regulated kinase; STLK, STE20-like serine/threonine-protein kinase; TBK, TANK-binding kinase; TTBK, tau-tubulin kinase; ULK, Unc-51 like autophagy activating kinase; Wnk, with-no-lysine (K) kinase. 


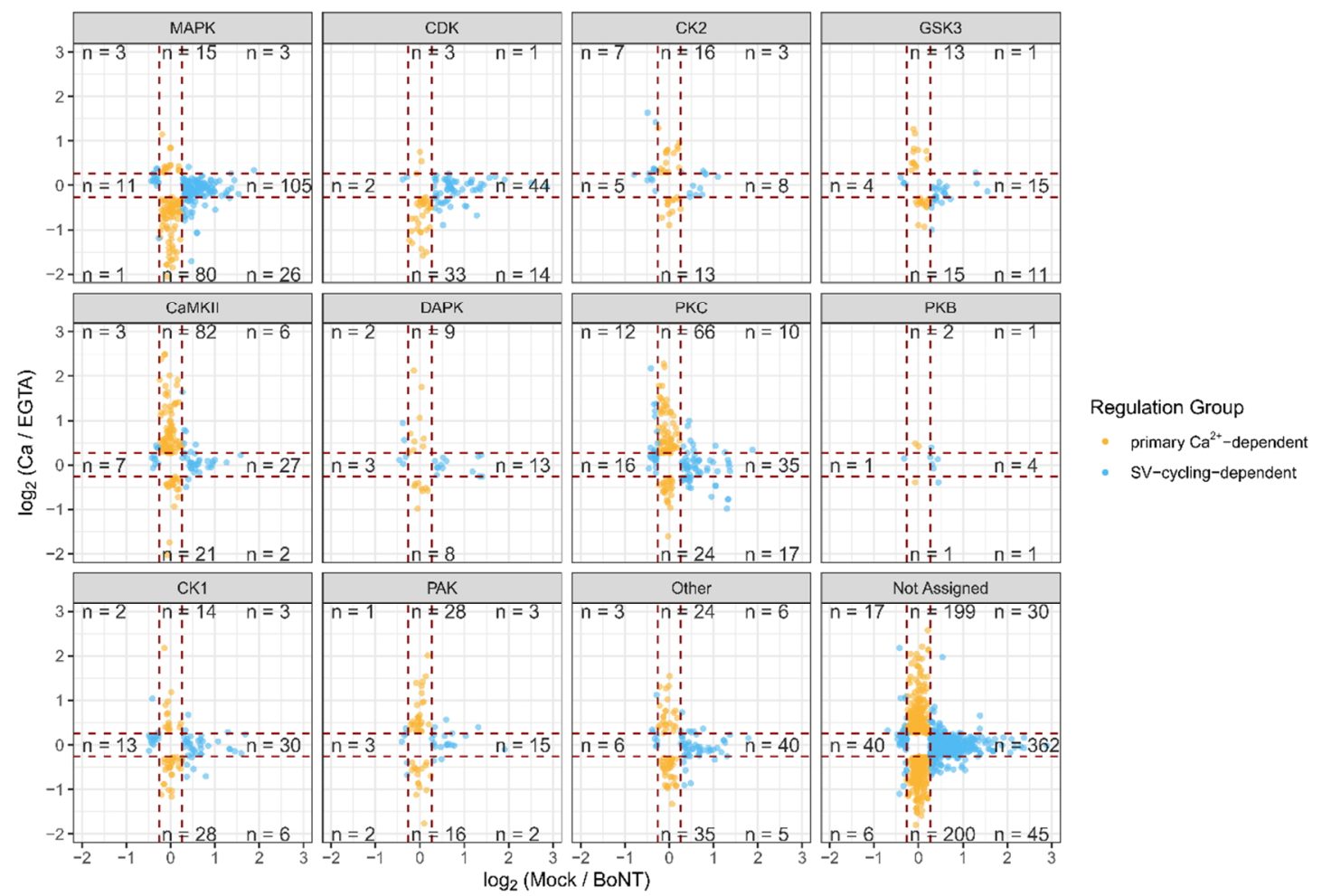

Figure 5.S3: Dependence of phosphorylation sites on calcium influx and SV cycling. An extended version of Figure 5.4A: categorization of phosphorylation events based on the magnitude of the log2 fold changes in Mock vs. BoNT experiment (x-axis) and log2 fold changes in Ca vs. EGTA experiment ( $y$-axis). Phosphorylation events were further segregated based on the known / predicted kinase class. The kinase classification (Netphorest Groups) follows one introduced by Netphorest (144) and uses its second-level group annotation. "Other" category contains phosphorylation evens attributed to smaller kinase classes. Only events quantified in both experiments are shown, and their q-values satisfy a q-value threshold of $<0.01$. The events are classified into two regulation groups: (i) "primary $\mathrm{Ca}^{2+}$-dependent" (sites with no significant or not-quantified SV cycling effect, i.e., absolute log2 (Mock/BoNT) < 0.263 and absolute log2 (Ca/EGTA) > 0.263), shown as orange dots; and (ii) "SV-cycling-dependent" (absolute log2 (Mock/BoNT) > 0.263). 


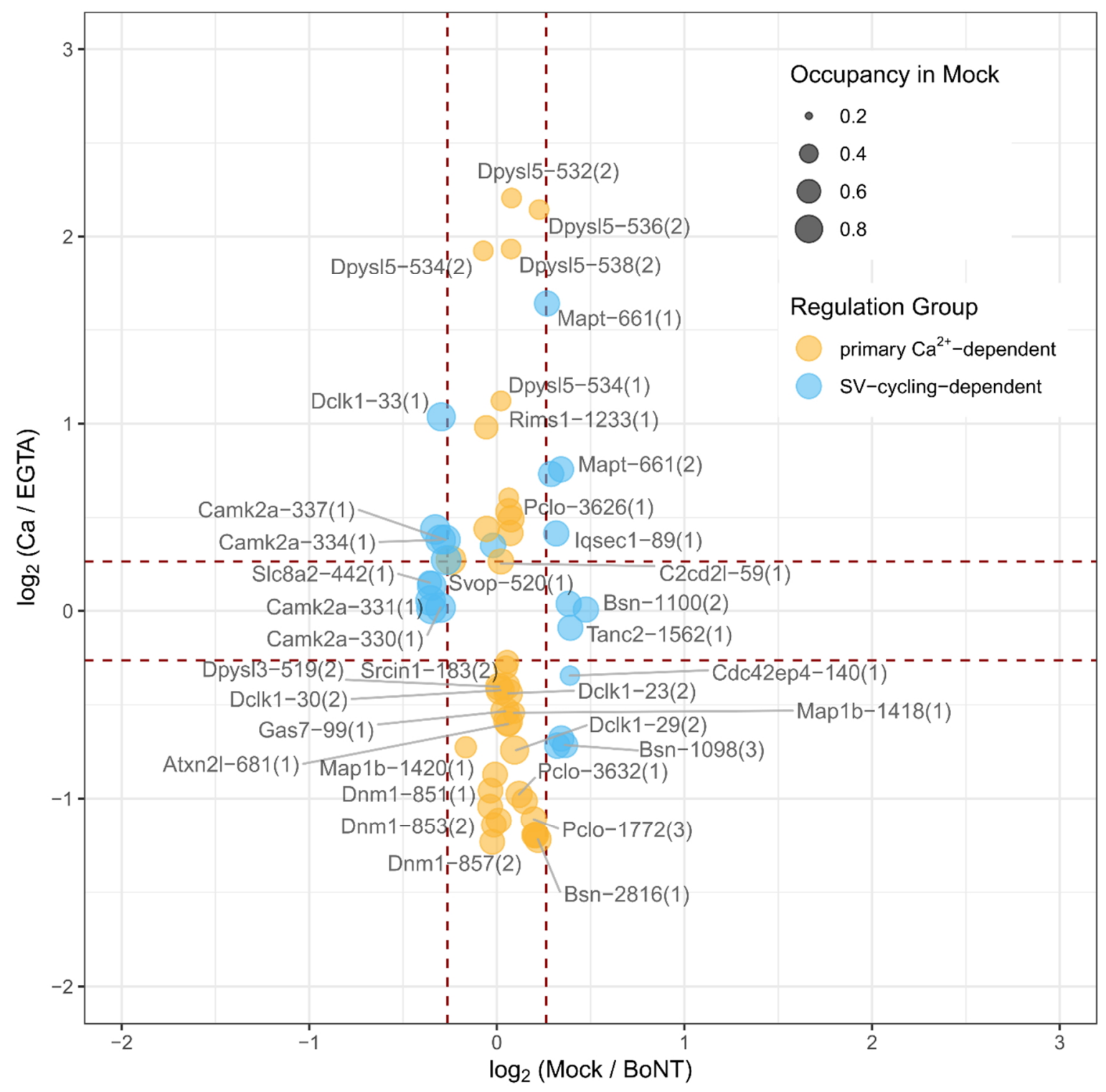

Figure 5.S4: Site occupancy assessment. Site occupancies were calculated using the 3D linear model as suggested by Hogrebe et al (300) and are based on the observed intensity changes in phosphorylated peptide/non-phosphorylated peptide pairs and total protein intensity in Mock/BoNT experiments. Only occupancy estimations with a $p$ value below 0.1 were considered. 


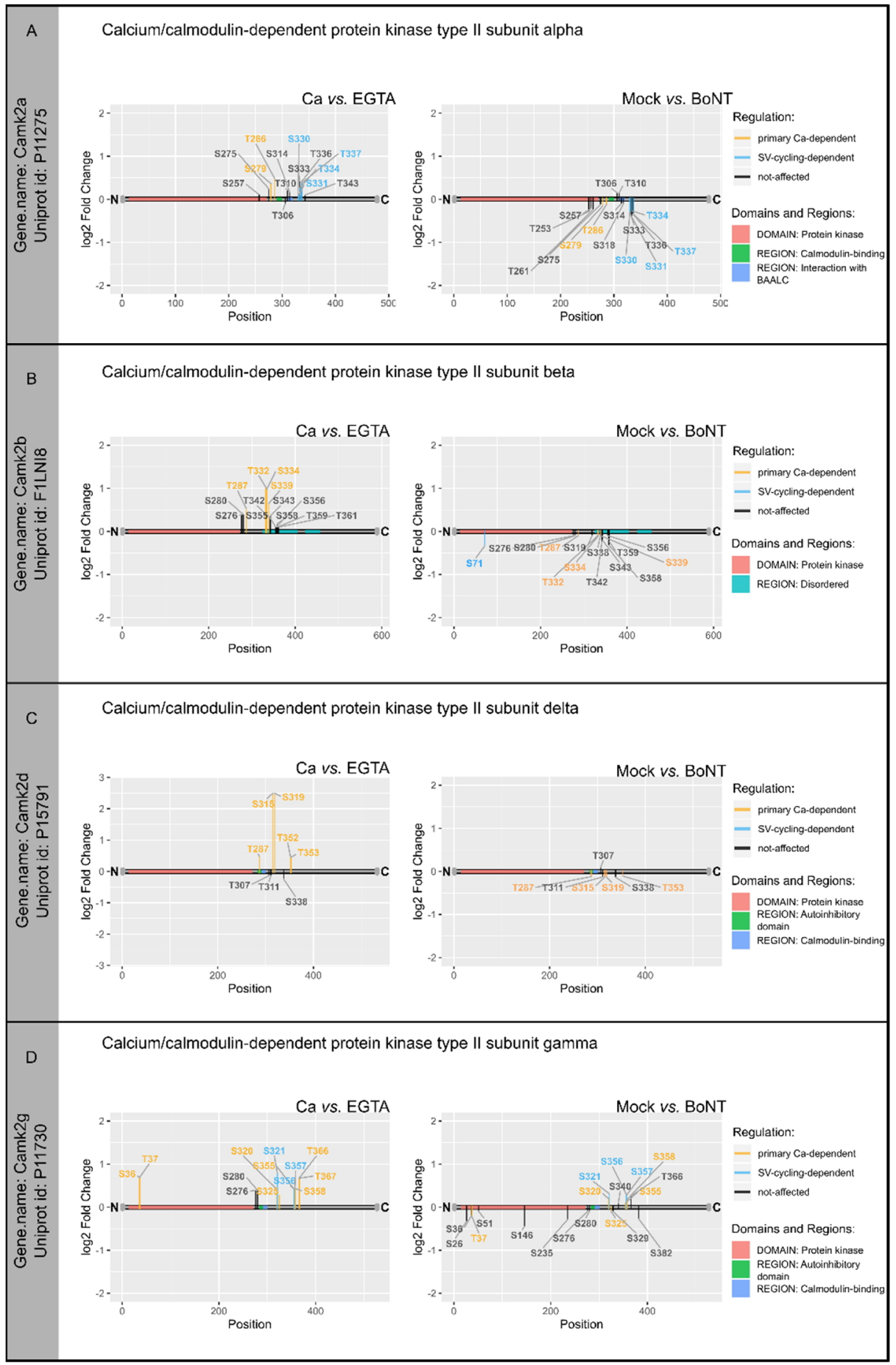

Figure 5.S5: Changes in phosphorylation site intensities for Calcium/calmodulin-dependent kinase 2 alpha, beta, gamma, and delta. X-axis shows positions of modified amino acids. A grey horizontal bar represents a protein sequence with its $N$ - and $C$ termini denoted as " $N$ " and " $C$ ". Colored segments mark positions of domains and regions on protein sequence as annotated in Uniprot (366). Changes in phosphorylation site intensities are expressed as $\log _{2}$ fold changes ( $y$-axis). Non-significant changes in phosphorylation site intensities are colored black. Significant changes are shown in orange or blue, depending on the classification of the site as "primarily Ca-dependent", or "SV-cycling-dependent", respectively. If a phosphorylation site was quantified on peptides that were singly, doubly or multiply phosphorylated (also known as "multiplicity"), the multiplicity with the highest magnitude of log2 fold change is depicted. Proteins are grouped by the gene name and Uniprot identifier. 


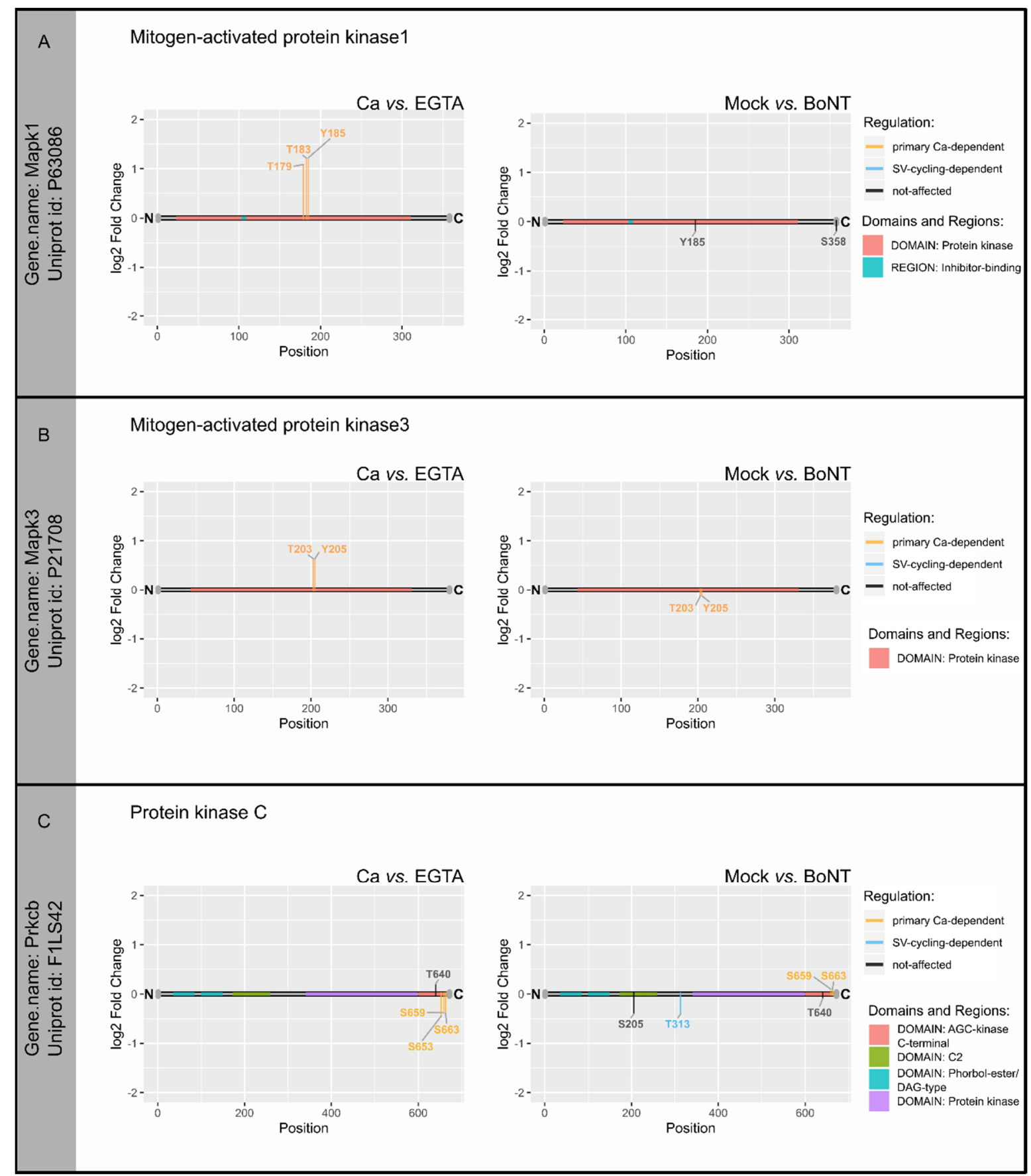

Figure 5.S6: Changes in phosphorylation site intensities for Mitogen-activated protein kinase 1, 3, and

Protein kinase $\boldsymbol{C}$ beta. $X$-axis shows positions of modified amino acids. A grey horizontal bar represents a protein sequence with its $\mathrm{N}$ - and $\mathrm{C}$ termini denoted as " $N$ " and "C". Colored segments mark positions of domains and regions on protein sequence as annotated in Uniprot (366). Changes in phosphorylation site intensities are expressed as log2 fold changes ( $y$-axis). Non-significant changes in phosphorylation site intensities are colored black. Significant changes are shown in orange or blue, depending on the classification of the site as "primarily Ca-dependent", or "SV-cycling-dependent", respectively. If a phosphorylation site was quantified on peptides that were singly, doubly or multiply phosphorylated (also known as "multiplicity"), the multiplicity with the highest magnitude of log2 fold change is depicted. Proteins are grouped by the gene name and Uniprot identifier. 
A

Ppp1r1a-46 (2)

Ppp1r1a-47(2)

Ppp1r1a-67(1)

Ppp1r1b-45(1)

Ppp1r1b-51(2)

Ppp1r2-20(1)

Ppp1r3d-54(2)

Ppp1r3e-33(1)

Ppp1r7-12(1)

Ppp1r7-24(1)

Ppp1r7-44(2)

Ppp1r7-47(1)

Ppp1r7-47(2)

Ppp1r9a-94(1)

Ppp1r9a-95(1)

Ppp1r9a-153(1

Ppp1r9a-160(1)

Ppp1r9a-929(1)

Ppp1r9b-100(1)

Ppp1r12a-299(1)

Ppp1r12a-596(2)

Ppp1r12a-601(1)

Ppp1r12a-601(2)

Ppp1r12a-894(1)

Ppp1r12c-508(1)

Ppp1r13b-562(1)

Ppp1r16b-477(1)

Ppp1r21-101(1)

Ppp2r5c-497(1)

Ppp3ca-462(1)

Ppp3ca-498(1)

Ppp6r2-629(1)

Pcp2-92(2)

Pcp2-98(2)

Pcp2-111(1)

Phactr1-67(1)

Phactr1-423(1)

Phactr2-154(1)

Phactr3-267(1)

Ptpn4-403(1)

Ptprd-1314(1)

Ssh3-642(1)

Synj1-833(1)

Synj1-1150(1)

Synj1-1163(1)

Synj1-1215(1)
B

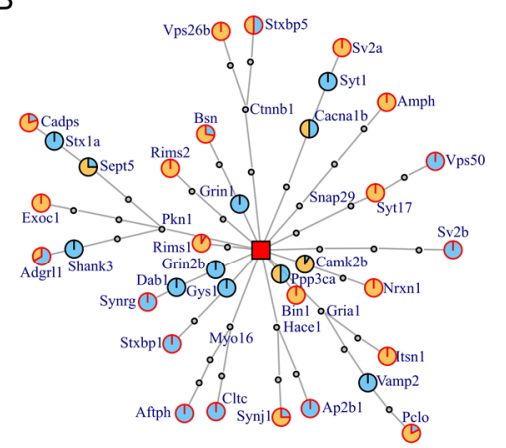

C

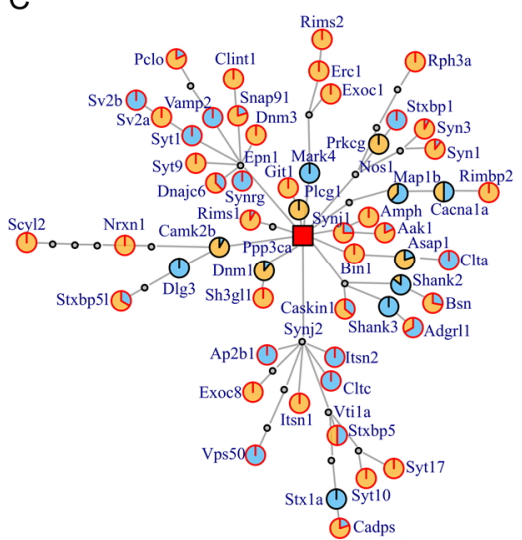

- Synaptosomal protein

Contain PP1-docking motif

No PP1-docking motif

(C) $=\%$ of $\mathrm{SV}$-cycling-dependent sites

$\square$ PP1 (Ppp1ca)

- Synaptosomal protein

Contain Calcineurin-docking motif

$\bigcirc$ No Calcineurin-docking motif

(1) $=\%$ of SV-cycling-dependent sites

$\square$ Calcineurin (Ppp3ca)

D

Number of phosphorylation events on proteins
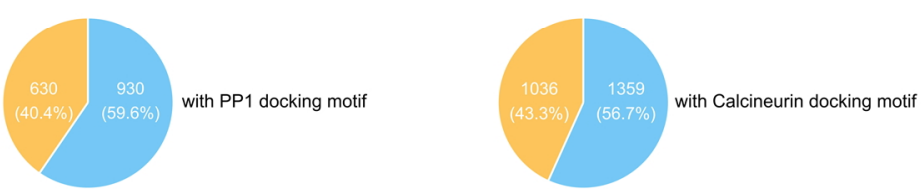

Regulation group

primary $\mathrm{Ca}^{2+}$-dependent $\quad \mathrm{SV}$-cycling-dependent

Figure 5.S7: Phosphatases and their putative substrates. (A) Significantly regulated phosphorylation events on phosphatases and phosphatase-related proteins in Ca/EGTA or Mock/BoNT experiment. Phosphorylation events are designated as gene name followed by the amino acid position and phosphorylation multiplicity (in brackets). (B and $\boldsymbol{C}$ ). Protein-Protein Interaction map between phosphatase (PP1 in B and Calcineurin in C) and selected phosphoproteins involved in endo/exocytosis and containing PP1 (B) or Calcineurin (C) docking motif (shown as blue/orange-colored circles with red edges). Docking motifs described in (371) were used for the analysis. Phosphoproteins that did not contain docking motifs are shown as blue/orange-colored circles with black edges. The blue/orange area within a circle correspond to a percentage of sites classified as "primary $\mathrm{Ca}^{2+-d e p e n d e n t " ~(o r a n g e) ~ o r ~ " S V-c y c l i n g-d e p e n d e n t " ~(b l u e) . ~ S y n a p t o s o m a l ~ p r o t e i n s ~ n o t ~ c o n t a i n i n g ~ s i g n i f i c a n t l y ~}$ regulated phosphorylation sites are shown as small grey circles. Phosphatases (PP1 or Calcineurin) are represented by red squares. Protein-protein interactions were extracted from String database (322) and included interactions between synaptosomal proteins which are experimentally supported or annotated in manually curated databases. (D) Number of "primary Ca ${ }^{2+}$-dependent" (orange) and "SV-cycling-dependent" (blue) phosphorylation events on proteins containing a PP1 (E) or Calcineurin (F) docking motif (371). 


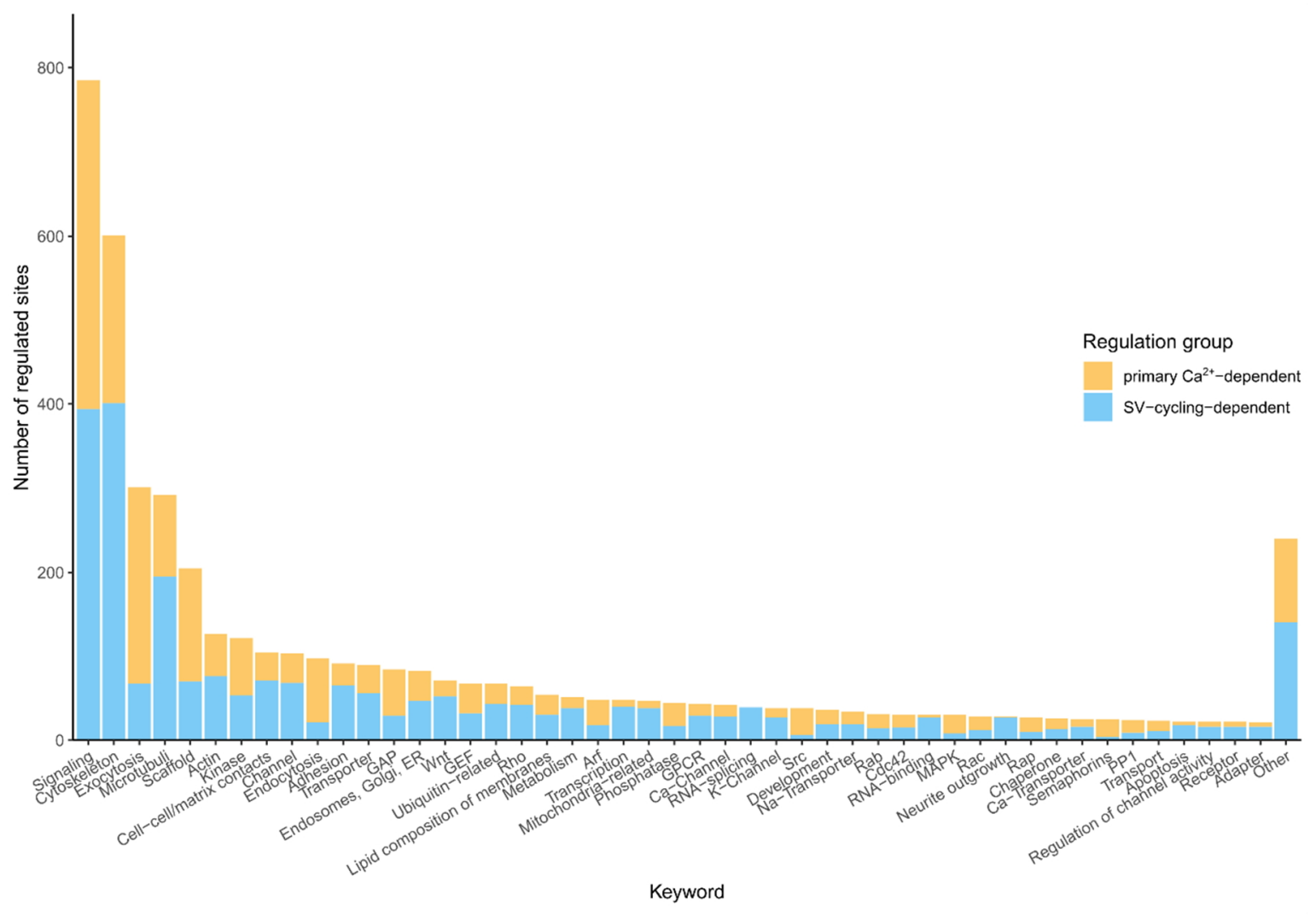

Figure 5.S8: Manual annotation of proteins carrying regulated phosphorylation sites. Key-words were assigned to proteins carrying regulated phosphorylation sites that specify a function / localization the protein might be related to. One protein can have one or more terms assigned. Number of significantly regulated phosphorylation sites for each term is shown as stacked bars with colors representing regulation group of the phosphorylation sites. Arf stays for ADP-ribosylation factor small GTPase; ER endoplasmatic reticulum; GAP $\sim$ GTPase activating protein; GEF GTP exchanging factor; GPCR G-protein coupled receptor; PP1 protein phosphatase-1 


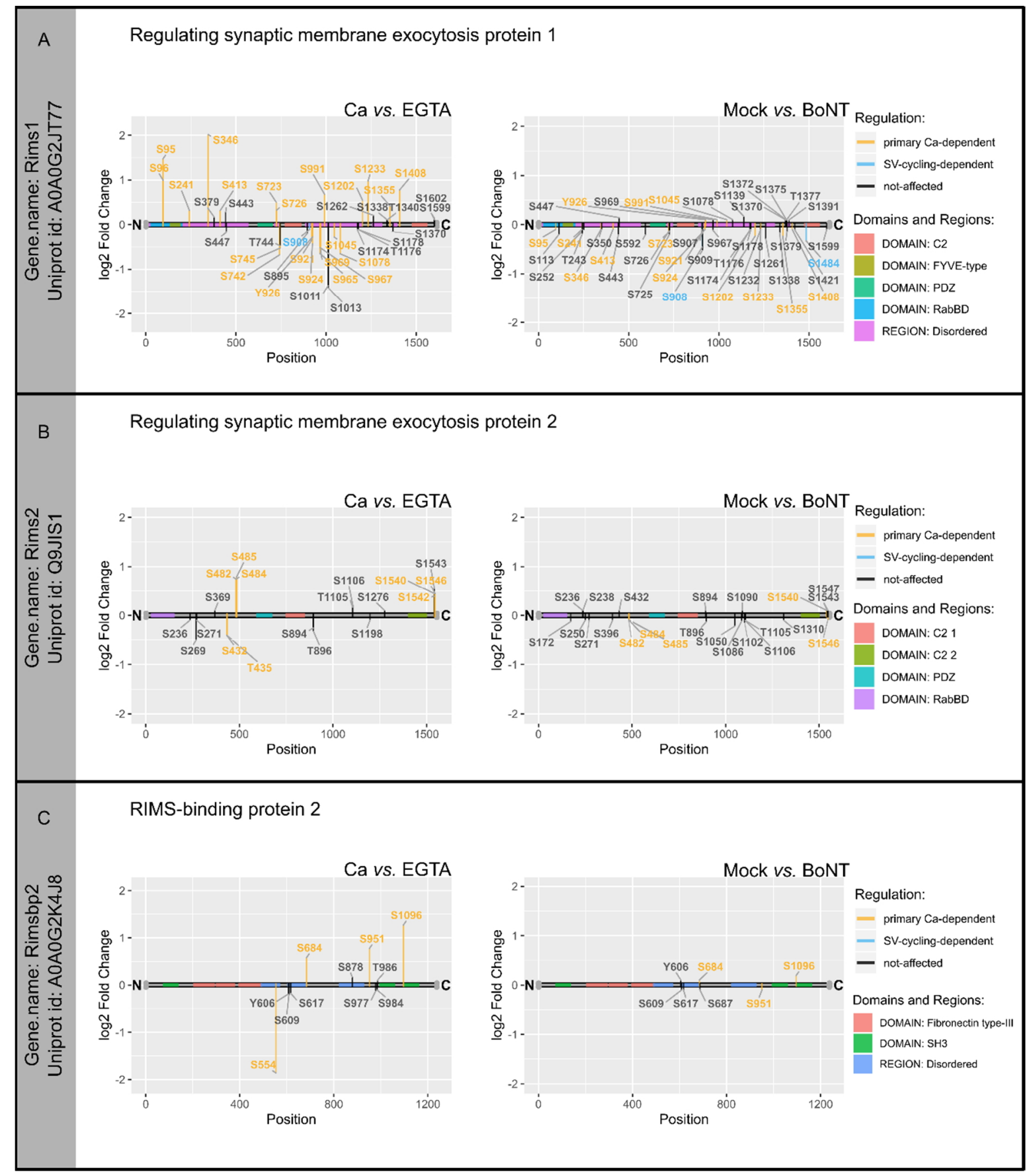

Figure 5.59: Changes in phosphorylation site intensities for Regulating synaptic membrane exocytosis (RIM) protein 1, 2, and RIM-binding protein 2. X-axis shows positions of modified amino acids. A grey horizontal bar represents a protein sequence with its $N$ - and $C$ termini denoted as " $N$ " and " $C$ ". Colored segments mark positions of domains and regions on protein sequence as annotated in Uniprot (366). Changes in phosphorylation site intensities are expressed as $\log _{2}$ fold changes ( $y$-axis). Non-significant changes in phosphorylation site intensities are colored black. Significant changes are shown in orange or blue, depending on the classification of the site as "primarily Ca-dependent", or "SV-cycling-dependent", respectively. If a phosphorylation site was quantified on peptides that were singly, doubly or multiply phosphorylated (also known as "multiplicity"), the multiplicity with the highest magnitude of log2 fold change is depicted. Proteins are grouped by the gene name and Uniprot identifier. 


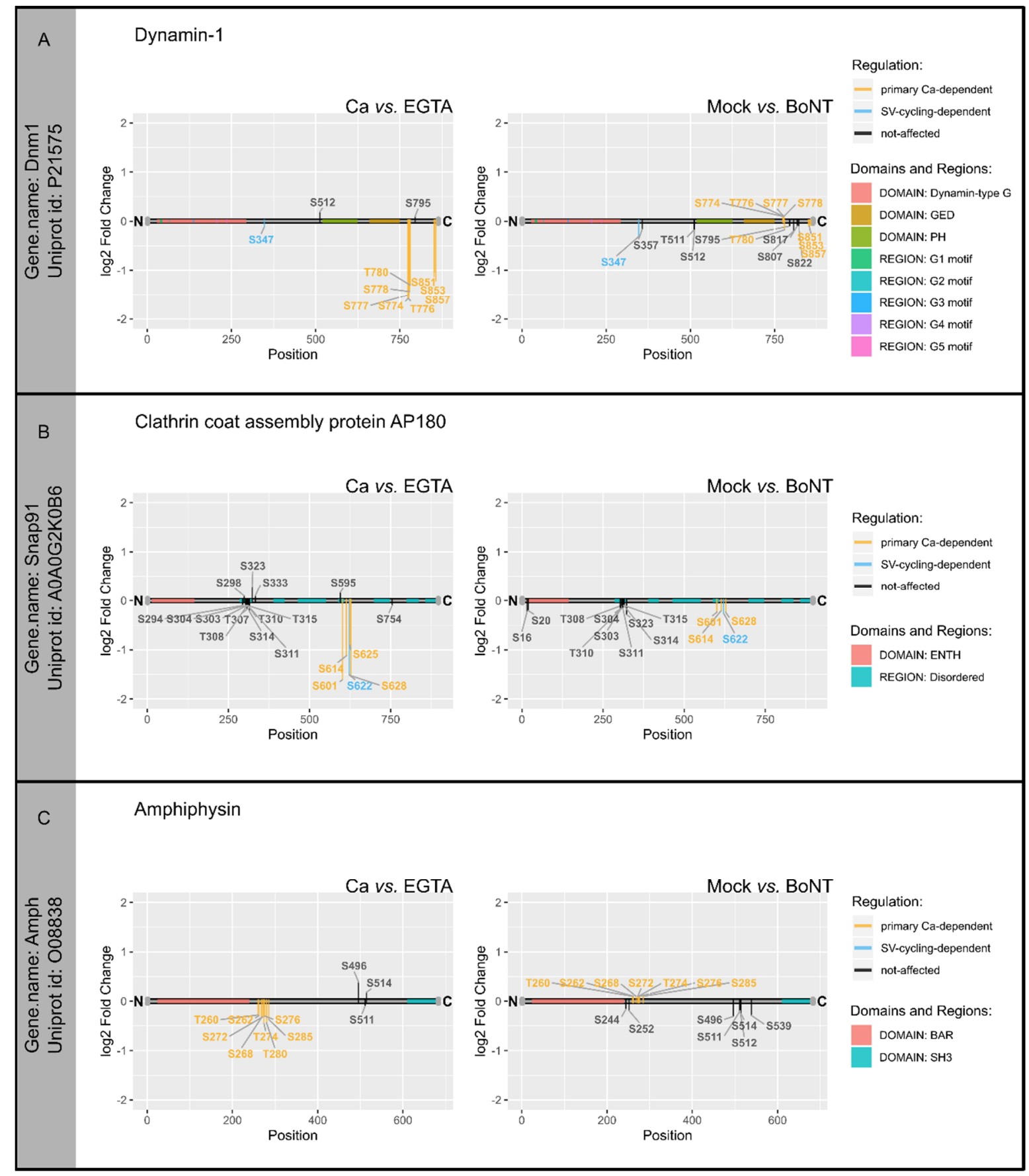

Figure 5.S10: Changes in phosphorylation site intensities for Dynamin-1, Clathrin coat assembly protein

AP180, and Amphiphysin. X-axis shows positions of modified amino acids. A grey horizontal bar represents a protein sequence with its $N$ - and C termini denoted as " $N$ " and "C". Colored segments mark positions of domains and regions on protein sequence as annotated in Uniprot (366). Changes in phosphorylation site intensities are expressed as $\log _{2}$ fold changes ( $y$-axis). Non-significant changes in phosphorylation site intensities are colored black. Significant changes are shown in orange or blue, depending on the classification of the site as "primarily Ca-dependent", or "SV-cycling-dependent", respectively. If a phosphorylation site was quantified on peptides that were singly, doubly or multiply phosphorylated (also known as "multiplicity"), the multiplicity with the highest magnitude of log2 fold change is depicted. Proteins are grouped by the gene name and Uniprot identifier. 


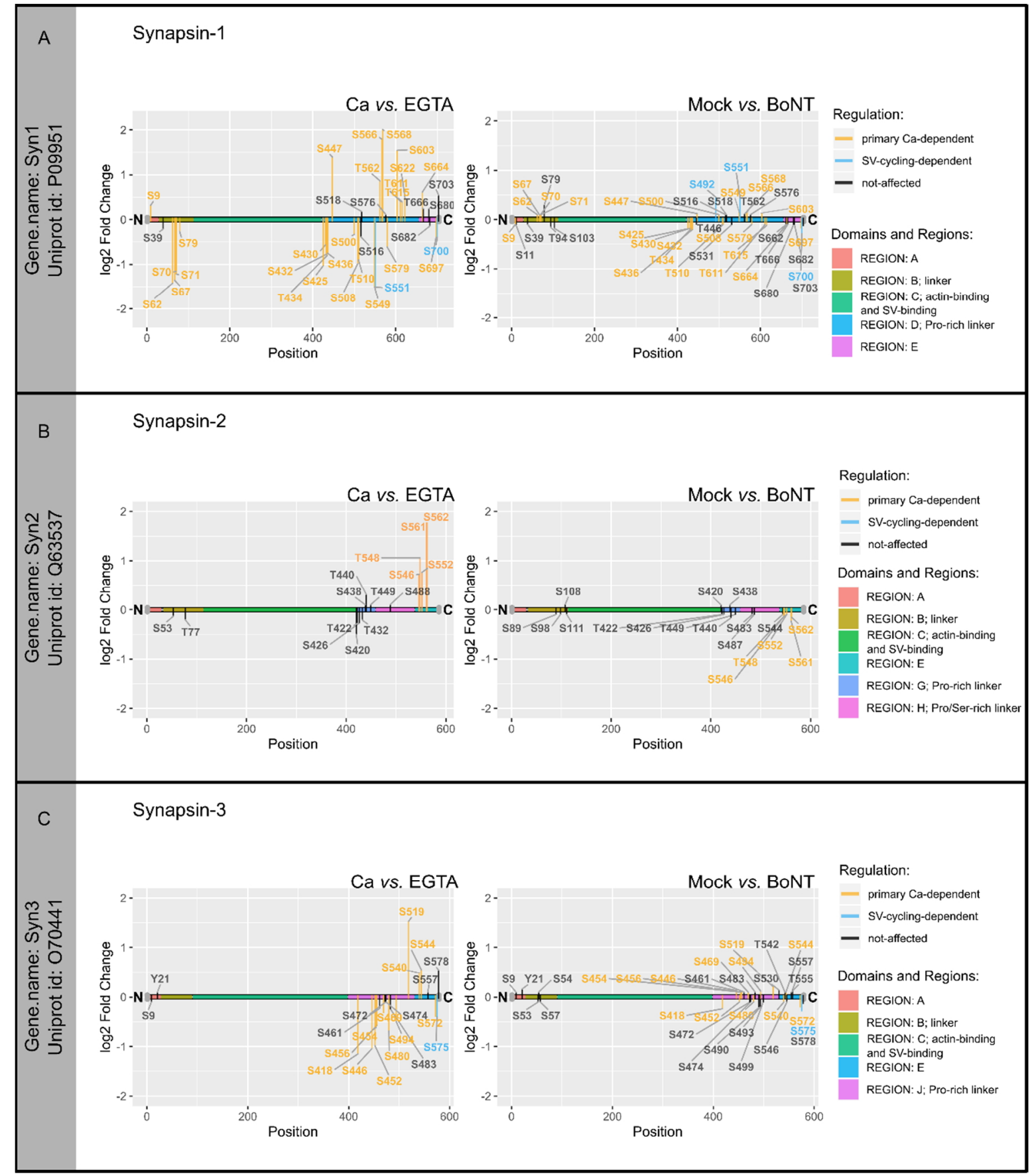

Figure 5.S11: Changes in phosphorylation site intensities for Synapsin-1, 2, and 3. X-axis shows positions of modified amino acids. A grey horizontal bar represents a protein sequence with its $N$ - and $C$ termini denoted as " $N$ " and " $C$ ". Colored segments mark positions of domains and regions on protein sequence as annotated in Uniprot (366). Changes in phosphorylation site intensities are expressed as $\log _{2}$ fold changes (y-axis). Non-significant changes in phosphorylation site intensities are colored black. Significant changes are shown in orange or blue, depending on the classification of the site as "primarily Ca-dependent", or "SV-cyclingdependent", respectively. If a phosphorylation site was quantified on peptides that were singly, doubly or multiply phosphorylated (also known as "multiplicity"), the multiplicity with the highest magnitude of $\log _{2}$ fold change is depicted. Proteins are grouped by the gene name and Uniprot identifier. 


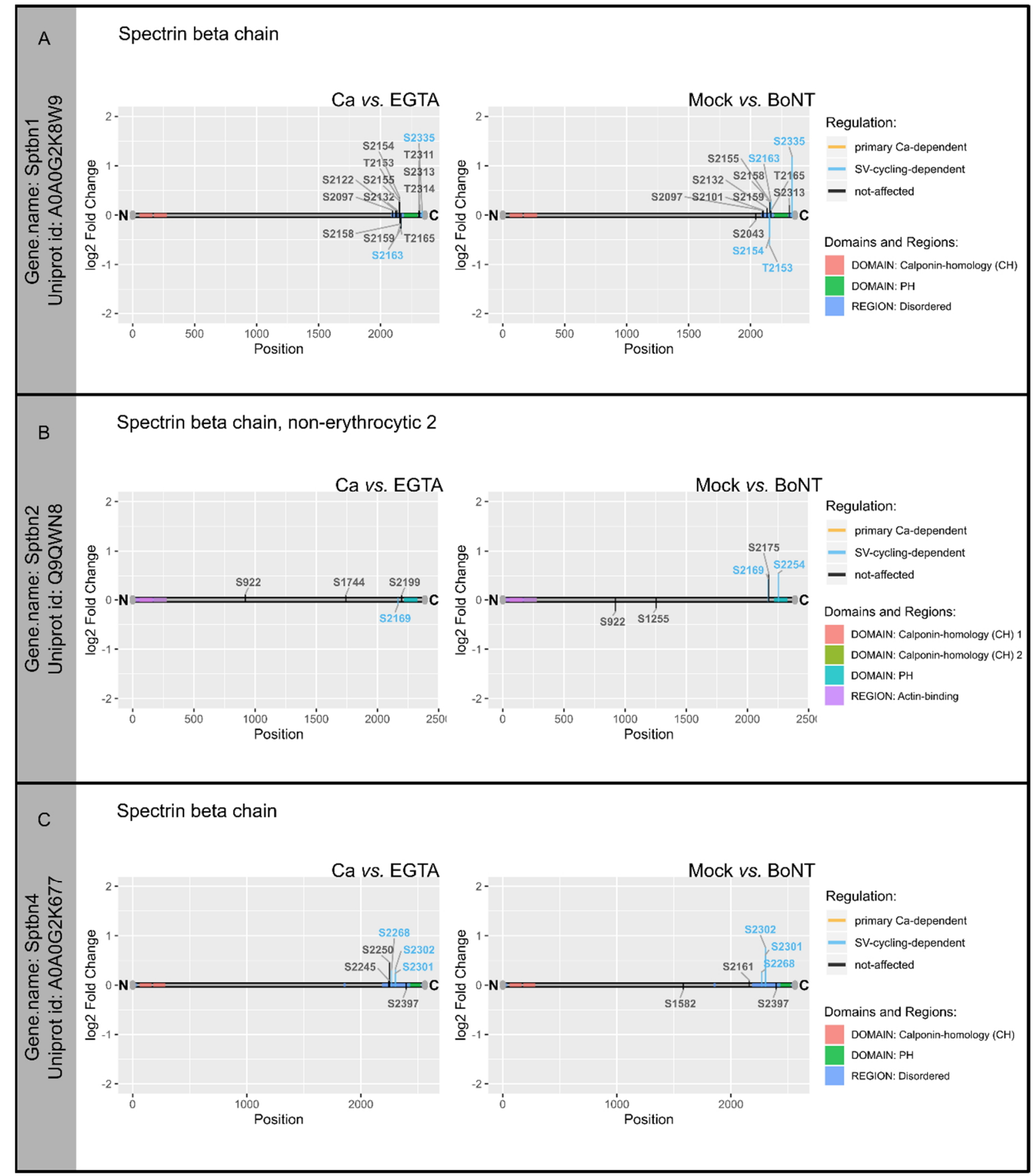

Figure 5.S12: Changes in phosphorylation site intensities for Spectrin beta chain. X-axis shows positions of modified amino acids. A grey horizontal bar represents a protein sequence with its $N$ - and $C$ termini denoted as " $N$ " and " $C$ ". Colored segments mark positions of domains and regions on protein sequence as annotated in Uniprot (366). Changes in phosphorylation site intensities are expressed as $\log _{2}$ fold changes (y-axis). Non-significant changes in phosphorylation site intensities are colored black. Significant changes are shown in orange or blue, depending on the classification of the site as "primarily Ca-dependent", or "SV-cyclingdependent", respectively. If a phosphorylation site was quantified on peptides that were singly, doubly or multiply phosphorylated (also known as "multiplicity"), the multiplicity with the highest magnitude of $\log _{2}$ fold change is depicted. Proteins are grouped by the gene name and Uniprot identifier. 


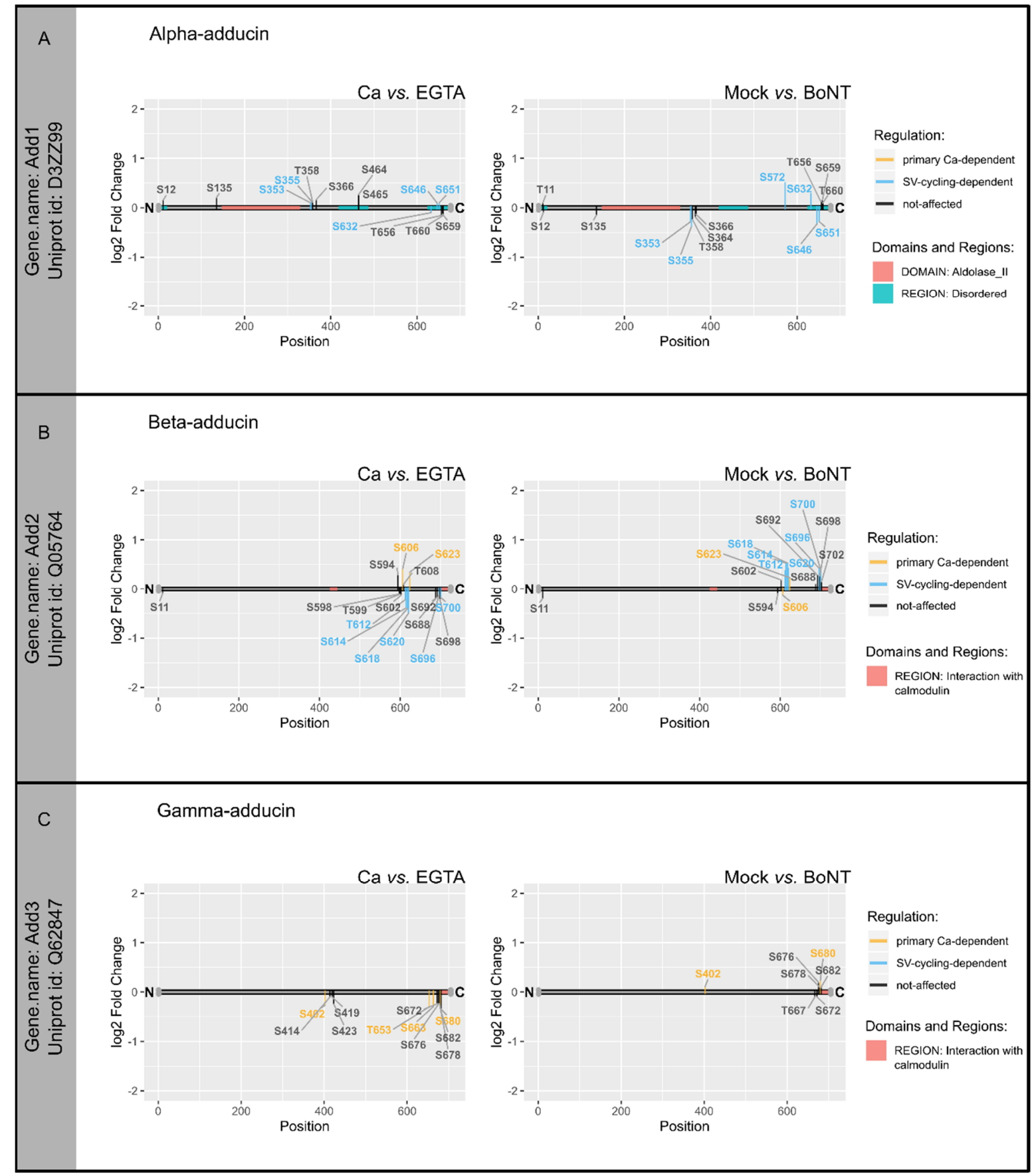

Figure 5.S13: Changes in phosphorylation site intensities for Adducin alpha, beta, and gamma. X-axis shows positions of modified amino acids. A grey horizontal bar represents a protein sequence with its $N$ and $C$ termini denoted as " $N$ " and " $C$ ". Colored segments mark positions of domains and regions on protein sequence as annotated in Uniprot (366). Changes in phosphorylation site intensities are expressed as $\log _{2}$ fold changes (y-axis). Non-significant changes in phosphorylation site intensities are colored black. Significant changes are shown in orange or blue, depending on the classification of the site as "primarily Ca-dependent", or "SV-cycling-dependent", respectively. If a phosphorylation site was quantified on peptides that were singly, doubly or multiply phosphorylated (also known as "multiplicity"), the multiplicity with the highest magnitude of $\log _{2}$ fold change is depicted. Proteins are grouped by the gene name and Uniprot identifier. 


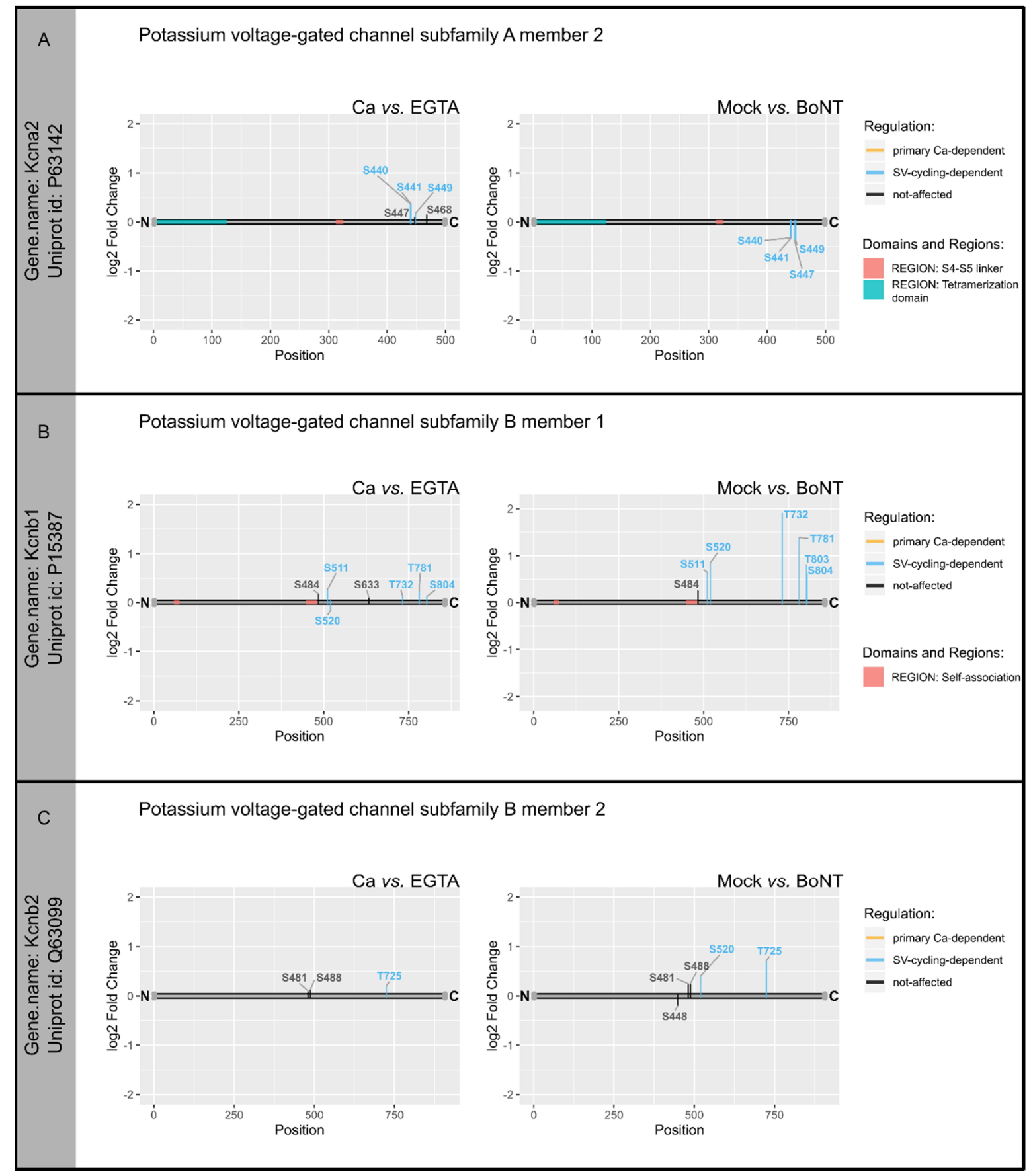

Figure 5.S14: Changes in phosphorylation site intensities for Potassium voltage-gated channel subfamily A member 2, and subfamily B member 1 and 2. X-axis shows positions of modified amino acids. A grey horizontal bar represents a protein sequence with its $N$ - and $C$ termini denoted as " $N$ " and "C". Colored segments mark positions of domains and regions on protein sequence as annotated in Uniprot (366). Changes in phosphorylation site intensities are expressed as $\log _{2}$ fold changes (y-axis). Non-significant changes in phosphorylation site intensities are colored black. Significant changes are shown in orange or blue, depending on the classification of the site as "primarily Ca-dependent", or "SV-cycling-dependent", respectively. If a phosphorylation site was quantified on peptides that were singly, doubly or multiply phosphorylated (also known as "multiplicity"), the multiplicity with the highest magnitude of $\log _{2}$ fold change is depicted. Proteins are grouped by the gene name and Uniprot identifier. 


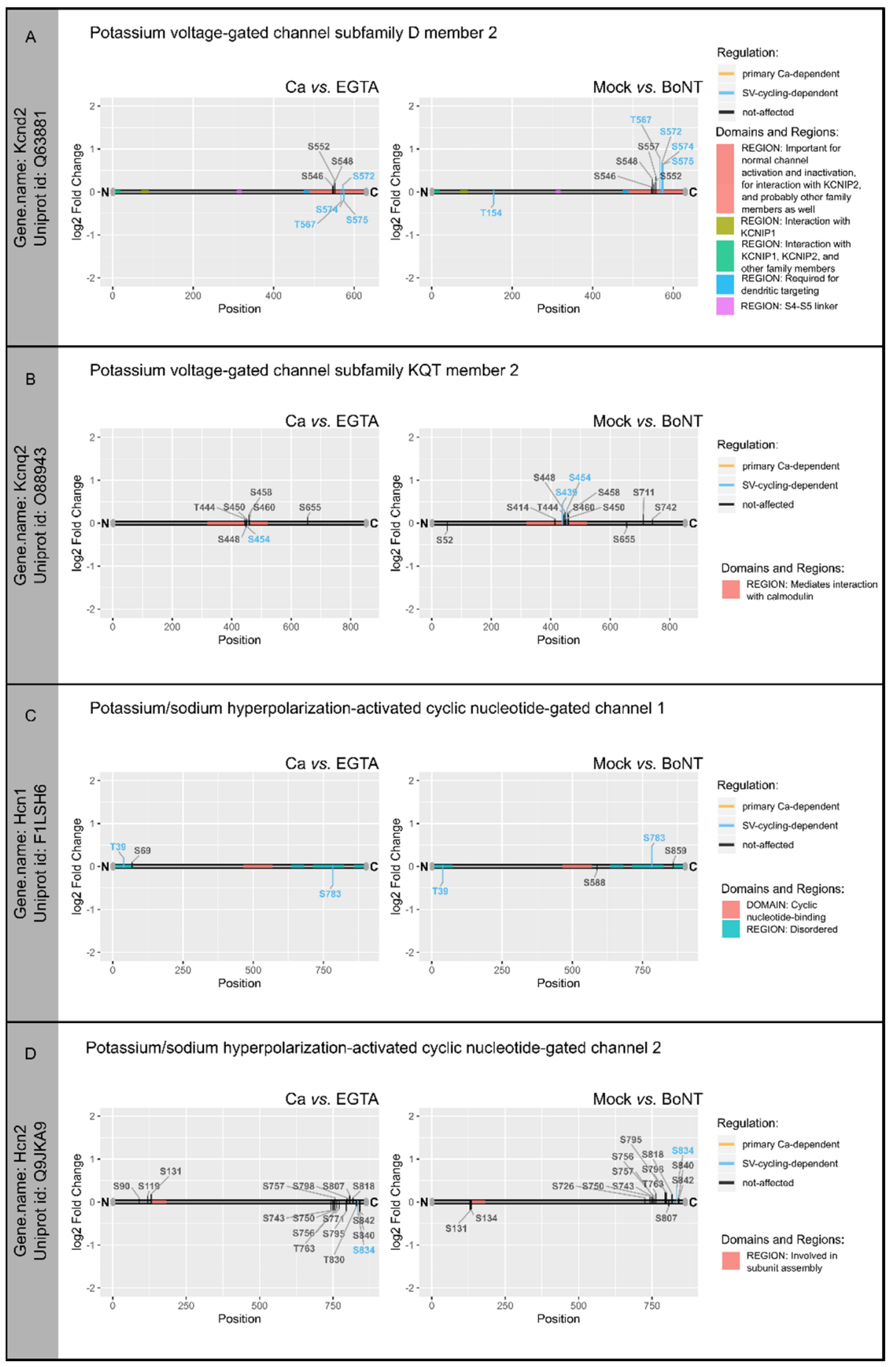

Figure 5.S15: Changes in phosphorylation site intensities for Potassium voltage-gated channel subfamily D member 2, subfamily KQT member 2, Potassium/sodium hyperpolarization-activated cyclic nucleotide-gated channel 1 and 2. $X$-axis shows positions of modified amino acids. A grey horizontal bar represents a protein sequence with its $N$ - and $C$ termini denoted as " $N$ " and " $C$ ". Colored segments mark positions of domains and regions on protein sequence as annotated in Uniprot (366). Changes in phosphorylation site intensities are expressed as $\log _{2}$ fold changes (y-axis). Non-significant changes in phosphorylation site intensities are colored black. Significant changes are shown in orange or blue, depending on the classification of the site as "primarily Ca-dependent", or "SV-cycling-dependent", respectively. If a phosphorylation site was quantified on peptides that were singly, doubly or multiply phosphorylated (also known 
as "multiplicity"), the multiplicity with the highest magnitude of $\log _{2}$ fold change is depicted. Proteins are grouped by the gene name and Uniprot identifier. 


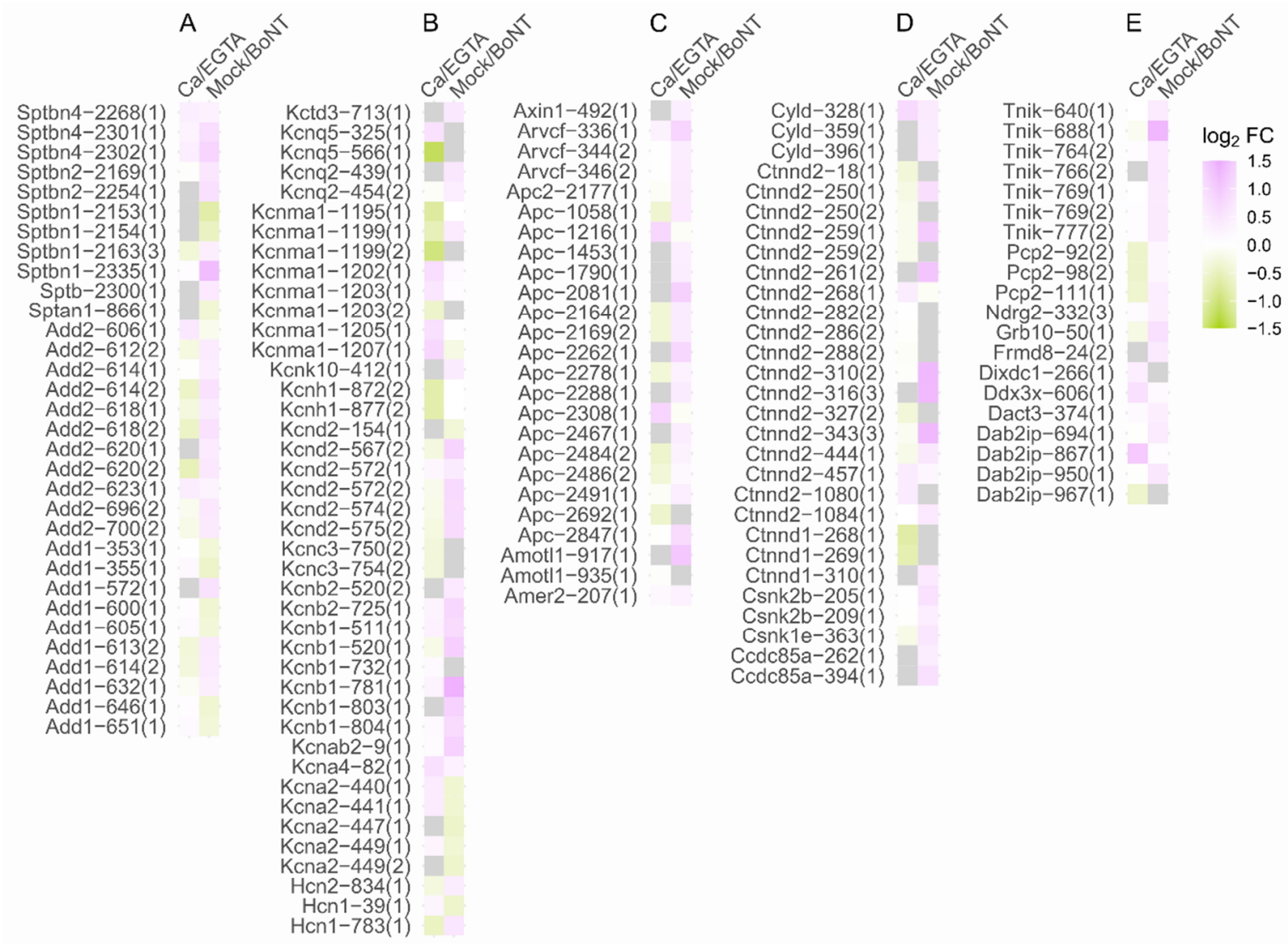

Figure 5.S16: Regulated phosphorylation sites on selected proteins. (A) Regulated sites on cytoskeletonrelated proteins spectrin and adducing. (B) Regulated sites on potassium-channel proteins. (C-E). Regulated sites on Wnt-pathway-related proteins. Phosphorylation events are designated as gene name followed by the amino acid position and phosphorylation multiplicity (in brackets). 


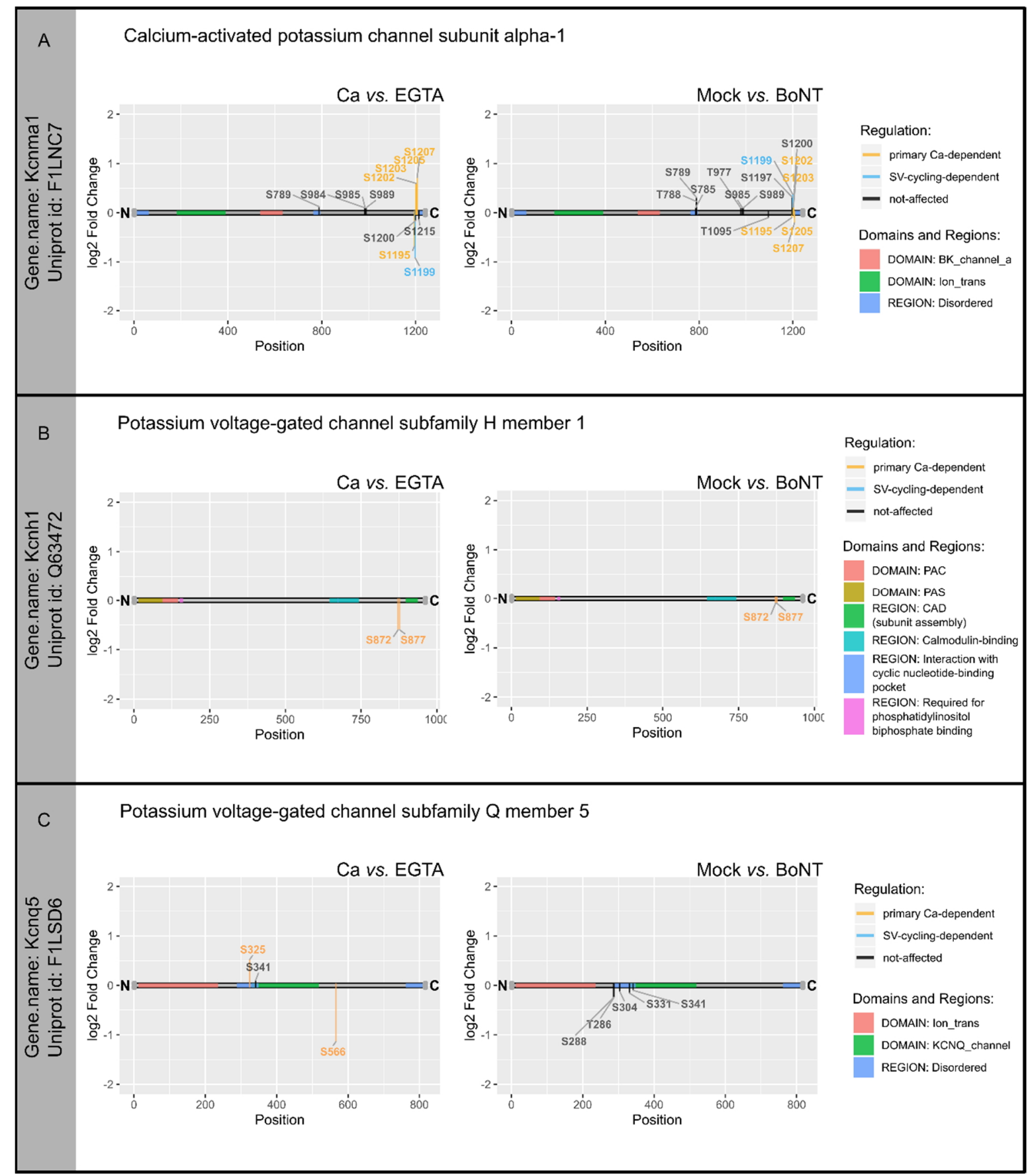

Figure 5.S17: Changes in phosphorylation site intensities for Calcium-activated potassium channel subunit alpha-1, Potassium voltage-gated channel subfamily H member 1, and Potassium voltage-gated channel subfamily $Q$ member 5. X-axis shows positions of modified amino acids. A grey horizontal bar represents a protein sequence with its $N$ - and $C$ termini denoted as " $N$ " and " $C$ ". Colored segments mark positions of domains and regions on protein sequence as annotated in Uniprot (366). Changes in phosphorylation site intensities are expressed as $\log _{2}$ fold changes ( $y$-axis). Non-significant changes in phosphorylation site intensities are colored black. Significant changes are shown in orange or blue, depending on the classification of the site as "primarily Ca-dependent", or "SV-cycling-dependent", respectively. If a phosphorylation site was quantified on peptides that were singly, doubly or multiply phosphorylated (also known as "multiplicity"), the multiplicity with the highest magnitude of $\log _{2}$ fold change is depicted. Proteins are grouped by the gene name and Uniprot identifier. 


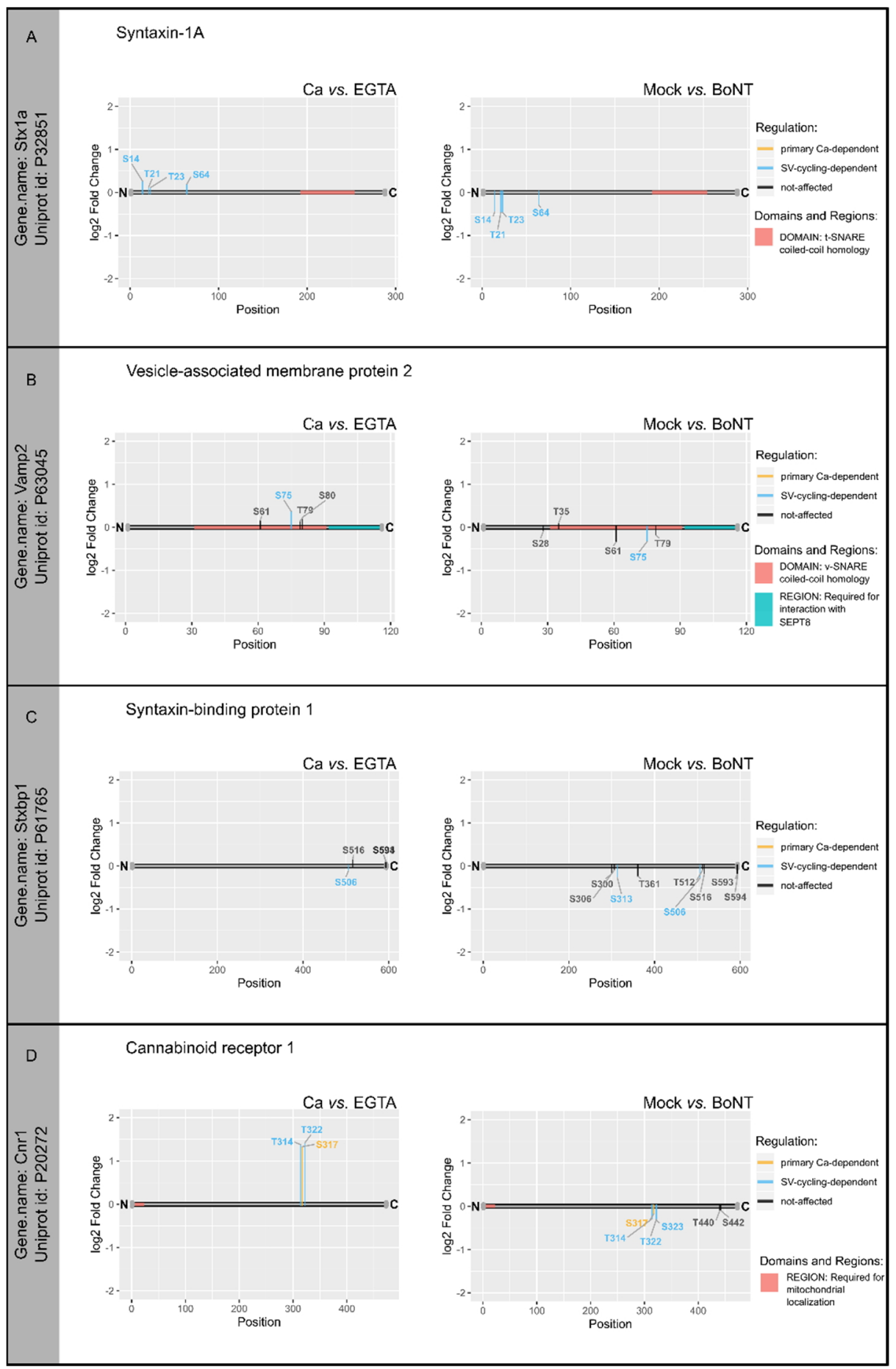

Figure 5.S18: Changes in phosphorylation site intensities for Syntaxin-1A, Vesicle-associated membrane protein 2, Syntaxin-binding protein 1, and Cannabinoid receptor 1. X-axis shows positions of modified amino acids. A grey horizontal bar represents a protein sequence with its $N$ - and $C$ termini denoted as " $N$ " and "C". Colored segments mark positions of domains and regions on protein sequence as annotated in Uniprot (366). Changes in phosphorylation site intensities are expressed as $\log _{2}$ fold changes ( $y$-axis). Nonsignificant changes in phosphorylation site intensities are colored black. Significant changes are shown in orange or blue, depending on the classification of the site as "primarily Ca-dependent", or "SV-cyclingdependent", respectively. If a phosphorylation site was quantified on peptides that were singly, doubly or multiply phosphorylated (also known as "multiplicity"), the multiplicity with the highest magnitude of $\log _{2}$ fold change is depicted. Proteins are grouped by the gene name and Uniprot identifier. 


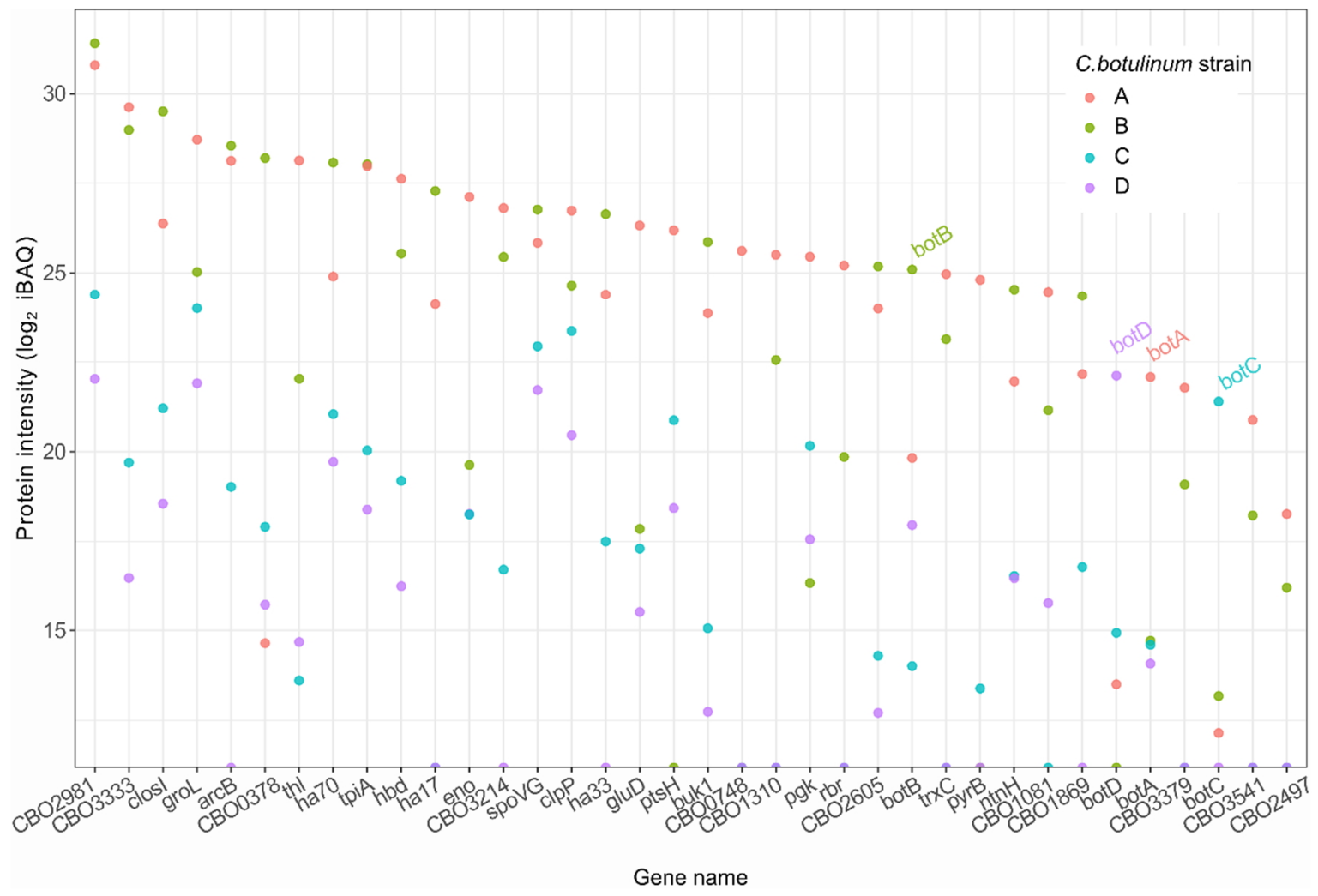

Figure 5.S19: Proteomic profiling of C. botulinum cell culture supernatants. Protein content in C. botulinum cell culture supernatants was assessed using iBAQ values (406). Top 30 most intense proteins, botulinum toxins (botA, botB, botC, botD), as well as putative protease (CBO3541) and putative phosphatases (CBO3379, $C B O 2497)$ that were identified in the cell culture supernatants are ranked based on iBAQ intensity. Colors encode C.botulinum strains: $A$, red; $B$, green; $C$, blue; $D$, violet. 
A

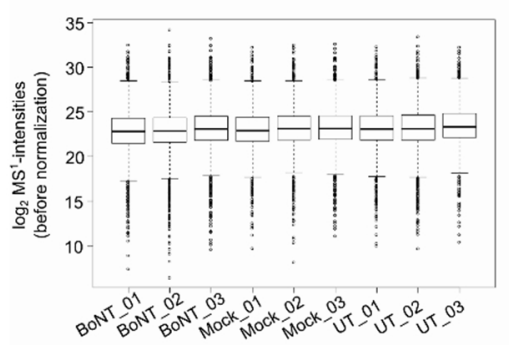

C

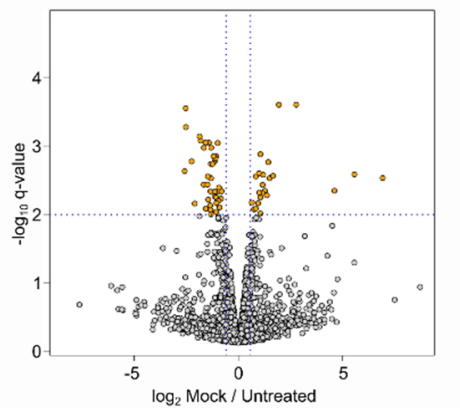

B

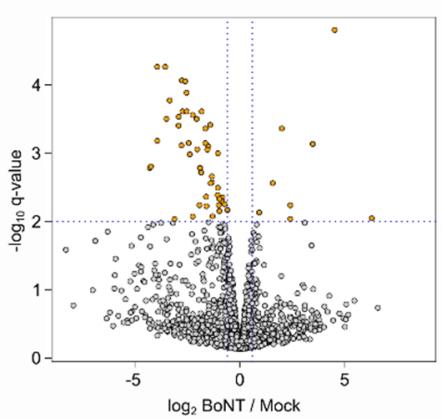

D

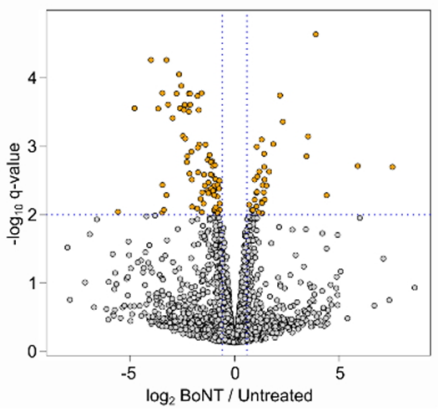

Figure 5.S20: Phosphoproteome analysis of BoNT-treated HeLa nuclear extract. Unspecific activity of C. botulinum cell culture supernatants was tested in nuclear extract of HeLa cells. Nuclear extract was treated for $1.5 \mathrm{~h}$ with a combination of the four C. botulinum cell culture supernatants, BoNT A-D (BoNT); heatinactivated BoNT A-D (Mock), or buffer (Untreated, UT). Afterwards, proteins were digested with trypsin and phosphorylated peptides were enriched using TiO2-beads. Precursor intensities in MS1 scans were used to assess intensities of phosphorylated sites. (A) Box-whisker plot of $\log _{2}$ phosphorylation site intensities before normalization. Boxes indicate $25 \%$ and $75 \%$ quantiles (interquartile range, IQR), vertical lines indicate medians, and whiskers indicate data within $1.5 \times I Q R$. (B-D) Differences in phosphorylation site intensities were assessed using limma package (311). Volcano plots of - $\log _{10} q$-values vs. $\log _{2}$ fold change: BoNT vs. Mock (b), Mock vs. Untreated (c), BoNT vs. Untreated (d). Orange dots represent phosphorylation events with a q-value of $<0.01$ and an absolute $\log _{2}$ FC of at least 0.586 . 
A

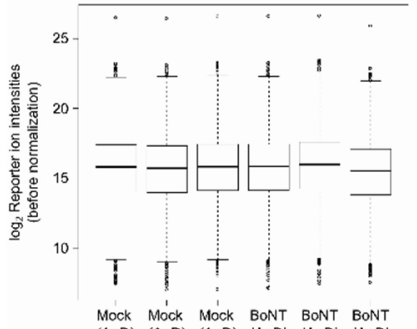

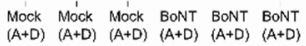

D

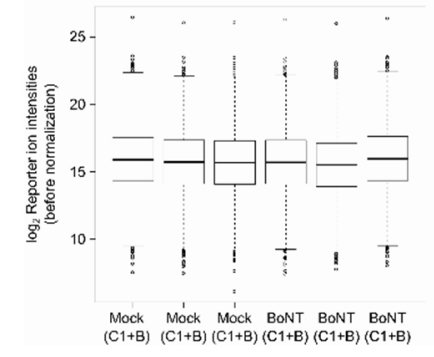

B

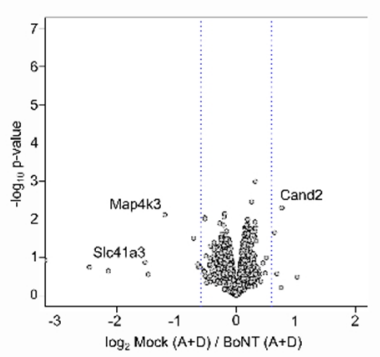

E

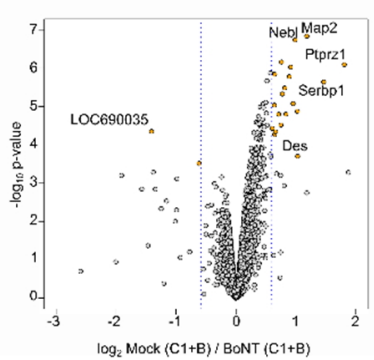

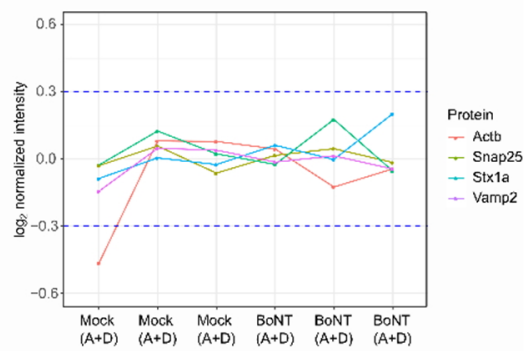

F

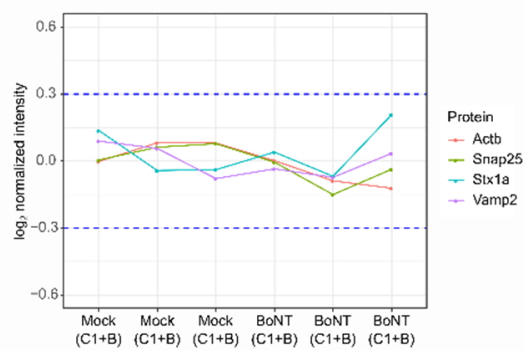

Figure 5.S21: Proteomics analysis of Mock- or BoNT-treated synaptosomes. Protein content in Mock- or BoNT-treated synaptosomes was assessed using TMT6-labeled peptides in the unbound fraction (notphosphorylated peptides) after phosphopeptide enrichment. Following $C$. botulinum cell culture supernatants were used: $(\boldsymbol{A}-\boldsymbol{C}) C$. botulinum $A+D,(\boldsymbol{D}-\boldsymbol{E})$ C. botulinum $C 1+B$. $(\boldsymbol{A}, \boldsymbol{D})$ Box-whisker plot of $\log _{2}$ reporter ion intensities before normalization. Boxes indicate $25 \%$ and $75 \%$ quantiles (interquartile range, IQR), vertical lines indicate medians, and whiskers indicate data within $1.5 \times I Q R$. (B, E) Differential protein content was assessed using limma package (311). Volcano plots of -log10 p-values vs. $\log _{2}$ Mock/BoNT. Orange dots: proteins with a Benjamini-Hochberg adjusted $p$-value of $<0.01$ and an absolute $\log _{2} F C$ of at least 0.586 . (C, E) $\log _{2}$ normalized reporter ion intensities of selected proteins, actin-beta (Actb), Snap25, syntaxin-1a (Stx1a), Vamp2. 


\subsubsection{Supplementary Tables}

Table 5.S1: Number of regulated phosphorylation events per predicted kinase group in Ca vs. EGTA experiment. "Sites in" show number of phosphorylation events that can be regulated by a respective kinase group as determined in the Ca vs. EGTA experiment. "Sites out" contains the number of phosphorylation events possibly regulated by other kinases. Similarly, "Sites in bcgr" and "Sites out bcgr" summarize the number of predicted substrate-kinase relations in the human proteome (used as background). Fisher's exact test was applied to test enrichment of substrate-kinase relationships as compared to predicted substrate-kinase relations in the human proteome. "p.val" and "p.adj" are raw and Benjamini-Hochberg adjusted p-values, respectively.

\begin{tabular}{|l|l|l|l|l|l|l|}
\hline Kinase group & Sites in & Sites out & Sites in bcgr & Sites out bcgr & p.val & p.adj \\
\hline MAPK & 175 & 728 & 6329 & 34101 & 0.003 & 0.009 \\
\hline CaMKII & 160 & 743 & 2764 & 37666 & 0.000 & 0.000 \\
\hline PKC & 141 & 762 & 5783 & 34647 & 0.269 & 0.377 \\
\hline CK1 & 80 & 823 & 4292 & 36138 & 0.100 & 0.201 \\
\hline PAK & 67 & 836 & 3317 & 37113 & 0.425 & 0.518 \\
\hline CDK & 60 & 843 & 3278 & 37152 & 0.122 & 0.221 \\
\hline CK2 & 46 & 857 & 2457 & 37973 & 0.258 & 0.371 \\
\hline GSK3 & 44 & 859 & 970 & 39460 & 0.000 & 0.000 \\
\hline DAPK & 29 & 874 & 2043 & 38387 & 0.011 & 0.026 \\
\hline MAP2K & 9 & 894 & 1967 & 38463 & 0.000 & 0.000 \\
\hline AMPK & 8 & 895 & 196 & 40234 & 0.091 & 0.196 \\
\hline PKB & 8 & 895 & 601 & 39829 & 0.162 & 0.283 \\
\hline CAMK & 7 & 896 & 261 & 40169 & 0.531 & 0.627 \\
\hline GRK & 7 & 896 & 798 & 39632 & 0.007 & 0.018 \\
\hline RSK & 6 & 897 & 276 & 40154 & 1.000 & 1.000 \\
\hline PLK & 6 & 897 & 929 & 39501 & 0.000 & 0.001 \\
\hline PDHK3_PDHK1 & 5 & 898 & 250 & 40180 & 1.000 & 1.000 \\
\hline CLK & 5 & 898 & 771 & 39659 & 0.001 & 0.004 \\
\hline MARK & 4 & 899 & 279 & 40151 & 0.537 & 0.627 \\
\hline SGK & 3 & 900 & 69 & 40361 & 0.208 & 0.315 \\
\hline BCKDK_PDHK4 & 3 & 900 & 74 & 40356 & 0.237 & 0.349 \\
\hline PKD & 3 & 900 & 193 & 40237 & 0.804 & 0.868 \\
\hline CaMKI & 2 & 901 & 36 & 40394 & 0.201 & 0.314 \\
\hline DMPK & 2 & 901 & 198 & 40232 & 0.335 & 0.446 \\
\hline p70S6K & 1 & 902 & 49 & 40381 & 1.000 & 1.000 \\
\hline PKGcGK & 1 & 902 & 414 & 40016 & 0.002 & 0.006 \\
\hline PKA & 1 & 902 & 507 & 39923 & 0.000 & 0.001 \\
\hline mTOR_ATM_ATR & 902 & 904 & 39526 & 0.000 & 0.000 \\
\hline Aurora & & & & & & \\
\hline & 1 & 425 & 0.006 \\
\hline
\end{tabular}


Table 5.S2: Enriched gene ontology biological function terms based on synapse-specific SynGO database (324). Proteins carrying regulated phosphorylation sites as determined in Ca vs. EGTA experiments were annotated using the synapse specific SynGO database. Significantly enriched (FDR < 0.001) GO biological function terms are presented together with the hierarchical structure. Note that only a part of proteins carrying regulated phosphorylation sites could be annotated using SynGO database (161 out of 463 unique genes).

\begin{tabular}{|c|c|c|}
\hline GO term ID & GO term name - hierarchical structure & FDR \\
\hline SYNGO:synprocess & process in the synapse & $5.58 \mathrm{E}-54$ \\
\hline SYNGO:presynprocess & F process in the presynapse & $9.91 \mathrm{E}-32$ \\
\hline GO:0099509 & F regulation of presynaptic cytosolic calcium levels & 8.86E-09 \\
\hline GO:0099626 & $\begin{array}{l}T_{\text {regulation of presynaptic cytosolic calcium levels }}\llcorner\text { voltage-gated calcium channel activity involved in } \\
\text { restion }\end{array}$ & 3.02E-05 \\
\hline GO:0099508 & $\begin{array}{l}\text { I } \\
\text { of presynaptic membrane potential }\end{array}$ & $2.38 \mathrm{E}-04$ \\
\hline GO:0099504 & T - synaptic vesicle cycle & $2.42 \mathrm{E}-27$ \\
\hline GO:0097091 & F synaptic vesicle clustering & $3.02 \mathrm{E}-05$ \\
\hline GO:0016079 & F- synaptic vesicle exocytosis & $4.57 \mathrm{E}-17$ \\
\hline GO:0099502 & | || $\mid F$ calcium-dependent activation of synaptic vesicle fusion & 8.08E-08 \\
\hline GO:0150037 & $\operatorname{l|l|l}_{\text {synaptic vesicle fusion }} \mid \quad L$ regulation of calcium-dependent activation of & $7.22 \mathrm{E}-08$ \\
\hline GO:2000300 & 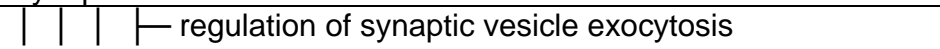 & $1.13 \mathrm{E}-05$ \\
\hline GO:0016081 & 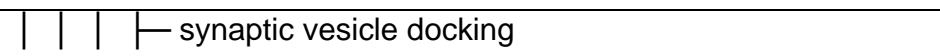 & $1.41 \mathrm{E}-06$ \\
\hline GO:0016082 & \begin{tabular}{l|l|l} 
& & L synaptic vesicle priming
\end{tabular} & $6.78 \mathrm{E}-09$ \\
\hline GO:0048488 & \begin{tabular}{l|l} 
& L synaptic vesicle endocytosis
\end{tabular} & $8.40 \mathrm{E}-08$ \\
\hline GO:0099525 & - presynaptic dense core vesicle exocytosis & 7.59E-04 \\
\hline SYNGO:postsynprocess & $F$ process in the postsynapse & 7.05E-11 \\
\hline GO:0099072 & $\begin{array}{l}\underset{\text { receptor levels }}{F} \text { regulation of postsynaptic membrane neurotransmitter } \\
\text {. }\end{array}$ & 2.16E-11 \\
\hline GO:0099645 & $\begin{array}{l}\text { If - neurotransmitter receptor localization to postsynaptic } \\
\text { specialization membrane }\end{array}$ & $1.38 \mathrm{E}-06$ \\
\hline GO:0098884 & 1 ட & 6.86E-04 \\
\hline GO:0099537 & $\mid \vdash$ trans-synaptic signaling & $3.84 \mathrm{E}-10$ \\
\hline GO:0099542 & | $\mid$ | $ட$ trans-synaptic signaling by endocannabinoid & 1.27E-04 \\
\hline GO:0007268 & | $\mid\llcorner$ chemical synaptic transmission & 7.22E-08 \\
\hline GO:0050804 & I $\quad$ - modulation of chemical synaptic transmission & $1.41 \mathrm{E}-06$ \\
\hline GO:0050808 & F synapse organization & $1.53 \mathrm{E}-24$ \\
\hline GO:0099173 & T & $7.98 \mathrm{E}-04$ \\
\hline GO:0099175 & \begin{tabular}{l|l} 
& - regulation of postsynapse organization
\end{tabular} & 7.69E-04 \\
\hline GO:0099010 & | $\mid$ L modification of postsynaptic structure & $1.10 \mathrm{E}-06$ \\
\hline GO:0098885 & L modification of postsynaptic actin cytoskeleton & 4.06E-06 \\
\hline GO:1905274 & $\prod_{\text {cytoskeleton }} L$ regulation of modification of postsynaptic actin & 2.61E-04 \\
\hline GO:0098918 & $\mid \vdash$ structural constituent of synapse & $2.45 \mathrm{E}-13$ \\
\hline GO:0098882 & \begin{tabular}{l|l|l} 
& $\mid$ & - structural constituent of active zone
\end{tabular} & $1.01 \mathrm{E}-07$ \\
\hline GO:0099186 & | $\mid$ L structural constituent of postsynapse & $1.80 \mathrm{E}-07$ \\
\hline GO:0098919 & L structural constituent of postsynaptic density & 6.09E-05 \\
\hline GO:0007416 & T & $2.38 \mathrm{E}-04$ \\
\hline GO:0099188 & ᄂ postsynaptic cytoskeleton organization & $1.38 \mathrm{E}-06$ \\
\hline GO:0098974 & F-postsynaptic actin cytoskeleton organization & 4.06E-06 \\
\hline
\end{tabular}


Table 5.S3 Number of regulated phosphorylation events per predicted kinase group in Mock vs. BoNT experiment. "Sites in" show number of phosphorylation events that can be regulated by a respective kinase group as determined in the Mock vs. BoNT experiment. "Sites out" contains the number of phosphorylation events possibly regulated by other kinases. Similarly, "Sites in bcgr" and "Sites out bcgr" summarize the number of predicted substrate-kinase relations in human proteome (used as background). Fisher's exact test was applied to test enrichment of substrate-kinase relationships as compared to predicted substrate-kinase relations in the human proteome. "p.val" and "p.adj" are raw and Benjamini-Hochberg adjusted p-values, respectively.

\begin{tabular}{|l|l|l|l|l|l|l|}
\hline Kinase group & Sites in & $\begin{array}{l}\text { Sites } \\
\text { out }\end{array}$ & $\begin{array}{l}\text { Sites in } \\
\text { bcgr }\end{array}$ & $\begin{array}{l}\text { Sites out } \\
\text { bcgr }\end{array}$ & p.val & p.adj \\
\hline MAPK & 229 & 628 & 6320 & 34052 & 0.000 & 0.000 \\
\hline PKC & 169 & 688 & 5933 & 34439 & 0.000 & 0.000 \\
\hline CK1 & 82 & 775 & 4486 & 35886 & 0.169 & 0.287 \\
\hline CDK & 78 & 779 & 3324 & 37048 & 0.347 & 0.452 \\
\hline CaMKII & 72 & 785 & 2812 & 37560 & 0.104 & 0.201 \\
\hline GSK3 & 40 & 817 & 984 & 39388 & 0.000 & 0.001 \\
\hline PAK & 36 & 821 & 3407 & 36965 & 0.000 & 0.000 \\
\hline CK2 & 32 & 825 & 2565 & 37807 & 0.001 & 0.004 \\
\hline DAPK & 24 & 833 & 2076 & 38296 & 0.001 & 0.004 \\
\hline CLK & 14 & 843 & 773 & 39599 & 0.704 & 0.804 \\
\hline PKB & 11 & 846 & 603 & 39769 & 0.775 & 0.868 \\
\hline GRK & 10 & 847 & 794 & 39578 & 0.104 & 0.201 \\
\hline DMPK & 9 & 848 & 171 & 40201 & 0.013 & 0.031 \\
\hline RSK & 9 & 848 & 245 & 40127 & 0.116 & 0.216 \\
\hline CAMK & 9 & 848 & 273 & 40099 & 0.202 & 0.314 \\
\hline AMPK & 3 & 854 & 208 & 40164 & 0.806 & 0.868 \\
\hline PDHK3_PDHK1 & 3 & 854 & 266 & 40106 & 0.387 & 0.493 \\
\hline PKGcGK & 3 & 854 & 404 & 39968 & 0.054 & 0.120 \\
\hline Aurora & 2 & 855 & 1078 & 39294 & 0.000 & 0.000 \\
\hline MAPKAPK & 1 & 856 & 18 & 40354 & 0.329 & 0.446 \\
\hline SLK & 1 & 856 & 23 & 40349 & 0.396 & 0.493 \\
\hline p70S6K & 1 & 856 & 50 & 40322 & 1.000 & 1.000 \\
\hline PKD & 1 & 856 & 191 & 40181 & 0.196 & 0.314 \\
\hline ROCK & 1 & 856 & 355 & 40017 & 0.008 & 0.021 \\
\hline PKA & 1 & 856 & 430 & 39942 & 0.002 & 0.006 \\
\hline NEK1_NEK5_NEK3_NEK4_NEK11_N & 1 & 856 & 601 & 39771 & 0.000 & 0.000 \\
\hline EK2 & 1 & 856 & 1982 & 38390 & 0.000 & 0.000 \\
\hline
\end{tabular}


Table 5.S4: Number of regulated phosphorylation events per predicted kinase group in Ca vs. EGTA and Mock vs. BoNT experiments. "CaEGTA" and "MockBoNT" show the number of the regulated phosphorylation events per kinase group in $\mathrm{Ca}^{2+}$ vs. EGTA and Mock vs. BoNT experiments, respectively. "Total CaEGTA" and "Total MockBoNT" show the total number of the regulated phosphorylation events with a predicted substratekinase relationship in respective experiment. Fisher's exact test was used to test the difference in the occurrence of predicted substrate-kinase relationships in Ca vs. EGTA and Mock vs. BoNT experiments. "p.val" is a p-value of the respective test. "p.adj.BH" is the p-value after Benjamini-Hochberg correction.

\begin{tabular}{|l|l|l|l|l|l|l|}
\hline $\begin{array}{l}\text { Kinase } \\
\text { group }\end{array}$ & CaEGTA & $\begin{array}{l}\text { Total } \\
\text { CaEGTA }\end{array}$ & MockBoTN & $\begin{array}{l}\text { Total } \\
\text { MockBoTN }\end{array}$ & p.val & p.adj \\
\hline CaMKII & 160 & 903 & 72 & 857 & 0.000 & 0.000 \\
\hline MAPK & 175 & 903 & 229 & 857 & 0.004 & 0.039 \\
\hline PAK & 67 & 903 & 36 & 857 & 0.008 & 0.051 \\
\hline MAP2K & 9 & 903 & 1 & 857 & 0.022 & 0.101 \\
\hline PLK & 6 & 903 & 0 & 857 & 0.031 & 0.101 \\
\hline DMPK & 2 & 903 & 9 & 857 & 0.034 & 0.101 \\
\hline CLK & 5 & 903 & 14 & 857 & 0.037 & 0.101 \\
\hline PKC & 141 & 903 & 169 & 857 & 0.065 & 0.153 \\
\hline CDK & 60 & 903 & 78 & 857 & 0.078 & 0.164 \\
\hline CK2 & 46 & 903 & 32 & 857 & 0.204 & 0.387 \\
\hline AMPK & 8 & 903 & 3 & 857 & 0.227 & 0.393 \\
\hline RSK & 6 & 903 & 9 & 857 & 0.443 & 0.669 \\
\hline GRK & 7 & 903 & 10 & 857 & 0.470 & 0.669 \\
\hline PKB & 8 & 903 & 11 & 857 & 0.493 & 0.669 \\
\hline DAPK & 29 & 903 & 24 & 857 & 0.677 & 0.719 \\
\hline CAMK & 7 & 903 & 9 & 857 & 0.620 & 0.719 \\
\hline DYRK & 7 & 903 & 9 & 857 & 0.620 & 0.719 \\
\hline CK1 & 80 & 903 & 82 & 857 & 0.682 & 0.719 \\
\hline GSK3 & 44 & 903 & 40 & 857 & 0.911 & 0.911 \\
\hline
\end{tabular}




\section{Discussion}

In this thesis, I applied an MS-based quantification workflow to study phosphoproteome changes in synaptosomes. Specifically, the investigations were focused on identifying phosphorylation sites that require active SV cycling and to distinguish them from phosphorylation events that are primarily triggered by the increase in the cytoplasmic $\mathrm{Ca}^{2+}$ concentration due to the depolarization of the plasma membrane. To achieve this, I utilized synaptosomes that were a) electrically stimulated or b) chemically stimulated using $\mathrm{KCl}$. While the electrical stimulation remained ambiguous for the reasons discussed below, $\mathrm{KCl}$ stimulation in combination with BoNT-treatment allowed for the differentiation of "SVcycling-dependent" and "primarily $\mathrm{Ca}^{2+}$-dependent" sites, respectively.

\subsection{Electrical field-stimulation of synaptosomes}

Synaptosomes represent pinched-off axonal terminals which plasma membrane re-seals during the homogenization process. While axolemma contains $\mathrm{Na}^{+}$-channels that facilitate the propagation of the action potential, the diversity of $\mathrm{Na}^{+}$-channels in the terminals is less pronounced (25). Therefore, it was hypothesized that the depolarization spreads passively at the nerve terminal and not in the form of an action potential. Considering this, the electrical field-stimulation would probably not be able to evoke an action potential in synaptosomes as it does in the whole cell cultures. Earlier studies utilized harsh stimulation regimes resulting in the release of neurotransmitters from synaptosomes, accumulation of lactate, and $\mathrm{Ca}^{2+}$, but the underlying mechanisms of these changes were not further investigated (105-112). Here I applied a milder $10 \mathrm{~Hz}$ stimulation in order to achieve a $\mathrm{Ca}^{2+}$ dependent glutamate release, since $\mathrm{Ca}^{2+}$ is considered as a trigger of $\mathrm{SV}$ exocytosis in nerve terminals. Although the release was significantly lower in EGTA, monitoring of the cytoplasmic $\mathrm{Ca}^{2+}$ concentration using Fura-2 did not show any $\mathrm{Ca}^{2+}$-influx that could be attributed to the electrical stimulation. However, Fura-2 assay reflects $\mathrm{Ca}^{2+}$ concentration in the whole cytoplasm, but it would not capture local spikes of $\mathrm{Ca}^{2+}$ concentration at the active zone, which are already sufficient to trigger the neurotransmitter release (407). Earlier studies demonstrated $\mathrm{Ca}^{2+}$ accumulation in synaptosomes during the course of electrical stimulation $(111,112)$. The measurements were conducted using a ${ }^{45} \mathrm{Ca}$ isotope and showed a significant, although a relatively moderate increase $(\sim 4 \mathrm{nM}$ increase $v s$. up to $200 \mathrm{nM}$ when using $\mathrm{KCl}$ stimulation), which might not be captured by Fura-2 fluorimetry as applied in this study. However, application of $\mathrm{Ca}^{2+}$ channel blockers such as $\mathrm{Cd}^{2+}$ or $\omega$-conotoxin MVCII was not able to abolish the release. The effect was rather controversial: while the release was slightly decreased using $20 \mu \mathrm{M} \mathrm{Cd}^{2+}$ or $1-2 \mu \mathrm{M}$ of the $\omega$-conotoxin

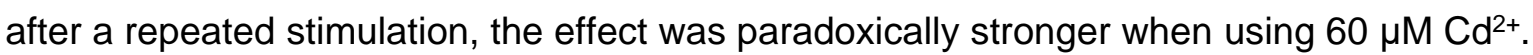


However, adding $\mathrm{Cd}^{2+}$ to the phosphate-containing buffer might cause a precipitation of a phosphate salt, which would reduce the net $\mathrm{Cd}^{2+}$ concentration and unpredictably affect the release. Another set of experiments utilized acridine orange to monitor the neurotransmitter release and SV recycling. Here, electrical stimulation did not cause an observable change in $\mathrm{AO}$ fluorescence. Nonetheless, the latter might be explained by that rapid recycling mechanisms efficiently dampen the increase in AO-fluorescence, since even in the case of a $\mathrm{KCl}$-evoked release, the initial spike in the AO-fluorescence returns to the basal levels within 2-3 seconds. Strikingly, treatment with BoNT A/D did not suppress the release. This fact raises the question, whether the observed glutamate liberation occurs via SV exocytosis. Other routs of the release are possible, e.g., leakage of the cytoplasmic glutamate via the glutamate transporter in the plasma membrane. However, the latter would not explain the significant suppression of the release in EGTA condition. Taken together, these observations do not favor the hypothesis that the electrical stimulation evokes glutamate release via exocytosis, though it is not possible to exclude that some glutamate (probably $<30 \%$ ) is released exocytotically.

Although exact mechanisms of the neurotransmitter release caused by electrical stimulation need to be further elucidated, electrical stimulation is advantageous for kinetics studies since it is free of mixing effects (such in the case of adding $\mathrm{KCl}$ ) and has, therefore, clearly defined start and end points. As a proof of principle, phosphoproteome changes in synaptosomes were measured at six time points, including a time point before stimulation and two time points after the stimulation. A selection of sites that showed a Pearson correlation of $>0.6$ between the two kinetic measurements and a minimum $50 \%$ intensity change at any time point has shown that changes affect proteins that are already known to regulate SV cycling, such as synapsin-1 or CaMKII, active zone proteins, SV proteins, proteins involved in endocytosis, etc. This implies that the changes might reflect adaptations of the synapse to the altered membrane potential and an increased energy demand due to the glutamate release caused by the electrical stimulation. In this regard, it is tempting to speculate, whether the electrical stimulation might appear useful in deciphering molecular mechanisms underlying the modulation of the neuronal excitability and short behavior effects such as those induced by transcranial direct current stimulation $(408,409)$.

\subsection{BoNT treatment of synaptosomes}

BoNTs are highly potent clostridial toxins that exert an exceptionally specific action in cleaving SNARE proteins. In this study, a combination of two BoNT types was applied, i.e., $A$ and $D$ or $C 1$ and $B$. Each pair of toxins cleaves plasma membrane-standing t-SNARE and vesicular v-SNARE, SNAP-25 and synaptobrevin-2 or syntaxin-1 and synaptobrevin-2, respectively. It has to be noted that the toxins are applied in their native form, which consists 
of a heavy and light chain. The heavy chain facilitates the toxin uptake into the nerve terminal, while the light chain exerts its catalytic activity (410). The toxin entry relies on endocytosis and can occur, therefore, only in a functional synaptosome. The latter is the probable reason for an overall unaltered content of SNARE proteins in BoNT- and Mocktreated synaptosomes, as discussed in section 5.13.2. Of note, BoNT-treated synaptosomes were compared against synaptosomes treated with heat-inactivated (Mock) toxins. This experimental detail is important as it allows to compensate for prolonged incubation times and unforeseen effects of adding $C$. botulinum cell culture supernatants to synaptosomal suspensions. Furthermore, an application of the four different toxin types $(A+D$ and $C 1+B)$ aimed to minimize unpredictable effects associated with a particular toxin type. As shown by previous studies $(121,122)$ and confirmed here (Figure 5.S1), glutamate liberation following $\mathrm{KCl}$-evoked depolarization is significantly reduced in BoNT-treated synaptosomes, while the $\mathrm{Ca}^{2+-i n f l u x}$ is unaffected. This fact allowed identifying phosphorylation events that are responsive to BoNT-treatment, i.e., dependent on active SV cycling, and separate phosphorylation events that are primarily triggered by $\mathrm{Ca}^{2+}$-influx.

\subsection{Categorization of phosphorylation events}

This study categorizes phosphorylation events in two groups, namely, "SV-cyclingdependent" and "primarily $\mathrm{Ca}^{2+}$-dependent". The categorization is based on the responsiveness of protein phosphorylation to BoNT-treatment and changes following depolarization under $\mathrm{Ca}$ or EGTA condition (Figure 5.4). This categorization follows a simplified assumption that there are three basic triggers: a) $\mathrm{Ca}^{2+}$-influx; b) membrane depolarization; and c) SV cycling. While $A$ is a generally accepted cause of the synaptic phosphoproteome changes given the abundance of $\mathrm{Ca}^{2+}$-regulated kinases and phosphatases such as CaMKII and calcineurin in the brain, $C$ was a working hypothesis, and $B$ could not be addressed in this study, since we lack means to control membrane potential in synaptosomes, apart from persistent depolarization using chemical agents. Accordingly, all conditions compared here are based on synaptosomes depolarized using $50 \mathrm{mM} \mathrm{KCl}$. Comparison of $\mathrm{KCl}$-stimulated synaptosomes under $\mathrm{Ca}$ or EGTA conditions shows, therefore, changes that are $\mathrm{Ca}^{2+}$ and/or SV-cycling-dependent, since EGTA prevents $\mathrm{Ca}^{2+}$-influx and, consequently, an active SV cycling. BoNT-treatment hampers SV cycling, but not the $\mathrm{Ca}^{2+}$-influx and thus allows to demarcate phosphorylation events that require SV cycling. Indeed, identification of BoNT-responsive phosphorylation sites supports the hypothesis that SV cycling is required for certain phosphorylation events to take place. A hypothesis is that SV cycling relies on multiple protein rearrangements, interaction with cytoskeleton elements and assembly/disassembly of multimeric protein complexes (3). These rearrangements might expose docking sites for kinases and 
phosphatases that can then exert their actions (Figure 6.1). As already discussed in section 5.6, PP1 is a probable mediator of SV-cycling-dependent changes, as it can be targeted to cytoskeleton elements via regulatory subunits. Accordingly, differentially phosphorylated sites were observed on Neurabin-1 and other PP1 regulators (Figure 5.S7), which may influence the synaptic localization of PP1 (238).

The classification of phosphorylation events into "primarily $\mathrm{Ca}^{2+}$-dependent" and "SVcycling-dependent" is indirectly corroborated by the fact that predicted CaMKII phosphorylation sites are significantly enriched among "primarily $\mathrm{Ca}^{2+}$-dependent" group. This observation is expected given the $\mathrm{Ca}^{2+}$-dependence of CaMKII. Moreover, phosphorylation sites on "dephosphins" which are known targets for calcineurin, a $\mathrm{Ca}^{2+} /$ calmodulin-dependent phosphatase, fall into the same group. It has to be noted, however, that such dichotomic classification is likely an oversimplification, which, as any other model, does not consider the whole palette of relationships between kinases, phosphatases and their substrates in the synapse. Thus, it is reported that CaMKII may also act as a scaffold, therefore, it is likely that protein rearrangements during SV cycling influence this type of CaMKII activity $(157,158)$. Kinases/phosphosphatases can activate or inhibit each other via respective (de)phosphorylation reactions. For instance, calcineurin regulates PP1 activity via dephosphorylation of its regulatory subunits (259), while PP1 is known to dephosphorylate CaMKII, thus inhibiting its kinase activity (411). Nonetheless, this classification proved useful, as it is first to suggest that the synaptic phosphoproteome is affected by SV cycling. Moreover, it can be used for selection of candidate sites that might regulate SV cycling. Thus, SV-cycling-dependent sites on syntaxin-1, synaptobrevin-2 and cannabinoid receptor-1 have drawn our attention, and their modulatory effect on effect on exo- and endocytosis could be demonstrated in cultured hippocampal neurons.

\subsection{Challenges and limitations of MS-based phosphoproteomics}

Technical advances in the field of mass spectrometry allowed for identification and quantification of thousands of phosphorylation sites (288). Nonetheless, analysis of PTMs poses additional experimental and analytical challenges. When analyzing phosphorylated peptides, one needs to consider that phosphorylated peptides are generally underrepresented in the proteome. Current state of the enrichment techniques, such as $\mathrm{TiO}_{2}$-based enrichment, allows to enrich phosphorylated peptides with high specificity (>90\%). However, none of the available techniques would cover all phosphorylated peptides. Moreover, different enrichment techniques have varying affinities toward certain types of peptides. For example, IMAC-based enrichment outperforms $\mathrm{TiO}_{2}$ in the enrichment of multiply phosphorylated peptides (412). Other analytical challenges arise during LC-MS/MS acquisition. Phosphorylation provides an additional negative charge to a 
peptide, which decreases its "flyability" in the MS if analyzed in the positive ion mode. Moreover, phosphorylation is a labile chemical modification that is first to be eliminated during the fragmentation process, which creates an additional site localization problem. The latter is further complicated by existing phosphorylation isoforms - peptides that carry the same number of phosphorylation groups on different positions. Such peptides are poorly resolved by conventional LC systems and often produce mixed spectra of co-fragmented precursors. The subsequent analysis usually considers only phosphorylation sites identified with a high localization probability ( $>75 \%)$. Onethe one hand, this approach decreases the number of analyzed phosphorylation sites, on the other hand, it increases the confidence of the identifications, since phosphorylation sites with a high localization probability are usually identified based on high-score PSMs with a comprehensive fragmentation pattern (413).

In this study, it was possible to alleviate some of the problems by using TMT labeling approach and extensive pre-fractionation using bRP. This allowed us to significantly increase coverage of the identified and quantified phosphorylation sites as compared to other studies (Figure 5.2) $(351,352)$. Furthermore, TMT-labeling reduced the missing value problem, which arises due to the semi-stochastic nature of MS acquisition. The latter increased the number of sites that could be compared among different experiments and conditions. However, higher sensitivity and better comparability come at the cost of a reduced dynamic range. Due to the co-fragmentation of several precursors, TMT-based quantification does not accurately depict intensity changes and tends to underestimate them (300). The latter was taken into account when determining the candidate sites, since phosphorylation sites that showed an intensity fold change of 1.2 were considered as significant, if satisfying other criteria. For kinetics analysis, the quantification was achieved using an SPS-MS3 approach, which allows for an accurate ratio estimation though at the cost of reduced sensitivity (301).

\subsection{Challenges and limitations of the functional analysis of phosphorylation sites}

Another type of challenges associates with the functional analysis of the phosphorylation sites. Thus, in the case of a protein expression analysis, it is legitimate to assume that the decrease in the protein amount correlates with a decreased functional activity of the protein. This, however, does not hold true for protein phosphorylation: a reduced/increased phosphorylation might result in both, a gain or loss of a protein function, and the degree of a change does not necessarily reflect the degree of a functional change. Moreover, since the function of the most phosphorylation sites remains unknown, it is not always possible to 
anticipate the functional consequences of a phosphorylation. Therefore, the functional analysis in phosphoproteomics is usually restricted to the analysis of general protein functions. A further complication is that a phosphorylation site might appear on a multiply phosphorylated peptide. It is common, therefore, to distinguish a single, double, or multiple phosphorylation states (multiplicity) and consider each multiplicity state of a site as a separate phosphorylation event. The differential analysis is often conducted at the level of phosphorylation events, because singly or multiply phosphorylated species of the same peptide might be regulated differently. However, when using global functional analysis of differentially phosphorylated proteins, it is currently not possible to account for these differences.

Here, the functional analysis was focused on identifying possible kinase-substrate relationships and the association of the identified regulating kinase groups with functional terms (Figure 5.3). For the kinase-substrate prediction, an experimental information available in PhosphoSitePlus database was utilized in the first place. For other sites, a kinase was predicted using an available NetworKIN algorithm, which utilizes the information about the consensus sequence motif known protein-protein interactions (323). Although a prediction in each particular case might be error-prone, however, it provides a global view on possible kinase-substrate interactions. In the following, application of different annotation sources such as general GO terms (317), as well as the neuron/synapse-specific annotations (Reactome pathways (319) and SynGO database (324), respectively), and manually assigned keywords (Figure 5.S8) demonstrated that a) pre-synaptic proteins are significantly enriched among differentially phosphorylated proteins; b) putative sites for CaMKII and MAPK are preferably enriched among "primary $\mathrm{Ca}^{2+}$-dependent" and "SVcycling-dependent" sites, respectively; c) while CaMKII preferentially regulates sites on proteins that are directly involved in $\mathrm{Ca}^{2+}$-dependent neurotransmitter release or its regulation, putative MAPK targets are mostly involved in the synapse organization, i.e., cytoskeleton protein, adhesion molecules, etc.

\subsection{Regulation of the kinase and phosphatase activity via phosphorylation}

Kinases and phosphatases control the phosphorylation status of their substrates. However, phosphorylation of the kinases, phosphatases or their direct regulators may further shed light on the activation status of these enzymes and hint at the intricate coordination of their activities. 


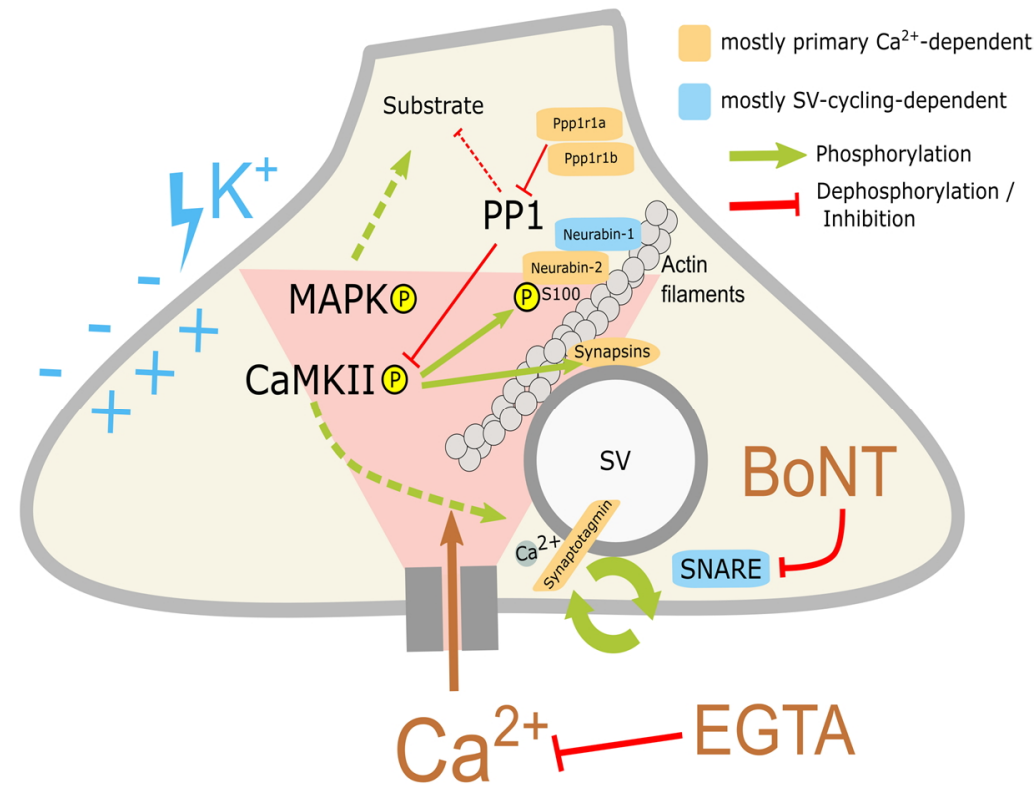

Figure 6.1: $\mathrm{KCl}$ stimulation of synaptosomes: proposed connection of protein phosphorylation and SV cycling activity. The scheme shows effects of BoNT and EGTA on SV cycling, as well as proposed balanced activities of CaMKII, MAPK and PP1. Following depolarization by $50 \mathrm{mM} \mathrm{KCl}$, $\mathrm{Ca}^{2+}$-influx triggers $\mathrm{SV}$ cycling via interaction with $\mathrm{Ca}^{2+}$ sensor synaptotagmin. EGTA prevents $\mathrm{Ca}^{2+-i n f l u x}$ and subsequently, SV exocytosis. BoNTs blocks exocytosis via cleavage of SNARE proteins, but do not compromise Ca ${ }^{2+}$-influx. EGTA prevents $\mathrm{Ca}^{2+}$-influx and subsequently, SV exocytosis. BoNTs blocks exocytosis via cleavage of SNARE proteins, but do not compromise $\mathrm{Ca}^{2+}$-influx. Increase in $\mathrm{Ca}^{2+}$ concentration activates CaMKII (via autophosphorylation) and MAPK (presumably via Ras activation). CaMKII is deactivated via dephosphorylation by PP1. Following Ca ${ }^{2+-i n f l u x, ~ P P 1 ~ c a n n o t ~ k e e p ~ p a c e ~ i n ~ d e p h o s p h o r y l a t i n g ~}$ CaMKII, which results in the sustained CaMKII activity and phosphorylation of its substrates. Comparison of synaptosomes stimulated under $\mathrm{Ca}$ and EGTA conditions implies dephosphorylation of putative MAPK substrates and even stronger dephosphorylation in the case of stalled SV cycling due to BoNT-treatment, which contradicts apparent MAPK activation. A suggested hypothesis is that PP1 dephosphorylates MAPK substrates, which further sequesters PP1 activity and disinhibits CaMKII. PP1 is targeted to F-actin via its regulatory subunits, neurabin-1 and neurabin-2. CaMKII presumably attenuates this targeting and the PP1 activity towards MAPK substrates via phosphorylating neurabin-2 at S100. (De)phosphorylation of PP1 inhibitors Ppp1r1a and Ppp1r1b, e.g., via PKA, calcineurin, etc., may further modulate PP1 activity. Active SV cycling might attenuate the dephosphorylation of MAPK targets probably due to the protein complex rearrangements and due to the detachment of SVS from actin filaments during SV cycling. Synapsin-1 phosphorylation may affect SV association with actin filaments. Accordingly, block of SV cycling by BoNT causes even stronger dephosphorylation of MAPK targets, which appear as an increased phosphorylation when comparing Mock vs. BoNT treated synaptosomes. Green arrows represent kinase/substrate interaction (phosphorylation). Blunt-end arrows represent dephosphorylation and/or inhibition, see in text. Dashed lines represent hypothetical interactions. Protein names on blue/orange background denote the prevalent phosphorylation type, "SV-cyclingdependent", or "primary $\mathrm{Ca}^{2+}$-dependent", respectively.

\subsubsection{CaMKII, MAPK, and PP1}

Remarkably, the phosphorylation sites on CaMKII and MAPK themselves fall into the group of "primarily $\mathrm{Ca}^{2+}$-dependent". Specifically, the phosphorylation of Camk2a-T286, Camk2bT287, Camk2d-T287, Mapk1-T183, Y185, Mapk3-T203, Y205 points out activation of these kinases following depolarization and $\mathrm{Ca}^{2+-e n t r y . ~ D e s p i t e ~ t h e ~ a p p a r e n t ~ a c t i v a t i o n ~ o f ~ M A P K, ~}$ 
the majority of MAPK substrates appear dephosphorylated. The latter can be explained by the competing phosphatase activity that counterbalance MAPK activation at the time point of observation. It was previously hypothesized that CaMKII and PP1 form a switch-like system in the synapse (414), where PP1 controls the phosphorylation status of CaMKII and, therefore, its activity. However, the catalytic activity of PP1 is a limiting factor. Once the rate of CaMKII autophosphorylation increases dramatically due to the $\mathrm{Ca}^{2+}$-influx, thedephosphorylation by PP1 cannot keep pace, which results in a burst of CaMKII activity. It is tempting to speculate whether this model can be further extended to incorporate MAPK. Thus, increased $\mathrm{Ca}^{2+}$ concentration might activate MAPK via Ras activation (167). On the other hand, we observe the dephosphorylation of MAPK-targets when comparing $\mathrm{Ca}$ and EGTA conditions. If we assume that PP1 is at least partially responsible for the dephosphorylation of MAPK targets, this might further sequester the activity of PP1 towards CaMKII, thus keeping CaMKII longer in an active state, which results in an increased phosphorylation of CaMKII substrates. Furthermore, it seems that inhibition of SV cycling via BoNT further increases phosphatase activity towards MAPK-substrates (Figure 5.2F). Following hypothesis provides a solution to this conundrum (Figure 6.1): PP1 is targeted to F-actin via its regulatory subunits, neurabin-1 and neurabin-2. $\mathrm{Ca}^{2+-i n f l u x ~ l e a d s ~ t o ~ a u t o-~}$ phosphorylation of CaMKII and MAPK activation. PP1 counteracts MAPK activity via dephosphorylation of its substrates, which further disinhibits CaMKII and results in the phosphorylation of CaMKII substrates. CaMKII presumably attenuates the PP1 activity towards MAPK substrates via phosphorylating neurabin-2 at $\mathrm{S} 100$ and thus reducing the PP1 interaction with F-actin. Note that cytoskeleton-associated proteins are enriched among MAPK targets (Figure 5.3A). Active SV cycling might further attenuate the dephosphorylation of MAPK targets probably due to the protein complex rearrangements and due to the detachment of SVs from actin filaments during SV cycling. Accordingly, block of SV cycling by BoNT causes even stronger dephosphorylation of MAPK targets, which appear as an increased phosphorylation when comparing Mock vs. BoNT treated synaptosomes (Figure 5.2F).

\subsubsection{PKA, CDK5, and GSK3}

The action of PP1 can be further fine-tuned by the activity of PKA. Thus, PP1 inhibitors Ppp1r1a and Ppp1r1b attenuate PP1 activity following phosphorylation by PKA (245-247). Accordingly, Ppp1r1a-S46, S47 and Ppp1r1b-S45 sites are significantly upregulated in Ca condition, which implies the activation of the PP1 inhibitors. The CDK5-mediated phosphorylation at Ppp1r1a-S67 was previously shown to inhibit PKA activity $(246,247)$. Since this site appear dephosphorylated, it implies PKA activation following $\mathrm{KCl}$ depolarization. Furthermore, PKA activation may be an adaptation towards increased 
energy demand because of depolarization, since the kinase is involved in the regulation of metabolic pathways. Remarkably, the catalytic subunit of PKA itself is differentially phosphorylated at Prkaa1-S486. A stretch of $S$ and T residues at the C-terminus of PKA is a probable target of GSK3 (415), which attenuates PKA activity. The dephosphorylation of the Prkaa1 at these residues implies disinhibition of PKA. Phosphorylation of CDK5 or GSK3 themselves does not allow to deduce the activity status of the kinases. However, of note is the "SV-cycling-dependent" phosphorylation of a CDK5 regulator p39 (Cdk5r2-T84). Phosphorylation at this site has been previously shown to affect localization of CDK5 (416). Given that predicted CDK targets, similarly to MAPK targets, appear strongly dephosphorylated following BoNT-treatment (Figure 5.2F), it is tempting to speculate, whether the inhibition of SV cycling influences the localization of CDK5 and thus modulates its activity.

\subsubsection{CK1 and CK2}

Based on the phosphorylation pattern of CK1 isoforms, it is not possible to infer the activity status of CK1. However, increased phosphorylation of $\epsilon$-CK1 (Csnk1e) at S363 implies inhibition of CK1 activity following BoNT-treatment, since C-terminal phosphorylation of the $\delta$ isoform (Csnk1d) by PKC or autophosphorylation has been shown to attenuate kinase activity $(417,418)$. The phosphorylation of Csnk1e-S363 has been previously identified by other studies; however, its functional role has not been elucidated yet.$(419,420)$

Not much can be said about the activity $\mathrm{CK} 2$ following $\mathrm{KCl}$-depolarization. A regulatory beta subunit of CK2 appear dephosphorylated under BoNT-treatment (Csnk2b-S205, S209). The phosphorylation at S209 has been previously observed in mitotic cells, but its functional role remained unknown, though it was hypothesized that it can affect the localization of CK2 (421).

\subsubsection{PAK1}

In our data, Pak1 appears significantly dephosphorylated at several sites (T167, S218, $\mathrm{S} 219, \mathrm{~S} 222)$ in stimulated synaptosomes in the presence of $\mathrm{Ca}^{2+}$. As shown by Shin et al, phosphorylation at $\mathrm{S} 222$ by CK2 is required for a complete PAK1 activation (422). The dephosphorylation of PAK1 at the aforementioned residues implies $\mathrm{Ca}^{2+}$-dependent deactivation of PAK1 following $2 \mathrm{~min}$ of $\mathrm{KCl}$-depolarization. Moreover, absence of differentially regulated sites on a PAK1-substrate Raf1 implies that the observed MAPK activation is not mediated via PAK1/Raf1 activation. 


\subsubsection{PKC}

Increased phosphorylation of PKC at Prkcb-T640 (T642) implies catalytically active state of the kinase following $\mathrm{KCl}$-depolarization in the presence of $\mathrm{Ca}^{2+}$. Dephosphorylation of further "primarily $\mathrm{Ca}^{2+}$-dependent" sites at the C-terminus of an unconventional Prkcb sequence (Uniprot accession F1LS42, sites S659, S653, and S663) implies regulation of its intracellular localization. The dephosphorylation is presumably mediated by PP2 (184).

\subsubsection{PP2}

The activity of PP2 might be linked to the regulation of PP1 and PKA activity via dephosphorylation of PP1 inhibitor Ppp1r1b (254). It was previously shown that PKAmediated phosphorylation of B56ठ subunit (Ppp2r5d) has an activating effect on PP2A. PP2A dephosphorylates T75 on PP1 inhibitory subunit Ppp1r1b leading to PP1 inhibition (254) Though the proposed phosphorylation of the regulatory subunit Ppp2r5d was not observed in this study, the phosphorylation of another regulatory subunit, Ppp2r5c, at $\$ 497$ is strongly upregulated following $\mathrm{KCl}$-depolarization in the presence of $\mathrm{Ca}^{2+}$. It is tempting to speculate whether this phosphorylation has a similar activating function for PP2.

\subsubsection{Calcineurin}

The activity of calcineurin following $\mathrm{KCl}$-depolarization is indirectly corroborated by the dephosphorylated state of the proteins involved in endocytosis, also known as dephosphins. Remarkably, the majority of the differentially phosphorylated sites on dephosphins belong to the "primary $\mathrm{Ca}^{2+}$-dependent" group. The latter reflects the high affinity of calcineurin towards $\mathrm{Ca}^{2+} /$ calmodulin, which is required to adequately adjust the endocytosis rate in response to the depolarization by $\mathrm{KCl}$ (257). Calcineurin might further participate in the regulation of PP1 activity via dephosphorylating Ppp1r1a and Ppp1r1b (259). Two sites of an a catalytic subunit close to an autoinhibitory segment are differentially regulated (Figure 5.S7), i.e., Ppp3ca-S498 ("primary $\mathrm{Ca}^{2+}$-dependent") and Ppp3ca-S462 ("SVcycling-dependent"). Although the functional role of these sites is unknown, it is tempting to speculate that these phosphorylation events may affect the catalytic activity and/or the localization of the enzyme.

\subsection{Primary $\mathrm{Ca}^{2+}$-dependent sites and SV-cycling-dependent phosphorylation}

Functional analysis of differentially phosphorylated proteins revealed that certain functional groups carry phosphorylation sites that are categorized either as "primary $\mathrm{Ca}^{2+}$-dependent" or "SV-cycling-dependent" (Figure 5.5 and Figure 5.S16). Unfortunately, the functional role of the most phosphorylation events is unknown and can only be speculated. However, it 
appears that certain functional groups tend to be phosphorylated following the one or another type. Some of the prominent functional groups are discussed below.

\subsubsection{Active zone and exocytosis-related proteins}

The categorization of phosphorylation sites into "primary Ca ${ }^{2+}$-dependent" and "SV-cyclingdependent" demonstrated that "primary $\mathrm{Ca}^{2+}$-dependent" sites are preferably clustered on proteins that are located near the active zone and, except a few cases discussed below, are directly involved in the process of exo- and endocytosis. Primary $\mathrm{Ca}^{2+}$-dependent phosphorylation of these proteins may be explained by the fact that they are located in a vicinity of $\mathrm{Ca}^{2+}$-channel and, consequently, represent the closest targets for $\mathrm{Ca}^{2+}$-activated kinases and phosphatases.

Large proteins of the active zone such as bassoon, piccolo, and RIM-proteins are strongly phosphorylated. Since these proteins are known to organize the active zone, it is tempting to speculate, whether their (de)phosphorylation provides a necessary milieu for various protein-protein interactions near the plasma membrane or acts as a $\mathrm{Ca}^{2+-b u f f e r}$. A very recent study indicates that bassoon might regulate the SV distribution between the readily releasable and reserved pools of SVs (423). Furthermore, bassoon deletion alters CDK5/calcineurin and PKA signaling in the presynapse, which may further affect phosphorylation of other exocytosis-related proteins. The phosphorylation of active zone proteins may indeed affect SV pool distributions. Thus, phosphorylation of a close cooperating partner of bassoon, Erc2, at $\mathrm{S} 45$ has been previously shown to impede the reloading of the RRP (424). Interestingly, our data show a strong upregulation of S45 phosphorylation on a related protein, Erc1, and only a sub-threshold dephosphorylation of Erc2-S45. Moreover, an active-zone associated kinase, Brsk1, which is responsible for Erc2 phosphorylation, is dephosphorylated at multiple sites following $\mathrm{KCl}$-depolarization in the presence of $\mathrm{Ca}^{2+}$. Although the functional role of Brsk1 phosphorylation sites is not known, these observations imply that SV pools and the neurotransmitter release might be tightly controlled via phosphorylation of the active zone proteins.

\subsection{2 $\mathrm{K}^{+}$-channels}

A substantial group of SV-cycling-dependent phosphorylation sites constitutes sites on $\mathrm{K}^{+}$channel proteins (Figure 5.S14-16) that are differentially affected by BoNT-treatment. Remarkable is the dephosphorylation of several C-terminal sites of Kcnd2 following BoNT treatment. This region is important for channel activation and inactivation (425), and the amino acid phosphorylation in this region is governed by PKC and MAPK (426), which may change the activation voltage and conductivity of the channel (427). Furthermore, Cterminal phosphorylation of another subunit, Kcna2, has been associated with the trafficking 
and cell surface expression of $\mathrm{K}_{\mathrm{v}} 1.2$ channels (428). In contrast to Kcnd2, Kcna2-S440, S441, S447, S449 show increased phosphorylation following BoNT-treatment. It is tempting to speculate if the C-terminal phosphorylation of Kcna2 represents a compensatory mechanism to alleviate clamp-depolarization via increasing the presence of $\mathrm{K}^{+}$-channels at the plasma membrane. Intriguingly, several sites on Kcnb1, a Kv2.1 forming subunit, are dephosphorylated following BoNT treatment. Similarly to Kcna2 and Kcnd2, its phosphorylation has been implicated in the regulation of channel trafficking and gating properties $(429,430)$. Moreover, Kcnb1 phosphorylation and membrane insertion have been proposed as a step accompanying neuronal apoptosis (431). The study of Pal et al (432) reported reduced plasma membrane expression of $\mathrm{K}_{\mathrm{v}} 2.1$ in neurons expressing BoNT of type $\mathrm{C} 1$ or $\mathrm{E}$. Authors hypothesized that the reduced channel expression at the plasma membrane may be due to the reduced direct interaction of SNARE proteins, as this interaction has been demonstrated previously (433-435). However, our data imply that the link might be indirect. It is tempting to speculate that the channel trafficking is regulated via phosphorylation, similar to Kcna2, and this phosphorylation depends on active SV cycling. The SV cycling dependence of the phosphorylation is governed by SNARE: BoNT-exposure leads to a stalled SV cycling and/or distorted direct interaction with SNARE, which may change the availability of phosphorylation sites for kinase/phosphatase activity as discussed above and, consequently, results in an altered plasma membrane presentation of the channel.

\subsubsection{Cytoskeleton-associated proteins}

Cytoskeleton-associated proteins comprise a large group of proteins that interact with microtubuli and/or actin filaments or other structural proteins and play important roles in the synapse organization and membrane trafficking. Especially the role of actin filaments in the synapse was extensively investigated. It was proposed that SV interaction with actin via phosphoprotein synapsin-1 might regulate the release probability of SVs. Thus, synapsin-1 (de)phosphorylation, e.g., phosphorylation at $\mathrm{S} 603$ following $\mathrm{KCl}$-depolarization reduces SVs binding to actin and facilitates their mobility (385). Notably, proteins constituting actinspectrin network such as spectrin $\beta$-chain and adducin $\alpha, \beta$ contain preferably SV-cyclingdependent phosphorylation sites (Figure 5.S16). Although the exact function of these phosphorylation events is not known, it hints at the re-organization of the synapse in response to the active SV cycling. Considering the aspect of the synapse remodeling, it is interesting to note, that several members of the Wnt pathway show SV-cycling-dependent phosphorylations, including Adenomatous polyposis coli protein (Apc), TRAF2 and NCKinteracting kinase (Tnik), $\delta$-catenin (Ctnnd1, Ctnnd2) and others. Given that Wnt pathway is implicated in synapse development and maintenance (436), it is tempting to speculate 
whether these phosphorylation events reflect remodeling processes associated with SV cycling. A previous study has identified CDK5 as a mediator of Ctnnd2 phosphorylation that affects its subcellular localization and the neuronal activity. Accordingly, dephosphorylation of Cttnd2 following BoNT-treatment implies its reduced membrane localization, which might affect the cadherin-cytoskeletal interactions (437) and the stability of synaptic junctions $(438,439)$.

Remarkable is the dephosphorylation of microtubule-associated proteins $1 \mathrm{a}, 1 \mathrm{~b}, 2$, and tau (Map1a, Map1b, Map2, and Mapt, respectively) following BoNT treatment. Given that the phosphorylation status of these proteins might affect the microtubule stability, it is reasonable to speculate that SV cycling activity might additionally influence the synapse stability via phosphorylation of microtubule-associated proteins. The latter hypothesis aligns well with the observed SV-cycling-dependent phosphorylation of other cytoskeleton- and Wnt-related proteins. PAK, CK1, GSK3, and CDK5 have been previously suggested as candidate kinases that are capable of phosphorylating Map2 and Mapt (220, 440), while PP2A and PP1 have being shown to dephosphorylate them at distinct positions $(441,442)$.

\subsubsection{Phosphorylation of SNARE and its implication for exocytosis}

In contrast to other exocytosis-related proteins, phosphorylation sites on the three core SNARE proteins, syntaxin-1, synaptobrevin-2, and SNAP25, as well as the closely associated Munc18-1 were exclusively SV-cycling-dependent. This notice has further strengthened the hypothesis that active SV cycling is necessary to provide an access for kinases and phosphatases to their substrates, as it is likely that the pre-assembled SNARE complexes are less accessible for kinases and phosphatases. These proteins may become modified after the disassembly, e.g., after the exocytosis was complete. Remarkably, in contrast to the majority of SV-cycling-dependent phosphorylation sites, BoNT-treatment led to an increased phosphorylation of the three SNARE proteins and Munc18. The latter implies an increased kinase activity that may affect the SNARE complex formation (229, 353-358). In this work, expression of non-phosphorylatable or pseudo-phosphorylated variants of syntaxin-1 and synaptobrevin-2, as well as on the cannabinoid receptor-1 - a receptor known for its regulatory role in the exocytosis - in the cultured hippocampal neurons have shown modulatory effects of these modifications on the SV cycling. Interestingly, a recent study has investigated a non-phosphorylatable Stx1a-D17A using a similar pHluorin-based approach (229). According to authors, Stx1a-D17A prevents Stx1a phosphorylation at $\mathrm{S} 14$ by CK2. The analysis of exocytosis has shown an increased exocytosis in Stx1a-D17A expressing mutants under long (900 AP) stimulation. In contrast, our analysis revealed that pseudo-phosphorylated Stx1a-T21E/T23E increases exocytosis following short (60 AP) stimulation (Figure 5.6). This discrepancy implies that despite close 
localization, the phosphorylations at S14 and T21/T23 sites might have a different impact on the exocytosis rate and, therefore, represent a possible molecular mechanism for finetuning of the neurotransmitter release.

\subsection{Conclusions and Outlook}

In this work, an optimized MS-based phosphoproteomics was applied to deepen our knowledge about the synaptic phosphoproteome and its dynamics in response to stimulation. Electrical field-stimulation of synaptosomes has been characterized in respect to $\mathrm{Ca}^{2+}$-dependent neurotransmitter release and its utility for kinetics studies has been demonstrated. Using BoNT-treated synaptosomes it was possible to demonstrate SV cycling dependence of phosphorylation events in the synapse and dissect primary $\mathrm{Ca}^{2+}$ dependent and SV-cycling-dependent phosphorylation events, respectively. Based on this categorization, selected SV-cycling-dependent phosphorylation sites on syntaxin-1, synaptobrevin-2, and cannabinoid receptor-1 were tested for their modulatory effects on the SV cycling. The effects they exert on exo- and endocytosis in cultured hippocampal cells confirmed the utility of this categorization.

Several aspects can be proposed for the future work. Firstly, using super-resolution microscopy it would be important to confirm the hypothesis that stalled SV cycling affects localization of kinases and phosphatases such as PP1, leading to the observation of SVcycling-dependent phosphorylation events. Secondly, use of selective inhibitors of kinases and phosphatases may further help to unravel the complex enzyme-substrate interactions. Thirdly, other PTMs such as ubiquitination and glycosylation are likely to play important role in the synapse physiology. Studying interplay of glycosylation and phosphorylation may further broaden our understanding of the molecular mechanisms governing neurotransmitter release and synaptic functioning. 


\section{$7 \quad$ References}

1. Ryan, T. J., and Grant, S. G. (2009) The origin and evolution of synapses. Nature Reviews Neuroscience 10, 701-712

2. Guzman, R. E., Alekov, A. K., Filippov, M., Hegermann, J., and Fahlke, C. (2014) Involvement of $\mathrm{CIC}-3$ chloride/proton exchangers in controlling glutamatergic synaptic strength in cultured hippocampal neurons. Frontiers in cellular neuroscience 8, 143

3. Jahn, R., and Fasshauer, D. (2012) Molecular machines governing exocytosis of synaptic vesicles. Nature 490, 201-207

4. Takamori, S., Holt, M., Stenius, K., Lemke, E. A., Grønborg, M., Riedel, D., Urlaub, H., Schenck, S., Brügger, B., and Ringler, P. (2006) Molecular anatomy of a trafficking organelle. Cell 127, 831-846

5. Couteaux, R., and Pecot-Dechavassine, M. (1970) Synaptic vesicles and pouches at the level of" active zones" of the neuromuscular junction. Comptes rendus hebdomadaires des seances de l'Academie des sciences. Serie D: Sciences naturelles $271,2346-2349$

6. Landis, D. M. (1988) Membrane and cytoplasmic structure at synaptic junctions in the mammalian central nervous system. Journal of electron microscopy technique 10, 129151

7. Südhof, T. C. (2012) The presynaptic active zone. Neuron 75, 11-25

8. Bloom, F. E., and Aghajanian, G. K. (1966) Cytochemistry of synapses: selective staining for electron microscopy. Science 154, 1575-1577

9. Collins, M. O., Husi, H., Yu, L., Brandon, J. M., Anderson, C. N., Blackstock, W. P., Choudhary, J. S., and Grant, S. G. (2006) Molecular characterization and comparison of the components and multiprotein complexes in the postsynaptic proteome. Journal of neurochemistry $97,16-23$

10. Ding, J. D., Kennedy, M. B., and Weinberg, R. J. (2013) Subcellular organization of camkii in rat hippocampal pyramidal neurons. Journal of Comparative Neurology 521, 3570 3583

11. Graziane, N., and Dong, Y. (2016) Electrophysiological analysis of synaptic transmission, Springer

12. Feiner, A.-S., and McEvoy, A. (1994) The Nernst equation. Journal of chemical education 71,493

13. Goldman, D. E. (1943) Potential, impedance, and rectification in membranes. The Journal of general physiology 27, 37-60

14. Hodgkin, A. L., and Katz, B. (1949) The effect of sodium ions on the electrical activity of the giant axon of the squid. The Journal of physiology 108, 37-77

15. Johnston, D., and Wu, S. M.-S. (1994) Foundations of cellular neurophysiology, MIT press

16. Kaplan, J. H. (2002) Biochemistry of Na, K-ATPase. Annual review of biochemistry 71, 511-535

17. Khananshvili, D. (2013) The SLC8 gene family of sodium-calcium exchangers (NCX)-Structure, function, and regulation in health and disease. Molecular aspects of medicine 34, 220-235

18. Dang, D., and Rao, R. (2016) Calcium-ATPases: gene disorders and dysregulation in cancer. Biochimica et Biophysica Acta (BBA)-Molecular Cell Research 1863, 1344-1350 19. Bonar, P. T., and Casey, J. R. (2008) Plasma membrane $\mathrm{Cl} / \mathrm{HCO}$-exchangers: structure, mechanism and physiology. Channels 2, 337-345

20. Virtanen, M. A., Uvarov, P., Hübner, C. A., and Kaila, K. (2020) NKCC1, an elusive molecular target in brain development: making sense of the existing data. Cells 9, 2607

21. Kaila, K., Price, T. J., Payne, J. A., Puskarjov, M., and Voipio, J. (2014) Cationchloride cotransporters in neuronal development, plasticity and disease. Nature Reviews Neuroscience 15, 637-654 
22. Catterall, W. A. (2011) Voltage-gated calcium channels. Cold Spring Harbor perspectives in biology 3, a003947

23. Reid, C. A., Bekkers, J. M., and Clements, J. D. (2003) Presynaptic Ca ${ }^{2+}$ channels: a functional patchwork. Trends in neurosciences 26, 683-687

24. Campiglio, M., and Flucher, B. E. (2015) The role of auxiliary subunits for the functional diversity of voltage-gated calcium channels. Journal of cellular physiology 230 , 2019-2031

25. Debanne, D., Campanac, E., Bialowas, A., Carlier, E., and Alcaraz, G. (2011) Axon physiology. Physiological reviews 91, 555-602

26. Leão, R. M., Kushmerick, C., Pinaud, R., Renden, R., Li, G.-L., Taschenberger, H., Spirou, G., Levinson, S. R., and Von Gersdorff, H. (2005) Presynaptic Na+ channels: locus, development, and recovery from inactivation at a high-fidelity synapse. Journal of Neuroscience 25, 3724-3738

27. Engel, D., and Jonas, P. (2005) Presynaptic action potential amplification by voltagegated $\mathrm{Na}^{+}$channels in hippocampal mossy fiber boutons. Neuron 45, 405-417

28. Kawaguchi, S.-y., and Sakaba, T. (2015) Control of inhibitory synaptic outputs by low excitability of axon terminals revealed by direct recording. Neuron 85, 1273-1288

29. González, C., Baez-Nieto, D., Valencia, I., Oyarzún, I., Rojas, P., Naranjo, D., and Latorre, R. (2012) $\mathrm{K}^{+}$channels: function-structural overview. Comprehensive physiology 2 , 2087-2149

30. Wang, H., Kunkel, D., Schwartzkroin, P. A., and Tempel, B. L. (1994) Localization of Kv1.1 and Kv1.2, two K channel proteins, to synaptic terminals, somata, and dendrites in the mouse brain. Journal of Neuroscience 14, 4588-4599

31. Dodson, P. D., Billups, B., Rusznák, Z., Szûcs, G., Barker, M. C., and Forsythe, I. D. (2003) Presynaptic rat Kv1.2 channels suppress synaptic terminal hyperexcitability following action potential invasion. Wiley Online Library

32. Ishikawa, T., Nakamura, Y., Saitoh, N., Li, W.-B., Iwasaki, S., and Takahashi, T. (2003) Distinct roles of Kv1 and Kv3 potassium channels at the calyx of Held presynaptic terminal. Journal of Neuroscience 23, 10445-10453

33. Dodson, P. D., and Forsythe, I. D. (2004) Presynaptic $\mathrm{K}^{+}$channels: electrifying regulators of synaptic terminal excitability. Trends in neurosciences 27, 210-217

34. Katz, B., and Miledi, R. (1967) The release of acetylcholine from nerve endings by graded electric pulses. Proceedings of the Royal Society of London. Series B. Biological Sciences 167, 23-38

35. Schneggenburger, R., and Neher, E. (2005) Presynaptic calcium and control of vesicle fusion. Current opinion in neurobiology 15, 266-274

36. Söllner, T., Whiteheart, S. W., Brunner, M., Erdjument-Bromage, H., Geromanos, S., Tempst, P., and Rothman, J. E. (1993) SNAP receptors implicated in vesicle targeting and fusion. Nature 362, 318-324

37. Chapman, E. R., An, S., Barton, N., and Jahn, R. (1994) SNAP-25, a t-SNARE which binds to both syntaxin and synaptobrevin via domains that may form coiled coils. Journal of Biological Chemistry 269, 27427-27432

38. Geppert, M., Goda, Y., Hammer, R. E., Li, C., Rosahl, T. W., Stevens, C. F., and Südhof, T. C. (1994) Synaptotagmin I: a major $\mathrm{Ca}^{2+}$ sensor for transmitter release at a central synapse. Cell 79, 717-727

39. Toonen, R. F., and Verhage, M. (2007) Munc18-1 in secretion: lonely Munc joins SNARE team and takes control. Trends in neurosciences 30, 564-572

40. Brose, N. (2008) Altered complexin expression in psychiatric and neurological disorders: cause or consequence? Molecules \& Cells (Springer Science \& Business Media BV) 25

41. Fernández-Chacón, R., Shin, O.-H., Königstorfer, A., Matos, M. F., Meyer, A. C., Garcia, J., Gerber, S. H., Rizo, J., Südhof, T. C., and Rosenmund, C. (2002) Structure/function analysis of $\mathrm{Ca}^{2+}$ binding to the C2A domain of synaptotagmin 1. Journal of Neuroscience 22, 8438-8446

42. Südhof, T. C. (2004) The synaptic vesicle cycle. Annu. Rev. Neurosci. 27, 509-547 
43. Fujita, Y., Shirataki, H., Sakisaka, T., Asakura, T., Ohya, T., Kotani, H., Yokoyama, S., Nishioka, H., Matsuura, Y., and Mizoguchi, A. (1998) Tomosyn: a syntaxin-1-binding protein that forms a novel complex in the neurotransmitter release process. Neuron 20, 905915

44. Scales, S. J., Hesser, B. A., Masuda, E. S., and Scheller, R. H. (2002) Amisyn, a novel syntaxin-binding protein that may regulate SNARE complex assembly. Journal of Biological Chemistry 277, 28271-28279

45. Edelmann, L., Hanson, P., Chapman, E., and Jahn, R. (1995) Synaptobrevin binding to synaptophysin: a potential mechanism for controlling the exocytotic fusion machine. The EMBO journal 14, 224-231

46. Südhof, T. C., and Rizo, J. (2011) Synaptic vesicle exocytosis. Cold Spring Harbor perspectives in biology 3, a005637

47. Gandhi, S. P., and Stevens, C. F. (2003) Three modes of synaptic vesicular recycling revealed by single-vesicle imaging. Nature 423, 607-613

48. Chanaday, N. L., Cousin, M. A., Milosevic, I., Watanabe, S., and Morgan, J. R. (2019) The synaptic vesicle cycle revisited: new insights into the modes and mechanisms. Journal of Neuroscience 39, 8209-8216

49. Heuser, J., and Reese, T. (1973) Evidence for recycling of synaptic vesicle membrane during transmitter release at the frog neuromuscular junction. The Journal of cell biology 57, 315-344

50. Beck, K. A., and Keen, J. H. (1991) Interaction of phosphoinositide cycle intermediates with the plasma membrane-associated clathrin assembly protein AP-2. Journal of Biological Chemistry 266, 4442-4447

51. Ahle, S., and Ungewickell, E. (1986) Purification and properties of a new clathrin assembly protein. The EMBO Journal 5, 3143-3149

52. Chen, H., Fre, S., Slepnev, V. I., Capua, M. R., Takei, K., Butler, M. H., Di Fiore, P. P., and De Camilli, P. (1998) Epsin is an EH-domain-binding protein implicated in clathrinmediated endocytosis. Nature 394, 793-797

53. Henne, W. M., Kent, H. M., Ford, M. G., Hegde, B. G., Daumke, O., Butler, P. J. G., Mittal, R., Langen, R., Evans, P. R., and McMahon, H. T. (2007) Structure and analysis of FCHo2 F-BAR domain: a dimerizing and membrane recruitment module that effects membrane curvature. Structure 15, 839-852

54. Shimada, A., Niwa, H., Tsujita, K., Suetsugu, S., Nitta, K., Hanawa-Suetsugu, K., Akasaka, R., Nishino, Y., Toyama, M., and Chen, L. (2007) Curved EFC/F-BAR-domain dimers are joined end to end into a filament for membrane invagination in endocytosis. Cell 129, 761-772

55. Edeling, M. A., Smith, C., and Owen, D. (2006) Life of a clathrin coat: insights from clathrin and AP structures. Nature reviews Molecular cell biology 7, 32-44

56. Sweitzer, S. M., and Hinshaw, J. E. (1998) Dynamin undergoes a GTP-dependent conformational change causing vesiculation. Cell 93, 1021-1029

57. Haffner, C., Takei, K., Chen, H., Ringstad, N., Hudson, A., Butler, M. H., Salcini, A. E., Di Fiore, P. P., and De Camilli, P. (1997) Synaptojanin 1: localization on coated endocytic intermediates in nerve terminals and interaction of its $170 \mathrm{kDa}$ isoform with Eps15. FEBS letters 419, 175-180

58. Schuske, K. R., Richmond, J. E., Matthies, D. S., Davis, W. S., Runz, S., Rube, D. A., van der Bliek, A. M., and Jorgensen, E. M. (2003) Endophilin is required for synaptic vesicle endocytosis by localizing synaptojanin. Neuron 40, 749-762

59. Chappell, T. G., Welch, W. J., Schlossman, D. M., Palter, K. B., Schlesinger, M. J., and Rothman, J. E. (1986) Uncoating ATPase is a member of the 70 kilodalton family of stress proteins. Cell 45, 3-13

60. Prasad, K., Barouch, W., Greene, L., and Eisenberg, E. (1993) A protein cofactor is required for uncoating of clathrin baskets by uncoating ATPase. Journal of Biological Chemistry 268, 23758-23761 
61. Wong, K. A., Wilson, J., Russo, A., Wang, L., Okur, M. N., Wang, X., Martin, N. P., Scappini, E., Carnegie, G. K., and O'Bryan, J. P. (2012) Intersectin (ITSN) family of scaffolds function as molecular hubs in protein interaction networks. PLoS One 7, e36023

62. Wang, L., Johnson, A., Hanna, M., and Audhya, A. (2016) Eps15 membrane-binding and-bending activity acts redundantly with Fcho1 during clathrin-mediated endocytosis. Molecular biology of the cell 27, 2675-2687

63. Salazar, M. A., Kwiatkowski, A. V., Pellegrini, L., Cestra, G., Butler, M. H., Rossman, K. L., Serna, D. M., Sondek, J., Gertler, F. B., and De Camilli, P. (2003) Tuba, a novel protein containing bin/amphiphysin/Rvs and Dbl homology domains, links dynamin to regulation of the actin cytoskeleton. Journal of Biological Chemistry 278, 49031-49043

64. Miki, H., Miura, K., and Takenawa, T. (1996) N-WASP, a novel actin-depolymerizing protein, regulates the cortical cytoskeletal rearrangement in a PIP2-dependent manner downstream of tyrosine kinases. The EMBO journal 15, 5326-5335

65. Saheki, Y., and De Camilli, P. (2012) Synaptic vesicle endocytosis. Cold Spring Harbor perspectives in biology 4, a005645

66. Peter, B. J., Kent, H. M., Mills, I. G., Vallis, Y., Butler, P. J. G., Evans, P. R., and McMahon, H. T. (2004) BAR domains as sensors of membrane curvature: the amphiphysin BAR structure. Science 303, 495-499

67. $\mathrm{Wu}, \mathrm{T}$., and Baumgart, T. (2014) BIN1 membrane curvature sensing and generation show autoinhibition regulated by downstream ligands and PI $(4,5)$ P2. Biochemistry 53, 7297-7309

68. Taylor, M. J., Perrais, D., and Merrifield, C. J. (2011) A high precision survey of the molecular dynamics of mammalian clathrin-mediated endocytosis. PLoS biol 9, e1000604 69. Ceccarelli, B., Hurlbut, W., and Mauro, A. (1973) Turnover of transmitter and synaptic vesicles at the frog neuromuscular junction. The Journal of cell biology 57, 499524

70. Staal, R. G., Mosharov, E. V., and Sulzer, D. (2004) Dopamine neurons release transmitter via a flickering fusion pore. Nature neuroscience 7, 341-346

71. Qin, X., Tsien, R. W., and Park, H. (2019) Real-time three-dimensional tracking of single synaptic vesicles reveals that synaptic vesicles undergoing kiss-and-run fusion remain close to their original fusion site before reuse. Biochemical and biophysical research communications 514, 1004-1008

72. Watanabe, S., Rost, B. R., Camacho-Pérez, M., Davis, M. W., Söhl-Kielczynski, B., Rosenmund, C., and Jorgensen, E. M. (2013) Ultrafast endocytosis at mouse hippocampal synapses. Nature 504, 242-247

73. Delvendahl, I., Vyleta, N. P., von Gersdorff, H., and Hallermann, S. (2016) Fast, temperature-sensitive and clathrin-independent endocytosis at central synapses. Neuron 90, $492-498$

74. Watanabe, S., Mamer, L. E., Raychaudhuri, S., Luvsanjav, D., Eisen, J., Trimbuch, T., Söhl-Kielczynski, B., Fenske, P., Milosevic, I., and Rosenmund, C. (2018) Synaptojanin and endophilin mediate neck formation during ultrafast endocytosis. Neuron 98, 1184-1197. e1186

75. Clayton, E. L., Evans, G. J., and Cousin, M. A. (2008) Bulk synaptic vesicle endocytosis is rapidly triggered during strong stimulation. Journal of Neuroscience 28, 6627-6632

76. Cousin, M. A. (2009) Activity-dependent bulk synaptic vesicle endocytosis-a fast, high capacity membrane retrieval mechanism. Molecular neurobiology 39, 185-189

77. Wu, W., Xu, J., Wu, X.-S., and Wu, L.-G. (2005) Activity-dependent acceleration of endocytosis at a central synapse. Journal of Neuroscience 25, 11676-11683

78. Clayton, E. L., Anggono, V., Smillie, K. J., Chau, N., Robinson, P. J., and Cousin, M. A. (2009) The phospho-dependent dynamin-syndapin interaction triggers activitydependent bulk endocytosis of synaptic vesicles. Journal of Neuroscience 29, 7706-7717 79. Cheung, G., and Cousin, M. A. (2019) Synaptic vesicle generation from activitydependent bulk endosomes requires a dephosphorylation-dependent dynamin-syndapin interaction. Journal of neurochemistry 151, 570-583 
80. Kononenko, N. L., Puchkov, D., Classen, G. A., Walter, A. M., Pechstein, A., Sawade, L., Kaempf, N., Trimbuch, T., Lorenz, D., and Rosenmund, C. (2014) Clathrin/AP2 mediate synaptic vesicle reformation from endosome-like vacuoles but are not essential for membrane retrieval at central synapses. Neuron 82, 981-988

81. Cheung, G., and Cousin, M. A. (2012) Adaptor protein complexes 1 and 3 are essential for generation of synaptic vesicles from activity-dependent bulk endosomes. Journal of Neuroscience 32, 6014-6023

82. Hoopmann, P., Punge, A., Barysch, S. V., Westphal, V., Bückers, J., Opazo, F., Bethani, I., Lauterbach, M. A., Hell, S. W., and Rizzoli, S. O. (2010) Endosomal sorting of readily releasable synaptic vesicles. Proceedings of the National Academy of Sciences 107, 19055-19060

83. Stadler, H., and Tsukita, S. (1984) Synaptic vesicles contain an ATP-dependent proton pump and show 'knob-like'protrusions on their surface. The EMBO journal 3, 33333337

84. Maycox, P., Deckwerth, T., Hell, J. W., and Jahn, R. (1988) Glutamate uptake by brain synaptic vesicles. Energy dependence of transport and functional reconstitution in proteoliposomes. Journal of Biological Chemistry 263, 15423-15428

85. Strata, P., and Harvey, R. (1999) Dale's principle. Brain research bulletin 50, 349350

86. Zeisel, A., Hochgerner, H., Lönnerberg, P., Johnsson, A., Memic, F., Van Der Zwan, J., Häring, M., Braun, E., Borm, L. E., and La Manno, G. (2018) Molecular architecture of the mouse nervous system. Cell 174, 999-1014. e1022

87. Upmanyu, N., Jin, J., Ganzella, M., Bösche, L., Malviya, V. N., Zhuleku, E., Politi, A., Ninov, M., Silbern, I., Urlaub, H., Riedel, D., Preobraschenski, J., Milosevic, I., Jahn, R., and Sambandan, S. (2021) Co-localization of different Neurotransmitter Transporters on the same Synaptic Vesicle is Bona-fide yet Sparse. bioRxiv

88. Fernández-Busnadiego, R., Asano, S., Oprisoreanu, A.-M., Sakata, E., Doengi, M., Kochovski, Z., Zürner, M., Stein, V., Schoch, S., and Baumeister, W. (2013) Cryo-electron tomography reveals a critical role of RIM1a in synaptic vesicle tethering. Journal of Cell Biology 201, 725-740

89. Imig, C., Min, S.-W., Krinner, S., Arancillo, M., Rosenmund, C., Südhof, T. C., Rhee, J., Brose, N., and Cooper, B. H. (2014) The morphological and molecular nature of synaptic vesicle priming at presynaptic active zones. Neuron 84, 416-431

90. Neher, E., and Brose, N. (2018) Dynamically primed synaptic vesicle states: key to understand synaptic short-term plasticity. Neuron 100, 1283-1291

91. Schrimpf, S. P., Meskenaite, V., Brunner, E., Rutishauser, D., Walther, P., Eng, J., Aebersold, R., and Sonderegger, P. (2005) Proteomic analysis of synaptosomes using isotope-coded affinity tags and mass spectrometry. Proteomics 5, 2531-2541

92. Hebb, C. O., and Whittaker, V. (1958) Intracellular distributions of acetylcholine and choline acetylase. The journal of physiology 142, 187-196

93. Gray, E., and Whittaker, V. (1962) The isolation of nerve endings from brain: an electron microscopic study of cell fragments derived by homogenization and centrifugation. Journal of anatomy 96,79

94. Nicholls, D. G. (1989) Release of glutamate, aspartate, and $y$-aminobutyric acid from isolated nerve terminals. Journal of neurochemistry 52, 331-341

95. Xue, J., Quan, A., and Robinson, P. J. (2018) Preparation of P2 or Percoll-Purified Synaptosomes from Mammalian Brain Tissue. Synaptosomes, pp. 85-105, Springer

96. Dunkley, P. R., and Robinson, P. J. (2018) Synaptosome Preparations: Which Procedure Should I Use? Synaptosomes, pp. 27-53, Springer

97. Dunkley, P. R., Heath, J. W., Harrison, S. M., Jarvie, P. E., Glenfield, P. J., and Rostas, J. A. (1988) A rapid Percoll gradient procedure for isolation of synaptosomes directly from an S1 fraction: homogeneity and morphology of subcellular fractions. Brain research $441,59-71$ 
98. Kauppinen, R. A., and Nicholls, D. G. (1986) Synaptosomal bioenergetics: the role of glycolysis, pyruvate oxidation and responses to hypoglycaemia. European journal of biochemistry 158, 159-165

99. Nicholls, D. G. (2003) Bioenergetics and transmitter release in the isolated nerve terminal. Neurochemical research 28, 1433-1441

100. McMahon, H. T., and Nicholls, D. G. (1991) Transmitter glutamate release from isolated nerve terminals: evidence for biphasic release and triggering by localized $\mathrm{Ca}^{2+}$. Journal of neurochemistry 56, 86-94

101. Komulainen, H., and Bondy, S. (1987) The estimation of free calcium within synaptosomes and mitochondria with fura-2; comparison to quin-2. Neurochemistry international 10, 55-64

102. Barrie, A. P., Nicholls, D. G., Sanchez-Prieto, J., and Sihra, T. S. (1991) An ion channel locus for the protein kinase $\mathrm{C}$ potentiation of transmitter glutamate release from guinea pig cerebrocortical synaptosomes. Journal of neurochemistry 57, 1398-1404

103. Cousin, M. A., and Robinson, P. J. (2000) Two mechanisms of synaptic vesicle recycling in rat brain nerve terminals. Journal of neurochemistry $75,1645-1653$

104. Zoccarato, F., Cavallini, L., and Alexandre, A. (1999) The pH-Sensitive Dye Acridine Orange as a Tool to MonitorExocytosis/Endocytosis in Synaptosomes. Journal of neurochemistry 72, 625-633

105. Bradford, H. F. (1970) Metabolic response of synaptosomes to electrical stimulation: release of amino acids. Brain Res 19, 239-247

106. Hawthorne, J. N., and Bleasdale, J. E. (1975) Phosphatidic acid metabolism, calcium ions and transmitter release from electrically stimulated synaptosomes. Mol Cell Biochem 8, 83-87

107. Bleasdale, J. E., and Hawthorne, J. N. (1975) The effect of electrical stimulation on the turnover of phosphatidic acid in synaptosomes from guinea-pig brain. J Neurochem 24, 373-379

108. Osborne, R. H., Bradford, H. F., and Jones, D. G. (1973) Patterns of amino acid release from nerve-endings isolated from spinal cord and medulla. $J$ Neurochem 21, 407419

109. Bradford, H. F., Bennett, G. W., and Thomas, A. J. (1973) Depolarizing stimuli and the release of physiologically active amino acids from suspensions of mammalian synaptosomes. J Neurochem 21, 495-505

110. Lahdesmaki, P., Pasula, M., and Oja, S. S. (1975) Effect of electrical stimulation and chlorpromazine on the uptake and release of taurine, gamma-aminobutyric acid and glutamic acid in mouse brain synaptosomes. J Neurochem 25, 675-680

111. Swanson, P. D., Anderson, L., and Stahl, W. L. (1974) Uptake of calcium ions by synaptosomes from rat brain. Biochim Biophys Acta 356, 174-183

112. Goncalves, M. L., Pinto, F., and Ribeiro, J. A. (1991) Effect of adenosine on ${ }^{45} \mathrm{Ca}^{2+}$ uptake by electrically stimulated rat brain synaptosomes. J Neurochem $56,1769-1773$

113. Osborne, R. H., and Bradford, H. F. (1973) Tetanus toxin inhibits amino acid release from nerve endings in vitro. Nat New Biol 244, 157-158

114. Nicholls, D. G. (2018) Synaptosomal Bioenergetics and Glutamate Release. Synaptosomes, pp. 109-129, Springer

115. Scott, I., and Nicholls, D. (1980) Energy transduction in intact synaptosomes. Influence of plasma-membrane depolarization on the respiration and membrane potential of internal mitochondria determined in situ. Biochemical Journal 186, 21-33

116. Tibbs, G. R., Dolly, J. O., and Nicholls, D. G. (1989) Dendrotoxin, 4-aminopyridine, and $\beta$-bungarotoxin act at common loci but by two distinct mechanisms to induce $\mathrm{Ca}^{2+}$ dependent release of glutamate from guinea-pig cerebrocortical synaptosomes. Journal of neurochemistry 52, 201-206

117. Tibbs, G., Barrie, A., Van Mieghem, F., McMahon, H. a., and Nicholls, D. (1989) Repetitive action potentials in isolated nerve terminals in the presence of 4-aminopyridine: effects on cytosolic free $\mathrm{Ca}^{2+}$ and glutamate release. Journal of neurochemistry 53, $1693-$ 1699 
118. Nicholls, D. G., Sihra, T. S., and Sanchez-Prieto, J. (1987) Calcium-dependent andindependent release of glutamate from synaptosomes monitored by continuous fluorometry. Journal of neurochemistry 49, 50-57

119. Turner, T. J., Lampe, R. A., and Dunlap, K. (1995) Characterization of presynaptic calcium channels with omega-conotoxin MVIIC and omega-grammotoxin SIA: role for a resistant calcium channel type in neurosecretion. Mol Pharmacol 47, 348-353

120. Montecucco, C., Schiavo, G., and Pantano, S. (2005) SNARE complexes and neuroexocytosis: how many, how close? Trends Biochem Sci 30, 367-372

121. Sanchez-Prieto, J., Sihra, T. S., Evans, D., Ashton, A., Dolly, J. O., and Nicholls, D. G. (1987) Botulinum toxin A blocks glutamate exocytosis from guinea-pig cerebral cortical synaptosomes. Eur J Biochem 165, 675-681

122. McMahon, H. T., Foran, P., Dolly, J. O., Verhage, M., Wiegant, V. M., and Nicholls, D. G. (1992) Tetanus toxin and botulinum toxins type A and B inhibit glutamate, gammaaminobutyric acid, aspartate, and met-enkephalin release from synaptosomes. Clues to the locus of action. J Biol Chem 267, 21338-21343

123. Rizzoli, S. O., and Betz, W. J. (2005) Synaptic vesicle pools. Nature Reviews Neuroscience 6, 57-69

124. Elmqvist, D., and Quastel, D. (1965) A quantitative study of end-plate potentials in isolated human muscle. The Journal of physiology 178, 505-529

125. Waters, J., and Smith, S. J. (2002) Vesicle pool partitioning influences presynaptic diversity and weighting in rat hippocampal synapses. The Journal of physiology 541, 811823

126. Hanse, E., and Gustafsson, B. (2001) Vesicle release probability and pre-primed pool at glutamatergic synapses in area CA1 of the rat neonatal hippocampus. The Journal of Physiology 531, 481-493

127. Neher, E., and Taschenberger, H. (2021) Non-negative matrix factorization as a tool to distinguish between synaptic vesicles in different functional states. Neuroscience 458, 182-202

128. Zhang, Q., Li, Y., and Tsien, R. W. (2009) The dynamic control of kiss-and-run and vesicular reuse probed with single nanoparticles. Science 323, 1448-1453

129. Granseth, B., Odermatt, B., Royle, S. J., and Lagnado, L. (2009) Comment on "The Dynamic Control of Kiss-and-Run and Vesicular Reuse Probed with Single Nanoparticles". Science 325, 1499-1499

130. Kamin, D., Lauterbach, M. A., Westphal, V., Keller, J., Schönle, A., Hell, S. W., and Rizzoli, S. O. (2010) High-and low-mobility stages in the synaptic vesicle cycle. Biophysical Journal 99, 675-684

131. Denker, A., and Rizzoli, S. O. (2010) Synaptic vesicle pools: an update. Frontiers in synaptic neuroscience 2, 135

132. Hayashi, M., Raimondi, A., O'Toole, E., Paradise, S., Collesi, C., Cremona, O., Ferguson, S. M., and De Camilli, P. (2008) Cell-and stimulus-dependent heterogeneity of synaptic vesicle endocytic recycling mechanisms revealed by studies of dynamin 1-null neurons. Proceedings of the National Academy of Sciences 105, 2175-2180

133. Citri, A., and Malenka, R. C. (2008) Synaptic plasticity: multiple forms, functions, and mechanisms. Neuropsychopharmacology 33, 18-41

134. Bairoch, A., and Apweiler, R. (2000) The SWISS-PROT protein sequence database and its supplement TrEMBL in 2000. Nucleic acids research 28, 45-48

135. Khoury, G. A., Baliban, R. C., and Floudas, C. A. (2011) Proteome-wide posttranslational modification statistics: frequency analysis and curation of the swiss-prot database. Scientific reports 1, 1-5

136. Cohen, P. (2002) The origins of protein phosphorylation. Nature cell biology 4, E127E130

137. Burnett, G., and Kennedy, E. P. (1954) The enzymatic phosphorylation of proteins. Journal of Biological Chemistry 211, 969-980

138. Cieśla, J., Frączyk, T., and Rode, W. (2011) Phosphorylation of basic amino acid residues in proteins: important but easily missed. Acta Biochimica Polonica 58 
139. Fuhs, S. R., and Hunter, T. (2017) pHisphorylation: the emergence of histidine phosphorylation as a reversible regulatory modification. Current opinion in cell biology 45, 8-16

140. Ohsako, S., Nakazawa, H., Sekihara, S.-i., Ikai, A., and Yamauchi, T. (1991) Role of threonine-286 as autophosphorylation site for appearance of $\mathrm{Ca}^{2+}$-independent activity of calmodulin-dependent protein kinase II a subunit. The Journal of Biochemistry 109, 137143

141. Rellos, P., Pike, A. C., Niesen, F. H., Salah, E., Lee, W. H., Von Delft, F., and Knapp, S. (2010) Structure of the CaMKIIס/calmodulin complex reveals the molecular mechanism of CaMKII kinase activation. PLoS biology 8, e1000426

142. Yaffe, M. B., and Elia, A. E. (2001) Phosphoserine/threonine-binding domains. Current opinion in cell biology 13, 131-138

143. Kaneko, T., Joshi, R., Feller, S. M., and Li, S. S. (2012) Phosphotyrosine recognition domains: the typical, the atypical and the versatile. Cell Communication and Signaling 10, $1-20$

144. Miller, M. L., Jensen, L. J., Diella, F., Jørgensen, C., Tinti, M., Li, L., Hsiung, M., Parker, S. A., Bordeaux, J., and Sicheritz-Ponten, T. (2008) Linear motif atlas for phosphorylation-dependent signaling. Science signaling 1, ra2-ra2

145. Manning, G., Whyte, D. B., Martinez, R., Hunter, T., and Sudarsanam, S. (2002) The protein kinase complement of the human genome. Science 298, 1912-1934

146. Soderling, T. R., and Derkach, V. A. (2000) Postsynaptic protein phosphorylation and LTP. Trends in neurosciences 23, 75-80

147. Myers, J. B., Zaegel, V., Coultrap, S. J., Miller, A. P., Bayer, K. U., and Reichow, S. L. (2017) The CaMKII holoenzyme structure in activation-competent conformations. Nature communications $8,1-15$

148. Kennedy, M. B. (2010) Calcium/calmodulin-dependent protein kinase II. Handbook of Cell Signaling, pp. 565-568, Elsevier

149. Patton, B. L., Miller, S. G., and Kennedy, M. B. (1990) Activation of type II calcium/calmodulin-dependent protein kinase by $\mathrm{Ca}^{2+} /$ calmodulin is inhibited by autophosphorylation of threonine within the calmodulin-binding domain. Journal of Biological Chemistry 265, 11204-11212

150. Barria, A., Muller, D., Derkach, V., Griffith, L. C., and Soderling, T. R. (1997) Regulatory phosphorylation of AMPA-type glutamate receptors by CaM-KII during longterm potentiation. Science 276, 2042-2045

151. Omkumar, R. V., Kiely, M. J., Rosenstein, A. J., Min, K.-T., and Kennedy, M. B. (1996) Identification of a phosphorylation site for calcium/calmodulindependent protein kinase II in the NR2B subunit of the N-methyl-D-aspartate receptor. Journal of Biological Chemistry 271, 31670-31678

152. Chen, H.-J., Rojas-Soto, M., Oguni, A., and Kennedy, M. B. (1998) A synaptic RasGTPase activating protein (p135 SynGAP) inhibited by CaM kinase II. Neuron 20, 895-904 153. Huttner, W., DeGennaro, L., and Greengard, P. (1981) Differential phosphorylation of multiple sites in purified protein I by cyclic AMP-dependent and calcium-dependent protein kinases. Journal of Biological Chemistry 256, 1482-1488

154. Greengard, P., Benfenati, F., and Valtorta, F. (1994) Synapsin I, an actin-binding protein regulating synaptic vesicle traffic in the nerve terminal. Advances in second messenger and phosphoprotein research 29, 31-45

155. Ceccaldi, P.-E., Grohovaz, F., Benfenati, F., Chieregatti, E., Greengard, P., and Valtorta, F. (1995) Dephosphorylated synapsin I anchors synaptic vesicles to actin cytoskeleton: an analysis by videomicroscopy. The Journal of cell biology 128, 905-912

156. Ninan, I., and Arancio, O. (2004) Presynaptic CaMKII is necessary for synaptic plasticity in cultured hippocampal neurons. Neuron 42, 129-141

157. Hojjati, M. R., Van Woerden, G. M., Tyler, W. J., Giese, K. P., Silva, A. J., PozzoMiller, L., and Elgersma, Y. (2007) Kinase activity is not required for aCaMKII-dependent presynaptic plasticity at CA3-CA1 synapses. Nature neuroscience 10, 1125-1127 
158. Okamoto, K.-I., Narayanan, R., Lee, S. H., Murata, K., and Hayashi, Y. (2007) The role of CaMKII as an F-actin-bundling protein crucial for maintenance of dendritic spine structure. Proceedings of the National Academy of Sciences 104, 6418-6423

159. Wortzel, I., and Seger, R. (2011) The ERK cascade: distinct functions within various subcellular organelles. Genes \& cancer 2, 195-209

160. Johnson, G. L., and Lapadat, R. (2002) Mitogen-activated protein kinase pathways mediated by ERK, JNK, and p38 protein kinases. Science 298, 1911-1912

161. Payne, D. M., Rossomando, A., Martino, P., Erickson, A., Her, J., Shabanowitz, J., Hunt, D., Weber, M., and Sturgill, T. (1991) Identification of the regulatory phosphorylation sites in pp42/mitogen-activated protein kinase (MAP kinase). The EMBO journal 10, 885892

162. Gonzalez, F. A., Raden, D. L., and Davis, R. J. (1991) Identification of substrate recognition determinants for human ERK1 and ERK2 protein kinases. Journal of Biological Chemistry 266, 22159-22163

163. Boulton, T. G., Nye, S. H., Robbins, D. J., Ip, N. Y., Radzlejewska, E., Morgenbesser, S. D., DePinho, R. A., Panayotatos, N., Cobb, M. H., and Yancopoulos, G. D. (1991) ERKs: a family of protein-serine/threonine kinases that are activated and tyrosine phosphorylated in response to insulin and NGF. Cell 65, 663-675

164. Fiore, R., Murphy, T., Sanghera, J., Pelech, S., and Baraban, J. M. (1993) Activation of p42 mitogen-activated protein kinase by glutamate receptor stimulation in rat primary cortical cultures. Journal of neurochemistry 61, 1626-1633

165. Kurino, M., Fukunaga, K., Ushio, Y., and Miyamoto, E. (1995) Activation of mitogenactivated protein kinase in cultured rat hippocampal neurons by stimulation of glutamate receptors. Journal of neurochemistry $65,1282-1289$

166. Xia, Z., Dudek, H., Miranti, C. K., and Greenberg, M. E. (1996) Calcium influx via the NMDA receptor induces immediate early gene transcription by a MAP kinase/ERKdependent mechanism. Journal of Neuroscience 16, 5425-5436

167. Rosen, L. B., Ginty, D. D., Weber, M. J., and Greenberg, M. E. (1994) Membrane depolarization and calcium influx stimulate MEK and MAP kinase via activation of Ras. Neuron 12, 1207-1221

168. Walker, S. A., Cullen, P. J., Taylor, J. A., and Lockyer, P. J. (2003) Control of Ras cycling by $\mathrm{Ca}^{2+}$. FEBS letters 546, 6-10

169. Thomas, G. M., and Huganir, R. L. (2004) MAPK cascade signalling and synaptic plasticity. Nature Reviews Neuroscience 5, 173-183

170. Adams, J. P., Anderson, A. E., Varga, A. W., Dineley, K. T., Cook, R. G., Pfaffinger, P. J., and Sweatt, J. D. (2000) The A-type potassium channel Kv4. 2 is a substrate for the mitogen-activated protein kinase ERK. Journal of neurochemistry 75, 2277-2287

171. Matsubara, M., Kusubata, M., Ishiguro, K., Uchida, T., Titani, K., and Taniguchi, H. (1996) Site-specific phosphorylation of synapsin I by mitogen-activated protein kinase and Cdk5 and its effects on physiological functions. Journal of Biological Chemistry 271, 2110821113

172. Patterson, S. L., Pittenger, C., Morozov, A., Martin, K. C., Scanlin, H., Drake, C., and Kandel, E. R. (2001) Some forms of cAMP-mediated long-lasting potentiation are associated with release of BDNF and nuclear translocation of phospho-MAP kinase. Neuron 32, 123-140

173. Davis, S., Vanhoutte, P., Pages, C., Caboche, J., and Laroche, S. (2000) The MAPK/ERK cascade targets both Elk-1 and cAMP response element-binding protein to control long-term potentiation-dependent gene expression in the dentate gyrus in vivo. Journal of Neuroscience 20, 4563-4572

174. Elkins, J. M., and Knapp, S. (2012) The Structure of the Full-Length Tetrameric PKA Regulatory RII $\beta$ Complex Reveals the Mechanism of Allosteric PKA Activation. Science signaling 5 , pe21-pe21

175. Smith, F. D., Esseltine, J. L., Nygren, P. J., Veesler, D., Byrne, D. P., Vonderach, M., Strashnov, I., Eyers, C. E., Eyers, P. A., and Langeberg, L. K. (2017) Local protein kinase A action proceeds through intact holoenzymes. Science 356, 1288-1293 
176. Lonart, G., Schoch, S., Kaeser, P. S., Larkin, C. J., Südhof, T. C., and Linden, D. J. (2003) Phosphorylation of RIM1a by PKA triggers presynaptic long-term potentiation at cerebellar parallel fiber synapses. Cell 115, 49-60

177. Kaeser, P. S., Kwon, H.-B., Blundell, J., Chevaleyre, V., Morishita, W., Malenka, R. C., Powell, C. M., Castillo, P. E., and Südhof, T. C. (2008) RIM1a phosphorylation at serine413 by protein kinase $A$ is not required for presynaptic long-term plasticity or learning. Proceedings of the National Academy of Sciences 105, 14680-14685

178. Nagy, G., Reim, K., Matti, U., Brose, N., Binz, T., Rettig, J., Neher, E., and Sørensen, J. B. (2004) Regulation of releasable vesicle pool sizes by protein kinase A-dependent phosphorylation of SNAP-25. Neuron 41, 417-429

179. Cho, R. W., Buhl, L. K., Volfson, D., Tran, A., Li, F., Akbergenova, Y., and Littleton, J. T. (2015) Phosphorylation of complexin by PKA regulates activity-dependent spontaneous neurotransmitter release and structural synaptic plasticity. Neuron 88, 749761

180. Park, A. J., Havekes, R., Choi, J. H., Luczak, V., Nie, T., Huang, T., and Abel, T. (2014) A presynaptic role for PKA in synaptic tagging and memory. Neurobiology of learning and memory 114, 101-112

181. Waltereit, R., and Weller, M. (2003) Signaling from cAMP/PKA to MAPK and synaptic plasticity. Molecular neurobiology 27, 99-106

182. Newton, A. C. (2018) Protein kinase C: perfectly balanced. Critical reviews in biochemistry and molecular biology 53, 208-230

183. Nakanishi, H., and Exton, J. H. (1992) Purification and characterization of the zeta isoform of protein kinase C from bovine kidney. Journal of Biological Chemistry 267, 1634716354

184. Keranen, L. M., Dutil, E. M., and Newton, A. C. (1995) Protein kinase C is regulated in vivo by three functionally distinct phosphorylations. Current Biology 5, 1394-1403

185. Wierda, K. D., Toonen, R. F., de Wit, H., Brussaard, A. B., and Verhage, M. (2007) Interdependence of PKC-dependent and PKC-independent pathways for presynaptic plasticity. Neuron 54, 275-290

186. Shimazaki, Y., Nishiki, T.-i., Omori, A., Sekiguchi, M., Kamata, Y., Kozaki, S., and Takahashi, M. (1996) Phosphorylation of 25-kDa synaptosome-associated protein: possible involvement in protein kinase $\mathrm{C}$-mediated regulation of neurotransmitter release. Journal of Biological Chemistry 271, 14548-14553

187. Barclay, J. W., Craig, T. J., Fisher, R. J., Ciufo, L. F., Evans, G. J., Morgan, A., and Burgoyne, R. D. (2003) Phosphorylation of Munc18 by protein kinase C regulates the kinetics of exocytosis. Journal of Biological Chemistry 278, 10538-10545

188. Hilfiker, S., Pieribone, V. A., Nordstedt, C., Greengard, P., and Czernik, A. J. (1999) Regulation of synaptotagmin I phosphorylation by multiple protein kinases. Journal of neurochemistry 73, 921-932

189. Cheng, K., and Ip, N. Y. (2003) Cdk5: a new player at synapses. Neurosignals 12, 180-190

190. Lew, J., Huang, Q.-Q., Qi, Z., Winkfein, R. J., Aebersold, R., Hunt, T., and Wang, J. H. (1994) A brain-specific activator of cyclin-dependent kinase 5. Nature 371, 423-426

191. Tsai, L.-H., Delalle, I., Caviness, V. S., Chae, T., and Harlow, E. (1994) p35 is a neural-specific regulatory subunit of cyclin-dependent kinase 5. Nature 371, 419-423

192. Tang, D., Yeung, J., Lee, K.-Y., Matsushita, M., Matsui, H., Tomizawa, K., Hatase, O., and Wang, J. H. (1995) An isoform of the neuronal cyclin-dependent kinase 5 (Cdk5) activator. Journal of Biological Chemistry 270, 26897-26903

193. Humbert, S., Dhavan, R., and Tsai, L. (2000) p39 activates cdk5 in neurons, and is associated with the actin cytoskeleton. Journal of cell science 113, 975-983

194. Wei, F. Y., Tomizawa, K., Ohshima, T., Asada, A., Saito, T., Nguyen, C., Bibb, J. A., Ishiguro, K., Kulkarni, A. B., and Pant, H. C. (2005) Control of cyclin-dependent kinase 5 (Cdk5) activity by glutamatergic regulation of p35 stability. Journal of neurochemistry 93, $502-512$ 
195. Zukerberg, L. R., Patrick, G. N., Nikolic, M., Humbert, S., Wu, C.-L., Lanier, L. M., Gertler, F. B., Vidal, M., Van Etten, R. A., and Tsai, L.-H. (2000) Cables links Cdk5 and c$\mathrm{Abl}$ and facilitates Cdk5 tyrosine phosphorylation, kinase upregulation, and neurite outgrowth. Neuron 26, 633-646

196. Sasaki, Y., Cheng, C., Uchida, Y., Nakajima, O., Ohshima, T., Yagi, T., Taniguchi, M., Nakayama, T., Kishida, R., and Kudo, Y. (2002) Fyn and Cdk5 mediate semaphorin-3A signaling, which is involved in regulation of dendrite orientation in cerebral cortex. Neuron $35,907-920$

197. Jovanovic, J. N., Sihra, T. S., Nairn, A. C., Hemmings, H. C., Greengard, P., and Czernik, A. J. (2001) Opposing changes in phosphorylation of specific sites in synapsin I during $\mathrm{Ca}^{2+}$-dependent glutamate release in isolated nerve terminals. Journal of Neuroscience 21, 7944-7953

198. Shuang, R., Zhang, L., Fletcher, A., Groblewski, G. E., Pevsner, J., and Stuenkel, E. L. (1998) Regulation of Munc-18/syntaxin 1A interaction by cyclin-dependent kinase 5 in nerve endings. Journal of Biological Chemistry 273, 4957-4966

199. Fletcher, A. I., Shuang, R., Giovannucci, D. R., Zhang, L., Bittner, M. A., and Stuenkel, E. L. (1999) Regulation of exocytosis by cyclin-dependent kinase 5 via phosphorylation of Munc18. Journal of Biological Chemistry 274, 4027-4035

200. Tomizawa, K., Ohta, J., Matsushita, M., Moriwaki, A., Li, S.-T., Takei, K., and Matsui, H. (2002) Cdk5/p35 regulates neurotransmitter release through phosphorylation and downregulation of $\mathrm{P} / \mathrm{Q}$-type voltage-dependent calcium channel activity. Journal of Neuroscience 22, 2590-2597

201. Floyd, S. R., Porro, E. B., Slepnev, V. I., Ochoa, G.-C., Tsai, L.-H., and De Camilli, P. (2001) Amphiphysin 1 binds the cyclin-dependent kinase (cdk) 5 regulatory subunit p35 and is phosphorylated by cdk5 and cdc2. Journal of Biological Chemistry 276, 8104-8110 202. Liang, S., Wei, F. Y., Wu, Y. M., Tanabe, K., Abe, T., Oda, Y., Yoshida, Y., Yamada, H., Matsui, H., and Tomizawa, K. (2007) Major Cdk5-dependent phosphorylation sites of amphiphysin 1 are implicated in the regulation of the membrane binding and endocytosis. Journal of neurochemistry 102, 1466-1476

203. Clayton, E. L., Sue, N., Smillie, K. J., O'Leary, T., Bache, N., Cheung, G., Cole, A. R., Wyllie, D. J., Sutherland, C., and Robinson, P. J. (2010) Dynamin I phosphorylation by GSK3 controls activity-dependent bulk endocytosis of synaptic vesicles. Nature neuroscience 13, 845-851

204. Lee, S. Y., Wenk, M. R., Kim, Y., Nairn, A. C., and De Camilli, P. (2004) Regulation of synaptojanin 1 by cyclin-dependent kinase 5 at synapses. Proceedings of the National Academy of Sciences 101, 546-551

205. Cousin, M. A., and Robinson, P. J. (2001) The dephosphins: dephosphorylation by calcineurin triggers synaptic vesicle endocytosis. Trends in neurosciences 24, 659-665

206. Tan, T. C., Valova, V. A., Malladi, C. S., Graham, M. E., Berven, L. A., Jupp, O. J., Hansra, G., McClure, S. J., Sarcevic, B., and Boadle, R. A. (2003) Cdk5 is essential for synaptic vesicle endocytosis. Nature cell biology 5, 701-710

207. Beurel, E., Grieco, S. F., and Jope, R. S. (2015) Glycogen synthase kinase-3 (GSK3): regulation, actions, and diseases. Pharmacology \& therapeutics 148, 114-131

208. ter Haar, E., Coll, J. T., Austen, D. A., Hsiao, H.-M., Swenson, L., and Jain, J. (2001) Structure of GSK3 $\beta$ reveals a primed phosphorylation mechanism. Nature structural biology 8, 593-596

209. Frame, S., Cohen, P., and Biondi, R. M. (2001) A common phosphate binding site explains the unique substrate specificity of GSK3 and its inactivation by phosphorylation. Molecular cell 7, 1321-1327

210. Song, B., Lai, B., Zheng, Z., Zhang, Y., Luo, J., Wang, C., Chen, Y., Woodgett, J. R., and Li, M. (2010) Inhibitory phosphorylation of GSK-3 by CaMKII couples depolarization to neuronal survival. Journal of Biological Chemistry 285, 41122-41134

211. Zhu, L.-Q., Liu, D., Hu, J., Cheng, J., Wang, S.-H., Wang, Q., Wang, F., Chen, J.G., and Wang, J.-Z. (2010) GSK-3 $\beta$ inhibits presynaptic vesicle exocytosis by 
phosphorylating P/Q-type calcium channel and interrupting snare complex formation. Journal of Neuroscience 30, 3624-3633

212. Hanger, D. P., Hughes, K., Woodgett, J. R., Brion, J. P., and Anderton, B. H. (1992) Glycogen synthase kinase-3 induces Alzheimer's disease-like phosphorylation of tau: generation of paired helical filament epitopes and neuronal localisation of the kinase. Neurosci Lett 147, 58-62

213. Cheong, J. K., and Virshup, D. M. (2011) Casein kinase 1: Complexity in the family. Int J Biochem Cell Biol 43, 465-469

214. Eide, E. J., and Virshup, D. M. (2001) Casein kinase I: another cog in the circadian clockworks. Chronobiology international 18, 389-398

215. Ebisawa, T. (2007) Circadian rhythms in the CNS and peripheral clock disorders: human sleep disorders and clock genes. Journal of pharmacological sciences 103, 150-154 216. Lampe, P. D., and Lau, A. F. (2004) The effects of connexin phosphorylation on gap junctional communication. The international journal of biochemistry \& cell biology 36, 1171 1186

217. Salinas, P. C. (2007) Modulation of the microtubule cytoskeleton: a role for a divergent canonical Wnt pathway. Trends in cell biology 17, 333-342

218. Purro, S. A., Galli, S., and Salinas, P. C. (2014) Dysfunction of Wnt signaling and synaptic disassembly in neurodegenerative diseases. Journal of molecular cell biology 6 , 75-80

219. Price, M. A. (2006) CKI, there's more than one: casein kinase I family members in Wnt and Hedgehog signaling. Genes \& development 20, 399-410

220. Flaherty, D., Soria, J., Tomasiewicz, H., and Wood, J. (2000) Phosphorylation of human tau protein by microtubule-associated kinases: GSK3 $\beta$ and cdk5 are key participants. Journal of neuroscience research 62, 463-472

221. Perez, D. I., Gil, C., and Martinez, A. (2011) Protein kinases CK1 and CK2 as new targets for neurodegenerative diseases. Medicinal research reviews 31, 924-954

222. Filhol, O., and Cochet, C. (2009) Protein kinase CK2 in health and disease. Cellular and Molecular Life Sciences 66, 1830-1839

223. Sanz-Clemente, A., Gray, J. A., Ogilvie, K. A., Nicoll, R. A., and Roche, K. W. (2013) Activated CaMKII couples GluN2B and casein kinase 2 to control synaptic NMDA receptors. Cell reports 3, 607-614

224. Agostinis, P., Goris, J., Pinna, L., and Merlevede, W. (1987) Regulation of casein kinase 2 by phosphorylation/dephosphorylation. Biochemical Journal 248, 785-789

225. Donella-Deana, A., Cesaro, L., Sarno, S., Ruzzene, M., Brunati, A. M., Marin, O., Vilk, G., Doherty-Kirby, A., Lajoie, G., and Litchfield, D. W. (2003) Tyrosine phosphorylation of protein kinase CK2 by Src-related tyrosine kinases correlates with increased catalytic activity. Biochemical Journal 372, 841-849

226. Bulat, V., Rast, M., and Pielage, J. (2014) Presynaptic CK2 promotes synapse organization and stability by targeting Ankyrin2. Journal of Cell Biology 204, 77-94

227. Kimura, R., and Matsuki, N. (2008) Protein kinase CK2 modulates synaptic plasticity by modification of synaptic NMDA receptors in the hippocampus. The Journal of physiology 586, 3195-3206

228. Risinger, C., and Bennett, M. K. (1999) Differential phosphorylation of syntaxin and synaptosome-associated protein of $25 \mathrm{kDa}$ (SNAP-25) isoforms. Journal of neurochemistry $72,614-624$

229. Shi, V., Craig, T. J., Bishop, P., Nakamura, Y., Rocca, D., Wilkinson, K. A., and Henley, J. M. (2021) Phosphorylation of Syntaxin-1a by casein kinase $2 \alpha$ regulates presynaptic vesicle exocytosis from the reserve pool. Journal of Neurochemistry 156, 614-623 230. Chung, H. J., Huang, Y. H., Lau, L.-F., and Huganir, R. L. (2004) Regulation of the NMDA receptor complex and trafficking by activity-dependent phosphorylation of the NR2B subunit PDZ ligand. Journal of Neuroscience 24, 10248-10259

231. Manser, E., Leung, T., Salihuddin, H., Zhao, Z.-S., and Lim, L. (1994) A brain serine/threonine protein kinase activated by Cdc42 and Rac1. Nature 367, 40-46 
232. Manser, E., Huang, H.-Y., Loo, T.-H., Chen, X.-Q., Dong, J.-M., Leung, T., and Lim, L. (1997) Expression of constitutively active alpha-PAK reveals effects of the kinase on actin and focal complexes. Molecular and cellular biology 17, 1129-1143

233. Sells, M. A., Boyd, J. T., and Chernoff, J. (1999) p21-activated kinase 1 (Pak1) regulates cell motility in mammalian fibroblasts. The Journal of cell biology 145, 837-849

234. Chaudhary, A., King, W., Mattaliano, M., Frost, J., Diaz, B., Morrison, D., Cobb, M., Marshall, M., and Brugge, J. (2000) Phosphatidylinositol 3-kinase regulates Raf1 through Pak phosphorylation of serine 338. Current Biology 10, 551-554

235. Sacco, F., Perfetto, L., Castagnoli, L., and Cesareni, G. (2012) The human phosphatase interactome: An intricate family portrait. FEBS Lett 586, 2732-2739

236. Tiganis, T., and Bennett, A. M. (2007) Protein tyrosine phosphatase function: the substrate perspective. Biochem J 402, 1-15

237. Woolfrey, K. M., and Dell'Acqua, M. L. (2015) Coordination of Protein Phosphorylation and Dephosphorylation in Synaptic Plasticity. J Biol Chem 290, 2860428612

238. Peti, W., Nairn, A. C., and Page, R. (2013) Structural basis for protein phosphatase 1 regulation and specificity. FEBS $J 280,596-611$

239. Morishita, W., Connor, J. H., Xia, H., Quinlan, E. M., Shenolikar, S., and Malenka, R. C. (2001) Regulation of synaptic strength by protein phosphatase 1. Neuron 32, 11331148

240. Hu, X. D., Huang, Q., Roadcap, D. W., Shenolikar, S. S., and Xia, H. (2006) Actinassociated neurabin-protein phosphatase-1 complex regulates hippocampal plasticity. $J$ Neurochem 98, 1841-1851

241. Hu, X. D., Huang, Q., Yang, X., and Xia, H. (2007) Differential regulation of AMPA receptor trafficking by neurabin-targeted synaptic protein phosphatase- 1 in synaptic transmission and long-term depression in hippocampus. J Neurosci 27, 4674-4686

242. Hsieh-Wilson, L. C., Benfenati, F., Snyder, G. L., Allen, P. B., Nairn, A. C., and Greengard, P. (2003) Phosphorylation of spinophilin modulates its interaction with actin filaments. J Biol Chem 278, 1186-1194

243. Grossman, S. D., Futter, M., Snyder, G. L., Allen, P. B., Nairn, A. C., Greengard, P., and Hsieh-Wilson, L. C. (2004) Spinophilin is phosphorylated by $\mathrm{Ca}^{2+} /$ calmodulindependent protein kinase II resulting in regulation of its binding to F-actin. $J$ Neurochem 90 , 317-324

244. McAvoy, T., Allen, P. B., Obaishi, H., Nakanishi, H., Takai, Y., Greengard, P., Nairn, A. C., and Hemmings, H. C., Jr. (1999) Regulation of neurabin I interaction with protein phosphatase 1 by phosphorylation. Biochemistry 38, 12943-12949

245. Svenningsson, P., Nishi, A., Fisone, G., Girault, J. A., Nairn, A. C., and Greengard, P. (2004) DARPP-32: an integrator of neurotransmission. Annu Rev Pharmacol Toxicol 44, 269-296

246. Nguyen, C., Hosokawa, T., Kuroiwa, M., Ip, N. Y., Nishi, A., Hisanaga, S., and Bibb, J. A. (2007) Differential regulation of the Cdk5-dependent phosphorylation sites of inhibitor1 and DARPP-32 by depolarization. J Neurochem 103, 1582-1593

247. Rodriguez, P., Mitton, B., Nicolaou, P., Chen, G., and Kranias, E. G. (2007) Phosphorylation of human inhibitor-1 at Ser67 and/or Thr75 attenuates stimulatory effects of protein kinase A signaling in cardiac myocytes. Am J Physiol Heart Circ Physiol 293, H762-769

248. Xu, Y., Xing, Y., Chen, Y., Chao, Y., Lin, Z., Fan, E., Yu, J. W., Strack, S., Jeffrey, P. D., and Shi, Y. (2006) Structure of the protein phosphatase 2A holoenzyme. Cell 127, 1239-1251

249. Wang, J., Xie, R., Kou, X., Liu, Y., Qi, C., Liu, R., You, W., Gao, J., and Gao, X. (2019) A protein phosphatase 2A deficit in the hippocampal CA1 area impairs memory extinction. Mol Brain 12, 51

250. Fukunaga, K., Muller, D., Ohmitsu, M., Bako, E., DePaoli-Roach, A. A., and Miyamoto, E. (2000) Decreased protein phosphatase 2A activity in hippocampal long-term potentiation. J Neurochem 74, 807-817 
251. Drewes, G., Mandelkow, E. M., Baumann, K., Goris, J., Merlevede, W., and Mandelkow, E. (1993) Dephosphorylation of tau protein and Alzheimer paired helical filaments by calcineurin and phosphatase-2A. FEBS Lett 336, 425-432

252. Gong, C. X., Grundke-lqbal, I., and lqbal, K. (1994) Dephosphorylation of Alzheimer's disease abnormally phosphorylated tau by protein phosphatase-2A. Neuroscience 61, 765-772

253. Xu, Y., Chen, Y., Zhang, P., Jeffrey, P. D., and Shi, Y. (2008) Structure of a protein phosphatase $2 \mathrm{~A}$ holoenzyme: insights into B55-mediated Tau dephosphorylation. Mol Cell 31, 873-885

254. Ahn, J. H., McAvoy, T., Rakhilin, S. V., Nishi, A., Greengard, P., and Nairn, A. C. (2007) Protein kinase A activates protein phosphatase $2 A$ by phosphorylation of the B56delta subunit. Proc Natl Acad Sci U S A 104, 2979-2984

255. Stewart, A. A., Ingebritsen, T. S., Manalan, A., Klee, C. B., and Cohen, P. (1982) Discovery of a $\mathrm{Ca}^{2+}$ - and calmodulin-dependent protein phosphatase: probable identity with calcineurin (CaM-BP80). FEBS Lett 137, 80-84

256. Stemmer, P. M., and Klee, C. B. (1994) Dual calcium ion regulation of calcineurin by calmodulin and calcineurin B. Biochemistry 33, 6859-6866

257. Groth, R. D., Dunbar, R. L., and Mermelstein, P. G. (2003) Calcineurin regulation of neuronal plasticity. Biochem Biophys Res Commun 311, 1159-1171

258. Sun, T., Wu, X. S., Xu, J., McNeil, B. D., Pang, Z. P., Yang, W., Bai, L., Qadri, S., Molkentin, J. D., Yue, D. T., and Wu, L. G. (2010) The role of calcium/calmodulin-activated calcineurin in rapid and slow endocytosis at central synapses. J Neurosci 30, 11838-11847 259. King, M. M., Huang, C. Y., Chock, P. B., Nairn, A. C., Hemmings, H. C., Jr., Chan, K. F., and Greengard, P. (1984) Mammalian brain phosphoproteins as substrates for calcineurin. J Biol Chem 259, 8080-8083

260. Goto, S., Yamamoto, H., Fukunaga, K., Iwasa, T., Matsukado, Y., and Miyamoto, E. (1985) Dephosphorylation of microtubule-associated protein 2, tau factor, and tubulin by calcineurin. J Neurochem 45, 276-283

261. Mandelkow, E. M., Biernat, J., Drewes, G., Gustke, N., Trinczek, B., and Mandelkow, E. (1995) Tau domains, phosphorylation, and interactions with microtubules. Neurobiol Aging 16, 355-362; discussion 362-353

262. Seki, K., Chen, H. C., and Huang, K. P. (1995) Dephosphorylation of protein kinase $C$ substrates, neurogranin, neuromodulin, and MARCKS, by calcineurin and protein phosphatases 1 and 2A. Arch Biochem Biophys 316, 673-679

263. Yamashita, M., and Fenn, J. B. (1984) Electrospray ion source. Another variation on the free-jet theme. The Journal of Physical Chemistry 88, 4451-4459

264. Karas, M., Bachmann, D., and Hillenkamp, F. (1985) Influence of the wavelength in high-irradiance ultraviolet laser desorption mass spectrometry of organic molecules. Analytical chemistry 57, 2935-2939

265. Tanaka, K., Waki, H., Ido, Y., Akita, S., Yoshida, Y., Yoshida, T., and Matsuo, T. (1988) Protein and polymer analyses up to $\mathrm{m} / \mathrm{z} 100000$ by laser ionization time-of-flight mass spectrometry. Rapid communications in mass spectrometry 2, 151-153

266. Griffiths, J. (2008) A brief history of mass spectrometry. Anal. Chem 80, 5678-5683

267. Scheltema, R. A., Hauschild, J.-P., Lange, O., Hornburg, D., Denisov, E., Damoc, E., Kuehn, A., Makarov, A., and Mann, M. (2014) The Q Exactive HF, a Benchtop mass spectrometer with a pre-filter, high-performance quadrupole and an ultra-high-field Orbitrap analyzer. Molecular \& Cellular Proteomics 13, 3698-3708

268. Domon, B., and Aebersold, R. (2006) Mass spectrometry and protein analysis. science $312,212-217$

269. Wilm, M. (2011) Principles of electrospray ionization. Molecular \& cellular proteomics 10

270. Michalski, A., Damoc, E., Hauschild, J.-P., Lange, O., Wieghaus, A., Makarov, A., Nagaraj, N., Cox, J., Mann, M., and Horning, S. (2011) Mass spectrometry-based proteomics using $\mathrm{Q}$ Exactive, a high-performance benchtop quadrupole Orbitrap mass spectrometer. Molecular \& cellular proteomics 10 
271. De Hoffmann, E., and Stroobant, V. (2007) Mass spectrometry: principles and applications, John Wiley \& Sons

272. Olsen, J. V., Macek, B., Lange, O., Makarov, A., Horning, S., and Mann, M. (2007) Higher-energy C-trap dissociation for peptide modification analysis. Nature methods 4, 709712

273. Steen, H., and Mann, M. (2004) The ABC's (and XYZ's) of peptide sequencing. Nature reviews Molecular cell biology 5, 699-711

274. Biemann, K. (1988) Contributions of mass spectrometry to peptide and protein structure. Biomedical \& environmental mass spectrometry 16, 99-111

275. Roepstorff, P. (1984) Proposal for a nomen clature for sequence ions in mass spectra of peptides. Biomed. Mass Spec. 11, 60

276. Zubarev, R. A., and Makarov, A. (2013) Orbitrap mass spectrometry. ACS Publications

277. Makarov, A. (2000) Electrostatic axially harmonic orbital trapping: a highperformance technique of mass analysis. Analytical chemistry 72, 1156-1162

278. Chait, B. T. (2006) Mass spectrometry: bottom-up or top-down? Science 314, 65-66

279. Andersson, L., and Porath, J. (1986) Isolation of phosphoproteins by immobilized metal $\left(\mathrm{Fe}^{3+}\right)$ affinity chromatography. Analytical biochemistry 154, 250-254

280. Larsen, M. R., Thingholm, T. E., Jensen, O. N., Roepstorff, P., and Jørgensen, T. J. (2005) Highly selective enrichment of phosphorylated peptides from peptide mixtures using titanium dioxide microcolumns. Molecular \& cellular proteomics 4, 873-886

281. Humphrey, S. J., Karayel, O., James, D. E., and Mann, M. (2018) High-throughput and high-sensitivity phosphoproteomics with the EasyPhos platform. Nature protocols 13, 1897-1916

282. Lenz, C., and Urlaub, H. (2014) Separation methodology to improve proteome coverage depth. Expert review of proteomics 11, 409-414

283. Song, C., Ye, M., Han, G., Jiang, X., Wang, F., Yu, Z., Chen, R., and Zou, H. (2010) Reversed-phase-reversed-phase liquid chromatography approach with high orthogonality for multidimensional separation of phosphopeptides. Analytical chemistry 82, 53-56

284. Wang, Y., Yang, F., Gritsenko, M. A., Wang, Y., Clauss, T., Liu, T., Shen, Y., Monroe, M. E., Lopez-Ferrer, D., and Reno, T. (2011) Reversed-phase chromatography with multiple fraction concatenation strategy for proteome profiling of human MCF10A cells. Proteomics 11, 2019-2026

285. McLafferty, F. W. (1981) Tandem mass spectrometry. Science 214, 280-287

286. Michalski, A., Cox, J., and Mann, M. (2011) More than 100,000 detectable peptide species elute in single shotgun proteomics runs but the majority is inaccessible to datadependent LC- MS/MS. Journal of proteome research 10, 1785-1793

287. Nesvizhskii, A. I. (2007) Protein identification by tandem mass spectrometry and sequence database searching. Mass Spectrometry Data Analysis in Proteomics, 87-119

288. Olsen, J. V., Blagoev, B., Gnad, F., Macek, B., Kumar, C., Mortensen, P., and Mann, M. (2006) Global, in vivo, and site-specific phosphorylation dynamics in signaling networks. Cell 127, 635-648

289. Elias, J. E., and Gygi, S. P. (2010) Target-decoy search strategy for mass spectrometry-based proteomics. Proteome bioinformatics, pp. 55-71, Springer

290. Nesvizhskii, A. I. (2010) A survey of computational methods and error rate estimation procedures for peptide and protein identification in shotgun proteomics. Journal of proteomics 73, 2092-2123

291. Nesvizhskii, A. I., and Aebersold, R. (2005) Interpretation of shotgun proteomic data. Molecular \& cellular proteomics 4, 1419-1440

292. Rappsilber, J., and Mann, M. (2002) What does it mean to identify a protein in proteomics? Trends in biochemical sciences 27, 74-78

293. Nesvizhskii, A. I., Keller, A., Kolker, E., and Aebersold, R. (2003) A statistical model for identifying proteins by tandem mass spectrometry. Analytical chemistry 75, 4646-4658

294. Vaudel, M., Sickmann, A., and Martens, L. (2010) Peptide and protein quantification: a map of the minefield. Proteomics 10, 650-670 
295. Tyanova, S., Temu, T., and Cox, J. (2016) The MaxQuant computational platform for mass spectrometry-based shotgun proteomics. Nature protocols 11, 2301-2319

296. Cox, J., Hein, M. Y., Luber, C. A., Paron, I., Nagaraj, N., and Mann, M. (2014) Accurate proteome-wide label-free quantification by delayed normalization and maximal peptide ratio extraction, termed MaxLFQ. Molecular \& cellular proteomics 13, 2513-2526 297. Bondarenko, P. V., Chelius, D., and Shaler, T. A. (2002) Identification and relative quantitation of protein mixtures by enzymatic digestion followed by capillary reversed-phase liquid chromatography- tandem mass spectrometry. Analytical chemistry 74, 4741-4749 298. Thompson, A., Schäfer, J., Kuhn, K., Kienle, S., Schwarz, J., Schmidt, G., Neumann, T., and Hamon, C. (2003) Tandem mass tags: a novel quantification strategy for comparative analysis of complex protein mixtures by MS/MS. Analytical chemistry 75, 18951904

299. Biringer, R. G., Horner, J. A., Viner, R., Hühmer, A. F., and Specht, A. (2011) Quantitation of TMT-Labeled Peptides Using Higher-Energy Collisional Dissociation on the Velos Pro Ion Trap Mass Spectrometer.

300. Hogrebe, A., von Stechow, L., Bekker-Jensen, D. B., Weinert, B. T., Kelstrup, C. D., and Olsen, J. V. (2018) Benchmarking common quantification strategies for large-scale phosphoproteomics. Nature communications 9, 1-13

301. McAlister, G. C., Nusinow, D. P., Jedrychowski, M. P., Wühr, M., Huttlin, E. L., Erickson, B. K., Rad, R., Haas, W., and Gygi, S. P. (2014) MultiNotch MS3 enables accurate, sensitive, and multiplexed detection of differential expression across cancer cell line proteomes. Analytical chemistry 86, 7150-7158

302. Huang, T., Choi, M., Tzouros, M., Golling, S., Pandya, N. J., Banfai, B., Dunkley, T., and Vitek, O. (2020) MSstatsTMT: Statistical detection of differentially abundant proteins in experiments with isobaric labeling and multiple mixtures. Molecular \& Cellular Proteomics 19, 1706-1723

303. Sticker, A., Goeminne, L., Martens, L., and Clement, L. (2020) Robust summarization and inference in proteome-wide label-free quantification. Molecular \& Cellular Proteomics 19, 1209-1219

304. Goeminne, L. J., Argentini, A., Martens, L., and Clement, L. (2015) Summarization vs peptide-based models in label-free quantitative proteomics: performance, pitfalls, and data analysis guidelines. Journal of proteome research 14, 2457-2465

305. Brönstrup, M. (2004) Absolute quantification strategies in proteomics based on mass spectrometry. Expert review of proteomics 1, 503-512

306. Aguilan, J. T., Kulej, K., and Sidoli, S. (2020) Guide for protein fold change and pvalue calculation for non-experts in proteomics. Molecular Omics 16, 573-582

307. Karpievitch, Y. V., Dabney, A. R., and Smith, R. D. (2012) Normalization and missing value imputation for label-free LC-MS analysis. BMC bioinformatics 13, 1-9

308. Herbrich, S. M., Cole, R. N., West Jr, K. P., Schulze, K., Yager, J. D., Groopman, J. D., Christian, P., Wu, L., O'Meally, R. N., and May, D. H. (2013) Statistical inference from multiple ITRAQ experiments without using common reference standards. Journal of proteome research 12, 594-604

309. Quackenbush, J. (2001) Computational analysis of microarray data. Nature reviews genetics 2, 418-427

310. Plubell, D. L., Wilmarth, P. A., Zhao, Y., Fenton, A. M., Minnier, J., Reddy, A. P., Klimek, J., Yang, X., David, L. L., and Pamir, N. (2017) Extended multiplexing of tandem mass tags (TMT) labeling reveals age and high fat diet specific proteome changes in mouse epididymal adipose tissue. Molecular \& Cellular Proteomics 16, 873-890

311. Smyth, G. K. (2005) Limma: linear models for microarray data. Bioinformatics and computational biology solutions using $R$ and Bioconductor, pp. 397-420, Springer

312. Kammers, K., Cole, R. N., Tiengwe, C., and Ruczinski, I. (2015) Detecting significant changes in protein abundance. EuPA open proteomics 7, 11-19

313. Benjamini, Y., and Hochberg, Y. (1995) Controlling the false discovery rate: a practical and powerful approach to multiple testing. Journal of the Royal statistical society: series $B$ (Methodological) 57, 289-300 
314. Storey, J. D. (2002) A direct approach to false discovery rates. Journal of the Royal Statistical Society: Series B (Statistical Methodology) 64, 479-498

315. JD, T. R. (2003) Statistical significance for genome-wide experiments. Proc Natl Acad Sci USA 100, 94409445

316. Munk, S., Refsgaard, J. C., and Olsen, J. V. (2016) Systems analysis for interpretation of phosphoproteomics data. Phospho-Proteomics, 341-360

317. Ashburner, M., Ball, C. A., Blake, J. A., Botstein, D., Butler, H., Cherry, J. M., Davis, A. P., Dolinski, K., Dwight, S. S., and Eppig, J. T. (2000) Gene ontology: tool for the unification of biology. Nature genetics 25, 25-29

318. Kanehisa, M., and Goto, S. (2000) KEGG: kyoto encyclopedia of genes and genomes. Nucleic acids research 28, 27-30

319. Fabregat, A., Jupe, S., Matthews, L., Sidiropoulos, K., Gillespie, M., Garapati, P., Haw, R., Jassal, B., Korninger, F., and May, B. (2018) The reactome pathway knowledgebase. Nucleic acids research 46, D649-D655

320. Finn, R. D., Attwood, T. K., Babbitt, P. C., Bateman, A., Bork, P., Bridge, A. J., Chang, H.-Y., Dosztányi, Z., El-Gebali, S., and Fraser, M. (2017) InterPro in 2017-beyond protein family and domain annotations. Nucleic acids research 45, D190-D199

321. Colaert, N., Helsens, K., Martens, L., Vandekerckhove, J., and Gevaert, K. (2009) Improved visualization of protein consensus sequences by iceLogo. Nature methods $6,786-$ 787

322. Szklarczyk, D., Gable, A. L., Lyon, D., Junge, A., Wyder, S., Huerta-Cepas, J., Simonovic, M., Doncheva, N. T., Morris, J. H., and Bork, P. (2019) STRING v11: proteinprotein association networks with increased coverage, supporting functional discovery in genome-wide experimental datasets. Nucleic acids research 47, D607-D613

323. Horn, H., Schoof, E. M., Kim, J., Robin, X., Miller, M. L., Diella, F., Palma, A., Cesareni, G., Jensen, L. J., and Linding, R. (2014) KinomeXplorer: an integrated platform for kinome biology studies. Nature methods 11, 603-604

324. Koopmans, F., van Nierop, P., Andres-Alonso, M., Byrnes, A., Cijsouw, T., Coba, M. P., Cornelisse, L. N., Farrell, R. J., Goldschmidt, H. L., and Howrigan, D. P. (2019) SynGO: an evidence-based, expert-curated knowledge base for the synapse. Neuron 103, 217-234. e214

325. Hornbeck, P. V., Zhang, B., Murray, B., Kornhauser, J. M., Latham, V., and Skrzypek, E. (2015) PhosphoSitePlus, 2014: mutations, PTMs and recalibrations. Nucleic acids research 43, D512-D520

326. Silbern, I., Pan, K.-T., Fiosins, M., Bonn, S., Rizzoli, S. O., Fornasiero, E. F., Urlaub, H., and Jahn, R. (2021) Protein Phosphorylation in Depolarized Synaptosomes: Dissecting Primary Effects of Calcium from Synaptic Vesicle Cycling. Molecular \& Cellular Proteomics 20

327. Del Castillo, J., and Katz, B. (1956) Biophysical aspects of neuro-muscular transmission. Prog Biophys Biophys Chem 6, 121-170

328. Chapman, E. R. (2002) Synaptotagmin: $\mathrm{A} \mathrm{Ca}^{2+}$ sensor that triggers exocytosis? Nature Reviews Molecular Cell Biology 3, 498-508

329. Fesce, R., Grohovaz, F., Valtorta, F., and Meldolesi, J. (1994) Neurotransmitter release: fusion or 'kiss-and-run'? Trends in cell biology 4, 1-4

330. Von Gersdorff, H., and Mathews, G. (1994) Dynamics of synaptic vesicle fusion and membrane retrieval in synaptic terminals. Nature 367, 735-739

331. Boucrot, E., Ferreira, A. P., Almeida-Souza, L., Debard, S., Vallis, Y., Howard, G., Bertot, L., Sauvonnet, N., and McMahon, H. T. (2015) Endophilin marks and controls a clathrin-independent endocytic pathway. Nature 517, 460-465

332. Gan, Q., and Watanabe, S. (2018) Synaptic vesicle endocytosis in different model systems. Frontiers in cellular neuroscience 12, 171

333. Edwards, R. H. (2007) The neurotransmitter cycle and quantal size. Neuron 55, 835858

334. Sihra, T. S. (1997) Protein phosphorylation and dephosphorylation in isolated nerve terminals (synaptosomes). Regulatory Protein Modification, pp. 67-119, Springer 
335. Gorelick, F. S., Wang, J., Lai, Y., Nairn, A., and Greengard, P. (1988) Autophosphorylation and activation of $\mathrm{Ca}^{2+} /$ calmodulin-dependent protein kinase II in intact nerve terminals. Journal of Biological Chemistry 263, 17209-17212

336. Walaas, S. I., Gorelick, F. S., and Greengard, P. (1989) Presence of calcium/calmodulin-dependent protein Kinase II in Nerve terminals of rat brain. Synapse 3, 356-362

337. Lisman, J., Schulman, H., and Cline, H. (2002) The molecular basis of CaMKII function in synaptic and behavioural memory. Nature Reviews Neuroscience 3, 175-190

338. De Camilli, P., Benfenati, F., Valtorta, F., and Greengard, P. (1990) The synapsins. Annual review of cell biology 6, 433-460

339. Robinson, P. J. (1992) Differential stimulation of protein kinase C activity by phorbol ester or calcium/phosphatidylserine in vitro and in intact synaptosomes. Journal of Biological Chemistry 267, 21637-21644

340. Robinson, P. J., Sontag, J.-M., Liu, J.-P., Fykse, E. M., Slaughter, C., McMahontt, H., and Südhof, T. C. (1993) Dynamin GTPase regulated by protein kinase C phosphorylation in nerve terminals. Nature 365, 163-166

341. Sihra, T., Bogonez, E., and Nicholls, D. (1992) Localized $\mathrm{Ca}^{2+}$ entry preferentially effects protein dephosphorylation, phosphorylation, and glutamate release. Journal of Biological Chemistry 267, 1983-1989

342. Liu, J.-P., Sim, A., and Robinson, P. J. (1994) Calcineurin inhibition of dynamin I GTPase activity coupled to nerve terminal depolarization. Science 265, 970-973

343. Nichols, R. A., Suplick, G. R., and Brown, J. M. (1994) Calcineurin-mediated protein dephosphorylation in brain nerve terminals regulates the release of glutamate. Journal of Biological Chemistry 269, 23817-23823

344. Bauerfeind, R., Takei, K., and De Camilli, P. (1997) Amphiphysin I is associated with coated endocytic intermediates and undergoes stimulation-dependent dephosphorylation in nerve terminals. Journal of Biological Chemistry 272, 30984-30992

345. Marks, B., and McMahon, H. T. (1998) Calcium triggers calcineurin-dependent synaptic vesicle recycling in mammalian nerve terminals. Current biology 8, 740-749

346. Cousin, M. A., Tan, T. C., and Robinson, P. J. (2001) Protein phosphorylation is required for endocytosis in nerve terminals: potential role for the dephosphins dynamin I and synaptojanin, but not AP180 or amphiphysin. Journal of neurochemistry 76, 105-116

347. Schmitz, S. K., King, C., Kortleven, C., Huson, V., Kroon, T., Kevenaar, J. T., Schut, D., Saarloos, I., Hoetjes, J. P., and de Wit, H. (2016) Presynaptic inhibition upon CB 1 or $m G$ lu2/3 receptor activation requires ERK/MAPK phosphorylation of Munc18-1. The EMBO journal 35, 1236-1250

348. Jovanovic, J. N., Benfenati, F., Siow, Y., Sihra, T. S., Sanghera, J. S., Pelech, S. L., Greengard, P., and Czernik, A. J. (1996) Neurotrophins stimulate phosphorylation of synapsin I by MAP kinase and regulate synapsin I-actin interactions. Proceedings of the National Academy of Sciences 93, 3679-3683

349. Impey, S., Obrietan, K., Wong, S. T., Poser, S., Yano, S., Wayman, G., Deloulme, J. C., Chan, G., and Storm, D. R. (1998) Cross talk between ERK and PKA is required for $\mathrm{Ca}^{2+}$ stimulation of CREB-dependent transcription and ERK nuclear translocation. Neuron $21,869-883$

350. Levenson, J. M., O'Riordan, K. J., Brown, K. D., Trinh, M. A., Molfese, D. L., and Sweatt, J. D. (2004) Regulation of histone acetylation during memory formation in the hippocampus. Journal of Biological Chemistry 279, 40545-40559

351. Kohansal-Nodehi, M., Chua, J. J., Urlaub, H., Jahn, R., and Czernik, D. (2016) Analysis of protein phosphorylation in nerve terminal reveals extensive changes in active zone proteins upon exocytosis. Elife 5, e14530

352. Engholm-Keller, K., Waardenberg, A. J., Müller, J. A., Wark, J. R., Fernando, R. N., Arthur, J. W., Robinson, P. J., Dietrich, D., Schoch, S., and Graham, M. E. (2019) The temporal profile of activity-dependent presynaptic phospho-signalling reveals long-lasting patterns of poststimulus regulation. PLoS biology 17, e3000170 
353. Gao, J., Hirata, M., Mizokami, A., Zhao, J., Takahashi, I., Takeuchi, H., and Hirata, M. (2016) Differential role of SNAP-25 phosphorylation by protein kinases A and C in the regulation of SNARE complex formation and exocytosis in PC12 cells. Cellular signalling 28, 425-437

354. Tian, J.-H., Das, S., and Sheng, Z.-H. (2003) $\mathrm{Ca}^{2+}$-dependent phosphorylation of syntaxin-1A by the death-associated protein (DAP) kinase regulates its interaction with Munc18. Journal of Biological Chemistry 278, 26265-26274

355. Rickman, C., and Duncan, R. R. (2010) Munc18/Syntaxin Interaction Kinetics Control Secretory Vesicle Dynamics 2. Journal of Biological Chemistry 285, 3965-3972

356. Shen, C., Rathore, S. S., Yu, H., Gulbranson, D. R., Hua, R., Zhang, C., Schoppa, N. E., and Shen, J. (2015) The trans-SNARE-regulating function of Munc18-1 is essential to synaptic exocytosis. Nature communications 6, 1-10

357. Li, J., Han, Y. R., Plummer, M. R., and Herrup, K. (2009) Cytoplasmic ATM in neurons modulates synaptic function. Current Biology 19, 2091-2096

358. Craig, T. J., Evans, G. J., and Morgan, A. (2003) Physiological regulation of Munc18/nSec1 phosphorylation on serine-313. Journal of neurochemistry 86, 1450-1457

359. Garcia, D., Brown, S., Hille, B., and Mackie, K. (1998) Protein kinase C disrupts cannabinoid actions by phosphorylation of the CB1 cannabinoid receptor. Journal of Neuroscience 18, 2834-2841

360. Choi, S. W., Gerencser, A. A., Lee, D. W., Rajagopalan, S., Nicholls, D. G., Andersen, J. K., and Brand, M. D. (2011) Intrinsic bioenergetic properties and stress sensitivity of dopaminergic synaptosomes. Journal of Neuroscience 31, 4524-4534

361. Blaustein, M. (1975) Effects of potassium, veratridine, and scorpion venom on calcium accumulation and transmitter release by nerve terminals in vitro. The Journal of physiology 247, 617-655

362. von Mollard, G. F., Südhof, T. C., and Jahn, R. (1991) A small GTP-binding protein dissociates from synaptic vesicles during exocytosis. Nature $349,79-81$

363. Wessel, D., and Flügge, U. (1984) A method for the quantitative recovery of protein in dilute solution in the presence of detergents and lipids. Analytical biochemistry 138, 141143

364. Humphrey, S. J., Azimifar, S. B., and Mann, M. (2015) High-throughput phosphoproteomics reveals in vivo insulin signaling dynamics. Nature biotechnology 33, 990-995

365. Cox, J., Neuhauser, N., Michalski, A., Scheltema, R. A., Olsen, J. V., and Mann, M. (2011) Andromeda: a peptide search engine integrated into the MaxQuant environment. Journal of proteome research 10, 1794-1805

366. Consortium, U. (2019) UniProt: a worldwide hub of protein knowledge. Nucleic acids research 47, D506-D515

367. Storey, J. D., and Tibshirani, R. (2003) Statistical significance for genomewide studies. Proceedings of the National Academy of Sciences 100, 9440-9445

368. Jiao, X., Sherman, B. T., Huang, D. W., Stephens, R., Baseler, M. W., Lane, H. C., and Lempicki, R. A. (2012) DAVID-WS: a stateful web service to facilitate gene/protein list analysis. Bioinformatics 28, 1805-1806

369. Jassal, B., Matthews, L., Viteri, G., Gong, C., Lorente, P., Fabregat, A., Sidiropoulos, K., Cook, J., Gillespie, M., and Haw, R. (2020) The reactome pathway knowledgebase. Nucleic acids research 48, D498-D503

370. Altschul, S. F., Gish, W., Miller, W., Myers, E. W., and Lipman, D. J. (1990) Basic local alignment search tool. Journal of molecular biology 215, 403-410

371. Roy, J., and Cyert, M. S. (2009) Cracking the phosphatase code: docking interactions determine substrate specificity. Science signaling 2, re9-re9

372. Csardi, G., and Nepusz, T. (2006) The igraph software package for complex network research. InterJournal, complex systems 1695, 1-9

373. Dignam, J. D., Lebovitz, R. M., and Roeder, R. G. (1983) Accurate transcription initiation by RNA polymerase II in a soluble extract from isolated mammalian nuclei. Nucleic acids research $11,1475-1489$ 
374. Truckenbrodt, S., Viplav, A., Jähne, S., Vogts, A., Denker, A., Wildhagen, H., Fornasiero, E. F., and Rizzoli, S. O. (2018) Newly produced synaptic vesicle proteins are preferentially used in synaptic transmission. The EMBO journal 37, e98044

375. Sankaranarayanan, S., De Angelis, D., Rothman, J. E., and Ryan, T. A. (2000) The use of pHluorins for optical measurements of presynaptic activity. Biophysical journal 79, 2199-2208

376. Keihani, S., Kluever, V., Mandad, S., Bansal, V., Rahman, R., Fritsch, E., Gomes, L. C., Gärtner, A., Kügler, S., and Urlaub, H. (2019) The long noncoding RNA neuroLNC regulates presynaptic activity by interacting with the neurodegeneration-associated protein TDP-43. Science advances 5, eaay2670

377. Götzke, H., Kilisch, M., Martínez-Carranza, M., Sograte-Idrissi, S., Rajavel, A., Schlichthaerle, T., Engels, N., Jungmann, R., Stenmark, P., and Opazo, F. (2019) The ALFA-tag is a highly versatile tool for nanobody-based bioscience applications. Nature communications 10, 1-12

378. Fornasiero, E. F., Raimondi, A., Guarnieri, F. C., Orlando, M., Fesce, R., Benfenati, F., and Valtorta, F. (2012) Synapsins contribute to the dynamic spatial organization of synaptic vesicles in an activity-dependent manner. Journal of Neuroscience 32, 1221412227

379. Klee, C., Crouch, T., and Krinks, M. (1979) Calcineurin: a calcium-and calmodulinbinding protein of the nervous system. Proceedings of the National Academy of Sciences $76,6270-6273$

380. Wang, Z.-W. (2008) Regulation of synaptic transmission by presynaptic CaMKII and BK channels. Molecular neurobiology 38, 153-166

381. Rosenberg, O. S., Deindl, S., Sung, R.-J., Nairn, A. C., and Kuriyan, J. (2005) Structure of the autoinhibited kinase domain of CaMKII and SAXS analysis of the holoenzyme. Cell 123, 849-860

382. Czernik, A. J., Pang, D. T., and Greengard, P. (1987) Amino acid sequences surrounding the cAMP-dependent and calcium/calmodulin-dependent phosphorylation sites in rat and bovine synapsin I. Proceedings of the National Academy of Sciences 84, 7518-7522

383. Wang, J., Walaas, S., and Greengard, P. (1988) Protein phosphorylation in nerve terminals: comparison of calcium/calmodulin-dependent and calcium/diacylglyceroldependent systems. Journal of Neuroscience 8, 281-288

384. Menegon, A., Dunlap, D. D., Castano, F., Benfenati, F., Czernik, A. J., Greengard, P., and Valtorta, F. (2000) Use of phosphosynapsin I-specific antibodies for image analysis of signal transduction in single nerve terminals. Journal of Cell Science 113, 3573-3582

385. Cesca, F., Baldelli, P., Valtorta, F., and Benfenati, F. (2010) The synapsins: key actors of synapse function and plasticity. Progress in neurobiology 91, 313-348

386. Chua, J. J., Kindler, S., Boyken, J., and Jahn, R. (2010) The architecture of an excitatory synapse. Journal of cell science 123, 819-823

387. Marsicano, G., Goodenough, S., Monory, K., Hermann, H., Eder, M., Cannich, A., Azad, S. C., Cascio, M. G., Gutiérrez, S. O., and Van der Stelt, M. (2003) CB1 cannabinoid receptors and on-demand defense against excitotoxicity. Science 302, 84-88

388. Kellogg, R., Mackie, K., and Straiker, A. (2009) Cannabinoid CB1 receptordependent long-term depression in autaptic excitatory neurons. Journal of neurophysiology $102,1160-1171$

389. Turner, K. M., Burgoyne, R. D., and Morgan, A. (1999) Protein phosphorylation and the regulation of synaptic membrane traffic. Trends in neurosciences 22, 459-464

390. Guitart, X., Egea, G., Solsona, C., and Marsal, J. (1987) Botulinum neurotoxin inhibits depolarization-stimulated protein phosphorylation in pure cholinergic synaptosomes. FEBS letters 219, 219-223

391. Boggio, E. M., Putignano, E., Sassoe-Pognetto, M., Pizzorusso, T., and Giustetto, M. (2007) Visual stimulation activates ERK in synaptic and somatic compartments of rat cortical neurons with parallel kinetics. PLoS One 2, e604 
392. Edbauer, D., Cheng, D., Batterton, M. N., Wang, C.-F., Duong, D. M., Yaffe, M. B., Peng, J., and Sheng, M. (2009) Identification and Characterization of Neuronal Mitogenactivated Protein Kinase Substrates Using a Specific Phosphomotif Antibody ${ }^{\star}$ S. Molecular \& Cellular Proteomics 8, 681-695

393. Park, J. M., Hu, J.-H., Milshteyn, A., Zhang, P.-W., Moore, C. G., Park, S., Datko, M. C., Domingo, R. D., Reyes, C. M., and Wang, X. J. (2013) A prolyl-isomerase mediates dopamine-dependent plasticity and cocaine motor sensitization. Cell 154, 637-650

394. Mao, L.-M., and Wang, J. Q. (2016) Synaptically localized mitogen-activated protein kinases: local substrates and regulation. Molecular neurobiology 53, 6309-6315

395. Ataman, B., Ashley, J., Gorczyca, M., Ramachandran, P., Fouquet, W., Sigrist, S. J., and Budnik, V. (2008) Rapid activity-dependent modifications in synaptic structure and function require bidirectional Wnt signaling. Neuron $57,705-718$

396. Cerpa, W., Godoy, J. A., Alfaro, I., Farías, G. G., Metcalfe, M. J., Fuentealba, R., Bonansco, C., and Inestrosa, N. C. (2008) Wnt-7a modulates the synaptic vesicle cycle and synaptic transmission in hippocampal neurons. Journal of Biological Chemistry 283, 59185927

397. Budnik, V., and Salinas, P. C. (2011) Wnt signaling during synaptic development and plasticity. Current opinion in neurobiology 21, 151-159

398. Ciani, L., Marzo, A., Boyle, K., Stamatakou, E., Lopes, D. M., Anane, D., McLeod, F., Rosso, S. B., Gibb, A., and Salinas, P. C. (2015) Wnt signalling tunes neurotransmitter release by directly targeting Synaptotagmin-1. Nature communications $6,1-13$

399. Allen, P. B., Greenfield, A. T., Svenningsson, P., Haspeslagh, D. C., and Greengard, P. (2004) Phactrs 1-4: A family of protein phosphatase 1 and actin regulatory proteins. Proceedings of the National Academy of Sciences 101, 7187-7192

400. Oliver, C. J., Terry-Lorenzo, R. T., Elliott, E., Bloomer, W. A. C., Li, S., Brautigan, D. L., Colbran, R. J., and Shenolikar, S. (2002) Targeting protein phosphatase 1 (PP1) to the actin cytoskeleton: the neurabin I/PP1 complex regulates cell morphology. Molecular and cellular biology 22, 4690-4701

401. Dubois, T., Kerai, P., Learmonth, M., Cronshaw, A., and Aitken, A. (2002) Identification of syntaxin-1A sites of phosphorylation by casein kinase I and casein kinase II. European journal of biochemistry 269, 909-914

402. Trinidad, J. C., Barkan, D. T., Gulledge, B. F., Thalhammer, A., Sali, A., Schoepfer, R., and Burlingame, A. L. (2012) Global identification and characterization of both OGlcNAcylation and phosphorylation at the murine synapse. Molecular \& Cellular Proteomics 11, 215-229

403. Brüning, F., Noya, S. B., Bange, T., Koutsouli, S., Rudolph, J. D., Tyagarajan, S. K., Cox, J., Mann, M., Brown, S. A., and Robles, M. S. (2019) Sleep-wake cycles drive daily dynamics of synaptic phosphorylation. Science 366

404. Perez-Riverol, Y., Csordas, A., Bai, J., Bernal-Llinares, M., Hewapathirana, S., Kundu, D. J., Inuganti, A., Griss, J., Mayer, G., and Eisenacher, M. (2019) The PRIDE database and related tools and resources in 2019: improving support for quantification data. Nucleic acids research 47, D442-D450

405. Popoff, M. R., and Poulain, B. (2010) Bacterial toxins and the nervous system: neurotoxins and multipotential toxins interacting with neuronal cells. Toxins 2, 683-737

406. Schwanhäusser, B., Busse, D., Li, N., Dittmar, G., Schuchhardt, J., Wolf, J., Chen, W., and Selbach, M. (2011) Global quantification of mammalian gene expression control. Nature 473, 337-342

407. Heidelberger, R., Heinemann, C., Neher, E., and Matthews, G. (1994) Calcium dependence of the rate of exocytosis in a synaptic terminal. Nature 371, 513-515

408. Stafford, J., Brownlow, M. L., Qualley, A., and Jankord, R. (2018) AMPA receptor translocation and phosphorylation are induced by transcranial direct current stimulation in rats. Neurobiology of learning and memory 150, 36-41

409. Stagg, C. J., and Nitsche, M. A. (2011) Physiological basis of transcranial direct current stimulation. The Neuroscientist 17, 37-53 
410. Montecucco, C., and Schiavo, G. (1994) Mechanism of action of tetanus and botulinum neurotoxins. Molecular microbiology 13, 1-8

411. Shields, S. M., Ingebritsen, T. S., and Kelly, P. T. (1985) Identification of protein phosphatase 1 in synaptic junctions: dephosphorylation of endogenous calmodulindependent kinase II and synapse-enriched phosphoproteins. Journal of Neuroscience 5, 3414-3422

412. Yue, X., Schunter, A., and Hummon, A. B. (2015) Comparing multistep immobilized metal affinity chromatography and multistep TiO2 methods for phosphopeptide enrichment. Anal Chem 87, 8837-8844

413. Refsgaard, J. C., Munk, S., and Jensen, L. J. (2016) Search databases and statistics: pitfalls and best practices in phosphoproteomics. Phospho-Proteomics, 323-339 414. Lisman, J. E., and Zhabotinsky, A. M. (2001) A model of synaptic memory: a CaMKII/PP1 switch that potentiates transmission by organizing an AMPA receptor anchoring assembly. Neuron 31, 191-201

415. Suzuki, T., Bridges, D., Nakada, D., Skiniotis, G., Morrison, S. J., Lin, J. D., Saltiel, A. R., and Inoki, K. (2013) Inhibition of AMPK catabolic action by GSK3. Molecular cell 50, 407-419

416. Asada, A., Saito, T., and Hisanaga, S.-i. (2012) Phosphorylation of p35 and p39 by Cdk5 determines the subcellular location of the holokinase in a phosphorylation-site-specific manner. Journal of cell science 125, 3421-3429

417. Rivers, A., Gietzen, K. F., Vielhaber, E., and Virshup, D. M. (1998) Regulation of Casein Kinase $I \varepsilon$ and Casein Kinase I $\delta$ by anin Vivo Futile Phosphorylation Cycle. Journal of Biological Chemistry 273, 15980-15984

418. Meng, Z., Bischof, J., lanes, C., Henne-Bruns, D., Xu, P., and Knippschild, U. (2016) CK1 $1 \delta$ kinase activity is modulated by protein kinase $\mathrm{C} \alpha(\mathrm{PKC \alpha})$-mediated site-specific phosphorylation. Amino Acids 48, 1185-1197

419. Robles, M. S., Humphrey, S. J., and Mann, M. (2017) Phosphorylation is a central mechanism for circadian control of metabolism and physiology. Cell metabolism 25, 118127

420. Sacco, F., Humphrey, S. J., Cox, J., Mischnik, M., Schulte, A., Klabunde, T., Schäfer, M., and Mann, M. (2016) Glucose-regulated and drug-perturbed phosphoproteome reveals molecular mechanisms controlling insulin secretion. Nature communications 7, 1-13

421. Litchfield, D., Lüscher, B., Lozeman, F., Eisenman, R., and Krebs, E. (1992) Phosphorylation of casein kinase II by p34cdc2 in vitro and at mitosis. Journal of Biological Chemistry 267, 13943-13951

422. Shin, Y. J., Kim, Y.-B., and Kim, J.-H. (2013) Protein kinase CK2 phosphorylates and activates p21-activated kinase 1. Molecular biology of the cell 24, 2990-2999

423. Montenegro-Venegas, C., Guhathakurta, D., Pina-Fernandez, E., Andres-Alonso, M., Plattner, F., Lazarevic, V., Gundelfinger, E. D., and Fejtova, A. (2021) Bassoon controls synaptic vesicle pools via regulation of presynaptic phosphorylation and CAMP homeostasis. bioRxiv

424. Mochida, S., Hida, Y., Tanifuji, S., Hagiwara, A., Hamada, S., Abe, M., Ma, H., Yasumura, M., Kitajima, I., and Sakimura, K. (2016) SAD-B phosphorylation of CAST controls active zone vesicle recycling for synaptic depression. Cell reports 16, 2901-2913

425. Han, W., Nattel, S., Noguchi, T., and Shrier, A. (2006) C-terminal domain of Kv4. 2 and associated KChIP2 interactions regulate functional expression and gating of Kv4. 2. Journal of Biological Chemistry 281, 27134-27144

426. Schrader, L. A., Ren, Y., Cheng, F., Bui, D., Sweatt, J. D., and Anderson, A. E. (2009) Kv4. 2 is a locus for PKC and ERK/MAPK cross-talk. Biochemical Journal 417, 705715

427. Schrader, L. A., Birnbaum, S. G., Nadin, B. M., Ren, Y., Bui, D., Anderson, A. E., and Sweatt, J. D. (2006) ERK/MAPK regulates the Kv4. 2 potassium channel by direct phosphorylation of the pore-forming subunit. American Journal of Physiology-Cell Physiology 
428. Yang, J.-W., Vacher, H., Park, K.-S., Clark, E., and Trimmer, J. S. (2007) Traffickingdependent phosphorylation of Kv1. 2 regulates voltage-gated potassium channel cell surface expression. Proceedings of the National Academy of Sciences 104, 20055-20060

429. Park, K.-S., Mohapatra, D. P., and Trimmer, J. S. (2007) Proteomic analyses of Kv2. 1 channel phosphorylation sites determining cell background-specific differences in function. Channels 1, 59-61

430. Redman, P. T., He, K., Hartnett, K. A., Jefferson, B. S., Hu, L., Rosenberg, P. A., Levitan, E. S., and Aizenman, E. (2007) Apoptotic surge of potassium currents is mediated by p38 phosphorylation of Kv2. 1. Proceedings of the National Academy of Sciences 104, 3568-3573

431. Pal, S., Hartnett, K. A., Nerbonne, J. M., Levitan, E. S., and Aizenman, E. (2003) Mediation of neuronal apoptosis by Kv2. 1-encoded potassium channels. Journal of Neuroscience 23, 4798-4802

432. Pal, S., Takimoto, K., Aizenman, E., and Levitan, E. S. (2006) Apoptotic surface delivery of $\mathrm{K}^{+}$channels. Cell Death \& Differentiation 13, 661-667

433. MacDonald, P. E., Wang, G., Tsuk, S., Dodo, C., Kang, Y., Tang, L., Wheeler, M. B., Cattral, M. S., Lakey, J. R., and Salapatek, A. M. F. (2002) Synaptosome-associated protein of 25 kilodaltons modulates Kv2. 1 voltage-dependent $\mathrm{K}^{+}$channels in neuroendocrine islet $\beta$-cells through an interaction with the channel $\mathrm{N}$ terminus. Molecular endocrinology 16, 2452-2461

434. Leung, Y. M., Kang, Y., Gao, X., Xia, F., Xie, H., Sheu, L., Tsuk, S., Lotan, I., Tsushima, R. G., and Gaisano, H. Y. (2003) Syntaxin 1A binds to the cytoplasmic C terminus of Kv2. 1 to regulate channel gating and trafficking. Journal of Biological Chemistry 278, 17532-17538

435. Kang, Y., Linial, M., Gaisano, H. Y., Michaelevski, I., Chikvashvili, D., Tsuk, S., Singer-Lahat, D., Fili, O., and Lotan, I. (2003) Direct interaction of target SNAREs with the Kv2. 1 channel: modal regulation of channel activation and inactivation gating. Journal of Biological Chemistry 278, 34320-34330

436. Teo, S., and Salinas, P. C. (2021) Wnt-Frizzled signaling regulates activity-mediated synapse formation. Frontiers in Molecular Neuroscience 14

437. Lu, Q., Paredes, M., Medina, M., Zhou, J., Cavallo, R., Peifer, M., Orecchio, L., and Kosik, K. S. (1999) $\delta$-Catenin, an adhesive junction-associated protein which promotes cell scattering. The Journal of cell biology 144, 519-532

438. Kosik, K. S., Donahue, C. P., Israely, I., Liu, X., and Ochiishi, T. (2005) $\delta$-Catenin at the synaptic-adherens junction. Trends in cell biology 15, 172-178

439. Ide, N., Hata, Y., Deguchi, M., Hirao, K., Yao, I., and Takai, Y. (1999) Interaction of S-SCAM with Neural Plakophilin-RelatedArmadillo-Repeat Protein/ס-Catenin. Biochemical and biophysical research communications 256, 456-461

440. Sánchez, C., Pérez, M., and Avila, J. (2000) GSK3ß-mediated phosphorylation of the microtubule-associated protein $2 \mathrm{C}$ (MAP2C) prevents microtubule bundling. European journal of cell biology 79, 252-260

441. Sanchez, C., Díaz-Nido, J., and Avila, J. (1998) Regulation of a site-specific phosphorylation of the microtubule-associated protein 2 during the development of cultured neurons. Neuroscience 87, 861-870

442. Taymans, J.-M., and Baekelandt, V. (2014) Phosphatases of a-synuclein, LRRK2, and tau: important players in the phosphorylation-dependent pathology of Parkinsonism. Frontiers in genetics 5, 382 


\section{Appendix}

Table 8.1: primary $\mathrm{Ca}^{2+}$-dependent sites, selection from Suppl. Data 01. UP.Accession Uniprot accession; Gene.name official gene symbol; AA modified amino acid; Pos position in the protein sequence; Mult multiplicity of phosphorylation (1 - singly phosphorylated, 2 - doubly phosphorylated, 3 - multiply phosphorylated); Seq.window $\sim \pm 7$ amino acids flanking phosphorylated residue; CaEGTA $\log _{2}$ intensity fold change in Ca vs. EGTA experiment, MockBoNT $\log _{2}$ intensity fold change in Mock vs. BoNT experiment

\begin{tabular}{|c|c|c|c|c|c|c|c|}
\hline UP.Accession & Gene.name & AA & Pos & Mult & Seq.window & CaEGTA & MockBoNT \\
\hline P0C1X8 & Aak1 & S & 638 & 1 & AGHRRILSDVTHSAV & -0.7362 & 0.1272 \\
\hline P0C1X8 & Aak1 & $S$ & 638 & 2 & AGHRRILSDVTHSAV & -1.3276 & 0.0638 \\
\hline $\mathrm{P} 0 \mathrm{C} 1 \mathrm{X} 8$ & Aak1 & S & 653 & 1 & FGVPASKSTQLLHAA & -1.2734 & 0.0707 \\
\hline $\mathrm{P} 0 \mathrm{C} 1 \mathrm{X} 8$ & Aak1 & S & 653 & 2 & FGVPASKSTQLLHAA & -1.3278 & \\
\hline P0C1X8 & Aak1 & S & 671 & 1 & ASLSKSKSATTTPSG & -0.7759 & 0.0095 \\
\hline $\mathrm{P} 0 \mathrm{C} 1 \mathrm{X} 8$ & Aak1 & S & 671 & 2 & ASLSKSKSATTTPSG & -1.4722 & 0.0747 \\
\hline $\mathrm{P} 0 \mathrm{C} 1 \mathrm{X} 8$ & Aak1 & S & 671 & 3 & ASLSKSKSATTTPSG & -1.2298 & -0.0267 \\
\hline P0C1X8 & Aak1 & $T$ & 675 & 3 & KSKSATTTPSGSPRT & -1.2337 & -0.0378 \\
\hline $\mathrm{P} 0 \mathrm{C} 1 \mathrm{X} 8$ & Aak1 & S & 677 & 2 & KSATTTPSGSPRTSQ & -0.7228 & 0.0769 \\
\hline $\mathrm{P} 0 \mathrm{C} 1 \mathrm{X} 8$ & Aak1 & S & 679 & 1 & ATTTPSGSPRTSQQN & 0.3564 & 0.0833 \\
\hline $\mathrm{P} 0 \mathrm{C} 1 \times 8$ & Aak1 & S & 679 & 3 & ATTTPSGSPRTSQQN & -1.2337 & -0.0340 \\
\hline $\mathrm{P} 0 \mathrm{C} 1 \mathrm{X} 8$ & Aak1 & S & 732 & 1 & LPEKLGGSAESLIPG & -0.3218 & 0.0157 \\
\hline $\mathrm{P} 0 \mathrm{C} 1 \mathrm{X} 8$ & Aak1 & S & 732 & 2 & LPEKLGGSAESLIPG & -0.8128 & \\
\hline $\mathrm{P} 0 \mathrm{C} 1 \times 8$ & Aak1 & $T$ & 743 & 2 & LIPGFQATQGDAFAT & -0.8286 & \\
\hline P0C1X8 & Aak1 & S & 847 & 1 & PVAQRLPSHTESVTS & -0.6066 & -0.0092 \\
\hline Q9QZM5 & Abi1 & S & 217 & 1 & TSPARLGSQHSPGRT & 0.3935 & -0.1388 \\
\hline Q9QZM5 & Abi1 & S & 226 & 1 & HSPGRTASLNQRPRT & 0.6219 & -0.1397 \\
\hline F1MON1 & Abl2 & S & 629 & 1 & PRKQRDKSPSSLLED & -0.6648 & \\
\hline F1MON1 & Abl2 & S & 631 & 1 & KQRDKSPSSLLEDAK & -0.7520 & \\
\hline F1MON1 & Abl2 & S & 799 & 1 & LQLERTVSTSSQPEE & 0.8680 & \\
\hline F1M8U2 & Ablim3 & S & 393 & 1 & MSPTFSRSPHHYYRS & -0.3155 & \\
\hline F1M8U2 & Ablim3 & S & 408 & 2 & GPESGRSSPYHSQLD & -0.5498 & -0.0424 \\
\hline F1M8U2 & Ablim3 & S & 412 & 2 & GRSSPYHSQLDVRSS & -0.5587 & -0.0495 \\
\hline A0A0G2K4T3 & Acap2 & $\mathrm{S}$ & 612 & 2 & ESLPSTVSANSLYEP & -0.4995 & -0.0115 \\
\hline A0A0G2K4T3 & Acap2 & S & 615 & 2 & PSTVSANSLYEPEGE & -0.4995 & -0.0115 \\
\hline MOR5P8 & Adam22 & $S$ & 726 & 3 & RFRPRSNSTETLSPA & -0.3061 & \\
\hline M0R5P8 & Adam22 & $T$ & 729 & 3 & PRSNSTETLSPAKSP & -0.2873 & 0.0317 \\
\hline MOR5P8 & Adam22 & S & 731 & 3 & SNSTETLSPAKSPSS & -0.2813 & 0.0340 \\
\hline MOR5P8 & Adam22 & $S$ & 735 & 1 & ETLSPAKSPSSSTGS & -0.3635 & 0.0610 \\
\hline M0R5P8 & Adam22 & $\mathrm{S}$ & 735 & 2 & ETLSPAKSPSSSTGS & -0.2898 & 0.0877 \\
\hline MOR5P8 & Adam22 & $\mathrm{S}$ & 735 & 3 & ETLSPAKSPSSSTGS & -0.2655 & 0.0113 \\
\hline MOR5P8 & Adam22 & $S$ & 739 & 1 & PAKSPSSSTGSIASS & 0.2935 & 0.2130 \\
\hline M0R5P8 & Adam22 & S & 742 & 1 & SPSSSTGSIASSRKY & 0.4259 & 0.0526 \\
\hline MOR5P8 & Adam22 & S & 767 & 1 & EKTVNRQSARLWETS & -0.7870 & -0.1530 \\
\hline Q04400 & Adcy 5 & $\mathrm{~T}$ & 62 & 1 & KRSGGAVTPQQQQRL & -0.3139 & 0.0146 \\
\hline MOR5U4 & Adcy 9 & S & 1274 & 2 & VDGSIGRSPTDEITN & -0.4237 & \\
\hline
\end{tabular}




\begin{tabular}{|c|c|c|c|c|c|c|c|}
\hline MOR5U4 & Adcy 9 & $\mathrm{~T}$ & 1276 & 2 & GSIGRSPTDEITNLV & -0.3969 & \\
\hline Q05764 & Add2 & S & 606 & 1 & TPASPVQSPTRAGTK & 0.4197 & -0.1341 \\
\hline Q05764 & Add2 & S & 623 & 1 & AVSPSKASEDAKKTE & 0.3441 & 0.2358 \\
\hline Q62847 & Add3 & $S$ & 402 & 2 & REKPRHKSDVEIPAT & -0.2680 & 0.1066 \\
\hline Q62847 & Add3 & $T$ & 653 & 3 & SRLTTSTTIENIEIT & -0.3005 & \\
\hline Q62847 & Add3 & S & 663 & 2 & NIEITIKSPDRTEEV & -0.2971 & \\
\hline Q62847 & Add3 & $S$ & 680 & 1 & PDGSPSKSPSKKKKK & -0.3335 & 0.2343 \\
\hline Q62847 & Add3 & $S$ & 680 & 2 & PDGSPSKSPSKKKKK & -0.3241 & 0.1066 \\
\hline 088917 & Adgrl1 & S & 1128 & 1 & PPGGAHGSLKTSAMR & 0.4962 & -0.1920 \\
\hline Q9Z173 & Adgrl3 & S & 1239 & 1 & RYSTGSQSRIRRMWN & 0.5008 & -0.0398 \\
\hline O35889 & Afdn & S & 181 & 1 & KEALRQASDKEERPS & 0.6297 & \\
\hline A0A0G2K847 & Agap1 & S & 345 & 1 & GLESRADSIGSGRAI & 1.5076 & \\
\hline Q8CGU4 & Agap2 & S & 1172 & 1 & PSPRRRSSAASLGRV & 0.3920 & 0.1942 \\
\hline A0A0G2JZ83 & Agap3 & $S$ & 478 & 1 & PSTAPGTSPRGANGL & -0.3562 & 0.1023 \\
\hline Q4KLH5 & Agfg1 & $\mathrm{T}$ & 179 & 2 & HLNKGTPTQSPVVGR & -0.3567 & \\
\hline Q4KLH5 & Agfg1 & S & 298 & 1 & SSSADFGSFSTSQSH & -0.5385 & \\
\hline Q5QD51 & Akap12 & $\mathrm{S}$ & 304 & 1 & KKTSFKKSKEDDLET & 0.6604 & -0.1102 \\
\hline P09117 & Aldoc & S & 39 & 1 & AADESVGSMAKRLSQ & 0.3393 & -0.1296 \\
\hline P0C5Y8 & Als2 & S & 477 & 2 & EGGSRRLSLPGLLSQ & -0.3327 & 0.0436 \\
\hline P0C5Y8 & Als2 & $S$ & 486 & 2 & PGLLSQVSPRLLRKA & -0.3327 & 0.0436 \\
\hline Q02356 & Ampd2 & S & 21 & 1 & YPFKKRASLQASAAA & 1.0988 & -0.1266 \\
\hline Q02356 & Ampd2 & S & 113 & 1 & QRLERQISQDVKLEP & 1.6248 & 0.0362 \\
\hline 008838 & Amph & $\mathrm{T}$ & 260 & 3 & GPLRIAKTPSPPEEA & -0.2793 & 0.0712 \\
\hline 008838 & Amph & S & 262 & 3 & LRIAKTPSPPEEASP & -0.2958 & 0.0399 \\
\hline 008838 & Amph & $S$ & 268 & 3 & PSPPEEASPLPSPTA & -0.3349 & 0.0120 \\
\hline 008838 & Amph & $S$ & 272 & 3 & EEASPLPSPTASPNH & -0.3122 & 0.0231 \\
\hline 008838 & Amph & $\mathrm{T}$ & 274 & 3 & ASPLPSPTASPNHTL & -0.4534 & 0.0175 \\
\hline 008838 & Amph & $S$ & 276 & 3 & PLPSPTASPNHTLAP & -0.3025 & 0.0945 \\
\hline 008838 & Amph & $\mathrm{T}$ & 280 & 3 & PTASPNHTLAPASPA & -0.4395 & \\
\hline 008838 & Amph & S & 285 & 3 & NHTLAPASPAPVRPR & -0.3690 & 0.0108 \\
\hline A0A0G2JZ56 & Ank2 & $S$ & 1800 & 1 & KIIRRYVSSDGTEKE & 0.3369 & 0.0694 \\
\hline F1M9N9 & Ank2 & $S$ & 1726 & 1 & LPTAKATSPLIEETP & -0.7095 & \\
\hline F1M9N9 & Ank2 & S & 1797 & 1 & SKMERHSSLTSSAKT & 0.5266 & 0.1878 \\
\hline F1M9N9 & Ank2 & $S$ & 1813 & 2 & RHSPVSPSSKNEKHS & -0.3515 & \\
\hline F1M9N9 & Ank2 & $\mathrm{T}$ & 3054 & 2 & PDTTPARTPTEEGTP & -0.5207 & \\
\hline Q5BJT1 & Ankrd34a & $\mathrm{T}$ & 315 & 1 & GPLSRRNTAPEAQES & 0.3036 & -0.0611 \\
\hline Q5BJT1 & Ankrd34a & $S$ & 448 & 2 & QPGGRAPSLPAPPHS & 0.2872 & -0.0622 \\
\hline Q5BJT1 & Ankrd34a & S & 460 & 2 & PHSGAPGSPRTKRKL & 0.2872 & -0.0622 \\
\hline Q5BJT1 & Ankrd34a & S & 472 & 1 & RKLVRRHSMQTEQIR & 0.4943 & -0.0365 \\
\hline D3ZKP1 & Ankrd34b & $S$ & 496 & 1 & KMLLRRQSLQTEQIK & 0.5918 & -0.0012 \\
\hline F1LWB9 & Ankrd50 & S & 1137 & 1 & SLTIRSNSSGGTGGG & 0.4376 & \\
\hline D4A0V6 & Anln & S & 633 & 1 & DSSLSAPSPKPGKFQ & -0.3938 & -0.1464 \\
\hline 035430 & Apba1 & $S$ & 286 & 1 & KQSMSSQSLDKAAED & 0.4552 & \\
\hline A0A0G2K8G0 & Apc & $\mathrm{T}$ & 1216 & 1 & PSFSFSKTPSVQGTK & 0.7146 & -0.0676 \\
\hline
\end{tabular}




\begin{tabular}{|c|c|c|c|c|c|c|c|}
\hline A0A0G2K8G0 & Apc & $S$ & 2278 & 1 & LKTPASKSPSEGPVA & -0.2873 & 0.2612 \\
\hline A0A0G2K8G0 & Apc & $S$ & 2308 & 1 & SPITRQTSHISGSNK & 0.7546 & -0.0802 \\
\hline A0A0G2K8G0 & Apc & $S$ & 2484 & 2 & SASFESLSPSSRPDS & -0.3699 & 0.2488 \\
\hline A0A0G2K8G0 & Apc & $S$ & 2486 & 2 & SFESLSPSSRPDSPT & -0.2808 & 0.1414 \\
\hline A0A0G2K8G0 & Apc & $S$ & 2692 & 1 & NNPRSGRSPTGNTPP & -0.3685 & \\
\hline Q3S4A4 & Arfgap1 & $S$ & 247 & 1 & FWGYKQQSEPASELG & 2.5740 & 0.2088 \\
\hline Q3S4A4 & Arfgap1 & S & 251 & 1 & KQQSEPASELGHSLN & 2.3481 & \\
\hline D4A631 & Arfgef1 & $S$ & 1076 & 1 & TVRGREGSLTGTKDQ & 0.7003 & 0.2252 \\
\hline D4A631 & Arfgef1 & $S$ & 1566 & 1 & LDAISQKSVDIHDSA & 0.7857 & 0.0497 \\
\hline Q6AY65 & Arfip2 & $S$ & 72 & 1 & TGSGRHPSHSTSPSG & -0.7034 & \\
\hline Q6AY65 & Arfip2 & $S$ & 72 & 2 & TGSGRHPSHSTSPSG & -0.6915 & \\
\hline Q6AY65 & Arfip2 & $S$ & 74 & 2 & SGRHPSHSTSPSGPG & -0.4811 & \\
\hline Q6AY65 & Arfip2 & $\mathrm{T}$ & 75 & 2 & GRHPSHSTSPSGPGD & -0.4554 & \\
\hline Q6AY65 & Arfip2 & $S$ & 76 & 1 & RHPSHSTSPSGPGDE & -0.3954 & 0.1709 \\
\hline Q6AY65 & Arfip2 & $S$ & 76 & 2 & RHPSHSTSPSGPGDE & -0.5794 & \\
\hline D4A6C5 & Arhgap1 & S & 27 & 1 & LNQLKLASIDEKNWP & 2.3853 & -0.2110 \\
\hline A0A0G2K1Z2 & Arhgap12 & $S$ & 163 & 1 & KFNSDSHSPKVSSQN & -0.4312 & -0.0212 \\
\hline D4A9G6 & Arhgap33 & $S$ & 588 & 1 & TTPKTPASPVERRKR & 0.2905 & 0.0048 \\
\hline F1LR18 & Arhgap39 & S & 294 & 1 & LIQPRKPSSDSQPSS & 0.4982 & 0.2614 \\
\hline F1LR18 & Arhgap39 & $S$ & 294 & 2 & LIQPRKPSSDSQPSS & 0.4835 & 0.1154 \\
\hline F1LR18 & Arhgap39 & $S$ & 297 & 1 & PRKPSSDSQPSSPRY & 0.4798 & 0.2303 \\
\hline F1LR18 & Arhgap39 & S & 599 & 1 & GPVVRAFSEDEALAQ & 0.3240 & -0.1117 \\
\hline F1LQX4 & Arhgap44 & $S$ & 598 & 1 & VSKSKELSPGSGQKG & -0.4125 & 0.0799 \\
\hline F1LQX4 & Arhgap44 & $S$ & 798 & 1 & LEHARRHSVTDKRDS & -0.6337 & -0.0634 \\
\hline Q5XI73 & Arhgdia & S & 34 & 1 & YKPPAQKSIQEIQEL & 1.3931 & -0.0808 \\
\hline F1LQS9 & Arhgef11 & $S$ & 563 & 1 & RYIGKPKSSSQSIKP & 1.4143 & 0.0625 \\
\hline F1LQS9 & Arhgef11 & $S$ & 1463 & 2 & KSLGGESSGGTTPVG & -0.3669 & -0.0500 \\
\hline F1LQS9 & Arhgef11 & $\mathrm{T}$ & 1467 & 2 & GESSGGTTPVGSFHT & -0.3503 & \\
\hline Q6P720 & Arhgef25 & $S$ & 491 & 1 & IEYQRRESQTNSLGR & 0.2863 & 0.2039 \\
\hline A0A0G2QC21 & Arhgef7 & $S$ & 135 & 1 & HRIKSFDSLGSQSSH & 0.4436 & -0.0644 \\
\hline A0A0G2QC21 & Arhgef7 & S & 673 & 1 & RKPERKPSDEEFAVR & 0.7728 & 0.1342 \\
\hline A0A0G2JVX9 & Arpp21 & $S$ & 32 & 1 & NGILKSESLDEEEKL & 1.2358 & 0.0077 \\
\hline A0A0G2JZQ0 & Asap1 & $\mathrm{T}$ & 789 & 2 & VALRKTETSHHLSLD & 1.9411 & 0.0180 \\
\hline A0A0G2JZQ0 & Asap1 & S & 794 & 2 & TETSHHLSLDRANIP & 1.7549 & 0.0134 \\
\hline A0A0G2JZQ0 & Asap1 & $S$ & 911 & 1 & RSHTGDLSPNVQSRD & -0.4919 & -0.1062 \\
\hline Q1AAU6 & Asap1 & $S$ & 855 & 2 & RTLSDPPSPLPHGPP & -0.4220 & 0.0734 \\
\hline A0A0G2K9U6 & Atg16/1 & S & 303 & 1 & SDAARRRSVSSIPVP & -0.3397 & -0.0117 \\
\hline D3ZFK6 & Atg16l1 & $S$ & 287 & 1 & TNIFGRRSVSSIPVP & -0.3397 & -0.0105 \\
\hline F1MAF8 & Atg2b & $S$ & 280 & 1 & RYYLRKDSLSLGVSS & 0.7071 & -0.0099 \\
\hline D4A8B3 & Atp2b2 & S & 1152 & 1 & NTFKSGASFQGALRR & 0.5471 & 0.2346 \\
\hline D4A8B3 & Atp2b2 & S & 1162 & 1 & GALRRQSSVTSQSQD & 0.2646 & 0.1806 \\
\hline D4A8B3 & Atp2b2 & $S$ & 1165 & 2 & RRQSSVTSQSQDVAS & 0.3672 & 0.0333 \\
\hline P11506 & Atp2b2 & $S$ & 1150 & 1 & VVKAFRSSLYEGLEK & 0.6029 & -0.1874 \\
\hline A0A0G2K9Q6 & Atp2b3 & $S$ & 1124 & 1 & VVSTFKRSGSFQGAV & 0.6882 & 0.0592 \\
\hline
\end{tabular}




\begin{tabular}{|c|c|c|c|c|c|c|c|}
\hline A0A0G2K9Q6 & Atp2b3 & S & 1126 & 1 & STFKRSGSFQGAVRR & 0.6155 & 0.0678 \\
\hline A0A0G2JYE0 & Atxn2l & S & 681 & 1 & KSTSTPTSPGPRTHS & -0.6020 & 0.0597 \\
\hline Q6GMN2 & Baiap2 & S & 455 & 1 & LQQGKSSSTGNLLDK & -0.5024 & -0.1230 \\
\hline A0A0G2K1L8 & Basp1 & $\mathrm{S}$ & 214 & 1 & PVASSEQSVAVKE & 0.4246 & \\
\hline Q05175 & Basp1 & $S$ & 130 & 1 & AGEASAESTGAADGA & 0.2954 & \\
\hline 008839 & Bin1 & $\mathrm{s}$ & 321 & 2 & NHEPEPASGASPGAT & -1.0804 & -0.2199 \\
\hline 008839 & Bin1 & S & 324 & 2 & PEPASGASPGATIPK & -0.9200 & -0.1813 \\
\hline 008839 & Bin1 & $T$ & 328 & 1 & SGASPGATIPKSPSQ & -1.0314 & 0.0324 \\
\hline 008839 & Bin1 & $T$ & 328 & 2 & SGASPGATIPKSPSQ & -0.9516 & \\
\hline 008839 & Bin1 & $\mathrm{S}$ & 332 & 1 & PGATIPKSPSQLRKG & -1.2854 & -0.0424 \\
\hline O08839 & Bin1 & S & 332 & 2 & PGATIPKSPSQLRKG & -1.0277 & -0.2360 \\
\hline 008839 & Bin1 & S & 334 & 1 & ATIPKSPSQLRKGPP & -0.9335 & -0.0554 \\
\hline O08839 & Bin1 & S & 334 & 2 & ATIPKSPSQLRKGPP & -0.6960 & \\
\hline O08839 & Bin1 & $T$ & 349 & 1 & VPPPPKHTPSKEMKQ & -0.7219 & 0.0673 \\
\hline Q5HZA7 & Bin1 & S & 255 & 1 & FTVKAQPSDSAPEKG & 0.5255 & 0.0424 \\
\hline Q5HZA7 & Bin1 & $\mathrm{S}$ & 257 & 1 & VKAQPSDSAPEKGNK & 0.4363 & -0.0039 \\
\hline B2DD29 & Brsk1 & S & 442 & 3 & GASTGLSSSPLSSPR & -0.3172 & \\
\hline B2DD29 & Brsk1 & S & 443 & 3 & ASTGLSSSPLSSPRS & -0.3207 & \\
\hline B2DD29 & Brsk1 & $\mathrm{S}$ & 446 & 3 & GLSSSPLSSPRSPVF & -0.2889 & \\
\hline B2DD29 & Brsk1 & S & 447 & 3 & LSSSPLSSPRSPVFS & -0.2889 & \\
\hline D3ZML2 & Brsk2 & S & 415 & 1 & GQRSRSISGASSGLS & 0.4132 & 0.0046 \\
\hline G3V984 & Bsn & S & 135 & 2 & VDSRTQRSGRSPSVS & -1.3293 & \\
\hline G3V984 & Bsn & $S$ & 138 & 2 & RTQRSGRSPSVSPDR & -1.2617 & \\
\hline G3V984 & Bsn & S & 140 & 2 & QRSGRSPSVSPDRGS & -1.3274 & \\
\hline G3V984 & Bsn & $S$ & 142 & 1 & SGRSPSVSPDRGSTP & 0.8377 & -0.0085 \\
\hline G3V984 & Bsn & $S$ & 233 & 1 & DMTTAPRSKSQQQLH & -0.9557 & -0.0035 \\
\hline G3V984 & Bsn & S & 233 & 2 & DMTTAPRSKSQQQLH & -1.4701 & \\
\hline G3V984 & Bsn & $S$ & 235 & 1 & TTAPRSKSQQQLHSP & -0.4854 & -0.0400 \\
\hline G3V984 & Bsn & $S$ & 235 & 2 & TTAPRSKSQQQLHSP & -1.7446 & -0.0285 \\
\hline G3V984 & Bsn & S & 235 & 3 & TTAPRSKSQQQLHSP & -2.0305 & -0.0749 \\
\hline G3V984 & Bsn & S & 241 & 1 & KSQQQLHSPALSPAH & -0.9993 & 0.0631 \\
\hline G3V984 & Bsn & $S$ & 241 & 2 & KSQQQLHSPALSPAH & -1.6988 & -0.0045 \\
\hline G3V984 & Bsn & S & 241 & 3 & KSQQQLHSPALSPAH & -1.7851 & -0.0857 \\
\hline G3V984 & Bsn & $S$ & 249 & 2 & PALSPAHSPAKQPLG & -1.6418 & 0.1175 \\
\hline G3V984 & Bsn & $S$ & 249 & 3 & PALSPAHSPAKQPLG & -0.9583 & -0.0888 \\
\hline G3V984 & Bsn & $\mathrm{S}$ & 263 & 1 & GKPEQERSRSPGATQ & -1.6065 & -0.0269 \\
\hline G3V984 & Bsn & $S$ & 265 & 1 & PEQERSRSPGATQSG & -1.2681 & -0.0251 \\
\hline G3V984 & Bsn & S & 283 & 1 & AEAARATSVPGPTQA & 0.3857 & 0.0342 \\
\hline G3V984 & Bsn & S & 821 & 1 & DSSEGGLSPLPPQPP & -0.6741 & -0.1626 \\
\hline G3V984 & Bsn & $S$ & 1084 & 2 & AQRRRERSKTPPSNL & -0.3376 & \\
\hline G3V984 & Bsn & S & 1084 & 3 & AQRRRERSKTPPSNL & -0.9354 & 0.0842 \\
\hline G3V984 & Bsn & S & 1089 & 3 & ERSKTPPSNLSPIED & -1.0130 & 0.1486 \\
\hline G3V984 & Bsn & $S$ & 1220 & 1 & TGPRSQGSFEYQDTQ & 1.4265 & \\
\hline G3V984 & Bsn & $Y$ & 1223 & 1 & RSQGSFEYQDTQDHD & 0.4604 & \\
\hline
\end{tabular}




\begin{tabular}{|c|c|c|c|c|c|c|c|}
\hline G3V984 & Bsn & $T$ & 1226 & 1 & GSFEYQDTQDHDYGG & 2.2291 & \\
\hline G3V984 & Bsn & S & 1353 & 2 & QEPLKLHSSPASPSL & -1.7652 & 0.0942 \\
\hline G3V984 & Bsn & S & 1354 & 2 & EPLKLHSSPASPSLA & -2.0586 & -0.0702 \\
\hline G3V984 & Bsn & $S$ & 1357 & 2 & KLHSSPASPSLASKE & -1.8484 & 0.0441 \\
\hline G3V984 & Bsn & S & 1484 & 3 & PSESPTFSPSKLGPR & -0.5046 & \\
\hline G3V984 & Bsn & $\mathrm{S}$ & 1512 & 1 & PSSDIPRSPGTPSPM & -1.1175 & 0.0101 \\
\hline G3V984 & Bsn & S & 2630 & 1 & RSRLSRHSDSGSDSK & -0.4549 & 0.1325 \\
\hline G3V984 & Bsn & S & 2632 & 1 & RLSRHSDSGSDSKHE & 0.4575 & 0.2069 \\
\hline G3V984 & Bsn & $\mathrm{s}$ & 2636 & 1 & HSDSGSDSKHEASAS & 0.3991 & 0.2241 \\
\hline G3V984 & Bsn & S & 2783 & 2 & VSRQPPKSPQVLYSP & -0.4416 & 0.0561 \\
\hline G3V984 & Bsn & $S$ & 2783 & 3 & VSRQPPKSPQVLYSP & -1.2145 & \\
\hline G3V984 & Bsn & $Y$ & 2788 & 2 & PKSPQVLYSPVSPLS & -0.4829 & \\
\hline G3V984 & Bsn & $Y$ & 2788 & 3 & PKSPQVLYSPVSPLS & -1.2421 & \\
\hline G3V984 & Bsn & S & 2789 & 2 & KSPQVLYSPVSPLSP & -0.5423 & \\
\hline G3V984 & Bsn & $\mathrm{s}$ & 2789 & 3 & KSPQVLYSPVSPLSP & -1.2370 & \\
\hline G3V984 & Bsn & S & 2792 & 2 & QVLYSPVSPLSPHRL & -0.6353 & \\
\hline G3V984 & Bsn & $\mathrm{S}$ & 2792 & 3 & QVLYSPVSPLSPHRL & -1.1586 & \\
\hline G3V984 & Bsn & $S$ & 2795 & 2 & YSPVSPLSPHRLLDT & -0.3497 & 0.0153 \\
\hline G3V984 & Bsn & S & 2795 & 3 & YSPVSPLSPHRLLDT & -1.2543 & \\
\hline G3V984 & Bsn & $\mathrm{S}$ & 2816 & 1 & RLNKAHVSPQKQFIA & -1.2191 & 0.2195 \\
\hline G3V984 & Bsn & $\mathrm{S}$ & 2842 & 1 & PMKTLQRSLSDPKPL & 0.3755 & -0.0443 \\
\hline G3V984 & Bsn & S & 2842 & 2 & PMKTLQRSLSDPKPL & -0.5480 & 0.1939 \\
\hline G3V984 & Bsn & $S$ & 2842 & 3 & PMKTLQRSLSDPKPL & 0.4822 & \\
\hline G3V984 & Bsn & $S$ & 2844 & 1 & KTLQRSLSDPKPLSP & 0.3129 & 0.0211 \\
\hline G3V984 & Bsn & $\mathrm{S}$ & 2844 & 2 & KTLQRSLSDPKPLSP & -0.6985 & 0.2481 \\
\hline G3V984 & Bsn & $\mathrm{s}$ & 2844 & 3 & KTLQRSLSDPKPLSP & 0.4822 & \\
\hline G3V984 & Bsn & $\mathrm{T}$ & 2852 & 2 & DPKPLSPTAEESAKE & -0.5246 & 0.1391 \\
\hline G3V984 & Bsn & $\mathrm{T}$ & 2852 & 3 & DPKPLSPTAEESAKE & 0.4447 & \\
\hline G3V984 & Bsn & S & 3006 & 1 & GEHRDYLSDSELNQL & -0.5627 & -0.1202 \\
\hline G3V984 & Bsn & S & 3445 & 1 & SRVASAYSGEKLSSH & -0.5073 & -0.0163 \\
\hline G3V984 & Bsn & S & 3494 & 2 & RARPPMRSQASEEES & 0.6707 & -0.0001 \\
\hline G3V984 & Bsn & S & 3497 & 1 & PPMRSQASEEESPVS & 0.8959 & -0.1090 \\
\hline G3V984 & Bsn & $\mathrm{S}$ & 3497 & 2 & PPMRSQASEEESPVS & 0.4470 & -0.0378 \\
\hline G3V984 & Bsn & $S$ & 3497 & 3 & PPMRSQASEEESPVS & 0.4998 & \\
\hline G3V984 & Bsn & S & 3501 & 3 & SQASEEESPVSPLGR & 0.4998 & \\
\hline G3V984 & Bsn & $\mathrm{S}$ & 3504 & 1 & SEEESPVSPLGRPRP & -0.5009 & 0.2177 \\
\hline G3V984 & Bsn & $\mathrm{S}$ & 3504 & 3 & SEEESPVSPLGRPRP & 0.4998 & \\
\hline G3V984 & Bsn & S & 3680 & 1 & TRPHPQASPAPAMQK & -0.3838 & 0.2030 \\
\hline G3V984 & Bsn & $\mathrm{S}$ & 3713 & 1 & PSAYHHASDSKKGSR & 0.8589 & -0.2329 \\
\hline G3V984 & Bsn & $S$ & 3715 & 1 & AYHHASDSKKGSRQA & 0.7428 & -0.2368 \\
\hline G3V984 & Bsn & S & 3853 & 1 & PRAEQAGSSKPAAKA & 0.3747 & 0.0188 \\
\hline Q5U2P5 & C2cd2l & $\mathrm{S}$ & 59 & 1 & SAAEPGGSLRELGVW & 0.2643 & 0.0216 \\
\hline D3ZLU0 & $\mathrm{C} 2 \mathrm{~cd} 4 \mathrm{c}$ & $\mathrm{S}$ & 156 & 2 & GFATLAESPHTRRKE & -0.2947 & \\
\hline D3ZLU0 & $\mathrm{C} 2 \mathrm{~cd} 4 \mathrm{c}$ & S & 168 & 2 & RKESLFHSEHGALAQ & -0.8254 & 0.0302 \\
\hline
\end{tabular}




\begin{tabular}{|c|c|c|c|c|c|c|c|}
\hline D3ZLU0 & $\mathrm{C} 2 \mathrm{~cd} 4 \mathrm{c}$ & $S$ & 178 & 2 & GALAQVGSPGASRRR & -0.6879 & 0.0314 \\
\hline D3ZLU0 & $\mathrm{C} 2 \mathrm{~cd} 4 \mathrm{c}$ & S & 206 & 1 & EVGGGLMSPSRYFSG & -0.5540 & \\
\hline D3ZLU0 & $\mathrm{C} 2 \mathrm{~cd} 4 \mathrm{c}$ & S & 262 & 1 & QSVGRHGSLSADDST & 1.5349 & -0.1817 \\
\hline D3ZLU0 & $\mathrm{C} 2 \mathrm{~cd} 4 \mathrm{C}$ & S & 264 & 1 & VGRHGSLSADDSTPD & 0.7152 & -0.1063 \\
\hline D3ZLU0 & $\mathrm{C} 2 \mathrm{~cd} 4 \mathrm{C}$ & S & 273 & 1 & DDSTPDTSPGVRRRL & -0.4055 & -0.0212 \\
\hline D3ZLU0 & $\mathrm{C} 2 \mathrm{~cd} 4 \mathrm{c}$ & $\mathrm{T}$ & 285 & 1 & RRLTRRATPEPGPES & 0.4295 & -0.0938 \\
\hline A0A0G2K543 & C2cd5 & $\mathrm{T}$ & 339 & 1 & KALLRQQTQSALEQR & 0.6985 & -0.0514 \\
\hline A0A0G2JXK1 & Cacna1a & S & 431 & 1 & RRATLKKSKTDLLNP & -0.4349 & \\
\hline A0A0G2JXK1 & Cacna1a & $\mathrm{s}$ & 1051 & 2 & GHSGLPPSPAKIGNS & -0.3845 & 0.2385 \\
\hline A0A0G2JXK1 & Cacna1a & S & 2078 & 1 & SPMKRSASVLGPKAR & 0.3270 & -0.2575 \\
\hline A0A0G2JXK1 & Cacna1a & $S$ & 2214 & 1 & HATHRQGSSSVSGSP & 0.4048 & 0.0451 \\
\hline F1LQ87 & Cacna1b & S & 2128 & 1 & SQERRQPSSSSSEKQ & 1.3201 & 0.0436 \\
\hline F1LQ87 & Cacna1b & S & 2243 & 1 & GRLSRGLSEHNALLQ & 0.7221 & 0.0316 \\
\hline F1LMS1 & Cacna1e & $\mathrm{T}$ & 846 & 1 & KGDIGGLTSVLDNQR & 0.4889 & \\
\hline G3V6K8 & Cacnb1 & $\mathrm{s}$ & 495 & 2 & AGTLRALSRQDTFDA & 0.4415 & \\
\hline G3V6K8 & Cacnb1 & $\mathrm{T}$ & 499 & 2 & RALSRQDTFDADTPG & 0.4734 & \\
\hline G3V6K8 & Cacnb1 & $\mathrm{T}$ & 504 & 2 & QDTFDADTPGSRNSA & 0.4240 & \\
\hline D4A055 & Cacnb4 & $S$ & 443 & 1 & RMRHSNHSTENSPIE & 0.3170 & \\
\hline Q8VHW5 & Cacng8 & S & 314 & 2 & YTLSRDPSKGSVAAG & -0.2903 & \\
\hline Q8VHW5 & Cacng8 & $\mathrm{S}$ & 317 & 2 & SRDPSKGSVAAGLAS & -0.2903 & \\
\hline F1LLX6 & Cadps & $\mathrm{S}$ & 36 & 2 & SGARLSPSRTSEGSA & -0.3829 & 0.0236 \\
\hline F1LLX6 & Cadps & S & 91 & 2 & GGRPSSPSPSVVSEK & -1.3491 & \\
\hline F1LLX6 & Cadps & $S$ & 93 & 2 & RPSSPSPSVVSEKEK & -1.2748 & \\
\hline F1LLX6 & Cadps & $S$ & 375 & 1 & LKRSHNASIIDMGEE & 0.6236 & -0.0708 \\
\hline F1LWT1 & Cadps2 & $\mathrm{T}$ & 731 & 1 & TETMNQATPARKLEE & 0.3195 & \\
\hline F1LZG4 & Camk2a & $\mathrm{T}$ & 331 & 1 & VKESSESTNTTIEDE & 0.2713 & -0.2447 \\
\hline P11275 & Camk2a & $S$ & 279 & 1 & SHRSTVASCMHRQET & 0.3630 & -0.1545 \\
\hline P11275 & Camk2a & $\mathrm{T}$ & 286 & 1 & SCMHRQETVDCLKKF & 0.4834 & -0.1216 \\
\hline F1LNI8 & Camk2b & $\mathrm{T}$ & 287 & 1 & SMMHRQETVECLKKF & 0.5280 & -0.0882 \\
\hline F1LNI8 & Camk2b & $\mathrm{T}$ & 332 & 1 & ADGVKPQTNSTKNSS & 1.0229 & -0.0367 \\
\hline F1LNI8 & Camk2b & S & 334 & 1 & GVKPQTNSTKNSSAI & 0.9768 & -0.0232 \\
\hline F1LNI8 & Camk2b & $\mathrm{s}$ & 339 & 1 & TNSTKNSSAITSPKG & 0.6501 & -0.0160 \\
\hline F1LUE2 & Camk2b & $\mathrm{S}$ & 363 & 1 & TNSTKNSSAITSPKG & 0.6501 & -0.0160 \\
\hline F1LUE2 & Camk2b & $S$ & 371 & 2 & AITSPKGSLPPAALE & 0.8827 & 0.0229 \\
\hline F1LUE2 & Camk2b & $\mathrm{T}$ & 381 & 1 & PAALEPQTTVIHNPV & 0.7402 & -0.0170 \\
\hline F1LUE2 & Camk2b & $\mathrm{T}$ & 381 & 2 & PAALEPQTTVIHNPV & 0.6928 & \\
\hline F1LUE2 & Camk2b & $\mathrm{T}$ & 382 & 1 & AALEPQTTVIHNPVD & 0.6101 & -0.0094 \\
\hline F1LUE2 & Camk2b & $\mathrm{T}$ & 382 & 2 & AALEPQTTVIHNPVD & 1.1483 & 0.0175 \\
\hline F1LUE2 & Camk2b & $\mathrm{T}$ & 400 & 1 & ESSDSTNTTIEDEDA & 0.4735 & \\
\hline F1LUE2 & Camk2b & $\mathrm{T}$ & 401 & 1 & SSDSTNTTIEDEDAK & 0.6492 & \\
\hline P08413 & Camk2b & $\mathrm{T}$ & 400 & 1 & ESSDSTNTTIEDEDA & 0.3922 & -0.1303 \\
\hline P15791 & Camk2d & $\mathrm{T}$ & 287 & 1 & SMMHRQETVDCLKKF & 0.4834 & -0.1216 \\
\hline P15791 & Camk2d & $\mathrm{S}$ & 315 & 2 & MLATRNFSAAKSLLK & 2.4892 & -0.1281 \\
\hline P15791 & Camk2d & S & 319 & 2 & RNFSAAKSLLKKPDG & 2.4892 & -0.1281 \\
\hline
\end{tabular}




\begin{tabular}{|c|c|c|c|c|c|c|c|}
\hline P15791 & Camk2d & $\mathrm{T}$ & 352 & 1 & TPALEPQTTVIHNPD & 0.4576 & \\
\hline P15791 & Camk2d & $\mathrm{T}$ & 353 & 1 & PALEPQTTVIHNPDG & 0.4516 & -0.1081 \\
\hline P11730 & Camk2g & S & 36 & 2 & RRCVKKTSTQEYAAK & 0.6906 & \\
\hline $\mathrm{P} 11730$ & Camk2g & $T$ & 37 & 1 & RCVKKTSTQEYAAKI & 0.7043 & -0.2138 \\
\hline $\mathrm{P} 11730$ & Camk2g & $S$ & 320 & 1 & NFSVGRQSSAPASPA & 0.4161 & 0.1941 \\
\hline P11730 & Camk2g & $\mathrm{s}$ & 325 & 2 & RQSSAPASPAASAAG & 0.2855 & -0.0143 \\
\hline P11730 & Camk2g & $S$ & 355 & 3 & GGVKKRKSSSSVHLM & 0.4245 & 0.0900 \\
\hline $\mathrm{P} 11730$ & Camk2g & $S$ & 358 & 2 & KKRKSSSSVHLMEPQ & 0.2650 & 0.1792 \\
\hline $\mathrm{P} 11730$ & Camk2g & $\mathrm{s}$ & 358 & 3 & KKRKSSSSVHLMEPQ & 0.4319 & 0.1192 \\
\hline P11730 & Camk2g & $T$ & 366 & 3 & VHLMEPQTTVVHNAT & 0.6735 & \\
\hline $\mathrm{P} 11730$ & Camk2g & $\mathrm{T}$ & 367 & 2 & HLMEPQTTVVHNATD & 0.3787 & \\
\hline P11730 & Camk2g & $\mathrm{T}$ & 367 & 3 & HLMEPQTTVVHNATD & 0.6735 & \\
\hline P97756 & Camkk1 & S & 74 & 1 & SLSARKFSLQERPAG & 1.5338 & -0.2187 \\
\hline A0A0G2K5T1 & Camsap1 & $S$ & 619 & 1 & TIMAKRPSEGSQPLV & 0.5449 & -0.0251 \\
\hline D3ZXQ2 & Camsap3 & $\mathrm{s}$ & 572 & 1 & SPKAVASSPAANNSE & -0.4486 & -0.1590 \\
\hline D3ZXQ2 & Camsap3 & S & 829 & 1 & LPHLRKFSPSQVPVQ & -0.4597 & 0.0060 \\
\hline P35565 & Canx & $S$ & 582 & 1 & EDEILNRSPRNRKPR & 0.2647 & -0.0729 \\
\hline Q9WU49 & Carhsp1 & $S$ & 30 & 1 & TPRARDRSPSPLRGN & -0.2699 & \\
\hline Q9WU49 & Carhsp1 & S & 30 & 2 & TPRARDRSPSPLRGN & -0.9148 & 0.1550 \\
\hline Q9WU49 & Carhsp1 & $\mathrm{S}$ & 32 & 2 & RARDRSPSPLRGNVV & -0.9148 & 0.1550 \\
\hline D3ZC15 & Carmil2 & S & 1314 & 1 & RPLRLQRSPVLKRRP & -1.0093 & -0.1249 \\
\hline Q8VHK2 & Caskin1 & S & 388 & 1 & PFAGGDRSGSLSNVA & 0.3151 & \\
\hline Q8VHK2 & Caskin1 & $S$ & 392 & 1 & GDRSGSLSNVAGGRS & 0.3390 & -0.0119 \\
\hline Q8VHK2 & Caskin1 & $S$ & 423 & 1 & ATVLSQKSVSESSPG & 1.6786 & 0.0597 \\
\hline Q8VHK2 & Caskin1 & $\mathrm{S}$ & 698 & 1 & TTSRQESSLSGRARH & 0.8719 & \\
\hline Q8VHK2 & Caskin1 & S & 727 & 1 & PGSPMSRSQEYLLDE & -0.8690 & \\
\hline Q8VHK2 & Caskin1 & $\mathrm{s}$ & 753 & 1 & RSSRHGHSVKRASVP & 1.4594 & -0.2100 \\
\hline Q8VHK2 & Caskin1 & $\mathrm{S}$ & 827 & 1 & SPRSLPQSPTHRGFA & -0.2758 & 0.1248 \\
\hline Q8VHK2 & Caskin1 & $S$ & 883 & 1 & RPKKRAHSLNRYAAS & -1.2060 & 0.0203 \\
\hline Q8VHK2 & Caskin1 & S & 988 & 1 & RAQRRRASDLAGSVD & -0.3619 & -0.0535 \\
\hline F1MAS1 & Cbarp & $S$ & 46 & 1 & ESSQRASSLDTRGSP & 0.5411 & 0.0656 \\
\hline F1MAS1 & Cbarp & $S$ & 245 & 2 & RGAGDEVSELPAPAR & -0.3577 & 0.0579 \\
\hline F1MAS1 & Cbarp & $\mathrm{s}$ & 253 & 2 & ELPAPARSPPRSPRA & -0.5350 & 0.2359 \\
\hline F1MAS1 & Cbarp & $S$ & 257 & 2 & PARSPPRSPRAWPRR & -0.5267 & 0.1857 \\
\hline D4A8V2 & Ccdc177 & $S$ & 299 & 2 & PLTARSFSLGDLSHS & -0.2851 & \\
\hline D4A8V2 & Ccdc177 & $\mathrm{s}$ & 306 & 2 & SLGDLSHSPQTAQHV & -0.2821 & \\
\hline Q498D0 & Ccdc28a & $S$ & 34 & 1 & NTIPASKSTGFSNPA & -0.5972 & -0.1475 \\
\hline Q5BK07 & Ccdc43 & S & 186 & 1 & RDSLRDESQRKKEQD & 0.5968 & \\
\hline D4AEK9 & Ccdc6 & $\mathrm{S}$ & 233 & 2 & EKLDQPVSAPPSPRD & -0.3070 & 0.0611 \\
\hline D4AEK9 & Ccdc6 & $S$ & 237 & 2 & QPVSAPPSPRDISME & -0.3070 & 0.0611 \\
\hline Q9JK72 & Ccs & $S$ & 267 & 1 & AGQGRKDSAQPPAHL & 0.3818 & 0.1564 \\
\hline O35832 & Cdk18 & $\mathrm{s}$ & 51 & 1 & RDEPGQLSPGVQYQQ & -0.3465 & -0.1181 \\
\hline Q91XU8 & Cds2 & $S$ & 59 & 1 & EVLNRALSNLSSRWK & 0.3561 & -0.1886 \\
\hline Q5FVI4 & Cend1 & $T$ & 59 & 1 & PAAPGPATTKKTPAK & 0.3375 & -0.1480 \\
\hline
\end{tabular}




\begin{tabular}{|c|c|c|c|c|c|c|c|}
\hline Q5FVI4 & Cend1 & $\mathrm{T}$ & 60 & 1 & AAPGPATTKKTPAKA & 0.3068 & -0.1635 \\
\hline D4A1G8 & Cep170b & S & 337 & 2 & GPGDDRHSTKSDLPV & -0.3546 & 0.0277 \\
\hline D4A1G8 & Cep170b & S & 340 & 2 & DDRHSTKSDLPVHTR & -0.3547 & 0.0332 \\
\hline D4A1G8 & Cep170b & $S$ & 371 & 1 & DPLAKTASVSGAPTE & 0.2877 & 0.0019 \\
\hline D4A1G8 & Cep170b & $S$ & 843 & 1 & IQLRSGRSPEPDPAP & -0.3516 & \\
\hline D4A1G8 & Cep170b & $\mathrm{s}$ & 1101 & 1 & QVLTRSNSLSTPRPT & 0.2985 & -0.0153 \\
\hline D4A1G8 & Cep170b & $S$ & 1103 & 1 & LTRSNSLSTPRPTRA & 0.5202 & -0.0230 \\
\hline D4A1G8 & Cep170b & $S$ & 1166 & 1 & MPRKRAGSFTGPSDS & 0.3079 & 0.0975 \\
\hline D4A1G8 & Cep170b & $T$ & 1168 & 1 & RKRAGSFTGPSDSET & 0.2993 & -0.0049 \\
\hline O35314 & Chgb & $\mathrm{s}$ & 93 & 1 & LRDPSDASVGRWASS & 0.4304 & \\
\hline E9PSL7 & Cit & $\mathrm{S}$ & 1999 & 1 & TELRRDKSPGRPLER & -0.7265 & 0.1714 \\
\hline A0A0G2JUI5 & Clasp1 & $S$ & 689 & 1 & ANPAGAGSRSSSPGK & 0.5121 & -0.1134 \\
\hline A0A0G2JZM8 & Clasp2 & S & 549 & 1 & KSSGSVASLPQSDRS & 0.3011 & -0.1364 \\
\hline A0A0G2JZM8 & Clasp2 & $S$ & 637 & 2 & AGALNPGSYASLEDT & 0.3416 & 0.0175 \\
\hline A0A0G2JZM8 & Clasp2 & $\mathrm{s}$ & 640 & 2 & LNPGSYASLEDTSDK & 0.3993 & 0.0343 \\
\hline A0A0G2JZM8 & Clasp2 & S & 770 & 2 & REASRESSRDTSPVR & 0.8892 & \\
\hline A0A0G2JZM8 & Clasp2 & $S$ & 1492 & 1 & IKRAQTGSAGADPTT & 0.7901 & -0.1096 \\
\hline A0A0G2JW94 & Clint1 & $S$ & 340 & 1 & LGLPMSRSQNADMVQ & -0.7637 & 0.0095 \\
\hline A0A0G2JZF2 & Clip2 & S & 50 & 1 & SPLHKQASGPSSAGA & 0.7926 & -0.0099 \\
\hline P20272 & Cnr1 & $\mathrm{S}$ & 317 & 1 & IQRGTQKSIIIHTSE & 1.1460 & -0.1828 \\
\hline P20272 & Cnr1 & S & 317 & 2 & IQRGTQKSIIIHTSE & 1.3301 & -0.1186 \\
\hline Q91ZN1 & Coro1a & S & 426 & 1 & PEPSSTLSSDTVSRL & 0.5743 & \\
\hline P80432 & Cox7c & $S$ & 17 & 1 & TTSVVRRSHYEEGPG & 0.4540 & -0.1365 \\
\hline Q62950 & Crmp1 & $S$ & 518 & 2 & KHAAPAPSAKSSPSK & -0.3473 & 0.0923 \\
\hline Q62950 & Crmp1 & $\mathrm{S}$ & 522 & 2 & PAPSAKSSPSKHQPP & -0.4149 & 0.0851 \\
\hline Q62950 & Crmp1 & $\mathrm{S}$ & 540 & 1 & NLHQSNFSLSGAQID & 0.7557 & -0.1175 \\
\hline Q62950 & Crmp1 & $\mathrm{s}$ & 540 & 2 & NLHQSNFSLSGAQID & 0.8291 & -0.1026 \\
\hline Q62950 & Crmp1 & $\mathrm{S}$ & 542 & 1 & HQSNFSLSGAQIDDN & 1.2058 & -0.1888 \\
\hline Q62950 & Crmp1 & $S$ & 542 & 2 & HQSNFSLSGAQIDDN & 0.9129 & -0.0936 \\
\hline Q157S1 & Crtc1 & S & 151 & 1 & TSWRRTNSDSALHQS & -0.7189 & 0.0269 \\
\hline D3ZZZ9 & Ctnnd1 & S & 268 & 1 & PQVRVGGSSVDLHRF & -0.6217 & \\
\hline D3ZZZ9 & Ctnnd1 & $S$ & 269 & 1 & QVRVGGSSVDLHRFH & -0.5609 & \\
\hline F1M787 & Ctnnd2 & $\mathrm{s}$ & 18 & 1 & SEKNSSLSPGLNTSN & -0.2771 & \\
\hline F1M787 & Ctnnd2 & $S$ & 268 & 1 & TKLQRGGSAPEGAAY & 0.3413 & -0.0802 \\
\hline F1M787 & Ctnnd2 & $S$ & 327 & 2 & VQSTISSSPIHQLSS & -0.2682 & \\
\hline F1M787 & Ctnnd2 & $\mathrm{s}$ & 457 & 1 & VPLQRTGSQHGPQNA & 0.3550 & 0.1573 \\
\hline F1M787 & Ctnnd2 & $S$ & 1080 & 1 & SPNNRSASAPASPRE & 0.3608 & \\
\hline D3ZGE6 & Cttn & $\mathrm{T}$ & 401 & 3 & QARAKKQTPPASPSP & -0.9357 & -0.0188 \\
\hline D3ZGE6 & Cttn & $\mathrm{S}$ & 405 & 3 & KKQTPPASPSPQPAE & -0.9635 & 0.0272 \\
\hline D3ZGE6 & Cttn & $S$ & 407 & 3 & QTPPASPSPQPAEDR & -0.8973 & -0.0033 \\
\hline D3ZGE6 & Cttn & $S$ & 417 & 3 & PAEDRPPSSPIYEDA & -0.9579 & 0.0485 \\
\hline D3ZGE6 & Cttn & $Y$ & 421 & 3 & RPPSSPIYEDAAPLK & -0.8682 & 0.0985 \\
\hline A0A0G2JTF2 & Dab2ip & $S$ & 867 & 1 & ELARRQMSLTEKGGQ & 0.9533 & 0.0521 \\
\hline A0A0G2JTF2 & Dab2ip & $\mathrm{s}$ & 967 & 1 & KQGPSPVSPNALDRT & -0.3708 & \\
\hline
\end{tabular}




\begin{tabular}{|c|c|c|c|c|c|c|c|}
\hline AOAOH2UHL9 & Dbn1 & $S$ & 241 & 1 & EHRRKQQSLEAEEAK & 0.5914 & 0.0879 \\
\hline A0AOH2UHL9 & Dbn1 & S & 383 & 1 & PTPIPTRSPSDSSTA & -0.2675 & 0.0271 \\
\hline AOAOH2UHL9 & Dbn1 & S & 383 & 2 & PTPIPTRSPSDSSTA & -0.4051 & \\
\hline AOAOH2UHL9 & Dbn1 & $S$ & 388 & 2 & TRSPSDSSTASTPIT & -0.4484 & \\
\hline AOAOH2UHL9 & Dbn1 & $\mathrm{T}$ & 392 & 2 & SDSSTASTPITEQIE & -0.4365 & \\
\hline Q9JHL4 & Dbnl & $\mathrm{S}$ & 277 & 1 & KQKERAMSTTSVSSS & 1.7864 & -0.0446 \\
\hline A0A1W2Q6Q2 & Dclk1 & S & 347 & 2 & SLRKQRISQHGGSST & -0.3659 & 0.0488 \\
\hline Q9WVP7 & Dclk1 & $S$ & 23 & 2 & STPRSGKSPSPSPTS & -0.4388 & 0.0613 \\
\hline Q9WVP7 & Dclk1 & $\mathrm{T}$ & 29 & 2 & KSPSPSPTSPGSLRK & -0.7406 & 0.0943 \\
\hline Q9WVP7 & Dclk1 & S & 30 & 2 & SPSPSPTSPGSLRKQ & -0.4227 & 0.0183 \\
\hline Q3ZAU5 & Ddhd1 & S & 11 & 1 & PGRGAPRSPERNGRG & -0.5791 & 0.2083 \\
\hline A0A0G2K719 & Ddx3x & S & 606 & 1 & ARDYRQSSGASSSSF & 0.5524 & 0.1227 \\
\hline A0A0G2JY22 & Dip2b & $S$ & 177 & 1 & AESWINRSIQGSSTS & 0.3971 & \\
\hline D3ZBZ6 & Disp2 & $S$ & 6 & 1 & MAPEASPERSCSL & 0.2660 & \\
\hline Q2VUH7 & Dixdc1 & $\mathrm{s}$ & 266 & 1 & NRTDETGSPLSRDWR & 0.3216 & \\
\hline P31016 & Dlg4 & S & 142 & 1 & EALKEAGSIVRLYVM & 0.4889 & -0.1967 \\
\hline P97837 & Dlgap2 & $\mathrm{s}$ & 983 & 1 & NDWKIMESPERKEER & 0.3887 & -0.2288 \\
\hline F1M3D2 & Dmwd & $\mathrm{s}$ & 487 & 1 & RSLSRSNSLPHPAGG & 0.2725 & -0.0063 \\
\hline D4AA13 & Dmxl1 & S & 2375 & 1 & QTTSKALSVEGEKQN & 1.0821 & \\
\hline F1M3W5 & Dmxl2 & $\mathrm{s}$ & 451 & 2 & MKLDHELSLDRESEA & 0.2673 & \\
\hline F1M3W5 & Dmxl2 & $\mathrm{S}$ & 931 & 1 & AKAAEGISSDSLLSV & -0.9400 & \\
\hline F1M3W5 & Dmxl2 & S & 931 & 2 & AKAAEGISSDSLLSV & -0.8829 & -0.1497 \\
\hline F1M3W5 & Dmxl2 & $\mathrm{S}$ & 932 & 2 & KAAEGISSDSLLSVP & -0.7190 & \\
\hline F1M3W5 & Dmxl2 & $\mathrm{s}$ & 934 & 2 & AEGISSDSLLSVPGQ & -0.8811 & -0.1497 \\
\hline F1M3W5 & Dmxl2 & S & 962 & 1 & SSMPHSSSIANLQTA & -1.1219 & -0.0819 \\
\hline F1M3W5 & Dmxl2 & $T$ & 1399 & 2 & TKRHLSRTISVSGST & 1.1677 & 0.0437 \\
\hline F1M3W5 & Dmxl2 & $\mathrm{s}$ & 1401 & 1 & RHLSRTISVSGSTAK & 1.1837 & -0.2097 \\
\hline F1M3W5 & Dmxl2 & $\mathrm{S}$ & 1401 & 2 & RHLSRTISVSGSTAK & 1.0069 & -0.0031 \\
\hline F1M3W5 & Dmxl2 & S & 1403 & 1 & LSRTISVSGSTAKDT & 1.1500 & -0.1927 \\
\hline F1M3W5 & Dmxl2 & S & 1405 & 2 & RTISVSGSTAKDTVT & 1.1854 & 0.0241 \\
\hline F1M3W5 & Dmxl2 & $\mathrm{T}$ & 1406 & 2 & TISVSGSTAKDTVTI & 1.1876 & \\
\hline F1M3W5 & Dmxl2 & $\mathrm{T}$ & 1410 & 2 & SGSTAKDTVTIGKDG & 1.1331 & \\
\hline F1M3W5 & Dmxl2 & $\mathrm{s}$ & 2397 & 1 & VVKPRRQSESIAAPP & 0.4485 & -0.1464 \\
\hline D3ZUU5 & Dnajb1 & $S$ & 16 & 1 & LGLARGASDDEIKRA & 0.2897 & -0.1746 \\
\hline A0A0G2JY26 & Dnajc6 & $S$ & 591 & 2 & TPRRATTSASASPTL & -0.5501 & 0.0367 \\
\hline A0A0G2JY26 & Dnajc6 & $\mathrm{s}$ & 595 & 2 & ATTSASASPTLRVGE & -0.5348 & 0.0227 \\
\hline A0A0G2JY26 & Dnajc6 & $S$ & 679 & 3 & GFGMGSKSAATSPTG & -0.9546 & \\
\hline A0A0G2JY26 & Dnajc6 & $\mathrm{T}$ & 682 & 2 & MGSKSAATSPTGSTH & -0.9566 & 0.1294 \\
\hline A0A0G2JY26 & Dnajc6 & $\mathrm{T}$ & 682 & 3 & MGSKSAATSPTGSTH & -0.9652 & \\
\hline A0A0G2JY26 & Dnajc6 & $S$ & 683 & 3 & GSKSAATSPTGSTHG & -0.9684 & \\
\hline P21575 & Dnm1 & $S$ & 774 & 1 & SVPAGRRSPTSSPTP & -0.8548 & -0.0382 \\
\hline P21575 & Dnm1 & $\mathrm{s}$ & 774 & 2 & SVPAGRRSPTSSPTP & -1.5821 & 0.0683 \\
\hline P21575 & Dnm1 & $\mathrm{T}$ & 776 & 2 & PAGRRSPTSSPTPQR & -1.5917 & 0.0676 \\
\hline P21575 & Dnm1 & $\mathrm{s}$ & 777 & 1 & AGRRSPTSSPTPQRR & 0.8453 & 0.0008 \\
\hline
\end{tabular}




\begin{tabular}{|c|c|c|c|c|c|c|c|}
\hline P21575 & Dnm1 & S & 777 & 2 & AGRRSPTSSPTPQRR & -1.5037 & 0.0691 \\
\hline P21575 & Dnm1 & S & 778 & 1 & GRRSPTSSPTPQRRA & 0.7520 & 0.0013 \\
\hline P21575 & Dnm1 & S & 778 & 2 & GRRSPTSSPTPQRRA & -1.4327 & 0.0340 \\
\hline P21575 & Dnm1 & $\mathrm{T}$ & 780 & 2 & RSPTSSPTPQRRAPA & -1.3000 & -0.1746 \\
\hline P21575 & Dnm1 & $S$ & 851 & 1 & PSRSGQASPSRPESP & -0.9563 & -0.0336 \\
\hline P21575 & Dnm1 & S & 851 & 2 & PSRSGQASPSRPESP & -1.1410 & -0.0158 \\
\hline P21575 & Dnm1 & $S$ & 853 & 2 & RSGQASPSRPESPRP & -1.0425 & -0.0360 \\
\hline P21575 & Dnm1 & $S$ & 857 & 1 & ASPSRPESPRPPFDL & -0.8711 & -0.0096 \\
\hline P21575 & Dnm1 & S & 857 & 2 & ASPSRPESPRPPFDL & -1.2327 & -0.0245 \\
\hline Q08877 & Dnm3 & S & 769 & 1 & WLQHSRRSPPPSPTT & 0.3444 & -0.0584 \\
\hline Q08877 & Dnm3 & $S$ & 769 & 2 & WLQHSRRSPPPSPTT & -0.4608 & -0.0372 \\
\hline Q08877 & Dnm3 & S & 773 & 2 & SRRSPPPSPTTQRRL & -0.4533 & -0.0451 \\
\hline P47942 & Dpysl2 & $\mathrm{T}$ & 514 & 3 & SVTPKTVTPASSAKT & -0.4724 & 0.1720 \\
\hline P47942 & Dpysl2 & S & 517 & 3 & PKTVTPASSAKTSPA & -0.5390 & 0.2232 \\
\hline P47942 & Dpysl2 & S & 518 & 3 & KTVTPASSAKTSPAK & -0.4917 & 0.1921 \\
\hline P47942 & Dpysl2 & S & 540 & 1 & NLHQSGFSLSGAQID & 1.2689 & -0.1140 \\
\hline P47942 & Dpysl2 & $\mathrm{S}$ & 540 & 2 & NLHQSGFSLSGAQID & 1.1650 & -0.0784 \\
\hline P47942 & Dpysl2 & S & 542 & 1 & HQSGFSLSGAQIDDN & 1.1028 & 0.0921 \\
\hline P47942 & Dpysl2 & S & 542 & 2 & HQSGFSLSGAQIDDN & 1.3511 & -0.0789 \\
\hline Q62952 & Dpysl3 & S & 518 & 2 & KGGTPAGSTRGSPTR & -0.4003 & 0.0504 \\
\hline Q62952 & Dpysl3 & $\mathrm{T}$ & 519 & 2 & GGTPAGSTRGSPTRP & -0.4037 & 0.0113 \\
\hline Q62952 & Dpysl3 & S & 522 & 2 & PAGSTRGSPTRPNPP & -0.4384 & 0.0831 \\
\hline Q62952 & Dpysl3 & $S$ & 539 & 1 & NLHQSGFSLSGTQVD & 0.9523 & -0.0892 \\
\hline Q62952 & Dpysl3 & S & 539 & 2 & NLHQSGFSLSGTQVD & 0.8274 & 0.0047 \\
\hline Q62952 & Dpysl3 & $S$ & 541 & 2 & HQSGFSLSGTQVDEG & 0.8577 & 0.0308 \\
\hline Q62951 & Dpys/4 & $\mathrm{S}$ & 532 & 2 & NLHQSGFSLSGSQAD & 0.3386 & -0.1611 \\
\hline Q62951 & Dpysl4 & S & 534 & 1 & HQSGFSLSGSQADDH & 0.4708 & 0.0449 \\
\hline Q62951 & Dpysl4 & $S$ & 536 & 1 & SGFSLSGSQADDHIA & 0.2995 & -0.0264 \\
\hline Q62951 & Dpysl4 & $S$ & 536 & 2 & SGFSLSGSQADDHIA & 0.3386 & -0.1276 \\
\hline Q9JHU0 & Dpysl5 & S & 532 & 1 & MRDLHESSFSLSGSQ & 0.6056 & 0.0632 \\
\hline Q9JHU0 & Dpysl5 & S & 532 & 2 & MRDLHESSFSLSGSQ & 2.2061 & 0.0784 \\
\hline Q9JHU0 & Dpysl5 & $\mathrm{S}$ & 532 & 3 & MRDLHESSFSLSGSQ & 0.9265 & \\
\hline Q9JHU0 & Dpysl5 & S & 534 & 1 & DLHESSFSLSGSQID & 1.1216 & 0.0222 \\
\hline Q9JHU0 & Dpysl5 & S & 534 & 2 & DLHESSFSLSGSQID & 1.9246 & -0.0721 \\
\hline Q9JHU0 & Dpysl5 & $S$ & 534 & 3 & DLHESSFSLSGSQID & 0.9265 & \\
\hline Q9JHU0 & Dpysl5 & $\mathrm{S}$ & 536 & 2 & HESSFSLSGSQIDDH & 2.1442 & 0.2249 \\
\hline Q9JHU0 & Dpysl5 & $S$ & 536 & 3 & HESSFSLSGSQIDDH & 1.2867 & \\
\hline Q9JHU0 & Dpysl5 & S & 538 & 2 & SSFSLSGSQIDDHVP & 1.9344 & 0.0760 \\
\hline Q9JHU0 & Dpysl5 & S & 538 & 3 & SSFSLSGSQIDDHVP & 0.9265 & \\
\hline B0K014 & Dtd1 & $S$ & 196 & 1 & RKEDRSASSGAEGDV & 0.2855 & \\
\hline Q9QXU8 & Dync1li1 & $\mathrm{T}$ & 512 & 2 & KPASVSPTTPPSPTE & -0.3507 & 0.1281 \\
\hline G3V7D4 & Efnb3 & S & 268 & 1 & SRHPGPGSFGRGGSL & 0.2958 & -0.0672 \\
\hline F1LVX2 & Ehbp1 & $S$ & 310 & 1 & TFVMIKDSPPQSTRR & 1.1739 & \\
\hline F1LVX2 & Ehbp1 & $S$ & 1009 & 1 & PEMKRQRSIQEDTER & 0.7812 & 0.0782 \\
\hline
\end{tabular}




\begin{tabular}{|c|c|c|c|c|c|c|c|}
\hline Q6AYK8 & Eif3d & S & 161 & 1 & SQKPRDSSVEVRSDW & 0.4008 & \\
\hline Q5RKG9 & Eif4b & S & 340 & 1 & KLNLKPRSTPKEDDS & 2.1244 & 0.2214 \\
\hline Q5RKG9 & Eif4b & S & 495 & 2 & SSNPPARSQSSDTEQ & 0.6665 & 0.0021 \\
\hline Q5RKG9 & Eif4b & $\mathrm{T}$ & 500 & 2 & ARSQSSDTEQPSPTS & 0.4319 & 0.0922 \\
\hline Q5RKG9 & Eif4b & S & 504 & 1 & SSDTEQPSPTSGGGK & -0.4848 & 0.1802 \\
\hline A0A0G2JY73 & Eif4g3 & $\mathrm{s}$ & 1186 & 1 & NTFLRGSSKDLLDNQ & 0.9698 & 0.2274 \\
\hline P60841 & Ensa & $S$ & 2 & 1 & MSQKQEEEN & 0.7105 & \\
\hline P60841 & Ensa & $S$ & 67 & 1 & KGQKYFDSGDYNMAK & 0.6643 & \\
\hline A0A0G2K0F3 & Epb4111 & $\mathrm{s}$ & 540 & 1 & ERERRLPSSPASPSP & -0.4341 & -0.0865 \\
\hline AOA0G2K0F3 & Epb4111 & $\mathrm{S}$ & 540 & 2 & ERERRLPSSPASPSP & -0.4486 & 0.0618 \\
\hline A0A0G2K0F3 & Epb4111 & S & 541 & 2 & RERRLPSSPASPSPK & -0.3278 & 0.2383 \\
\hline A0A0G2K0F3 & Epb4111 & $\mathrm{T}$ & 1252 & 1 & PRAPESDTGDEDQDQ & 0.4163 & -0.1111 \\
\hline AOAOG2K0F3 & Epb4111 & $\mathrm{s}$ & 1311 & 2 & AFEDFSRSLPELDRD & -0.3508 & 0.1155 \\
\hline AOA0G2K0F3 & Epb4111 & $T$ & 1394 & 1 & MLQSRVSTADSTQVD & 0.3181 & \\
\hline Q9WTP0 & Epb4111 & $\mathrm{S}$ & 540 & 1 & ERERRLPSSPASPSP & -0.4341 & -0.0865 \\
\hline Q9WTP0 & Epb4111 & $\mathrm{S}$ & 540 & 2 & ERERRLPSSPASPSP & -0.4486 & 0.0618 \\
\hline Q9WTP0 & Epb4111 & $\mathrm{s}$ & 541 & 2 & RERRLPSSPASPSPK & -0.3278 & 0.2256 \\
\hline D3ZM69 & Epb4112 & $S$ & 543 & 2 & RASSKRVSRSLDGAP & -0.3329 & \\
\hline D3ZM69 & Epb4112 & $\mathrm{S}$ & 545 & 2 & SSKRVSRSLDGAPIG & -0.3821 & \\
\hline G3V874 & Epb4113 & S & 463 & 1 & YATTKVISQTNLITT & -0.3907 & -0.0745 \\
\hline G3V874 & Epb4113 & $\mathrm{T}$ & 465 & 1 & TTKVISQTNLITTVT & -0.3476 & -0.0238 \\
\hline A0A0G2JYT1 & Erc1 & S & 17 & 1 & KVEPSSQSPGRSPRL & 0.3483 & 0.1966 \\
\hline A0A0G2JYT1 & Erc1 & $\mathrm{s}$ & 17 & 2 & KVEPSSQSPGRSPRL & -0.5493 & \\
\hline A0A0G2JYT1 & Erc1 & $S$ & 21 & 2 & SSQSPGRSPRLPRSP & -0.5493 & \\
\hline A0A0G2JYT1 & Erc1 & $\mathrm{S}$ & 37 & 1 & LGHRRTNSTGGSSGN & 0.6165 & -0.1003 \\
\hline A0A0G2JYT1 & Erc1 & $\mathrm{s}$ & 45 & 2 & TGGSSGNSVGGGSGK & 1.3096 & -0.0558 \\
\hline Q8K3M6 & Erc2 & $\mathrm{s}$ & 14 & 1 & TISNPEGSPSRSPRL & 0.3488 & -0.0778 \\
\hline Q8K3M6 & Erc2 & $\mathrm{S}$ & 18 & 1 & PEGSPSRSPRLPRSP & -0.7571 & -0.0054 \\
\hline Q8K3M6 & Erc2 & S & 321 & 1 & SKGLPSKSLEDDNER & 0.7321 & 0.0318 \\
\hline MOR5H1 & Et|4 & $\mathrm{T}$ & 59 & 1 & RNIPRRHTLGGPRSS & 0.4638 & -0.2082 \\
\hline MOR5H1 & Et/4 & $\mathrm{S}$ & 1088 & 1 & SGLTTTRSGDVIYTG & 0.5970 & \\
\hline MOR5H1 & Etl4 & $S$ & 1775 & 1 & TSKNRPGSLDKASKP & 1.9110 & 0.1575 \\
\hline 008719 & Evl & $\mathrm{s}$ & 335 & 1 & KPWERSNSVEKPVSS & 0.9272 & 0.2171 \\
\hline 008719 & Evl & $\mathrm{T}$ & 347 & 2 & VSSLLSRTPSVAKSP & 0.6636 & \\
\hline 008719 & Evl & $S$ & 353 & 2 & RTPSVAKSPEAKSPL & 0.8704 & \\
\hline A0A0G2JYT4 & Exoc1 & $\mathrm{s}$ & 470 & 2 & KLTGSTSSLNKLSVQ & -0.4472 & 0.0102 \\
\hline O54924 & Exoc8 & $\mathrm{S}$ & 28 & 1 & RLYVKQLSQQSDGDR & 0.3452 & \\
\hline Q4V7D4 & Fam126b & $\mathrm{S}$ & 321 & 1 & RTAITTASIRRHRWR & 0.5262 & -0.1123 \\
\hline Q4V7D4 & Fam126b & S & 402 & 1 & SNESPRDSVVGKQFL & 0.9025 & \\
\hline F8WFH6 & Fam131b & S & 75 & 1 & LPKLKRNSNAYGIGA & 0.3855 & -0.1059 \\
\hline F8WFH6 & Fam131b & $S$ & 130 & 1 & PAIQPQHSHEAVRRD & 0.3407 & 0.0422 \\
\hline F8WFH6 & Fam131b & $T$ & 344 & 1 & SRKVSDVTSSGVQSF & -0.3788 & \\
\hline F1LZ91 & Fam135b & $\mathrm{s}$ & 539 & 1 & DVDICRRSPGPEEGH & -0.2999 & \\
\hline D3ZTG3 & Fam171b & s & 346 & 2 & TTHINHISSVKVALK & 0.3446 & -0.0647 \\
\hline
\end{tabular}




\begin{tabular}{|c|c|c|c|c|c|c|c|}
\hline A0A0G2JTS9 & Fbxo42 & $S$ & 365 & 2 & PSGRAPLSPSLNSRP & -0.5242 & 0.0402 \\
\hline A0A0G2JTS9 & Fbxo42 & $S$ & 373 & 2 & PSLNSRPSPISATPP & -0.5242 & \\
\hline A0A0G2JTS9 & Fbxo42 & $S$ & 488 & 1 & SLVPRRGSLPDQKDL & 0.3052 & 0.0323 \\
\hline D3ZYR1 & Fcho2 & $S$ & 403 & 1 & VQMNRNSSNEELTKS & -0.4226 & \\
\hline F1LQJ2 & Fhod3 & $S$ & 367 & 1 & RRRSRRHSIQNIKSP & 0.6106 & -0.0418 \\
\hline Q5RKI5 & Flii & $S$ & 436 & 1 & RLRRRKDSAQDVQAK & 0.4074 & 0.1476 \\
\hline A0A0G2K749 & Fry & $S$ & 1876 & 1 & EERQLSRSTPSLNKM & -0.4531 & 0.0293 \\
\hline P18088 & Gad1 & $S$ & 55 & 1 & GFLQRTNSLEEKSRL & 1.5949 & -0.1172 \\
\hline P07936 & Gap43 & $S$ & 103 & 1 & SPKAEEPSKAGDAPS & 0.6367 & -0.1604 \\
\hline P07936 & Gap43 & $S$ & 122 & 1 & GEGDAAPSEEKAGSA & 0.4135 & -0.2050 \\
\hline P07936 & Gap43 & $S$ & 209 & 1 & DEAKPKESARQDEGK & 0.7132 & \\
\hline A0A0G2JT50 & Gas7 & $S$ & 99 & 1 & AHMSLRKSTGDSQNL & -0.5348 & 0.0439 \\
\hline Q9Z254 & Gipc1 & $S$ & 225 & 1 & FDMISQRSSGGHPGS & 0.3188 & -0.0482 \\
\hline Q9Z272 & Git1 & $S$ & 419 & 2 & TRNNRARSMDSSDLS & 0.5820 & 0.0628 \\
\hline Q9Z272 & Git1 & $S$ & 422 & 2 & NRARSMDSSDLSDGA & 0.6005 & \\
\hline Q9Z272 & Git1 & $S$ & 507 & 1 & TLMGPGGSTHRRDRQ & 0.2789 & 0.0946 \\
\hline Q9Z272 & Git1 & $S$ & 601 & 1 & SKLSRHGSGAESDYE & 0.6731 & -0.1728 \\
\hline Q9Z272 & Git1 & $S$ & 601 & 2 & SKLSRHGSGAESDYE & 0.2999 & -0.0849 \\
\hline Q9Z272 & Git1 & $S$ & 605 & 1 & RHGSGAESDYENTQS & 0.6262 & -0.1830 \\
\hline P08050 & Gja1 & $S$ & 325 & 2 & NRMGQAGSTISNSHA & -0.3637 & \\
\hline P08050 & Gja1 & $S$ & 368 & 2 & QRPSSRASSRASSRP & -0.4713 & \\
\hline P08050 & Gja1 & $S$ & 369 & 2 & RPSSRASSRASSRPR & -0.4873 & \\
\hline G3V8K2 & Gng3 & $\mathrm{T}$ & 10 & 1 & GETPVNSTMSIGQAR & 0.3304 & -0.1404 \\
\hline G3V8K2 & Gng3 & $S$ & 12 & 1 & TPVNSTMSIGQARKM & 0.4048 & -0.1214 \\
\hline Q6MG06 & Gnl1 & $S$ & 68 & 1 & RRLNQQPSQGLGPRG & 0.4925 & 0.0814 \\
\hline Q03555 & Gphn & $S$ & 337 & 1 & NILRASHSAVDITKV & -0.3981 & 0.1505 \\
\hline A0A0A0MY13 & Gpr158 & $S$ & 881 & 2 & PLVCKSASAHNLSSE & -0.4135 & -0.1182 \\
\hline A0A0A0MY13 & Gpr158 & $S$ & 886 & 2 & SASAHNLSSEKKPGH & -0.5357 & -0.0611 \\
\hline A0A0A0MY13 & Gpr158 & $S$ & 887 & 2 & ASAHNLSSEKKPGHP & -0.4445 & -0.2382 \\
\hline A0A0A0MY13 & Gpr158 & $S$ & 902 & 1 & RTSMLQKSLSVIASA & 0.5217 & -0.2165 \\
\hline D3ZF21 & Gprin3 & $S$ & 570 & 2 & GSEGPEVSPAPSPGR & -1.0652 & \\
\hline D3ZF21 & Gprin3 & $S$ & 574 & 2 & PEVSPAPSPGRKSTV & -1.0652 & \\
\hline P97879 & Grip1 & $S$ & 937 & 1 & SQLGRQASFQERSNS & 0.9979 & 0.1220 \\
\hline P97879 & Grip1 & $\mathrm{T}$ & 956 & 1 & SQTTRSNTLPSDVGR & 0.2678 & -0.0551 \\
\hline P26817 & Grk2 & $S$ & 487 & 1 & ADAFDIGSFDEEDTK & 0.6897 & -0.1126 \\
\hline P26817 & Grk2 & $S$ & 670 & 1 & KMKNKPRSPVVELSK & -0.4126 & -0.0658 \\
\hline A0A0G2JV93 & Grm3 & $S$ & 875 & 1 & QPTPQHHSTKRQGIC & 0.4652 & \\
\hline A0A0G2QC41 & Hdac6 & $S$ & 909 & 1 & ALLAQGQSSEQAAKG & 1.5552 & 0.0445 \\
\hline D4ADD3 & Hecw2 & $S$ & 1046 & 1 & QHLTRQRSHSAGEVG & 2.0798 & \\
\hline D4ADD3 & Hecw2 & $S$ & 1048 & 1 & LTRQRSHSAGEVGED & -0.5176 & \\
\hline D4ADD3 & Hecw2 & $S$ & 1181 & 1 & PVSSPQNSPGTQRAN & -0.4713 & 0.0664 \\
\hline P82995 & Hsp90aa1 & $S$ & 454 & 1 & KLGIHEDSQNRKKLS & 0.2657 & -0.1694 \\
\hline Q9WVR1 & Inpp5e & S & 235 & 1 & NRLVRAHSNLGPSRP & 0.6923 & \\
\hline D3ZKG7 & Inpp5f & $S$ & 905 & 2 & APRLGSRSQSASSTD & -0.7695 & \\
\hline
\end{tabular}




\begin{tabular}{|c|c|c|c|c|c|c|c|}
\hline D3ZKG7 & Inpp5f & S & 935 & 2 & ELGKGLESPLKKSPS & -0.8745 & 0.1800 \\
\hline D3ZKG7 & Inpp5f & S & 935 & 3 & ELGKGLESPLKKSPS & -0.4006 & \\
\hline D3ZKG7 & Inpp5f & S & 940 & 2 & LESPLKKSPSADNIH & -0.8348 & 0.1672 \\
\hline D3ZKG7 & Inpp5f & $S$ & 940 & 3 & LESPLKKSPSADNIH & -0.3992 & \\
\hline D3ZKG7 & Inpp5f & $S$ & 942 & 3 & SPLKKSPSADNIHTL & -0.3983 & \\
\hline D3ZKG7 & Inpp5f & $\mathrm{T}$ & 948 & 2 & PSADNIHTLTGFAKP & -0.8675 & 0.1806 \\
\hline D3ZKG7 & Inpp5f & $\mathrm{T}$ & 948 & 3 & PSADNIHTLTGFAKP & -0.4285 & \\
\hline Q9JMC1 & Inpp5j & $S$ & 69 & 1 & VGPRAAVSPPSERPR & -0.2664 & \\
\hline Q9JMC1 & Inpp5j & S & 320 & 2 & LPESGTRSPGLLSPT & -0.4294 & -0.0052 \\
\hline Q9JMC1 & Inpp5j & S & 325 & 2 & TRSPGLLSPTFRPGI & -0.4152 & \\
\hline Q9JMC1 & Inpp5j & S & 881 & 2 & GKSKRHRSRSPGLAR & -1.5195 & -0.0308 \\
\hline Q9JMC1 & Inpp5j & S & 883 & 2 & SKRHRSRSPGLARFP & -1.5195 & -0.0308 \\
\hline Q9JMC1 & Inpp5j & S & 909 & 1 & RGGSRSPSPQSRQLP & 1.3746 & 0.1394 \\
\hline A0A0G2JUG7 & Iqsec1 & $S$ & 87 & 2 & RRPRLQHSTSVLRKQ & 0.5414 & \\
\hline A0A0G2JUG7 & Iqsec1 & $\mathrm{T}$ & 88 & 1 & RPRLQHSTSVLRKQA & 0.4184 & 0.0725 \\
\hline A0A0G2JUG7 & Iqsec1 & $\mathrm{T}$ & 88 & 2 & RPRLQHSTSVLRKQA & 0.5414 & \\
\hline A0A0G2JUG7 & Iqsec1 & $\mathrm{S}$ & 103 & 2 & EEEAIKRSRSLSESY & 0.4101 & -0.0594 \\
\hline A0A0G2JUG7 & Iqsec1 & S & 105 & 1 & EAIKRSRSLSESYEL & 0.5338 & -0.1523 \\
\hline A0A0G2JUG7 & Iqsec1 & S & 105 & 2 & EAIKRSRSLSESYEL & 0.3977 & -0.0289 \\
\hline A0A0G2JUG7 & Iqsec1 & S & 109 & 2 & RSRSLSESYELSSDL & 0.4203 & 0.0033 \\
\hline A0A0G2JUG7 & Iqsec1 & $Y$ & 110 & 2 & SRSLSESYELSSDLQ & 0.3559 & \\
\hline A0A0G2JUG7 & Iqsec1 & S & 180 & 1 & SNMRMQFSFEGPEKV & 0.4728 & -0.1761 \\
\hline A0A0G2JUG7 & Iqsec1 & $S$ & 363 & 1 & TSCRSTPSLERPEPR & 0.4156 & 0.1319 \\
\hline A0A0G2JUG7 & Iqsec1 & S & 404 & 1 & RSSLKRQSAYERSLG & -0.6598 & -0.0497 \\
\hline A0A0G2JZX5 & Iqsec2 & $S$ & 168 & 1 & NFERLRSSASESRMS & 0.4132 & -0.0383 \\
\hline A0A0G2JZW3 & Itfg2 & $\mathrm{S}$ & 219 & 1 & TWNKDTGSPPASEEA & 0.3066 & \\
\hline P17105 & Itpka & S & 135 & 2 & SSSLLEDSEDDLLSD & -0.3497 & \\
\hline P17105 & Itpka & $S$ & 141 & 2 & DSEDDLLSDSESRSR & -0.4039 & \\
\hline F1LNT1 & Itpr1 & $S$ & 1613 & 1 & RNAARRDSVLAASRD & 0.3309 & -0.0059 \\
\hline Q9WVE9 & Itsn1 & S & 623 & 1 & QQLQKQRSIEAERLK & 0.4858 & \\
\hline Q9WVE9 & Itsn1 & S & 896 & 1 & TATGSSPSPVLGQGE & -0.3371 & \\
\hline Q9WVE9 & Itsn1 & $S$ & 970 & 1 & GPVRKSTSIDTGPTE & 0.5956 & -0.0420 \\
\hline Q9WVE9 & Itsn1 & S & 1129 & 2 & ANYVKLLSPGTSKIT & -0.4416 & -0.0088 \\
\hline Q9WVE9 & Itsn1 & $\mathrm{T}$ & 1132 & 2 & VKLLSPGTSKITPTE & -0.4362 & -0.0202 \\
\hline Q9WVE9 & Itsn1 & $\mathrm{T}$ & 1136 & 2 & SPGTSKITPTELPKT & -0.4624 & -0.0130 \\
\hline Q6AXU6 & JPT1 & $\mathrm{S}$ & 17 & 1 & VDPNSRNSSRVLRPP & 0.4275 & 0.1108 \\
\hline Q6AXU6 & JPT1 & $S$ & 65 & 1 & NPPSWAKSAGGREDS & 0.9287 & \\
\hline Q6AXU6 & JPT1 & S & 72 & 1 & SAGGREDSESPGTQR & 1.0504 & \\
\hline Q811T3 & Kcnc3 & $\mathrm{T}$ & 750 & 2 & LRLAPLATPPGSPRA & -0.2634 & \\
\hline Q811T3 & Kcnc3 & $S$ & 754 & 2 & PLATPPGSPRATRRA & -0.2634 & \\
\hline Q63472 & Kcnh1 & S & 872 & 2 & DNVGEARSPQDRSPI & -0.5987 & -0.0099 \\
\hline Q63472 & Kcnh1 & S & 877 & 2 & ARSPQDRSPILAEVK & -0.5987 & -0.0099 \\
\hline F1LNC7 & Kcnma1 & $S$ & 1195 & 1 & NAGQSRASLSHSSHS & -0.6826 & -0.0030 \\
\hline F1LNC7 & Kcnma1 & S & 1202 & 1 & SLSHSSHSSQSSSKK & 0.5978 & 0.1187 \\
\hline
\end{tabular}




\begin{tabular}{|c|c|c|c|c|c|c|c|}
\hline F1LNC7 & Kcnma1 & S & 1203 & 1 & LSHSSHSSQSSSKKS & 0.4688 & 0.0637 \\
\hline F1LNC7 & Kcnma1 & S & 1203 & 2 & LSHSSHSSQSSSKKS & -0.3771 & \\
\hline F1LNC7 & Kcnma1 & S & 1205 & 1 & HSSHSSQSSSKKSSS & 0.5777 & -0.0135 \\
\hline F1LNC7 & Kcnma1 & $S$ & 1207 & 1 & SHSSQSSSKKSSSVH & 0.7169 & -0.2007 \\
\hline F1LSD6 & Kcnq5 & $S$ & 325 & 1 & SIKSRQASVGDRRSP & 0.5278 & \\
\hline F1LSD6 & Kcnq5 & $\mathrm{S}$ & 566 & 1 & GCLSRSASANISRGL & -1.1393 & \\
\hline $\mathrm{D} 4 \mathrm{~A} 0 \times 3$ & Kiaa1107 & S & 218 & 1 & ERLASTGSVDETKEN & 0.4579 & 0.2013 \\
\hline $\mathrm{D} 4 \mathrm{~A} 0 \times 3$ & Kiaa1107 & $S$ & 218 & 2 & ERLASTGSVDETKEN & 0.5732 & \\
\hline $\mathrm{D} 4 \mathrm{~A} 0 \times 3$ & Kiaa1107 & $\mathrm{T}$ & 222 & 2 & STGSVDETKENGSVE & 0.5796 & \\
\hline $\mathrm{D} 4 \mathrm{~A} 0 \times 3$ & Kiaa1107 & S & 336 & 1 & TLAQTQGSQGESPHS & 0.4046 & 0.2514 \\
\hline F1M4A4 & Kif1a & $\mathrm{s}$ & 425 & 2 & ALSSRAASVSSLHER & 0.2692 & \\
\hline F1M4A4 & Kif1a & $S$ & 427 & 2 & SSRAASVSSLHERIL & 0.2833 & \\
\hline F1M4A4 & Kif1a & $S$ & 428 & 2 & SRAASVSSLHERILF & 0.2754 & \\
\hline D3ZCG2 & Kif21a & S & 1227 & 1 & EKKVPEPSPVTRRKA & -0.5134 & -0.0754 \\
\hline G3V6L4 & Kif5c & $\mathrm{s}$ & 918 & 1 & NMARRAHSAQIAKPI & 0.9338 & -0.1909 \\
\hline B2GV74 & Klc2 & S & 582 & 2 & SRMKRASSLNFLNKS & 0.7248 & 0.1003 \\
\hline Q5PQM2 & Klc4 & $\mathrm{s}$ & 565 & 1 & IRDVLRRSSELLVRK & -0.4273 & 0.0318 \\
\hline F1LY04 & Ksr2 & $\mathrm{s}$ & 313 & 1 & TALHRSKSHEFQLGN & -0.5562 & -0.0298 \\
\hline D3ZGM3 & LOC100909750 & S & 909 & 1 & KAGKPAQSPSQDVAG & -0.4034 & 0.0039 \\
\hline D3ZGM3 & LOC100909750 & $\mathrm{s}$ & 911 & 1 & GKPAQSPSQDVAGEA & -0.4092 & 0.0325 \\
\hline Q9Z252 & Lin7b & $\mathrm{S}$ & 201 & 1 & RQQHHSYSSLESRG & 0.6229 & -0.0449 \\
\hline D3ZBH5 & Lmtk2 & S & 576 & 2 & AEDSHAASIPGSPFN & -0.4634 & \\
\hline D3ZBH5 & Lmtk2 & $\mathrm{S}$ & 580 & 2 & HAASIPGSPFNIFSD & -0.4634 & \\
\hline F1LT49 & Lrrc47 & $\mathrm{s}$ & 429 & 1 & RKQKKRQSVSGLHRY & 0.2906 & -0.1208 \\
\hline Q4V7E8 & Lrrfip2 & S & 125 & 1 & ASATTPLSGNSSRRG & 0.4261 & 0.1845 \\
\hline Q5HZA4 & Lysmd1 & $\mathrm{s}$ & 194 & 1 & DTGLYPSSPRMQQRA & -0.4872 & -0.1054 \\
\hline 008873 & Madd & $\mathrm{s}$ & 708 & 1 & SSTTASSSPSTIVHG & -0.3223 & -0.0596 \\
\hline 008873 & Madd & $\mathrm{S}$ & 812 & 1 & QKLLRPNSLKLASDS & 0.7722 & -0.0574 \\
\hline 008873 & Madd & S & 900 & 1 & KSSVIKHSPTVKREP & -0.4991 & -0.1397 \\
\hline O08873 & Madd & S & 1224 & 2 & KPKEKPASSPVRSSE & -0.3080 & \\
\hline 008873 & Madd & S & 1225 & 2 & PKEKPASSPVRSSED & -0.3132 & -0.2056 \\
\hline 008873 & Madd & S & 1229 & 2 & PASSPVRSSEDVSQR & -0.3586 & -0.2083 \\
\hline MoR8T1 & Magi1 & $\mathrm{s}$ & 644 & 1 & QPLERKDSQNSSQHS & 1.2018 & 0.1383 \\
\hline P34926 & Map1a & $S$ & 2204 & 2 & SSLPQLPSPSSPGGP & -0.6192 & 0.0517 \\
\hline P34926 & Map1a & $S$ & 2206 & 2 & LPQLPSPSSPGGPLL & -0.6310 & 0.0409 \\
\hline P34926 & Map1a & $\mathrm{s}$ & 2207 & 2 & PQLPSPSSPGGPLLS & -0.4927 & \\
\hline P15205 & Map1b & $\mathrm{s}$ & 601 & 1 & GKVESKPSVTEKEVP & 1.0930 & -0.2449 \\
\hline P15205 & Map1b & $\mathrm{T}$ & 1262 & 2 & PPSPIEKTPLGERSV & -1.5088 & \\
\hline P15205 & Map1b & $\mathrm{T}$ & 1262 & 3 & PPSPIEKTPLGERSV & -0.9297 & 0.1316 \\
\hline P15205 & Map1b & $S$ & 1371 & 2 & ENERSSISPMDEPVP & -0.2671 & 0.1807 \\
\hline P15205 & Map1b & $S$ & 1380 & 2 & MDEPVPDSESPIEKV & -0.3210 & 0.1043 \\
\hline P15205 & Map1b & $\mathrm{s}$ & 1382 & 2 & EPVPDSESPIEKVLS & -0.2677 & 0.1361 \\
\hline P15205 & Map1b & $S$ & 1389 & 2 & SPIEKVLSPLRSPPL & -0.4273 & 0.0860 \\
\hline P15205 & Map1b & $\mathrm{s}$ & 1389 & 3 & SPIEKVLSPLRSPPL & -0.8947 & \\
\hline
\end{tabular}




\begin{tabular}{|c|c|c|c|c|c|c|c|}
\hline P15205 & Map1b & S & 1393 & 2 & KVLSPLRSPPLIGSE & -0.3423 & 0.0284 \\
\hline P15205 & Map1b & S & 1393 & 3 & KVLSPLRSPPLIGSE & -0.9990 & \\
\hline P15205 & Map1b & S & 1401 & 3 & PPLIGSESAYEDFLS & -1.0091 & \\
\hline P15205 & Map1b & $Y$ & 1403 & 2 & LIGSESAYEDFLSAD & -0.4042 & 0.0187 \\
\hline P15205 & Map1b & $S$ & 1408 & 2 & SAYEDFLSADDKALG & -0.4335 & \\
\hline P15205 & Map1b & $\mathrm{S}$ & 1418 & 1 & DKALGRRSESPFEGK & -0.5421 & 0.0910 \\
\hline P15205 & Map1b & S & 1420 & 1 & ALGRRSESPFEGKNG & -0.7258 & -0.1655 \\
\hline P15205 & Map1b & $S$ & 1494 & 2 & RKLGGDGSPTQVDVS & -0.3031 & 0.2281 \\
\hline P15205 & Map1b & $\mathrm{s}$ & 1505 & 2 & VDVSQFGSFKEDTKM & -0.3130 & 0.2266 \\
\hline P15205 & Map1b & $\mathrm{T}$ & 1626 & 1 & PKTAKSRTPVQDHRS & -0.9447 & 0.2229 \\
\hline P15205 & Map1b & $S$ & 1908 & 1 & KTERTIKSPCDSGYS & -0.3411 & 0.0121 \\
\hline P0C5W1 & Map1s & $S$ & 683 & 1 & RKPPPPASPGSSDSS & -0.5918 & 0.1209 \\
\hline F1MAQ5 & Map2 & $S$ & 685 & 1 & DDLTLSRSLGLGGRS & -0.3638 & \\
\hline F1MAQ5 & Map2 & $\mathrm{T}$ & 749 & 3 & DDYLPPTTPAVEKIP & -0.5705 & \\
\hline F1MAQ5 & Map2 & $\mathrm{s}$ & 892 & 2 & SENLSGESGSFYEGT & -0.3513 & \\
\hline F1MAQ5 & Map2 & S & 1740 & 1 & KAQAKVGSLDNAHHV & 0.6089 & 0.1765 \\
\hline F1LP57 & Map2k4 & $\mathrm{T}$ & 51 & 1 & PHIERLRTHSIESSG & 1.5600 & -0.0711 \\
\hline F1LP57 & Map2k4 & $\mathrm{s}$ & 53 & 1 & IERLRTHSIESSGKL & 1.5446 & 0.0113 \\
\hline Q4KSH7 & Map2k7 & S & 61 & 1 & RSPSSESSPQHPTPP & 0.6976 & 0.2135 \\
\hline D3ZG83 & Map3k10 & $\mathrm{s}$ & 714 & 1 & GRFPRGLSPTGRPGG & -0.4770 & \\
\hline $\mathrm{D} 3 \mathrm{ZZH} 6$ & Map3k5 & $\mathrm{S}$ & 1035 & 2 & DENFEDHSAPPSPEE & -0.7522 & \\
\hline $\mathrm{D} 3 Z Z \mathrm{H} 6$ & Map3k5 & S & 1039 & 2 & EDHSAPPSPEEKDSG & -0.7522 & \\
\hline A0A0G2JW88 & Map4 & $S$ & 2123 & 1 & KAQAKVGSLDNVGHL & 0.8840 & -0.2160 \\
\hline P63086 & Mapk1 & $\mathrm{T}$ & 179 & 2 & ADPDHDHTGFLTEYV & 1.0856 & \\
\hline P63086 & Mapk1 & $\mathrm{T}$ & 183 & 2 & HDHTGFLTEYVATRW & 1.2139 & \\
\hline P63086 & Mapk1 & $\mathrm{Y}$ & 185 & 2 & HTGFLTEYVATRWYR & 1.2236 & \\
\hline P21708 & Mapk3 & $\mathrm{T}$ & 203 & 2 & HDHTGFLTEYVATRW & 0.6364 & -0.1289 \\
\hline P21708 & Mapk3 & $\mathrm{Y}$ & 205 & 2 & HTGFLTEYVATRWYR & 0.6364 & -0.1289 \\
\hline E9PSK7 & Mapk8ip3 & $\mathrm{T}$ & 287 & 2 & SVPSAAVTPLNESLQ & -0.3905 & \\
\hline E9PSK7 & Mapk8ip3 & S & 544 & 1 & WTEMIRASREHPSVQ & 1.0121 & \\
\hline E9PSK7 & Mapk8ip3 & $S$ & 740 & 1 & EPKSTHPSPEKKKAK & 0.3152 & -0.1555 \\
\hline D4A1Q2 & Mapt & $\mathrm{T}$ & 421 & 2 & TRIPAKTTPSPKTPP & -0.3117 & 0.2430 \\
\hline D4A1Q2 & Mapt & $\mathrm{s}$ & 423 & 2 & IPAKTTPSPKTPPGS & -0.3336 & 0.2398 \\
\hline D4A1Q2 & Mapt & $S$ & 430 & 2 & SPKTPPGSGEPPKSG & -0.3070 & 0.1898 \\
\hline D4A1Q2 & Mapt & $S$ & 550 & 1 & KHVPGGGSVQIVYKP & 0.5125 & -0.1126 \\
\hline D4A1Q2 & Mapt & $\mathrm{s}$ & 597 & 2 & DFKDRVQSKIGSLDN & 1.4973 & -0.1821 \\
\hline D4A1Q2 & Mapt & $\mathrm{T}$ & 631 & 2 & RENAKAKTDHGAEIV & 0.2979 & -0.0877 \\
\hline D4A1Q2 & Mapt & $\mathrm{T}$ & 648 & 1 & SPVVSGDTSPRHLSN & 0.3757 & 0.1656 \\
\hline Q5XI50 & 7-Mar & $\mathrm{s}$ & 302 & 1 & TFFSRRSSQDSLNTR & 0.4258 & -0.0198 \\
\hline P30009 & Marcks & $S$ & 138 & 2 & AEDGAAPSPSSETPK & 0.3653 & -0.0819 \\
\hline P30009 & Marcks & $S$ & 140 & 2 & DGAAPSPSSETPKKK & 0.3519 & -0.0778 \\
\hline P30009 & Marcks & $\mathrm{T}$ & 143 & 2 & APSPSSETPKKKKKR & 0.3548 & -0.0858 \\
\hline P30009 & Marcks & $S$ & 156 & 2 & KRFSFKKSFKLSGFS & 2.2814 & -0.1232 \\
\hline P30009 & Marcks & $\mathrm{s}$ & 156 & 3 & KRFSFKKSFKLSGFS & 2.1628 & \\
\hline
\end{tabular}




\begin{tabular}{|c|c|c|c|c|c|c|c|}
\hline P30009 & Marcks & S & 160 & 2 & FKKSFKLSGFSFKKS & 2.2087 & -0.1044 \\
\hline P30009 & Marcks & S & 160 & 3 & FKKSFKLSGFSFKKS & 2.1628 & \\
\hline Q9EPH2 & Marcksl1 & $\mathrm{T}$ & 85 & 1 & GEVAPKETPKKKKKF & 0.2748 & -0.0953 \\
\hline 008678 & Mark1 & $S$ & 463 & 2 & DTARRLGSTTVGSKS & -0.3653 & -0.0135 \\
\hline 008678 & Mark1 & $S$ & 468 & 2 & LGSTTVGSKSEVTAS & -0.3414 & \\
\hline 008678 & Mark1 & S & 666 & 1 & KFVRRDPSEGEASGR & 1.7072 & \\
\hline A0A1B0GWN5 & Mark3 & $S$ & 416 & 1 & PRGTASRSTFHGQPR & 0.2891 & -0.0964 \\
\hline Q810W7 & Mast1 & $S$ & 1254 & 2 & IVRPRPKSAEPPRSP & -0.9991 & \\
\hline Q810W7 & Mast1 & S & 1260 & 2 & KSAEPPRSPLLKRVQ & -0.9991 & \\
\hline Q63406 & Mcf21 & S & 966 & 2 & RALEQSHSLPLPTPA & 0.2633 & \\
\hline A0A0G2KAL9 & Mff & $\mathrm{T}$ & 112 & 2 & DLERPPPTPQSEEIR & -0.7970 & \\
\hline A0A0G2KAL9 & Mff & S & 115 & 2 & RPPPTPQSEEIRAVG & -0.6719 & \\
\hline A0A0G2KAL9 & Mff & S & 146 & 1 & GQLVRNDSIVTPSPP & 0.4463 & -0.0720 \\
\hline A0A0G2KAL9 & Mff & $\mathrm{T}$ & 149 & 2 & VRNDSIVTPSPPQAR & -0.3660 & -0.2130 \\
\hline A0A0G2KAL9 & Mff & S & 151 & 2 & NDSIVTPSPPQARVC & -0.3522 & -0.1127 \\
\hline A0A0G2K6P5 & Mfsd4a & S & 273 & 1 & AEKEDTSSLAHKFQP & 0.4445 & \\
\hline D3ZGN7 & Mical3 & $\mathrm{S}$ & 976 & 1 & HALLKGRSEEELEAS & 0.3058 & \\
\hline D3ZGN7 & Mical3 & $\mathrm{T}$ & 1404 & 2 & GDQPPLLTPKSPSDK & -1.3259 & \\
\hline D3ZGN7 & Mical3 & S & 1407 & 1 & PPLLTPKSPSDKELR & -0.7991 & 0.0322 \\
\hline D3ZGN7 & Mical3 & S & 1407 & 2 & PPLLTPKSPSDKELR & -1.2930 & \\
\hline Q5XIS8 & Mief1 & S & 59 & 1 & RAISAPTSPTRLSHS & 0.3628 & \\
\hline Q63327 & Mobp & S & 13 & 1 & AKEGPRLSKNQKFSE & 0.3604 & -0.2624 \\
\hline $\mathrm{P} 62775$ & Mtpn & $\mathrm{T}$ & 31 & 1 & KGEDVNRTLEGGRKP & 0.4133 & \\
\hline D4A3S6 & Mtss1I & S & 419 & 1 & MVLTRGLSLEHQKSS & 1.3906 & 0.1037 \\
\hline Q9JLT0 & Myh10 & $S$ & 1939 & 1 & GPISFSSSRSGRRQL & 1.2267 & -0.1313 \\
\hline Q9JLT0 & Myh10 & $S$ & 1952 & 2 & QLHIEGASLELSDDD & 0.8169 & 0.1984 \\
\hline Q9JLT0 & Myh10 & S & 1956 & 2 & EGASLELSDDDTESK & 0.8770 & 0.1922 \\
\hline Q9JLT0 & Myh10 & $\mathrm{T}$ & 1960 & 2 & LELSDDDTESKTSDV & 0.7400 & \\
\hline Q9QYF3 & Myo5a & S & 1624 & 2 & TGLRKRTSSIADEGT & 0.6037 & -0.0037 \\
\hline Q9QYF3 & Myo5a & S & 1625 & 2 & GLRKRTSSIADEGTY & 0.4837 & -0.0717 \\
\hline Q9QYF3 & Myo5a & $\mathrm{T}$ & 1631 & 2 & SSIADEGTYTLDSIL & 0.5006 & 0.0168 \\
\hline D4A5I4 & Nav1 & S & 787 & 1 & WRRERPESCDDSSKG & 0.8499 & \\
\hline D4A5I4 & Nav1 & S & 952 & 1 & RSNIQYRSLPRPAKS & -0.3649 & -0.2530 \\
\hline D4A514 & Nav1 & $S$ & 1085 & 2 & ANLDKVNSNSLDLPS & 0.8901 & \\
\hline D4A5I4 & Nav1 & S & 1087 & 1 & LDKVNSNSLDLPSSS & 0.5835 & 0.0615 \\
\hline D4A5I4 & Nav1 & $\mathrm{S}$ & 1087 & 2 & LDKVNSNSLDLPSSS & 0.9337 & \\
\hline D4A514 & Nav1 & $S$ & 1229 & 1 & GLRYQLQSQEETKER & 0.7471 & -0.0383 \\
\hline D4A5I4 & Nav1 & S & 1532 & 1 & SPKLQHGSTETASPS & 0.3691 & 0.0837 \\
\hline A0A0G2K0M8 & Ncam1 & $\mathrm{T}$ & 846 & 2 & ATSKPSPTPTPTPAG & -0.4029 & 0.1376 \\
\hline A0A0G2K0M8 & Ncam1 & $S$ & 856 & 2 & PTPAGAASPLAAVAA & -0.4102 & 0.1460 \\
\hline F1LWN1 & Ncoa7 & S & 184 & 2 & ATVSPSSSDAEYDKL & -0.3126 & -0.0293 \\
\hline A0A0G2JXP3 & Nedd4I & S & 434 & 2 & IRRPRSLSSPTVTLS & -0.3085 & \\
\hline P97526 & Nf1 & $S$ & 2524 & 1 & KLLGTRKSFDHLISD & 0.3829 & -0.0194 \\
\hline Q5BKC9 & Ngef & S & 687 & 1 & KMEDPQRSQNKDRRK & 0.8370 & 0.1487 \\
\hline
\end{tabular}




\begin{tabular}{|c|c|c|c|c|c|c|c|}
\hline A0A0G2JZL4 & Nhsl1 & $S$ & 1352 & 1 & KVLGRKDSEDDHTRN & 1.9045 & \\
\hline Q6AY81 & Npdc1 & S & 235 & 1 & ATAKGPTSPTTPRIS & 0.4154 & -0.2588 \\
\hline Q6AY81 & Npdc1 & S & 242 & 1 & SPTTPRISPGDERLA & 0.3279 & \\
\hline O35764 & Nptxr & $S$ & 251 & 1 & EKERAALSHGSHQQR & 0.3072 & \\
\hline A0A0G2JT23 & Nrbp2 & $\mathrm{T}$ & 407 & 2 & EEAQKAKTPTPEPFD & -0.4976 & -0.1189 \\
\hline A0A0G2JT23 & Nrbp2 & $\mathrm{T}$ & 409 & 2 & AQKAKTPTPEPFDSE & -0.4918 & -0.1210 \\
\hline P47245 & Nrdc & $S$ & 85 & 1 & ARLGADESEEEGRSL & 0.3507 & \\
\hline Q63372 & Nrxn1 & $S$ & 1485 & 1 & YRNRDEGSYHVDESR & 0.2736 & \\
\hline O35987 & Nsfl1c & $\mathrm{s}$ & 176 & 1 & AGERRRHSGQDVHVV & -0.3928 & -0.0222 \\
\hline Q2LC84 & Numb & $\mathrm{T}$ & 437 & 1 & FQAGHRRTPSEADRW & 0.8087 & \\
\hline Q2LC84 & Numb & $S$ & 635 & 1 & PKQRTNPSPTNPFSS & -0.3916 & 0.0844 \\
\hline Q2LC84 & Numb & $\mathrm{T}$ & 637 & 1 & QRTNPSPTNPFSSDA & -0.3634 & \\
\hline A1L1I3 & Numbl & $S$ & 411 & 1 & PGHKRTPSEAERWLE & 0.9620 & \\
\hline P33535 & Oprm1 & $T$ & 370 & 1 & STRVRQNTREHPSTA & 0.3778 & -0.0487 \\
\hline Q9ERC5 & Otof & $\mathrm{S}$ & 237 & 2 & GLDPDSVSLASVTAL & -0.4323 & \\
\hline Q9ERC5 & Otof & $\mathrm{S}$ & 240 & 2 & PDSVSLASVTALTSN & -0.4323 & \\
\hline AOA0G2K7Y2 & Oxr1 & $S$ & 15 & 1 & WLKKKSQSVDITAPG & 1.8243 & -0.1610 \\
\hline A0A0G2K7Y2 & Oxr1 & $S$ & 90 & 1 & KKDGRRMSFQKPKGT & 0.3349 & -0.0441 \\
\hline A0A0G2K7Y2 & Oxr1 & $T$ & 203 & 1 & PARVVSSTSEEEEAF & 0.4467 & \\
\hline A0A0G2K7Y2 & Oxr1 & $S$ & 204 & 1 & ARVVSSTSEEEEAFT & 0.5749 & -0.1767 \\
\hline A0A0G2K7Y2 & Oxr1 & S & 510 & 1 & RKLWKTHSMQQAKQQ & 0.9990 & -0.0228 \\
\hline A0A0G2K7Y2 & Oxr1 & $S$ & 529 & 1 & QQVAQRESKHRGAPA & 0.5968 & 0.0356 \\
\hline D3ZUC9 & Oxsr1 & $\mathrm{s}$ & 359 & 1 & AAISQLRSPRVKDSL & -0.8200 & -0.0337 \\
\hline O88588 & Pacs1 & $S$ & 408 & 1 & FEGMSQSSSQTEIGS & -0.6444 & 0.0015 \\
\hline O88588 & Pacs1 & $S$ & 409 & 1 & EGMSQSSSQTEIGSL & -0.5958 & 0.0706 \\
\hline 088588 & Pacs1 & $\mathrm{s}$ & 449 & 1 & WIKNQDDSLTETDTL & 0.7954 & -0.0387 \\
\hline 088588 & Pacs1 & $\mathrm{s}$ & 495 & 2 & LQGSASPSKVEGTHT & -0.3152 & \\
\hline O88588 & Pacs1 & $S$ & 517 & 1 & PLKERQLSKPLSERT & 3.9793 & \\
\hline D3ZJG4 & Pacs2 & $S$ & 328 & 1 & RSHREPPSPADVPEK & -0.3468 & -0.1059 \\
\hline Q9ZoW5 & Pacsin 1 & $T$ & 335 & 2 & ATGAVESTSQAGDRG & -0.5315 & 0.0397 \\
\hline Q9ZOW5 & Pacsin 1 & $\mathrm{~T}$ & 335 & 3 & ATGAVESTSQAGDRG & -0.4324 & -0.1086 \\
\hline Q9ZoW5 & Pacsin 1 & $S$ & 336 & 1 & TGAVESTSQAGDRGS & -0.6145 & -0.0869 \\
\hline Q9ZOW5 & Pacsin 1 & $\mathrm{~s}$ & 336 & 2 & TGAVESTSQAGDRGS & -0.5315 & -0.0329 \\
\hline Q9Z0W5 & Pacsin1 & $S$ & 336 & 3 & TGAVESTSQAGDRGS & -0.5510 & -0.1060 \\
\hline Q9ZoW5 & Pacsin 1 & $S$ & 343 & 3 & SQAGDRGSVSSYDRG & -0.5359 & -0.1000 \\
\hline Q9ZOW5 & Pacsin 1 & $\mathrm{~s}$ & 346 & 3 & GDRGSVSSYDRGQAY & -0.5783 & -0.0912 \\
\hline P35465 & Pak1 & $\mathrm{T}$ & 167 & 2 & NVKTVSETPAVPPVS & -0.4176 & \\
\hline P35465 & Pak1 & $\mathrm{T}$ & 218 & 2 & TPTRDVATSPISPTE & -0.6000 & -0.0892 \\
\hline P35465 & Pak1 & $S$ & 219 & 2 & PTRDVATSPISPTEN & -0.4502 & -0.0329 \\
\hline P35465 & Pak1 & $S$ & 222 & 2 & DVATSPISPTENNTT & -0.4202 & -0.0316 \\
\hline Q62829 & Pak3 & $S$ & 2 & 1 & MSDSLDNEE & 0.7232 & -0.0747 \\
\hline Q62829 & Pak3 & $\mathrm{s}$ & 4 & 1 & MSDSLDNEEKP & 0.7206 & -0.0785 \\
\hline D4A1J3 & Palm3 & $S$ & 524 & 1 & QEKDGEGSLDRESKT & 0.3757 & \\
\hline G3V7T3 & Pank4 & $\mathrm{s}$ & 15 & 1 & GGGSGGDSLDKSITL & 0.4974 & -0.0493 \\
\hline
\end{tabular}




\begin{tabular}{|c|c|c|c|c|c|c|c|}
\hline A1A5Q1 & Parp9 & $S$ & 342 & 3 & LDKIKLSSDYQVVQV & -0.5958 & \\
\hline A1A5Q1 & Parp9 & $Y$ & 344 & 3 & KIKLSSDYQVVQVTK & -0.5958 & \\
\hline A1A5Q1 & Parp9 & $\mathrm{T}$ & 350 & 3 & DYQVVQVTKGFKLSC & -0.5958 & \\
\hline A0A0G2K6T9 & Pcdh1 & $S$ & 923 & 2 & GRHYRSNSPLPSIQL & -0.6286 & \\
\hline D3ZE55 & Pcdh8 & $S$ & 802 & 1 & GGASAPGSPDETARG & 0.2784 & -0.1365 \\
\hline F1LS01 & Pcdh9 & $T$ & 954 & 2 & AFHLKPDTPVSVKKH & -0.5631 & 0.1087 \\
\hline F1LS01 & Pcdh9 & $S$ & 957 & 2 & LKPDTPVSVKKHHVI & -0.6447 & \\
\hline F1M7V4 & Pclo & $S$ & 1458 & 2 & YKLPSPTSPLSPHSN & 0.5874 & 0.1347 \\
\hline F1M7V4 & Pclo & $\mathrm{s}$ & 1461 & 1 & PSPTSPLSPHSNKSS & 1.3287 & 0.1011 \\
\hline F1M7V4 & Pclo & $\mathrm{s}$ & 1461 & 2 & PSPTSPLSPHSNKSS & 0.5376 & \\
\hline F1M7V4 & Pclo & $S$ & 1889 & 1 & GGMKPSMSDTNLAEA & -0.4294 & -0.0304 \\
\hline Q9JKS6 & Pclo & $\mathrm{T}$ & 111 & 1 & PGLSKSRTTDTFRSE & -0.6717 & 0.1376 \\
\hline Q9JKS6 & Pclo & S & 125 & 2 & EQKLPGRSPSTISLK & -0.9418 & -0.0526 \\
\hline Q9JKS6 & Pclo & $S$ & 130 & 2 & GRSPSTISLKESKSR & -0.9429 & -0.0431 \\
\hline Q9JKS6 & Pclo & $\mathrm{s}$ & 136 & 1 & ISLKESKSRTDFKEE & -1.7957 & -0.0628 \\
\hline Q9JKS6 & Pclo & S & 321 & 1 & AGLEKTSSSQQPGPK & 1.0283 & -0.0291 \\
\hline Q9JKS6 & Pclo & $S$ & 329 & 1 & SQQPGPKSLAQTPGH & 0.4233 & \\
\hline Q9JKS6 & Pclo & $S$ & 591 & 1 & LAAAIPSSPQPTPKA & -1.3396 & 0.0671 \\
\hline Q9JKS6 & Pclo & S & 857 & 3 & QEQSRRFSLNLGGIT & -0.8680 & 0.0650 \\
\hline Q9JKS6 & Pclo & $\mathrm{S}$ & 869 & 2 & GITDAPKSQPTTPQE & -1.1603 & 0.0867 \\
\hline Q9JKS6 & Pclo & $\mathrm{T}$ & 872 & 2 & DAPKSQPTTPQETVT & -1.0963 & 0.0963 \\
\hline Q9JKS6 & Pclo & $\mathrm{T}$ & 872 & 3 & DAPKSQPTTPQETVT & -0.8893 & 0.0650 \\
\hline Q9JKS6 & Pclo & $\mathrm{T}$ & 873 & 1 & APKSQPTTPQETVTG & -0.2956 & 0.0188 \\
\hline Q9JKS6 & Pclo & $T$ & 873 & 2 & APKSQPTTPQETVTG & -1.1926 & 0.0854 \\
\hline Q9JKS6 & Pclo & $T$ & 873 & 3 & APKSQPTTPQETVTG & -0.8812 & 0.0650 \\
\hline Q9JKS6 & Pclo & $\mathrm{S}$ & 922 & 1 & SKQAPTPSQSPAAQG & -0.7166 & 0.2026 \\
\hline Q9JKS6 & Pclo & $\mathrm{s}$ & 1162 & 1 & ALPEKKPSEEEKAIS & 0.4600 & -0.0914 \\
\hline Q9JKS6 & Pclo & $\mathrm{S}$ & 1274 & 1 & AQPQAEGSSKDGQGE & 0.8448 & 0.1120 \\
\hline Q9JKS6 & Pclo & $S$ & 1344 & 1 & LKGLKKDSFSQESSP & 0.9949 & 0.0444 \\
\hline Q9JKS6 & Pclo & S & 1344 & 2 & LKGLKKDSFSQESSP & 0.7988 & 0.0834 \\
\hline Q9JKS6 & Pclo & $S$ & 1344 & 3 & LKGLKKDSFSQESSP & 0.6065 & \\
\hline Q9JKS6 & Pclo & S & 1346 & 1 & GLKKDSFSQESSPSS & 1.2388 & 0.0368 \\
\hline Q9JKS6 & Pclo & $\mathrm{s}$ & 1346 & 2 & GLKKDSFSQESSPSS & 0.7737 & 0.0833 \\
\hline Q9JKS6 & Pclo & $S$ & 1349 & 2 & KDSFSQESSPSSPSD & 0.5097 & -0.0632 \\
\hline Q9JKS6 & Pclo & S & 1451 & 1 & PELVDDLSPRRASYD & -0.4573 & 0.1366 \\
\hline Q9JKS6 & Pclo & $\mathrm{s}$ & 1562 & 2 & HRRLTRKSSTSFDDD & -0.3512 & \\
\hline Q9JKS6 & Pclo & $S$ & 1563 & 1 & RRLTRKSSTSFDDDA & 0.7005 & -0.1513 \\
\hline Q9JKS6 & Pclo & S & 1563 & 2 & RRLTRKSSTSFDDDA & 1.2929 & \\
\hline Q9JKS6 & Pclo & $\mathrm{T}$ & 1564 & 2 & RLTRKSSTSFDDDAG & 0.8613 & -0.0266 \\
\hline Q9JKS6 & Pclo & $S$ & 1565 & 2 & LTRKSSTSFDDDAGR & 1.7622 & -0.0168 \\
\hline Q9JKS6 & Pclo & $S$ & 1597 & 1 & LKFRETKSQESEELV & 0.8230 & \\
\hline Q9JKS6 & Pclo & $\mathrm{s}$ & 1597 & 2 & LKFRETKSQESEELV & 0.6233 & -0.1401 \\
\hline Q9JKS6 & Pclo & $S$ & 1600 & 2 & RETKSQESEELVVAG & 0.6233 & -0.1351 \\
\hline Q9JKS6 & Pclo & $T$ & 1772 & 3 & RRRERPKTPPSNLSP & -1.1106 & 0.1970 \\
\hline
\end{tabular}




\begin{tabular}{|c|c|c|c|c|c|c|c|}
\hline Q9JKS6 & Pclo & $S$ & 1775 & 3 & ERPKTPPSNLSPIED & -1.1967 & 0.2002 \\
\hline Q9JKS6 & Pclo & S & 1778 & 3 & KTPPSNLSPIEDASP & -1.1974 & 0.2073 \\
\hline Q9JKS6 & Pclo & S & 1784 & 1 & LSPIEDASPTEELRQ & 0.4405 & -0.0546 \\
\hline Q9JKS6 & Pclo & S & 1784 & 3 & LSPIEDASPTEELRQ & -1.1929 & 0.2073 \\
\hline Q9JKS6 & Pclo & $S$ & 2326 & 1 & TYRLPSGSLPVSTHP & 0.8145 & -0.0113 \\
\hline Q9JKS6 & Pclo & $\mathrm{s}$ & 3561 & 1 & PTVQLAPSPPKSPKV & -0.6130 & 0.0390 \\
\hline Q9JKS6 & Pclo & $S$ & 3561 & 2 & PTVQLAPSPPKSPKV & -1.4290 & -0.0484 \\
\hline Q9JKS6 & Pclo & $S$ & 3565 & 2 & LAPSPPKSPKVLYSP & -1.4290 & -0.0484 \\
\hline Q9JKS6 & Pclo & $\mathrm{s}$ & 3624 & 1 & TAKMMQRSMSDPKPL & 0.5316 & 0.0640 \\
\hline Q9JKS6 & Pclo & S & 3626 & 1 & KMMQRSMSDPKPLSP & 0.4941 & 0.0753 \\
\hline Q9JKS6 & Pclo & $S$ & 3632 & 1 & MSDPKPLSPTADESS & -0.9768 & 0.1186 \\
\hline Q9JKS6 & Pclo & $S$ & 3795 & 1 & TRPSRVESQHGVERP & 0.8349 & -0.1093 \\
\hline Q9JKS6 & Pclo & S & 4034 & 1 & RTTETRRSQEVTDFL & 0.4750 & -0.1052 \\
\hline Q9JKS6 & Pclo & $S$ & 4155 & 1 & KFSPIQESRDLEPDY & 1.1757 & -0.0865 \\
\hline Q9JKS6 & Pclo & $\mathrm{s}$ & 4235 & 3 & PYSSGSRSRPSSRPS & -1.1264 & -0.2367 \\
\hline Q9JKS6 & Pclo & S & 4242 & 3 & SRPSSRPSSVYGLDL & -1.1308 & \\
\hline Q9JKS6 & Pclo & $S$ & 4243 & 3 & RPSSRPSSVYGLDLS & -1.0949 & -0.2024 \\
\hline Q9JKS6 & Pclo & $S$ & 4293 & 1 & TSLPISQSRGRIPIV & -0.8673 & -0.2072 \\
\hline Q9JKS6 & Pclo & S & 4341 & 2 & TRDQFGSSHSLPEVQ & -0.3079 & -0.0599 \\
\hline Q9JKS6 & Pclo & $\mathrm{S}$ & 4343 & 2 & DQFGSSHSLPEVQQH & -0.2837 & -0.0610 \\
\hline Q9JKS6 & Pclo & S & 4561 & 1 & PKVDKAKSPGVDPKQ & -1.2484 & -0.0666 \\
\hline Q9JKS6 & Pclo & S & 4801 & 1 & PSVIKSRSHGIFPDP & -0.7649 & -0.0786 \\
\hline Q9JKS6 & Pclo & $S$ & 4831 & 2 & PGSSKSSSEGHLRSH & -0.5241 & \\
\hline Q9JKS6 & Pclo & $S$ & 4837 & 2 & SSEGHLRSHGPSRSQ & -0.8698 & -0.1027 \\
\hline Q9JKS6 & Pclo & $\mathrm{S}$ & 4841 & 2 & HLRSHGPSRSQSKTS & -0.7898 & -0.0213 \\
\hline A0A0G2K0X1 & Pcm1 & S & 68 & 1 & TNDISPESSPGVGRR & 0.5319 & \\
\hline D3ZXP8 & Pcp2 & $\mathrm{s}$ & 92 & 2 & QKRPGTLSPQPLLSP & -0.3779 & 0.1603 \\
\hline D3ZXP8 & Pcp2 & $\mathrm{S}$ & 98 & 2 & LSPQPLLSPQDPAAL & -0.3779 & 0.1603 \\
\hline Q01066 & Pde1b & $S$ & 465 & 1 & SFQWRQPSLDVDVGD & 0.4867 & -0.0336 \\
\hline A0A0G2K876 & Pde2a & S & 885 & 1 & FTIRGLPSNNSLDFL & -0.4167 & 0.0330 \\
\hline A0A0G2K876 & Pde2a & S & 888 & 1 & RGLPSNNSLDFLDEE & 0.6885 & -0.0814 \\
\hline P54748 & Pde4a & S & 333 & 1 & KKLVHTGSLNTNVPR & 0.8807 & -0.0738 \\
\hline P14646 & Pde4b & $\mathrm{s}$ & 197 & 1 & HGAPNKRSPAASQAP & -0.7462 & -0.1836 \\
\hline P14646 & Pde4b & $S$ & 319 & 1 & KKLMHSSSLNNTSIS & 0.3080 & 0.0930 \\
\hline P14270 & Pde4d & S & 192 & 1 & SFLYRSDSDYDLSPK & 0.5242 & -0.1299 \\
\hline P26284 & Pdha1 & $\mathrm{s}$ & 293 & 1 & TYRYHGHSMSDPGVS & -0.2879 & -0.1225 \\
\hline P26284 & Pdha1 & $S$ & 293 & 2 & TYRYHGHSMSDPGVS & -0.4282 & -0.0391 \\
\hline P26284 & Pdha1 & S & 295 & 1 & RYHGHSMSDPGVSYR & -0.3564 & -0.0983 \\
\hline P26284 & Pdha1 & $\mathrm{S}$ & 295 & 2 & RYHGHSMSDPGVSYR & -0.5864 & 0.0487 \\
\hline P26284 & Pdha1 & $S$ & 300 & 2 & SMSDPGVSYRTREEI & -0.5043 & 0.0011 \\
\hline O55173 & Pdpk1 & $T$ & 33 & 1 & PSMVRSQTEPSSSPG & 0.5675 & \\
\hline D3ZC81 & Pex10 & $\mathrm{s}$ & 259 & 1 & NLSHRRSSLEDRAVC & 0.2727 & \\
\hline P30835 & Pfkl & $S$ & 775 & 1 & HVTRRTLSIDKGF & 0.7158 & 0.0210 \\
\hline P47860 & Pfkp & $T$ & 313 & 1 & GHVQRGGTPSAFDRI & 0.2776 & -0.2613 \\
\hline
\end{tabular}




\begin{tabular}{|c|c|c|c|c|c|c|c|}
\hline P62024 & Phactr1 & $S$ & 67 & 1 & IRRVRSKSDTPYLAE & -0.4952 & -0.1166 \\
\hline P62024 & Phactr1 & S & 423 & 1 & MKVCRKDSLAIKLSN & 0.7915 & -0.2379 \\
\hline Q6RFY2 & Phactr3 & S & 267 & 1 & ATKHRQDSFQGRECR & 1.3140 & -0.1256 \\
\hline A0A0G2K9C8 & Phkb & $S$ & 693 & 1 & SKVKRQSSTADAPEL & 0.3004 & -0.0851 \\
\hline D3ZNS1 & Phldb1 & $S$ & 325 & 1 & GGHERPPSPGLRGLL & -0.4931 & 0.0793 \\
\hline D3ZNS1 & Phldb1 & $\mathrm{s}$ & 534 & 1 & SLAPRKGSFSGRLSP & 0.7066 & -0.1720 \\
\hline D3ZNS1 & Phldb1 & $S$ & 579 & 2 & IPRERKNSITEISDN & -0.2877 & \\
\hline D3ZNS1 & Phldb1 & $S$ & 584 & 2 & KNSITEISDNEDDLL & -0.2877 & \\
\hline D3ZYT8 & Pikfyve & $\mathrm{s}$ & 487 & 2 & KDIKFDDSDTEQIAE & -0.2938 & \\
\hline D3ZYT8 & Pikfyve & $\mathrm{s}$ & 505 & 2 & DNLANSASPSKRTSV & -0.2938 & \\
\hline $\mathrm{F} 1 \mathrm{M} 8 \mathrm{H} 6$ & Pip5k1c & $\mathrm{S}$ & 475 & 1 & LGPTAAFSASQIPSE & -0.4642 & \\
\hline $\mathrm{F} 1 \mathrm{M} 8 \mathrm{H} 6$ & Pip5k1c & $S$ & 554 & 1 & RYRRRTQSSGQDGRP & -0.3895 & 0.0441 \\
\hline $\mathrm{F} 1 \mathrm{M} 8 \mathrm{H} 6$ & Pip5k1c & S & 555 & 1 & YRRRTQSSGQDGRPQ & -0.4020 & -0.0083 \\
\hline F1LXD6 & Pitpnm2 & $S$ & 630 & 1 & SSRHLSRSNIDIPRS & -0.4303 & -0.0319 \\
\hline F1M2K6 & Pkp4 & $\mathrm{s}$ & 213 & 1 & RAMRRVSSVPSRAQS & 0.4495 & 0.0223 \\
\hline F1M2K6 & Pkp4 & S & 289 & 1 & TAVRRVGSVTSRQTS & 0.4468 & 0.0744 \\
\hline F1M2K6 & Pkp4 & $S$ & 405 & 2 & QDLRSAVSPDLHITP & -0.8731 & \\
\hline F1M2K6 & Pkp4 & $T$ & 411 & 2 & VSPDLHITPIYEGRT & -0.8731 & \\
\hline P10687 & Plcb1 & S & 1199 & 2 & KVNLKSPSSEEVQGE & -0.3128 & 0.1559 \\
\hline P10686 & Plcg1 & $\mathrm{S}$ & 1248 & 1 & HVRAREGSFEARYQQ & 0.6989 & 0.1438 \\
\hline D4AAX6 & Plch2 & S & 575 & 1 & SKIKKVASVEEGDES & 0.4315 & -0.2224 \\
\hline Q91Z79 & Ppfia3 & S & 507 & 2 & RPPSYSRSLPGSALE & -0.8654 & -0.0379 \\
\hline Q91Z79 & Ppfia3 & $S$ & 511 & 2 & YSRSLPGSALELRYS & -0.8654 & -0.0379 \\
\hline F1M863 & Ppfia4 & $S$ & 657 & 1 & TPKLTSRSAAQDLDR & -0.4810 & \\
\hline F1M863 & Ppfia4 & $\mathrm{S}$ & 721 & 1 & KLGHPTLSQEEGKSA & 0.7515 & 0.0906 \\
\hline Q6DGG0 & Ppid & $S$ & 198 & 1 & GIFPKDGSGDSHPDF & 0.3916 & \\
\hline Q5M821 & Ppm1h & $\mathrm{s}$ & 118 & 1 & TSTPNRNSKRRSSLP & 0.3829 & -0.1996 \\
\hline Q5M821 & Ppm1h & $\mathrm{S}$ & 123 & 1 & RNSKRRSSLPNGEGL & 0.3477 & -0.2264 \\
\hline Q10728 & Ppp1r12a & $S$ & 299 & 1 & SEKRDKKSPLIESTA & -0.4110 & 0.0283 \\
\hline Q10728 & Ppp1r12a & $T$ & 596 & 2 & STSTTAKTPPGSSPA & -0.8074 & -0.0874 \\
\hline Q10728 & Ppp1r12a & S & 601 & 1 & AKTPPGSSPAGTQSS & -0.6787 & 0.0661 \\
\hline Q10728 & Ppp1r12a & $S$ & 601 & 2 & AKTPPGSSPAGTQSS & -0.7456 & -0.0911 \\
\hline P19103 & Ppp1r1a & $\mathrm{s}$ & 46 & 2 & LVLTSDQSSPEVDED & 0.3068 & \\
\hline P19103 & Ppp1r1a & $S$ & 47 & 2 & VLTSDQSSPEVDEDR & 0.3068 & \\
\hline P19103 & Ppp1r1a & $S$ & 67 & 1 & LKSTLSMSPRQRKKM & -0.5112 & 0.2069 \\
\hline Q6J4I0 & Ppp1r1b & $\mathrm{s}$ & 45 & 1 & LFRVSEHSSPEEESS & 0.5083 & -0.0750 \\
\hline Q6J4I0 & Ppp1r1b & $S$ & 51 & 2 & HSSPEEESSPHQRTS & -0.3223 & \\
\hline P50411 & Ppp1r2 & S & 20 & 1 & GILKNKTSTTSSVVA & 0.7712 & 0.1819 \\
\hline F1LYZ8 & Ppp1r21 & $\mathrm{S}$ & 101 & 1 & GESSSQLSQEQKSVF & 0.9893 & 0.0334 \\
\hline P0C7L8 & Ppp1r3e & $S$ & 33 & 1 & YYRSQRPSLEEESEE & 0.2933 & \\
\hline Q5HZV9 & Ppp1r7 & S & 12 & 1 & RGAGQQQSQEMMEVD & 0.6518 & \\
\hline Q5HZV9 & Ppp1r7 & $\mathrm{s}$ & 24 & 1 & EVDRRVESEESGDEE & 0.4007 & -0.2439 \\
\hline Q5HZV9 & Ppp1r7 & $S$ & 44 & 2 & GGIVADLSQQSLKDG & -0.4705 & -0.1460 \\
\hline Q5HZV9 & Ppp1r7 & s & 47 & 1 & VADLSQQSLKDGVER & 0.4434 & -0.2049 \\
\hline
\end{tabular}




\begin{tabular}{|c|c|c|c|c|c|c|c|}
\hline Q5HzV9 & Ppp1r7 & $S$ & 47 & 2 & VADLSQQSLKDGVER & -0.4705 & -0.1460 \\
\hline O35274 & Ppp1r9b & S & 100 & 1 & LSLPRASSLNENVDH & 1.6476 & -0.0739 \\
\hline D4A1A5 & Ppp2r5c & S & 497 & 1 & RPLVRRKSELPQDPH & 1.4669 & -0.0572 \\
\hline P63329 & Ppp3ca & $S$ & 498 & 1 & PSDANLNSINKALAS & 0.3204 & \\
\hline P80386 & Prkab1 & $S$ & 108 & 1 & SKLPLTRSQNNFVAI & -0.3738 & -0.1349 \\
\hline F1LS42 & Prkcb & $\mathrm{S}$ & 653 & 2 & VIRNIDQSEFEGFSF & -0.4484 & \\
\hline F1LS42 & Prkcb & S & 659 & 2 & QSEFEGFSFVNSEFL & -0.3817 & 0.0756 \\
\hline F1LS42 & Prkcb & $S$ & 663 & 2 & EGFSFVNSEFLKPEV & -0.3900 & 0.0785 \\
\hline P63319 & Prkcg & $\mathrm{T}$ & 689 & 2 & HPDARSPTSPVPVPV & -0.2726 & \\
\hline D3ZFB6 & Prrt2 & $\mathrm{T}$ & 74 & 2 & LAPETTETPVETPET & -0.2690 & 0.1713 \\
\hline D3ZFB6 & Prrt2 & $\mathrm{T}$ & 78 & 2 & TTETPVETPETVQAT & -0.2690 & 0.1718 \\
\hline D3ZFB6 & Prrt2 & $S$ & 242 & 1 & AHGGHPGSPRGSLSR & 0.3057 & -0.0474 \\
\hline D3ZWQ0 & Prrt3 & $S$ & 794 & 3 & GSVGPAPSLSELDLR & -0.3372 & 0.0663 \\
\hline D3ZWQ0 & Prrt3 & S & 796 & 3 & VGPAPSLSELDLRPP & -0.3372 & 0.0694 \\
\hline D3ZWQ0 & Prrt3 & $\mathrm{s}$ & 804 & 3 & ELDLRPPSPINLSRS & -0.3372 & 0.0663 \\
\hline Q4FZT9 & Psmd2 & S & 363 & 1 & NRFGGSGSQVDSARM & 0.4927 & -0.1119 \\
\hline A0A0G2JZ88 & RGD1306556 & $\mathrm{T}$ & 1677 & 1 & EPPRVERTPVGHPQR & -0.7101 & -0.1514 \\
\hline A0A0G2JZ88 & RGD1306556 & $\mathrm{T}$ & 1721 & 2 & SWTPQPKTPKSPFQP & -1.5606 & \\
\hline A0A0G2JZ88 & RGD1306556 & S & 1724 & 2 & PQPKTPKSPFQPGVL & -1.5606 & \\
\hline D4A3X1 & RGD1308601 & $\mathrm{S}$ & 67 & 1 & MELRRDSSESQLAST & -0.5254 & -0.1338 \\
\hline D3ZBU7 & RGD1310819 & $\mathrm{S}$ & 204 & 2 & IKILVSSSRPQSPDH & -0.4348 & -0.0988 \\
\hline D3ZBU7 & RGD1310819 & S & 208 & 2 & VSSSRPQSPDHMSDA & -0.3989 & \\
\hline D3ZBU7 & RGD1310819 & $\mathrm{S}$ & 968 & 1 & PAAGPDTSPGESERL & -0.4527 & -0.0561 \\
\hline D4A5F4 & RGD1311575 & $\mathrm{s}$ & 1129 & 1 & PMLQSRHSLDGSKLT & 2.0431 & -0.1827 \\
\hline A0A0G2K1W1 & Rab11fip5 & S & 251 & 1 & RGSHGTSSLEAVPGQ & 0.3793 & \\
\hline Q6NYB7 & Rab1A & $\mathrm{T}$ & 195 & 1 & SNVKIQSTPVKQSGG & 0.3121 & -0.2403 \\
\hline BOBN19 & Rab22a & $\mathrm{s}$ & 187 & 1 & FKLRRQPSEPKRSCC & 0.3382 & -0.1721 \\
\hline P63012 & Rab3a & $\mathrm{S}$ & 7 & 1 & MASATDSRYGQKES & 0.5243 & -0.1631 \\
\hline A0A0G2K1B4 & Rab3ip & $\mathrm{T}$ & 249 & 2 & LVLSSSPTSPTQEPL & -0.2803 & \\
\hline G3V631 & Rabgef1 & S & 143 & 1 & PSINRQTSIETDRVT & 0.4360 & 0.1663 \\
\hline Q5FVT1 & Ralbp1 & S & 29 & 3 & SGLTRTPSSEEISPT & -0.2654 & \\
\hline Q5FVT1 & Ralbp1 & S & 34 & 3 & TPSSEEISPTKFPGL & -0.3125 & \\
\hline 055007 & Ralgapa1 & $\mathrm{s}$ & 796 & 1 & QPLPRSSSTSDILEP & -0.3373 & 0.0302 \\
\hline O55007 & Ralgapa1 & $S$ & 860 & 2 & TMTRRGSSPGSLEIP & -0.3146 & 0.1805 \\
\hline P86410 & Ralgapb & $\mathrm{T}$ & 416 & 2 & VQHQASSTSPLSSPN & -0.4095 & -0.0371 \\
\hline F1LV89 & Rap1gap & $\mathrm{s}$ & 524 & 1 & NTVSTSHSGSFTPNN & -0.4142 & 0.1456 \\
\hline F1LV89 & Rap1gap & $S$ & 557 & 2 & SRFGRRGSALGIGAV & -0.9825 & -0.0490 \\
\hline F1LV89 & Rap1gap & S & 574 & 2 & SLIVPGKSPTRKKSG & -0.9825 & -0.0577 \\
\hline F1LV89 & Rap1gap & $\mathrm{s}$ & 605 & 2 & EVQEKRESPPAGQKT & -0.5546 & 0.0315 \\
\hline F1LV89 & Rap1gap & $\mathrm{T}$ & 612 & 2 & SPPAGQKTPDSGHVS & -0.5390 & \\
\hline F1LV89 & Rap1gap & $S$ & 615 & 1 & AGQKTPDSGHVSQEP & -0.4297 & 0.0044 \\
\hline F1LV89 & Rap1gap & $\mathrm{s}$ & 615 & 2 & AGQKTPDSGHVSQEP & -0.5004 & 0.0325 \\
\hline F1LV89 & Rap1gap & $S$ & 721 & 1 & SSPGLTRSPHPDAVK & -0.9933 & \\
\hline AOA0G2K0S7 & Rap1gap2 & $\mathrm{s}$ & 558 & 1 & TVKNQSRSPIKRRSG & -0.7596 & 0.1606 \\
\hline
\end{tabular}




\begin{tabular}{|c|c|c|c|c|c|c|c|}
\hline A0A0G2K0S7 & Rap1gap2 & $S$ & 572 & 1 & GLFPRLHSGSEGQGD & 1.0875 & \\
\hline AOA0G2K0S7 & Rap1gap2 & $\mathrm{S}$ & 574 & 1 & FPRLHSGSEGQGDSR & 1.2779 & \\
\hline AOA0G2K0S7 & Rap1gap2 & $S$ & 727 & 1 & DKLSHASSSAGH & 0.6419 & -0.0360 \\
\hline A0A0G2K0S7 & Rap1gap2 & $S$ & 728 & 1 & KLSHASSSAGH & 0.9734 & 0.0219 \\
\hline A0A0A0MP77 & Rapgef2 & $S$ & 1113 & 1 & RQNQSRESLEQAQSR & 1.1977 & 0.0371 \\
\hline D3ZHK4 & Rb1cc1 & $S$ & 257 & 2 & TNTSLLTSFHKSVEH & -0.5214 & \\
\hline D3ZHK4 & Rb1cc1 & S & 261 & 2 & LLTSFHKSVEHVAPD & -0.5214 & \\
\hline D3ZL11 & Rbsn & $S$ & 635 & 2 & FDEEDLSSPIEGAVS & -0.4426 & \\
\hline D3ZL11 & Rbsn & $S$ & 642 & 2 & SPIEGAVSPAAVEAF & -0.4426 & \\
\hline A0A0G2JZY3 & Reps1 & $S$ & 93 & 1 & QVKKAPGSHDAAQPR & 0.9242 & 0.0417 \\
\hline A0A0G2JZY3 & Reps1 & $S$ & 430 & 1 & GDHTNPTSPLLVKPS & -0.3629 & \\
\hline A0A0G2JZY3 & Reps1 & $S$ & 430 & 2 & GDHTNPTSPLLVKPS & -0.4985 & \\
\hline A0A0G2JZY3 & Reps1 & $T$ & 492 & 2 & SGASPDNTAPPPPPP & -0.3711 & 0.0837 \\
\hline A0A0G2K1L4 & Reps2 & $S$ & 407 & 1 & KTHSRASSLDLNKVF & 0.5464 & 0.1208 \\
\hline A0A0G2K326 & Rgs12 & $S$ & 477 & 1 & GAPVKQSSAVNSSPR & 1.7546 & 0.0396 \\
\hline A0A0G2K4J8 & Rimbp2 & S & 554 & 1 & STGPGRRSPSPSRIL & -1.8636 & \\
\hline A0A0G2K4J8 & Rimbp2 & $S$ & 684 & 1 & DGGRRRPSGTSHNAL & 0.5820 & 0.0562 \\
\hline A0A0G2K4J8 & Rimbp2 & $S$ & 951 & 1 & EHVSRRYSHSGGGPQ & 0.7729 & -0.1000 \\
\hline A0A0G2K4J8 & Rimbp2 & $S$ & 1096 & 1 & RRSGRGHSVPTRRMV & 1.2722 & 0.1918 \\
\hline A0A0G2JT77 & Rims1 & $S$ & 95 & 1 & VCVPRKPSSEEGGPE & 1.4727 & -0.0646 \\
\hline A0A0G2JT77 & Rims1 & $S$ & 96 & 1 & CVPRKPSSEEGGPER & 0.9897 & \\
\hline A0A0G2JT77 & Rims1 & S & 241 & 2 & RLQERSRSQTPLSTA & 0.3231 & -0.0551 \\
\hline A0A0G2JT77 & Rims1 & $S$ & 346 & 1 & RRLEKGRSQDYSDRP & 2.0172 & -0.0242 \\
\hline A0A0G2JT77 & Rims1 & $S$ & 413 & 1 & ARHERRHSDVALPHT & 0.3195 & -0.0491 \\
\hline A0A0G2JT77 & Rims1 & S & 723 & 1 & SSHPPLESSSSSFES & 0.5020 & -0.1555 \\
\hline A0A0G2JT77 & Rims1 & $S$ & 723 & 2 & SSHPPLESSSSSFES & 0.2918 & \\
\hline A0A0G2JT77 & Rims1 & $S$ & 726 & 2 & PPLESSSSSFESQKM & 0.3260 & \\
\hline A0A0G2JT77 & Rims1 & S & 742 & 2 & RPSISVISPTSPGAL & -0.6754 & \\
\hline A0A0G2JT77 & Rims1 & $S$ & 745 & 2 & ISVISPTSPGALKDA & -0.5236 & \\
\hline A0A0G2JT77 & Rims1 & $S$ & 921 & 2 & RSQRISDSDISDYEV & -0.3378 & -0.0353 \\
\hline A0A0G2JT77 & Rims1 & S & 921 & 3 & RSQRISDSDISDYEV & -0.2802 & \\
\hline A0A0G2JT77 & Rims1 & $S$ & 924 & 2 & RISDSDISDYEVDDG & -0.4309 & -0.0358 \\
\hline A0A0G2JT77 & Rims1 & $S$ & 924 & 3 & RISDSDISDYEVDDG & -0.2802 & \\
\hline A0A0G2JT77 & Rims1 & $Y$ & 926 & 2 & SDSDISDYEVDDGIG & -0.2682 & 0.0200 \\
\hline A0A0G2JT77 & Rims1 & Y & 926 & 3 & SDSDISDYEVDDGIG & -0.2717 & \\
\hline A0A0G2JT77 & Rims1 & $S$ & 965 & 2 & QRTTHHRSRSVSPHR & -0.6436 & \\
\hline A0A0G2JT77 & Rims1 & S & 967 & 2 & TTHHRSRSVSPHRGD & -0.5165 & \\
\hline A0A0G2JT77 & Rims1 & $S$ & 969 & 2 & HHRSRSVSPHRGDDQ & -0.5674 & \\
\hline A0A0G2JT77 & Rims1 & $S$ & 991 & 1 & PNVPLQRSLDEIHPT & 0.6805 & 0.0579 \\
\hline A0A0G2JT77 & Rims1 & S & 1045 & 1 & PRAKRGRSAESLHMT & -0.2783 & 0.0225 \\
\hline A0A0G2JT77 & Rims1 & S & 1078 & 2 & RPDTSLHSPERERHS & -0.6512 & \\
\hline A0A0G2JT77 & Rims1 & $S$ & 1202 & 1 & QVPVRSGSIEQASLV & 0.5469 & -0.2321 \\
\hline A0A0G2JT77 & Rims1 & $S$ & 1233 & 1 & QTTGSGSSQELDHEQ & 0.9811 & -0.0565 \\
\hline A0A0G2JT77 & Rims1 & $\mathrm{s}$ & 1355 & 1 & GGKKRRSSLSAKVVA & 0.4156 & -0.2329 \\
\hline
\end{tabular}




\begin{tabular}{|c|c|c|c|c|c|c|c|}
\hline A0A0G2JT77 & Rims1 & S & 1408 & 2 & RKMVRQPSRESTDGS & 0.7779 & -0.0024 \\
\hline Q9JIS1 & Rims2 & S & 432 & 2 & PQRTTNHSPPTPRRS & -0.4042 & \\
\hline Q9JIS1 & Rims2 & $\mathrm{T}$ & 435 & 2 & TTNHSPPTPRRSPIP & -0.4138 & \\
\hline Q9JIS1 & Rims2 & $S$ & 482 & 2 & ETMLRNDSLSSDQSE & 0.7483 & -0.0822 \\
\hline Q9JIS1 & Rims2 & $S$ & 484 & 2 & MLRNDSLSSDQSESV & 0.7508 & -0.0708 \\
\hline Q9JIS1 & Rims2 & $\mathrm{S}$ & 485 & 2 & LRNDSLSSDQSESVR & 0.7912 & -0.0439 \\
\hline Q9JIS1 & Rims2 & S & 1540 & 2 & APLTRRASQSSLESS & 0.4463 & 0.0158 \\
\hline Q9JIS1 & Rims2 & $S$ & 1542 & 2 & LTRRASQSSLESSTG & 0.3900 & \\
\hline Q9JIS1 & Rims2 & $\mathrm{s}$ & 1546 & 2 & ASQSSLESSTGPSYS & 0.4419 & -0.0556 \\
\hline P47709 & Rph3a & S & 274 & 1 & QGLRRANSVQASRPA & 0.7613 & -0.0214 \\
\hline Q9JK11 & Rtn4 & $\mathrm{s}$ & 425 & 1 & EGRNEDASFPSTPEP & 0.2691 & 0.1528 \\
\hline Q9JK11 & Rtn4 & $S$ & 425 & 2 & EGRNEDASFPSTPEP & -0.8392 & -0.0996 \\
\hline Q9JK11 & Rtn4 & $S$ & 428 & 2 & NEDASFPSTPEPVKD & -0.7237 & -0.1071 \\
\hline Q9JK11 & Rtn4 & $\mathrm{T}$ & 429 & 1 & EDASFPSTPEPVKDS & -0.2717 & \\
\hline Q9JK11 & Rtn4 & $\mathrm{T}$ & 429 & 2 & EDASFPSTPEPVKDS & -0.8198 & -0.0852 \\
\hline F1LRZ9 & Rundc3a & S & 374 & 2 & SKLFRRHSFMSTEPL & -0.3006 & -0.1246 \\
\hline F1LRZ9 & Rundc3a & $\mathrm{s}$ & 377 & 2 & FRRHSFMSTEPLSAE & -0.3006 & -0.1464 \\
\hline Q5BJU3 & Samd14 & $\mathrm{s}$ & 151 & 1 & SAPSSDSSPSFVRRY & -0.3363 & \\
\hline P04774 & Scn1a & S & 620 & 1 & RHGERRNSNLSQTSR & 0.3643 & 0.0989 \\
\hline F1M9G9 & Scn2a & $\mathrm{s}$ & 531 & 1 & RKSASEDSIRKKGFR & 0.6118 & -0.0418 \\
\hline F1M9G9 & Scn2a & $\mathrm{S}$ & 553 & 3 & LTYEKRFSSPHQSLL & -0.3738 & \\
\hline F1M9G9 & Scn2a & S & 554 & 3 & TYEKRFSSPHQSLLS & -0.4317 & \\
\hline F1M9G9 & Scn2a & $\mathrm{S}$ & 558 & 3 & RFSSPHQSLLSIRGS & -0.5601 & \\
\hline F1M9G9 & Scn2a & $\mathrm{s}$ & 561 & 3 & SPHQSLLSIRGSLFS & -0.4317 & \\
\hline F1M9G9 & Scn2a & S & 623 & 1 & RHGERRPSNVSQASR & 0.2891 & -0.0487 \\
\hline F1M9G9 & Scn2a & $\mathrm{s}$ & 629 & 1 & PSNVSQASRASRGIP & 0.5120 & -0.1655 \\
\hline F1M9G9 & Scn2a & $\mathrm{Y}$ & 689 & 2 & RKRRSSSYHVSMDLL & -0.5081 & \\
\hline F1M9G9 & Scn2a & $\mathrm{S}$ & 692 & 2 & RSSSYHVSMDLLEDP & -0.6492 & -0.1148 \\
\hline A0A0G2JVC2 & Scyl2 & S & 681 & 1 & QGKQKRGSLTLEEKQ & 0.2911 & 0.0096 \\
\hline A0A096MJN4 & 4-Sep & S & 413 & 1 & RNKLTRESGTDFPIP & -0.3381 & -0.0250 \\
\hline D3ZDH8 & 5-Sep & S & 17 & 1 & RLVEQLLSPRTQAQR & -1.9315 & \\
\hline D3ZDH8 & 5-Sep & S & 336 & 2 & TQDSRMESPIPILPL & -0.6566 & \\
\hline D3ZDH8 & 5-Sep & $\mathrm{T}$ & 345 & 1 & IPILPLPTPDSETEK & -0.4997 & \\
\hline D3ZDH8 & 5-Sep & $\mathrm{T}$ & 345 & 2 & IPILPLPTPDSETEK & -0.6524 & \\
\hline A0A0U1RRT8 & 6-Sep & $S$ & 411 & 1 & ELLQSQGSQAGGSQT & 0.3933 & -0.0665 \\
\hline Q9WVC0 & 7-Sep & $\mathrm{s}$ & 422 & 1 & RILEQQNSSRTLEKN & 0.4303 & -0.1912 \\
\hline P0DJJ3 & Sgip1 & $S$ & 142 & 2 & TVDELKASIGNIALS & -0.6045 & -0.1055 \\
\hline P0DJJ3 & Sgip1 & S & 149 & 2 & SIGNIALSPSPVRKS & -1.1080 & -0.0493 \\
\hline PODJJ3 & Sgip1 & $\mathrm{s}$ & 151 & 2 & GNIALSPSPVRKSPR & -1.1335 & \\
\hline P0DJJ3 & Sgip1 & $S$ & 160 & 1 & VRKSPRRSPGAIKRN & -0.6349 & -0.1676 \\
\hline P0DJJ3 & Sgip1 & $S$ & 289 & 2 & IEKLPSISDLDSIFG & -0.2668 & 0.0222 \\
\hline PODJJ3 & Sgip1 & $\mathrm{s}$ & 371 & 1 & PGPRHVPSPLNLEEV & -0.4934 & -0.0077 \\
\hline D3ZAS2 & Sgsm1 & $S$ & 229 & 3 & LCIQKRHSSGSMDDR & 0.8637 & \\
\hline D3ZAS2 & Sgsm1 & $S$ & 230 & 3 & CIQKRHSSGSMDDRP & 0.8637 & \\
\hline
\end{tabular}




\begin{tabular}{|c|c|c|c|c|c|c|c|}
\hline D3ZAS2 & Sgsm1 & $S$ & 232 & 1 & QKRHSSGSMDDRPSI & 0.8959 & -0.0640 \\
\hline D3ZAS2 & Sgsm1 & S & 232 & 3 & QKRHSSGSMDDRPSI & 0.8637 & \\
\hline D3ZAS2 & Sgsm1 & $S$ & 406 & 2 & QGSSESTSSDKEDDE & 0.6522 & \\
\hline D3ZAS2 & Sgsm1 & $S$ & 407 & 2 & GSSESTSSDKEDDEA & 0.6508 & \\
\hline 070593 & Sgta & $S$ & 302 & 1 & IRSQVVRSRTPSASH & -0.5427 & \\
\hline Q80W98 & Sgtb & $S$ & 299 & 1 & RSRSFSSSTEEHS & 0.3234 & \\
\hline Q80W98 & Sgtb & $T$ & 300 & 1 & SRSFSSSTEEHS & 0.3110 & \\
\hline Q80W98 & Sgtb & $S$ & 304 & 2 & SSSTEEHS & 0.5086 & \\
\hline O35964 & Sh3gl1 & $S$ & 288 & 1 & PKITASSSFRSGDKP & -0.6301 & -0.0967 \\
\hline Q925Q9 & Sh3kbp1 & $S$ & 274 & 1 & PIKLRPRSIEVENDF & 0.3344 & 0.0139 \\
\hline A0A0G2JX92 & Sh3pxd2a & $S$ & 1014 & 1 & RLAERAASQGSESPL & 0.3855 & -0.0660 \\
\hline Q9QX74 & Shank2 & $\mathrm{T}$ & 903 & 1 & GPLRRQETENKYETD & 0.8834 & 0.1716 \\
\hline Q7TP36 & Shroom2 & $S$ & 918 & 1 & STMETSRSPSPQFAP & -0.3903 & 0.1520 \\
\hline Q7TP36 & Shroom2 & $S$ & 920 & 1 & METSRSPSPQFAPQK & -0.3751 & 0.0945 \\
\hline Q7TP36 & Shroom2 & $S$ & 996 & 1 & IKIVHSESQPEKESR & 1.3409 & 0.0149 \\
\hline A0A0G2KAW2 & Sipa111 & $S$ & 288 & 1 & PLKRRSKSETGDSSI & 0.4454 & \\
\hline A0A0G2KAW2 & Sipa111 & $\mathrm{T}$ & 1190 & 1 & ASIDRQNTQSDIGGS & 0.9166 & 0.0176 \\
\hline A0A0G2KAW2 & Sipa111 & $S$ & 1192 & 1 & IDRQNTQSDIGGSGK & 0.9247 & \\
\hline A0A0G2KAW2 & Sipa111 & $S$ & 1384 & 1 & SKSQGGSSPLTRENS & -0.5853 & 0.0600 \\
\hline E9PTX9 & Slc12a2 & $S$ & 73 & 1 & KPLGPTPSQSRFQVD & -0.6815 & 0.0464 \\
\hline O54701 & SIc24a2 & $\mathrm{s}$ & 334 & 1 & PRLQRGGSSASLHNS & 0.2874 & -0.1903 \\
\hline O54701 & Slc24a2 & $\mathrm{S}$ & 337 & 2 & QRGGSSASLHNSLMR & 0.6074 & 0.0371 \\
\hline O54701 & Slc24a2 & $S$ & 341 & 2 & SSASLHNSLMRNSIF & 0.5503 & 0.0358 \\
\hline Q921A2 & Slc2a13 & S & 6 & 1 & MSRKASEDVEYTL & 0.3491 & -0.1206 \\
\hline Q921A2 & SIc2a13 & $S$ & 624 & 3 & EYIRVKGSNYHLSDN & -0.9079 & \\
\hline Q921A2 & Slc2a13 & $S$ & 629 & 3 & KGSNYHLSDNDASDV & -0.9308 & \\
\hline Q921A2 & SIc2a13 & S & 634 & 3 & HLSDNDASDVE & -0.9308 & \\
\hline Q6QIX3 & Slc30a3 & $\mathrm{T}$ & 35 & 2 & LRLKSLFTEPSEPLP & 2.1253 & -0.1362 \\
\hline Q6QIX3 & Slc30a3 & $S$ & 38 & 2 & KSLFTEPSEPLPEGP & 2.1744 & -0.1389 \\
\hline D4A517 & Slc39a10 & $\mathrm{T}$ & 538 & 1 & KWFMKQSTEESTIGR & 0.2640 & \\
\hline $\mathrm{AOAOH} 2 \mathrm{UHB} 7$ & Slc4a4 & $\mathrm{S}$ & 261 & 1 & THRNLTSSSLNDISD & -0.7861 & 0.1430 \\
\hline A0AOH2UHB7 & Slc4a4 & $S$ & 261 & 2 & THRNLTSSSLNDISD & -0.8475 & -0.1600 \\
\hline $\mathrm{AOAOH} 2 \mathrm{UHB} 7$ & Slc4a4 & S & 262 & 2 & HRNLTSSSLNDISDK & -0.7890 & -0.2629 \\
\hline Q9R1N3 & Slc4a7 & $S$ & 238 & 1 & SRIPLVRSFADIGKK & -0.8533 & -0.0336 \\
\hline P31662 & Slc6a17 & $S$ & 13 & 2 & KVTQREHSNEHVTES & 0.3012 & -0.0643 \\
\hline P31662 & Slc6a17 & $\mathrm{S}$ & 20 & 2 & SNEHVTESVADLLAL & 0.3383 & -0.0506 \\
\hline P31662 & Slc6a17 & $\mathrm{S}$ & 665 & 1 & GRMMKDISNLEENDE & -0.7038 & 0.0260 \\
\hline P31662 & Slc6a17 & $S$ & 682 & 2 & FILSKVPSEAPSPMP & -0.5428 & 0.0727 \\
\hline P31662 & SIc6a17 & $\mathrm{s}$ & 686 & 2 & KVPSEAPSPMPTHRS & -0.5428 & 0.0847 \\
\hline P31662 & Slc6a17 & $S$ & 715 & 2 & HPNGRYGSGYLLAST & -1.2328 & \\
\hline P31662 & Slc6a17 & $T$ & 722 & 2 & SGYLLASTPESEL & -1.0214 & -0.0431 \\
\hline P31662 & Slc6a17 & S & 725 & 2 & LLASTPESEL & -0.6762 & -0.0715 \\
\hline P26431 & Slc9a1 & $S$ & 606 & 2 & GKIPSAVSTVSMQNI & -0.9830 & \\
\hline P26431 & Slc9a1 & $\mathrm{T}$ & 607 & 2 & KIPSAVSTVSMQNIH & -0.9009 & -0.0935 \\
\hline
\end{tabular}




\begin{tabular}{|c|c|c|c|c|c|c|c|}
\hline P26431 & Slc9a1 & S & 609 & 2 & PSAVSTVSMQNIHPK & -0.9693 & -0.0406 \\
\hline P26431 & Slc9a1 & $T$ & 784 & 2 & PGTDDVFTPGPSDSP & -0.8272 & \\
\hline P26431 & Slc9a1 & S & 790 & 2 & FTPGPSDSPGSQRIQ & -0.7406 & -0.0930 \\
\hline D3ZJR6 & Smcr8 & $S$ & 488 & 1 & SVLSKSDSQASLTVP & 0.4111 & -0.1000 \\
\hline D3ZJR6 & Smcr8 & $S$ & 488 & 3 & SVLSKSDSQASLTVP & -0.3019 & \\
\hline D3ZJR6 & Smcr8 & $\mathrm{s}$ & 491 & 3 & SKSDSQASLTVPLSP & -0.3019 & \\
\hline D3ZJR6 & Smcr8 & S & 497 & 3 & ASLTVPLSPHVVRSK & -0.3019 & \\
\hline A0A0G2K0B6 & Snap91 & S & 601 & 1 & SSPPRGASPVPESSL & -0.7133 & 0.0110 \\
\hline AOA0G2K0B6 & Snap91 & S & 601 & 2 & SSPPRGASPVPESSL & -1.6128 & -0.0318 \\
\hline AOA0G2K0B6 & Snap91 & $\mathrm{S}$ & 601 & 3 & SSPPRGASPVPESSL & -1.1729 & -0.2366 \\
\hline A0A0G2K0B6 & Snap91 & $\mathrm{s}$ & 614 & 1 & SLTADLLSVDAFAAP & -0.6628 & 0.0201 \\
\hline AOA0G2K0B6 & Snap91 & S & 614 & 2 & SLTADLLSVDAFAAP & -1.0138 & -0.0410 \\
\hline A0A0G2K0B6 & Snap91 & S & 614 & 3 & SLTADLLSVDAFAAP & -1.1190 & -0.2085 \\
\hline A0A0G2K0B6 & Snap91 & $S$ & 625 & 2 & FAAPSPASTASPAKA & -0.9133 & \\
\hline AOA0G2K0B6 & Snap91 & S & 628 & 2 & PSPASTASPAKAESS & -1.5278 & \\
\hline AOA0G2K0B6 & Snap91 & $\mathrm{S}$ & 628 & 3 & PSPASTASPAKAESS & -1.2359 & -0.2366 \\
\hline AOA0G2JXH9 & Snx11 & S & 197 & 1 & WAQEERQSTSHLAKG & 0.2744 & -0.1758 \\
\hline P57769 & Snx16 & S & 29 & 1 & NRNQRSSSFGSVSTS & 0.2668 & -0.0638 \\
\hline B2RYP4 & Snx2 & $\mathrm{S}$ & 117 & 1 & APRIESKSISAPVIF & -0.8319 & \\
\hline D4A0A1 & Soga3 & S & 748 & 1 & IRGSPRDSDAESDAG & 0.3034 & \\
\hline $\mathrm{F} 1 \mathrm{M} 820$ & Sorbs1 & S & 54 & 3 & SYRETPSSSPVSPQE & -0.3778 & 0.2501 \\
\hline F1M820 & Sorbs1 & S & 250 & 2 & DDDSDLHSPRYSFSE & -0.3521 & 0.0407 \\
\hline F1M820 & Sorbs1 & $S$ & 254 & 1 & DLHSPRYSFSEDTKS & 0.7750 & \\
\hline F1M820 & Sorbs1 & S & 254 & 2 & DLHSPRYSFSEDTKS & -0.3350 & 0.0246 \\
\hline F1M820 & Sorbs1 & $S$ & 256 & 1 & HSPRYSFSEDTKSPL & 0.8683 & \\
\hline F1M820 & Sorbs1 & $\mathrm{S}$ & 256 & 2 & HSPRYSFSEDTKSPL & -0.3374 & \\
\hline F1M820 & Sorbs1 & S & 670 & 1 & LGASQEGSEHIPKHT & 0.2737 & \\
\hline F1M820 & Sorbs1 & $S$ & 1209 & 1 & PSRSATVSPQQPQAQ & -0.6414 & 0.1675 \\
\hline B2RYN7 & Spast & $S$ & 222 & 1 & KTVMKSGSTGLSGHH & 1.1860 & 0.0135 \\
\hline D3ZEX7 & Spire1 & S & 458 & 2 & QPPQRRHSIEKETPT & 0.7841 & 0.0221 \\
\hline Q3C2P9 & Spred1 & S & 239 & 1 & LKSIRHVSFQDEDEI & 0.5939 & \\
\hline Q5HZA2 & Spry2 & $S$ & 111 & 1 & AALSRSVSTVSSGSR & 0.6266 & \\
\hline Q9QXY2 & Srcin1 & $\mathrm{T}$ & 64 & 1 & EYPREYRTLGGGGSG & -0.4775 & -0.1177 \\
\hline Q9QXY2 & Srcin1 & $S$ & 79 & 1 & GSGGRRFSNVGLVHT & 0.3116 & -0.0698 \\
\hline Q9QXY2 & Srcin1 & $S$ & 175 & 3 & AAKLSYASAESLETM & -0.3806 & \\
\hline Q9QXY2 & Srcin1 & $\mathrm{S}$ & 178 & 2 & LSYASAESLETMSEA & -0.2710 & 0.0553 \\
\hline Q9QXY2 & Srcin 1 & $S$ & 183 & 2 & AESLETMSEAELPLG & -0.3012 & 0.0481 \\
\hline Q9QXY2 & Srcin1 & S & 366 & 3 & SPSRSRLSYAGGRPP & -0.4813 & -0.1158 \\
\hline Q9QXY2 & Srcin1 & S & 374 & 3 & YAGGRPPSYAGSPVH & -0.4858 & -0.0681 \\
\hline Q9QXY2 & Srcin 1 & $S$ & 378 & 3 & RPPSYAGSPVHHAAE & -0.4413 & -0.0479 \\
\hline Q9QXY2 & Srcin1 & S & 393 & 2 & RLGGAPTSQGVSPSP & -0.8241 & -0.1394 \\
\hline Q9QXY2 & Srcin1 & S & 397 & 1 & APTSQGVSPSPSAIL & -0.5616 & -0.1322 \\
\hline Q9QXY2 & Srcin1 & $S$ & 397 & 2 & APTSQGVSPSPSAIL & -0.6345 & -0.1736 \\
\hline Q9QXY2 & Srcin1 & S & 399 & 2 & TSQGVSPSPSAILER & -0.5259 & \\
\hline
\end{tabular}




\begin{tabular}{|c|c|c|c|c|c|c|c|}
\hline Q9QXY2 & Srcin1 & $S$ & 401 & 2 & QGVSPSPSAILERRD & -0.6874 & -0.0950 \\
\hline Q9QXY2 & Srcin1 & S & 469 & 3 & YKRGSVRSLSTYSAA & -0.7115 & \\
\hline Q9QXY2 & Srcin1 & S & 471 & 2 & RGSVRSLSTYSAAAL & -1.3351 & \\
\hline Q9QXY2 & Srcin1 & $S$ & 471 & 3 & RGSVRSLSTYSAAAL & -0.7664 & \\
\hline Q9QXY2 & Srcin1 & $T$ & 472 & 2 & GSVRSLSTYSAAALQ & -1.3950 & \\
\hline Q9QXY2 & Srcin 1 & $T$ & 472 & 3 & GSVRSLSTYSAAALQ & -0.7925 & \\
\hline Q9QXY2 & Srcin1 & $S$ & 474 & 2 & VRSLSTYSAAALQSD & -1.3818 & \\
\hline Q9QXY2 & Srcin1 & $S$ & 474 & 3 & VRSLSTYSAAALQSD & -0.9562 & \\
\hline Q9QXY2 & Srcin1 & $\mathrm{s}$ & 480 & 2 & YSAAALQSDLEDSLY & -0.8938 & 0.0015 \\
\hline Q9QXY2 & Srcin1 & $\mathrm{s}$ & 519 & 1 & LADVSAPSGGPPPPH & -0.3956 & -0.0253 \\
\hline Q9QXY2 & Srcin1 & $\mathrm{S}$ & 527 & 1 & GGPPPPHSPYSGPPS & -0.4985 & -0.0086 \\
\hline Q9QXY2 & Srcin1 & $S$ & 527 & 3 & GGPPPPHSPYSGPPS & -0.5016 & \\
\hline Q9QXY2 & Srcin1 & $Y$ & 529 & 3 & PPPPHSPYSGPPSRG & -0.4765 & \\
\hline Q9QXY2 & Srcin1 & $S$ & 530 & 3 & PPPHSPYSGPPSRGS & -0.5306 & \\
\hline Q9QXY2 & Srcin 1 & $\mathrm{~s}$ & 534 & 3 & SPYSGPPSRGSPVRQ & -0.5080 & \\
\hline Q9QXY2 & Srcin1 & S & 537 & 3 & SGPPSRGSPVRQSFR & -0.5645 & \\
\hline Q9QXY2 & Srcin1 & $S$ & 547 & 1 & RQSFRKDSGSSSVFA & 0.2939 & 0.1041 \\
\hline Q9QXY2 & Srcin1 & $S$ & 549 & 1 & SFRKDSGSSSVFAES & 0.4027 & 0.2027 \\
\hline Q9QXY2 & Srcin1 & S & 551 & 2 & RKDSGSSSVFAESPG & -0.3149 & -0.1370 \\
\hline Q9QXY2 & Srcin1 & $\mathrm{S}$ & 654 & 2 & VCGSGSRSSGATPVS & -0.3337 & \\
\hline Q9QXY2 & Srcin1 & $\mathrm{T}$ & 915 & 2 & LSGPAEGTPLTPKSG & -0.4000 & 0.2483 \\
\hline Q9QXY2 & Srcin1 & $\mathrm{T}$ & 918 & 2 & PAEGTPLTPKSGNPT & -0.5858 & \\
\hline Q9QXY2 & Srcin1 & $S$ & 941 & 1 & RNMDKAVSVEAAERD & 1.8802 & \\
\hline Q9QXY2 & Srcin1 & $S$ & 1077 & 1 & VFIKKAESEELEIQK & 1.2660 & -0.0746 \\
\hline Q9QXY2 & Srcin1 & $\mathrm{S}$ & 1094 & 1 & VKLRRAVSEVVRPAS & 1.1119 & -0.2221 \\
\hline D4A208 & Srgap2 & $T$ & 989 & 2 & TSPVVAPTSEPSSPL & -0.5533 & 0.1469 \\
\hline D4A208 & Srgap2 & $\mathrm{s}$ & 990 & 2 & SPVVAPTSEPSSPLH & -0.5762 & 0.1763 \\
\hline D4A208 & Srgap2 & $\mathrm{S}$ & 994 & 2 & APTSEPSSPLHTQLL & -0.5865 & 0.1820 \\
\hline F1M5M9 & Srgap3 & $S$ & 948 & 2 & CGSTRHSSLGDHKSL & 1.3223 & -0.0092 \\
\hline F1M5M9 & Srgap3 & S & 954 & 2 & SSLGDHKSLEAEALA & 1.3223 & -0.0092 \\
\hline F1M5M9 & Srgap3 & S & 1078 & 2 & SSSSGVGSPAVTPTE & -0.9918 & \\
\hline F1M5M9 & Srgap3 & $\mathrm{T}$ & 1082 & 2 & GVGSPAVTPTEKMFP & -0.9918 & \\
\hline Q5XIS1 & Ssh3 & $\mathrm{s}$ & 642 & 1 & RKVIRQASVDDSREE & 0.4781 & \\
\hline Q08013 & Ssr3 & $S$ & 105 & 1 & KEVTRKLSEADNRKM & 0.2809 & -0.1897 \\
\hline D4A3D9 & Stk32c & $S$ & 10 & 2 & SGAERRGSSAAAPPG & 0.4574 & 0.0092 \\
\hline D4A3D9 & Stk32c & $\mathrm{s}$ & 18 & 2 & SAAAPPGSPPPGRAR & 0.4525 & 0.0092 \\
\hline $\mathrm{P} 13668$ & Stmn1 & $S$ & 16 & 1 & KELEKRASGQAFELI & 1.3643 & 0.0961 \\
\hline $\mathrm{P} 13668$ & Stmn1 & S & 31 & 2 & LSPRSKESVPEFPLS & 0.4947 & \\
\hline $\mathrm{P} 13668$ & Stmn1 & $\mathrm{S}$ & 38 & 2 & SVPEFPLSPPKKKDL & 0.5087 & \\
\hline A0A0G2JT26 & Strip2 & $S$ & 326 & 2 & VKSMRAASPPSYTLD & 0.6614 & \\
\hline G3V7P1 & Stx12 & $S$ & 142 & 1 & ARAGSRLSAEDRQRE & 0.3469 & -0.0691 \\
\hline Q9WU70 & Stxbp5 & $\mathrm{s}$ & 1059 & 1 & LFGGGAQSLDREELF & 0.9383 & 0.0859 \\
\hline D3ZU84 & Stxbp5I & $S$ & 493 & 2 & SQLPSSRSLSGSTNT & -0.5027 & -0.0735 \\
\hline D3ZU84 & Stxbp5I & $\mathrm{s}$ & 497 & 1 & SSRSLSGSTNTVSSE & -0.3126 & -0.0733 \\
\hline
\end{tabular}




\begin{tabular}{|c|c|c|c|c|c|c|c|}
\hline D3ZU84 & Stxbp5I & $S$ & 497 & 2 & SSRSLSGSTNTVSSE & -0.4067 & -0.1413 \\
\hline D3ZU84 & Stxbp5I & $\mathrm{T}$ & 500 & 2 & SLSGSTNTVSSEGVT & -0.3213 & \\
\hline D3ZU84 & Stxbp5I & S & 502 & 2 & SGSTNTVSSEGVTKD & -0.3182 & -0.1253 \\
\hline D3ZU84 & Stxbp5I & $S$ & 503 & 2 & GSTNTVSSEGVTKDS & -0.3509 & -0.0382 \\
\hline D3ZU84 & Stxbp5I & $S$ & 675 & 1 & CSSGKRLSSADVSKV & 0.5052 & 0.0447 \\
\hline D3ZSC1 & Susd5 & $\mathrm{s}$ & 237 & 1 & EKAQEDASDETPKQD & 0.2658 & \\
\hline Q02563 & Sv2a & $S$ & 42 & 1 & DRVQDEYSRRSYSRF & 0.6222 & \\
\hline Q02563 & Sv2a & $S$ & 45 & 1 & QDEYSRRSYSRFEEE & -0.3599 & -0.0408 \\
\hline P09951 & Syn1 & $\mathrm{s}$ & 9 & 1 & NYLRRRLSDSNFMAN & 0.3192 & -0.0788 \\
\hline P09951 & Syn1 & $\mathrm{s}$ & 62 & 1 & STAAPVASPAAPSPG & -0.5561 & -0.0318 \\
\hline P09951 & Syn1 & $S$ & 62 & 2 & STAAPVASPAAPSPG & -1.4201 & 0.0531 \\
\hline P09951 & Syn1 & $S$ & 62 & 3 & STAAPVASPAAPSPG & -1.0097 & \\
\hline P09951 & Syn1 & S & 67 & 2 & VASPAAPSPGSSGGG & -1.3581 & 0.0372 \\
\hline P09951 & Syn1 & $S$ & 67 & 3 & VASPAAPSPGSSGGG & -1.0860 & \\
\hline P09951 & Syn1 & $\mathrm{s}$ & 70 & 2 & PAAPSPGSSGGGGFF & -1.1595 & 0.0202 \\
\hline P09951 & Syn1 & S & 70 & 3 & PAAPSPGSSGGGGFF & -1.1327 & \\
\hline P09951 & Syn1 & $S$ & 71 & 2 & AAPSPGSSGGGGFFS & -1.2159 & 0.0163 \\
\hline P09951 & Syn1 & $S$ & 71 & 3 & AAPSPGSSGGGGFFS & -1.0399 & \\
\hline P09951 & Syn1 & S & 79 & 3 & GGGGFFSSLSNAVKQ & -0.2704 & \\
\hline P09951 & Syn1 & $\mathrm{S}$ & 425 & 3 & LPRQRDASPGRGSHS & -1.0298 & -0.1863 \\
\hline P09951 & Syn1 & S & 430 & 3 & DASPGRGSHSQTPSP & -0.5680 & -0.1792 \\
\hline P09951 & Syn1 & S & 432 & 2 & SPGRGSHSQTPSPGA & -0.7130 & -0.1195 \\
\hline P09951 & Syn1 & $S$ & 432 & 3 & SPGRGSHSQTPSPGA & -0.7759 & -0.2208 \\
\hline P09951 & Syn1 & $T$ & 434 & 2 & GRGSHSQTPSPGALP & -0.4780 & -0.0788 \\
\hline P09951 & Syn1 & $T$ & 434 & 3 & GRGSHSQTPSPGALP & -0.8073 & -0.1732 \\
\hline P09951 & Syn1 & $S$ & 436 & 1 & GSHSQTPSPGALPLG & -0.7530 & -0.0951 \\
\hline P09951 & Syn1 & $\mathrm{s}$ & 436 & 2 & GSHSQTPSPGALPLG & -0.6050 & -0.0617 \\
\hline P09951 & Syn1 & $\mathrm{S}$ & 436 & 3 & GSHSQTPSPGALPLG & -0.5554 & -0.2015 \\
\hline P09951 & Syn1 & $S$ & 447 & 1 & LPLGRQTSQQPAGPP & 1.3955 & 0.1226 \\
\hline P09951 & Syn1 & S & 500 & 1 & GLGPPAGSPLPQRLP & -0.3305 & 0.0446 \\
\hline P09951 & Syn1 & S & 508 & 1 & PLPQRLPSPTAAPQQ & -0.9879 & -0.0614 \\
\hline P09951 & Syn1 & $\mathrm{T}$ & 510 & 1 & PQRLPSPTAAPQQSA & -0.8974 & -0.0016 \\
\hline P09951 & Syn1 & $\mathrm{s}$ & 549 & 1 & PAARPPASPSPQRQA & -0.6319 & 0.0417 \\
\hline P09951 & Syn1 & $S$ & 549 & 2 & PAARPPASPSPQRQA & -1.5076 & 0.1425 \\
\hline P09951 & Syn1 & $\mathrm{T}$ & 562 & 1 & QAGPPQATRQASISG & 0.8627 & \\
\hline P09951 & Syn1 & $\mathrm{s}$ & 566 & 1 & PQATRQASISGPAPP & 1.7972 & 0.1393 \\
\hline P09951 & Syn1 & $S$ & 568 & 1 & ATRQASISGPAPPKV & 2.0113 & 0.1755 \\
\hline P09951 & Syn1 & S & 579 & 1 & PPKVSGASPGGQQRQ & -0.6100 & -0.0092 \\
\hline P09951 & Syn1 & $\mathrm{S}$ & 603 & 1 & AGPIRQASQAGPGPR & 1.5423 & 0.1331 \\
\hline P09951 & Syn1 & $T$ & 611 & 1 & QAGPGPRTGPPTTQQ & 0.4087 & -0.0796 \\
\hline P09951 & Syn1 & $T$ & 615 & 1 & GPRTGPPTTQQPRPS & 0.3944 & -0.1212 \\
\hline P09951 & Syn1 & $\mathrm{s}$ & 622 & 1 & TTQQPRPSGPGPAGR & 0.4911 & \\
\hline P09951 & Syn1 & $S$ & 664 & 1 & PQLNKSQSLTNAFNL & 0.6129 & -0.0265 \\
\hline P09951 & Syn1 & s & 697 & 1 & TIRSLRKSFASLFSD & -0.3972 & -0.1752 \\
\hline
\end{tabular}




\begin{tabular}{|c|c|c|c|c|c|c|c|}
\hline Q63537 & Syn2 & $S$ & 546 & 1 & PQLNKSQSLTNAFSF & 0.5257 & -0.1399 \\
\hline Q63537 & Syn2 & S & 546 & 2 & PQLNKSQSLTNAFSF & 0.7289 & -0.0236 \\
\hline Q63537 & Syn2 & $\mathrm{T}$ & 548 & 1 & LNKSQSLTNAFSFSE & 0.5301 & -0.1225 \\
\hline Q63537 & Syn2 & $\mathrm{T}$ & 548 & 2 & LNKSQSLTNAFSFSE & 1.0623 & -0.0048 \\
\hline Q63537 & Syn2 & $S$ & 552 & 2 & QSLTNAFSFSESSFF & 0.7680 & -0.0813 \\
\hline Q63537 & Syn2 & $\mathrm{s}$ & 561 & 1 & SESSFFRSSANEDEA & 1.7778 & -0.2310 \\
\hline Q63537 & Syn2 & $S$ & 562 & 1 & ESSFFRSSANEDEAK & 1.7805 & -0.1544 \\
\hline O70441 & Syn3 & $S$ & 418 & 1 & PQTKSAKSPGQGQLG & -1.1508 & -0.2370 \\
\hline O70441 & Syn3 & $\mathrm{s}$ & 446 & 1 & GGPRQAQSPQPPRSR & -1.0423 & 0.1327 \\
\hline O70441 & Syn3 & S & 446 & 2 & GGPRQAQSPQPPRSR & -0.6201 & 0.0504 \\
\hline O70441 & Syn3 & $S$ & 452 & 1 & QSPQPPRSRSPSQQR & -0.4836 & 0.0018 \\
\hline 070441 & Syn3 & $S$ & 452 & 2 & QSPQPPRSRSPSQQR & -0.9806 & -0.0274 \\
\hline O70441 & Syn3 & S & 454 & 2 & PQPPRSRSPSQQRLS & -0.6167 & 0.0267 \\
\hline O70441 & Syn3 & $S$ & 456 & 2 & PPRSRSPSQQRLSPQ & -0.5685 & 0.0127 \\
\hline O70441 & Syn3 & $\mathrm{s}$ & 469 & 1 & PQGQQPVSPQSGSPQ & -0.3256 & 0.0555 \\
\hline O70441 & Syn3 & S & 480 & 1 & GSPQQQRSPGSPQLS & -0.6843 & -0.0649 \\
\hline O70441 & Syn3 & $S$ & 494 & 1 & SRASGGSSPNQASKP & -0.4893 & 0.1196 \\
\hline O70441 & Syn3 & $S$ & 519 & 1 & PVQGRSTSQQGEEPQ & 1.5310 & 0.2067 \\
\hline O70441 & Syn3 & S & 540 & 1 & PHLNKSQSLTNSLST & 0.3386 & 0.0337 \\
\hline O70441 & Syn3 & $\mathrm{S}$ & 540 & 2 & PHLNKSQSLTNSLST & 0.4842 & -0.1003 \\
\hline 070441 & Syn3 & S & 544 & 2 & KSQSLTNSLSTSDTS & 0.5491 & 0.0548 \\
\hline O70441 & Syn3 & S & 572 & 1 & TIRNLRKSFASLFSD & -0.3972 & -0.2101 \\
\hline Q9QUH6 & Syngap1 & $S$ & 1073 & 1 & PPLQRGKSQQLTVSA & 0.2739 & 0.0222 \\
\hline D4ABN3 & Synj1 & $S$ & 833 & 1 & DLDLLNASFQDESKI & 0.8075 & 0.0036 \\
\hline D4ABN3 & Synj1 & $\mathrm{S}$ & 1150 & 1 & GGVGAPPSPGVTRRE & -0.6263 & -0.0192 \\
\hline D4ABN3 & Synj1 & $\mathrm{S}$ & 1163 & 1 & REMEAPKSPGTARKD & -1.0053 & 0.1503 \\
\hline 008625 & Syt10 & $\mathrm{T}$ & 163 & 1 & ARVQRQTTDPTSSSR & 0.3970 & 0.0102 \\
\hline Q62807 & Syt17 & $\mathrm{S}$ & 110 & 2 & YSLTRRISSLDSRRP & 0.5948 & -0.1017 \\
\hline Q62807 & Syt17 & $S$ & 114 & 2 & RRISSLDSRRPSSPL & 0.5948 & -0.1017 \\
\hline Q62807 & Syt17 & S & 118 & 2 & SLDSRRPSSPLIDIK & 0.4552 & -0.0204 \\
\hline Q62807 & Syt17 & $S$ & 119 & 2 & LDSRRPSSPLIDIKP & 0.4552 & -0.0178 \\
\hline Q62746 & Syt6 & $\mathrm{T}$ & 164 & 1 & TKLQRQTTEPASSTR & 1.3254 & \\
\hline Q62746 & Syt6 & $\mathrm{s}$ & 217 & 1 & PELYKQKSVDGDEAK & 0.7705 & -0.0306 \\
\hline Q925C0 & Syt9 & $\mathrm{T}$ & 159 & 1 & VRVQRQVTEPTPSAR & 0.5742 & -0.1631 \\
\hline Q925C0 & Syt9 & S & 207 & 1 & PELYKQRSLDNDDGR & 0.4633 & \\
\hline Q812E4 & Syt15 & $\mathrm{s}$ & 211 & 1 & AESGRSYSLDLDNQN & 1.4037 & -0.0106 \\
\hline P37805 & Tagln3 & $S$ & 163 & 1 & QQNRRGFSEEQLRQG & 1.7788 & -0.1153 \\
\hline A0A0G2K9JO & Tanc2 & S & 84 & 2 & IMEGVSRSLPSSPLL & -0.4663 & -0.0720 \\
\hline A0A0G2K9J0 & Tanc2 & $\mathrm{S}$ & 88 & 2 & VSRSLPSSPLLTHQS & -0.4663 & -0.0720 \\
\hline D3ZSY8 & Tbc1d10b & $T$ & 135 & 2 & SPVPGPGTPTRTPSR & -0.4053 & \\
\hline A0A0G2JWM9 & Tbc1d22b & S & 110 & 1 & YKVIKSSSDAQLSRN & 0.2666 & 0.0153 \\
\hline A0A0G2K5B0 & Tbc1d24 & $\mathrm{s}$ & 475 & 2 & CLISHSVSSDPADRL & -0.4576 & \\
\hline A0A0G2K5B0 & Tbc1d24 & $S$ & 476 & 2 & LISHSVSSDPADRLS & -0.4576 & \\
\hline A0A0G2K5B0 & Tbc1d24 & $\mathrm{s}$ & 483 & 2 & SDPADRLSPFLAARH & -0.4632 & \\
\hline
\end{tabular}




\begin{tabular}{|c|c|c|c|c|c|c|c|}
\hline Q9R1K2 & Tenm2 & S & 142 & 1 & GIKSRRSSGLSSREN & 0.3138 & \\
\hline P04177 & Th & S & 19 & 1 & KGFRRAVSEQDAKQA & 1.9169 & -0.2432 \\
\hline P04177 & Th & S & 31 & 1 & KQAEAVTSPRFIGRR & 0.2676 & -0.1239 \\
\hline Q3ZB99 & T.jp2 & $\mathrm{T}$ & 451 & 2 & DITAAGVTEANKEPR & -0.4056 & 0.1017 \\
\hline Q3ZB99 & T.jp2 & $S$ & 459 & 2 & EANKEPRSQEESPVP & -0.3925 & \\
\hline Q3ZB99 & T.jp2 & S & 463 & 2 & EPRSQEESPVPQPRT & -0.3815 & 0.1000 \\
\hline D3ZE26 & Tmcc2 & $S$ & 166 & 1 & PSIKRGASLHSSGGS & 1.1831 & -0.1256 \\
\hline D3ZE26 & Tmcc2 & $S$ & 169 & 1 & KRGASLHSSGGSGGR & 0.9653 & 0.0872 \\
\hline P62329 & Tmsb4x & $\mathrm{T}$ & 23 & 1 & SKLKKTETQEKNPLP & 2.6314 & \\
\hline Q52KJ9 & Tmx1 & S & 245 & 1 & EADEEDVSEEETENR & 0.3320 & \\
\hline D3ZF26 & Tnks1bp1 & S & 1642 & 1 & KLRSRNRSAEEGEVT & 0.3376 & \\
\hline Q5XI21 & Tom1 & S & 376 & 1 & FALTRGSSLADQRKG & 0.8166 & 0.1267 \\
\hline A0A0G2K9L2 & Tom112 & S & 160 & 2 & MADLDALSPIHTPQR & -0.5287 & 0.0281 \\
\hline A0A0G2K9L2 & Tom112 & $\mathrm{T}$ & 164 & 2 & DALSPIHTPQRSVPE & -0.5287 & 0.0281 \\
\hline A0A0G2K9L2 & Tom112 & S & 394 & 1 & FAQTRGNSLAEQRKT & 1.4109 & 0.1900 \\
\hline Q5PQX1 & Tor1aip1 & S & 157 & 1 & LRDAQSLSEDRGEDE & 0.4179 & 0.1302 \\
\hline A0A0G2K8M7 & Tpd52l1 & $\mathrm{S}$ & 132 & 1 & FGDMRSHSIGYSIRH & 0.7744 & -0.1634 \\
\hline Q6РCT3 & Tpd5212 & S & 180 & 1 & RNSATFKSFEDRVGT & 0.4152 & 0.2387 \\
\hline A0A0G2K2D6 & Tppp & $\mathrm{T}$ & 32 & 2 & PTKAANKTPPKSPGD & -0.5796 & 0.1563 \\
\hline A0A0G2K2D6 & Tppp & S & 36 & 1 & ANKTPPKSPGDPAKA & -0.4514 & 0.1090 \\
\hline A0A0G2K2D6 & Tppp & S & 36 & 2 & ANKTPPKSPGDPAKA & -0.5796 & 0.1563 \\
\hline D3ZE49 & Trappc12 & S & 314 & 1 & EKSPDSTSPSYSTRM & -1.3229 & \\
\hline D3ZQG6 & Trim2 & $S$ & 424 & 1 & FKLKVIRSADVSPTT & 0.2932 & -0.1190 \\
\hline D3ZQG6 & Trim2 & S & 456 & 2 & KAVKRPASMYSTGKR & -0.4262 & \\
\hline D3ZQG6 & Trim2 & $S$ & 459 & 2 & KRPASMYSTGKRKEN & -0.4262 & \\
\hline $\mathrm{F} 1 \mathrm{M} 0 \mathrm{Z} 1$ & Trio & $\mathrm{T}$ & 2432 & 1 & ALGSTSGTSQDGNTK & 0.8762 & \\
\hline F1M0Z1 & Trio & S & 2459 & 2 & KTRPGAVSPLNSPLS & -0.4473 & 0.0960 \\
\hline F1M0Z1 & Trio & $S$ & 2463 & 2 & GAVSPLNSPLSTTFP & -0.3520 & 0.0279 \\
\hline F1M0Z1 & Trio & $S$ & 2466 & 2 & SPLNSPLSTTFPSPF & -0.3333 & 0.0459 \\
\hline F1LP64 & Trip12 & S & 1111 & 1 & SGLARAASKDTISNN & 0.4878 & 0.0135 \\
\hline Q9Z136 & Tsc1 & $S$ & 515 & 1 & YRDSLSGSQRKTHSA & 0.5402 & 0.0371 \\
\hline D3ZLW4 & Tsc2 & $\mathrm{T}$ & 1383 & 2 & LGDLGDKTDIGRLSP & -0.2735 & \\
\hline D3ZLW4 & Tsc2 & S & 1432 & 1 & LPSSSPRSPSGLRPR & -0.7143 & 0.0609 \\
\hline D3ZSP7 & Ttc3 & $S$ & 1080 & 1 & EDHNRRNSDSAGPFA & 0.4487 & -0.0824 \\
\hline P69897 & Tubb5 & $\mathrm{T}$ & 285 & 2 & SQQYRALTVPELTQQ & 0.5897 & \\
\hline P69897 & Tubb5 & $\mathrm{T}$ & 290 & 2 & ALTVPELTQQVFDAK & 0.5897 & \\
\hline Q5U300 & Uba1 & $S$ & 820 & 1 & ELQSANASVDDSRLE & 0.3938 & -0.1507 \\
\hline Q5M7A4 & Uba5 & S & 380 & 1 & VPKKREDSVSEVTVE & 0.2857 & -0.0599 \\
\hline P0C627 & Ubxn2b & S & 56 & 2 & STATAFKSPRTPPLR & -1.0543 & 0.0177 \\
\hline P0C627 & Ubxn2b & $\mathrm{T}$ & 59 & 2 & TAFKSPRTPPLRLYS & -1.0543 & 0.0177 \\
\hline P0C627 & Ubxn2b & S & 216 & 1 & RLRFKAFSGEGQKLG & 1.2309 & -0.0154 \\
\hline A0A0G2K012 & Ubxn6 & S & 119 & 1 & EKTSKGKSPQLALRQ & -0.3633 & \\
\hline A0A0G2K012 & Ubxn6 & $S$ & 161 & 1 & RGPTSQDSIRNQVRK & 1.2591 & -0.1995 \\
\hline MOR8V0 & Uhrf1bp1I & S & 443 & 1 & KGTPRTPSVSSQPSK & 0.6849 & 0.0193 \\
\hline
\end{tabular}




\begin{tabular}{|c|c|c|c|c|c|c|c|}
\hline D3ZMG0 & Ulk1 & $S$ & 614 & 2 & LPDFLQRSPLPPILG & -1.4063 & -0.0388 \\
\hline D3ZMG0 & Ulk1 & $S$ & 622 & 2 & PLPPILGSPTKAGPS & -1.4063 & -0.0388 \\
\hline A0A0G2K952 & Usp32 & $S$ & 1452 & 1 & LRLPQIGSKNKLSSS & -0.5960 & -0.1154 \\
\hline A0A0G2K952 & Usp32 & $\mathrm{S}$ & 1459 & 1 & SKNKLSSSKENLDAS & -0.7847 & -0.0541 \\
\hline D3ZVQ0 & Usp5 & $S$ & 785 & 1 & RSAADSISESVPVGP & 0.3767 & -0.1899 \\
\hline Q8CF97 & Vcpip1 & $S$ & 999 & 2 & RSRESSPSHGLLKLG & -0.3421 & \\
\hline Q8CF97 & Vcpip1 & $S$ & 1030 & 1 & AFQGKGHSLGTASSN & 0.3755 & 0.0891 \\
\hline Q8CF97 & Vcpip1 & $S$ & 1127 & 1 & DKHLRDQSTEQTPSD & 0.8465 & 0.0101 \\
\hline Q8CF97 & Vcpip1 & $\mathrm{T}$ & 1128 & 1 & KHLRDQSTEQTPSDL & 0.8463 & \\
\hline B1WBS4 & Vps26b & $\mathrm{T}$ & 325 & 1 & TSLGEVRTPGQLSDN & -0.7940 & \\
\hline D3ZP60 & Vwa5b1 & $S$ & 664 & 1 & CSRATTASDPTGTTR & 0.3102 & -0.2014 \\
\hline D4A7U5 & Vwa5b2 & $S$ & 759 & 1 & ALAGRSLSSPSGRAN & 0.4956 & -0.1182 \\
\hline M0R7F3 & Wasf3 & $S$ & 235 & 1 & ASSEGSLSPDTRSHT & -0.2794 & \\
\hline D3ZQ02 & Wdr37 & $S$ & 22 & 1 & KQKRKSHSLSIRRTN & 1.1715 & -0.0608 \\
\hline D3ZQ02 & Wdr37 & $S$ & 24 & 1 & KRKSHSLSIRRTNSS & 0.8269 & -0.1423 \\
\hline G3V9M3 & Wdr47 & $S$ & 332 & 1 & TSHDKRISDLGNKTS & 2.0374 & -0.0433 \\
\hline Q9ERH3 & Wdr7 & $\mathrm{T}$ & 926 & 1 & TRPPRPGTPDLSKAR & 0.3022 & -0.1843 \\
\hline Q9ERH3 & Wdr7 & $S$ & 1150 & 1 & KLLTRPRSSSQIPEG & -0.5159 & \\
\hline Q9ERH3 & Wdr7 & S & 1152 & 1 & LTRPRSSSQIPEGFG & -0.3029 & 0.0833 \\
\hline E9PT53 & Wfs1 & $S$ & 237 & 1 & MLERLVSSESKNYIA & 0.9339 & \\
\hline D3ZUD3 & Wipf2 & $S$ & 235 & 1 & PPVKPPPSPVNVRTG & -0.6033 & -0.0338 \\
\hline D3ZUD3 & Wipf2 & S & 385 & 1 & LRNGHRDSITTVRSF & 0.5466 & -0.0444 \\
\hline D3ZMJ7 & Wnk2 & $S$ & 1801 & 1 & HRSSRAGSLGPETPS & 0.8352 & -0.0836 \\
\hline M0R8P6 & Wwc3 & $S$ & 961 & 1 & CRLYRSDSDSSTLPR & 0.5380 & \\
\hline Q5GH59 & Xkr4 & $S$ & 551 & 1 & RSISNNRSVASDRDQ & 0.5327 & -0.0546 \\
\hline A0A0G2K951 & Zfp704 & $S$ & 11 & 1 & RRLAKRPSLGSRRGG & 1.0477 & 0.0104 \\
\hline Q5PQS2 & Znf365 & $S$ & 139 & 1 & AVDIRADSLDAPRSS & 0.2689 & \\
\hline A0A0G2JW65 & & S & 726 & 2 & RLCRKFSSPPPLAVS & 0.3923 & 0.2053 \\
\hline A0A0G2JW65 & & $S$ & 733 & 2 & SPPPLAVSRTSSPVR & 0.5924 & 0.2053 \\
\hline A0A0G2JW65 & & $S$ & 745 & 2 & PVRARKLSLTSSLNS & 1.2352 & 0.0945 \\
\hline A0A0G2JZ27 & & $\mathrm{T}$ & 477 & 2 & TEELGARTPGAGGSV & -0.4906 & \\
\hline A0A0G2JZ27 & & $S$ & 483 & 2 & RTPGAGGSVHLLGRG & -0.4906 & \\
\hline A0A0G2K021 & & $\mathrm{T}$ & 23 & 1 & SKLKKTETQEKNPLP & 2.6314 & \\
\hline M0R970 & & $S$ & 42 & 3 & LLPSSVRSAPSSAPS & -1.2281 & \\
\hline M0R970 & & $S$ & 46 & 1 & SVRSAPSSAPSTPLS & -0.3855 & 0.0298 \\
\hline M0R970 & & $S$ & 46 & 2 & SVRSAPSSAPSTPLS & -0.6711 & 0.0310 \\
\hline M0R970 & & $\mathrm{S}$ & 46 & 3 & SVRSAPSSAPSTPLS & -1.2388 & \\
\hline M0R970 & & S & 49 & 3 & SAPSSAPSTPLSTDA & -1.2238 & \\
\hline M0R970 & & $T$ & 50 & 2 & APSSAPSTPLSTDAP & -0.6770 & \\
\hline M0R970 & & $\mathrm{T}$ & 50 & 3 & APSSAPSTPLSTDAP & -1.2473 & \\
\hline MOR970 & & $S$ & 71 & 1 & KDRPRKKSAPETLTL & -0.7994 & \\
\hline PoCD96 & & S & 28 & 1 & VKDATEDSITEDDKR & 0.3519 & \\
\hline
\end{tabular}


Table 8.2: SV-cycling-dependent sites, selection from Suppl. Data 01. UP.Accession Uniprot accession; Gene.name official gene symbol; AA modified amino acid; Pos position in the protein sequence; Mult multiplicity of phosphorylation (1 - singly phosphorylated, 2 - doubly phosphorylated, 3 - multiply phosphorylated); Seq.window $\sim \pm 7$ amino acids flanking phosphorylated residue; CaEGTA $\log _{2}$ intensity fold change in Ca vs. EGTA experiment, MockBoNT $\log _{2}$ intensity fold change in Mock vs. BoNT experiment

\begin{tabular}{|c|c|c|c|c|c|c|c|}
\hline UP.Accession & Gene.name & AA & Pos & Mult & Seq.window & CaEGTA & MockBoNT \\
\hline $\mathrm{P} 0 \mathrm{C} 1 \mathrm{X} 8$ & Aak1 & $T$ & 621 & 2 & GQKVGSLTPPSSPKT & -0.4166 & 0.2843 \\
\hline P0C1X8 & Aak1 & S & 625 & 2 & GSLTPPSSPKTQRAG & -0.425 & 0.3318 \\
\hline Q63120 & Abcc2 & $T$ & 61 & 2 & RTKRSSITKFYLAKQ & & -0.4556 \\
\hline Q63120 & Abcc2 & $\mathrm{Y}$ & 64 & 2 & RSSITKFYLAKQVFV & & -0.4556 \\
\hline Q9QZM5 & Abi1 & $\mathrm{S}$ & 220 & 1 & ARLGSQHSPGRTASL & -0.0489 & 0.7182 \\
\hline Q9QZM5 & Abi1 & $S$ & 287 & 2 & GTMTRQISRHNSTTS & & 0.4094 \\
\hline F1LYA6 & Abi2 & S & 150 & 1 & NMAPSQQSPVRTASV & -0.0261 & 0.311 \\
\hline A0A0G2JTB5 & Ablim2 & $S$ & 413 & 1 & HYTPTSRSPQHYSRP & 0.1171 & 1.5765 \\
\hline A0A0G2JTB5 & Ablim2 & $\mathrm{S}$ & 540 & 1 & DADTRTNSPDLDSQS & -0.044 & 0.282 \\
\hline Q6KC51 & Ablim2 & S & 383 & 1 & HYTPTSRSPQHYSRP & 0.0409 & 1.6334 \\
\hline Q6KC51 & Ablim2 & $S$ & 392 & 1 & QHYSRPGSESGRSTP & -0.1411 & 1.1417 \\
\hline E9PST5 & Acin1 & $\mathrm{S}$ & 216 & 1 & FSEEKGESDDEKPRK & & 0.5293 \\
\hline P35745 & Acyp2 & S & 42 & 1 & VGWVKNTSKGTVTGQ & & -0.3131 \\
\hline D4A3N4 & Adcy 1 & $S$ & 397 & 1 & SEKLRNRSSFSTNVV & -0.0711 & -0.3943 \\
\hline M0R5U4 & Adcy 9 & $\mathrm{~S}$ & 678 & 1 & KTQNGLLSPPAEEKL & & -0.3337 \\
\hline D3ZZ99 & Add1 & $S$ & 353 & 1 & PGKYKAKSRSPGTPA & 0.0195 & -0.2836 \\
\hline D3ZZ99 & Add1 & $S$ & 355 & 1 & KYKAKSRSPGTPAGE & 0.1345 & -0.3877 \\
\hline D3ZZ99 & Add1 & $\mathrm{S}$ & 572 & 1 & ERTEQTFSPAKSVSF & & 0.5208 \\
\hline D3ZZ99 & Add1 & $S$ & 632 & 1 & VERKQKGSEENLDET & -0.0875 & 0.3007 \\
\hline D3ZZ99 & Add1 & $S$ & 646 & 1 & TREQKEKSPPDQSAV & 0.0526 & -0.3296 \\
\hline D3ZZ99 & Add1 & $\mathrm{S}$ & 651 & 1 & EKSPPDQSAVPNTPP & 0.0995 & -0.2767 \\
\hline Q63028 & Add1 & $\mathrm{s}$ & 600 & 1 & TREQKEKSPPDQSAV & 0.0526 & -0.3296 \\
\hline Q63028 & Add1 & $S$ & 605 & 1 & EKSPPDQSAVPNTPP & 0.0995 & -0.2767 \\
\hline Q63028 & Add1 & S & 613 & 2 & AVPNTPPSTPVKLEG & -0.2729 & 0.3956 \\
\hline Q63028 & Add1 & $\mathrm{T}$ & 614 & 2 & VPNTPPSTPVKLEGG & -0.2572 & 0.376 \\
\hline Q05764 & Add2 & $\mathrm{T}$ & 612 & 2 & QSPTRAGTKSPAVSP & -0.2254 & 0.3831 \\
\hline Q05764 & Add2 & $\mathrm{S}$ & 614 & 1 & PTRAGTKSPAVSPSK & -0.0329 & 0.318 \\
\hline Q05764 & Add2 & $\mathrm{s}$ & 614 & 2 & PTRAGTKSPAVSPSK & -0.3943 & 0.5275 \\
\hline Q05764 & Add2 & $S$ & 618 & 1 & GTKSPAVSPSKASED & -0.2495 & 0.3624 \\
\hline Q05764 & Add2 & S & 618 & 2 & GTKSPAVSPSKASED & -0.4012 & 0.5318 \\
\hline Q05764 & Add2 & $\mathrm{S}$ & 620 & 1 & KSPAVSPSKASEDAK & & 0.3774 \\
\hline Q05764 & Add2 & $S$ & 620 & 2 & KSPAVSPSKASEDAK & -0.4998 & 0.4356 \\
\hline Q05764 & Add2 & S & 696 & 2 & GPLSPEGSPSKSPSK & -0.13 & 0.4278 \\
\hline Q05764 & Add2 & $S$ & 700 & 2 & PEGSPSKSPSKKKKK & -0.1791 & 0.3976 \\
\hline $\mathrm{COHL} 12$ & Adgrb1 & $S$ & 1253 & 1 & TGTFKRPSLPEEEKM & 0.1392 & 0.2759 \\
\hline $\mathrm{COHL} 12$ & Adgrb1 & S & 1462 & 1 & VATLSVSSLERRKSR & & 0.5488 \\
\hline A0A0G2K6N2 & Adgrb2 & $S$ & 1469 & 1 & SSAKEKPSPPGGRPG & 0.1498 & 0.4184 \\
\hline D4A831 & Adgrb3 & S & 1411 & 1 & GSTISMSSLERRKSR & & 0.3928 \\
\hline
\end{tabular}




\begin{tabular}{|c|c|c|c|c|c|c|c|}
\hline O88917 & Adgrl1 & S & 1250 & 2 & YNTLIAESVGFNPSS & -0.2419 & 0.3891 \\
\hline O88917 & Adgrl1 & S & 1263 & 2 & SSPPVFNSPGSYREP & -0.1787 & 0.3431 \\
\hline Q9Z173 & Adgrl3 & $\mathrm{S}$ & 1535 & 1 & PPNKDGASPEGTSKG & -0.2666 & 0.8795 \\
\hline P22909 & Adra2a & S & 297 & 2 & ALDLEESSSSEHAER & & -0.3294 \\
\hline O35889 & Afdn & $S$ & 1179 & 1 & KNRADHRSSPNVANQ & 0.0209 & 0.2983 \\
\hline O35889 & Afdn & $\mathrm{S}$ & 1180 & 1 & NRADHRSSPNVANQP & -0.1098 & 0.3649 \\
\hline O35889 & Afdn & S & 1180 & 2 & NRADHRSSPNVANQP & -0.2731 & 0.6676 \\
\hline O35889 & Afdn & S & 1189 & 1 & NVANQPPSPGGKSPY & -0.0989 & 0.4754 \\
\hline O35889 & Afdn & $\mathrm{S}$ & 1189 & 2 & NVANQPPSPGGKSPY & -0.4384 & 0.3518 \\
\hline D3ZQQ1 & Aftph & S & 619 & 1 & LGTPRTHSVSSAASK & & 0.2695 \\
\hline Q8CGU4 & Agap2 & S & 650 & 1 & LFANRRGSDSEKRSL & 0.3995 & 1.3185 \\
\hline Q8CGU4 & Agap2 & S & 1171 & 1 & TPSPRRRSSAASLGR & 0.3938 & 0.2865 \\
\hline Q8CGU4 & Agap2 & $S$ & 1175 & 1 & RRRSSAASLGRVDTT & 0.1775 & 0.265 \\
\hline Q4KLH5 & Agfg1 & S & 167 & 2 & LKSLLGESAPALHLN & -0.335 & 0.3218 \\
\hline Q4KLH5 & Agfg1 & $T$ & 177 & 2 & ALHLNKGTPTQSPVV & -0.1556 & 0.2633 \\
\hline Q4KLH5 & Agfg1 & S & 181 & 1 & NKGTPTQSPVVGRSQ & -0.157 & 0.413 \\
\hline Q4KLH5 & Agfg1 & S & 181 & 2 & NKGTPTQSPVVGRSQ & -0.225 & 0.2785 \\
\hline Q4KLH5 & Agfg1 & $\mathrm{S}$ & 293 & 1 & DNFPKSSSADFGSFS & -0.5138 & 0.2967 \\
\hline G3V8G1 & Agtpbp1 & S & 51 & 1 & KILHLAQSQEKTRRE & & -0.337 \\
\hline B5DFN2 & Ahcyl1 & S & 37 & 1 & SAASYTDSSDDEVSP & 0.0082 & -0.3822 \\
\hline B5DFN2 & Ahcyl1 & S & 38 & 1 & AASYTDSSDDEVSPR & -0.1325 & -0.4847 \\
\hline B5DFN2 & Ahcyl1 & $S$ & 38 & 2 & AASYTDSSDDEVSPR & & -0.2664 \\
\hline D3ZTF0 & Ajm1 & S & 109 & 1 & SLTTAPASPPVLQRR & 0.1982 & 0.3637 \\
\hline D3ZTF0 & Ajm1 & S & 129 & 1 & RAVRVEGSPCREPSY & & 0.4908 \\
\hline D3ZTF0 & Ajm1 & $S$ & 509 & 2 & RYAALSLSETSLTEK & -0.2384 & 0.7389 \\
\hline D3ZTF0 & Ajm1 & $\mathrm{T}$ & 511 & 1 & AALSLSETSLTEKGR & & 0.987 \\
\hline D3ZTF0 & Ajm1 & S & 512 & 2 & ALSLSETSLTEKGRT & -0.2539 & 0.927 \\
\hline D3ZTF0 & Ajm1 & $S$ & 542 & 1 & ITDNDLRSAERPTAK & 0.2587 & 0.4055 \\
\hline P29410 & Ak2 & S & 151 & 1 & IHPKSGRSYHEEFNP & & -0.3554 \\
\hline 088884 & Akap1 & S & 101 & 1 & TRQVRRRSESSGNLP & 0.0407 & 0.2835 \\
\hline F1LPP6 & Akap5 & S & 90 & 1 & LVTHRKPSESAEKQK & 0.1454 & 0.3793 \\
\hline F1LPP6 & Akap5 & S & 223 & 1 & LRMRTPGSEKEAKVI & & 1.0626 \\
\hline Q9WVC7 & Akap6 & S & 1323 & 1 & TVLTKSLSKDSSFSS & & 0.3086 \\
\hline P05065 & Aldoa & $S$ & 100 & 1 & PFPQVIKSKGGVVGI & & -0.3353 \\
\hline Q6AY07 & Aldoart2 & S & 100 & 1 & PFPQVIKSKGGVVGI & & -0.4312 \\
\hline A0A0G2JWK6 & Amer2 & S & 207 & 1 & RTARRPDSPGQDAPR & 0.1457 & 0.2764 \\
\hline F1LZQ6 & Amotl1 & S & 917 & 1 & LSTAPSNSPVLKHPA & & 0.9939 \\
\hline F1LZQ6 & Amotl1 & S & 935 & 1 & TVEKQENSPGHGKSS & -0.0381 & 2.3019 \\
\hline O09178 & Ampd3 & S & 68 & 1 & KELAEQKSVETAKRK & & 0.4151 \\
\hline D3Z9Z0 & Ank1 & S & 1520 & 1 & VHARITDSPSVRQVL & & 0.9026 \\
\hline F1M9N9 & Ank2 & S & 1808 & 1 & SAKTERHSPVSPSSK & -0.1284 & 0.2814 \\
\hline F1M9N9 & Ank2 & S & 1808 & 2 & SAKTERHSPVSPSSK & -0.3732 & 0.193 \\
\hline F1M9N9 & Ank2 & S & 1820 & 1 & SSKNEKHSPVSPSAK & -0.2626 & 0.3652 \\
\hline F1M9N9 & Ank2 & $S$ & 1820 & 2 & SSKNEKHSPVSPSAK & -0.4212 & 0.1747 \\
\hline
\end{tabular}




\begin{tabular}{|c|c|c|c|c|c|c|c|}
\hline F1M9N9 & Ank2 & $S$ & 1823 & 1 & NEKHSPVSPSAKAER & -0.27 & 0.2966 \\
\hline F1M9N9 & Ank2 & S & 1823 & 2 & NEKHSPVSPSAKAER & -0.424 & 0.179 \\
\hline F1M9N9 & Ank2 & S & 1843 & 1 & SGKPEKHSPGSPSTK & -0.0773 & 0.3542 \\
\hline F1M9N9 & Ank2 & $\mathrm{T}$ & 1909 & 1 & PGAPSIRTPEKQAPG & & 0.3738 \\
\hline F1M9N9 & Ank2 & $S$ & 1917 & 1 & PEKQAPGSATGKHEK & -0.0109 & 0.5676 \\
\hline F1M9N9 & Ank2 & $\mathrm{S}$ & 1929 & 1 & HEKHLPVSPGKTEKQ & -0.3547 & 0.2779 \\
\hline F1M9N9 & Ank2 & S & 2373 & 1 & KPQGVIRSPQGLELP & -0.3272 & 0.4456 \\
\hline F1M9N9 & Ank2 & $S$ & 2630 & 1 & SESRKASSSSESEPE & & 0.4566 \\
\hline F1M9N9 & Ank2 & $\mathrm{T}$ & 3060 & 1 & RTPTEEGTPTSEQNP & & 0.4022 \\
\hline A0A0G2K1Q7 & Ank3 & S & 1390 & 1 & EKADRRQSFTSLALR & 0.2522 & 0.4187 \\
\hline O70511 & Ank3 & S & 1526 & 1 & KSGSLSSSPSNTPSA & & 1.0513 \\
\hline O70511 & Ank3 & $S$ & 1676 & 1 & LISSPLKSVVSPTKS & 0.2295 & 1.3718 \\
\hline O70511 & Ank3 & $S$ & 1679 & 1 & SPLKSVVSPTKSAAD & 0.2847 & 1.2892 \\
\hline A0A0G2K2G7 & Anks1a & $S$ & 557 & 1 & EEEDGSRSRAPPTSK & -0.0162 & 0.7755 \\
\hline P0C6S7 & Anks1b & $\mathrm{T}$ & 969 & 2 & REPSGNHTPPQLSPS & -0.1997 & 0.3141 \\
\hline A0A0G2K5K1 & Ano8 & S & 634 & 1 & DGLLEEGSPTMVEKG & & 0.4784 \\
\hline P62944 & Ap2b1 & $\mathrm{T}$ & 2 & 1 & MTDSKYFTT & 1.6363 & -0.4876 \\
\hline B5DFK6 & Ap3d1 & $\mathrm{s}$ & 688 & 1 & FYIKSSPSPQKRYQD & & 0.5064 \\
\hline B5DFK6 & Ap3d1 & S & 1176 & 1 & LLVKKGESSVSVDGK & & -0.3163 \\
\hline A0A0G2K8G0 & Apc & $\mathrm{s}$ & 1058 & 1 & QLNSGRQSPSQNERW & -0.334 & 0.4083 \\
\hline A0A0G2K8G0 & Apc & $\mathrm{T}$ & 1453 & 1 & MPPSRSKTPPPPPPP & & 0.3195 \\
\hline A0A0G2K8G0 & Apc & S & 1790 & 1 & TKKKKPTSPVKPMPQ & & 0.3443 \\
\hline A0A0G2K8G0 & Apc & $\mathrm{s}$ & 2081 & 1 & KGEGERQSPRKVGSV & & 0.8325 \\
\hline A0A0G2K8G0 & Apc & $\mathrm{s}$ & 2164 & 2 & KSGVSLGSPFHLTPD & -0.3071 & 0.3523 \\
\hline A0A0G2K8G0 & Apc & $\mathrm{T}$ & 2169 & 2 & LGSPFHLTPDQEEKP & -0.3071 & 0.3523 \\
\hline A0A0G2K8G0 & Apc & $\mathrm{s}$ & 2262 & 1 & RNSSSSTSPVSKKGP & & 0.7192 \\
\hline A0A0G2K8G0 & Apc & $\mathrm{s}$ & 2288 & 1 & EGPVATTSPRGTKPA & & 0.3654 \\
\hline A0A0G2K8G0 & Apc & $\mathrm{S}$ & 2467 & 1 & TFIKEAPSPTLRRKL & & 0.3413 \\
\hline A0A0G2K8G0 & Apc & S & 2491 & 1 & SPSSRPDSPTRSQAQ & -0.0715 & 0.3231 \\
\hline A0A0G2K8G0 & Apc & S & 2847 & 1 & TESSGAQSPKRHSGS & -0.025 & 0.6356 \\
\hline A0A0G2JZW4 & Apc2 & S & 2177 & 1 & ALPLRGSSPEDSPAG & -0.0619 & 0.4015 \\
\hline D4A631 & Arfgef1 & S & 52 & 1 & KVETEKQSPPHGEAK & 0.2498 & -0.3889 \\
\hline D3ZF86 & Arfgef3 & $\mathrm{s}$ & 348 & 1 & RYSESNFSVDDQDLS & & -0.3558 \\
\hline D4A6C5 & Arhgap1 & S & 50 & 1 & KSDDSKSSSPEPVTH & & -0.515 \\
\hline D4A6C5 & Arhgap1 & $S$ & 50 & 2 & KSDDSKSSSPEPVTH & 0.1073 & -0.3684 \\
\hline D4A6C5 & Arhgap1 & $\mathrm{s}$ & 51 & 1 & SDDSKSSSPEPVTHL & 0.0995 & -0.4342 \\
\hline D4A6C5 & Arhgap1 & $\mathrm{s}$ & 51 & 2 & SDDSKSSSPEPVTHL & 0.0885 & -0.3749 \\
\hline F1LXQ7 & Arhgap21 & S & 863 & 1 & PLIRRQLSHDQESVG & -0.0131 & 0.2949 \\
\hline F1LXQ7 & Arhgap21 & $\mathrm{s}$ & 863 & 2 & PLIRRQLSHDQESVG & -0.1411 & 0.3204 \\
\hline F1LXQ7 & Arhgap21 & $S$ & 868 & 1 & QLSHDQESVGPPSLD & & 0.3498 \\
\hline F1LXQ7 & Arhgap21 & $S$ & 1105 & 1 & PKTQSPHSPKEESER & -0.1403 & 0.4604 \\
\hline F1LXQ7 & Arhgap21 & $\mathrm{s}$ & 1564 & 1 & SSTKKSTSPETRHTE & & 0.4353 \\
\hline F1MAK3 & Arhgap32 & $S$ & 606 & 1 & PKSLLVSSPSTKLLT & & 0.955 \\
\hline F1MAK3 & Arhgap32 & $\mathrm{s}$ & 720 & 2 & GTLRSAKSEESLTSL & -0.0522 & 0.8496 \\
\hline
\end{tabular}




\begin{tabular}{|c|c|c|c|c|c|c|c|}
\hline F1MAK3 & Arhgap32 & S & 723 & 2 & RSAKSEESLTSLHAV & -0.1294 & 0.8496 \\
\hline F1MAK3 & Arhgap32 & S & 870 & 1 & TASSEPVSPVQEKLS & -0.1166 & 0.8862 \\
\hline F1MAK3 & Arhgap32 & $S$ & 966 & 1 & ERDVINRSPTQLGKM & 0.0279 & 1.0432 \\
\hline F1LR18 & Arhgap39 & S & 295 & 1 & IQPRKPSSDSQPSSP & 0.502 & 0.3069 \\
\hline F1LR18 & Arhgap39 & S & 301 & 1 & SSDSQPSSPRYGYEP & -0.6042 & 0.4461 \\
\hline F1LR18 & Arhgap39 & S & 301 & 2 & SSDSQPSSPRYGYEP & -0.5876 & 0.0327 \\
\hline A0A0G2K7N9 & Arhgap5 & $S$ & 968 & 1 & SEDVFLPSPRDCFPY & & -0.297 \\
\hline D3Z8W6 & Arhgef33 & S & 146 & 1 & ASAPAQGSPFRSINV & -0.057 & 0.6548 \\
\hline D3Z8W6 & Arhgef33 & S & 692 & 1 & PLPKSATSPASSSGA & 0.0522 & 0.5878 \\
\hline A0A0G2QC21 & Arhgef7 & S & 497 & 1 & LSASPRMSGFIYQGK & & -0.3815 \\
\hline D4A3E3 & Arid1a & S & 90 & 1 & RSHHAPMSPGSSGGG & & 0.5337 \\
\hline B1WBW4 & Armc10 & S & 43 & 1 & RRLRPSRSAEDLTEG & 0.1986 & -0.3318 \\
\hline Q642B5 & Armcx2 & S & 46 & 1 & AKPKNRASVGTGTRA & -0.17 & 0.4169 \\
\hline B4F7F3 & Arvcf & S & 336 & 1 & PERGSLGSLDRVVRR & 0.2414 & 0.7682 \\
\hline B4F7F3 & Arvcf & S & 344 & 2 & LDRVVRRSPSVDSTR & 0.0188 & 0.3441 \\
\hline B4F7F3 & Arvcf & S & 346 & 2 & RVVRRSPSVDSTRKE & -0.0233 & 0.378 \\
\hline Q1AAU6 & Asap1 & $\mathrm{S}$ & 851 & 1 & PGHKRTLSDPPSPLP & 0.187 & 0.4997 \\
\hline Q1AAU6 & Asap1 & S & 851 & 2 & PGHKRTLSDPPSPLP & -0.422 & 0.0734 \\
\hline P55926 & Asic1 & $S$ & 477 & 1 & QKEAKRSSADKGVAL & & -0.3044 \\
\hline A0A0G2JYU3 & Astn2 & S & 445 & 1 & RSKGLLKSPVNKTAL & & 0.5346 \\
\hline Q3KRE0 & Atad3 & S & 41 & 1 & RGAGDRPSPKDKWSN & 0.1656 & -0.3219 \\
\hline P06685 & Atp1a1 & $\mathrm{T}$ & 226 & 1 & TGESEPQTRSPDFTN & 0.1105 & -0.3153 \\
\hline P06685 & Atp1a1 & $\mathrm{s}$ & 484 & 1 & IVEIPFNSTNKYQLS & & -0.5541 \\
\hline P06685 & Atp1a1 & $S$ & 499 & 1 & IHKNPNASEPKHLLV & 0.1783 & -0.399 \\
\hline P06686 & Atp1a2 & $\mathrm{T}$ & 224 & 1 & TGESEPQTRSPEFTH & 0.1491 & -0.3117 \\
\hline P06686 & Atp1a2 & $\mathrm{T}$ & 380 & 1 & SDKTGTLTQNRMTVA & & -0.3575 \\
\hline P06686 & Atp1a2 & $\mathrm{S}$ & 450 & 1 & RDTAGDASESALLKC & 0.1372 & -0.4037 \\
\hline P06686 & Atp1a2 & $S$ & 482 & 1 & VAEIPFNSTNKYQLS & & -0.3886 \\
\hline P06687 & Atp1a3 & $\mathrm{s}$ & 456 & 1 & CIELSSGSVKLMRER & & -0.3373 \\
\hline P06687 & Atp1a3 & S & 474 & 1 & VAEIPFNSTNKYQLS & & -0.4808 \\
\hline P11505 & Atp2b1 & $S$ & 1126 & 1 & RVVNAFRSSLYEGLE & & -0.3588 \\
\hline P11505 & Atp2b1 & $\mathrm{s}$ & 1127 & 1 & VVNAFRSSLYEGLEK & 0.6029 & -0.2675 \\
\hline P11505 & Atp2b1 & $\mathrm{S}$ & 1177 & 1 & DAPTKRNSSPPPSPN & 0.0681 & 0.5024 \\
\hline P11505 & Atp2b1 & $S$ & 1178 & 1 & APTKRNSSPPPSPNK & -0.0308 & 0.4503 \\
\hline P11505 & Atp2b1 & $\mathrm{s}$ & 1178 & 2 & APTKRNSSPPPSPNK & -0.0295 & 0.2883 \\
\hline P11506 & Atp2b2 & $\mathrm{S}$ & 1149 & 1 & RVVKAFRSSLYEGLE & & -0.3605 \\
\hline P11506 & Atp2b2 & $\mathrm{S}$ & 1160 & 2 & EGLEKPESRTSIHNF & 0.0489 & 0.3714 \\
\hline P11506 & Atp2b2 & $\mathrm{T}$ & 1162 & 2 & LEKPESRTSIHNFMA & 0.0489 & 0.3986 \\
\hline $\mathrm{P} 11506$ & Atp2b2 & $\mathrm{s}$ & 1163 & 2 & EKPESRTSIHNFMAH & 0.0489 & 0.3933 \\
\hline P11506 & Atp2b2 & $\mathrm{T}$ & 1188 & 1 & HIPLIDDTDLEEDAA & -0.2512 & 0.3946 \\
\hline P11506 & Atp2b2 & $\mathrm{T}$ & 1188 & 2 & HIPLIDDTDLEEDAA & -0.1747 & 0.7144 \\
\hline P11506 & Atp2b2 & $\mathrm{S}$ & 1200 & 1 & DAALKQNSSPPSSLN & -0.2951 & 0.4472 \\
\hline P11506 & Atp2b2 & $\mathrm{S}$ & 1200 & 2 & DAALKQNSSPPSSLN & -0.1841 & 0.7924 \\
\hline P11506 & Atp2b2 & $\mathrm{s}$ & 1201 & 1 & AALKQNSSPPSSLNK & -0.0302 & 0.3971 \\
\hline
\end{tabular}




\begin{tabular}{|c|c|c|c|c|c|c|c|}
\hline P11506 & Atp2b2 & S & 1201 & 2 & AALKQNSSPPSSLNK & -0.1723 & 0.7622 \\
\hline P11506 & Atp2b2 & S & 1204 & 2 & KQNSSPPSSLNKNNS & -0.0407 & 1.0315 \\
\hline P11506 & Atp2b2 & $S$ & 1205 & 2 & QNSSPPSSLNKNNSA & -0.0842 & 0.876 \\
\hline P15999 & Atp5f1a & $T$ & 236 & 1 & QKRFNDGTDEKKKLY & & -0.2968 \\
\hline P15999 & Atp5f1a & $\mathrm{s}$ & 533 & 1 & IRSDGKISEQSDAKL & & -0.3436 \\
\hline Q6P503 & Atp6v1d & S & 216 & 1 & KKIIKEKSEKDLERR & & -0.2978 \\
\hline D4A3X6 & Atp8a2 & $S$ & 52 & 1 & DEMSRATSVGDQLEA & & -0.2657 \\
\hline A0A0G2JXZ3 & Atrx & S & 770 & 1 & GKRKRKNSTSGSDFD & & 0.4591 \\
\hline Q63540 & Atxn1 & S & 213 & 1 & AGLVNPGSPPPTQQN & & 0.3126 \\
\hline F1M049 & Atxn2 & S & 529 & 1 & LQDQRQNSPAGNKEN & & 1.4942 \\
\hline A0A0G2JYE0 & Atxn2l & S & 337 & 1 & RQGSGRESPSLVSRE & & 0.5694 \\
\hline O70239 & Axin1 & S & 492 & 1 & SPGPGHRSPDSGHVA & & 0.3119 \\
\hline Q6GMN2 & Baiap2 & $S$ & 324 & 2 & RKAAQPKSLSPPQSQ & -0.0515 & 0.4707 \\
\hline Q6GMN2 & Baiap2 & S & 326 & 1 & AAQPKSLSPPQSQSK & 0.011 & 0.4687 \\
\hline Q6GMN2 & Baiap2 & S & 326 & 2 & AAQPKSLSPPQSQSK & -0.0515 & 0.4707 \\
\hline A0A0G2K1L8 & Basp1 & $\mathrm{T}$ & 31 & 1 & KKAEGAGTEEEGTQK & 0.3282 & -0.3434 \\
\hline A0A0G2K1L8 & Basp1 & $\mathrm{T}$ & 36 & 1 & AGTEEEGTQKESEPQ & 0.2704 & -0.2825 \\
\hline A0A0G2K1L8 & Basp1 & S & 40 & 1 & EEGTQKESEPQAAAD & 0.2935 & -0.3751 \\
\hline A0A0G2K1L8 & Basp1 & S & 91 & 1 & PKAEPEKSEGAAEEQ & 0.0254 & -0.3736 \\
\hline A0A0G2K1L8 & Basp1 & S & 165 & 1 & AAPAASDSKPSSAEP & 0.1208 & -0.3252 \\
\hline A0A0G2K1L8 & Basp1 & S & 168 & 1 & AASDSKPSSAEPAPS & 0.2988 & -0.2913 \\
\hline A0A0G2K1L8 & Basp1 & $S$ & 169 & 1 & ASDSKPSSAEPAPSS & & -0.2708 \\
\hline G3V661 & Baz1b & S & 311 & 1 & TKRKNTGSPDRKPSK & & 1.3454 \\
\hline Q9QYN5 & Bcl10 & S & 138 & 1 & NNLSRSNSDESNFSE & 0.0218 & 0.3024 \\
\hline B1WC16 & Bclaf1 & $S$ & 263 & 1 & TPSQHSHSIQHSPER & -0.1014 & 1.9144 \\
\hline B1WC16 & Bclaf1 & S & 267 & 1 & HSHSIQHSPERSGSG & -0.1371 & 1.5539 \\
\hline B1WC16 & Bclaf1 & S & 496 & 1 & TVKKEVQSPEQVKSE & 0.1246 & 0.528 \\
\hline O88881 & Begain & $S$ & 213 & 1 & LEKPDPGSLSSRMSD & & 0.4141 \\
\hline 088881 & Begain & S & 474 & 1 & GGGGGGGSPGKNAEG & -0.1786 & 0.8912 \\
\hline 088881 & Begain & S & 474 & 2 & GGGGGGGSPGKNAEG & & 1.7859 \\
\hline 088881 & Begain & S & 484 & 1 & KNAEGRASPLYASYK & & 0.7657 \\
\hline 088881 & Begain & S & 484 & 2 & KNAEGRASPLYASYK & & 1.7194 \\
\hline 088881 & Begain & S & 521 & 1 & AGGDLSLSPSRSADP & -0.0441 & 0.9255 \\
\hline Q5U3Z5 & Bri3bp & $S$ & 51 & 1 & RGTADKNSHRRATSS & & -0.2724 \\
\hline Q5U3Z5 & Bri3bp & S & 250 & 1 & VLENLDRSNEK & 0.1366 & -0.2672 \\
\hline B2DD29 & Brsk1 & S & 275 & 1 & VEPEKRLSLEQIQKH & & -0.4987 \\
\hline G3V984 & Bsn & S & 90 & 1 & LGSQRATSPTPKQAS & -1.7085 & 0.4685 \\
\hline G3V984 & Bsn & S & 245 & 1 & QLHSPALSPAHSPAK & -0.6205 & 0.28 \\
\hline G3V984 & Bsn & S & 245 & 2 & QLHSPALSPAHSPAK & -1.6773 & 0.0297 \\
\hline G3V984 & Bsn & S & 245 & 3 & QLHSPALSPAHSPAK & -1.7895 & -0.084 \\
\hline G3V984 & Bsn & S & 548 & 1 & PQRAAGASPLKQKGP & -0.9047 & 0.3409 \\
\hline G3V984 & Bsn & $\mathrm{T}$ & 1086 & 1 & RRRERSKTPPSNLSP & & 0.695 \\
\hline G3V984 & Bsn & $\mathrm{T}$ & 1086 & 2 & RRRERSKTPPSNLSP & 0.0378 & 0.383 \\
\hline G3V984 & Bsn & $\mathrm{T}$ & 1086 & 3 & RRRERSKTPPSNLSP & -0.7174 & 0.3222 \\
\hline
\end{tabular}




\begin{tabular}{|c|c|c|c|c|c|c|c|}
\hline G3V984 & Bsn & $S$ & 1092 & 3 & KTPPSNLSPIEDASP & -0.6794 & 0.3433 \\
\hline G3V984 & Bsn & S & 1098 & 1 & LSPIEDASPTEELRQ & 0.3503 & -0.0204 \\
\hline G3V984 & Bsn & S & 1098 & 3 & LSPIEDASPTEELRQ & -0.714 & 0.3634 \\
\hline G3V984 & Bsn & $\mathrm{T}$ & 1100 & 2 & PIEDASPTEELRQAA & 0.0072 & 0.4748 \\
\hline G3V984 & Bsn & $S$ & 1469 & 1 & YSYFTGSSPPLSPST & 0.3313 & 0.1816 \\
\hline G3V984 & Bsn & S & 1469 & 2 & YSYFTGSSPPLSPST & -0.2452 & 0.5949 \\
\hline G3V984 & Bsn & $S$ & 1469 & 3 & YSYFTGSSPPLSPST & -0.605 & 0.4956 \\
\hline G3V984 & Bsn & $S$ & 1473 & 3 & TGSSPPLSPSTPSES & -0.7101 & 0.4691 \\
\hline G3V984 & Bsn & S & 1475 & 3 & SSPPLSPSTPSESPT & -0.8634 & 0.4853 \\
\hline G3V984 & Bsn & $\mathrm{T}$ & 1476 & 2 & SPPLSPSTPSESPTF & -0.1421 & 0.5702 \\
\hline G3V984 & Bsn & $\mathrm{T}$ & 1476 & 3 & SPPLSPSTPSESPTF & -0.9133 & 0.1017 \\
\hline G3V984 & Bsn & S & 1478 & 2 & PLSPSTPSESPTFSP & -0.1754 & 0.5627 \\
\hline G3V984 & Bsn & S & 1478 & 3 & PLSPSTPSESPTFSP & -0.6575 & \\
\hline G3V984 & Bsn & S & 1480 & 2 & SPSTPSESPTFSPSK & -0.2179 & 0.6476 \\
\hline G3V984 & Bsn & S & 1480 & 3 & SPSTPSESPTFSPSK & -0.4521 & 0.5195 \\
\hline G3V984 & Bsn & S & 2004 & 1 & FQGPGRDSAVDLSSL & -0.6739 & -0.2832 \\
\hline G3V984 & Bsn & $\mathrm{S}$ & 2634 & 1 & SRHSDSGSDSKHEAS & 0.3301 & 0.3351 \\
\hline G3V984 & Bsn & S & 2850 & 1 & LSDPKPLSPTAEESA & -0.552 & 0.3295 \\
\hline G3V984 & Bsn & S & 2850 & 2 & LSDPKPLSPTAEESA & -0.6354 & 0.2229 \\
\hline G3V984 & Bsn & S & 2850 & 3 & LSDPKPLSPTAEESA & 0.4901 & \\
\hline G3V984 & Bsn & S & 3349 & 1 & LAPAAISSKRSKHRK & & -0.3366 \\
\hline A0A0G2K2V4 & C2cd2I & S & 465 & 1 & VDGKLADSPSRSPSK & 0.4169 & 0.4068 \\
\hline AOA0G2K2V4 & C2cd2I & $S$ & 465 & 2 & VDGKLADSPSRSPSK & -0.1881 & 0.5541 \\
\hline AOA0G2K2V4 & C2cd2I & S & 467 & 2 & GKLADSPSRSPSKVE & -0.1783 & 0.7078 \\
\hline AOA0G2K2V4 & C2cd2I & $S$ & 469 & 2 & LADSPSRSPSKVEVT & -0.1976 & 0.5344 \\
\hline A0A0G2K2V4 & C2cd2I & $\mathrm{S}$ & 471 & 2 & DSPSRSPSKVEVTEK & -0.1654 & 0.5251 \\
\hline AOA0G2K2V4 & C2cd2I & S & 641 & 1 & KDHKDNSSPANSSRW & 0.1776 & 1.3209 \\
\hline Q5U2P5 & C2cd2I & $S$ & 464 & 2 & RVDGKLDSPSRSPSK & -0.024 & 0.4357 \\
\hline Q5U2P5 & C2cd2I & S & 468 & 2 & KLDSPSRSPSKVEVT & -0.0441 & 0.4041 \\
\hline A0A0G2K543 & C2cd5 & S & 260 & 1 & AFLPACNSPSKEMKE & & -0.3675 \\
\hline A0A0G2JXK1 & Cacna1a & S & 462 & 1 & FARASIKSAKLENST & & -0.2896 \\
\hline A0A0G2JXK1 & Cacna1a & $\mathrm{T}$ & 1092 & 1 & SNPGPPKTPENSLIV & & 0.3216 \\
\hline A0A0G2JXK1 & Cacna1a & $\mathrm{T}$ & 2070 & 1 & NLSTISDTSPMKRSA & & 0.9945 \\
\hline A0A0G2JXK1 & Cacna1a & S & 2071 & 1 & LSTISDTSPMKRSAS & & 1.1996 \\
\hline F1LQ87 & Cacna1b & $S$ & 48 & 1 & QRVLYKQSIAQRART & & -0.5709 \\
\hline F1LQ87 & Cacna1b & $\mathrm{S}$ & 2029 & 1 & TQPAPNASPMKRSIS & & 0.3127 \\
\hline P22002 & Cacna1c & $S$ & 1720 & 1 & KEAVSAASEDDIFRR & & 0.5861 \\
\hline P22002 & Cacna1c & S & 1771 & 1 & NNQADTESPSHEKLV & -0.1062 & 0.9452 \\
\hline F1LMS1 & Cacna1e & S & 837 & 1 & ERISRGGSLKGDIGG & 0.4629 & 0.5399 \\
\hline F1LMS1 & Cacna1e & $S$ & 837 & 2 & ERISRGGSLKGDIGG & -0.3298 & 0.3979 \\
\hline F1LMS1 & Cacna1e & S & 854 & 2 & SVLDNQRSPLSLGKR & -0.3185 & 0.402 \\
\hline F1LMS1 & Cacna1e & S & 925 & 2 & ASRSRSASQERSLDE & 0.2621 & 0.3112 \\
\hline F1LMS1 & Cacna1e & $S$ & 929 & 2 & RSASQERSLDEGVSI & 0.2507 & 0.2996 \\
\hline F1LMS1 & Cacna1e & S & 2036 & 2 & TYKSRRRSYHSSLRL & & -0.3036 \\
\hline
\end{tabular}




\begin{tabular}{|c|c|c|c|c|c|c|c|}
\hline Q9WUB8 & Cacna1g & $S$ & 467 & 1 & VRAGLLSSPVARSGQ & 0.3392 & -0.3373 \\
\hline Q9WUB8 & Cacna1g & S & 1087 & 1 & AAHHEMKSPPSARSS & & 0.4983 \\
\hline Q9WUB8 & Cacna1g & S & 1165 & 1 & SDHRHRGSLEREAKS & 0.0252 & 0.3752 \\
\hline Q9WUB8 & Cacna1g & $S$ & 1173 & 1 & LEREAKSSFDLPDTL & 0.0968 & 0.4288 \\
\hline Q9WUB8 & Cacna1g & $S$ & 2268 & 1 & DSTAASPSPKKDTLS & & 0.3984 \\
\hline D4A055 & Cacnb4 & $\mathrm{S}$ & 16 & 1 & GAADGPHSPSSQVAR & -0.0451 & 0.6545 \\
\hline D4A055 & Cacnb4 & S & 447 & 1 & SNHSTENSPIERRSL & -0.1905 & 1.5926 \\
\hline D4A055 & Cacnb4 & $S$ & 474 & 1 & RKSRNRLSSSSQHSR & -0.384 & 0.3269 \\
\hline Q71RJ2 & Cacng2 & $\mathrm{s}$ & 243 & 2 & RSRSSSRSTEPSHSR & & 0.621 \\
\hline Q71RJ2 & Cacng2 & S & 249 & 2 & RSTEPSHSRDASPVG & -0.2552 & 0.6311 \\
\hline Q71RJ2 & Cacng2 & $\mathrm{s}$ & 253 & 1 & PSHSRDASPVGVKGF & 0.1258 & 0.6082 \\
\hline Q71RJ2 & Cacng2 & $S$ & 253 & 2 & PSHSRDASPVGVKGF & -0.2491 & 0.7494 \\
\hline Q71RJ2 & Cacng2 & $S$ & 310 & 1 & IQKDSKDSLHANTAN & 0.0921 & 0.8288 \\
\hline Q8VHW5 & Cacng8 & $S$ & 290 & 1 & ASPSRDASPGGPGGP & 0.0071 & 0.3539 \\
\hline Q8VHW5 & Cacng8 & $\mathrm{s}$ & 357 & 1 & GSERDRGSSAGFLTL & & 0.3693 \\
\hline F1LLX6 & Cadps & S & 89 & 1 & AGGGRPSSPSPSVVS & -0.9209 & -0.3369 \\
\hline F1LLX6 & Cadps & $\mathrm{s}$ & 89 & 2 & AGGGRPSSPSPSVVS & -1.2942 & \\
\hline P18418 & Calr & $\mathrm{s}$ & 44 & 1 & WVESKHKSDFGKFVL & & -0.3521 \\
\hline F1LZG4 & Camk2a & S & 330 & 1 & GVKESSESTNTTIED & 0.4369 & -0.3282 \\
\hline F1LZG4 & Camk2a & $\mathrm{T}$ & 334 & 1 & SSESTNTTIEDEDTK & 0.382 & -0.2993 \\
\hline P11275 & Camk2a & $\mathrm{S}$ & 330 & 1 & KNDGVKESSESTNTT & 0.0176 & -0.2998 \\
\hline P11275 & Camk2a & S & 331 & 1 & NDGVKESSESTNTTI & 0.0552 & -0.3531 \\
\hline P11275 & Camk2a & $\mathrm{T}$ & 334 & 1 & VKESSESTNTTIEDE & 0.2713 & -0.2704 \\
\hline P11275 & Camk2a & $\mathrm{T}$ & 337 & 1 & SSESTNTTIEDEDTK & 0.382 & -0.2737 \\
\hline P11275 & Camk2a & $\mathrm{T}$ & 337 & 3 & SSESTNTTIEDEDTK & 0.0105 & -0.348 \\
\hline F1LNI8 & Camk2b & $\mathrm{s}$ & 71 & 1 & ICRLLKHSNIVRLHD & & -0.3379 \\
\hline F1M3F8 & Camk2g & $\mathrm{s}$ & 351 & 1 & GGVKKRKSSSSVHLM & 0.1935 & 0.2825 \\
\hline P11730 & Camk2g & $\mathrm{S}$ & 321 & 1 & FSVGRQSSAPASPAA & 0.789 & 0.3125 \\
\hline P11730 & Camk2g & S & 321 & 2 & FSVGRQSSAPASPAA & 0.2855 & 0.0337 \\
\hline P11730 & Camk2g & S & 356 & 2 & GVKKRKSSSSVHLME & 0.3179 & 0.2999 \\
\hline P11730 & Camk2g & S & 356 & 3 & GVKKRKSSSSVHLME & 0.4245 & 0.0986 \\
\hline P11730 & Camk2g & S & 357 & 2 & VKKRKSSSSVHLMEP & 0.3317 & 0.2813 \\
\hline $\mathrm{P} 11730$ & Camk2g & $\mathrm{s}$ & 357 & 3 & VKKRKSSSSVHLMEP & 0.4245 & 0.115 \\
\hline Q63092 & Camkv & $\mathrm{T}$ & 430 & 2 & PATDRSATPATDGRA & -0.1151 & 0.5235 \\
\hline Q63092 & Camkv & $\mathrm{T}$ & 438 & 1 & PATDGRATPATEEST & 0.1299 & 0.4969 \\
\hline Q63092 & Camkv & $\mathrm{T}$ & 438 & 2 & PATDGRATPATEEST & -0.0878 & 0.4049 \\
\hline D4AEC2 & Camsap2 & $\mathrm{s}$ & 952 & 1 & SPRPTHPSPQSSTRK & & 0.3894 \\
\hline P35565 & Canx & S & 56 & 2 & SDTSTPPSPKVTYKA & & -0.3402 \\
\hline Q8VHK2 & Caskin1 & $\mathrm{s}$ & 432 & 1 & SESSPGDSPVKPPEG & -0.0285 & 0.4727 \\
\hline Q8VHK2 & Caskin1 & $S$ & 790 & 1 & PTKAQPGSPQALGGP & -0.2383 & 0.3081 \\
\hline Q8VHK2 & Caskin1 & $S$ & 1243 & 1 & PVSKIQGSPTPASKK & -0.4762 & 0.2915 \\
\hline Q8VHK2 & Caskin1 & $\mathrm{s}$ & 1258 & 1 & VPLPGPGSPEVKRAH & 0.0555 & 0.3358 \\
\hline Q8VHK2 & Caskin1 & $S$ & 1400 & 1 & DGQGPRPSSIEEKST & 0.2598 & -0.2716 \\
\hline F1MAS1 & Cbarp & $\mathrm{s}$ & 52 & 1 & SSLDTRGSPKRHHFQ & & 0.6212 \\
\hline
\end{tabular}




\begin{tabular}{|c|c|c|c|c|c|c|c|}
\hline A0A0G2JUY5 & Ccdc120 & $S$ & 253 & 1 & FRAVSGGSPERRAPW & 0.1734 & 0.3017 \\
\hline D4AEK9 & Ccdc6 & S & 410 & 2 & TRPSPRRSSSPDKFK & & 0.595 \\
\hline D4AEK9 & Ccdc6 & S & 411 & 2 & RPSPRRSSSPDKFKR & & 0.5461 \\
\hline D3ZMD4 & Ccdc85a & $\mathrm{T}$ & 262 & 1 & HKPRASGTPDHPKAL & & 0.3389 \\
\hline D3ZMD4 & Ccdc85a & $S$ & 394 & 1 & RPAQEDSSPHHRNVY & & 0.5722 \\
\hline Q7TT49 & Cdc42bpb & $\mathrm{s}$ & 1685 & 2 & HSTPSNSSNPSGPPS & -0.245 & 0.6788 \\
\hline Q7TT49 & Cdc42bpb & $S$ & 1688 & 1 & PSNSSNPSGPPSPNS & & 0.5325 \\
\hline Q7TT49 & Cdc42bpb & $S$ & 1692 & 2 & SNPSGPPSPNSPHRS & -0.224 & 0.4523 \\
\hline Q7TT49 & Cdc42bpb & $\mathrm{s}$ & 1695 & 1 & SGPPSPNSPHRSQLP & -0.1153 & 0.9262 \\
\hline Q7TT49 & Cdc42bpb & $\mathrm{s}$ & 1695 & 2 & SGPPSPNSPHRSQLP & -0.2052 & 0.6635 \\
\hline B1WC33 & Cdc42ep4 & $S$ & 140 & 1 & LPKSLSSSPVKKADT & -0.3445 & 0.3895 \\
\hline B1WC33 & Cdc42ep4 & $S$ & 154 & 1 & TRDGSPKSPHRNGAT & & 0.6902 \\
\hline Q63315 & Cdh22 & S & 717 & 1 & ASSSERHSLPRGPSS & 0.0405 & 0.3153 \\
\hline F1M491 & Cdk5r2 & $T$ & 84 & 1 & KKGSKKVTPKPASTG & & 0.7062 \\
\hline F1LQC8 & Cdk7 & $\mathrm{s}$ & 165 & 1 & GLAKSFGSPNRAYTH & & -0.2982 \\
\hline D3ZG85 & Cdkl5 & S & 343 & 1 & QTHHRSNSKDIQNLS & & 0.9937 \\
\hline D3ZG85 & Cdkl5 & $S$ & 407 & 1 & NNIPHLLSPKEAKSK & 0.13 & 1.7226 \\
\hline D3ZG85 & Cdkl5 & $S$ & 691 & 1 & GVYHDPHSDDGTAPK & & 0.5594 \\
\hline Q5XIM5 & Cdv3 & S & 51 & 1 & SRPGDGGSLGSGARS & 0.2791 & 0.4654 \\
\hline Q9QYP2 & Celsr2 & $\mathrm{S}$ & 1854 & 1 & FACSRKPSPDPALTT & & -0.289 \\
\hline Q5FVI4 & Cend1 & S & 7 & 1 & MESRGKSASSPKPD & 0.0425 & 0.4552 \\
\hline Q5FVI4 & Cend1 & S & 7 & 2 & MESRGKSASSPKPD & 0.0837 & 0.6331 \\
\hline Q5FVI4 & Cend 1 & $S$ & 9 & 2 & ESRGKSASSPKPDTK & 0.0771 & 0.6403 \\
\hline Q5FVI4 & Cend1 & $S$ & 10 & 2 & SRGKSASSPKPDTKV & 0.0761 & 0.6452 \\
\hline Q5FVI4 & Cend1 & $T$ & 15 & 2 & ASSPKPDTKVPQATA & 0.0853 & 0.6006 \\
\hline Q5FVI4 & Cend 1 & $\mathrm{~T}$ & 21 & 1 & DTKVPQATAEAKATP & & 0.4018 \\
\hline B2RZ23 & Cenpa & $\mathrm{s}$ & 15 & 1 & GTPRRRPSSPAPGPS & & 1.2181 \\
\hline B2RZ23 & Cenpa & $\mathrm{S}$ & 16 & 1 & TPRRRPSSPAPGPSQ & & 1.0186 \\
\hline B2RZ23 & Cenpa & $S$ & 41 & 1 & TPTRRPSSPAPGPSR & & 0.4291 \\
\hline A0A0G2K315 & Cep170 & S & 675 & 1 & DSKALLHSGSNSSKE & & 0.384 \\
\hline A0A0G2K315 & Cep170 & S & 677 & 1 & KALLHSGSNSSKEKS & 0.5197 & 0.2966 \\
\hline D4A1G8 & Cep170b & $S$ & 360 & 1 & KHEDGTQSDSEDPLA & -0.0714 & 0.3794 \\
\hline D4A1G8 & Cep170b & $\mathrm{s}$ & 362 & 1 & EDGTQSDSEDPLAKT & -0.0819 & 0.4551 \\
\hline D4A1G8 & Cep170b & $S$ & 862 & 1 & LTFARQESFTKEPTS & 0.2803 & 0.2647 \\
\hline D4A1G8 & Cep170b & $S$ & 965 & 1 & LSGKNGPSPTTPQTP & -0.1159 & 0.3807 \\
\hline D4A1G8 & Cep170b & $\mathrm{T}$ & 967 & 1 & GKNGPSPTTPQTPGP & -0.1058 & 0.4321 \\
\hline D4A1G8 & Cep170b & $S$ & 1254 & 1 & TSEEEYGSHHSSPKH & -0.0698 & 1.0695 \\
\hline D4A1G8 & Cep170b & S & 1257 & 1 & EEYGSHHSSPKHTRS & -0.122 & 0.9923 \\
\hline D3ZZ61 & Cep68 & $\mathrm{S}$ & 416 & 1 & GKNASAGSPQLKTKE & 0.0758 & 1.1451 \\
\hline M0RC65 & $\mathrm{Cfl}$ & $S$ & 23 & 1 & NDMKVRKSSTQEEIK & & -0.328 \\
\hline D3ZUX5 & Chchd3 & $S$ & 46 & 1 & SSPSGSKSQRYSSVY & & -0.3045 \\
\hline D4A7N1 & Chchd6 & $\mathrm{s}$ & 147 & 1 & TEKHLKASLPKKKAT & & -0.3371 \\
\hline D4A7N1 & Chchd6 & $T$ & 179 & 1 & AELSRRDTFYKEQQE & & -0.2706 \\
\hline A0A0G2KA92 & Chd2 & $\mathrm{s}$ & 1100 & 1 & AQTNDSDSDTESKRQ & & 0.5401 \\
\hline
\end{tabular}




\begin{tabular}{|c|c|c|c|c|c|c|c|}
\hline E9PU01 & Chd4 & $S$ & 1528 & 1 & KKMSQPGSPSPKTPT & & -0.2817 \\
\hline O35314 & Chgb & S & 155 & 1 & KEAKIRHSEERGGKE & 0.3859 & -0.4208 \\
\hline Q5U2T8 & Cir1 & $S$ & 380 & 1 & KSRSHQHSPERKGSD & 0.1408 & 0.765 \\
\hline Q5U2T8 & Cir1 & $S$ & 426 & 1 & ARSRPHQSPSEEQKG & 0.0231 & 0.5659 \\
\hline E9PSL7 & Cit & $S$ & 1458 & 1 & FCRDKVSSPGLQSKE & -0.0053 & -0.3088 \\
\hline P07335 & Ckb & $S$ & 309 & 1 & LGKHEKFSEVLKRLR & & -0.4259 \\
\hline A0A0G2JZF2 & Clip2 & $S$ & 203 & 1 & SVKTGNESGSNLSDS & & -0.3834 \\
\hline A0A0G2JZF2 & Clip2 & $S$ & 205 & 1 & KTGNESGSNLSDSGS & & -0.4307 \\
\hline A0A0G2K5Q8 & Clk2 & $\mathrm{s}$ & 528 & 1 & VQSSRKLSPAEPKNY & -0.5594 & -0.2701 \\
\hline D4A626 & CImn & $\mathrm{S}$ & 419 & 1 & KDGRRSNSLPVKKSV & 0.0946 & 0.5547 \\
\hline D4A626 & CImn & S & 536 & 1 & EKGPSPPSPRDNTIL & 0.0769 & 0.828 \\
\hline D4A626 & Clmn & S & 722 & 1 & EGLDFKPSPPLSKIS & & 0.8159 \\
\hline P08081 & Clta & $S$ & 105 & 1 & SEVDRLQSEPESIRK & 0.3735 & -0.3541 \\
\hline P11442 & Cltc & $T$ & 238 & 1 & IEVGTPPTGNQPFPK & & -0.3279 \\
\hline P11442 & Cltc & $\mathrm{S}$ & 576 & 1 & ALKNNRPSEGPLQTR & 0.0742 & -0.4081 \\
\hline D4A1C0 & Cnnm1 & $\mathrm{T}$ & 454 & 2 & KAPTTRGTPQTPKDD & -0.1275 & 0.4618 \\
\hline D4A1C0 & Cnnm1 & $T$ & 457 & 2 & TTRGTPQTPKDDPVL & -0.1335 & 0.4606 \\
\hline A0A0G2K717 & Cnot2 & $S$ & 165 & 1 & MSSSGLGSPNRSSPS & & 0.9298 \\
\hline D3ZUV9 & Cnot3 & $\mathrm{S}$ & 299 & 1 & TDSEVSQSPAKNGSK & 0.212 & 0.6891 \\
\hline P20272 & Cnr1 & $T$ & 314 & 2 & VRMIQRGTQKSIIIH & 1.377 & -0.29 \\
\hline P20272 & Cnr1 & $\mathrm{T}$ & 322 & 1 & QKSIIIHTSEDGKVQ & 1.2867 & -0.2432 \\
\hline P20272 & Cnr1 & $T$ & 322 & 2 & QKSIIIHTSEDGKVQ & 1.4183 & -0.3059 \\
\hline P20272 & Cnr1 & $\mathrm{s}$ & 323 & 2 & KSIIIHTSEDGKVQV & & -0.3373 \\
\hline D3ZKK3 & Cnst & $S$ & 356 & 1 & KIVPVEQSPGKKGFP & & 0.3851 \\
\hline D3ZUI5 & Cobl & $\mathrm{S}$ & 219 & 1 & REMLRKSSLGNDETD & & -0.3671 \\
\hline D3ZUI5 & Cobl & $\mathrm{s}$ & 838 & 1 & GKSATHNSPAAVHRN & -0.1341 & 0.5028 \\
\hline D4ACN6 & Col4a3bp & $\mathrm{s}$ & 380 & 2 & SRSPSMSSIDLVSAS & & -0.2663 \\
\hline P22734 & Comt & $\mathrm{S}$ & 260 & 1 & AIYQGPSSPDKS & 0.1362 & -0.2711 \\
\hline O35142 & Copb2 & S & 860 & 1 & PDGKPASSPVIMASQ & & 0.3938 \\
\hline Q920J3 & Coro6 & S & 418 & 1 & LDVRPPASPRRSQSA & & 0.6628 \\
\hline P10888 & Cox4i1 & $S$ & 26 & 1 & VCLRAHGSVVKSEDY & & -0.3241 \\
\hline P10888 & Cox4i1 & $\mathrm{s}$ & 58 & 1 & HVKLLSASQKALKEK & 0.3069 & -0.3512 \\
\hline P63041 & Cplx1 & $\mathrm{s}$ & 93 & 1 & MEANSEGSLTRPKKA & 0.1562 & 0.3125 \\
\hline D3ZGN2 & Cpne5 & $S$ & 575 & 1 & PPAAPVQSPPQSPAH & & -0.2694 \\
\hline P36201 & Crip2 & $\mathrm{s}$ & 104 & 1 & RTEERKTSGPPKGPS & & 0.5172 \\
\hline Q62950 & Crmp1 & $\mathrm{s}$ & 521 & 2 & APAPSAKSSPSKHQP & -0.5016 & 0.2967 \\
\hline Q8VHF5 & Cs & $\mathrm{S}$ & 225 & 1 & RNLYREGSSIGAIDS & & -0.3248 \\
\hline Q8VHF5 & Cs & $\mathrm{s}$ & 226 & 1 & NLYREGSSIGAIDSK & & -0.3629 \\
\hline P18395 & Csde1 & S & 514 & 1 & VRLLGRNSNSKRLLG & & -0.5896 \\
\hline Q9JJ76 & Csnk1e & $S$ & 363 & 1 & IQQAGNTSPRAISRA & -0.1775 & 0.4624 \\
\hline P67874 & Csnk2b & $S$ & 205 & 1 & QLQLQAASNFKSPVK & 0.0096 & 0.5441 \\
\hline P67874 & Csnk2b & $\mathrm{s}$ & 209 & 1 & QAASNFKSPVKTIR & -0.0258 & 0.3192 \\
\hline $\mathrm{D} 4 \mathrm{~A} 6 \mathrm{H} 8$ & Ctnna2 & $S$ & 462 & 1 & GSQKKHISPVQALSE & & 0.6749 \\
\hline D3ZZZ9 & Ctnnd1 & $\mathrm{T}$ & 310 & 1 & GTARRTGTPSDPRRR & & 0.411 \\
\hline
\end{tabular}




\begin{tabular}{|c|c|c|c|c|c|c|c|}
\hline F1M787 & Ctnnd2 & $S$ & 250 & 1 & PAPPRGGSPLTTTQG & -0.1991 & 0.5833 \\
\hline F1M787 & Ctnnd2 & S & 250 & 2 & PAPPRGGSPLTTTQG & -0.1388 & 1.6764 \\
\hline F1M787 & Ctnnd2 & $S$ & 259 & 1 & LTTTQGGSPTKLQRG & -0.1302 & 0.95 \\
\hline F1M787 & Ctnnd2 & $S$ & 259 & 2 & LTTTQGGSPTKLQRG & -0.1416 & 1.6852 \\
\hline F1M787 & Ctnnd2 & $\mathrm{T}$ & 261 & 2 & TTQGGSPTKLQRGGS & & 1.0462 \\
\hline F1M787 & Ctnnd2 & S & 282 & 2 & YAAPRGSSPKQSPSR & -0.0146 & 2.8917 \\
\hline F1M787 & Ctnnd2 & $S$ & 286 & 2 & RGSSPKQSPSRLAKS & -0.0267 & 2.8538 \\
\hline F1M787 & Ctnnd2 & S & 288 & 2 & SSPKQSPSRLAKSYS & -0.0718 & 2.3735 \\
\hline F1M787 & Ctnnd2 & $S$ & 310 & 2 & VVSSAGLSPIRVTSP & -0.043 & 1.2637 \\
\hline F1M787 & Ctnnd2 & $S$ & 316 & 3 & LSPIRVTSPPTVQST & & 1.2466 \\
\hline F1M787 & Ctnnd2 & $S$ & 343 & 3 & IGTYATLSPTKRLVH & -0.0974 & 1.2625 \\
\hline F1M787 & Ctnnd2 & $S$ & 444 & 1 & RTSTAPSSPGVDSVP & -0.1597 & 0.4478 \\
\hline F1M787 & Ctnnd2 & $S$ & 1084 & 1 & RSASAPASPREMISL & 0.0068 & 0.4045 \\
\hline D3ZGE6 & Cttn & S & 47 & 1 & GAKTVQGSGHQEHIN & -0.0415 & 0.6655 \\
\hline D3ZGE6 & Cttn & S & 150 & 1 & GKTEKHASQKDYSSG & & 0.3057 \\
\hline Q66HL2 & Cttn & $S$ & 261 & 1 & EKLQLHESQKDYAKG & & 0.3524 \\
\hline Q2IBD4 & Cttnbp2 & $S$ & 472 & 1 & SPPSRDVSPTSRDNL & 0.0231 & 0.9691 \\
\hline Q2IBD4 & Cttnbp2 & $S$ & 554 & 1 & PGLSQTPSPPHPQLR & & 0.3173 \\
\hline A0A0G2K652 & Cul9 & $S$ & 939 & 1 & STQGQDGSPESLIRS & & -0.8514 \\
\hline Q5EB81 & Cyb5r1 & $S$ & 169 & 1 & NIQPNKKSPPELRVA & & -0.2813 \\
\hline Q66H62 & Cyld & $S$ & 328 & 1 & RGVGDKGSSSHNKPK & 0.6781 & 0.4006 \\
\hline Q66H62 & Cyld & $S$ & 359 & 1 & FYTLNGSSVDSQQQS & & 0.3492 \\
\hline Q66H62 & Cyld & S & 396 & 1 & SSDFGHSSPPPQPPS & & 0.3815 \\
\hline Q8CJH2 & Dab1 & S & 400 & 1 & TNDSARSSPQSDKPR & & 0.5227 \\
\hline A0A0G2JTF2 & Dab2ip & $S$ & 694 & 1 & RSSGVQPSPARSSSY & -0.0335 & 0.3794 \\
\hline A0A0G2JTF2 & Dab2ip & S & 950 & 1 & SSSSKGDSPELKPRA & -0.0266 & 0.4404 \\
\hline M0R4J7 & Dact3 & $S$ & 374 & 1 & RGRSVEQSPPRERPR & 0.117 & 0.2985 \\
\hline Q5YLM1 & Dagla & $S$ & 733 & 1 & SKSQSEMSLEGFSEG & -0.0799 & 0.492 \\
\hline Q5YLM1 & Dagla & S & 808 & 1 & GFRSIRGSPSLHAVL & 0.2031 & 0.5574 \\
\hline Q5YLM1 & Dagla & $\mathrm{T}$ & 1025 & 1 & TDKIRTSTPTGHGAS & & 1.2612 \\
\hline Q5YLM1 & Dagla & $\mathrm{T}$ & 1025 & 2 & TDKIRTSTPTGHGAS & -0.2622 & 1.4076 \\
\hline Q5YLM1 & Dagla & $S$ & 1032 & 1 & TPTGHGASPTKQDDL & -0.1666 & 1.1749 \\
\hline Q5YLM1 & Dagla & $S$ & 1032 & 2 & TPTGHGASPTKQDDL & -0.2559 & 1.3079 \\
\hline A0A0H2UHL9 & Dbn1 & $S$ & 385 & 1 & PIPTRSPSDSSTAST & & 0.3074 \\
\hline A0AOH2UHL9 & Dbn1 & $S$ & 385 & 2 & PIPTRSPSDSSTAST & -0.3499 & \\
\hline D4A8L4 & Dcaf6 & $S$ & 693 & 1 & DSATRRASDNPELPS & -0.0093 & -0.4293 \\
\hline Q9WVP7 & Dclk1 & $S$ & 33 & 1 & PSPTSPGSLRKQRDL & 1.0367 & -0.2965 \\
\hline Q9ESI7 & Dcx & $S$ & 306 & 1 & GPMRRSKSPADSGND & & 0.4896 \\
\hline P49621 & Dgkb & $S$ & 95 & 1 & SNKFPHSSPNVKSKP & 0.1773 & 0.3436 \\
\hline A0A0G2K1M2 & Dlg1 & $S$ & 143 & 2 & HVSEKSLSEIENVHG & -0.2103 & 0.7444 \\
\hline A0A0G2K1M2 & Dlg1 & $S$ & 155 & 1 & VHGFVSHSHISPIKP & -0.0082 & 0.7214 \\
\hline A0A0G2K1M2 & Dlg1 & S & 158 & 1 & FVSHSHISPIKPTEA & -0.0599 & 0.7082 \\
\hline $\mathrm{A} 0 \mathrm{~A} 0 \mathrm{G} 2 \mathrm{~K} 1 \mathrm{M} 2$ & Dlg1 & $S$ & 158 & 2 & FVSHSHISPIKPTEA & -0.2168 & 0.7444 \\
\hline Q63622 & Dlg2 & $S$ & 175 & 1 & EALKEAGSIVRLYVR & 0.4889 & -0.2633 \\
\hline
\end{tabular}




\begin{tabular}{|c|c|c|c|c|c|c|c|}
\hline Q63622 & Dlg2 & $T$ & 317 & 2 & NGTLEYKTSLPPISP & -0.7041 & 0.7973 \\
\hline Q63622 & Dlg2 & S & 323 & 2 & KTSLPPISPGRYSPI & -0.45 & 0.7773 \\
\hline Q63622 & Dlg2 & $\mathrm{S}$ & 328 & 2 & PISPGRYSPIPKHML & -0.5513 & 0.788 \\
\hline Q63622 & Dlg2 & S & 360 & 1 & KLCDKPASPRHYSPV & 0.0378 & 1.0697 \\
\hline Q63622 & Dlg2 & S & 365 & 1 & PASPRHYSPVECDKS & 0.0712 & 0.5461 \\
\hline Q63622 & Dlg2 & $\mathrm{S}$ & 406 & 1 & STATRQPSVTLQRAI & 0.1254 & 0.5397 \\
\hline Q62936 & Dlg3 & S & 632 & 1 & KGVTSNTSDSESSSK & 0.1838 & 0.2989 \\
\hline G3V849 & Dlgap1 & $T$ & 557 & 1 & CITTYKKTPPPVPPR & 0.1487 & 0.2705 \\
\hline G3V849 & Dlgap1 & $\mathrm{S}$ & 947 & 1 & APLIRERSLESSQRQ & 0.2245 & 1.5822 \\
\hline P97837 & Dlgap2 & S & 455 & 2 & EESGESDSSPKTSPT & 0.2056 & 0.546 \\
\hline P97837 & Dlgap2 & $\mathrm{s}$ & 456 & 2 & ESGESDSSPKTSPTV & -0.1062 & 0.5503 \\
\hline P97837 & Dlgap2 & $T$ & 459 & 2 & ESDSSPKTSPTVALR & & 0.6499 \\
\hline P97837 & Dlgap2 & S & 637 & 1 & ISVTAQSSTESTQDA & -0.0628 & 0.8563 \\
\hline P97837 & Dlgap2 & S & 670 & 1 & GLYNSMDSLDSNKAM & & 0.4486 \\
\hline P97837 & Dlgap2 & $\mathrm{S}$ & 1012 & 1 & FPITREKSLDLPDRQ & 0.4817 & 0.3042 \\
\hline G3V7T8 & Dlgap3 & S & 165 & 1 & APEARSESPSRIRHL & & 0.7221 \\
\hline G3V7T8 & Dlgap3 & S & 428 & 1 & RFASRRSSSVDTARI & 0.1263 & 0.4894 \\
\hline G3V7T8 & Dlgap3 & $\mathrm{S}$ & 559 & 2 & ISITAQSSTDSAHES & -0.1514 & 0.5736 \\
\hline G3V7T8 & Dlgap3 & $\mathrm{S}$ & 562 & 2 & TAQSSTDSAHESFTA & -0.1143 & 0.3586 \\
\hline G3V927 & Dlgap4 & S & 388 & 2 & GGGSPKPSPKTAARR & & 0.5816 \\
\hline G3V927 & Dlgap4 & S & 405 & 1 & YLRATQQSLGEQSNP & 0.2321 & 0.3517 \\
\hline G3V927 & Dlgap4 & $S$ & 410 & 1 & QQSLGEQSNPRRSLD & -0.0316 & 0.4476 \\
\hline G3V927 & Dlgap4 & S & 415 & 1 & EQSNPRRSLDRLDSV & 0.0804 & 0.3018 \\
\hline G3V927 & Dlgap4 & S & 581 & 2 & ISVTVQSSTESAQDT & & 0.5611 \\
\hline G3V927 & Dlgap4 & $\mathrm{T}$ & 582 & 2 & SVTVQSSTESAQDTY & & 0.5611 \\
\hline G3V927 & Dlgap4 & S & 683 & 1 & PVPKEEPSPATKFQS & & 0.4703 \\
\hline B2GUY4 & Dmtn & S & 26 & 1 & RDSSVPGSPSNIVAK & -0.0389 & 0.5556 \\
\hline B2GUY4 & Dmtn & $S$ & 152 & 1 & PIYKQRESVGGSPQS & 1.525 & 0.1124 \\
\hline B2GUY4 & Dmtn & S & 152 & 2 & PIYKQRESVGGSPQS & & 0.3096 \\
\hline B2GUY4 & Dmtn & S & 156 & 1 & QRESVGGSPQSKHLI & 0.101 & 0.9248 \\
\hline B2GUY4 & Dmtn & S & 156 & 2 & QRESVGGSPQSKHLI & & 0.3096 \\
\hline Q5XIP0 & Dnajb4 & S & 38 & 1 & FHPDKNKSPQAEEKF & 0.3709 & -0.2913 \\
\hline P60905 & Dnajc5 & $\mathrm{T}$ & 27 & 1 & LGLDKNATSDDIKKS & & -0.3799 \\
\hline P60905 & Dnajc5 & $S$ & 28 & 1 & GLDKNATSDDIKKSY & 0.2311 & -0.4726 \\
\hline A0A0G2JY26 & Dnajc6 & $S$ & 687 & 2 & AATSPTGSTHGTPTH & -0.4863 & 0.6633 \\
\hline A0A0G2JY26 & Dnajc6 & S & 687 & 3 & AATSPTGSTHGTPTH & -0.8134 & \\
\hline A0A0G2JY26 & Dnajc6 & $\mathrm{T}$ & 688 & 2 & ATSPTGSTHGTPTHQ & -0.5397 & 0.6465 \\
\hline A0A0G2JY26 & Dnajc6 & $\mathrm{T}$ & 688 & 3 & ATSPTGSTHGTPTHQ & -0.8183 & \\
\hline A0A0G2JY26 & Dnajc6 & S & 795 & 1 & QSDRGKGSTNLEGKQ & 0.4131 & 0.4129 \\
\hline P21575 & Dnm1 & S & 347 & 1 & FEKRIEGSGDQIDTY & -0.1217 & -0.3109 \\
\hline Q1LZ53 & Dnmt3a & S & 102 & 1 & EPQPEEGSPAAGQKG & & 0.6826 \\
\hline D3ZZW1 & Dock1 & S & 1857 & 1 & KTTRKQTSVDSGIVQ & & 0.3614 \\
\hline F1M4N6 & Dock3 & S & 1766 & 1 & APSSTRGSPSLPDKY & 0.1728 & 0.7939 \\
\hline F1M4N6 & Dock3 & $S$ & 2049 & 1 & RGLHRKASLPPGSAK & 0.391 & 0.4659 \\
\hline
\end{tabular}




\begin{tabular}{|c|c|c|c|c|c|c|c|}
\hline MOR6K4 & Dock4 & $S$ & 1697 & 1 & APSSARASPLLSDKH & 0.2314 & 0.5762 \\
\hline MOR6K4 & Dock4 & $S$ & 1758 & 1 & LSAPEKASPARHTTS & & 1.381 \\
\hline MOR6K4 & Dock4 & $S$ & 1774 & 1 & SPSPAGRSPLKSSVQ & & 0.7551 \\
\hline A0A0G2K3H2 & Dock7 & $\mathrm{S}$ & 440 & 1 & WSERRNSSLVGRRSL & & -0.3789 \\
\hline P47942 & Dpysl2 & $\mathrm{T}$ & 521 & 2 & TPASSAKTSPAKQQA & -0.2769 & 0.3849 \\
\hline P47942 & Dpysl2 & $\mathrm{T}$ & 521 & 3 & TPASSAKTSPAKQQA & -0.4307 & 0.12 \\
\hline P47942 & Dpysl2 & S & 522 & 2 & PASSAKTSPAKQQAP & -0.3987 & 0.4764 \\
\hline P47942 & Dpysl2 & $S$ & 522 & 3 & PASSAKTSPAKQQAP & -0.5053 & 0.2483 \\
\hline P18901 & Drd1 & $\mathrm{T}$ & 446 & 1 & THSGQHST & -0.0244 & 0.3928 \\
\hline Q9EPA0 & Drp2 & $\mathrm{T}$ & 910 & 1 & PREKGQTTPDTEAAD & & 0.3554 \\
\hline A0A0G2JVM6 & Dtnb & $S$ & 531 & 1 & QAAQATGSPHTSPTH & 0.366 & 0.1311 \\
\hline A0A0G2JVM6 & Dtnb & $S$ & 531 & 2 & QAAQATGSPHTSPTH & -0.091 & 0.7233 \\
\hline A0A0G2JVM6 & Dtnb & $\mathrm{T}$ & 534 & 2 & QATGSPHTSPTHGGG & -0.0532 & 0.6229 \\
\hline A0A0G2JVM6 & Dtnb & $S$ & 535 & 2 & ATGSPHTSPTHGGGR & -0.1073 & 0.7922 \\
\hline Q62698 & Dync1li2 & $S$ & 432 & 1 & KKTGSPGSPSAGGVQ & & 0.3343 \\
\hline P23965 & Eci1 & $\mathrm{T}$ & 249 & 1 & KSMMRKATADNLIKQ & & -0.3029 \\
\hline Q68FR9 & Eef1d & $S$ & 133 & 1 & APQTQHVSPMRQVEP & 0.0986 & 0.355 \\
\hline F1LTW9 & Efr3b & $S$ & 216 & 1 & EAESRSPSPLQAPEK & 0.1502 & -0.2942 \\
\hline D4ACF1 & Eif4enif1 & $S$ & 77 & 1 & LYPASGRSSPVESLK & & 0.8623 \\
\hline D4ACF1 & Eif4enif1 & $S$ & 78 & 1 & YPASGRSSPVESLKK & & 0.9083 \\
\hline D3ZU13 & Eif4g1 & $S$ & 1185 & 1 & RTPATKRSFSKEVEE & & 0.3592 \\
\hline A0A0G2JY73 & Eif4g3 & S & 322 & 1 & PEPPRTSSPTTLPPL & & 0.4022 \\
\hline A0A0G2JY73 & Eif4g3 & $S$ & 526 & 1 & QDKAEAESDGQTEET & 0.1311 & 0.4639 \\
\hline D3ZZ44 & Elfn1 & $S$ & 650 & 1 & SSSGSARSPRTFRAE & & 1.3782 \\
\hline $\mathrm{D} 3 \mathrm{ZH} 36$ & Elfn2 & S & 619 & 1 & HPLQRQLSADAAVTR & 0.258 & 0.5523 \\
\hline $\mathrm{D} 3 \mathrm{ZH} 36$ & Elfn2 & $S$ & 747 & 1 & DSTYSQLSPRHYYSG & & 0.5929 \\
\hline $\mathrm{D} 3 \mathrm{ZH} 36$ & Elfn2 & $S$ & 757 & 2 & HYYSGYSSSPEYSSE & -0.2092 & 0.6845 \\
\hline $\mathrm{D} 3 \mathrm{ZH} 36$ & Elfn2 & S & 758 & 1 & YYSGYSSSPEYSSES & & 0.7351 \\
\hline D3ZH36 & Elfn2 & $S$ & 758 & 2 & YYSGYSSSPEYSSES & -0.2092 & 0.6845 \\
\hline D4A4R1 & Eml3 & $S$ & 156 & 1 & SDTPRRNSSSSSSPS & -0.0615 & 0.596 \\
\hline Q5XHX3 & Enah & S & 430 & 1 & TEQKEDRSEDAEPVT & & 0.5454 \\
\hline P04764 & Eno1 & $S$ & 419 & 1 & RIEEELGSKAKFAGR & 0.2513 & -0.3777 \\
\hline D3ZIP3 & Epb41 & $\mathrm{T}$ & 60 & 1 & LKASNGDTPTHEDLT & & 0.9663 \\
\hline D3ZIP3 & Epb41 & $\mathrm{T}$ & 62 & 1 & ASNGDTPTHEDLTKN & & 0.5724 \\
\hline D3ZIP3 & Epb41 & $S$ & 675 & 1 & EIKKHHASISELKKN & & 0.5452 \\
\hline AOA0G2KOF3 & Epb4111 & $\mathrm{T}$ & 488 & 1 & ELKPEQETTPRHKQE & 0.0457 & 1.3429 \\
\hline A0A0G2KOF3 & Epb41I1 & $\mathrm{T}$ & 489 & 1 & LKPEQETTPRHKQEF & 0.1911 & 1.3175 \\
\hline A0A0G2K0F3 & Epb4111 & $S$ & 544 & 2 & RLPSSPASPSPKGTP & -0.2134 & 0.4522 \\
\hline A0A0G2K0F3 & Epb4111 & $S$ & 546 & 1 & PSSPASPSPKGTPEK & & 0.3303 \\
\hline A0A0G2KOF3 & Epb41I1 & S & 546 & 2 & PSSPASPSPKGTPEK & -0.0964 & 0.7109 \\
\hline A0A0G2K0F3 & Epb4111 & $\mathrm{T}$ & 550 & 1 & ASPSPKGTPEKASEA & & 0.3186 \\
\hline AOA0G2KOF3 & Epb4111 & $\mathrm{T}$ & 550 & 2 & ASPSPKGTPEKASEA & 0.0431 & 0.7691 \\
\hline A0A0G2K0F3 & Epb41/1 & $\mathrm{S}$ & 915 & 1 & WMGKTEKSPPARRKK & & 0.5032 \\
\hline A0A0G2K0F3 & Epb41/1 & $S$ & 1223 & 1 & GGRLRFASPPGPQRA & & 0.5394 \\
\hline
\end{tabular}




\begin{tabular}{|c|c|c|c|c|c|c|c|}
\hline Q9WTP0 & Epb4111 & S & 544 & 2 & RLPSSPASPSPKGTP & -0.2106 & 0.42 \\
\hline Q9WTP0 & Epb4111 & S & 546 & 1 & PSSPASPSPKGTPEK & & 0.3303 \\
\hline Q9WTP0 & Epb4111 & $S$ & 546 & 2 & PSSPASPSPKGTPEK & -0.0939 & 0.7109 \\
\hline Q9WTP0 & Epb4111 & $T$ & 550 & 1 & ASPSPKGTPEKASER & & 0.3186 \\
\hline Q9WTP0 & Epb4111 & $\mathrm{T}$ & 550 & 2 & ASPSPKGTPEKASER & 0.0413 & 0.6506 \\
\hline G3V874 & Epb4113 & $Y$ & 82 & 2 & AAAKQLEYQQFEDDK & -0.2417 & 0.5565 \\
\hline G3V874 & Epb4113 & $\mathrm{s}$ & 91 & 1 & QFEDDKLSQKSSSSK & 0.1 & 0.9216 \\
\hline G3V874 & Epb4113 & S & 91 & 2 & QFEDDKLSQKSSSSK & -0.2808 & 0.4869 \\
\hline G3V874 & Epb4113 & S & 94 & 2 & DDKLSQKSSSSKLSR & -0.2804 & 0.4771 \\
\hline G3V874 & Epb4113 & S & 508 & 1 & RHEGKTDSERTDTAA & & 0.6801 \\
\hline G3V874 & Epb4113 & S & 578 & 1 & WEKRLSTSPVRLAAR & 0.0255 & 0.8575 \\
\hline A0A0G2JW20 & Epb4114a & $S$ & 217 & 1 & KKAKNENSPDPQRSK & 0.0184 & 0.6067 \\
\hline A0A0G2JW20 & Epb4114a & S & 356 & 1 & RSRHRSRSRSPDIQA & 0.0146 & 0.6173 \\
\hline A0A0G2JW20 & Epb4114a & S & 358 & 1 & RHRSRSRSPDIQAKE & 0.0064 & 0.5379 \\
\hline $\mathrm{A} 0 \mathrm{~A} 0 \mathrm{G} 2 \mathrm{~K} 3 \mathrm{H} 4$ & Epb4114b & S & 569 & 1 & SPKIQQVSSPQKSEV & 0.037 & 0.5616 \\
\hline A0A0G2K3H4 & Epb4114b & $\mathrm{S}$ & 570 & 1 & PKIQQVSSPQKSEVK & 0.0087 & 0.8881 \\
\hline $\mathrm{A} 0 \mathrm{~A} 0 \mathrm{G} 2 \mathrm{~K} 3 \mathrm{H} 4$ & Epb4114b & $\mathrm{S}$ & 578 & 1 & PQKSEVKSPLSPGAK & 0.1702 & 0.8089 \\
\hline A0A0G2K3H4 & Epb4114b & S & 581 & 1 & SEVKSPLSPGAKSPS & & 0.9305 \\
\hline D3ZZK3 & Epha4 & $S$ & 887 & 1 & KLIRNPNSLKRTGPE & & -0.3347 \\
\hline M0R9T2 & Erbin & S & 377 & 1 & AETNAGNSPVTTNRK & -0.1844 & 0.9043 \\
\hline D3ZJ32 & Esyt2 & S & 667 & 1 & SSSSLLASPSHIAAK & & 0.7415 \\
\hline M0R5H1 & Etl4 & $S$ & 187 & 1 & RGSRTRASLPVVRST & & -0.3747 \\
\hline M0R5H1 & Etl4 & S & 1856 & 1 & SIASTPLSPQAGRSV & -0.1866 & 0.3155 \\
\hline M0R5H1 & Etl4 & S & 1881 & 1 & NGSFKFQSPPHAGKG & -0.0731 & 0.8613 \\
\hline M0R5H1 & Etl4 & $S$ & 1917 & 1 & SSPPSPASPTSLNQG & & 0.2887 \\
\hline 008719 & Evl & S & 249 & 1 & PEDASGGSSPSGTSK & -0.0859 & 0.2846 \\
\hline 008719 & Evl & S & 250 & 1 & EDASGGSSPSGTSKS & -0.0266 & 0.3064 \\
\hline Q924K2 & Faf1 & $\mathrm{T}$ & 268 & 1 & RLTVGRRTSPVQTRE & 0.1308 & -0.2732 \\
\hline Q924K2 & Faf1 & S & 269 & 1 & LTVGRRTSPVQTREQ & 0.1306 & -0.2893 \\
\hline 088407 & Faim2 & S & 7 & 1 & MTQGKLSVANKAPG & & -0.4446 \\
\hline 088407 & Faim2 & $S$ & 37 & 2 & AVPSAPPSYEEATSG & & -0.3195 \\
\hline F1M8D5 & Fam117b & $\mathrm{S}$ & 160 & 1 & PGGADKGSNNSSRSQ & & 0.5954 \\
\hline Q4V7D4 & Fam126b & S & 294 & 1 & PFDAPDSSQEGQKVL & & -0.2878 \\
\hline Q4V7D4 & Fam126b & $S$ & 398 & 1 & SAIKSNESPRDSVVG & -0.1683 & 0.2772 \\
\hline F1LUC1 & Fam13c & S & 562 & 1 & FFKQTGRSPQKEDRM & & 0.7413 \\
\hline D4A7Y4 & Fam171a1 & S & 793 & 1 & LEGSGRRSGGQLPSL & & 0.3411 \\
\hline D4AAI7 & Fam219a & S & 55 & 1 & LKNSSMGSPVNQQPK & & 0.5469 \\
\hline F1LYQ8 & Farp1 & S & 427 & 1 & LEPGPRQSPALSKSP & 0.044 & 1.0366 \\
\hline $\mathrm{P} 12785$ & Fasn & S & 2191 & 1 & EMSSKAGSDTELAAP & 0.1277 & -0.2873 \\
\hline D3Z858 & Fbrsl1 & $S$ & 372 & 1 & TRVKENHSPSKEDGA & 0.269 & 0.9251 \\
\hline A0A0G2K9A9 & Fbxo41 & S & 391 & 1 & YAVSRHGSSPSTGAS & & 0.4889 \\
\hline A0A0G2K9A9 & Fbxo41 & S & 392 & 1 & AVSRHGSSPSTGASS & 0.0042 & 0.6014 \\
\hline A0A0G2K9A9 & Fbxo41 & S & 522 & 1 & SARPEGGSGRGRRME & 0.2361 & 0.9419 \\
\hline A0A0G2K9A9 & Fbxo41 & S & 532 & 1 & GRRMERGSPSRSNEV & & 0.3033 \\
\hline
\end{tabular}




\begin{tabular}{|c|c|c|c|c|c|c|c|}
\hline A0A0G2K7L6 & Fgd6 & S & 382 & 1 & ALTRESNSDRQDSVS & & 0.3998 \\
\hline A0A0G2K7L6 & Fgd6 & S & 387 & 1 & SNSDRQDSVSSQKAV & & 0.3571 \\
\hline Q8R5L7 & Fgf14 & $\mathrm{S}$ & 210 & 1 & VAMYREPSLHDVGET & 0.2267 & -0.2903 \\
\hline P84817 & Fis1 & $S$ & 29 & 1 & QSEQAAGSVSKSTQF & & -0.2643 \\
\hline Q5U2R3 & Frmd8 & S & 24 & 2 & SHRSSVSSVGARAAD & & 0.3918 \\
\hline A0A0G2JZZ5 & Frs2 & $\mathrm{S}$ & 374 & 1 & VWEARKLSRDEDDNL & $-3.00 \mathrm{E}-04$ & 0.2811 \\
\hline P85845 & Fscn1 & S & 38 & 1 & FKVNASASSLKKKQI & & -0.3438 \\
\hline P85845 & Fscn1 & S & 39 & 1 & KVNASASSLKKKQIW & 0.1778 & -0.419 \\
\hline P19132 & Fth1 & $\mathrm{S}$ & 5 & 1 & MTTASPSQVRQN & 0.1511 & -0.3871 \\
\hline Q6AY21 & G3bp2 & S & 225 & 1 & LEELEEKSATPPPTE & & 0.4768 \\
\hline Q6AY21 & G3bp2 & $T$ & 227 & 1 & ELEEKSATPPPTEPA & 0.2216 & 0.6916 \\
\hline A0A0G2JZD5 & Gab1 & S & 445 & 1 & FPSDRSSSLEGFHNQ & & 0.383 \\
\hline Q9EQH1 & Gab2 & $S$ & 220 & 1 & SQGTRQKSDTAVQKL & & 0.2723 \\
\hline O88871 & Gabbr2 & $T$ & 762 & 1 & QNRRFQFTQNQKKED & 0.9433 & -0.3868 \\
\hline P07936 & Gap43 & $T$ & 36 & 2 & DKAHKAATKIQASFR & 2.1757 & -0.4287 \\
\hline P07936 & Gap43 & $\mathrm{S}$ & 41 & 2 & AATKIQASFRGHITR & 2.1757 & -0.4137 \\
\hline P07936 & Gap43 & S & 110 & 1 & SKAGDAPSEEKKGEG & 0.4297 & -0.3087 \\
\hline P07936 & Gap43 & $T$ & 131 & 1 & EKAGSAETESAAKAT & 0.4235 & -0.2704 \\
\hline $\mathrm{F} 1 \mathrm{M} 8 \times 9$ & Gbf1 & $\mathrm{S}$ & 1786 & 1 & ASSSSPGSPVASSPS & & 0.3083 \\
\hline Q9JKB7 & Gda & S & 196 & 1 & KETERFVSEMLQKNY & & 0.2762 \\
\hline P08050 & Gja1 & S & 328 & 1 & GQAGSTISNSHAQPF & -0.4974 & 0.3542 \\
\hline P08050 & Gja1 & $S$ & 328 & 2 & GQAGSTISNSHAQPF & -0.339 & 0.094 \\
\hline P08050 & Gja1 & S & 330 & 1 & AGSTISNSHAQPFDF & & 0.3293 \\
\hline Q80XF7 & Gjc2 & S & 428 & 2 & RVGSEKGSTGSRDGK & -0.0714 & 0.4472 \\
\hline Q80XF7 & Gjc2 & $S$ & 431 & 2 & SEKGSTGSRDGKATV & -0.0422 & 0.4448 \\
\hline G3V8D7 & Gjd4 & $\mathrm{T}$ & 355 & 1 & GSAPHLRTKKSEWV & & 0.3407 \\
\hline D4ADD7 & Glrx5 & S & 151 & 1 & IDEKDQDSK & 0.1474 & -0.288 \\
\hline P10860 & Glud1 & $Y$ & 193 & 1 & VKINPKNYTDNELEK & & -0.3432 \\
\hline P10860 & Glud1 & $\mathrm{T}$ & 194 & 1 & KINPKNYTDNELEKI & & -0.3695 \\
\hline P10860 & Glud1 & S & 450 & 1 & LKNLNHVSYGRLTFK & 0.1651 & -0.4203 \\
\hline P10860 & Glud1 & $Y$ & 451 & 1 & KNLNHVSYGRLTFKY & 0.2511 & -0.3656 \\
\hline A0A0G2JX25 & Gmpr2 & S & 26 & 1 & PKRSTLKSRSEVDLT & & -0.2783 \\
\hline P54311 & Gnb1 & S & 136 & 1 & REGNVRVSRELAGHT & 0.2705 & -0.2875 \\
\hline P00507 & Got2 & $S$ & 306 & 1 & EEAKRVESQLKILIR & & -0.3027 \\
\hline Q03555 & Gphn & $\mathrm{T}$ & 278 & 2 & RDTASLSTTPSESPR & -0.1013 & 0.6838 \\
\hline Q03555 & Gphn & $\mathrm{T}$ & 279 & 1 & DTASLSTTPSESPRA & & 0.2748 \\
\hline Q03555 & Gphn & $\mathrm{T}$ & 279 & 2 & DTASLSTTPSESPRA & -0.2092 & 0.4356 \\
\hline Q03555 & Gphn & S & 281 & 2 & ASLSTTPSESPRAQA & -0.0856 & 0.5745 \\
\hline Q03555 & Gphn & S & 283 & 1 & LSTTPSESPRAQATS & -0.0888 & 0.4123 \\
\hline Q03555 & Gphn & S & 283 & 2 & LSTTPSESPRAQATS & -0.0699 & 0.5778 \\
\hline Q6P6V0 & Gpi & S & 441 & 1 & EALMKGKSPEEARKE & 0.3396 & -0.2919 \\
\hline A0A0A0MY13 & Gpr158 & S & 806 & 1 & TGRPKEESLKNRVFS & 1.0942 & -0.3173 \\
\hline A0A0A0MY13 & Gpr158 & S & 1042 & 1 & EVEQNPASFSKEKSH & & 0.3678 \\
\hline D4AAS1 & Gpr162 & S & 255 & 1 & DTRGKRRSSLDGSES & 0.2924 & -0.3003 \\
\hline
\end{tabular}




\begin{tabular}{|c|c|c|c|c|c|c|c|}
\hline D4AAS1 & Gpr162 & $S$ & 256 & 1 & TRGKRRSSLDGSESA & 0.364 & -0.2815 \\
\hline Q920R4 & Gprasp1 & $\mathrm{s}$ & 345 & 1 & YSDVTSGSVDKNKKD & 0.2876 & 0.3185 \\
\hline Q3KRC4 & Gprc5c & $\mathrm{S}$ & 434 & 1 & GKISQDQSPKNKTRW & & 0.3891 \\
\hline D3ZF21 & Gprin3 & S & 209 & 1 & RAVRIHSSPSADRPE & -0.0075 & 0.6179 \\
\hline D3ZF21 & Gprin3 & S & 211 & 1 & VRIHSSPSADRPEGE & & 1.007 \\
\hline A0A0G2JT06 & Gps1 & $\mathrm{s}$ & 275 & 1 & EQRGERDSQTQAILT & & -0.2637 \\
\hline Q3KR56 & Gramd1a & $S$ & 11 & 1 & TTPHSGRSSPSSSPS & 0.0398 & 0.3074 \\
\hline Q3KR56 & Gramd1a & $S$ & 12 & 1 & TPHSGRSSPSSSPSL & & 0.3596 \\
\hline D3ZYJ5 & Gramd1b & $\mathrm{s}$ & 174 & 1 & ILRKRSRSPTPQNQD & & 0.4235 \\
\hline D3ZYJ5 & Gramd1b & $\mathrm{s}$ & 194 & 1 & EKGSDHSSDKSPSTP & & 0.5058 \\
\hline D3ZYJ5 & Gramd1b & $\mathrm{T}$ & 200 & 1 & SSDKSPSTPEQGVQR & 0.0652 & 0.4058 \\
\hline P0CE43 & Grb10 & $S$ & 50 & 1 & SRGQPQASPRQKMQR & -0.1969 & 0.5651 \\
\hline Q63226 & Grid2 & S & 861 & 1 & WWSRRKGSRVPSKED & & -0.453 \\
\hline Q63226 & Grid2 & $S$ & 865 & 1 & RKGSRVPSKEDDKEI & 0.1362 & -0.3414 \\
\hline P35439 & Grin1 & $\mathrm{s}$ & 897 & 1 & SFKRRRSSKDTSTGG & 0.3389 & 0.5485 \\
\hline Q00959 & Grin2a & $\mathrm{s}$ & 1025 & 1 & MESLRQDSLNQNPVS & 0.0237 & 0.9425 \\
\hline Q00959 & Grin2a & $S$ & 1198 & 1 & FTLKDKGSPHSEGSD & 0.1332 & 1.1745 \\
\hline Q00959 & Grin2a & $S$ & 1198 & 2 & FTLKDKGSPHSEGSD & -0.0944 & 1.3655 \\
\hline Q00959 & Grin2a & $\mathrm{s}$ & 1201 & 1 & KDKGSPHSEGSDRYR & 0.285 & 1.2557 \\
\hline Q00959 & Grin2a & $S$ & 1201 & 2 & KDKGSPHSEGSDRYR & -0.0351 & 1.3571 \\
\hline Q00959 & Grin2a & $S$ & 1204 & 1 & GSPHSEGSDRYRQNS & -0.041 & 0.4678 \\
\hline Q00959 & Grin2a & $\mathrm{S}$ & 1204 & 2 & GSPHSEGSDRYRQNS & -0.1383 & 1.3708 \\
\hline Q00959 & Grin2a & $S$ & 1291 & 1 & LRINRQHSYDNILDK & 0.167 & 0.5227 \\
\hline Q00960 & Grin2b & $S$ & 1058 & 2 & GHDDLIRSDVSDIST & -0.1746 & 0.4664 \\
\hline Q00960 & Grin2b & $\mathrm{s}$ & 1061 & 2 & DLIRSDVSDISTHTV & -0.2037 & 0.4795 \\
\hline Q00960 & Grin2b & $T$ & 1067 & 2 & VSDISTHTVTYGNIE & -0.2198 & 0.4625 \\
\hline Q00960 & Grin2b & $S$ & 1255 & 2 & AGNLYDISEDNSLQE & -0.0801 & 0.4668 \\
\hline Q00960 & Grin2b & $S$ & 1259 & 1 & YDISEDNSLQELDQP & -0.0223 & 0.4291 \\
\hline Q00960 & Grin2b & $S$ & 1259 & 2 & YDISEDNSLQELDQP & -0.0801 & 0.4668 \\
\hline Q00960 & Grin2b & $S$ & 1284 & 1 & SSTKYPQSPTNSKAQ & 0.1661 & 0.3254 \\
\hline P97879 & Grip1 & $\mathrm{S}$ & 660 & 1 & IRKDEDNSDEQESSG & 0.0218 & -0.292 \\
\hline P23385 & Grm1 & $\mathrm{s}$ & 973 & 2 & AHFSPPSSPSMVVHR & -0.0922 & 0.2988 \\
\hline AOAOH2UHW6 & Grm5 & $S$ & 773 & 1 & PAAARPRSPSPISTL & & 0.85 \\
\hline AOAOH2UHW6 & Grm5 & $S$ & 773 & 2 & PAAARPRSPSPISTL & -0.1019 & 0.3406 \\
\hline AOAOH2UHW6 & Grm5 & $S$ & 775 & 1 & AARPRSPSPISTLSH & -0.1317 & 0.8925 \\
\hline AOAOH2UHW6 & Grm5 & $S$ & 778 & 2 & PRSPSPISTLSHLAG & -0.0557 & 0.4347 \\
\hline A0A0G2JY75 & Gtf3c2 & $S$ & 263 & 1 & PKSPKVSSPTKPKKT & & 0.652 \\
\hline Q9WVI4 & Gucy1a2 & $\mathrm{s}$ & 41 & 1 & LCWNGSRSPPGPPGS & 0.077 & 0.3243 \\
\hline A2RRU1 & Gys1 & $\mathrm{s}$ & 698 & 1 & PEWPRRASCSSSTGG & & 0.645 \\
\hline F1LSH6 & Hen1 & $T$ & 39 & 1 & AADKRLGTPPGGGAA & 0.1947 & -0.3718 \\
\hline F1LSH6 & Hon1 & S & 783 & 1 & PKNEVHKSTQALHNT & -0.4338 & 0.4701 \\
\hline Q9JKA9 & Hcn2 & $S$ & 834 & 1 & TTRTAAPSPDRRDSA & -0.238 & 0.3377 \\
\hline D3ZLS5 & Hectd1 & $S$ & 632 & 1 & SALAGPSSDDENEEE & & -0.3952 \\
\hline D3ZLS5 & Hectd1 & $\mathrm{s}$ & 1571 & 1 & MNLSRSSSDNNTNTL & & 0.3521 \\
\hline
\end{tabular}




\begin{tabular}{|c|c|c|c|c|c|c|c|}
\hline D4ADZ6 & Helz & $\mathrm{S}$ & 1127 & 1 & NSSRQQQSPPKVKSL & & 0.6081 \\
\hline A0A0G2JTT6 & Herc1 & S & 2718 & 1 & DEVGRRQSLTSPDSQ & 0.0721 & 0.2862 \\
\hline P05708 & Hk1 & $S$ & 337 & 1 & TRGKFNTSDVSAIEK & & -0.3745 \\
\hline $\mathrm{P} 17425$ & Hmgcs1 & S & 495 & 2 & TATEHIPSPAKKVPR & & 0.2645 \\
\hline $\mathrm{Q} 66 \mathrm{H} 40$ & Hmgn3 & S & 6 & 1 & MPKRKSPENAEGK & -0.9825 & 1.3039 \\
\hline P04256 & Hnrnpa1 & S & 2 & 2 & MSKSESPKE & & -0.3931 \\
\hline P04256 & Hnrnpa1 & $\mathrm{s}$ & 6 & 2 & MSKSESPKEPEQL & & -0.449 \\
\hline Q9QX80 & Hnrnpab & S & 260 & 1 & RNRGNRGSGGGQGST & & 0.8605 \\
\hline Q8VHV7 & Hnrnph1 & S & 104 & 1 & LKHTGPNSPDTANDG & 0.0688 & -0.2817 \\
\hline D4ABT8 & Hnrnpul2 & $\mathrm{S}$ & 183 & 1 & EQGDDQDSEKSKPAG & & 1.0485 \\
\hline D4ABT8 & Hnrnpul2 & $\mathrm{S}$ & 191 & 1 & EKSKPAGSDGERRGV & & 0.8338 \\
\hline Q6P747 & Hp1bp3 & $\mathrm{T}$ & 51 & 1 & AVNSTRETPPKSKLA & & 0.6863 \\
\hline P84076 & Hpca & S & 175 & 1 & EFIRGAKSDPSIVRL & 0.2369 & -0.2748 \\
\hline G3V8S6 & $\mathrm{Hrc}$ & S & 549 & 1 & RKEDDHSSQEGDEDL & 0.147 & -0.2956 \\
\hline D4A6D9 & Hs1bp3 & S & 294 & 1 & ESRGPTSSPEHRDAS & -0.0875 & 0.5874 \\
\hline P34058 & Hsp90ab1 & $S$ & 255 & 1 & PKIEDVGSDEEDDSG & 0.1514 & -0.4052 \\
\hline P34058 & Hsp90ab1 & $\mathrm{S}$ & 261 & 1 & GSDEEDDSGKDKKKK & & -0.4754 \\
\hline 088600 & Hspa4 & S & 692 & 1 & IKTRFQESEERPKLF & & -0.5763 \\
\hline P63018 & Hspa8 & $S$ & 511 & 1 & TNDKGRLSKEDIERM & & -0.3821 \\
\hline P63039 & Hspd1 & S & 232 & 1 & SPYFINTSKGQKCEF & & -0.3565 \\
\hline Q66HA8 & Hsph1 & S & 429 & 1 & RNHAAPFSKVLTFLR & & -0.3226 \\
\hline Q99NA5 & Idh3a & $S$ & 193 & 1 & YARNNHRSNVTAVHK & 0.2602 & -0.3128 \\
\hline F1LY69 & Ift140 & S & 357 & 1 & MWKKVPVSPSGRGAE & & -0.2754 \\
\hline D3ZB51 & Igsf9b & S & 797 & 1 & LRAPSESSDDQGQPA & 0.1015 & 0.3699 \\
\hline D3ZB51 & $\operatorname{lgsf9b}$ & $S$ & 1202 & 2 & VLQPSRLSPLTQSPL & 0.0648 & 0.332 \\
\hline D3ZB51 & Igsf9b & S & 1207 & 2 & RLSPLTQSPLSSRTG & & 0.4713 \\
\hline D3ZB51 & Igsf9b & S & 1258 & 1 & STPSSTGSPSQSSRS & -0.061 & 0.7262 \\
\hline D3ZB51 & $\operatorname{lgsf9b}$ & S & 1258 & 2 & STPSSTGSPSQSSRS & & 0.4697 \\
\hline D4A4I9 & $\| 16$ & S & 680 & 2 & GEPRRSASPETPASP & & 0.6474 \\
\hline D4A4I9 & $\| 16$ & S & 686 & 2 & ASPETPASPGKHPLL & -0.0514 & 0.5868 \\
\hline Q63257 & $\| 14 r$ & $S$ & 335 & 1 & AKTKPLQSPEKAGWY & & -0.271 \\
\hline D3ZGJ1 & Ildr2 & S & 560 & 1 & ESNSRGGSLETPSKL & 0.1167 & 1.0006 \\
\hline D3ZGJ1 & Ildr2 & $\mathrm{T}$ & 563 & 1 & SRGGSLETPSKLGAQ & & 1.1005 \\
\hline Q3KR86 & Immt & $S$ & 34 & 1 & LRPCRRYSTSSSSGV & & -0.3302 \\
\hline Q3KR86 & Immt & S & 106 & 1 & LPKKPIQSGPLKISS & & -0.335 \\
\hline Q3KR86 & Immt & S & 302 & 1 & KKVQAAQSEAKVVSQ & & -0.2907 \\
\hline Q5BJY3 & Ino80c & S & 26 & 1 & NSKKRPASPSHNSSG & & 0.9074 \\
\hline D3ZKG7 & Inpp5f & S & 667 & 1 & VSQYQRRSLEDLEKI & & -0.3991 \\
\hline Q9JMC1 & Inpp5j & S & 689 & 1 & KAPSGGPSPSGRESH & -0.0672 & -0.2751 \\
\hline D4A4J1 & Insyn2 & $\mathrm{T}$ & 107 & 1 & TKSTGVQTSPDLRKC & & 0.4678 \\
\hline D4A4J1 & Insyn2 & S & 108 & 1 & KSTGVQTSPDLRKCY & 0.0482 & 0.4209 \\
\hline M0R8E8 & Ipo11 & S & 343 & 1 & SKNFEDSSPETLEAH & & -0.3202 \\
\hline A0A0G2JUG7 & Iqsec1 & S & 89 & 1 & PRLQHSTSVLRKQAE & 0.4161 & 0.3166 \\
\hline A0A0G2JUG7 & Iqsec1 & $\mathrm{S}$ & 399 & 1 & SDRSDRSSLKRQSAY & & 0.3813 \\
\hline
\end{tabular}




\begin{tabular}{|c|c|c|c|c|c|c|c|}
\hline A0A0G2JUG7 & Iqsec1 & S & 416 & 1 & SLGGQQGSPKHGPHG & -0.1883 & 0.9406 \\
\hline A0A0G2JZXX5 & Iqsec2 & S & 918 & 1 & VNGTLARSSLEDTYG & 0.1769 & 0.4169 \\
\hline A0A0G2JZXX5 & Iqsec2 & $\mathrm{S}$ & 919 & 1 & NGTLARSSLEDTYGA & 0.1833 & 0.4008 \\
\hline Q76M68 & Iqsec3 & S & 131 & 1 & SRVQTPQSPHQHPVA & -0.1201 & 0.9334 \\
\hline Q76M68 & Iqsec3 & S & 255 & 1 & ERPGAVGSPRAGPLR & 0.125 & 0.5617 \\
\hline Q76M68 & Iqsec3 & $\mathrm{S}$ & 1053 & 1 & RLQTFQHSPKLGVER & 0.0657 & 0.7027 \\
\hline D4AAZ8 & Irf2bp1 & S & 384 & 1 & TPRRRKASPEPEGET & 0.057 & 0.7495 \\
\hline D4AAZ8 & Irf2bp1 & S & 436 & 1 & VAEALGHSPKDPGGG & & 0.2974 \\
\hline Q5EIC4 & Irf2bpl & $\mathrm{S}$ & 534 & 1 & SLRKRKASPEPPDSA & & 0.6097 \\
\hline P35570 & Irs1 & S & 1077 & 1 & AFTRVNLSPNHNQSA & -0.3393 & 0.4759 \\
\hline F1MAL5 & Irs2 & S & 1092 & 1 & PEGARVTSPTSGLKR & & 0.6348 \\
\hline M0R7A6 & Itsn2 & S & 231 & 1 & TASLSGNSPKTGTSE & & 0.7424 \\
\hline Q6AXU6 & JPT1 & $S$ & 14 & 1 & FKGVDPNSRNSSRVL & & 0.6165 \\
\hline Q3SWS9 & Jakmip1 & S & 382 & 1 & ASLKRHTSLNDLSLT & & -0.2803 \\
\hline F1M065 & Jam2 & $\mathrm{S}$ & 236 & 1 & ETSFQKGSPASKATT & & -0.2638 \\
\hline D3ZQ55 & Jph1 & $\mathrm{S}$ & 475 & 1 & ASPKQSHSPQPSSPK & & 0.5756 \\
\hline D3ZQ55 & Jph1 & S & 475 & 2 & ASPKQSHSPQPSSPK & 0.0904 & 0.5755 \\
\hline D3ZQ55 & Jph1 & $\mathrm{S}$ & 479 & 2 & QSHSPQPSSPKSMKK & 0.0892 & 0.6189 \\
\hline D3ZQ55 & Jph1 & $\mathrm{S}$ & 480 & 2 & SHSPQPSSPKSMKKQ & 0.0897 & 0.5649 \\
\hline Q69FB3 & Jph4 & S & 238 & 1 & RAGGRRSSLGSKRGS & & 0.5123 \\
\hline Q5FWS6 & Kazn & S & 344 & 1 & STPSDINSPRHRTHS & & 0.7647 \\
\hline P63142 & Kcna2 & $S$ & 440 & 1 & TSCPKIPSSPDLKKS & 0.3699 & -0.3181 \\
\hline P63142 & Kcna2 & S & 441 & 1 & SCPKIPSSPDLKKSR & 0.3712 & -0.3235 \\
\hline P63142 & Kcna2 & S & 447 & 1 & SSPDLKKSRSASTIS & & -0.3806 \\
\hline P63142 & Kcna2 & $S$ & 449 & 1 & PDLKKSRSASTISKS & 0.1878 & -0.3146 \\
\hline P63142 & Kcna2 & S & 449 & 2 & PDLKKSRSASTISKS & & -0.3699 \\
\hline P15385 & Kcna4 & S & 82 & 1 & DPQGSRGSREEEATR & 0.5451 & 0.271 \\
\hline P62483 & Kcnab2 & $S$ & 9 & 1 & YPESTTGSPARLSLR & 0.086 & 0.8231 \\
\hline P15387 & Kcnb1 & S & 511 & 1 & FETKEQGSPEKARSS & 0.2656 & 0.6487 \\
\hline P15387 & Kcnb1 & S & 520 & 1 & EKARSSSSPQHLNVQ & -0.1684 & 0.8552 \\
\hline P15387 & Kcnb1 & $\mathrm{T}$ & 732 & 1 & YTTASARTPPRSPEK & 0.1278 & 1.9096 \\
\hline $\mathrm{P} 15387$ & Kcnb1 & $\mathrm{T}$ & 781 & 1 & PKSLHGSTSPKFSTG & 0.2328 & 1.3851 \\
\hline P15387 & Kcnb1 & $\mathrm{T}$ & 803 & 1 & FESSPLPTSPKFLRP & & 0.8207 \\
\hline P15387 & Kcnb1 & $S$ & 804 & 1 & ESSPLPTSPKFLRPN & 0.1213 & 0.6368 \\
\hline Q63099 & Kcnb2 & S & 520 & 2 & QEVSQKDSHEQLNNT & & 0.4021 \\
\hline Q63099 & Kcnb2 & $\mathrm{T}$ & 725 & 1 & NRGSAPQTPPSTARP & 0.2071 & 0.715 \\
\hline Q63881 & Kcnd2 & $\mathrm{T}$ & 154 & 1 & RLQDDADTDNTGESA & & -0.2845 \\
\hline Q63881 & Kcnd2 & $\mathrm{T}$ & 567 & 2 & QIRCVERTPLSNSRS & -0.2318 & 0.7884 \\
\hline Q63881 & Kcnd2 & S & 572 & 1 & ERTPLSNSRSSLNAK & 0.16 & 0.3646 \\
\hline Q63881 & Kcnd2 & S & 572 & 2 & ERTPLSNSRSSLNAK & -0.1508 & 0.6743 \\
\hline Q63881 & Kcnd2 & S & 574 & 2 & TPLSNSRSSLNAKME & -0.1693 & 0.6477 \\
\hline Q63881 & Kcnd2 & S & 575 & 2 & PLSNSRSSLNAKMEE & -0.2245 & 0.6417 \\
\hline Q9JIS4 & Kcnk10 & S & 412 & 1 & TGRFKASSQESINNR & & 0.3617 \\
\hline F1LNC7 & Kcnma1 & S & 1199 & 1 & SRASLSHSSHSSQSS & -0.5141 & 0.3786 \\
\hline
\end{tabular}




\begin{tabular}{|c|c|c|c|c|c|c|c|}
\hline F1LNC7 & Kcnma1 & S & 1199 & 2 & SRASLSHSSHSSQSS & -0.921 & \\
\hline 088943 & Kcnq2 & S & 439 & 1 & VAAKGKGSPQAQTVR & & 0.445 \\
\hline 088943 & Kcnq2 & S & 454 & 2 & RSPSADQSLDDSPSK & -0.0646 & 0.3127 \\
\hline D3ZNXO & Kctd3 & $S$ & 713 & 1 & CAVCERKSPGTEGRC & & 0.4171 \\
\hline Q99PF5 & Khsrp & $S$ & 481 & 1 & KLFVIRGSPQQIDHA & 0.0546 & 0.6391 \\
\hline D4A0X3 & Kiaa1107 & S & 340 & 1 & TQGSQGESPHSVKSS & 0.2092 & 0.7452 \\
\hline D4A0X3 & Kiaa1107 & $S$ & 696 & 1 & HQRESPESDTGSATT & & 0.3869 \\
\hline D4A0X3 & Kiaa1107 & $S$ & 748 & 1 & DFLGRSSSDTSTPEE & & 0.2633 \\
\hline Q9EQG6 & Kidins220 & S & 1586 & 1 & GVRSNESSPNHSLHN & -0.103 & 1.0013 \\
\hline O35787 & Kif1c & S & 1086 & 1 & PRMRRQRSAPDLKES & & 0.3114 \\
\hline D3ZCG2 & Kif21a & S & 1217 & 1 & GSISRQPSVSEKKVP & 1.0174 & 0.1144 \\
\hline D3ZCG2 & Kif21a & S & 1217 & 2 & GSISRQPSVSEKKVP & 0.3082 & 0.4612 \\
\hline Q9WV63 & Kif2a & $S$ & 139 & 1 & NGSVSDISPVQAAKK & -0.2295 & 0.2709 \\
\hline A0A0G2JTL4 & Kit & S & 588 & 1 & EFPRNRLSFGKTLGA & & -0.4256 \\
\hline P37285 & Klc1 & S & 162 & 1 & KKYDDDISPSEDKDS & 0.313 & -0.3568 \\
\hline B2GV74 & Klc2 & S & 589 & 1 & SLNFLNKSVEEPVQP & 0.2886 & 0.2706 \\
\hline B2GV74 & Klc2 & $\mathrm{S}$ & 589 & 2 & SLNFLNKSVEEPVQP & 0.7237 & 0.1032 \\
\hline D4A8Q2 & Kndc1 & S & 843 & 1 & KAAASPSSPRGPDGH & 0.3051 & 0.5996 \\
\hline Q56R18 & Kpna3 & S & 60 & 1 & QEESLEDSDVDADFK & 0.0986 & -0.4449 \\
\hline Q56R16 & Kpna5 & S & 3 & 1 & MASPGKDNYR & -0.0323 & 0.8306 \\
\hline F1LXC7 & LOC100362814 & S & 763 & 1 & RDTAQDGSTIKTAKS & 0.0495 & 0.4493 \\
\hline F1LXC7 & LOC100362814 & S & 1145 & 1 & AVASLRRSTSDIGSK & -0.1365 & 0.688 \\
\hline F1LXC7 & LOC100362814 & $\mathrm{S}$ & 1147 & 1 & ASLRRSTSDIGSKTR & 0.0845 & 0.5212 \\
\hline F1LXC7 & LOC100362814 & S & 1151 & 1 & RSTSDIGSKTRMAES & & 0.5637 \\
\hline F1LXC7 & LOC100362814 & S & 1263 & 1 & RSRYQQSSPSRLPRQ & 0.0903 & 0.9548 \\
\hline A0A0G2K5L2 & LOC100911440 & $\mathrm{S}$ & 95 & 1 & DFFRHQLSKDGQKLT & 0.126 & -0.3088 \\
\hline D3ZF45 & Larp4b & S & 718 & 1 & RQPGRRPSPPAAGKR & 0.0927 & 0.8565 \\
\hline D3ZF45 & Larp4b & $\mathrm{T}$ & 732 & 2 & RLNREQNTPPKSP & & 1.0525 \\
\hline D3ZF45 & Larp4b & $S$ & 736 & 1 & EQNTPPKSP & 0.0411 & 0.6187 \\
\hline D3ZF45 & Larp4b & S & 736 & 2 & EQNTPPKSP & & 1.0525 \\
\hline Q99MZ8 & Lasp1 & S & 129 & 1 & YHEEFEKSRMGPSGG & 0.1749 & -0.2813 \\
\hline E9PTQ0 & Ldb2 & $\mathrm{S}$ & 263 & 1 & KRRKRKNSTSSTSNS & & 1.2455 \\
\hline Q641X2 & Leo1 & S & 642 & 1 & LKAKKLNSDEEGESS & 0.2473 & 0.8283 \\
\hline F1M392 & Limch1 & S & 231 & 1 & GSSDGRGSDSESDLP & -0.0853 & 0.4377 \\
\hline F1M392 & Limch1 & $\mathrm{T}$ & 255 & 1 & DDMSARRTSHGEPKS & 0.3128 & 0.3917 \\
\hline F1M392 & Limch1 & S & 256 & 1 & DMSARRTSHGEPKSA & 0.3126 & 0.4513 \\
\hline F1M392 & Limch1 & S & 523 & 1 & SVAAGTGSPSKTSTP & $-3.00 \mathrm{E}-04$ & 0.3024 \\
\hline F1M392 & Limch1 & S & 603 & 1 & GVATVHGSPVQVKQG & & 0.5205 \\
\hline P48679 & Lmna & $\mathrm{T}$ & 27 & 2 & PLSPTRITRLQEKED & & 0.4232 \\
\hline P48679 & Lmna & $S$ & 407 & 1 & GRASSHSSQSQGGGS & & 0.8217 \\
\hline P48679 & Lmna & $S$ & 414 & 1 & SQSQGGGSVTKKRKL & & 0.4709 \\
\hline A0A0G2K8R3 & Lmo7 & S & 439 & 1 & ITRRKNFSPTPGYRA & 0.1371 & 0.8794 \\
\hline A0A0G2K8R3 & Lmo7 & $S$ & 699 & 1 & KWKDRRKSYTSDLQK & 0.1008 & 1.2291 \\
\hline A0A0G2K8R3 & Lmo7 & $\mathrm{T}$ & 1075 & 1 & MRISINQTPGTKHDF & & -0.5247 \\
\hline
\end{tabular}




\begin{tabular}{|c|c|c|c|c|c|c|c|}
\hline A0A0G2K8R3 & Lmo7 & $S$ & 1231 & 1 & SQFFEQGSSDSVAPD & & 0.3283 \\
\hline A0A0G2K8R3 & Lmo7 & $S$ & 1632 & 1 & SVKTSPGSPSPRSHS & & 0.8009 \\
\hline A0A0G2K8R3 & Lmo7 & $S$ & 1639 & 1 & SPSPRSHSPSMSQSG & 0.1053 & 0.7284 \\
\hline A0A0G2K8R3 & Lmo7 & $S$ & 1641 & 1 & SPRSHSPSMSQSGSQ & 0.0749 & 0.2933 \\
\hline AOA0G2KOD3 & Lmod1 & $S$ & 119 & 1 & ALGPRQDSDVGKEPK & 0.5695 & 0.5925 \\
\hline D3ZBH5 & Lmtk2 & S & 756 & 1 & ASPLTEGSPNGPPDS & -0.0234 & 0.574 \\
\hline A0JN29 & Lnpk & $S$ & 177 & 1 & TAAQRNLSPAPANSN & & 0.3444 \\
\hline P0C7J6 & Lrfn1 & $S$ & 576 & 1 & RIKGTSRSPPRVSHV & 0.146 & -0.3239 \\
\hline PoCC10 & Lrrc4b & $\mathrm{T}$ & 707 & 1 & SKENVQETQI & & 0.5626 \\
\hline D3ZXT1 & Lrrc4c & $S$ & 631 & 1 & PLLIRMNSKDNVQET & & 0.9182 \\
\hline P70587 & Lrrc7 & $\mathrm{T}$ & 848 & 1 & PWQNWTRTPSPFEDR & & 1.5626 \\
\hline P70587 & Lrrc7 & S & 850 & 1 & QNWTRTPSPFEDRTA & 0.0995 & 1.1023 \\
\hline P70587 & Lrrc7 & $T$ & 864 & 2 & AFPSKLETTPTTSPL & & 0.9157 \\
\hline P70587 & Lrrc7 & $T$ & 865 & 2 & FPSKLETTPTTSPLP & -0.1933 & 1.5365 \\
\hline P70587 & Lrrc7 & $\mathrm{T}$ & 868 & 2 & KLETTPTTSPLPERK & -0.1452 & 1.4506 \\
\hline P70587 & Lrrc7 & S & 869 & 1 & LETTPTTSPLPERKD & & 0.927 \\
\hline P70587 & Lrrc7 & $S$ & 869 & 2 & LETTPTTSPLPERKD & -0.284 & 1.3311 \\
\hline P70587 & Lrrc7 & $S$ & 933 & 1 & SKSTERLSPLMKDIK & & 0.8728 \\
\hline P70587 & Lrrc7 & $S$ & 947 & 2 & KSNKFKKSQSIDEID & 0.0292 & 0.6428 \\
\hline P70587 & Lrrc7 & $S$ & 949 & 2 & NKFKKSQSIDEIDVG & 0.0761 & 0.9449 \\
\hline P70587 & Lrrc7 & $\mathrm{T}$ & 957 & 2 & IDEIDVGTYKVYNIP & 0.0617 & 0.9874 \\
\hline P70587 & Lrrc7 & S & 977 & 1 & SGSDHLGSHERPDKF & 0.1289 & 0.5473 \\
\hline P70587 & Lrrc7 & $S$ & 1343 & 1 & SQSLQHRSREQQPYE & 0.1097 & 0.6686 \\
\hline Q32Q07 & Lrrn1 & $S$ & 664 & 1 & KRKNYHHSLKKYMQK & & -0.3904 \\
\hline BOBNI4 & Lsm11 & S & 15 & 1 & ARSARAGSPASPPSP & 0.0866 & 0.3378 \\
\hline BOBNI4 & Lsm11 & $S$ & 21 & 1 & GSPASPPSPRLDVSS & -0.0346 & 0.414 \\
\hline B2GV58 & Lsm14a & $S$ & 182 & 1 & PQLAQGRSSPQLDPL & 0.1115 & 0.5272 \\
\hline B2GV58 & Lsm14a & S & 183 & 1 & QLAQGRSSPQLDPLR & 0.1884 & 0.6929 \\
\hline B2GV58 & Lsm14a & $S$ & 183 & 2 & QLAQGRSSPQLDPLR & & 0.6043 \\
\hline B2GV58 & Lsm14a & $S$ & 192 & 2 & QLDPLRKSPTMEQAV & & 0.4288 \\
\hline B2GV58 & Lsm14a & $\mathrm{T}$ & 194 & 2 & DPLRKSPTMEQAVQT & & 0.6008 \\
\hline B2GV58 & Lsm14a & $S$ & 216 & 1 & PAPVGRRSPVPARPL & -0.1682 & 0.7171 \\
\hline Q4QQV6 & Lsp1 & $S$ & 196 & 1 & KLADRTESLNRSIQK & & -0.3265 \\
\hline Q9ESV1 & Luzp1 & S & 395 & 1 & KHTSREQSPQHKRER & 0.1089 & 1.2286 \\
\hline Q9ESV1 & Luzp1 & $S$ & 660 & 1 & SGREKPDSDDDLDIE & 0.1913 & 1.0994 \\
\hline Q8CFC9 & Lzts1 & $S$ & 141 & 1 & SGAILHSSPESTNHQ & & 0.643 \\
\hline G3V8V8 & Lzts3 & $\mathrm{T}$ & 678 & 1 & GAAGGSSTPTPQHGE & & 1.0682 \\
\hline Q4V7D3 & Maco1 & $S$ & 331 & 1 & NASGVVNSSPRSHSA & & 0.2946 \\
\hline Q4V7D3 & Maco1 & $S$ & 332 & 1 & ASGVVNSSPRSHSAT & & 0.5973 \\
\hline Q5PPP4 & Mageh1 & S & 31 & 1 & KIQASEASKTPMAAS & & -0.449 \\
\hline P34926 & Map1a & S & 319 & 1 & KIKHRADSKESLKAA & 0.2086 & 0.8289 \\
\hline P34926 & Map1a & $S$ & 319 & 2 & KIKHRADSKESLKAA & 0.0041 & 0.8811 \\
\hline P34926 & Map1a & S & 322 & 1 & HRADSKESLKAAPKT & 0.1299 & 2.0976 \\
\hline P34926 & Map1a & $\mathrm{s}$ & 322 & 2 & HRADSKESLKAAPKT & 0.0041 & 0.8811 \\
\hline
\end{tabular}




\begin{tabular}{|c|c|c|c|c|c|c|c|}
\hline P34926 & Map1a & S & 384 & 1 & SERVRGESSEALKAE & & 0.2635 \\
\hline P34926 & Map1a & S & 500 & 2 & RGEKELSSEPRTPPA & 0.065 & 1.1628 \\
\hline P34926 & Map1a & $T$ & 504 & 1 & ELSSEPRTPPAQKGA & 0.14 & 1.227 \\
\hline P34926 & Map1a & $\mathrm{T}$ & 504 & 2 & ELSSEPRTPPAQKGA & 0.0544 & 1.1268 \\
\hline P34926 & Map1a & $S$ & 526 & 1 & GHRELALSSPEDLTQ & 0.3737 & 0.5571 \\
\hline P34926 & Map1a & S & 526 & 2 & GHRELALSSPEDLTQ & -0.0882 & 0.3851 \\
\hline P34926 & Map1a & $\mathrm{s}$ & 527 & 2 & HRELALSSPEDLTQD & -0.0921 & 0.362 \\
\hline P34926 & Map1a & $\mathrm{T}$ & 532 & 2 & LSSPEDLTQDFEELK & -0.0595 & 0.3736 \\
\hline P34926 & Map1a & S & 690 & 1 & TQEALKASPKSREAL & -0.1029 & 1.0668 \\
\hline P34926 & Map1a & S & 889 & 2 & QGLDYVPSAGTISPT & -0.0988 & 0.4177 \\
\hline P34926 & Map1a & $\mathrm{T}$ & 892 & 2 & DYVPSAGTISPTSSL & -0.1153 & 0.3766 \\
\hline P34926 & Map1a & $S$ & 897 & 2 & AGTISPTSSLEEDKG & -0.1597 & 0.4076 \\
\hline P34926 & Map1a & S & 990 & 1 & DSTVKMASPPPSGPP & 0.0378 & 0.4339 \\
\hline P34926 & Map1a & S & 990 & 2 & DSTVKMASPPPSGPP & -0.1144 & 0.3931 \\
\hline P34926 & Map1a & $\mathrm{T}$ & 1002 & 2 & GPPSAAHTPFHQSPV & -0.0628 & 0.4515 \\
\hline P34926 & Map1a & $\mathrm{S}$ & 1007 & 2 & AHTPFHQSPVEDKSE & -0.0872 & 0.3761 \\
\hline P34926 & Map1a & $\mathrm{S}$ & 1037 & 1 & PGVSKEDSEEQTVKP & 0.3003 & 0.309 \\
\hline P34926 & Map1a & S & 1134 & 3 & RFTDQSLSPEDAESL & -0.24 & 0.2685 \\
\hline P34926 & Map1a & $S$ & 1140 & 3 & LSPEDAESLSVLSVV & & 0.2925 \\
\hline P34926 & Map1a & S & 1145 & 2 & AESLSVLSVVSPDTT & -0.389 & 0.4073 \\
\hline P34926 & Map1a & S & 1148 & 1 & LSVLSVVSPDTTKQE & & 0.4256 \\
\hline P34926 & Map1a & $S$ & 1148 & 2 & LSVLSVVSPDTTKQE & -0.2953 & 0.3864 \\
\hline P34926 & Map1a & S & 1209 & 1 & SKQLSPESLGTLQFG & 0.1079 & 0.513 \\
\hline P34926 & Map1a & S & 1280 & 1 & LSVDRKHSPGEITGP & & 0.7194 \\
\hline P34926 & Map1a & $T$ & 1285 & 1 & KHSPGEITGPGGHFM & -0.2177 & 0.7951 \\
\hline P34926 & Map1a & S & 1594 & 1 & RALGLEESPAEGSKA & 0.1575 & 0.3812 \\
\hline P34926 & Map1a & S & 1622 & 1 & VQGWRETSPTRGEPV & 0.1647 & 0.7525 \\
\hline P34926 & Map1a & S & 1757 & 2 & EEDKLTRSPFEIISP & -0.1988 & 0.7932 \\
\hline P34926 & Map1a & S & 1757 & 3 & EEDKLTRSPFEIISP & -0.2899 & 0.1386 \\
\hline P34926 & Map1a & S & 1763 & 2 & RSPFEIISPPASPPE & -0.1393 & 0.8352 \\
\hline P34926 & Map1a & $S$ & 1763 & 3 & RSPFEIISPPASPPE & -0.2899 & 0.1386 \\
\hline P34926 & Map1a & S & 1767 & 2 & EIISPPASPPEMTGQ & -0.1422 & 0.8313 \\
\hline P34926 & Map1a & S & 1767 & 3 & EIISPPASPPEMTGQ & -0.2899 & 0.1386 \\
\hline P34926 & Map1a & $S$ & 1895 & 1 & AGAPDSSSFSPKVPE & 0.0428 & 0.5827 \\
\hline P34926 & Map1a & S & 1897 & 1 & APDSSSFSPKVPEAG & 0.0645 & 0.6665 \\
\hline P34926 & Map1a & S & 2588 & 1 & PGRAKPASPARRLDI & 0.1624 & 2.3808 \\
\hline P34926 & Map1a & S & 2600 & 1 & LDIRGKRSPTPGKGP & 0.1672 & 1.915 \\
\hline P34926 & Map1a & S & 2620 & 1 & RTVPRPRSTPSQVTS & & 0.471 \\
\hline P34926 & Map1a & S & 2635 & 1 & AEEKDGHSPMSKGLV & $6.00 \mathrm{E}-04$ & 1.0045 \\
\hline P34926 & Map1a & $S$ & 2638 & 1 & KDGHSPMSKGLVNGL & & 1.684 \\
\hline P15205 & Map1b & S & 825 & 1 & AERSLMSSPEDLTKD & & -0.4105 \\
\hline P15205 & Map1b & S & 1248 & 2 & ERLSPAKSPSLSPSP & -0.8973 & 0.5216 \\
\hline P15205 & Map1b & S & 1250 & 2 & LSPAKSPSLSPSPPS & -0.9995 & 0.3002 \\
\hline P15205 & Map1b & S & 1250 & 3 & LSPAKSPSLSPSPPS & -0.3374 & 0.2418 \\
\hline
\end{tabular}




\begin{tabular}{|c|c|c|c|c|c|c|c|}
\hline P15205 & Map1b & S & 1252 & 2 & PAKSPSLSPSPPSPI & -0.3078 & 0.7224 \\
\hline P15205 & Map1b & S & 1252 & 3 & PAKSPSLSPSPPSPI & -0.3683 & 0.2098 \\
\hline P15205 & Map1b & $\mathrm{S}$ & 1254 & 1 & KSPSLSPSPPSPIEK & 0.1402 & 0.3543 \\
\hline P15205 & Map1b & $S$ & 1254 & 2 & KSPSLSPSPPSPIEK & -0.3868 & 0.417 \\
\hline P15205 & Map1b & $S$ & 1254 & 3 & KSPSLSPSPPSPIEK & -0.3923 & 0.1987 \\
\hline P15205 & Map1b & $\mathrm{s}$ & 1257 & 1 & SLSPSPPSPIEKTPL & 0.1246 & 0.6239 \\
\hline P15205 & Map1b & S & 1257 & 2 & SLSPSPPSPIEKTPL & -0.3665 & 0.5831 \\
\hline P15205 & Map1b & S & 1257 & 3 & SLSPSPPSPIEKTPL & -0.3613 & 0.214 \\
\hline P15205 & Map1b & S & 1315 & 2 & EKTLEVVSPSQSVTG & -0.2821 & 0.39 \\
\hline P15205 & Map1b & $\mathrm{S}$ & 1317 & 2 & TLEVVSPSQSVTGSA & -0.2542 & 0.3806 \\
\hline P15205 & Map1b & S & 1317 & 3 & TLEVVSPSQSVTGSA & -0.1496 & 0.4017 \\
\hline P15205 & Map1b & $T$ & 1327 & 2 & VTGSAGHTPYYQSPT & -0.2689 & \\
\hline P15205 & Map1b & $T$ & 1327 & 3 & VTGSAGHTPYYQSPT & -0.1712 & 0.3592 \\
\hline P15205 & Map1b & S & 1332 & 2 & GHTPYYQSPTDEKSS & -0.2688 & 0.3714 \\
\hline P15205 & Map1b & S & 1432 & 2 & KNGKQGFSDKESPVS & -0.2866 & 0.3337 \\
\hline P15205 & Map1b & $\mathrm{S}$ & 1436 & 2 & QGFSDKESPVSDLTS & -0.2845 & 0.3229 \\
\hline P15205 & Map1b & S & 1439 & 2 & SDKESPVSDLTSDLY & & 0.306 \\
\hline P15205 & Map1b & S & 1765 & 2 & LASEKVQSLEGEKLS & 0.9949 & 0.4504 \\
\hline P15205 & Map1b & $\mathrm{S}$ & 1772 & 1 & SLEGEKLSPKSDISP & -0.0931 & 0.6115 \\
\hline P15205 & Map1b & S & 1772 & 2 & SLEGEKLSPKSDISP & 0.1044 & 0.7262 \\
\hline P15205 & Map1b & S & 1772 & 3 & SLEGEKLSPKSDISP & -0.5095 & 0.3437 \\
\hline P15205 & Map1b & S & 1775 & 2 & GEKLSPKSDISPLTP & -0.215 & 0.5987 \\
\hline P15205 & Map1b & $S$ & 1775 & 3 & GEKLSPKSDISPLTP & -0.5329 & 0.2574 \\
\hline P15205 & Map1b & S & 1778 & 1 & LSPKSDISPLTPRES & 0.0537 & 0.6479 \\
\hline P15205 & Map1b & $S$ & 1778 & 3 & LSPKSDISPLTPRES & -0.4912 & 0.2399 \\
\hline P15205 & Map1b & $\mathrm{T}$ & 1781 & 2 & KSDISPLTPRESSPT & -0.3642 & 0.5559 \\
\hline P15205 & Map1b & $\mathrm{T}$ & 1781 & 3 & KSDISPLTPRESSPT & -0.6129 & 0.1619 \\
\hline P15205 & Map1b & $S$ & 1786 & 1 & PLTPRESSPTYSPGF & -0.1252 & 0.5334 \\
\hline P15205 & Map1b & S & 1786 & 2 & PLTPRESSPTYSPGF & -0.1729 & 0.5829 \\
\hline P15205 & Map1b & $\mathrm{T}$ & 1788 & 1 & TPRESSPTYSPGFSD & -0.1108 & 0.4497 \\
\hline P15205 & Map1b & $Y$ & 1789 & 2 & PRESSPTYSPGFSDS & -0.1014 & 0.3249 \\
\hline P15205 & Map1b & $\mathrm{S}$ & 1790 & 2 & RESSPTYSPGFSDST & -0.177 & 0.5903 \\
\hline P15205 & Map1b & S & 1811 & 1 & TAAYQTSSSPPIDAA & 0.0731 & 0.4688 \\
\hline P15205 & Map1b & S & 1848 & 1 & SRDLTTSSVEKDNGG & 0.4906 & 0.4713 \\
\hline P15205 & Map1b & $S$ & 2249 & 1 & GTKTKSSSPVKKGDG & & 0.9206 \\
\hline P15205 & Map1b & $\mathrm{S}$ & 2264 & 1 & KSKPSAASPKPGALK & & 0.391 \\
\hline P0C5W1 & Map1s & $S$ & 686 & 1 & PPPASPGSSDSSARS & & 0.2829 \\
\hline F1MAQ5 & Map2 & S & 136 & 2 & ETVNLPPSPPPSPAS & -0.0851 & 0.7255 \\
\hline F1MAQ5 & Map2 & S & 140 & 2 & LPPSPPPSPASEQTA & -0.0878 & 0.7252 \\
\hline F1MAQ5 & Map2 & $\mathrm{T}$ & 146 & 2 & PSPASEQTAALEEAS & -0.083 & 0.6827 \\
\hline F1MAQ5 & Map2 & S & 217 & 2 & QDMEGEKSPASPFAQ & -0.2969 & 0.8906 \\
\hline F1MAQ5 & Map2 & S & 220 & 1 & EGEKSPASPFAQTFG & 0.162 & 1.1851 \\
\hline F1MAQ5 & Map2 & $S$ & 220 & 2 & EGEKSPASPFAQTFG & -0.2982 & 0.8674 \\
\hline F1MAQ5 & Map2 & $\mathrm{T}$ & 225 & 1 & PASPFAQTFGTNLED & & 1.2368 \\
\hline
\end{tabular}




\begin{tabular}{|c|c|c|c|c|c|c|c|}
\hline F1MAQ5 & Map2 & $\mathrm{T}$ & 225 & 2 & PASPFAQTFGTNLED & -0.3321 & 1.0293 \\
\hline F1MAQ5 & Map2 & S & 307 & 2 & WEGKQFDSPMPSPFH & -1.0731 & 0.5945 \\
\hline F1MAQ5 & Map2 & S & 311 & 1 & QFDSPMPSPFHGGSF & & 0.5853 \\
\hline F1MAQ5 & Map2 & $S$ & 311 & 2 & QFDSPMPSPFHGGSF & -1.0651 & 0.5939 \\
\hline F1MAQ5 & Map2 & $S$ & 363 & 1 & SATSKESSKDEEPQK & 0.4983 & 0.3211 \\
\hline F1MAQ5 & Map2 & S & 601 & 1 & SRGNAQESLDTVSPK & 0.3645 & 0.7655 \\
\hline F1MAQ5 & Map2 & $S$ & 601 & 2 & SRGNAQESLDTVSPK & -0.0367 & 0.6826 \\
\hline F1MAQ5 & Map2 & $\mathrm{T}$ & 604 & 1 & NAQESLDTVSPKNQQ & 0.2868 & 1.2891 \\
\hline F1MAQ5 & Map2 & S & 606 & 1 & QESLDTVSPKNQQDE & -0.0553 & 1.3368 \\
\hline F1MAQ5 & Map2 & S & 606 & 2 & QESLDTVSPKNQQDE & -0.0027 & 0.6826 \\
\hline F1MAQ5 & Map2 & $S$ & 624 & 1 & LAKASQPSPPAHEAG & -0.0181 & 0.6916 \\
\hline F1MAQ5 & Map2 & S & 624 & 2 & LAKASQPSPPAHEAG & -0.1217 & 0.7763 \\
\hline F1MAQ5 & Map2 & S & 652 & 1 & SELPEEPSSPQERMF & -0.0174 & 0.2919 \\
\hline F1MAQ5 & Map2 & $S$ & 652 & 2 & SELPEEPSSPQERMF & -0.1443 & 0.8909 \\
\hline F1MAQ5 & Map2 & S & 653 & 1 & ELPEEPSSPQERMFT & 0.0112 & 1.4392 \\
\hline F1MAQ5 & Map2 & S & 653 & 2 & ELPEEPSSPQERMFT & -0.1011 & 0.6293 \\
\hline F1MAQ5 & Map2 & $\mathrm{S}$ & 724 & 2 & FGRGHDLSPLASDIL & -0.2709 & 0.4337 \\
\hline F1MAQ5 & Map2 & S & 724 & 3 & FGRGHDLSPLASDIL & -0.4564 & \\
\hline F1MAQ5 & Map2 & $\mathrm{T}$ & 734 & 2 & ASDILTNTSGSMDEG & -0.069 & 1.0824 \\
\hline F1MAQ5 & Map2 & S & 735 & 2 & SDILTNTSGSMDEGD & -0.0946 & 1.3404 \\
\hline F1MAQ5 & Map2 & S & 737 & 1 & ILTNTSGSMDEGDDY & & 1.3672 \\
\hline F1MAQ5 & Map2 & S & 737 & 2 & ILTNTSGSMDEGDDY & -0.0902 & 1.3262 \\
\hline F1MAQ5 & Map2 & $Y$ & 744 & 2 & SMDEGDDYLPPTTPA & -0.1351 & 1.6955 \\
\hline F1MAQ5 & Map2 & S & 821 & 1 & GTRSRLASVSADAEV & 0.004 & 0.5763 \\
\hline F1MAQ5 & Map2 & $S$ & 823 & 1 & RSRLASVSADAEVAR & & 0.75 \\
\hline F1MAQ5 & Map2 & $S$ & 880 & 1 & KYTVPLPSPVQDSEN & & 0.5487 \\
\hline F1MAQ5 & Map2 & S & 880 & 2 & KYTVPLPSPVQDSEN & -0.304 & \\
\hline F1MAQ5 & Map2 & $S$ & 885 & 1 & LPSPVQDSENLSGES & -0.0477 & 0.6378 \\
\hline F1MAQ5 & Map2 & $S$ & 889 & 1 & VQDSENLSGESGSFY & & 0.4883 \\
\hline F1MAQ5 & Map2 & S & 935 & 1 & FTAEKEASPPSSADK & 0.1243 & 0.6756 \\
\hline F1MAQ5 & Map2 & $S$ & 938 & 1 & EKEASPPSSADKSGL & 0.017 & 0.41 \\
\hline F1MAQ5 & Map2 & $S$ & 1064 & 1 & GQMASGMSVDAGKTI & 0.1825 & 0.2911 \\
\hline F1MAQ5 & Map2 & S & 1158 & 2 & DEGKKETSPETSLIQ & -0.4837 & 0.6873 \\
\hline F1MAQ5 & Map2 & $\mathrm{T}$ & 1161 & 2 & KKETSPETSLIQDEV & -0.5437 & 0.5186 \\
\hline F1MAQ5 & Map2 & $S$ & 1441 & 1 & KTGRGRISTPERKVA & & 1.8966 \\
\hline F1MAQ5 & Map2 & $\mathrm{T}$ & 1442 & 1 & TGRGRISTPERKVAK & & 2.2479 \\
\hline F1MAQ5 & Map2 & $S$ & 1482 & 1 & KSEVQAHSPSRKLIL & 0.0547 & 2.5098 \\
\hline F1MAQ5 & Map2 & S & 1484 & 1 & EVQAHSPSRKLILKP & & 2.9912 \\
\hline F1MAQ5 & Map2 & $\mathrm{T}$ & 1534 & 1 & DKVTDGITKSPEKRS & 0.2384 & 1.6831 \\
\hline F1MAQ5 & Map2 & $S$ & 1536 & 1 & VTDGITKSPEKRSSL & 0.1977 & 1.7006 \\
\hline F1MAQ5 & Map2 & S & 1542 & 1 & KSPEKRSSLPRPSSI & & 0.7764 \\
\hline F1MAQ5 & Map2 & S & 1557 & 1 & LPPRRGVSGDREENS & & 0.664 \\
\hline F1MAQ5 & Map2 & $T$ & 1603 & 2 & TPGSTAITPGTPPSY & -0.2198 & 0.6952 \\
\hline F1MAQ5 & Map2 & $\mathrm{T}$ & 1606 & 1 & STAITPGTPPSYSSR & 0.0578 & 1.15 \\
\hline
\end{tabular}




\begin{tabular}{|c|c|c|c|c|c|c|c|}
\hline F1MAQ5 & Map2 & $\mathrm{T}$ & 1647 & 1 & KKVAIIRTPPKSPAT & 0.3389 & 1.8782 \\
\hline F1MAQ5 & Map2 & $\mathrm{T}$ & 1647 & 2 & KKVAIIRTPPKSPAT & -0.7688 & 1.3314 \\
\hline F1MAQ5 & Map2 & $T$ & 1647 & 3 & KKVAIIRTPPKSPAT & -0.4699 & 1.0471 \\
\hline F1MAQ5 & Map2 & $S$ & 1651 & 2 & IIRTPPKSPATPKQL & -0.7783 & 1.3275 \\
\hline F1MAQ5 & Map2 & S & 1651 & 3 & IIRTPPKSPATPKQL & -0.4699 & 1.0471 \\
\hline F1MAQ5 & Map2 & $\mathrm{T}$ & 1654 & 2 & TPPKSPATPKQLRLI & -0.6769 & 1.2807 \\
\hline F1MAQ5 & Map2 & $T$ & 1654 & 3 & TPPKSPATPKQLRLI & -0.4699 & 1.0471 \\
\hline F1MAQ5 & Map2 & $S$ & 1677 & 1 & NVKSKIGSTDNIKYQ & & 0.3817 \\
\hline F1MAQ5 & Map2 & $\mathrm{T}$ & 1678 & 1 & VKSKIGSTDNIKYQP & & 0.4194 \\
\hline F1MAQ5 & Map2 & $T$ & 1778 & 1 & DHGAEIITQSPSRSS & 0.0979 & 0.4053 \\
\hline F1MAQ5 & Map2 & $S$ & 1780 & 1 & GAEIITQSPSRSSVA & 0.2277 & 0.5734 \\
\hline F1MAQ5 & Map2 & $S$ & 1788 & 1 & PSRSSVASPRRLSNV & 0.1705 & 1.5676 \\
\hline F7FMK8 & Map3k13 & $S$ & 308 & 1 & SKELSDKSTKMSFAG & & -0.3227 \\
\hline A0A0G2JW88 & Map4 & $T$ & 1729 & 2 & GKAAVGLTGNDIATP & & 1.2225 \\
\hline A0A0G2JW88 & Map4 & $\mathrm{T}$ & 1735 & 2 & LTGNDIATPPNKELP & -0.1093 & 1.1903 \\
\hline A0A0G2JW88 & Map4 & $S$ & 1744 & 1 & PNKELPPSPEKKAKP & 0.227 & 0.6645 \\
\hline A0A0G2JW88 & Map4 & $\mathrm{S}$ & 1744 & 2 & PNKELPPSPEKKAKP & -0.1093 & 1.1984 \\
\hline A0A0G2JW88 & Map4 & $S$ & 1837 & 1 & KVAEKRTSPSKPSSA & & 1.0699 \\
\hline A0A0G2JW88 & Map4 & $S$ & 1862 & 1 & PTISKATSPSTLVST & 0.0812 & 0.5536 \\
\hline A0A0G2JW88 & Map4 & $S$ & 1875 & 1 & STGSSSRSPSTTLPK & -0.0109 & 1.8811 \\
\hline A0A0G2JW88 & Map4 & $\mathrm{s}$ & 1991 & 1 & SVRSKVGSTENMKHQ & & 0.2856 \\
\hline A0A0G2K7W4 & Map4k4 & $\mathrm{S}$ & 547 & 1 & FRKTNHSSPEAQAKQ & -0.0315 & 0.6377 \\
\hline A0A0G2K7W4 & Map4k4 & $S$ & 683 & 1 & PVLSRRDSPLQGSGQ & 0.3996 & 0.3338 \\
\hline A0A0G2K7W4 & Map4k4 & S & 745 & 1 & HPGSQSGSGERFRVR & -0.3702 & 0.4629 \\
\hline Q63560 & Map6 & $S$ & 432 & 1 & PAWMVRRSEGHEQTT & 0.1363 & 0.2711 \\
\hline Q63560 & Map6 & $\mathrm{T}$ & 438 & 1 & RSEGHEQTTAAHAQG & 0.0938 & 0.3016 \\
\hline Q63560 & Map6 & S & 681 & 1 & PGSLKGQSPTAPGPP & -0.1066 & 0.5079 \\
\hline Q63560 & Map6 & $\mathrm{T}$ & 683 & 1 & SLKGQSPTAPGPPKD & -0.2227 & 0.3062 \\
\hline D4A644 & Map7d1 & $S$ & 118 & 1 & SQRSSQPSPTAVPAS & 0.0152 & 0.2799 \\
\hline D4A644 & Map7d1 & $S$ & 512 & 1 & KEPAAPASPAPSPVP & & 0.3677 \\
\hline D4A4L4 & Map7d2 & $\mathrm{S}$ & 293 & 1 & VKPTYIGSPVKYYFP & & 1.1932 \\
\hline D4A1Q2 & Mapt & $T$ & 420 & 2 & ATRIPAKTTPSPKTP & -0.3579 & 0.4128 \\
\hline D4A1Q2 & Mapt & $\mathrm{T}$ & 426 & 2 & KTTPSPKTPPGSGEP & -0.2737 & 0.2633 \\
\hline D4A1Q2 & Mapt & $S$ & 440 & 2 & PPKSGERSGYSSPGS & -0.2262 & 0.3952 \\
\hline D4A1Q2 & Mapt & $S$ & 443 & 2 & SGERSGYSSPGSPGT & -0.1114 & 0.4426 \\
\hline D4A1Q2 & Mapt & $\mathrm{S}$ & 444 & 1 & GERSGYSSPGSPGTP & 0.029 & 0.264 \\
\hline D4A1Q2 & Mapt & $\mathrm{S}$ & 444 & 2 & GERSGYSSPGSPGTP & -0.0674 & 0.3581 \\
\hline D4A1Q2 & Mapt & $S$ & 447 & 1 & SGYSSPGSPGTPGSR & -0.0458 & 0.3565 \\
\hline D4A1Q2 & Mapt & $\mathrm{s}$ & 447 & 2 & SGYSSPGSPGTPGSR & -0.0858 & 0.5366 \\
\hline D4A1Q2 & Mapt & $T$ & 450 & 2 & SSPGSPGTPGSRSRT & 0.0047 & 0.4525 \\
\hline D4A1Q2 & Mapt & $T$ & 457 & 2 & TPGSRSRTPSLPTPP & 0.0359 & 0.3317 \\
\hline D4A1Q2 & Mapt & $\mathrm{T}$ & 462 & 1 & SRTPSLPTPPTREPK & 0.1999 & 0.2945 \\
\hline D4A1Q2 & Mapt & $\mathrm{T}$ & 462 & 2 & SRTPSLPTPPTREPK & 0.0111 & 0.3661 \\
\hline D4A1Q2 & Mapt & $\mathrm{T}$ & 476 & 1 & KKVAVVRTPPKSPSA & -0.0793 & 0.395 \\
\hline
\end{tabular}




\begin{tabular}{|c|c|c|c|c|c|c|c|}
\hline D4A1Q2 & Mapt & $\mathrm{T}$ & 476 & 2 & KKVAVVRTPPKSPSA & -0.3485 & 0.4482 \\
\hline D4A1Q2 & Mapt & S & 480 & 1 & VVRTPPKSPSASKSR & -0.5137 & 0.0657 \\
\hline D4A1Q2 & Mapt & $S$ & 480 & 2 & VVRTPPKSPSASKSR & -0.2851 & 0.4541 \\
\hline D4A1Q2 & Mapt & S & 482 & 2 & RTPPKSPSASKSRLQ & -0.3706 & 0.3437 \\
\hline D4A1Q2 & Mapt & $S$ & 601 & 1 & RVQSKIGSLDNITHV & 1.1247 & -0.2846 \\
\hline D4A1Q2 & Mapt & S & 601 & 2 & RVQSKIGSLDNITHV & 1.3087 & -0.2154 \\
\hline D4A1Q2 & Mapt & $\mathrm{T}$ & 606 & 1 & IGSLDNITHVPGGGN & 1.0863 & -0.2846 \\
\hline D4A1Q2 & Mapt & $\mathrm{T}$ & 606 & 2 & IGSLDNITHVPGGGN & 1.3874 & -0.2011 \\
\hline D4A1Q2 & Mapt & S & 641 & 2 & GAEIVYKSPVVSGDT & -0.0052 & 0.3144 \\
\hline D4A1Q2 & Mapt & S & 641 & 3 & GAEIVYKSPVVSGDT & -0.3495 & 0.2923 \\
\hline D4A1Q2 & Mapt & $\mathrm{S}$ & 645 & 1 & VYKSPVVSGDTSPRH & 0.5949 & 0.2113 \\
\hline D4A1Q2 & Mapt & S & 645 & 3 & VYKSPVVSGDTSPRH & -0.3889 & 0.3061 \\
\hline D4A1Q2 & Mapt & S & 649 & 2 & PVVSGDTSPRHLSNV & -0.2519 & 0.3945 \\
\hline D4A1Q2 & Mapt & S & 649 & 3 & PVVSGDTSPRHLSNV & -0.3556 & 0.2949 \\
\hline D4A1Q2 & Mapt & S & 661 & 1 & SNVSSTGSIDMVDSP & 1.64 & 0.2673 \\
\hline D4A1Q2 & Mapt & S & 661 & 2 & SNVSSTGSIDMVDSP & 0.7571 & 0.3422 \\
\hline D4A1Q2 & Mapt & $\mathrm{S}$ & 667 & 2 & GSIDMVDSPQLATLA & 0.7323 & 0.2892 \\
\hline P30009 & Marcks & S & 163 & 1 & SFKLSGFSFKKSKKE & 1.3679 & -0.3377 \\
\hline P30009 & Marcks & S & 163 & 2 & SFKLSGFSFKKSKKE & 1.4437 & -0.1571 \\
\hline P30009 & Marcks & S & 163 & 3 & SFKLSGFSFKKSKKE & 2.1628 & \\
\hline P30009 & Marcks & S & 167 & 2 & SGFSFKKSKKEAGEG & 1.0535 & -0.4045 \\
\hline Q9EPH2 & Marcksl1 & $S$ & 48 & 1 & TPKGEGESPPVNGAD & & -0.271 \\
\hline Q9EPH2 & Marcksl1 & S & 104 & 1 & PFKLSGLSFKRNRKE & 1.2165 & -0.3161 \\
\hline Q8VHF0 & Mark3 & S & 400 & 1 & LSNSTGQSPHHKGQR & -0.0331 & 0.4364 \\
\hline A0A0G2JTD8 & Mark4 & $S$ & 413 & 1 & NKGQRTSSSTYHRQR & & -0.2683 \\
\hline M0R3L1 & Mast4 & S & 1197 & 1 & SPTPQPTSPQRSPSP & & 0.4528 \\
\hline P43244 & Matr3 & S & 188 & 1 & KRHFRRDSFDDRGPS & & 0.3433 \\
\hline B0BN72 & Mcrip1 & $S$ & 21 & 1 & KRNSSPRSPTNSSEI & 0.0555 & 0.3154 \\
\hline P04636 & Mdh2 & $\mathrm{T}$ & 235 & 1 & GRIQEAGTEVVKAKA & & -0.4713 \\
\hline D4A2R5 & Mex3c & S & 342 & 1 & MKTQRRGSQPSTPRL & & 0.334 \\
\hline F1M5V7 & Mex3d & S & 488 & 1 & NTGTRRSSGGGGAAT & -0.0343 & 0.2662 \\
\hline Q5XIQ4 & Mgrn1 & S & 429 & 1 & KGKTQSKSPDSTLRS & 0.0306 & 0.4694 \\
\hline D3ZGN7 & Mical3 & S & 685 & 1 & AGKRRKTSQSEEEEP & 0.0586 & 0.4214 \\
\hline D3ZGN7 & Mical3 & $S$ & 1152 & 1 & RGPSQVSSPSQPPQK & -0.1209 & 0.5963 \\
\hline D3ZGN7 & Mical3 & S & 1165 & 2 & QKQAVLFSPAHSPGA & -0.4619 & 0.5124 \\
\hline D3ZGN7 & Mical3 & S & 1169 & 1 & VLFSPAHSPGAEEAK & & 0.5516 \\
\hline D3ZGN7 & Mical3 & S & 1169 & 2 & VLFSPAHSPGAEEAK & -0.4619 & 0.511 \\
\hline D3ZGN7 & Mical3 & S & 1218 & 1 & EEQKTVHSPIRSQPV & 0.149 & 0.5484 \\
\hline A0A0G2KAE6 & Miga2 & S & 111 & 1 & CSSRRVQSPSSKSND & 0.2615 & 0.7271 \\
\hline A0A0G2KAE6 & Miga2 & S & 113 & 1 & SRRVQSPSSKSNDTL & & 0.6858 \\
\hline A0A0G2KAE6 & Miga2 & $\mathrm{T}$ & 206 & 1 & QRGDGGSTPTPGDSL & -0.0036 & 0.6699 \\
\hline D3ZJ47 & Minar1 & S & 389 & 1 & SFFNRNPSEEKLRYP & & 0.7683 \\
\hline Q5BJQ2 & Mindy1 & S & 394 & 1 & SHGAEGGSGSPEKQL & & -0.3628 \\
\hline Q5BJQ2 & Mindy 1 & $S$ & 396 & 1 & GAEGGSGSPEKQLQV & 0.3415 & -0.2916 \\
\hline
\end{tabular}




\begin{tabular}{|c|c|c|c|c|c|c|c|}
\hline F1LN69 & Mink1 & $S$ & 547 & 1 & RMNKQQNSPLAKTKP & 0.1088 & 1.2738 \\
\hline F1LN69 & Mink1 & S & 793 & 1 & ASTKLDSSPVLSPGN & & 0.9743 \\
\hline D4ABB2 & Mlc1 & S & 207 & 1 & PCKKKKGSISDGTNI & 0.2159 & -0.3517 \\
\hline A0A096MK47 & Mlip & S & 442 & 1 & LSQAQPPSPPALASS & & 0.4385 \\
\hline A0A096MK47 & Mlip & $S$ & 719 & 1 & PVEHSSDSPSRPSQT & & 0.6964 \\
\hline Q63327 & Mobp & $\mathrm{T}$ & 73 & 1 & QKTSRRATSPQKPKH & & -0.2735 \\
\hline A0A0G2JUR5 & Mprip & S & 2091 & 1 & YDIMKSKSNPDFLKK & 0.1276 & -0.3012 \\
\hline A0A0G2JXC5 & Mrtfa & $S$ & 681 & 1 & QQPAPASSPVKRESS & -0.3274 & 0.6281 \\
\hline BOBN30 & Mtch1 & $\mathrm{s}$ & 61 & 1 & PRPAAQPSARRMDGG & 0.2964 & 0.5915 \\
\hline D4A5D4 & Mtcl1 & S & 1339 & 1 & GGTTPVSSPSRSLRS & 0.0841 & 1.0308 \\
\hline D3ZYM5 & Mtss1 & $\mathrm{s}$ & 329 & 1 & SSGSHSHSPSSHYRY & -0.549 & 1.0083 \\
\hline A0A0G2K4U7 & Mtus2 & S & 329 & 1 & CREERSTSPVDRKEL & & 0.8352 \\
\hline D3ZNK1 & Mtx3 & $S$ & 283 & 1 & MDGNLRQSPQLLPRK & 0.0797 & 0.3154 \\
\hline D4A2D3 & Mycbp2 & S & 2701 & 1 & ASSPRSSSPQDKNLP & & 0.6439 \\
\hline Q62812 & Myh9 & $\mathrm{s}$ & 1917 & 1 & AMNREVSSLKNKLRR & & -0.3914 \\
\hline Q9Z1N3 & Myo9a & S & 1898 & 1 & FKENKEPSPKAKRKR & & 0.2973 \\
\hline F1M6E5 & Nacad & $\mathrm{s}$ & 1159 & 1 & ARQRVSLSPHSANPK & & 0.7176 \\
\hline Q769K2 & Napepld & $\mathrm{s}$ & 27 & 1 & TLRKRQNSVQNSGGS & 0.8793 & 0.4153 \\
\hline D4A514 & Nav1 & S & 1039 & 1 & ISLKSIGSPESTPKN & & 0.9623 \\
\hline D4A5I4 & Nav1 & $\mathrm{s}$ & 1042 & 1 & KSIGSPESTPKNQAS & -0.0036 & 0.7802 \\
\hline D4A5I4 & Nav1 & $\mathrm{S}$ & 1456 & 1 & LRIKRQNSSDSISSL & -0.0292 & 0.469 \\
\hline AOA0G2KOM8 & Ncam1 & S & 903 & 1 & EAATAPASPKSKAPS & -0.0699 & 0.2801 \\
\hline D3ZXN9 & Ncor2 & $\mathrm{s}$ & 2162 & 1 & HSEGGKRSPEPSKTV & -0.0962 & 0.8452 \\
\hline D3ZXN9 & Ncor2 & $\mathrm{T}$ & 2350 & 1 & GRSDHPLTSPGGGGK & & 0.6664 \\
\hline D3ZXN9 & Ncor2 & S & 2351 & 1 & RSDHPLTSPGGGGKA & & 0.8226 \\
\hline Q8VBU2 & Ndrg2 & $\mathrm{s}$ & 332 & 3 & LSRSRTASLTSAASI & 0.0756 & 0.2964 \\
\hline D3ZS58 & Ndufa2 & $\mathrm{s}$ & 76 & 1 & FGQEKNVSLNNLSAA & & -0.3524 \\
\hline D4A0T0 & Ndufb 10 & $\mathrm{~T}$ & 24 & 1 & TPAPSPQTSIPNPIT & & -0.2828 \\
\hline B5DEL8 & Ndufs5 & $\mathrm{T}$ & 93 & 1 & LMKEGKYTPPPHHLG & & -0.306 \\
\hline A0A0G2JXP3 & Nedd4I & S & 617 & 1 & RNNIFEESYRRIMSV & & -0.3238 \\
\hline G3V7S2 & Nefm & S & 30 & 1 & SFSRVSGSPSSGFRS & 0.1381 & 0.556 \\
\hline G3V7S2 & Nefm & S & 44 & 1 & SQSWSRGSPSTVSSS & 0.0568 & 0.6427 \\
\hline G3V7S2 & Nefm & $\mathrm{s}$ & 501 & 2 & EEPEVEKSPVKSPEA & & -0.5221 \\
\hline G3V7S2 & Nefm & S & 505 & 2 & VEKSPVKSPEAKEEE & & -0.5221 \\
\hline G3V7S2 & Nefm & $S$ & 665 & 1 & PDKKKAESPVKEKAV & 0.2273 & -0.3141 \\
\hline P97526 & Nf1 & $\mathrm{s}$ & 2169 & 1 & SYRDRSFSPGSYERE & & -0.4147 \\
\hline P97526 & Nf1 & $\mathrm{s}$ & 2798 & 1 & SGRTRHGSASQVQKQ & -0.0797 & 0.3417 \\
\hline P97685 & Nfasc & S & 1226 & 2 & EETEGNESSEATSPV & -0.0705 & 0.2775 \\
\hline P97685 & Nfasc & $\mathrm{s}$ & 1226 & 3 & EETEGNESSEATSPV & -0.1068 & 0.4249 \\
\hline P97685 & Nfasc & S & 1227 & 3 & ETEGNESSEATSPVN & -0.1259 & 0.3693 \\
\hline P97685 & Nfasc & $\mathrm{T}$ & 1230 & 3 & GNESSEATSPVNAIY & -0.1195 & 0.4132 \\
\hline P97685 & Nfasc & $\mathrm{s}$ & 1231 & 3 & NESSEATSPVNAIYS & -0.1237 & 0.4114 \\
\hline O70185 & Nfib & $\mathrm{T}$ & 327 & 1 & EKPLFSSTSPQDSSP & & 0.8925 \\
\hline Q8K1Q0 & Nmt1 & $S$ & 47 & 1 & SHNRGGLSPANDTGA & -0.2121 & 0.2725 \\
\hline
\end{tabular}




\begin{tabular}{|c|c|c|c|c|c|c|c|}
\hline P41777 & Nolc1 & S & 318 & 1 & KKSVGAQSPKKAAAQ & & 1.0521 \\
\hline A0A0G2K3Q5 & Nrcam & S & 1299 & 2 & NESSEAPSPVNAMNS & -0.0648 & 0.2837 \\
\hline D3ZYH3 & Nudt10 & $S$ & 148 & 1 & EKLKLGGSPTNGNSA & & -0.2665 \\
\hline D3ZC82 & Nufip2 & S & 212 & 1 & KGADNDGSGSESGYT & 0.0839 & 0.7277 \\
\hline D3ZC82 & Nufip2 & S & 214 & 1 & ADNDGSGSESGYTTP & 0.0796 & 1.0742 \\
\hline Q5XIG4 & Ociad1 & $\mathrm{T}$ & 146 & 1 & SGQSSFGTSPAADNI & & 0.3055 \\
\hline Q5XIG4 & Ociad1 & $S$ & 198 & 1 & EDSPKRKSVTYEELR & 0.0034 & 0.2695 \\
\hline A0A0G2JXN8 & Osbpl8 & S & 341 & 1 & NDQEHDESDNEVLGK & 0.244 & -0.7943 \\
\hline A0A0G2JXN8 & Osbpl8 & S & 798 & 1 & KVAKGYSSPEPDVQD & & -0.4212 \\
\hline A0A0G2JXN8 & Osbpl8 & S & 806 & 1 & PEPDVQDSSGSEAQS & 0.0327 & -0.2796 \\
\hline A0A0G2JXN8 & Osbpl8 & S & 807 & 1 & EPDVQDSSGSEAQSV & 0.1164 & -0.3829 \\
\hline A0A0G2JXN8 & Osbpl8 & S & 809 & 1 & DVQDSSGSEAQSVKP & & -0.3937 \\
\hline Q5U2R6 & P33monox & S & 175 & 2 & KHPASAQSSPSSTPH & -0.0226 & 0.8477 \\
\hline Q5U2R6 & P33monox & S & 176 & 2 & HPASAQSSPSSTPHS & -0.0741 & 0.6252 \\
\hline Q5U2R6 & P33monox & S & 183 & 1 & SPSSTPHSSPKQKSR & 0.0647 & 0.3823 \\
\hline Q5U2R6 & P33monox & $S$ & 183 & 2 & SPSSTPHSSPKQKSR & -0.1133 & 0.5283 \\
\hline Q5U2R6 & P33monox & $\mathrm{S}$ & 184 & 1 & PSSTPHSSPKQKSRG & 0.1006 & 0.3976 \\
\hline Q5U2R6 & P33monox & S & 184 & 2 & PSSTPHSSPKQKSRG & -0.0992 & 0.3274 \\
\hline O88588 & Pacs1 & $S$ & 18 & 1 & PGGAGGGSSQRGSGV & 0.0965 & 0.4125 \\
\hline O88588 & Pacs1 & $\mathrm{T}$ & 44 & 1 & PSQPQQPTPPKLAQA & 0.0415 & 0.2858 \\
\hline 088588 & Pacs1 & S & 493 & 1 & ADLQGSASPSKVEGT & -0.4489 & 0.3697 \\
\hline O88588 & Pacs1 & $S$ & 493 & 2 & ADLQGSASPSKVEGT & -0.3288 & -0.0594 \\
\hline Q62829 & Pak3 & S & 156 & 1 & GYIAAHQSNTKTASE & -0.2857 & 0.2895 \\
\hline D3ZQ51 & Pak6 & S & 346 & 1 & TGPLPGRSSPAGSPR & & 0.5311 \\
\hline D3ZQ51 & Pak6 & $S$ & 351 & 1 & GRSSPAGSPRTRHAQ & & 0.7991 \\
\hline Q920Q0 & Palm & S & 112 & 3 & PAAPKENSAAPSPIR & -0.0381 & 0.753 \\
\hline Q920Q0 & Palm & S & 116 & 1 & KENSAAPSPIRPHST & 0.0588 & 0.5973 \\
\hline Q920Q0 & Palm & $S$ & 116 & 2 & KENSAAPSPIRPHST & -0.1043 & 1.3008 \\
\hline Q920Q0 & Palm & S & 116 & 3 & KENSAAPSPIRPHST & -0.0842 & 0.8466 \\
\hline Q920Q0 & Palm & S & 122 & 2 & PSPIRPHSTSPAKEE & -0.1067 & 0.9557 \\
\hline Q920Q0 & Palm & S & 122 & 3 & PSPIRPHSTSPAKEE & -0.0675 & 0.8756 \\
\hline Q920Q0 & Palm & $\mathrm{T}$ & 123 & 2 & SPIRPHSTSPAKEEQ & -0.1005 & 0.9157 \\
\hline Q920Q0 & Palm & $\mathrm{T}$ & 123 & 3 & SPIRPHSTSPAKEEQ & -0.0657 & 0.8795 \\
\hline Q920Q0 & Palm & S & 124 & 2 & PIRPHSTSPAKEEQK & -0.1066 & 0.9924 \\
\hline Q920Q0 & Palm & S & 124 & 3 & PIRPHSTSPAKEEQK & -0.0627 & 0.8887 \\
\hline Q920Q0 & Palm & $\mathrm{T}$ & 141 & 2 & TMVNAQQTPLGTPKE & -0.127 & 0.5277 \\
\hline Q920Q0 & Palm & $\mathrm{T}$ & 145 & 1 & AQQTPLGTPKENRKS & 0.0876 & 0.4222 \\
\hline Q920Q0 & Palm & $\mathrm{T}$ & 145 & 2 & AQQTPLGTPKENRKS & -0.127 & 0.5299 \\
\hline Q920Q0 & Palm & $\mathrm{T}$ & 153 & 2 & PKENRKSTPVRSPGG & -0.1521 & 1.1867 \\
\hline Q920Q0 & Palm & S & 157 & 1 & RKSTPVRSPGGSTMM & -0.067 & 0.5122 \\
\hline Q920Q0 & Palm & S & 157 & 2 & RKSTPVRSPGGSTMM & -0.2534 & 1.3446 \\
\hline Q920Q0 & Palm & S & 161 & 2 & PVRSPGGSTMMKAAM & -0.2241 & 1.2552 \\
\hline Q920Q0 & Palm & $\mathrm{T}$ & 162 & 2 & VRSPGGSTMMKAAMY & -0.2643 & 1.3414 \\
\hline D4A1J3 & Palm3 & $S$ & 600 & 1 & SGAKDDVSPEEQGKS & 0.117 & -0.3701 \\
\hline
\end{tabular}




\begin{tabular}{|c|c|c|c|c|c|c|c|}
\hline Q4KM62 & Palmd & S & 371 & 2 & VSPRTELSPSRASPG & -0.1669 & 1.247 \\
\hline Q4KM62 & Palmd & S & 373 & 2 & PRTELSPSRASPGKS & & 1.1199 \\
\hline Q4KM62 & Palmd & $S$ & 376 & 2 & ELSPSRASPGKSGPQ & -0.1537 & 1.1186 \\
\hline $\mathrm{P} 14925$ & Pam & S & 961 & 1 & DEDDGTESEEEYSAP & & -0.361 \\
\hline A0A0G2QC22 & Paxx & S & 116 & 1 & FDLSKVPSPEAAPRL & & -0.2677 \\
\hline A0A0G2K6T9 & Pcdh1 & S & 852 & 1 & DLYAPKPSGKAAKGS & & -0.4479 \\
\hline A0A0G2K6T9 & Pcdh1 & $S$ & 934 & 1 & SIQLQPQSPSASKKH & -0.281 & 0.3254 \\
\hline A0A0G2K6T9 & Pcdh1 & S & 934 & 2 & SIQLQPQSPSASKKH & -0.6178 & \\
\hline D3ZPNO & Pcdh17 & S & 1037 & 1 & QEVPSASSSPTKACI & & 0.3218 \\
\hline D3ZPNO & Pcdh17 & S & 1080 & 1 & PTDSQYPSPSKQPRD & & 0.2965 \\
\hline A0A0G2K8I5 & Pcdh19 & S & 1134 & 1 & KEGRDKESPAVKRLK & & 0.624 \\
\hline Q68HB8 & Pcdh7 & S & 1000 & 2 & ARHYKSSSPLPTVQL & -0.2128 & 0.5728 \\
\hline Q68HB8 & Pcdh7 & S & 1011 & 1 & TVQLHPQSPTAGKKH & -0.0605 & 0.5065 \\
\hline Q68HB8 & Pcdh7 & S & 1011 & 2 & TVQLHPQSPTAGKKH & -0.2128 & 0.5434 \\
\hline F1LS01 & Pcdh9 & S & 941 & 1 & DLAKHYKSASPQPAF & & 0.3522 \\
\hline F1LS01 & Pcdh9 & $S$ & 943 & 1 & AKHYKSASPQPAFHL & & 0.4713 \\
\hline F1LS01 & Pcdh9 & $\mathrm{S}$ & 943 & 2 & AKHYKSASPQPAFHL & -0.6157 & 0.1051 \\
\hline Q767H7 & Pcdhac2 & S & 838 & 1 & LTGQSRHSAGNLIIL & & -0.363 \\
\hline Q9JKS6 & Pclo & $S$ & 231 & 1 & EVIQQDSSPKSVSSQ & 0.1306 & 0.3183 \\
\hline Q9JKS6 & Pclo & S & 617 & 1 & PVPSQQASPKKEPPS & 0.1001 & 0.3134 \\
\hline Q9JKS6 & Pclo & S & 826 & 1 & TKPVPKGSPTPSGTR & -0.6311 & 0.3068 \\
\hline Q9JKS6 & Pclo & $S$ & 924 & 1 & QAPTPSQSPAAQGPA & -0.3338 & 0.4796 \\
\hline Q9JKS6 & Pclo & $\mathrm{s}$ & 1352 & 1 & FSQESSPSSPSDLAK & -0.3646 & 0.3483 \\
\hline Q9JKS6 & Pclo & $S$ & 1352 & 3 & FSQESSPSSPSDLAK & 0.3633 & \\
\hline Q9JKS6 & Pclo & $S$ & 1353 & 1 & SQESSPSSPSDLAKL & -0.4177 & 0.2906 \\
\hline Q9JKS6 & Pclo & $\mathrm{s}$ & 1353 & 3 & SQESSPSSPSDLAKL & 0.4177 & \\
\hline Q9JKS6 & Pclo & $\mathrm{S}$ & 1469 & 1 & DSSESENSPVVRRKR & -0.0645 & 0.4955 \\
\hline Q9JKS6 & Pclo & $\mathrm{T}$ & 1478 & 1 & VVRRKRRTSIGSSSS & & -0.2775 \\
\hline Q9JKS6 & Pclo & $\mathrm{s}$ & 4150 & 1 & DPKMSKFSPIQESRD & -0.0348 & -0.3057 \\
\hline Q9JKS6 & Pclo & S & 4791 & 2 & SSQNSQQSPKPSVIK & & -0.2716 \\
\hline Q9JKS6 & Pclo & $S$ & 4795 & 2 & SQQSPKPSVIKSRSH & & -0.2764 \\
\hline A0A0G2K0X1 & Pcm1 & $\mathrm{s}$ & 1059 & 1 & WKNHRPVSADGNYRP & & 0.6117 \\
\hline A0A0G2K0X1 & Pcm1 & $\mathrm{S}$ & 1382 & 1 & TFKTRKASAQASLAS & & 0.5649 \\
\hline A0A0G2K0X1 & Pcm1 & $S$ & 1534 & 1 & DIVSRHISESDEKEG & 0.1563 & 0.2688 \\
\hline A0A0G2K0X1 & Pcm1 & $\mathrm{s}$ & 1536 & 1 & VSRHISESDEKEGEN & & 0.323 \\
\hline D3ZSQ1 & Pcnx3 & $\mathrm{S}$ & 287 & 1 & QPLDRRGSGDPLPQK & 0.0543 & 0.5115 \\
\hline D3ZSQ1 & Pcnx3 & $\mathrm{T}$ & 370 & 1 & DTVIGAGTPPGQTEP & & 0.5011 \\
\hline D3ZSQ1 & Pcnx3 & $\mathrm{s}$ & 1953 & 1 & GPDGEPASGSPKGGT & -0.0588 & 0.6739 \\
\hline D3ZSQ1 & Pcnx3 & $\mathrm{s}$ & 1955 & 1 & DGEPASGSPKGGTPK & -0.0587 & 0.5242 \\
\hline D3ZXP8 & Pcp2 & $S$ & 111 & 1 & ALSFRRNSSPQPQTQ & -0.3521 & 0.3134 \\
\hline P19836 & Pcyt1a & $s$ & 319 & 1 & QAISPKQSPSSSPTH & & 0.7063 \\
\hline Q9QYJ6 & Pde10a & $\mathrm{S}$ & 69 & 1 & NKAEDEPSPKEVSRY & & -0.3378 \\
\hline Q62920 & Pdlim5 & $\mathrm{S}$ & 228 & 1 & AEGQRRGSQGDIKQQ & 0.2009 & 1.037 \\
\hline Q6TRW4 & Pds5b & $\mathrm{T}$ & 1380 & 1 & GRGRPSKTPSPSQPK & & 0.3828 \\
\hline
\end{tabular}




\begin{tabular}{|c|c|c|c|c|c|c|c|}
\hline F1M785 & Pdzd2 & $S$ & 1043 & 1 & TDTQSPRSPENHTSP & & 0.2648 \\
\hline F1M785 & Pdzd2 & S & 1360 & 1 & SEARLSQSPQKADCR & & 0.785 \\
\hline F1M785 & Pdzd2 & $S$ & 1763 & 1 & KGVTVPKSPPSRQKS & & 0.5109 \\
\hline D3ZXY2 & Pdzd8 & $S$ & 518 & 1 & KEETQPLSHSPKRTP & 0.0958 & 0.6704 \\
\hline D4A563 & Peak1 & $S$ & 725 & 1 & RSYSSTHSSPAKIQR & & 1.0415 \\
\hline D4A563 & Peak1 & $S$ & 726 & 1 & SYSSTHSSPAKIQRP & & 1.0524 \\
\hline P31044 & Pebp1 & $S$ & 153 & 1 & RGKFKVESFRKKYHL & & -0.5176 \\
\hline Q642G4 & Pex14 & $S$ & 271 & 1 & ISPVSNESPSSSPGK & & 0.3494 \\
\hline P47860 & Pfkp & $S$ & 156 & 1 & AKNGEIDSDTVKKHA & & -0.2923 \\
\hline A0A0G2K8Q4 & Phactr2 & $S$ & 154 & 1 & TGSKASSSPSASSTS & 0.0268 & 0.7899 \\
\hline Q5XIH7 & Phb2 & $S$ & 91 & 1 & RARPRKISSPTGSKD & 0.1382 & -0.3309 \\
\hline Q5XIH7 & Phb2 & $S$ & 92 & 1 & ARPRKISSPTGSKDL & 0.2532 & -0.3355 \\
\hline Q5XIH7 & Phb2 & $S$ & 293 & 1 & SFTRGSDSLIKGKK & 0.1982 & -0.4209 \\
\hline Q5U2N3 & Pitpnm1 & $S$ & 1203 & 1 & VDFLRKQSQLLRSRG & & -0.2683 \\
\hline A0A0U1RRV0 & Pja1 & $S$ & 137 & 1 & EERGKVESPPAARCS & 0.0863 & 1.0583 \\
\hline P11980 & Pkm & $S$ & 127 & 1 & RTGLIKGSGTAEVEL & & -0.3358 \\
\hline F1M2K6 & Pkp4 & $\mathrm{S}$ & 220 & 1 & SVPSRAQSPSYVTST & -0.1028 & 1.2715 \\
\hline F1M2K6 & Pkp4 & $T$ & 225 & 1 & AQSPSYVTSTGVSPS & & 0.6982 \\
\hline F1M2K6 & Pkp4 & $S$ & 230 & 1 & YVTSTGVSPSRGSLR & 0.0038 & 1.0499 \\
\hline F1M2K6 & Pkp4 & $\mathrm{s}$ & 230 & 2 & YVTSTGVSPSRGSLR & -0.1104 & 0.4883 \\
\hline F1M2K6 & Pkp4 & $\mathrm{s}$ & 235 & 2 & GVSPSRGSLRTSLGS & 0.0552 & 0.3575 \\
\hline F1M2K6 & Pkp4 & $\mathrm{S}$ & 272 & 2 & LPAQRAASPYSQRPA & -0.5116 & 0.9758 \\
\hline F1M2K6 & Pkp4 & $Y$ & 274 & 2 & AQRAASPYSQRPASP & & 0.9204 \\
\hline F1M2K6 & Pkp4 & S & 275 & 2 & QRAASPYSQRPASPT & & 1.0011 \\
\hline F1M2K6 & Pkp4 & $S$ & 280 & 1 & PYSQRPASPTAVRRV & 0.0762 & 0.7457 \\
\hline $\mathrm{F} 1 \mathrm{M} 2 \mathrm{~K} 6$ & Pkp4 & $S$ & 280 & 2 & PYSQRPASPTAVRRV & -0.4608 & 0.9783 \\
\hline F1M2K6 & Pkp4 & $\mathrm{T}$ & 282 & 1 & SQRPASPTAVRRVGS & & 0.7303 \\
\hline F1M2K6 & Pkp4 & $\mathrm{T}$ & 282 & 2 & SQRPASPTAVRRVGS & -0.5663 & 1.0497 \\
\hline F1M2K6 & Pkp4 & $S$ & 326 & 1 & DAQTRVASPSQGQVG & & 0.4254 \\
\hline F1M2K6 & Pkp4 & S & 326 & 2 & DAQTRVASPSQGQVG & -0.1125 & 1.3523 \\
\hline $\mathrm{F} 1 \mathrm{M} 2 \mathrm{~K} 6$ & Pkp4 & $\mathrm{S}$ & 328 & 1 & QTRVASPSQGQVGSS & & 0.343 \\
\hline F1M2K6 & Pkp4 & $S$ & 328 & 2 & QTRVASPSQGQVGSS & -0.0056 & 1.014 \\
\hline F1M2K6 & Pkp4 & S & 335 & 1 & SQGQVGSSSPKRSGM & -0.0376 & 0.5921 \\
\hline F1M2K6 & Pkp4 & $S$ & 335 & 2 & SQGQVGSSSPKRSGM & -0.0461 & 0.8155 \\
\hline $\mathrm{F} 1 \mathrm{M} 2 \mathrm{~K} 6$ & Pkp4 & $S$ & 336 & 1 & QGQVGSSSPKRSGMT & -0.1325 & 0.5707 \\
\hline F1M2K6 & Pkp4 & $\mathrm{S}$ & 336 & 2 & QGQVGSSSPKRSGMT & -0.0811 & 1.5788 \\
\hline F1M2K6 & Pkp4 & $\mathrm{S}$ & 426 & 2 & YYSPVYRSPNHGTVE & -0.0959 & 0.425 \\
\hline F1M2K6 & Pkp4 & $S$ & 1090 & 2 & YPGSSKPSPIYISSY & -0.0481 & 0.8912 \\
\hline F1M2K6 & Pkp4 & $\mathrm{s}$ & 1098 & 1 & PIYISSYSSPAREQN & & 0.6185 \\
\hline F1M2K6 & Pkp4 & $S$ & 1098 & 2 & PIYISSYSSPAREQN & -0.0898 & 0.8633 \\
\hline F1M2K6 & Pkp4 & $S$ & 1099 & 1 & IYISSYSSPAREQNR & & 0.8775 \\
\hline F1M2K6 & Pkp4 & S & 1099 & 2 & IYISSYSSPAREQNR & -0.0497 & 0.9659 \\
\hline D4A8C5 & Plcb4 & $S$ & 901 & 1 & KANVTPQSSSELRPT & & 0.4779 \\
\hline D4AAX6 & Plch2 & $s$ & 1089 & 1 & SDSSSPDSPGSPKVA & & 0.883 \\
\hline
\end{tabular}




\begin{tabular}{|c|c|c|c|c|c|c|c|}
\hline D4AAX6 & Plch2 & S & 1092 & 1 & SSPDSPGSPKVAPCQ & & 0.8988 \\
\hline Q6S3A5 & Plec & S & 4276 & 1 & GFRSRSSSVGSSSSY & -0.0511 & 0.9176 \\
\hline E9PTG5 & Plekha5 & $S$ & 574 & 1 & TYRSEVSSPIQRGDV & & 0.5355 \\
\hline E9PTG5 & Plekha5 & S & 1020 & 1 & GVPLRTKSPTPESST & & 0.7177 \\
\hline E9PTG5 & Plekha5 & S & 1109 & 1 & SPSSVRDSPSRVPQT & 0.1383 & 0.8306 \\
\hline A0A0G2K6Z8 & Plekha7 & S & 827 & 2 & PQLRKVVSPLQSPTK & -0.1439 & 0.9998 \\
\hline A0A0G2K6Z8 & Plekha7 & $S$ & 831 & 2 & KVVSPLQSPTKATPQ & -0.1398 & 0.9483 \\
\hline A0A0G2K6Z8 & Plekha7 & $\mathrm{T}$ & 833 & 2 & VSPLQSPTKATPQAE & -0.1418 & 1.0862 \\
\hline D4ACN8 & Plgrkt & S & 141 & 1 & EKARREQSKFFSDK & & -0.279 \\
\hline $\mathrm{P} 60203$ & Plp1 & S & 114 & 1 & TICGKGLSATVTGGQ & & -0.3084 \\
\hline $\mathrm{P} 60203$ & Plp1 & $\mathrm{T}$ & 116 & 1 & CGKGLSATVTGGQKG & 0.2759 & -0.3338 \\
\hline P60203 & Plp1 & $\mathrm{T}$ & 118 & 1 & KGLSATVTGGQKGRG & & -0.2985 \\
\hline P60203 & Plp1 & S & 134 & 1 & RGQHQAHSLERVCHC & 0.2179 & -0.2817 \\
\hline Q66H88 & Plpp6 & S & 24 & 1 & GGSSVPGSPAHGGGG & 0.0198 & 0.3577 \\
\hline Q66H88 & Plpp6 & S & 61 & 1 & ARLRASDSPVHRRGS & 0.2864 & 0.4922 \\
\hline G3V864 & Plppr4 & S & 474 & 1 & DSEPEGQSPPRSIEM & & 0.465 \\
\hline G3V864 & Plppr4 & $\mathrm{S}$ & 547 & 1 & EETQENISTSPKSSS & & 1.0588 \\
\hline G3V864 & Plppr4 & $\mathrm{T}$ & 548 & 1 & ETQENISTSPKSSSA & -0.1218 & 0.7693 \\
\hline G3V864 & Plppr4 & $S$ & 549 & 1 & TQENISTSPKSSSAR & & 0.6398 \\
\hline G3V864 & Plppr4 & S & 593 & 1 & KQQGVLQSSPKNAEG & 0.2711 & 0.4085 \\
\hline G3V864 & Plppr4 & S & 594 & 1 & QQGVLQSSPKNAEGS & 0.2294 & 0.3739 \\
\hline D3ZQN3 & Pnma8b & $S$ & 609 & 1 & TPEKKAGSGASTEDH & 0.1397 & 0.9204 \\
\hline D3ZQN3 & Pnma8b & S & 612 & 1 & KKAGSGASTEDHAGN & & 0.968 \\
\hline D3ZQN3 & Pnma8b & $\mathrm{T}$ & 613 & 1 & KAGSGASTEDHAGNN & 0.1925 & 1.1651 \\
\hline O88794 & Pnpo & $S$ & 164 & 1 & YFHSRPKSSQIGAVV & & -0.3642 \\
\hline D3ZGH9 & Pom12112 & S & 220 & 1 & RSLHAQSSDPKCKRS & 1.9696 & 0.5387 \\
\hline F1M8A4 & Ppfia2 & S & 1125 & 1 & LTTTSGQSRKMTTDV & & -0.5336 \\
\hline Q5M821 & Ppm1h & $S$ & 489 & 1 & KDRGWRISNDRLGSG & & -0.3028 \\
\hline Q10728 & Ppp1r12a & S & 894 & 1 & DSVSRYDSSSTSSSD & & -0.2786 \\
\hline A0A0G2K4R1 & Ppp1r12c & S & 508 & 1 & PSRTEPGSPVKPNVL & & 0.2732 \\
\hline A0A0G2KB61 & Ppp1r13b & S & 562 & 1 & DKGSRPQSPRKGPQT & & 0.4791 \\
\hline D4AA72 & Ppp1r16b & S & 477 & 1 & WSGFKEQSPQTLLEL & & 0.2995 \\
\hline D3ZZ26 & Ppp1r3d & S & 54 & 2 & IIQRRSRSLPTSPER & & 0.5061 \\
\hline O35867 & Ppp1r9a & S & 94 & 1 & TRGKGRPSSPQKRMK & 0.1146 & 1.3275 \\
\hline O35867 & Ppp1r9a & S & 95 & 1 & RGKGRPSSPQKRMKP & 0.0408 & 1.3767 \\
\hline O35867 & Ppp1r9a & S & 153 & 1 & FERSGHESGQNNRHS & -0.715 & 0.9538 \\
\hline O35867 & Ppp1r9a & S & 160 & 1 & SGQNNRHSPKKEKAG & -0.4636 & 1.0204 \\
\hline O35867 & Ppp1r9a & S & 929 & 1 & FTFNDDFSPSSTSSA & -0.0939 & 0.9554 \\
\hline P63329 & Ppp3ca & S & 462 & 1 & DEAIKGFSPQHKITS & -0.0663 & 0.5859 \\
\hline A0A0G2JV49 & Ppp6r2 & S & 629 & 1 & GQGRKTGSPTARNVP & & 0.5263 \\
\hline F1M0J7 & Prickle2 & S & 546 & 1 & TEQTPRGSMESLALS & & 0.2899 \\
\hline P54645 & Prkaa1 & S & 486 & 1 & DEITEAKSGTATPQR & -0.0098 & -0.2748 \\
\hline P68182 & Prkacb & S & 322 & 2 & FIPKFRGSGDTSNFD & -0.0456 & -0.3046 \\
\hline P68182 & Prkacb & S & 339 & 2 & EEEEIRVSITEKCGK & -0.0561 & -0.2979 \\
\hline
\end{tabular}




\begin{tabular}{|c|c|c|c|c|c|c|c|}
\hline F1LS42 & Prkcb & $\mathrm{T}$ & 313 & 1 & RAKIGQGTKAPEEKT & & -0.4059 \\
\hline Q4V8C7 & Prkra & S & 18 & 1 & PPLQREDSGTFSLGK & & 0.8352 \\
\hline Q6AXY7 & Prpf38b & S & 488 & 1 & EKRKREHSPSREKSR & 0.342 & -0.3833 \\
\hline 008618 & Prpsap2 & $S$ & 198 & 1 & ASAKRAQSFAERLRL & & -0.3605 \\
\hline D3ZPT0 & Prr36 & $S$ & 123 & 1 & AGQGSISSPGRASSG & & 0.6994 \\
\hline Q6MG48 & Prrc2a & $\mathrm{s}$ & 1007 & 1 & TPAGGNLSPAPRLRR & & 0.5441 \\
\hline G3V8J5 & Psd & $S$ & 1010 & 1 & PRAQRPGSEARAGAG & 0.0478 & 0.4836 \\
\hline D3ZUW0 & Psd3 & $S$ & 48 & 1 & ISNSSEFSAKETKAL & & -0.3396 \\
\hline D3ZUW0 & Psd3 & $\mathrm{s}$ & 58 & 1 & ETKALYNSIKNEKLE & & -0.471 \\
\hline Q812D1 & Psip1 & $\mathrm{s}$ & 176 & 1 & TASNVKASPKRGRPA & & 0.7637 \\
\hline O88761 & Psmd1 & $\mathrm{T}$ & 311 & 1 & AGAVAGKTPDASPEP & 0.5604 & -0.3511 \\
\hline O88761 & Psmd1 & $S$ & 315 & 1 & AGKTPDASPEPKDQT & & -0.428 \\
\hline G3V6B9 & Ptpn4 & S & 403 & 1 & TPNHRNSSFTQEGTR & & 0.4841 \\
\hline M0RB22 & Ptprd & $S$ & 1314 & 1 & ESESRKSSLPNSKEV & 0.219 & -0.3075 \\
\hline Q64605 & Ptprs & $\mathrm{s}$ & 1292 & 1 & PDSKRKDSEPRTKCL & 0.406 & -0.2804 \\
\hline D3ZYS1 & Purg & S & 160 & 2 & RQKHSAPSPPVSVGS & 0.0605 & 0.4403 \\
\hline D3ZYS1 & Purg & $S$ & 160 & 3 & RQKHSAPSPPVSVGS & 0.002 & 0.5899 \\
\hline D3ZYS1 & Purg & $S$ & 164 & 1 & SAPSPPVSVGSEEHP & & 0.3481 \\
\hline D3ZYS1 & Purg & S & 164 & 2 & SAPSPPVSVGSEEHP & 0.1155 & 0.4158 \\
\hline D3ZYS1 & Purg & $\mathrm{S}$ & 164 & 3 & SAPSPPVSVGSEEHP & 0.002 & 0.5899 \\
\hline D3ZYS1 & Purg & S & 167 & 2 & SPPVSVGSEEHPHSV & 0.0395 & 0.4379 \\
\hline D3ZYS1 & Purg & S & 167 & 3 & SPPVSVGSEEHPHSV & 0.002 & 0.619 \\
\hline A0A0G2K4E8 & R3hdm2 & $S$ & 393 & 1 & SILTRGDSIGSSKGG & 0.5469 & 0.4799 \\
\hline A0A0G2K4E8 & R3hdm2 & $S$ & 396 & 1 & TRGDSIGSSKGGSAG & & 0.4837 \\
\hline D4A3C2 & RGD1304884 & $\mathrm{S}$ & 74 & 1 & DQDRRSSSNESFSSN & & -0.3188 \\
\hline A0A096MJX5 & RGD1305178 & $S$ & 261 & 1 & EKPEPERSPPNRKRP & -0.091 & 0.4736 \\
\hline A0A0G2KAX2 & RGD1305455 & $\mathrm{s}$ & 495 & 1 & RPLSRKSSPSSPAVR & -0.0333 & 0.3096 \\
\hline A0A0G2KAX2 & RGD1305455 & $\mathrm{T}$ & 541 & 2 & VMERRAITPPVASPV & 0.1518 & 0.2999 \\
\hline A0A0G2KAX2 & RGD1305455 & $S$ & 546 & 2 & AITPPVASPVGRPLY & 0.1518 & 0.2999 \\
\hline D3Z9D0 & RGD1306271 & S & 1470 & 2 & FPVVDDLSSGDTKER & -0.0801 & 0.4883 \\
\hline D3Z9D0 & RGD1306271 & S & 1471 & 2 & PVVDDLSSGDTKERH & -0.0764 & 0.4446 \\
\hline D3Z9D0 & RGD1306271 & $S$ & 1830 & 2 & PGLGYPTSSTEDLQP & -0.0906 & 0.5082 \\
\hline A0A096MJT6 & RGD1307100 & $\mathrm{s}$ & 1301 & 2 & QDKSVGQSPLRSPLK & & 0.5055 \\
\hline A0A096MJT6 & RGD1307100 & $S$ & 1305 & 2 & VGQSPLRSPLKRQAS & & 0.5055 \\
\hline A0A096MJT6 & RGD1307100 & $S$ & 1355 & 1 & SDENVLDSPKQRRSF & 0.1532 & 0.3258 \\
\hline A0A096MJT6 & RGD1307100 & $\mathrm{s}$ & 2368 & 1 & RGSVVQESPVTKSGH & & 0.2934 \\
\hline D3ZBU7 & RGD1310819 & $S$ & 213 & 1 & PQSPDHMSDASVSSR & -0.0408 & 0.4542 \\
\hline D3ZBU7 & RGD1310819 & S & 213 & 2 & PQSPDHMSDASVSSR & -0.403 & -0.0558 \\
\hline D3ZBU7 & RGD1310819 & $\mathrm{S}$ & 484 & 1 & APPERDISPSKDNMS & -0.266 & 0.269 \\
\hline D3ZBU7 & RGD1310819 & $S$ & 486 & 1 & PERDISPSKDNMSPP & & 0.3043 \\
\hline D3ZBU7 & RGD1310819 & $S$ & 486 & 2 & PERDISPSKDNMSPP & -0.2146 & 0.7633 \\
\hline D3ZBU7 & RGD1310819 & $\mathrm{s}$ & 491 & 1 & SPSKDNMSPPKRIMA & -0.041 & 0.3924 \\
\hline D3ZBU7 & RGD1310819 & $S$ & 491 & 2 & SPSKDNMSPPKRIMA & -0.2306 & 0.708 \\
\hline D3ZBU7 & RGD1310819 & $\mathrm{s}$ & 505 & 1 & APPERDKSPSEGDVA & -0.2045 & 0.4019 \\
\hline
\end{tabular}




\begin{tabular}{|c|c|c|c|c|c|c|c|}
\hline D3ZBU7 & RGD1310819 & $S$ & 507 & 1 & PERDKSPSEGDVAPP & -0.1093 & 0.3525 \\
\hline D3ZBU7 & RGD1310819 & S & 568 & 1 & APPERDKSPSEGDVT & -0.2171 & 0.425 \\
\hline D3ZBU7 & RGD1310819 & S & 570 & 1 & PERDKSPSEGDVTPP & -0.2244 & 0.3889 \\
\hline D3ZBU7 & RGD1310819 & $S$ & 570 & 2 & PERDKSPSEGDVTPP & & 0.6705 \\
\hline D3ZBU7 & RGD1310819 & $\mathrm{T}$ & 575 & 1 & SPSEGDVTPPKRIMA & 0.0566 & 0.7534 \\
\hline D3ZBU7 & RGD1310819 & $\mathrm{T}$ & 575 & 2 & SPSEGDVTPPKRIMA & -0.1408 & 0.7054 \\
\hline D3ZBU7 & RGD1310819 & S & 876 & 1 & KLRSTSLSLKYRDSS & & -0.3704 \\
\hline D3ZBU7 & RGD1310819 & $S$ & 937 & 1 & QGKGKTRSPEQPGTK & & 0.2808 \\
\hline D3ZY47 & RGD1559896 & $\mathrm{s}$ & 176 & 1 & AQSSRSSSLDALGPA & 0.2902 & 0.4504 \\
\hline D3ZY47 & RGD1559896 & S & 199 & 1 & WKINAERSREGHEAE & & 0.4914 \\
\hline D3ZQK5 & RGD1561149 & $\mathrm{s}$ & 891 & 1 & TEPRSPQSPASKASF & & 0.4943 \\
\hline A0A0G2K1W1 & Rab11fip5 & $S$ & 152 & 1 & AGRDSIQSPKLLTHK & 0.0179 & 0.2949 \\
\hline A0A0G2K1W1 & Rab11fip5 & $S$ & 163 & 1 & LTHKRTYSDEASQLR & 0.1323 & 0.2971 \\
\hline A0A0G2K1W1 & Rab11fip5 & $S$ & 333 & 1 & NEVGRRGSVGEKGSP & 0.4369 & 0.4866 \\
\hline A0A0G2K1W1 & Rab11fip5 & $\mathrm{s}$ & 333 & 2 & NEVGRRGSVGEKGSP & & 0.6022 \\
\hline A0A0G2K1W1 & Rab11fip5 & S & 333 & 3 & NEVGRRGSVGEKGSP & -0.1 & 0.646 \\
\hline A0A0G2K1W1 & Rab11fip5 & $\mathrm{s}$ & 339 & 1 & GSVGEKGSPSLGASP & 0.1151 & 0.7497 \\
\hline A0A0G2K1W1 & Rab11fip5 & $\mathrm{s}$ & 339 & 2 & GSVGEKGSPSLGASP & -0.0031 & 0.7484 \\
\hline A0A0G2K1W1 & Rab11fip5 & S & 339 & 3 & GSVGEKGSPSLGASP & -0.0967 & 0.5842 \\
\hline $\mathrm{A} 0 \mathrm{~A} 0 \mathrm{G} 2 \mathrm{~K} 1 \mathrm{~W} 1$ & Rab11fip5 & $\mathrm{s}$ & 341 & 1 & VGEKGSPSLGASPHH & 0.0803 & 0.8531 \\
\hline A0A0G2K1W1 & Rab11fip5 & $\mathrm{S}$ & 341 & 2 & VGEKGSPSLGASPHH & 0.0305 & 0.6426 \\
\hline A0A0G2K1W1 & Rab11fip5 & S & 345 & 1 & GSPSLGASPHHSSTG & -0.0968 & 1.6046 \\
\hline A0A0G2K1W1 & Rab11fip5 & $\mathrm{S}$ & 345 & 2 & GSPSLGASPHHSSTG & -0.0283 & 0.8233 \\
\hline A0A0G2K1W1 & Rab11fip5 & $\mathrm{s}$ & 345 & 3 & GSPSLGASPHHSSTG & -0.0967 & 0.646 \\
\hline A0A0G2K1W1 & Rab11fip5 & S & 349 & 2 & LGASPHHSSTGEEKA & -0.0228 & 0.9334 \\
\hline A0A0G2K1W1 & Rab11fip5 & $\mathrm{s}$ & 350 & 2 & GASPHHSSTGEEKAK & -0.1915 & 1.0046 \\
\hline A0A0G2K1W1 & Rab11fip5 & $\mathrm{s}$ & 752 & 1 & RLFTPTNSQVEEDKE & & 0.7316 \\
\hline A0A0G2K1W1 & Rab11fip5 & $\mathrm{S}$ & 825 & 1 & DVVGETSSPGSRDCL & 0.0169 & 0.287 \\
\hline A0A0G2K4A0 & Rab11fip5 & S & 521 & 1 & TAAPVEASPDRKQSR & & 0.7077 \\
\hline 055007 & Ralgapa1 & S & 733 & 2 & QAPMRQRSATTTGSP & 0.0563 & 0.4453 \\
\hline 055007 & Ralgapa1 & S & 739 & 1 & RSATTTGSPGTEKAR & 0.1958 & 0.4503 \\
\hline 055007 & Ralgapa1 & S & 739 & 2 & RSATTTGSPGTEKAR & 0.0812 & 0.351 \\
\hline P86410 & Ralgapb & $\mathrm{s}$ & 414 & 1 & SKVQHQASSTSPLSS & & 0.2708 \\
\hline F1LV89 & Rap1gap & $S$ & 526 & 1 & VSTSHSGSFTPNNPD & -0.6723 & 0.2861 \\
\hline F1LV89 & Rap1gap & $S$ & 585 & 1 & KKSGPFGSRRSSAIG & -0.6494 & -0.3096 \\
\hline A0A0G2JTA7 & Rasal2 & $\mathrm{s}$ & 1068 & 1 & QSSSSRESPVPKVRA & -0.063 & 1.3749 \\
\hline MOR3Z8 & Rbm15 & $S$ & 51 & 1 & TMKGKERSPVKPKRS & & 1.0201 \\
\hline D3ZRC3 & Rbm26 & S & 127 & 1 & FSRRLNHSPPQSSSR & 0.0643 & 0.7476 \\
\hline F1M1R4 & Rbm27 & $\mathrm{s}$ & 120 & 1 & VRKKKYPSPQKSRSE & & 0.5336 \\
\hline D3ZTA8 & Rbm33 & $S$ & 814 & 1 & DAKAKPLSPGAQPKE & & 0.5375 \\
\hline P84586 & Rbmxrtl & $\mathrm{T}$ & 163 & 1 & GPPPKRSTPSGPVRS & & 0.3862 \\
\hline P84586 & Rbmxrtl & $\mathrm{s}$ & 205 & 1 & SRRDVYLSPRDDGYS & & 0.8208 \\
\hline D3ZL11 & Rbsn & $\mathrm{T}$ & 211 & 2 & ASKDSLSTHTSPSQS & & 0.3403 \\
\hline D3ZL11 & Rbsn & $\mathrm{T}$ & 213 & 2 & KDSLSTHTSPSQSPN & -0.042 & 0.3155 \\
\hline
\end{tabular}




\begin{tabular}{|c|c|c|c|c|c|c|c|}
\hline D3ZJ01 & Relch & S & 455 & 1 & SFIPKEKSSDSFPTK & -1.098 & -0.4295 \\
\hline A0A0G2K285 & Reps2 & S & 349 & 1 & PSQAAESSPTKKDVP & -0.0531 & 0.3905 \\
\hline A0A0G2JT77 & Rims1 & S & 908 & 1 & RHIHGESSSKKLQRS & -0.0783 & -0.4577 \\
\hline A0A0G2JT77 & Rims1 & $S$ & 1484 & 1 & VEVIRARSLTQKPGS & & -0.3284 \\
\hline Q498D5 & Rmdn2 & $S$ & 121 & 1 & RVTVHQVSPQHRARK & 0.0596 & 0.6587 \\
\hline Q66H15 & Rmdn3 & $\mathrm{S}$ & 46 & 1 & QRHGRSQSLPNSLDY & 0.1008 & 0.3152 \\
\hline Q3ZAU6 & Rnf14 & S & 30 & 1 & DEFRKAESVQGGETR & & -0.302 \\
\hline A0A0G2K6H9 & Rnf19b & $S$ & 629 & 1 & PRVHGAPSPSAHKSL & & 0.4807 \\
\hline A0A0G2JZA1 & Robo2 & $\mathrm{s}$ & 1393 & 1 & STERKGSSLERQQAA & & 0.2691 \\
\hline Q62868 & Rock2 & S & 1374 & 1 & MKIQQNQSIRRPSRQ & 0.7522 & -0.2732 \\
\hline $\mathrm{P} 11250$ & Rpl34 & $\mathrm{s}$ & 12 & 1 & LTYRRRLSYNTASNK & & -0.3626 \\
\hline P19945 & Rplp0 & $S$ & 304 & 2 & KVEAKEESEESDEDM & -0.2591 & 0.4654 \\
\hline P19945 & Rplp0 & $S$ & 307 & 2 & AKEESEESDEDMGFG & -0.2591 & 0.4654 \\
\hline P02401 & Rplp2 & S & 102 & 1 & KDEKKEESEESDDDM & & -0.2784 \\
\hline P27952 & Rps2 & $\mathrm{s}$ & 281 & 1 & VKTHTRVSVQRTQAP & & -0.3445 \\
\hline F1M853 & Rrbp1 & S & 135 & 1 & PQEKLASSPKDKKKK & & 1.0266 \\
\hline F1M853 & Rrbp1 & $\mathrm{s}$ & 635 & 1 & QGKKVEGSPNQAKKV & 0.1716 & 0.3228 \\
\hline Q5M9F3 & Rrp1 & $\mathrm{T}$ & 24 & 1 & LAGNEQVTRDRALRK & & -0.3488 \\
\hline Q9JK11 & Rtn4 & $\mathrm{T}$ & 14 & 1 & SSLVSSSTDSPPRPP & 0.0238 & 0.5055 \\
\hline Q9JK11 & Rtn4 & $\mathrm{s}$ & 16 & 1 & LVSSSTDSPPRPPPA & 0.1238 & 0.5827 \\
\hline Q9JK11 & Rtn4 & $\mathrm{S}$ & 169 & 1 & AAPKRRGSGSVDETL & -0.0347 & 0.3408 \\
\hline Q9JK11 & Rtn4 & $\mathrm{T}$ & 175 & 2 & GSGSVDETLFALPAA & 0.0858 & 0.3396 \\
\hline BOLPN4 & Ryr2 & $\mathrm{S}$ & 2353 & 1 & DPSRDGPSPTSGSSK & 0.2401 & -0.2665 \\
\hline P48303 & S1pr1 & $\mathrm{s}$ & 354 & 1 & PGMEFSRSKSDNSSH & -0.3075 & 0.4039 \\
\hline P48303 & S1pr1 & S & 356 & 1 & MEFSRSKSDNSSHPQ & -0.3917 & 0.4463 \\
\hline 088453 & Safb & $\mathrm{s}$ & 366 & 1 & RAPTAAPSPEPRDSK & & 0.4847 \\
\hline Q6AXV4 & Samm50 & $\mathrm{s}$ & 253 & 1 & VRKESGHSLKSSLSH & & -0.4191 \\
\hline Q6AY30 & Sccpdh & $\mathrm{S}$ & 215 & 1 & GSLRKLRSVSNLKPV & & -0.3997 \\
\hline Q6AY30 & Sccpdh & $\mathrm{s}$ & 217 & 1 & LRKLRSVSNLKPVPV & & -0.3173 \\
\hline P04774 & Scn1a & S & 480 & 1 & PSAAGRLSDSSSEAS & 0.1632 & -0.3171 \\
\hline P04774 & Scn1a & S & 623 & 1 & ERRNSNLSQTSRSSR & & 0.6249 \\
\hline F1M9G9 & Scn2a & $\mathrm{s}$ & 599 & 2 & DFADDEHSTFEDNDS & -0.3621 & 0.3266 \\
\hline F1M9G9 & Scn2a & $\mathrm{T}$ & 1909 & 1 & TDVTPSTTSPPSYDS & 0.0261 & -0.3357 \\
\hline P54900 & Scn2b & $\mathrm{T}$ & 198 & 1 & LSTDDLKTEEEGKTD & 0.0321 & -0.4232 \\
\hline P54900 & Scn2b & $\mathrm{T}$ & 204 & 1 & KTEEEGKTDGEGNAE & 0.2036 & -0.2693 \\
\hline P08104 & Scn3a & $\mathrm{s}$ & 531 & 1 & PKSESEDSVKRRSFL & & 0.5584 \\
\hline B2RZD1 & Sec61b & $\mathrm{s}$ & 17 & 1 & NVGSSGRSPSKAVAA & -0.0446 & 0.7379 \\
\hline Q7TP42 & Sec62 & S & 542 & 1 & GDHGPEGSGGERHSD & 0.1722 & -0.467 \\
\hline D3ZDH8 & 5-Sep & $\mathrm{s}$ & 234 & 1 & YQFPECDSDEDEDFK & 0.1146 & -0.4956 \\
\hline A0A0U1RRT8 & 6-Sep & $S$ & 192 & 1 & KSDAISKSELAKFKI & & -0.2783 \\
\hline A0A0G2K7G7 & 8-Sep & $S$ & 194 & 1 & KADTISKSELHKFKI & & -0.2682 \\
\hline Q6AXS5 & Serbp1 & $\mathrm{s}$ & 327 & 1 & KGFVLHKSKSEEAHA & -0.0046 & 0.5384 \\
\hline Q6AXS5 & Serbp1 & $S$ & 329 & 1 & FVLHKSKSEEAHAED & -0.067 & 0.6978 \\
\hline D3ZN16 & Sergef & $T$ & 300 & 1 & DYGQLGRTLGSPEAQ & & -0.2739 \\
\hline
\end{tabular}




\begin{tabular}{|c|c|c|c|c|c|c|c|}
\hline D3ZMS1 & Sf3b2 & $S$ & 326 & 1 & PQRVRAASSESSGDR & & 0.7117 \\
\hline D3ZFJ3 & Sh3bp1 & S & 539 & 1 & SELPKPASPKVSRSP & & 0.264 \\
\hline D3ZAF9 & Sh3pxd2b & $S$ & 569 & 1 & SKLRPAKSQEKALLD & & 0.3694 \\
\hline Q9WV48 & Shank1 & $\mathrm{T}$ & 176 & 1 & KQLAKLHTKTGLKKF & & -0.483 \\
\hline Q9WV48 & Shank1 & $\mathrm{T}$ & 178 & 1 & LAKLHTKTGLKKFLE & & -0.3957 \\
\hline Q9WV48 & Shank1 & $S$ & 413 & 1 & DVVPFQESPKYAARR & 0.2009 & -0.4255 \\
\hline Q9WV48 & Shank1 & $S$ & 540 & 1 & GSGGPGGSLGSRGRR & -0.1501 & 0.6929 \\
\hline Q9WV48 & Shank1 & S & 1071 & 1 & SPTSGAPSPSHHSSS & -0.1488 & 0.6168 \\
\hline Q9WV48 & Shank1 & $S$ & 1138 & 1 & GSIPSASSPTSPALP & & 0.7033 \\
\hline Q9WV48 & Shank1 & $S$ & 1174 & 1 & IKAPSTSSSGRSSQG & 0.4313 & 1.0409 \\
\hline Q9WV48 & Shank1 & $S$ & 1386 & 1 & QPPPRPPSPRYDAPP & -0.2316 & 1.15 \\
\hline Q9WV48 & Shank1 & $S$ & 1442 & 1 & QEKALTASPPAARRS & 0.0029 & 0.6738 \\
\hline Q9WV48 & Shank1 & $S$ & 1517 & 1 & PAGTRGSSTEDGPGV & 0.2059 & 0.4387 \\
\hline Q9QX74 & Shank2 & $S$ & 67 & 1 & ATSHRSLSPQLLQQT & & 0.3504 \\
\hline Q9QX74 & Shank2 & $S$ & 648 & 1 & KQSNVEDSPEKTCSI & & 0.8127 \\
\hline Q9QX74 & Shank2 & $S$ & 666 & 1 & TIIVKEPSTSSSGKS & & 0.4654 \\
\hline Q9QX74 & Shank2 & $\mathrm{S}$ & 1334 & 2 & SGPRRAPSPVVSPTE & -0.6184 & 0.4954 \\
\hline Q9QX74 & Shank2 & $S$ & 1338 & 1 & RAPSPVVSPTELSKE & 0.142 & 1.2085 \\
\hline Q9QX74 & Shank2 & $S$ & 1338 & 2 & RAPSPVVSPTELSKE & -0.5811 & 0.4236 \\
\hline Q9QX74 & Shank2 & $T$ & 1340 & 2 & PSPVVSPTELSKEIL & -0.3391 & 0.2917 \\
\hline Q9JLU4 & Shank3 & $\mathrm{s}$ & 986 & 1 & GSFAREPSPTHRGPR & & 0.9703 \\
\hline Q9JLU4 & Shank3 & $\mathrm{S}$ & 1135 & 1 & RSPTPVHSPDADRPG & 0.1547 & 0.7152 \\
\hline Q9JLU4 & Shank3 & $S$ & 1189 & 1 & PTREERKSPEDKKSM & 0.6594 & 1.3883 \\
\hline Q9JLU4 & Shank3 & S & 1511 & 1 & QLNKDTRSLGEEPVG & 0.4582 & 0.5669 \\
\hline D4A4MO & Shisa6 & $T$ & 284 & 1 & ILSSATQTPTHEKPR & & 0.3713 \\
\hline D3ZPJ0 & Shisa7 & $S$ & 287 & 1 & NLHYNVNSPKHRAAT & & 0.3748 \\
\hline A0MZ67 & Shtn1 & S & 381 & 1 & MSMIRKRSHPSGGST & -0.0872 & 0.5201 \\
\hline A0A0G2KAW2 & Sipa111 & $S$ & 1184 & 1 & VSRRSPASIDRQNTQ & & 0.4437 \\
\hline A0A0G2KAW2 & Sipa111 & $S$ & 1249 & 1 & QDPVVHLSPNKQGHS & 0.1598 & 0.4796 \\
\hline A0A0G2KAW2 & Sipa111 & S & 1708 & 2 & VQMKSYSSKDSSPTL & & 0.497 \\
\hline A0A0G2KAW2 & Sipa111 & $\mathrm{S}$ & 1712 & 1 & SYSSKDSSPTLASKV & -0.0039 & 0.6222 \\
\hline A0A0G2KAW2 & Sipa111 & $S$ & 1712 & 2 & SYSSKDSSPTLASKV & & 0.4987 \\
\hline O35412 & Sipa111 & S & 1752 & 1 & YSSSKDSSPTLASKV & -0.0039 & 0.638 \\
\hline F1LYG2 & Sipa113 & $S$ & 94 & 1 & LREQSNPSPSQDTDG & 0.115 & 1.4359 \\
\hline F1LYG2 & Sipa113 & $S$ & 1248 & 1 & QDAAGKDSPNRHSKG & 0.2684 & 1.3602 \\
\hline Q63633 & Slc12a5 & $\mathrm{S}$ & 49 & 1 & DGNPKESSPFINSTD & 0.1081 & 0.3706 \\
\hline Q63633 & Slc12a5 & $\mathrm{S}$ & 963 & 1 & ITDESRGSIRRKNPA & 0.0109 & 0.3932 \\
\hline Q63633 & Slc12a5 & $S$ & 1045 & 1 & AQKNKGPSPVSSEGI & 0.0114 & 0.4533 \\
\hline G3V6N7 & Slc12a6 & $\mathrm{s}$ & 32 & 1 & IPGLSDTSPDLSSRS & & 0.6532 \\
\hline P53987 & Slc16a1 & $S$ & 210 & 1 & GKVEKLKSKESLQEA & 0.1076 & -0.2984 \\
\hline P53987 & Slc16a1 & $S$ & 230 & 1 & NTDLIGGSPKGEKLS & 0.0376 & -0.4259 \\
\hline Q63344 & Slc16a7 & S & 219 & 1 & KSKSKVGSRQDSSTK & & -0.3855 \\
\hline G3V6R0 & Slc1a2 & $S$ & 240 & 1 & IEMTKTQSIYDDTKN & & 0.3606 \\
\hline Q63488 & SIc20a2 & $s$ & 256 & 1 & ESALSRASDESLRKV & 0.334 & -0.3235 \\
\hline
\end{tabular}




\begin{tabular}{|c|c|c|c|c|c|c|c|}
\hline O70594 & SIc22a5 & $S$ & 548 & 1 & TQKDGGESPTVLKST & & -0.3112 \\
\hline Q9WTW8 & Slc23a2 & S & 638 & 1 & GKSENRRSSDKDSQA & & -0.2762 \\
\hline F1LX07 & Slc25a12 & S & 640 & 1 & LYLPKFKSPGVAAAQ & & -0.2891 \\
\hline Q05962 & SIc25a4 & $S$ & 22 & 1 & GGIAAAVSKTAVAPI & & -0.4643 \\
\hline Q05962 & SIc25a4 & $S$ & 149 & 1 & AADVGKGSSQREFNG & & -0.4036 \\
\hline D3Z9I5 & Slc26a6 & $\mathrm{s}$ & 751 & 1 & HRKSGPKSPVLATKL & & -0.2687 \\
\hline A0A0G2JYF0 & Slc4a10 & $S$ & 1116 & 1 & SRFPSKSSPS & & 0.8109 \\
\hline P23347 & Slc4a2 & $T$ & 170 & 2 & TDRKAERTSPSPPTQ & -0.0587 & 0.3886 \\
\hline P23347 & Slc4a2 & $\mathrm{s}$ & 171 & 2 & DRKAERTSPSPPTQT & -0.0458 & 0.2983 \\
\hline P23347 & Slc4a2 & $\mathrm{s}$ & 173 & 2 & KAERTSPSPPTQTPH & 0.0396 & 0.2938 \\
\hline P23348 & Slc4a3 & $\mathrm{S}$ & 198 & 1 & VGLPSDQSPQRSGSS & & 0.4417 \\
\hline AOAOH2UHB7 & Slc4a4 & $S$ & 70 & 2 & ERISENYSDKSDVEN & -0.5709 & 0.3678 \\
\hline AOAOH2UHB7 & Slc4a4 & S & 73 & 1 & SENYSDKSDVENADE & -0.377 & 0.2654 \\
\hline AOAOH2UHB7 & Slc4a4 & $S$ & 73 & 2 & SENYSDKSDVENADE & -0.5699 & 0.3697 \\
\hline $\mathrm{AOAOH} 2 \cup \mathrm{HB} 7$ & Slc4a4 & $T$ & 259 & 2 & AMTHRNLTSSSLNDI & -0.5834 & -0.2714 \\
\hline AOAOH2UHB7 & Slc4a4 & S & 1031 & 1 & KKKKKKGSLDSDNDD & 0.1417 & -0.3837 \\
\hline Q08469 & Slc6a15 & $S$ & 701 & 1 & IYRKQSGSPTLDTAP & & 0.4144 \\
\hline Q9WVR6 & Slc7a8 & $S$ & 23 & 1 & PDRGSDTSPEAEASS & 0.1919 & -0.3639 \\
\hline Q9WVR6 & Slc7a8 & S & 30 & 1 & SPEAEASSGGGGVAL & & -0.276 \\
\hline Q01728 & Slc8a1 & $\mathrm{S}$ & 452 & 1 & DGTANAGSDYEFTEG & 0.1091 & -0.3494 \\
\hline P48768 & Slc8a2 & S & 442 & 1 & DGSAKAGSDYEYSEG & 0.1502 & -0.3575 \\
\hline P26431 & Slc9a1 & S & 776 & 1 & IRSKEPSSPGTDDVF & -0.7918 & 0.3181 \\
\hline P26431 & Slc9a1 & $S$ & 776 & 2 & IRSKEPSSPGTDDVF & -0.9138 & -0.0871 \\
\hline D4A4W6 & Slirp & $T$ & 104 & 1 & KVLHGAQTSDEEKDF & 0.18 & -0.3184 \\
\hline G3V7I8 & Slk & $T$ & 183 & 1 & VSAKNTRTIQRRDSF & 0.0018 & -0.2706 \\
\hline A0A0G2K9D6 & Smarcc1 & $S$ & 327 & 2 & NARKRKHSPSPPPPT & & 0.3434 \\
\hline A0A0G2K9D6 & Smarcc1 & $\mathrm{s}$ & 329 & 2 & RKRKHSPSPPPPTAT & & 0.3434 \\
\hline D4ACJ3 & Smg7 & $\mathrm{S}$ & 759 & 1 & VIPALGKSPPHHSGF & & 0.7575 \\
\hline P60881 & Snap25 & $T$ & 200 & 1 & DEANQRATKMLGSG & -0.1795 & -0.2988 \\
\hline A0A0G2K0B6 & Snap91 & S & 622 & 2 & VDAFAAPSPASTASP & -1.5075 & -0.0341 \\
\hline A0A0G2K0B6 & Snap91 & S & 622 & 3 & VDAFAAPSPASTASP & -1.1858 & -0.2638 \\
\hline B5DF41 & Snph & $\mathrm{T}$ & 197 & 2 & GVAKEEGTGESAGGS & 0.0863 & 0.4155 \\
\hline B5DF41 & Snph & $\mathrm{s}$ & 200 & 2 & KEEGTGESAGGSPAR & & 0.4176 \\
\hline B5DF41 & Snph & $S$ & 204 & 1 & TGESAGGSPARSLTR & 0.2067 & 0.6629 \\
\hline B5DF41 & Snph & $S$ & 204 & 2 & TGESAGGSPARSLTR & 0.105 & 0.4299 \\
\hline D4A0A1 & Soga3 & $\mathrm{s}$ & 759 & 1 & SDAGKKESDDDSRPP & 0.2769 & 0.7951 \\
\hline D4A0A1 & Soga3 & $\mathrm{s}$ & 763 & 1 & KKESDDDSRPPHRKR & & 0.6468 \\
\hline F1M820 & Sorbs 1 & S & 55 & 1 & YRETPSSSPVSPQES & -0.2658 & \\
\hline $\mathrm{F} 1 \mathrm{M} 820$ & Sorbs1 & $\mathrm{S}$ & 55 & 2 & YRETPSSSPVSPQES & -0.1553 & 0.9709 \\
\hline F1M820 & Sorbs 1 & $S$ & 55 & 3 & YRETPSSSPVSPQES & -0.3288 & 0.2984 \\
\hline F1M820 & Sorbs 1 & $S$ & 58 & 2 & TPSSSPVSPQESPKH & -0.2139 & 0.457 \\
\hline F1M820 & Sorbs1 & $\mathrm{s}$ & 58 & 3 & TPSSSPVSPQESPKH & -0.3134 & 0.3785 \\
\hline F1M820 & Sorbs 1 & $S$ & 62 & 1 & SPVSPQESPKHENKS & 0.045 & 0.7486 \\
\hline $\mathrm{F} 1 \mathrm{M} 820$ & Sorbs1 & s & 62 & 2 & SPVSPQESPKHENKS & -0.1607 & 0.8301 \\
\hline
\end{tabular}




\begin{tabular}{|c|c|c|c|c|c|c|c|}
\hline F1M820 & Sorbs1 & S & 62 & 3 & SPVSPQESPKHENKS & -0.3228 & 0.3039 \\
\hline F1M820 & Sorbs1 & S & 470 & 1 & LSGLKRPSSSASTKV & 0.185 & 0.4853 \\
\hline F1M820 & Sorbs1 & S & 794 & 1 & SREGSGGSVHGDFPK & 0.1419 & 0.4102 \\
\hline F1M840 & Sorbs1 & $S$ & 449 & 1 & LSGLKRPSSSASTKD & 0.185 & 0.4853 \\
\hline F1LPM3 & Sorbs2 & $S$ & 254 & 1 & RPLSKSHSDNGTDAF & -0.0452 & 1.1714 \\
\hline F1LPM3 & Sorbs2 & S & 397 & 1 & FISSSPSSPSRAQGG & -0.0996 & 0.3464 \\
\hline 035413 & Sorbs2 & S & 254 & 1 & RPLSKSHSDNGTDAF & -0.0452 & 1.1714 \\
\hline 035413 & Sorbs2 & $S$ & 329 & 1 & RPVSVYQSSIDRSLE & 0.1688 & 0.6405 \\
\hline 035413 & Sorbs2 & S & 330 & 1 & PVSVYQSSIDRSLER & 0.2873 & 0.3921 \\
\hline 035413 & Sorbs2 & $\mathrm{S}$ & 354 & 1 & DFRKRRKSEPAVGPP & 0.0763 & 0.8467 \\
\hline 035413 & Sorbs2 & S & 397 & 1 & FISSSPSSPSRAQGG & -0.0996 & 0.3464 \\
\hline 035413 & Sorbs2 & S & 645 & 2 & LCPKRRFSIESLLEE & -0.0949 & 0.8274 \\
\hline 035413 & Sorbs2 & S & 648 & 2 & KRRFSIESLLEEETQ & -0.0643 & 0.8153 \\
\hline 035413 & Sorbs2 & $T$ & 654 & 2 & ESLLEEETQVRHPSQ & -0.0847 & 0.8444 \\
\hline P27867 & Sord & S & 169 & 1 & IYACRRGSVSLGNKV & & -0.2644 \\
\hline D4A3T0 & Sos1 & $\mathrm{S}$ & 1152 & 1 & PESAPAESSPSKIMS & 0.2735 & 0.5802 \\
\hline D4A3T0 & Sos1 & S & 1153 & 1 & ESAPAESSPSKIMSK & 0.1023 & 0.9093 \\
\hline A0A0G2K5D7 & Specc1 & S & 450 & 1 & PFKSSKGSPTGSSPN & 0.1357 & 0.3739 \\
\hline A0A0G2K5D7 & Specc1 & $\mathrm{S}$ & 450 & 2 & PFKSSKGSPTGSSPN & -0.3398 & 0.2655 \\
\hline A0A0G2K5D7 & Specc1 & $T$ & 452 & 1 & KSSKGSPTGSSPNNA & -0.1293 & 0.3848 \\
\hline A0A0G2K5D7 & Specc1 & $T$ & 452 & 2 & KSSKGSPTGSSPNNA & -0.3292 & 0.2954 \\
\hline A0A0G2K5D7 & Specc1 & S & 455 & 1 & KGSPTGSSPNNASEL & & 0.4626 \\
\hline A0A0G2K5D7 & Specc1 & $S$ & 455 & 2 & KGSPTGSSPNNASEL & -0.3125 & 0.3078 \\
\hline Q63638 & Speg & S & 439 & 1 & VPLRKARSLEQPKSE & 0.2329 & 0.3141 \\
\hline Q63638 & Speg & $S$ & 2114 & 1 & PRMARAASSEAAPHH & & 0.5188 \\
\hline Q63638 & Speg & $\mathrm{S}$ & 2115 & 1 & RMARAASSEAAPHHQ & 0.3067 & 0.5491 \\
\hline A0A0G2K1Y8 & Sptan1 & S & 866 & 1 & EDVKAKLSELNQKWE & & -0.3129 \\
\hline A0A140UHX6 & Sptb & $S$ & 2300 & 1 & SIRVKAQSLPLPSLA & & 0.4083 \\
\hline A0A0G2K8W9 & Sptbn1 & $\mathrm{T}$ & 2153 & 1 & NGAAEQRTSSKESSP & & -0.6163 \\
\hline A0A0G2K8W9 & Sptbn1 & S & 2154 & 1 & GAAEQRTSSKESSPV & & -0.4493 \\
\hline A0A0G2K8W9 & Sptbn1 & S & 2163 & 3 & KESSPVPSPTSDRKA & -0.2911 & 0.2973 \\
\hline A0A0G2K8W9 & Sptbn1 & $\mathrm{S}$ & 2335 & 1 & VTITSESSPGKREKD & 0.0568 & 1.1886 \\
\hline Q9QWN8 & Sptbn2 & S & 2169 & 1 & NVPEGPGSGTGDESS & -0.0387 & 0.4445 \\
\hline Q9QWN8 & Sptbn2 & S & 2254 & 1 & YCVLRRGSLGFYKDA & & 0.556 \\
\hline A0A0G2K677 & Sptbn4 & S & 2268 & 1 & RRPERQESADHEGPH & 0.306 & 0.2645 \\
\hline A0A0G2K677 & Sptbn4 & $\mathrm{S}$ & 2301 & 1 & RRIERQESSEQETPT & 0.2373 & 0.6105 \\
\hline A0A0G2K677 & Sptbn4 & S & 2302 & 1 & RIERQESSEQETPTR & 0.3234 & 0.7665 \\
\hline Q9QXY2 & Srcin1 & S & 333 & 3 & TRRLNNLSPASHLAS & -0.1483 & 0.3618 \\
\hline Q9QXY2 & Srcin1 & S & 336 & 3 & LNNLSPASHLASSSP & & 0.2941 \\
\hline Q9QXY2 & Srcin 1 & $S$ & 341 & 3 & PASHLASSSPPPGLP & -0.172 & 0.3109 \\
\hline Q9QXY2 & Srcin1 & S & 342 & 3 & ASHLASSSPPPGLPS & -0.172 & 0.3249 \\
\hline Q9QXY2 & Srcin1 & S & 900 & 2 & DFEIPPPSPPLNLHE & -0.5007 & 0.2825 \\
\hline Q9QXY2 & Srcin1 & $S$ & 1152 & 1 & AAQGPAGSPDKGKHG & 0.0423 & 1.315 \\
\hline Q66H19 & Srfbp1 & S & 215 & 1 & KAVATPHSPGKPSEK & & 0.5274 \\
\hline
\end{tabular}




\begin{tabular}{|c|c|c|c|c|c|c|c|}
\hline F1M5M9 & Srgap3 & S & 908 & 1 & LSRGRIESPEKRRMA & 0.128 & 0.4347 \\
\hline BOBNJ1 & Sri & S & 172 & 1 & KLRALTDSFRRRDSG & & -0.4264 \\
\hline A0A0G2K2M9 & Srrm2 & $\mathrm{S}$ & 332 & 1 & PSTKQSSSPYEDKDK & & 1.1428 \\
\hline A0A0G2K2M9 & Srrm2 & S & 350 & 1 & SAVRPSPSPERSSTG & & 0.7735 \\
\hline A0A0G2K2M9 & Srrm2 & $S$ & 881 & 2 & SSTPPRQSPSRSSSP & & 1.1106 \\
\hline A0A0G2K2M9 & Srrm2 & $\mathrm{S}$ & 883 & 2 & TPPRQSPSRSSSPQP & & 1.1392 \\
\hline A0A0G2K2M9 & Srrm2 & S & 1068 & 2 & PKGGLSRSSSPVTEL & & 1.2588 \\
\hline A0A0G2K2M9 & Srrm2 & S & 1070 & 2 & GGLSRSSSPVTELTA & & 1.3986 \\
\hline A0A0G2K2M9 & Srrm2 & $T$ & 1076 & 2 & SSPVTELTARSPVKQ & & 1.3691 \\
\hline A0A0G2K2M9 & Srrm2 & S & 1096 & 1 & STDPKLKSGMSPEQN & & 1.4426 \\
\hline A0A0G2K2M9 & Srrm2 & S & 1099 & 1 & PKLKSGMSPEQNKTK & 0.0882 & 1.3542 \\
\hline A0A0G2K2M9 & Srrm2 & S & 1153 & 1 & CVLRDKFSPTQDRPE & 0.1319 & 0.8797 \\
\hline A0A0G2K2M9 & Srrm2 & S & 1181 & 1 & KERSGAGSPPGTRDQ & 0.1522 & 1.036 \\
\hline A0A0G2K2M9 & Srrm2 & S & 1461 & 2 & ARSHSPSSPERNNKS & & 1.3326 \\
\hline A0A0G2K2M9 & Srrm2 & $\mathrm{S}$ & 1524 & 1 & ESDSSPDSKPKARTP & & 1.3037 \\
\hline A0A0G2K2M9 & Srrm2 & S & 1580 & 1 & AQSGTDSSPEHKIPA & & 0.6539 \\
\hline A0A0G2K2M9 & Srrm2 & S & 1781 & 2 & RGGSGYHSRSPTRQE & -0.1464 & 0.4232 \\
\hline A0A0G2K2M9 & Srrm2 & $\mathrm{S}$ & 1783 & 2 & GSGYHSRSPTRQESS & -0.1411 & 0.391 \\
\hline A0A0G2K2M9 & Srrm2 & $T$ & 1785 & 2 & GYHSRSPTRQESSRT & & 0.4232 \\
\hline A0A0G2K2M9 & Srrm2 & S & 2023 & 2 & RAARGKRSLTRSPPA & -0.0746 & 0.5968 \\
\hline A0A0G2K2M9 & Srrm2 & $T$ & 2025 & 2 & ARGKRSLTRSPPAIR & -0.0908 & 0.5629 \\
\hline A0A0G2K2M9 & Srrm2 & $S$ & 2027 & 2 & GKRSLTRSPPAIRRR & -0.0448 & 0.5852 \\
\hline A0A0G2K2M9 & Srrm2 & $\mathrm{T}$ & 2365 & 1 & LDRARSRTPPSAPSQ & -0.0056 & 0.7931 \\
\hline A0A0G2K2M9 & Srrm2 & S & 2538 & 1 & VALKRVPSPTPVPKE & & 0.3945 \\
\hline A0A0G2K2M9 & Srrm2 & $S$ & 2659 & 1 & PVERRQPSPQPSPRD & 0.0341 & 0.7066 \\
\hline A0A0G2K2M9 & Srrm2 & S & 2682 & 2 & WRGQRGDSHSPGHKR & 0.0546 & 1.2625 \\
\hline A0A0G2K2M9 & Srrm2 & S & 2684 & 1 & GQRGDSHSPGHKRRK & 0.1517 & 1.7834 \\
\hline A0A0G2K2M9 & Srrm2 & $S$ & 2684 & 2 & GQRGDSHSPGHKRRK & 0.0546 & 1.2625 \\
\hline O35814 & Stip1 & S & 481 & 1 & AQYNRHDSPEDVKRR & 0.3348 & -0.2802 \\
\hline P32851 & Stx1a & S & 14 & 1 & ELRTAKDSDDDDDVT & 0.2636 & -0.457 \\
\hline P32851 & Stx1a & $\mathrm{T}$ & 21 & 1 & SDDDDDVTVTVDRDR & 0.1289 & -0.4686 \\
\hline P32851 & Stx1a & $\mathrm{T}$ & 23 & 1 & DDDDVTVTVDRDRFM & 0.1136 & -0.4612 \\
\hline P32851 & Stx1a & S & 64 & 1 & KHSAILASPNPDEKT & 0.1898 & -0.2923 \\
\hline P61765 & Stxbp1 & S & 313 & 1 & SLKDFSSSKRMNTGE & & -0.2679 \\
\hline P61765 & Stxbp1 & S & 506 & 1 & YPYISTRSSASFSTT & -0.1092 & -0.2855 \\
\hline Q9WU70 & Stxbp5 & S & 783 & 1 & FSRSRSSSVTSIDKE & 0.1375 & -0.3779 \\
\hline D3ZU84 & Stxbp5I & S & 724 & 1 & YNRSRSSSISSIDKD & & -0.3086 \\
\hline D3ZU84 & Stxbp5I & S & 726 & 1 & RSRSSSISSIDKDSK & & -0.2952 \\
\hline D3ZU84 & Stxbp5I & S & 727 & 1 & SRSSSISSIDKDSKE & 0.1187 & -0.29 \\
\hline F1LM47 & Sucla2 & S & 281 & 1 & DAKINFDSNSAYRQK & & -0.3344 \\
\hline F1LM47 & Sucla2 & S & 283 & 1 & KINFDSNSAYRQKKI & & -0.3302 \\
\hline Q68FU8 & Sugp1 & S & 253 & 1 & QAATQKVSPPEDEEA & & -0.366 \\
\hline Q63564 & Sv2b & S & 425 & 1 & FQDEEYKSKMKVFFG & & -0.3946 \\
\hline Q9Z217 & Svop & $\mathrm{s}$ & 520 & 1 & KGRALQESSHREWGQ & 0.1335 & -0.3475 \\
\hline
\end{tabular}




\begin{tabular}{|c|c|c|c|c|c|c|c|}
\hline F1LUC0 & Sybu & S & 46 & 1 & QQQQNKVSPASESPF & & 0.5011 \\
\hline F1LUC0 & Sybu & S & 199 & 1 & PSHKSGSSPPSPREK & & 0.913 \\
\hline F1LUC0 & Sybu & $S$ & 199 & 2 & PSHKSGSSPPSPREK & 0.078 & 0.777 \\
\hline F1LUC0 & Sybu & $S$ & 202 & 2 & KSGSSPPSPREKDLV & 0.0852 & 0.7439 \\
\hline F1LUC0 & Sybu & $\mathrm{s}$ & 220 & 1 & CRNPLSPSNIHPSYA & & 0.3372 \\
\hline P09951 & Syn1 & S & 492 & 1 & PQGQQHLSGLGPPAG & & 0.4578 \\
\hline P09951 & Syn1 & $S$ & 551 & 1 & ARPPASPSPQRQAGP & -0.5697 & 0.4381 \\
\hline P09951 & Syn1 & S & 551 & 2 & ARPPASPSPQRQAGP & -1.5076 & 0.1425 \\
\hline P09951 & Syn1 & S & 700 & 1 & SLRKSFASLFSD & -0.3996 & -0.2879 \\
\hline 070441 & Syn3 & S & 575 & 1 & NLRKSFASLFSD & -0.3996 & -0.2879 \\
\hline Q9QUH6 & Syngap1 & S & 109 & 1 & LGRSRRKSVPGGKQY & & 0.3815 \\
\hline Q9QUH6 & Syngap1 & S & 780 & 1 & MARGLNSSMDMARLP & 0.1113 & 0.6477 \\
\hline Q9QUH6 & Syngap1 & S & 1005 & 1 & ASILHSHSYSDEFGP & & 0.7916 \\
\hline Q9QUH6 & Syngap1 & S & 1023 & 1 & DFTRRQLSLQDNLQH & & 0.3034 \\
\hline Q9QUH6 & Syngap1 & S & 1108 & 2 & PARPRQQSLSKEGSI & & 1.3125 \\
\hline Q9QUH6 & Syngap1 & $S$ & 1114 & 1 & QSLSKEGSIGGSGGS & -0.0058 & 1.1895 \\
\hline Q9QUH6 & Syngap1 & $\mathrm{S}$ & 1114 & 2 & QSLSKEGSIGGSGGS & & 1.148 \\
\hline Q9QUH6 & Syngap1 & S & 1118 & 1 & KEGSIGGSGGSGGGG & 0.0408 & 1.5074 \\
\hline Q9QUH6 & Syngap1 & $S$ & 1118 & 2 & KEGSIGGSGGSGGGG & -0.1898 & 1.3935 \\
\hline Q9QUH6 & Syngap1 & S & 1121 & 1 & SIGGSGGSGGGGGGG & -0.0576 & 2.1683 \\
\hline Q9QUH6 & Syngap1 & S & 1121 & 2 & SIGGSGGSGGGGGGG & -0.1898 & 1.3935 \\
\hline Q9QUH6 & Syngap1 & $S$ & 1204 & 1 & EYEEEIHSLKERLHM & 0.1044 & 1.0705 \\
\hline D4ABN3 & Synj1 & S & 1215 & 1 & PQSQARVSAGRLTPE & & 0.2673 \\
\hline Q9Z327 & Synpo & S & 258 & 1 & RHLEKVASEEEEVPL & 0.3631 & 1.3814 \\
\hline Q9Z327 & Synpo & $S$ & 537 & 1 & RRLVGQRSPVVERRP & -0.0995 & 0.6348 \\
\hline Q9Z327 & Synpo & S & 572 & 1 & SQVRSPPSYSTLYPS & 0.0895 & 1.5041 \\
\hline Q9Z327 & Synpo & S & 767 & 1 & GFRVASLSPARTPPA & -0.0426 & 0.3492 \\
\hline Q9Z327 & Synpo & $S$ & 835 & 1 & TVSPRAASPAKPSSL & -0.1744 & 2.0667 \\
\hline Q9JKC9 & Synrg & S & 1090 & 1 & GSHKRSLSLGDKEIS & & 0.4131 \\
\hline P21707 & Syt1 & $\mathrm{T}$ & 201 & 1 & ETKVHRKTLNPVFNE & & -0.3867 \\
\hline G3V917 & Tanc1 & S & 1658 & 1 & SSSGSSGSPSSSVKM & -0.0813 & 0.6321 \\
\hline A0A0G2K9J0 & Tanc2 & S & 1562 & 1 & RSNSSVGSPTRQGYQ & -0.0899 & 0.3918 \\
\hline A0A0G2K9J0 & Tanc2 & S & 1646 & 1 & IYRSQSGSPVRYQQE & 0.0565 & 0.8037 \\
\hline A0A0G2K9J0 & Tanc2 & $S$ & 1665 & 1 & QLPGRPKSPLSKMAQ & & 0.2908 \\
\hline A0A0G2K9J0 & Tanc2 & S & 2063 & 1 & DSRQGQTSPIKPKRP & -0.3338 & 1.0515 \\
\hline 16L9G6 & Tardbp & S & 183 & 1 & KLPNSKQSPDEPLRS & & -0.385 \\
\hline D3ZG21 & Tcf20 & S & 592 & 1 & ASEKAGSSPTQGAQN & & 0.2705 \\
\hline D4A206 & Tcof1 & S & 1254 & 1 & AAGTSAGSPEKASRT & & 0.4492 \\
\hline F1LZ38 & Tenm4 & S & 54 & 1 & YTSSSADSEEGKGPQ & & 0.3268 \\
\hline G3V9L1 & Tex2 & S & 732 & 1 & LPAHSRHSSPSGHLS & -0.2524 & 0.6628 \\
\hline G3V9L1 & Tex2 & S & 733 & 1 & PAHSRHSSPSGHLSH & -0.107 & 0.9689 \\
\hline G3V9L1 & Tex2 & S & 733 & 2 & PAHSRHSSPSGHLSH & -0.221 & 0.4828 \\
\hline G3V9L1 & Tex2 & S & 741 & 2 & PSGHLSHSRSSSKGS & -0.3184 & 0.4887 \\
\hline G3V9L1 & Tex2 & S & 799 & 1 & PVQSAESSPTAGKKL & 0.196 & -0.3013 \\
\hline
\end{tabular}




\begin{tabular}{|c|c|c|c|c|c|c|c|}
\hline P24155 & Thop1 & $S$ & 634 & 1 & FKQEGVLSPKVGMDY & & -0.369 \\
\hline Q5M7V8 & Thrap3 & S & 248 & 2 & DLSPRERSPALKSPL & & 0.59 \\
\hline A0A0G2K2P5 & Tjp1 & S & 329 & 1 & PSDHSTQSPQQPSNG & -0.0417 & 0.4571 \\
\hline D3ZE26 & Tmcc2 & S & 119 & 1 & QGLSDQDSPDEKERS & 0.0295 & 0.4392 \\
\hline D3ZE26 & Tmcc2 & $S$ & 126 & 1 & SPDEKERSPEMHRVS & & 0.6401 \\
\hline F1LYB3 & Tmem132c & $\mathrm{S}$ & 1012 & 1 & PRQEPANSPTSKMKK & & 0.3471 \\
\hline A9CMA6 & Tmem163 & S & 7 & 1 & MERAPGSERRSPPG & 0.7944 & 0.3026 \\
\hline A9CMA6 & Tmem163 & $S$ & 56 & 1 & RQPRISESGQFSDGF & 0.0511 & -0.3009 \\
\hline Q68FV0 & Tmem178a & $\mathrm{s}$ & 291 & 1 & TKIAHLKSGRDSTV & & -0.4927 \\
\hline D3Z9V8 & Tmem25 & S & 275 & 1 & KGPSRRPSLISSDSN & & -0.2773 \\
\hline D3ZYW8 & Tmem266 & $\mathrm{T}$ & 473 & 1 & TPVGSVQTSPELEHR & 0.0815 & 0.3044 \\
\hline Q5XIP9 & Tmem43 & $S$ & 10 & 1 & ANYSSTGSRKEHVKV & 0.1752 & -0.2957 \\
\hline F1LUG5 & Tmem94 & $S$ & 517 & 1 & HGRSKHPSGSNVSFS & & 0.5031 \\
\hline F1LUG5 & Tmem94 & S & 522 & 1 & HPSGSNVSFSRDTEG & & 0.312 \\
\hline Q62733 & Tmpo & $\mathrm{s}$ & 155 & 1 & LREQGAESRSSTPLP & & 0.6678 \\
\hline A0A0G2K490 & Tnik & S & 640 & 1 & VEMPRQNSDPTSENP & -0.0044 & 0.3816 \\
\hline A0A0G2K490 & Tnik & $\mathrm{s}$ & 688 & 1 & PALARKNSPGNGSAL & -0.0972 & 1.295 \\
\hline A0A0G2K490 & Tnik & $\mathrm{s}$ & 764 & 2 & RTRVRANSKSEGSPV & 0.0626 & 0.4119 \\
\hline A0A0G2K490 & Tnik & S & 766 & 2 & RVRANSKSEGSPVLP & & 0.4305 \\
\hline A0A0G2K490 & Tnik & $\mathrm{s}$ & 769 & 1 & ANSKSEGSPVLPHEP & 0.0054 & 0.3869 \\
\hline A0A0G2K490 & Tnik & $\mathrm{S}$ & 769 & 2 & ANSKSEGSPVLPHEP & 0.0599 & 0.3958 \\
\hline A0A0G2K490 & Tnik & S & 777 & 2 & PVLPHEPSKVKPEES & 0.056 & 0.3895 \\
\hline D3ZF26 & Tnks1bp1 & $\mathrm{S}$ & 1125 & 1 & RVSGAGLSPSRKSGR & & 0.382 \\
\hline D3ZF26 & Tnks1bp1 & $\mathrm{s}$ & 1422 & 1 & PSRCLARSPPSGSQS & -0.156 & 0.4485 \\
\hline A0A0G2K6R0 & Tnrc6b & S & 609 & 1 & GSEVGGQSTGSNHKA & -0.0764 & 0.5212 \\
\hline Q75Q39 & Tomm70 & $\mathrm{T}$ & 88 & 1 & KRNSERKTPEGRASP & 0.1018 & -0.396 \\
\hline B3DMA0 & Tp53i11 & $\mathrm{s}$ & 14 & 1 & PPLMKKHSQTDLVSR & 0.3236 & 0.3123 \\
\hline A0A0G2K2D6 & Tppp & $\mathrm{S}$ & 191 & 1 & DTSKFTGSHKERFDQ & 1.0396 & -0.4102 \\
\hline Q5PPN5 & Tppp3 & S & 134 & 1 & DTSKYTGSHKERFDE & & -0.4649 \\
\hline A0A0G2K2H9 & Trerf1 & S & 497 & 1 & DGRAQPGSPESSGQT & & 0.5101 \\
\hline F1M0Z1 & Trio & S & 2433 & 1 & LGSTSGTSQDGNTKD & 0.8539 & 0.36 \\
\hline F1LP64 & Trip12 & S & 1049 & 1 & GPRRPKYSPPRDDDK & 0.0598 & 0.3367 \\
\hline F1LP64 & Trip12 & $\mathrm{s}$ & 1069 & 1 & KSPTTTQSPKSSFLA & 0.2596 & 0.9591 \\
\hline Q9WUD2 & Trpv2 & S & 82 & 1 & FDRDRLFSVVSRGVP & & -0.2877 \\
\hline Q3B8N7 & Tsc22d4 & $\mathrm{T}$ & 183 & 2 & PGKAKVETPPLSASP & & 0.3262 \\
\hline Q3B8N7 & Tsc22d4 & $\mathrm{s}$ & 189 & 2 & ETPPLSASPPQQRPP & & 0.336 \\
\hline D3ZAU7 & Ttbk1 & $\mathrm{T}$ & 635 & 2 & SETSQPPTPGSPSHS & & 0.5114 \\
\hline D3ZAU7 & Ttbk1 & S & 638 & 1 & SQPPTPGSPSHSPLH & 0.0454 & 0.3348 \\
\hline D3ZAU7 & Ttbk1 & $\mathrm{s}$ & 1033 & 1 & TKKGTIISPSRHAMP & & 0.5022 \\
\hline D3ZAU7 & Ttbk1 & $S$ & 1246 & 1 & QSLSRKESSSPSHQA & & 0.6882 \\
\hline A0A0G2JYZ1 & Ttbk2 & $S$ & 1148 & 1 & SPVVPRRSPSASPRS & -0.051 & 0.5099 \\
\hline A0A0G2JXJ6 & Ttc7b & $\mathrm{s}$ & 419 & 1 & ALSEVASSLQSSAPK & 0.2169 & -0.3689 \\
\hline D4ACG4 & Ttוl7 & $S$ & 441 & 1 & LQVRKQISQEEHENR & 0.1512 & 0.2649 \\
\hline 088808 & Tub & $\mathrm{s}$ & 209 & 1 & EDENSSSSSQLNSNT & & 0.3518 \\
\hline
\end{tabular}




\begin{tabular}{|c|c|c|c|c|c|c|c|}
\hline Q5XIF6 & Tuba4a & $\mathrm{T}$ & 223 & 1 & NLDIERPTYTNLNRL & & -0.2965 \\
\hline Q5XIF6 & Tuba4a & $Y$ & 224 & 1 & LDIERPTYTNLNRLI & & -0.3993 \\
\hline P69897 & Tubb5 & $S$ & 168 & 2 & DRIMNTFSVVPSPKV & & -0.4233 \\
\hline P69897 & Tubb5 & $S$ & 172 & 2 & NTFSVVPSPKVSDTV & & -0.4233 \\
\hline Q4QQV0 & Tubb6 & $S$ & 172 & 1 & NTFSVMPSPKVSDTV & & -0.36 \\
\hline A0A0G2JYC6 & Ubap2I & S & 467 & 1 & STSAPQMSPGSSDNQ & -0.103 & 0.9797 \\
\hline A0A0G2JYC6 & Ubap2l & $S$ & 493 & 1 & KQQKKKTSLTSKIPA & & 1.0771 \\
\hline A0A0G2JYC6 & Ubap2l & $S$ & 608 & 1 & RYPSSISSSPQKDLT & & 1.4308 \\
\hline A0A0G2JYC6 & Ubap2l & S & 609 & 1 & YPSSISSSPQKDLTQ & 0.0451 & 1.2136 \\
\hline Q5U203 & Ube2f & $S$ & 26 & 1 & ASASTSDSTRRVSVR & & -0.3773 \\
\hline A0A096MJHO & Ube2j1 & $S$ & 25 & 1 & APKNTSMSPRQRRAQ & & 0.2901 \\
\hline D3ZNQ6 & Ube2m & $\mathrm{T}$ & 20 & 1 & EEESAGGTKGSSKKA & & -0.452 \\
\hline D3ZNQ6 & Ube2m & $S$ & 23 & 1 & SAGGTKGSSKKASAA & & -0.5817 \\
\hline D3ZXV0 & Ube2ql1 & S & 66 & 1 & PGGSGDASPGPGKGK & -0.0595 & 0.4639 \\
\hline Q9ES53 & Ufd1 1 & S & 231 & 1 & GFRAFSGSGNRLDGK & & 0.5986 \\
\hline D3ZMR2 & Uhrf1bp1 & $S$ & 442 & 1 & GKSPLDKSPTQGRQA & & -0.2997 \\
\hline MOR8V0 & Uhrf1bp1I & $S$ & 21 & 1 & SRFTKNLSPDKINLS & & -0.4473 \\
\hline D3ZT03 & Upf2 & S & 37 & 1 & PVSSKEKSKDDLKIT & & -0.2677 \\
\hline $\mathrm{D} 4 \mathrm{ABC7}$ & Uprt & $S$ & 25 & 1 & VNSTSSPSPERLLAE & 0.0366 & 0.4071 \\
\hline A0A0G2K8Q8 & Uqcr10 & S & 3 & 1 & MASPTVTSRL & & -0.28 \\
\hline Q68FY0 & Uqcrc1 & S & 137 & 1 & AYLIKALSKDLPKVV & & -0.3509 \\
\hline P20788 & Uqcrfs1 & $S$ & 99 & 1 & RRAEVLDSTKSSKES & & -0.2758 \\
\hline Q5U2N2 & Usp14 & $S$ & 393 & 1 & PNANDKNSPPKEIKY & 0.1018 & -0.2776 \\
\hline D3ZLQ8 & Usp20 & S & 409 & 2 & GHTKLSSSPPRASPV & -0.1821 & 0.3324 \\
\hline D3ZLQ8 & Usp20 & $S$ & 414 & 2 & SSSPPRASPVRMGPS & & 0.3324 \\
\hline A0A0G2K2V3 & Usp31 & S & 1040 & 1 & TSTSKPSSPRVNQAR & & 0.6219 \\
\hline D3ZL57 & Usp6nl & S & 701 & 1 & RGYGSSGSPKSGKFI & 0.0823 & 0.4119 \\
\hline P63045 & Vamp2 & $S$ & 75 & 1 & DALQAGASQFETSAA & 0.3676 & -0.3335 \\
\hline Q8CF97 & Vcpip1 & S & 997 & 1 & TTRSRESSPSHGLLK & 0.1288 & 0.9072 \\
\hline Q9Z2L0 & Vdac1 & S & 104 & 1 & LTFDSSFSPNTGKKN & 0.2171 & -0.4889 \\
\hline P81155 & Vdac2 & $S$ & 252 & 1 & ISAKVNNSSLIGVGY & & -0.3346 \\
\hline Q793F9 & Vps4a & S & 97 & 1 & SEGKGSDSDSEGDNP & 0.0858 & -0.2931 \\
\hline F1LSG8 & Vps50 & S & 494 & 1 & KFLEQSRSPSVSPSK & 0.0656 & -0.272 \\
\hline F1LSG8 & Vps50 & S & 561 & 1 & YQDYDSDSDVPEELK & & -0.3219 \\
\hline Q80X08 & Washc2 & $S$ & 157 & 1 & LDIKAGNSDSEEEDA & & -0.3098 \\
\hline Q80X08 & Washc2 & $S$ & 159 & 1 & IKAGNSDSEEEDANE & & -0.3108 \\
\hline Q80X08 & Washc2 & S & 387 & 1 & VSEESPPSPKPGKKI & -0.1085 & 0.4089 \\
\hline A0A0G2K9M4 & Wdfy3 & $\mathrm{s}$ & 3345 & 1 & ARAHPQGSHSHPNPT & -0.0577 & 0.4726 \\
\hline D3Z9L5 & Wdr11 & S & 1216 & 1 & DLLNELGSPKEEATE & 0.2733 & -0.3862 \\
\hline A0A0G2KAW5 & Wdr48 & $S$ & 590 & 1 & YLQPHASSGAKTLKK & & -0.3924 \\
\hline A0A0G2K1X3 & Wdr60 & $S$ & 63 & 1 & LKAAQSGSPKEEKKL & & 0.497 \\
\hline E9PT53 & Wfs1 & S & 32 & 1 & ARLNATTSLEQDKIE & 0.7501 & 0.3472 \\
\hline Q810W9 & Whrn & S & 698 & 1 & GPFPRVQSPPHLKSP & 0.033 & 0.6167 \\
\hline D3ZMJ7 & Wnk2 & $S$ & 1677 & 1 & RGVRDSGSPHKRPGQ & -0.5206 & 0.4881 \\
\hline
\end{tabular}




\begin{tabular}{|c|c|c|c|c|c|c|c|}
\hline D3ZMJ7 & Wnk2 & $S$ & 1677 & 2 & RGVRDSGSPHKRPGQ & & 0.7641 \\
\hline Q8CG07 & Wrnip1 & S & 153 & 1 & AAAAGSASPRSWDET & & 0.461 \\
\hline F7EYF1 & Wwp2 & $S$ & 100 & 1 & ATATGEQSPGARNRH & & 0.5267 \\
\hline Q5GH56 & Xkr7 & $S$ & 154 & 1 & AFRTKEGSPELVPRP & 0.2582 & -0.2963 \\
\hline D4ABN8 & Xrn1 & $S$ & 1331 & 1 & KLLKRNESPGTSEAQ & & 0.4878 \\
\hline P62961 & Ybx1 & $S$ & 163 & 1 & YQQNYQNSESGEKNE & & 0.9245 \\
\hline P62961 & Ybx1 & $S$ & 172 & 1 & SGEKNEGSESAPEGQ & & 0.9007 \\
\hline P62961 & Ybx1 & S & 174 & 1 & EKNEGSESAPEGQAQ & & 0.6687 \\
\hline P62260 & Ywhae & $S$ & 46 & 1 & VEERNLLSVAYKNVI & & -0.4141 \\
\hline P61983 & Ywhag & $S$ & 71 & 1 & SSIEQKTSADGNEKK & -0.0366 & -0.2946 \\
\hline P63102 & Ywhaz & $S$ & 63 & 1 & RSSWRVVSSIEQKTE & & -0.4757 \\
\hline Q5S3S9 & Zbtb16 & $S$ & 302 & 2 & LHYGREESGEQLSPP & & -0.2652 \\
\hline Q5S3S9 & Zbtb16 & $S$ & 307 & 2 & EESGEQLSPPVEAGQ & & -0.2652 \\
\hline A0A0G2K782 & Zc2hc1a & $S$ & 222 & 1 & GNKPQTLSPSHRALA & & 0.2855 \\
\hline Q2THW7 & Zdhhc5 & S & 372 & 1 & AAMPHSSSAKLSRGD & -0.1931 & 0.2845 \\
\hline Q2THW7 & Zdhhc5 & $S$ & 380 & 1 & AKLSRGDSLKEPTSI & 0.3274 & 0.3116 \\
\hline Q2THW7 & Zdhhc5 & $\mathrm{S}$ & 694 & 1 & PGQPPLSSPTRGGVK & -0.1021 & 0.4341 \\
\hline Q2THW6 & Zdhhc8 & $S$ & 335 & 1 & LKTPRPGSAESALSV & & 0.3482 \\
\hline Q2THW6 & Zdhhc8 & $S$ & 672 & 1 & PARQGLPSPPGTPRS & -0.0516 & 0.5167 \\
\hline Q2THW6 & Zdhhc8 & $S$ & 679 & 1 & SPPGTPRSPSYSGSK & 0.0234 & 0.8915 \\
\hline D3ZJG8 & Zfp592 & S & 1087 & 1 & VKHAGGHSPQVNHLK & & 0.9861 \\
\hline D4A069 & Zmym4 & $\mathrm{S}$ & 121 & 1 & RRASHHESDNENEIQ & & 0.2959 \\
\hline Q5PQS2 & Znf365 & $S$ & 370 & 1 & AlHEQAESPREFFRP & & 0.6991 \\
\hline Q7TM96 & Znf622 & S & 143 & 1 & IKAQPSTSPKKAPFM & 0.3419 & 1.8787 \\
\hline O35986 & Zranb2 & $S$ & 290 & 1 & RDRKRSRSRPSSPAV & & 0.5467 \\
\hline Q5RK33 & Zrsr1 & $S$ & 418 & 1 & RGREEGSSPGPQSQS & -0.0298 & 1.2219 \\
\hline A0A0G2JW01 & & S & 51 & 1 & PGSSIPGSPGHTIYA & & 0.6732 \\
\hline A0A0G2JW01 & & $S$ & 51 & 2 & PGSSIPGSPGHTIYA & -0.3508 & 0.2072 \\
\hline A0A0G2JW01 & & $S$ & 106 & 1 & EDKQDRQSLGESPRT & 0.0642 & 0.8125 \\
\hline A0A0G2JW01 & & S & 110 & 1 & DRQSLGESPRTLSPT & 0.1727 & 1.424 \\
\hline A0A0G2JW01 & & $\mathrm{S}$ & 142 & 1 & TSQGSINSPVYSRHS & 0.1219 & 1.243 \\
\hline A0A0G2JW01 & & $S$ & 289 & 1 & LLASRYDSPLHSASH & 0.1056 & 0.6009 \\
\hline A0A0G2JW01 & & S & 293 & 1 & RYDSPLHSASHAPSS & & 0.4622 \\
\hline A0A0G2JZ27 & & $S$ & 416 & 1 & QLSSPNHSPSQSPNQ & -0.0629 & 0.374 \\
\hline A0A0G2JZ27 & & $S$ & 424 & 1 & PSQSPNQSPRIKKRP & 0.0211 & 0.3824 \\
\hline D3ZJKK8 & & $T$ & 5 & 2 & GYEKTDDVSEKT & 0.0279 & -0.4103 \\
\hline D3ZJKR8 & & $\mathrm{S}$ & 9 & 1 & YEKTDDVSEKTSLAD & & -0.6089 \\
\hline D3ZJKR8 & & $S$ & 9 & 2 & YEKTDDVSEKTSLAD & & -0.3025 \\
\hline D3ZJKK8 & & $T$ & 12 & 1 & TDDVSEKTSLADQEE & & -0.5936 \\
\hline D3ZJKR8 & & $S$ & 13 & 1 & DDVSEKTSLADQEEV & 0.3241 & -0.6942 \\
\hline D3ZJK8 & & $S$ & 13 & 2 & DDVSEKTSLADQEEV & 0.0469 & -0.3796 \\
\hline D3ZZH5 & & S & 98 & 1 & RSISIQTSPSLRKHF & & 0.3588 \\
\hline M0RDY2 & & S & 36 & 1 & GGAERRGSVPANPGA & & -0.2736 \\
\hline 008654 & & $\mathrm{~s}$ & 270 & 1 & EDKMKIHSPSPHKQV & -0.3446 & \\
\hline
\end{tabular}




\begin{tabular}{|l|r|r|r|r|l|r|r|}
\hline 008654 & & S & 270 & 2 & EDKMKIHSPSPHKQV & & -0.3365 \\
\hline 008654 & & S & 272 & 2 & KMKIHSPSPHKQVPS & & -0.3365 \\
\hline Q63003 & S & 251 & 1 & IPGQQEESPQQEPSS & 0.0547 & 0.4518 \\
\hline Q63003 & S & 690 & 1 & HGAINAPSAPDASPP & 0.0172 & 0.3693 \\
\hline
\end{tabular}


Table 8.3: Phosphorylation events from clusters 1 and 2 as presented in Figure 4.2. UP.Accession Uniprot accession; Gene.name official gene symbol; AA modified amino acid; Pos position in the protein sequence; Mult multiplicity of phosphorylation (1 - singly phosphorylated, 2 - doubly phosphorylated, 3 - multiply phosphorylated); Seq. window $\sim \pm 7$ amino acids flanking phosphorylated residue; Correlation $\sim$ Pearson correlation of the two kinetics measuremtns; 0s-180s $\sim$ log 2 intensity fold change relatively to the time point $0 \mathrm{~s}$

\begin{tabular}{|c|c|c|c|c|c|c|c|c|c|c|c|c|c|}
\hline UP.Accession & Gene.name & AA & Pos & Mult & Seq.window & Correlation & Cluster & Os & $10 \mathrm{~s}$ & $30 \mathrm{~s}$ & $60 \mathrm{~s}$ & $125 \mathrm{~s}$ & $180 \mathrm{~s}$ \\
\hline $\mathrm{P} 0 \mathrm{C} 1 \mathrm{X8}$ & Aak1 & $S$ & 653 & 1 & FGVPASKSTQLLHAA & 0.8329 & 1 & 0 & 0.3484 & 0.9029 & 0.7033 & -0.7212 & 0.7664 \\
\hline D4ABZ3 & Aatk & $S$ & 1282 & 1 & VPLRAGHSPDSSAPE & 0.6379 & 1 & 0 & -0.1953 & 0.2320 & 0.4731 & 0.0898 & 0.5942 \\
\hline O35889 & Afdn & $\mathrm{T}$ & 1218 & 2 & NLCTEEQTPPPRPEA & 0.7205 & 1 & 0 & 0.6180 & 0.4817 & 0.6412 & 0.0359 & 0.1577 \\
\hline D3ZTF0 & Ajm1 & $S$ & 109 & 1 & SLTTAPASPPVLQRR & 0.9139 & 1 & 0 & 0.1952 & 0.5913 & 0.6182 & -0.2823 & 0.4416 \\
\hline 008838 & Amph & $\mathrm{T}$ & 260 & 1 & GPLRIAKTPSPPEEA & 0.9762 & 1 & 0 & 0.1017 & 0.9036 & 0.9678 & -1.3263 & 0.9902 \\
\hline 008838 & Amph & $S$ & 268 & 2 & PSPPEEASPLPSPTA & 0.9653 & 1 & 0 & 0.1794 & 0.5000 & 0.5287 & -0.2957 & 0.6518 \\
\hline 008838 & Amph & $S$ & 272 & 2 & EEASPLPSPTASPNH & 0.9344 & 1 & 0 & 0.2170 & 0.6283 & 0.6231 & -0.4083 & 0.7693 \\
\hline 008838 & Amph & S & 285 & 2 & NHTLAPASPAPVRPR & 0.9025 & 1 & 0 & 0.1211 & 0.5420 & 0.7015 & -0.3835 & 0.7686 \\
\hline 008838 & Amph & $S$ & 514 & 2 & TELASSESPQAAELE & 0.7890 & 1 & 0 & 0.3489 & 0.6241 & 0.6383 & -0.2141 & 0.5301 \\
\hline D3ZKP1 & Ankrd34b & $S$ & 496 & 1 & KMLLRRQSLQTEQIK & 0.9942 & 1 & 0 & 0.0252 & 0.0910 & 0.1238 & -0.0770 & 0.9197 \\
\hline O35430 & Apba1 & $S$ & 243 & 1 & LHHYDERSDGESDSP & 0.8522 & 1 & 0 & 0.1176 & 0.3247 & 0.6128 & -0.0579 & 0.5227 \\
\hline A0A0G2K8G0 & Apc & $S$ & 2486 & 2 & SFESLSPSSRPDSPT & 0.9448 & 1 & 0 & 0.3354 & 0.6024 & 0.6341 & -0.1940 & 0.4879 \\
\hline F1LXQ7 & Arhgap21 & $\mathrm{S}$ & 863 & 1 & PLIRRQLSHDQESVG & 0.6667 & 1 & 0 & -0.2246 & -0.0528 & 0.1372 & -0.0456 & 0.9882 \\
\hline A0A0G2JZC6 & Arhgef11 & $S$ & 1512 & 2 & KSLGGESSGGTTPVG & 0.6319 & 1 & 0 & 0.1685 & 0.3459 & 0.3854 & 0.1082 & 0.6566 \\
\hline A0A0G2JZC6 & Arhgef11 & $\mathrm{T}$ & 1516 & 2 & GESSGGTTPVGSFHT & 0.6319 & 1 & 0 & 0.1685 & 0.3459 & 0.3854 & 0.1082 & 0.6566 \\
\hline F1MAF8 & Atg2b & $\mathrm{S}$ & 918 & 2 & PYDEDSGSEEETLQY & 0.9513 & 1 & 0 & 0.2575 & 0.7401 & 0.4667 & -1.1758 & 0.3843 \\
\hline F1MAF8 & Atg2b & $\mathrm{T}$ & 922 & 2 & DSGSEEETLQYFSAV & 0.9524 & 1 & 0 & 0.3110 & 0.7598 & 0.4968 & -1.1563 & 0.4156 \\
\hline P11506 & Atp2b2 & $T$ & 1162 & 2 & LEKPESRTSIHNFMA & 0.9794 & 1 & 0 & 0.0918 & 0.4700 & 0.3464 & -0.6172 & 0.1886 \\
\hline P11506 & Atp2b2 & $\mathrm{S}$ & 1163 & 2 & EKPESRTSIHNFMAH & 0.9794 & 1 & 0 & 0.0918 & 0.4700 & 0.3464 & -0.6172 & 0.1886 \\
\hline Q9ET45 & Bnip3 & $\mathrm{S}$ & 60 & 1 & SKSSHCDSPPRSQTP & 0.7248 & 1 & 0 & -0.1064 & 0.2760 & 0.7738 & -0.0968 & 0.5544 \\
\hline G3V984 & Bsn & $S$ & 2783 & 2 & VSRQPPKSPQVLYSP & 0.8756 & 1 & 0 & 0.0330 & 0.7025 & 0.3758 & -0.6481 & 0.5464 \\
\hline
\end{tabular}




\begin{tabular}{|c|c|c|c|c|c|c|c|c|c|c|c|c|c|}
\hline G3V984 & Bsn & $S$ & 2795 & 2 & YSPVSPLSPHRLLDT & 0.9751 & 1 & 0 & -0.0765 & 0.4895 & 0.3099 & -0.6269 & 0.3473 \\
\hline D4A055 & Cacnb4 & S & 443 & 1 & RMRHSNHSTENSPIE & 0.7825 & 1 & 0 & -0.1095 & 0.0778 & 0.2981 & 0.7322 & 0.4831 \\
\hline $\mathrm{F} 1 \mathrm{M} 3 \mathrm{~F} 8$ & Camk2g & S & 445 & 1 & AGMQPQPSLCSSAMR & 0.8362 & 1 & 0 & 0.2348 & 0.6925 & 0.7774 & -0.1144 & 0.6306 \\
\hline Q8VHK2 & Caskin1 & $\mathrm{S}$ & 648 & 1 & PAAAECQSPKMTTFQ & 0.6593 & 1 & 0 & 0.0278 & 0.5852 & 0.5210 & -0.2237 & 0.3729 \\
\hline Q5FVI4 & Cend1 1 & $\mathrm{~S}$ & 89 & 2 & PTVPAAPSSPDTTSE & 0.9695 & 1 & 0 & 0.0477 & 0.9312 & 0.8392 & -1.8572 & 0.9093 \\
\hline Q99P82 & Cldn11 & $\mathrm{S}$ & 198 & 1 & YYSSGSSSPTHAKSA & 0.7909 & 1 & 0 & -0.4443 & 0.7533 & 1.1596 & -0.3599 & 1.6203 \\
\hline D4A1C0 & Cnnm1 & $\mathrm{T}$ & 454 & 2 & KAPTTRGTPQTPKDD & 0.9496 & 1 & 0 & 0.0704 & 0.7693 & 0.5749 & -1.0438 & 0.5889 \\
\hline D4A1C0 & Cnnm1 & $\mathrm{T}$ & 457 & 2 & TTRGTPQTPKDDPVL & 0.9534 & 1 & 0 & 0.0095 & 0.6651 & 0.4538 & -0.8450 & 0.4689 \\
\hline P47875 & Csrp1 & S & 192 & 1 & GAGALVHSE & 0.8257 & 1 & 0 & 0.3405 & 0.5804 & 0.6103 & -0.1891 & 0.5209 \\
\hline Q5YLM1 & Dagla & S & 808 & 1 & GFRSIRGSPSLHAVL & 0.8435 & 1 & 0 & 0.0933 & 0.4079 & 0.4848 & -0.2582 & 0.6276 \\
\hline Q6AYH5 & Dctn2 & $\mathrm{T}$ & 199 & 1 & GGKSTGGTPPDSSLV & 0.8685 & 1 & 0 & 0.0491 & 0.5790 & 0.3357 & -0.6309 & 0.1984 \\
\hline Q6AYH5 & Dctn2 & $\mathrm{T}$ & 199 & 2 & GGKSTGGTPPDSSLV & 0.8737 & 1 & 0 & 0.3587 & 0.7908 & 0.6528 & -0.6387 & 0.6465 \\
\hline P60905 & Dnajc5 & S & 12 & 1 & RQRSLSTSGESLYHV & 0.8097 & 1 & 0 & 0.0532 & 0.6908 & 0.7346 & -0.2313 & 0.9672 \\
\hline A0A0G2JY26 & Dnajc6 & S & 679 & 3 & GFGMGSKSAATSPTG & 0.6262 & 1 & 0 & -0.0750 & 0.1251 & 0.0491 & 0.2764 & 0.6351 \\
\hline A0A0G2JY26 & Dnajc6 & $\mathrm{T}$ & 682 & 3 & MGSKSAATSPTGSTH & 0.6262 & 1 & 0 & -0.0750 & 0.1251 & 0.0491 & 0.2764 & 0.6351 \\
\hline G3V874 & Epb4113 & S & 91 & 1 & QFEDDKLSQKSSSSK & 0.8119 & 1 & 0 & 0.5162 & 0.6138 & 0.6170 & -0.1935 & 0.6839 \\
\hline G3V874 & Epb4113 & $\mathrm{S}$ & 91 & 2 & QFEDDKLSQKSSSSK & 0.7330 & 1 & 0 & 0.3859 & 0.6600 & 0.6247 & -0.1743 & 0.3462 \\
\hline G3V874 & Epb4113 & $\mathrm{S}$ & 91 & 3 & QFEDDKLSQKSSSSK & 0.8004 & 1 & 0 & 0.5118 & 0.7903 & 0.7710 & -0.2875 & 0.6256 \\
\hline G3V874 & Epb4113 & $\mathrm{S}$ & 94 & 3 & DDKLSQKSSSSKLSR & 0.9071 & 1 & 0 & 0.5813 & 0.8175 & 0.8445 & -0.2528 & 0.6595 \\
\hline G3V874 & Epb4113 & $\mathrm{S}$ & 95 & 2 & DKLSQKSSSSKLSRS & 0.7370 & 1 & 0 & 0.3592 & 0.6258 & 0.5918 & -0.0771 & 0.3364 \\
\hline G3V874 & Epb4113 & S & 95 & 3 & DKLSQKSSSSKLSRS & 0.8004 & 1 & 0 & 0.5118 & 0.7903 & 0.7710 & -0.2875 & 0.6256 \\
\hline G3V874 & Epb4113 & $\mathrm{S}$ & 96 & 2 & KLSQKSSSSKLSRSP & 0.7169 & 1 & 0 & 0.5210 & 0.6853 & 0.6537 & -0.0302 & 0.3195 \\
\hline G3V874 & Epb4113 & $S$ & 96 & 3 & KLSQKSSSSKLSRSP & 0.8004 & 1 & 0 & 0.5118 & 0.7903 & 0.7710 & -0.2875 & 0.6256 \\
\hline G3V874 & Epb4113 & $\mathrm{S}$ & 97 & 3 & LSQKSSSSKLSRSPL & 0.8509 & 1 & 0 & 0.4932 & 0.7308 & 0.7345 & -0.3609 & 0.5650 \\
\hline A0A0G2JZX5 & lasec2 & $S$ & 207 & 1 & YFEGKPASLDEGAMA & 0.7103 & 1 & 0 & 0.4222 & 0.6133 & 0.5836 & 0.1486 & 0.6285 \\
\hline F1M9N8 & LOC100361087 & $\mathrm{S}$ & 183 & 1 & DGQEDGESERNSDGS & 0.6921 & 1 & 0 & 0.5544 & 1.0945 & 1.3906 & 0.5379 & 1.6896 \\
\hline A0A0G2KA27 & Madd & $S$ & 861 & 1 & RNHSTSFSLSNLTLP & 0.6844 & 1 & 0 & -0.0435 & 0.3984 & 0.5114 & 0.2270 & 0.7258 \\
\hline G3V9B3 & Mag & $\mathrm{S}$ & 545 & 2 & RKKNVTESPSFSAGD & 0.9004 & 1 & 0 & 0.1837 & 0.6037 & 0.2520 & -0.3694 & 0.2542 \\
\hline P34926 & Map1a & $\mathrm{s}$ & 1985 & 2 & SAEKETSSPASPQNL & 0.9481 & 1 & 0 & 0.2245 & 0.8120 & 0.7608 & -0.8020 & 0.6691 \\
\hline
\end{tabular}




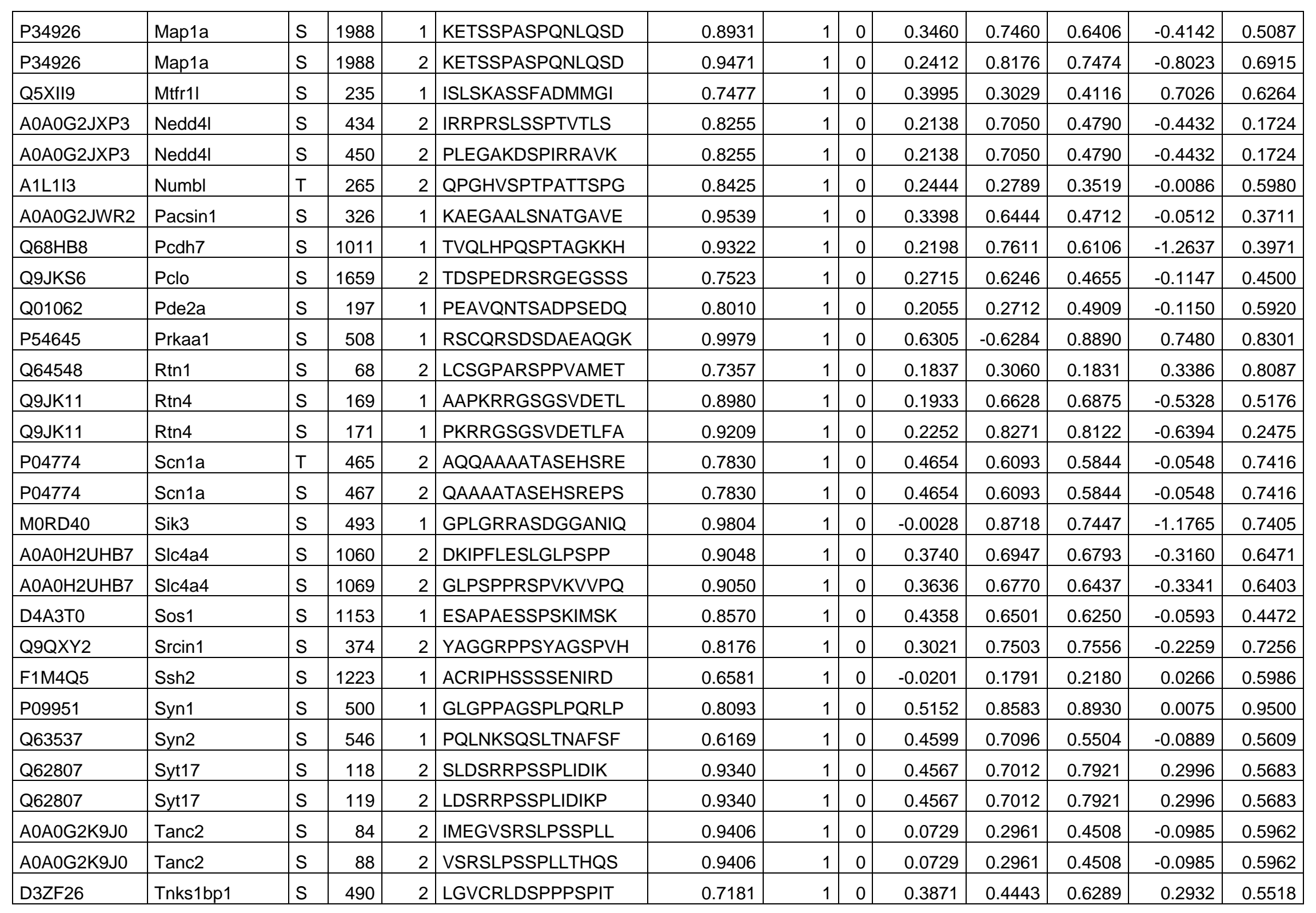




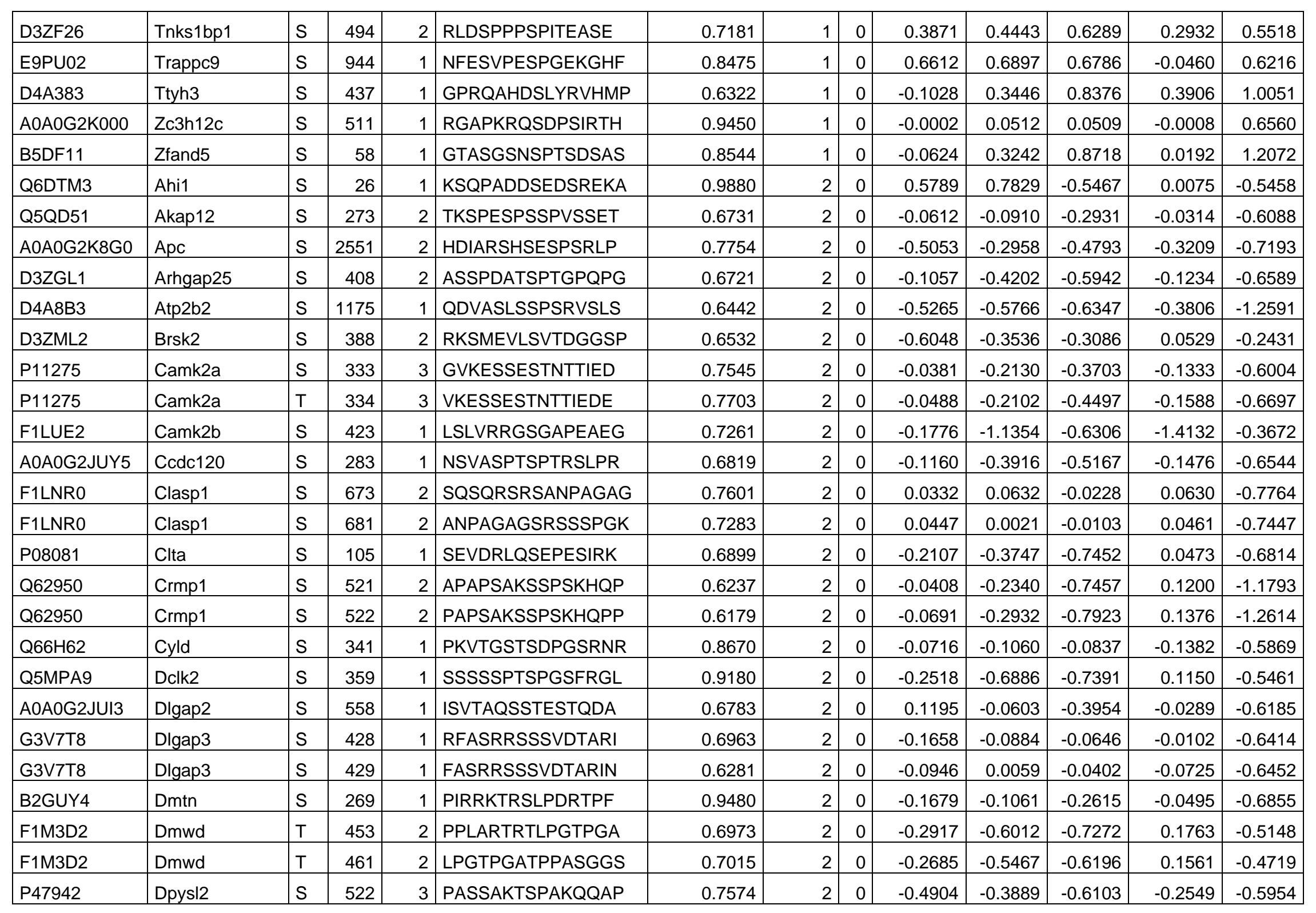




\begin{tabular}{|c|c|c|c|c|c|c|c|c|c|c|c|c|c|}
\hline $\mathrm{F} 1 \mathrm{M} 470$ & Eif4e2 & $S$ & 2 & 1 & MSLKDDDSG & 0.7030 & 2 & 0 & -0.3556 & -0.2974 & -0.7585 & -0.1927 & -0.1768 \\
\hline G3V874 & Epb41l3 & S & 743 & 1 & HVMNVHASGDASHTA & 0.6517 & 2 & 0 & -0.0977 & -0.3541 & -0.5894 & -0.0338 & -0.3871 \\
\hline MoR5H1 & Etl4 & $\mathrm{S}$ & 1914 & 1 & SSSSSPPSPASPTSL & 0.6574 & 2 & 0 & 0.0445 & -0.1740 & -0.2675 & -0.2421 & -0.6158 \\
\hline P19627 & Gnaz & S & 27 & 2 & DRHLRSESQRQRREI & 0.6419 & 2 & 0 & -0.1650 & -0.3595 & -0.3858 & 0.0407 & -0.6579 \\
\hline A0A0A0MY13 & Gpr158 & $\mathrm{S}$ & 946 & 1 & RETNRKYSNSDNTET & 0.8179 & 2 & 0 & -0.5831 & -1.0844 & -1.4053 & -0.0650 & -0.9936 \\
\hline D3ZEI4 & Hepacam & $S$ & 378 & 2 & ATGRTHTSPPRAPSS & 0.7531 & 2 & 0 & -0.2588 & -0.3967 & -0.6362 & -0.2011 & -1.1869 \\
\hline D3ZEI4 & Hepacam & $S$ & 385 & 2 & SPPRAPSSPGRSRSS & 0.7244 & 2 & 0 & -0.2753 & -0.3881 & -0.6857 & -0.1720 & -1.2058 \\
\hline A0A0G2K427 & Ivns1abp & $\mathrm{S}$ & 322 & 2 & IFLHGRNSPQSSPTS & 0.7603 & 2 & 0 & -0.2699 & -0.6448 & -0.8064 & 0.1779 & -0.6204 \\
\hline A0A0G2K427 & Ivns1abp & $S$ & 325 & 2 & HGRNSPQSSPTSTPK & 0.7549 & 2 & 0 & -0.2768 & -0.6374 & -0.8121 & 0.1781 & -0.6305 \\
\hline P63142 & Kcna2 & S & 447 & 2 & SSPDLKKSRSASTIS & 0.7870 & 2 & 0 & -0.1857 & -0.2570 & -0.3712 & 0.1342 & -0.5894 \\
\hline B2GV74 & Klc2 & $S$ & 508 & 1 & GRGDRRGSRDVAGGA & 0.6632 & 2 & 0 & -0.3152 & -0.1327 & -0.2805 & -0.0934 & -1.0479 \\
\hline M0R439 & Klhdc7a & $S$ & 344 & 1 & GGTLQVASPQPVPSP & 0.6987 & 2 & 0 & -0.7683 & 0.2002 & -0.1303 & 0.3033 & 0.1190 \\
\hline P34926 & Map1a & $S$ & 898 & 2 & GTISPTSSLEEDKGF & 0.8050 & 2 & 0 & -0.0135 & -0.2187 & -0.5093 & -0.0853 & -0.8699 \\
\hline D4A1Q2 & Mapt & $S$ & 597 & 2 & DFKDRVQSKIGSLDN & 0.7760 & 2 & 0 & -0.3588 & -0.3463 & -0.3323 & 0.0230 & -0.6621 \\
\hline D4A1Q2 & Mapt & $\mathrm{S}$ & 601 & 2 & RVQSKIGSLDNITHV & 0.7760 & 2 & 0 & -0.3588 & -0.3463 & -0.3323 & 0.0230 & -0.6621 \\
\hline 055164 & Mpdz & $S$ & 1802 & 2 & FHSERRPSQSSQVSE & 0.7465 & 2 & 0 & -0.7232 & -0.6492 & -0.9424 & -0.0879 & -1.3628 \\
\hline 055164 & Mpdz & $S$ & 1808 & 2 & PSQSSQVSESSLSSF & 0.7111 & 2 & 0 & -0.3463 & -0.2345 & -0.4552 & 0.0880 & -0.7732 \\
\hline Q78PB6 & Ndel1 & $S$ & 215 & 2 & SAVQASLSLPATPVG & 0.6106 & 2 & 0 & -0.1845 & -0.3153 & -0.6464 & -0.2417 & -0.7313 \\
\hline Q78PB6 & Ndel1 & $\mathrm{T}$ & 219 & 2 & ASLSLPATPVGKGTE & 0.6106 & 2 & 0 & -0.1845 & -0.3153 & -0.6464 & -0.2417 & -0.7313 \\
\hline Q9Z2L9 & Ndrg4 & $S$ & 304 & 2 & LSGGAVPSASMTRLA & 0.8339 & 2 & 0 & -0.5683 & -0.9128 & -0.8568 & -0.0231 & -0.6934 \\
\hline Q9JKS6 & Pclo & $S$ & 1493 & 2 & DEYKQEDSQGSGEEE & 0.7125 & 2 & 0 & -0.1394 & -0.2907 & -0.5229 & -0.0529 & -0.6850 \\
\hline Q9JKS6 & Pclo & $S$ & 1496 & 1 & KQEDSQGSGEEEDFI & 0.9376 & 2 & 0 & -0.0697 & -0.3094 & -0.6392 & 0.0073 & -0.7048 \\
\hline Q8VIE2 & Pde7b & $S$ & 439 & 1 & NQHRRRGSGQDPAGT & 0.9585 & 2 & 0 & -0.2309 & -0.9516 & -0.9231 & 0.2477 & -0.7538 \\
\hline Q5HZV9 & Ppp1r7 & $S$ & 44 & 1 & GGIVADLSQQSLKDG & 0.8220 & 2 & 0 & -0.3390 & -0.8632 & -0.9801 & 0.0288 & -0.8236 \\
\hline D3ZFB6 & Prrt2 & $\mathrm{S}$ & 242 & 3 & AHGGHPGSPRGSLSR & 0.8124 & 2 & 0 & -0.4686 & -0.6362 & -0.7668 & 0.2201 & -0.5856 \\
\hline D3ZFB6 & Prrt2 & $S$ & 246 & 3 & HPGSPRGSLSRHPSS & 0.7303 & 2 & 0 & -0.3685 & -0.5793 & -0.6996 & 0.1901 & -0.4393 \\
\hline
\end{tabular}




\begin{tabular}{|c|c|c|c|c|c|c|c|c|c|c|c|c|c|}
\hline D3ZWQ0 & Prrt3 & $S$ & 922 & 2 & PWRHGLSSVDSLPLD & 0.8150 & 2 & 0 & -0.2734 & -0.6660 & -0.2904 & 0.1071 & -0.4665 \\
\hline D3ZWQ0 & Prrt3 & $S$ & 925 & 2 & HGLSSVDSLPLDELP & 0.8150 & 2 & 0 & -0.2734 & -0.6660 & -0.2904 & 0.1071 & -0.4665 \\
\hline B2RYS6 & Prtfdc1 & $S$ & 4 & 1 & MAGSSEKAPDS & 0.6417 & 2 & 0 & -0.2360 & -0.3810 & -0.4887 & 0.0070 & -0.8161 \\
\hline D3ZX42 & Rabgap1 & $S$ & 42 & 1 & TPSTNNGSDDEKTGL & 0.6013 & 2 & 0 & -0.3125 & -0.4930 & -0.8848 & -0.1710 & -0.7855 \\
\hline Q9JIR3 & Rims3 & $S$ & 104 & 1 & SRVTRQGSRESTDGS & 0.8703 & 2 & 0 & -0.1770 & -0.1635 & -0.4750 & -0.0145 & -0.6315 \\
\hline A0A0G2K8W9 & Sptbn1 & $S$ & 2154 & 2 & GAAEQRTSSKESSPV & 0.7640 & 2 & 0 & -0.0760 & -0.3884 & -0.5853 & -0.0334 & -0.7279 \\
\hline F1M5M9 & Srgap3 & $S$ & 868 & 2 & AAIPRRRSGGDTHSP & 0.9202 & 2 & 0 & -0.3753 & -0.2930 & -0.5677 & -0.1765 & -0.9100 \\
\hline F1M5M9 & Srgap3 & $S$ & 874 & 2 & RSGGDTHSPPRGLGP & 0.9253 & 2 & 0 & -0.4110 & -0.2464 & -0.4878 & -0.2985 & -2.1900 \\
\hline P09951 & Syn1 & $S$ & 549 & 2 & PAARPPASPSPQRQA & 0.8239 & 2 & 0 & -0.0428 & -0.2003 & -0.4153 & -0.0721 & -0.7641 \\
\hline P09951 & Syn1 & $\mathrm{S}$ & 551 & 1 & ARPPASPSPQRQAGP & 0.6980 & 2 & 0 & -0.0511 & -0.1973 & -0.4142 & -0.1319 & -0.7201 \\
\hline P09951 & Syn1 & $S$ & 551 & 2 & ARPPASPSPQRQAGP & 0.8239 & 2 & 0 & -0.0428 & -0.2003 & -0.4153 & -0.0721 & -0.7641 \\
\hline Q75Q39 & Tomm70 & $S$ & 84 & 2 & ASGLKRNSERKTPEG & 0.6673 & 2 & 0 & 0.0415 & -0.2841 & -0.5373 & -0.0863 & -0.6836 \\
\hline Q75Q39 & Tomm70 & $\mathrm{T}$ & 88 & 1 & KRNSERKTPEGRASP & 0.7021 & 2 & 0 & -0.3184 & -0.5587 & -0.8622 & -0.2392 & -1.4362 \\
\hline Q6PCT3 & Tpd5212 & $\mathrm{S}$ & 175 & 2 & SMPVMRNSATFKSFE & 0.8708 & 2 & 0 & -0.1138 & -0.2171 & -0.3164 & -0.2647 & -0.6551 \\
\hline D3ZAU7 & Ttbk1 & $S$ & 433 & 2 & RGVGVPSSPVRAPPD & 0.6075 & 2 & 0 & -0.2771 & -0.3465 & -0.5729 & -0.0980 & -0.6538 \\
\hline Q499N6 & Ubxn1 & $S$ & 188 & 2 & GTVGSRSSPPATDPG & 0.8777 & 2 & 0 & -0.5727 & -0.9535 & -1.0804 & -0.1067 & -0.9013 \\
\hline Q499N6 & Ubxn1 & $S$ & 200 & 2 & DPGPVPSSPRQEPPT & 0.8635 & 2 & 0 & -0.5093 & -0.8739 & -0.9821 & -0.0902 & -0.8259 \\
\hline A0A0G2JX77 & Wdr44 & $S$ & 567 & 2 & YNTEGRVSPSPSQES & 0.7470 & 2 & 0 & -0.1132 & -0.1857 & -0.2768 & 0.0670 & -0.5854 \\
\hline P62961 & Ybx1 & $S$ & 172 & 1 & SGEKNEGSESAPEGQ & 0.7897 & 2 & 0 & -0.1406 & -0.2921 & -0.7509 & -0.3135 & -1.0044 \\
\hline D3ZTR5 & Zbed5 & $S$ & 46 & 1 & AAPSAVGSPAAAPRQ & 0.7487 & 2 & 0 & 0.0040 & -0.5867 & -0.3639 & 0.1331 & -0.4413 \\
\hline F1LWK7 & & $S$ & 412 & 2 & PGSSIPGSPGHTIYA & 0.6644 & 2 & 0 & -0.2834 & -0.2378 & -0.6225 & -0.0130 & -0.7902 \\
\hline
\end{tabular}

